1

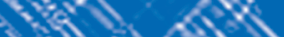

X.

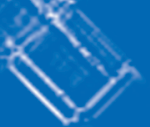

Les pêches

côtières bretonnes

Méthodes d'analyse et aménagement

Catherine Talidec, Jean Boncœur, Jean-Pierre Boude, coordinateurs
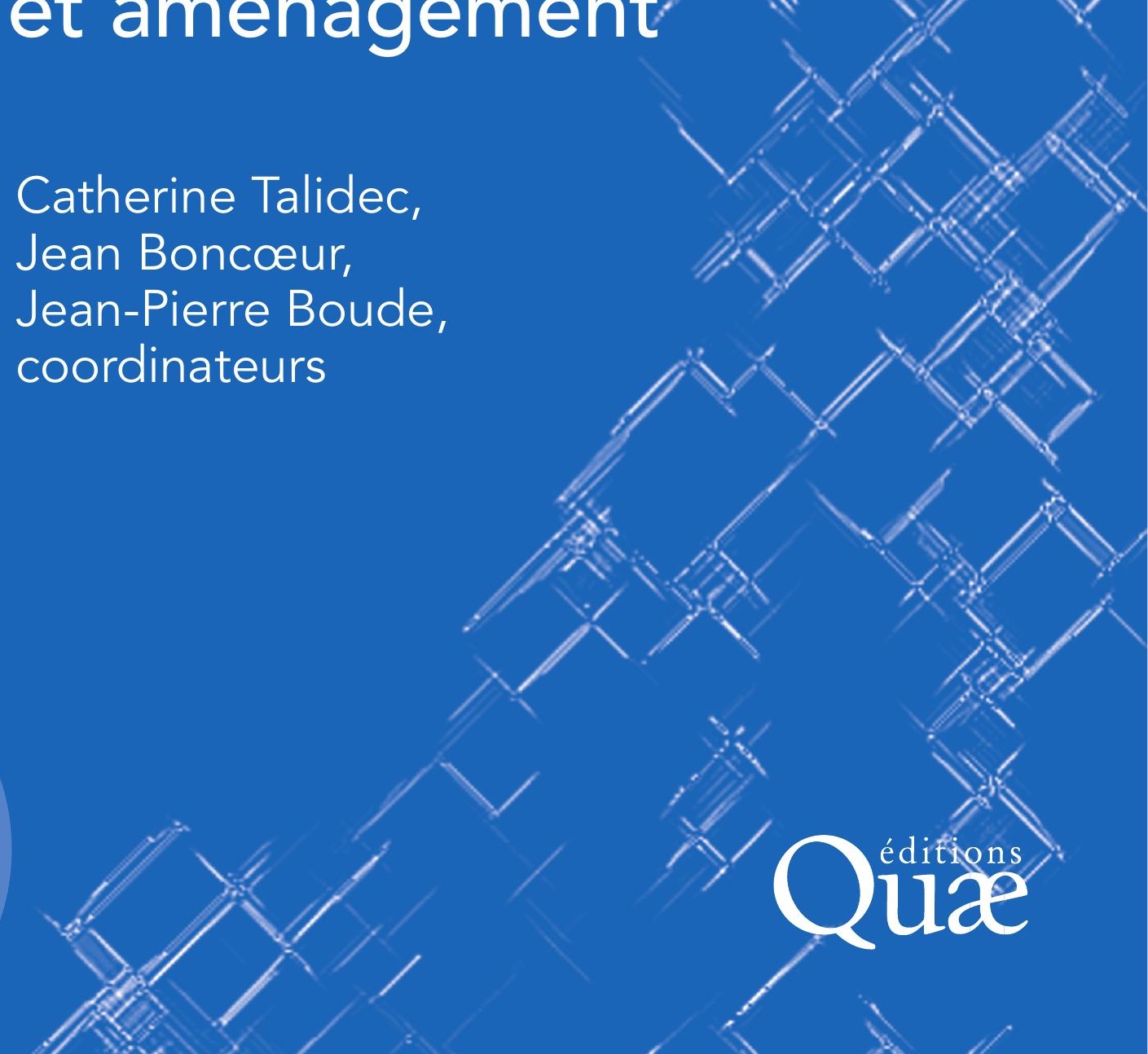



\section{Les pêches côtières bretonnes}

Méthodes d'analyse et aménagement 



\section{Les pêches côtières bretonnes}

Méthodes d'analyse et aménagement

Catherine Talidec, Jean Boncœur, Jean-Pierre Boude, coordinateurs

Éditions Quæ

c/o Inra, RD 10, 78026 Versailles Cedex 


\section{Collection Update Sciences \& Technologies}

Concevoir et construire la décision

Démarches en agriculture, agroalimentaire et espace rural

Elisabeth de Turckheim, Bernard Hubert, Antoine Messéan, coordinateurs 2009, 360 p.

La démarche qualité dans la recherche publique et l'enseignement supérieur.

Claude Granier, Léandre-Yves Mas, Luc Finot, Bernard Arnoux, Nathalie Pasqualini, Vincent Dollé, coordinateurs

2009, 376 pages

Homme et animal, la question des frontières

Valérie Camos, Frank Cézilly, Pierre Guenancia et Jean-Pierre Sylvestre, coordinateurs 2009, 216 p.

Le golfe du Lion. Un observatoire de l'environnement en Méditerranée André Monaco, Wolfgang Ludwig, Mireille Provansal, Bernard Picon, coordinateurs 2009, 344 p.

Politiques agricoles et territoires

Francis Aubert, Vincent Piveteau, Bertrand Schmitt, coordinateurs 2009, 224 p.

La mise à l'épreuve. Le transfert des connaissances scientifiques en questions Christophe Albaladejo, Philippe Geslin, Danièle Magda, Pascal Salembier, coordinateurs 2009, 280 p.

Le code de la propriété intellectuelle interdit la photocopie à usage collectif sans autorisation des ayants droit. Le non-respect de cette disposition met en danger l'édition, notamment scientifique, et est sanctionné pénalement. Toute reproduction, même partielle, du présent ouvrage est interdite sans autorisation du Centre français d'exploitation du droit de copie (CFC), 20 rue des GrandsAugustins, Paris 6e. 


\section{Avant-propos}

Cet ouvrage présente les résultats d'un projet de recherche qui s'est inscrit dans le cadre du programme « Conforter les pôles bretons de recherche et d'innovation » du contrat de plan État-Région 2000-2006 passé entre l'Ifremer et la Région Bretagne. Le projet s'intitulait « Recherche sur l'aménagement des activités de pêche sur les côtes bretonnes ».

Trois organismes se sont investis dans ce programme et ont collaboré à la réalisation des résultats :

- les départements d'économie maritime et des sciences et technologies halieutiques de l'Ifremer Brest et Lorient ;

- le centre de droit et d'économie de la mer (Cedem) de l'université de Bretagne occidentale de Brest ;

- le pôle halieutique d'Agrocampus Ouest de Rennes

Le département d'économie maritime de l'Ifremer et le Cedem se sont depuis regroupés en un laboratoire commun, l'UMR Amure.

L'objet de l'étude est la pêche côtière professionnelle et plaisancière de la région Bretagne au moyen d'une approche pluridisciplinaire intégrant les dimensions bio-économiques, juridiques et environnementales.

Une partie des travaux présentés dans l'ouvrage a fait l'objet de présentations orales suivies de débats avec un public composé de représentants professionnels de la pêche et de l'administration des pêches, de chercheurs et d'élus, lors d'un séminaire international organisé à Brest sur la «Régulation de l'accès aux ressources marines vivantes dans la zone côtière, les expériences internationales et perspectives pour la Bretagne ». 


\section{Remerciements}

Les auteurs remercient le réseau des observateurs de l'Ifremer pour la collecte des données d'enquêtes d'activités et économiques : Émilie Leblond, pour la coordination de ce réseau, Sylvain Bermell, pour la cartographie des pêcheries, et l'ensemble des acteurs du Système d'informations halieutiques de l'Ifremer.

Ils remercient également l'Observatoire économique régional des pêches de Bretagne, ainsi que tous les partenaires fournisseurs d'informations, en particulier la direction des pêches maritimes et de l'aquaculture.

De vifs remerciements sont adressés également à l'ensemble des patrons-pêcheurs et à leurs représentants pour leur aimable coopération dans le cadre des enquêtes, ainsi qu'à Shieldaig Export Limited, organisation écossaise de caseyeurs à langoustines, pour leur accueil.

Enfin, l'édition de cet ouvrage n'aurait pu se faire sans l'assistance d'Émilie Marc pour le travail de préparation des fichiers électroniques. Qu'elle en soit chaleureusement remerciée. 


\section{Responsables Scientifiques}

Catherine Talidec

Ifremer Lorient, responsable du département Sciences et technologies halieutiques

Catherine.talidec@ifremer.fr

www.ifremer.fr

Jean Boncœur

Université de Bretagne occidentale, Brest/UMR Amure, professeur

Jean.boncoeur@univ-brest.fr

www.umr.amure.fr

Jean-Pierre Boude

Agrocampus Ouest, Rennes/pôle halieutique, professeur

Boude@rhoazon.inra.fr

www.agrocampus-ouest.fr/halieutique

\section{Contributeurs}

Ont également contribué à la réalisation de cet ouvrage :

Pour l'Ifremer
Olivier Guyader
André Forest
Spyros Fifas
Yvon Morizur
Martial Laurans
Isabelle Peronnet
Olivier Thébaud
Alain Biseau
Claire Macher
Michel Bertignac
Fabienne Daurès
Sébastien Demanèche
Patrick Berthou
Johanna Herfaut

Pour l'université de Bretagne occidentale (UBO)

Olivier Curtil

Pascal Le Floc'h

Frédérique Alban

Pour Agrocampus Ouest, pôle halieutique

Marie Lesueur 



\section{Introduction}

Cet ouvrage résulte d'une collaboration d'halieutes biologistes, économistes et juristes, autour d'un projet de recherche qui porte sur les scénarios d'aménagement des activités de pêche dans la bande côtière bretonne. Quatre parties le composent.

Le premier chapitre pose la problématique générale de l'aménagement des pêcheries dans la bande côtière bretonne en abordant la question-clé des droits d'accès à la ressource et le besoin de les réguler.

L'état des connaissances acquises ou complétées au cours du projet fait l'objet du second chapitre.

Les espèces majeures intéressant la pêche côtière sont présentées selon leur état d'exploitation, ainsi que les flottilles bretonnes et les revenus qu'elles génèrent. L'apport de l'analyse juridique enrichit l'approche traditionnellement biologique et économique des pêches : le cadre juridique de la pêche côtière est décrit en abordant ses spécificités et les contraintes environnementales auxquelles elle est confrontée. Certains usages des ressources vivantes marines font l'objet d'analyses plus fines (pêche récréative, pêche à pied...). Des exemples de scénarios de gestion incluant leur évaluation bio-économique sont proposés dans une troisième partie.

Le dernier chapitre traite des développements méthodologiques qui ont été réalisés pour la collecte et l'analyse de données relatives aux entreprises de pêche, les méthodes de mesure des résultats économiques de la pêche et l'estimation des effets économiques induits. 



\section{Sommaire}

Avant-propos

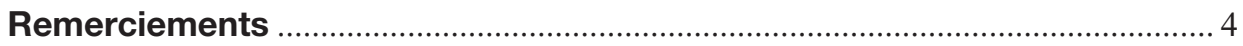

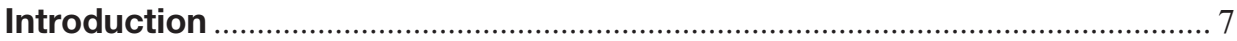

Chapitre 1 - L'aménagement des pêcheries dans la bande côtière bretonne, quelques points de repère et questions clés ................................... 11

Le besoin de régulation de l'accès.................................................................... 12

Les instruments de régulation de l'accès............................................................. 16

L'aménagement des pêcheries côtières ............................................................... 20

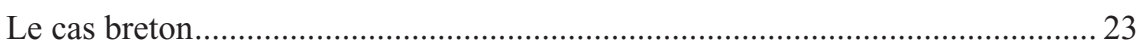

Chapitre 2 - Production de connaissances à l'échelle régionale .................. 29

Les espèces majeures de la bande côtière bretonne................................................ 29

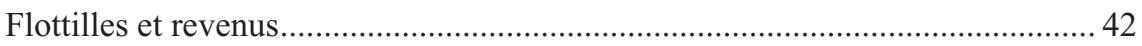

Cadre juridique : réflexions sur les spécificités de la pêche

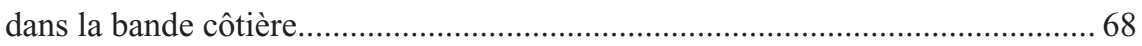

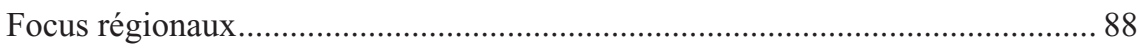

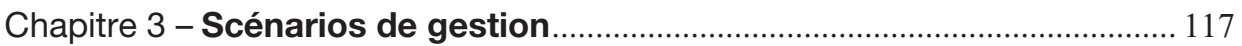

Régulation de l'accès de la pêcherie de coquilles Saint-Jacques

de la baie de Saint-Brieuc (1962-2006) Évolution et éléments de diagnostic... 117

Langoustine du golfe de Gascogne.................................................................. 122 
Chapitre 4 - Méthodologies développées …………………………………..... 157

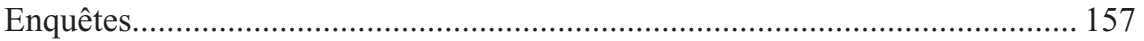

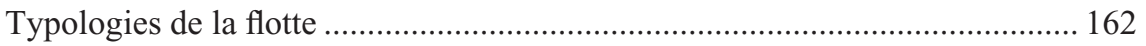

Effets économiques induits de la pêche côtière.................................................. 174

Mesure des résultats économiques de la pêche professionnelle bretonne : comparaison des méthodes à base de données comptables et d'enquêtes de terrain

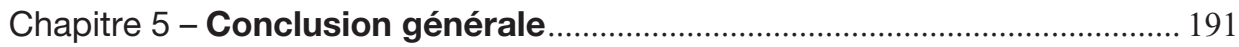

Annexe: Questionnaire d'enquête téléphonique

sur la pêche récréative

Références bibliographiques. 


\section{Chapitre 1}

\section{L'aménagement des pêcheries dans la bande côtière bretonne, quelques points de repère et questions clés}

Longtemps considérées comme pratiquement infinies et inépuisables, les ressources halieutiques marines sont, aujourd'hui, rangées sans ambiguïté dans la sphère économique des ressources rares ${ }^{1}$. Il en résulte une reconnaissance, largement partagée, du fait que leur exploitation doit s'accompagner de leur gestion. Malgré cet apparent consensus, la pêche rencontre, un peu partout dans le monde, d'importantes difficultés qui font ressortir les insuffisances des politiques publiques menées dans ce domaine. Les ressorts fondamentaux de ces difficultés sont aujourd'hui bien établis, et il apparaît de plus en plus clairement que les insuffisances des politiques publiques résident, dans une large mesure, dans un volet particulier de l'aménagement des pêcheries, concernant la régulation de l'accès aux ressources. L'observation des évolutions en cours indique que la situation n'est pas figée. Cependant les obstacles ne se laissent pas vaincre aisément, car ils mettent en jeu de multiples facteurs, non seulement techniques, mais aussi économiques, sociaux, politiques, voire culturels.

L'objet de cette introduction est tout d'abord de rappeler les termes élémentaires de la problématique de l'aménagement des pêcheries, puis de préciser les spécificités de son application au contexte de la bande côtière, notamment en Bretagne. La première section expose les raisons rendant nécessaire, dans le contexte actuel, la mise en place de mécanismes efficaces de régulation de l'accès aux ressources halieutiques. La deuxième section présente une typologie des différents instruments utilisables à cette fin. La troisième section s'interroge sur les spécificités de l'aménagement des pêcheries dans la mer côtière, et la quatrième section aborde plus spécifiquement le cas breton.

\footnotetext{
${ }^{1}$ Selon la terminologie économique usuelle, est réputée rare toute ressource qui, dans des circonstances données, n'est pas disponible en quantité suffisante pour satisfaire pleinement les besoins humains (quelle que soit la nature de ceux-ci).
} 


\section{Le besoin de régulation de l'accès}

Cette première section débute par un rappel de quelques données de cadrage concernant l'évolution des pêcheries à l'échelle mondiale. Elle présente ensuite une grille de lecture des tendances observées, et montre en quoi les mesures de conservation formant traditionnellement le socle de l'aménagement des pêcheries ne suffisent pas à enrayer la dégradation de l'état des ressources.

\section{Éléments de cadrage}

Les estimations de la FAO font état d'une multiplication par cinq du tonnage débarqué par les pêches mondiales depuis le milieu du XXe siècle. Ce développement considérable doit être rapproché des innovations techniques majeures qu'a connues le secteur sur la période, dans des domaines comme les matériaux, les engins de pêche, la propulsion, la conservation à bord, la navigation, la détection. Sous des formes différentes, ces innovations ont affecté toutes les formes de pêche, côtière comme hauturière, « artisanale » comme « industrielle ». Elles sont loin d'avoir épuisé leurs effets, comme en témoigne le développement spectaculaire de l'électronique embarquée sur des unités de toutes tailles.

Cependant, le rythme de croissance des débarquements, très soutenu dans les années cinquante et soixante ( $6 \%$ par an en moyenne), a connu depuis lors une forte décélération, ne dépassant pas $0,8 \%$ par an en moyenne sur la période 1990-2004. Ce taux est inférieur à celui de la croissance démographique mondiale (de l'ordre de 1,5\% par an), ce qui signifie que les disponibilités par habitant en produits de la pêche sont en recul depuis une quinzaine d'années. Selon les conclusions d'un exercice prospectif conduit par la FAO, il n'y a pas lieu d'espérer un retournement de tendance à moyen terme, la production halieutique mondiale devant fluctuer d'ici 2030 autour de 93 millions de tonnes, correspondant approximativement au niveau atteint dans la dernière décennie du $\mathrm{XX}^{\mathrm{e}}$ siècle (FAO, 2004).

Ce phénomène global recouvre des réalités différenciées selon les zones et les espèces. Il s'accompagne de transformations importantes dans la structure des populations exploitées et dans celle des captures. Les stocks considérés comme surexploités ou « en récupération » (terme désignant en fait des stocks que l'on a cessé d'exploiter car ils se sont effondrés) représentaient au début de la dernière décennie plus du tiers du total des stocks recensés par la FAO, alors que leur part était marginale au milieu du siècle dernier. Dans le même temps, la part des stocks sous-exploités ou modérément exploités, hégémonique en 1950, dépassait à peine $40 \%$ du total quarante ans plus tard. La taille moyenne et le niveau trophique moyen des captures régressent, et les espèces « nobles » sont particulièrement touchées par la raréfaction des ressources.

Certes, les tendances négatives qui affectent aujourd'hui les pêcheries mondiales sont statistiquement contrebalancées par le développement spectaculaire de l'aquaculture. Selon la FAO, le tonnage de la production aquacole mondiale représentait $62 \%$ de celui de la production halieutique en 2004, contre seulement $11 \%$ en 1980 . Ce ratio pourrait être proche de $90 \%$ en 2030 (FAO, 2004). Mais il existe de fortes incertitudes en ce domaine. Outre des contraintes environnementales susceptibles de limiter le développement de l'aquaculture, dans de nombreux cas le talon d'Achille de ce développement 
pourrait bien être la pêche elle-même, gros fournisseur d'aliments consommés par le cheptel des fermes aquacoles ${ }^{2}$.

\section{Le problème économique de la pêche}

Souvent imputée à la technique, la surexploitation des ressources halieutiques a des causes avant tout économiques, qui sont aujourd'hui bien établies. La première est la tendance à la surcapacité que suscite naturellement l'exploitation concurrentielle d'une ressource commune, et qui se renforce parallèlement à la rareté de la ressource. Une cause subsidiaire est l'existence de politiques publiques exacerbant cette tendance.

L'analyse part du constat que les stocks halieutiques marins forment, par nature, des ressources communes, catégorie définie par la réunion de deux caractéristiques quelque peu contradictoires (Berkes et al. 1989). D'une part, les ressources communes sont soustractives: contrairement, par exemple, à l'éclairage public ou à la connaissance scientifique, le poisson utilisé (capturé) par les uns n'est plus disponible pour les autres. Cette première caractéristique engendre une rivalité potentielle entre utilisateurs de la ressource. D'autre part, les ressources communes sont indivises : contrairement, par exemple, à des terres cultivables ou à des gisements miniers, les poissons dans la mer peuvent difficilement faire l'objet d'une allocation a priori entre exploitants. En l'absence de règles spécifiques, ils peuvent être capturés par quiconque, et c'est l'acte de capture qui vaut appropriation (ce qui correspond à la catégorie juridique de res nullius, « chose n'appartenant à personne»). L'indivision naturelle des ressources halieutiques marines tient à leur caractère « fugitif», terme évocateur désignant à la fois leur mobilité spatiale et leur caractère mal connu (les stocks halieutiques font, au mieux, l'objet d'estimations statistiques).

$\mathrm{Du}$ fait du caractère commun de la ressource, la capture d'un pêcheur dépend non seulement de son propre effort de pêche (c'est-à-dire du capital et du travail qu'il met en œuvre pour pêcher), mais aussi de l'effort exercé par les autres pêcheurs exploitant le même stock: l'augmentation de l'effort de ces derniers est de nature à réduire son volume de capture, dans la mesure où elle accroît la rareté de la ressource dont il dispose pour son propre compte. Qualifié d'externalité dans la littérature économique, ce phénomène crée une divergence entre rationalité individuelle et rationalité collective. En effet, tout exploitant soucieux de maximiser son revenu dans un environnement concurrentiel est incité à augmenter son effort de pêche tant que cet accroissement lui rapporte plus qu'il ne lui coûte, c'est-à-dire jusqu'au point où le supplément de produit que lui procure une unité d'effort supplémentaire devient juste égal au coût de cette unité d'effort. Mais, du fait de l'externalité entre pêcheurs, ce supplément de produit, appelé produit marginal privé de l'effort de pêche, est supérieur au produit marginal social de l'effort de pêche, c'est-à-dire à l'impact d'une unité d'effort supplémentaire sur le volume total de capture réalisé par l'ensemble des pêcheurs exploitant le même stock ${ }^{3}$. Par suite, à l'échelle de la pêcherie,

\footnotetext{
${ }^{2}$ Actuellement, un tiers environ du tonnage débarqué par la pêche mondiale (végétaux exclus) est utilisé pour d'autres usages que l'alimentation humaine directe et sert essentiellement à la fabrication de farines et d'huiles destinées à l'alimentation des animaux d'élevage.

${ }^{3}$ Lorsqu'un exploitant augmente son effort, il en résulte, toutes choses égales par ailleurs, une diminution de la capture des autres, de sorte que la variation de capture globale (produit marginal social) est égale à l'augmentation de capture pour celui qui augmente son effort (produit marginal privé), diminuée de la baisse des captures subie par les autres exploitants (externalité négative).
} 
l'agrégation des comportements individuels semblables à celui qui vient d'être décrit crée une situation où le revenu net issu de l'exploitation du stock (appelé « rente halieutique ») n'est pas maximisé : tendant à s'égaliser au produit marginal privé de l'effort de pêche, le coût unitaire de l'effort est normalement supérieur à son produit marginal social, ce qui signifie que la rente globale augmenterait si les pêcheurs diminuaient leur effort. La difficulté est qu'aucun d'entre eux, considéré isolément, n'est incité à le faire: tout pêcheur qui diminuerait son effort sans avoir l'assurance que les autres en font autant s'exposerait à une détérioration de sa propre situation, non seulement en termes relatifs, mais aussi en termes absolus.

Le mécanisme qui vient d'être décrit fonctionne avec un nombre donné de pêcheurs. Dans le cas d'un accès libre et gratuit à la ressource, il est relayé par le fait que l'existence d'une rente positive attire de nouveaux pêcheurs, et cela jusqu'à ce que la totalité de la rente susceptible d'être extraite de la ressource soit dissipée: l'équilibre normal d'une pêcherie en accès libre et gratuit est atteint lorsque le coût de l'effort de pêche absorbe intégralement la valeur du poisson débarqué ${ }^{4}$.

Partielle ou totale, la dissipation de la rente halieutique traduit un gaspillage de moyens à l'échelle de la société : trop de capital et de travail sont engagés pour l'exploitation d'une ressource naturelle donnée, et le surcoût résultant de cette surcapacité érode tout ou partie du revenu net que procurerait son exploitation rationnelle. Outre le gaspillage social de moyens qu'il représente, le phénomène de surcapacité présente deux conséquences négatives majeures. Tout d'abord, il favorise la surexploitation des stocks halieutiques, c'est-à-dire l'adoption de niveaux de prélèvement excessifs par rapport aux capacités de renouvellement des ressources (surexploitation de recrutement), mais aussi, à travers la « course au poisson » qu'il suscite, l'adoption de méthodes de pêche induisant une exploitation trop précoce des cohortes constituant les stocks (surexploitation de croissance). Ces différents types de surexploitation ont naturellement pour effet d'aggraver le gaspillage social engendré par la surcapacité. Dans des cas extrêmes, ils peuvent conduire à l'effondrement des stocks et à la disparition pure et simple de la pêcherie. En second lieu, la surcapacité attise les conflits d'usage: lorsque les capacités de capture sont globalement excessives par rapport à la ressource à exploiter, chaque exploitant est objectivement une menace pour les autres.

Les responsables de l'aménagement d'une pêcherie doivent affronter le fait que la tendance à la surcapacité est un phénomène inhérent à la nature même du problème qu'ils ont à traiter. Pourtant, cette tendance se trouve fréquemment exacerbée par des politiques publiques qui subventionnent l'effort de pêche. Selon une étude de l'OCDE, le montant des transferts publics en faveur de la pêche s'élevait en moyenne, au sein des pays membres de cette organisation, à $17 \%$ de la valeur débarquée en $1997^{5}$ (OCDE, 2000). Avec un ratio de $15 \%$, l’Union européenne (UE) se situait apparemment plutôt en dessous de la moyenne, quoique nettement au-dessus de pays comme l'Australie (9\%), l'Islande (4\%) ou la Nouvelle-Zélande (4\%). Mais, parmi l'ensemble des transferts recensés, la part des paiements directs aux pêcheurs et des aides à la réduction des coûts y atteignait la moitié du total (l'autre fraction représentant les « services généraux »), contre $11 \%$ en

\footnotetext{
${ }^{4}$ Ce coût inclut la rémunération du travail et du capital à des taux conformes à leur coût d'opportunité.

5 Des comparaisons avec des sources nationales suggèrent que les chiffres publiés par l'OCDE sous-estiment fortement dans certains cas fortement la réalité.
} 
moyenne dans le reste de l'OCDE. Depuis lors, dans le cadre de la réforme de la politique commune de la pêche (PCP) intervenue en 2002, les aides publiques ayant pour conséquence d'accroître la capacité de capture des navires sont en principe interdites au sein de l'UE.

Le problème économique de la pêche a une dimension historique (Troadec, 1994). En effet, la tendance à la surcapacité varie en raison directe de l'écart entre produit marginal privé et produit marginal social de l'effort de pêche, qui se creuse en même temps que s'accroît la rareté de la ressource ${ }^{6}$, sous l'effet conjugué de la pression de la demande et du développement de l'efficacité des techniques de pêche. Les systèmes traditionnels de régulation des activités halieutiques y résistent d'autant moins qu'ils reposent souvent sur des bases sociales en voie de délitement.

\section{L'insuffisance des mesures de conservation}

L'aménagement des pêcheries peut être défini comme une régulation publique de l'exploitation de ressources communes par des personnes privées. Deux missions complémentaires, correspondant à deux grandes catégories de mesures, lui sont assignées: une mission de conservation des ressources et une mission de régulation de l'accès aux ressources.

La fonction des mesures de conservation est de préserver la capacité productive des populations exploitées (production par recrue), ainsi que leur capacité reproductive (biomasse féconde). Ces mesures visent en premier lieu à assurer une certaine sélectivité des captures (mesures dites «techniques » portant sur les caractéristiques des engins, les tailles minimales au débarquement, ou encore l'interdiction de pêcher dans certaines zones et/ou à certaines périodes de l'année). Elles peuvent aussi tendre à limiter le volume global des prélèvements, à travers l'imposition de totaux admissibles de capture (TAC) ou de limitations portant sur certains paramètres de l'effort de pêche global (nombre annuel de jours ou d'heures de pêche par exemple). Les mesures de conservation constituent classiquement le socle de l'aménagement des pêcheries.

D'usage moins universel, un second ensemble de mesures a pour objet de réguler l'accès des exploitants individuels à la ressource commune. Ces mesures consistent à délimiter l'ensemble des pêcheurs autorisés à exploiter la ressource ainsi que la part à laquelle chacun peut prétendre dans cette exploitation. Alors que les mesures de conservation présentent un caractère général et impersonnel, les mesures de régulation de l'accès nécessitent d'identifier précisément chaque exploitant.

La littérature récente sur l'état des pêcheries (Hilborn et al. 2003 ; Garcia et Grainger, 2005) fait clairement ressortir le fait que les mesures de conservation ne suffisent pas au maintien d'un bon état biologique des stocks exploités. En effet, elles s'attaquent aux manifestations mais non aux causes profondes de la surexploitation. Lorsque l'aménagement d'une pêcherie se limite pour l'essentiel à des mesures de conservation, la portée de ces mesures est généralement battue en brèche par le développement de la surcapacité. Les difficultés économiques et sociales qui accompagnent ce développement créent une pression sociale en faveur de l'adoption de niveaux de prélèvement excessifs

\footnotetext{
${ }^{6}$ L'accroissement de la rareté de la ressource, au sens économique du terme, ne signifie pas nécessairement une diminution de sa disponibilité physique. Il traduit une tension croissante entre cette disponibilité et la demande.
} 
et d'un contrôle laxiste des mesures adoptées, et cette pression a facilement raison des recommandations de prudence émanant des milieux scientifiques.

\section{Les instruments de régulation de l'accès}

Dans les conditions qui viennent d'être décrites, on ne peut attendre du seul renforcement des mesures de conservation qu'il résolve les difficultés de la pêche. Bien que le diagnostic ne soit pas toujours énoncé de façon très claire dans le débat public, on voit un peu partout s'imposer l'idée que ces mesures doivent être complétées par des mécanismes efficaces de régulation de l'accès individuel à la ressource. Le problème essentiel consiste en effet à « internaliser» les effets externes croisés que suscite l'exploitation d'une ressource commune, c'est-à-dire à instaurer des mécanismes conduisant chaque exploitant à prendre en compte les effets négatifs que son action inflige aux autres (conceptuellement, cette question est très proche des problèmes rencontrés en matière de gestion environnementale). Le débat sur la nature de ces mécanismes n'est pas clos, mais il semble se décanter progressivement. Après avoir dressé une typologie sommaire des instruments de régulation de l'accès à la ressource, on examinera les principaux effets que produit le recours à l'un ou l'autre de ces instruments.

\section{Une typologie des instruments}

Réguler l'accès à une ressource commune implique d'exercer un certain contrôle sur l'activité de ceux qui exploitent cette ressource. Pour caractériser un dispositif de régulation de l'accès, il est donc nécessaire de définir deux paramètres : la variable sur laquelle s'exerce ce contrôle et la méthode de cet exercice. Une typologie sommaire des mesures de régulation de l'accès à la ressource peut être fondée sur le croisement de ces deux paramètres (Troadec et Boncœur, 2003).

En pêche, le choix de la variable de contrôle n'est pas trivial. Par nature, la ressource elle-même ne peut remplir cette fonction: on peut difficilement envisager de marquer des poissons dans la mer comme on le fait pour du bétail terrestre. Du fait de la mobilité de la majorité des ressources exploitées par la pêche, l'espace n'est que rarement une variable de contrôle. Aussi le choix se porte-t-il généralement sur l'effort de pêche ou sur les captures. Dans le premier cas, on cherche à contrôler les différentes variables déterminant le taux de mortalité qu'un pêcheur inflige à un stock donné (temps de pêche et ensemble des facteurs déterminant la « puissance de pêche », incluant les caractéristiques du navire, de l'équipage et des engins de pêche ${ }^{7}$ ). Dans le second cas, ce sont directement les prélèvements opérés par chaque pêcheur que l'on cherche à contrôler (quotas individuels de capture).

En matière de méthode de contrôle, la distinction principale oppose les méthodes dites « administratives » aux méthodes dites « économiques ». Les premières reposent sur des normes qui sont imposées aux acteurs: fixation d'une puissance motrice maximale ou d'un quota de capture pour chaque navire titulaire d'une licence de pêche par exemple. Les secondes reposent sur des incitations : plutôt que d'imposer un comportement par des

\footnotetext{
7 Les autorisations d'accès à la ressource fondées sur un contrôle des paramètres de l'effort de pêche sont généralement appelées « licences » dans la littérature internationale sur l'aménagement des pêcheries (Copes, 1997; OCDE, 1997).
} 
règles contraignantes, il s'agit de mettre en place des mécanismes qui incitent les acteurs à se comporter dans le sens jugé souhaitable du point de vue des intérêts de la collectivité. Illustrées dans le domaine de la gestion environnementale par le principe «pollueur payeur », les incitations, au sens économique du terme, sont censées réconcilier le calcul économique individuel avec celui de la collectivité, lorsqu'il y a divergence entre les deux (ce qui est typiquement le cas en présence d'effets externes). Deux grands types de méthodes économiques peuvent être envisagés pour réguler l'accès à une ressource commune: la taxation et l'allocation de droits d'usage transférables. Dans le premier cas, la taxe doit être fixée à un taux incitant chaque pêcheur à limiter son prélèvement sur la ressource commune au niveau souhaité par la collectivité (même principe que l'écotaxe). Dans le second cas, les autorités en charge de l'aménagement de la pêcherie délivrent des quotas d'effort ou de capture que les exploitants peuvent ensuite échanger entre eux à des prix librement négociés (à l'instar des droits à polluer).

Les deux dimensions du problème de la régulation de l'accès sont orthogonales: le choix de la variable de contrôle ne préjuge pas de celui de la méthode de contrôle, et inversement. Ainsi, une taxe visant à réguler le taux d'exploitation d'un stock peut être assise sur l'effort ou sur les captures. De même, le contrôle administratif de l'accès à la ressource peut, a priori, s'appuyer aussi bien sur des quotas de captures (non transférables) que sur des limitations individuelles d'effort de pêche. Enfin, qu'elles portent sur les captures ou sur l'effort de pêche, les autorisations individuelles se muent en instruments économiques dès lors qu'elles deviennent transférables.

\section{Retours d'expérience}

La méta analyse réalisée par Sutinen voici dix ans (OCDE, 1997) a bien mis en évidence les difficultés que soulève toute tentative pour porter un jugement d'ensemble sur la façon dont fonctionnent les régimes de régulation de l'accès aux ressources halieutiques. Les données sont incomplètes et hétérogènes, et la comparabilité des situations est affaiblie par la multiplicité des facteurs non contrôlés. Cependant, la littérature sur le sujet connaît un rapide développement, et permet de faire ressortir quelques observations dont la portée dépasse le cadre strict de la monographie (cf. par exemple Shotton, 2000; OCDE, 2006).

En ce qui concerne la variable de contrôle, il apparaît souvent délicat de faire appel à l'effort de pêche. En effet, la puissance de pêche des navires revêt un caractère multidimensionnel, doublé d'une forte substituabilité entre les éléments la composant. En outre, sa définition évolue en permanence sous l'effet du progrès technique. Par suite, les autorités qui tentent de réguler l'accès à la ressource sur la base de l'effort de pêche doivent affronter un dilemme inconfortable. Si elles décident de ne contrôler qu'une partie des déterminants de la puissance de pêche, elles se condamnent généralement à ne pas contrôler grand-chose. L'issue classique est alors de restreindre toujours plus la seule composante aisément contrôlable de l'effort de pêche, qui est le temps de pêche. Cette solution, qui débouche sur ce que la langue anglaise appelle de façon imagée le derby fishing, présente d'importants inconvénients en termes d'efficacité économique, de conditions de travail et de qualité des produits. Si les responsables de l'aménagement d'une pêcherie tentent d'opérer un contrôle exhaustif de la puissance de pêche des navires, ils s'engagent dans une opération pouvant entraîner des coûts de gestion considérables, mais présentant surtout l'inconvénient de figer la structure technique des armements au mépris de toute rationalité économique (il suffit pour s'en convaincre d'imaginer ce que 
représenterait, dans tout autre secteur productif, une tentative de l'administration pour contrôler l'ensemble des paramètres de «l'effort de production » des entreprises).

Ces considérations plaident clairement en faveur d'un contrôle de l'accès fondé sur les captures. Toutefois, cette solution nécessite que soient remplies certaines conditions. La première concerne la capacité du régulateur à contrôler effectivement les quantités débarquées. Cette condition est critique: si elle n'est pas satisfaite, une tentative pour réguler l'accès via les captures risque d'entraîner des résultats d'autant plus catastrophiques que ce mode de régulation constitue en soi une incitation à la dissimulation des captures. Un autre problème peut survenir dans le cas de pêcheries utilisant des engins peu sélectifs. Dans le cas d'une pêcherie plurispécifique, la fixation de quotas de capture individuels par espèce est susceptible d'accroître les rejets en mer, les quotas pour les différentes espèces capturées par un navire n'étant généralement pas atteints de façon simultanée. Par ailleurs, au sein d'une même espèce, le pêcheur soucieux de valoriser au mieux son quota peut être incité à rejeter les captures dont la valeur économique n'est pas maximale (highgrading). L'expérience des pays pratiquant le contrôle de l'accès à la ressource par l'intermédiaire de quotas individuels de capture suggère toutefois que ce genre de problème n'est pas insurmontable. L'adaptation d'un système de quotas individuels de capture à une pêcherie plurispécifique, en particulier, peut être facilitée par des mécanismes d'échange de quotas.

En matière de méthode de contrôle, les pays qui s'engagent dans l'instauration de mécanismes contraignants de régulation de l'accès commencent généralement par adopter des instruments de type administratif (licences non transférables). Ce choix se situe en effet dans la continuité des méthodes en usage dans le domaine de la conservation des ressources. Cependant, ce type d'instrument présente, dans le domaine de la régulation de l'accès, des inconvénients qui conduisent un nombre croissant de pays à adopter des instruments de type économique (l'Islande, la Nouvelle-Zélande, l'Australie, le Canada et les Pays-Bas ont joué un rôle pionnier en ce domaine). Les instruments administratifs se voient notamment reprocher leur rigidité et leur faible pouvoir incitatif, eu égard à la nécessité de développer l'intérêt individuel pour la conservation de la ressource commune (stewardship).

Dans le secteur des pêches, le passage à des instruments économiques de régulation de l'accès à la ressource prend la forme de systèmes de droits individuels transférables plutôt que d'une taxe. Plusieurs raisons expliquent ce phénomène, parmi lesquelles figure sans doute en bonne place l'impopularité de l'instauration d'un prélèvement fiscal pour accéder à une ressource que les pêcheurs ont naturellement tendance à considérer comme leur appartenant. Par ailleurs, la régulation de l'accès par le biais d'une taxe soulève des difficultés techniques, en particulier concernant la fixation du taux de la taxe (le problème de l'assiette renvoie, quant à lui, à la question de la variable de contrôle). Mais le principal inconvénient de la taxation est peut-être que, pas plus que les méthodes administratives, ce mécanisme n'incite les pêcheurs à s'intéresser à la conservation de la ressource. En revanche, un système de droits individuels transférables peut jouer ce rôle, dans la mesure où les droits d'accès que détient un pêcheur se voient reconnaître une valeur monétaire explicite, inscrite à l'actif du bilan de son entreprise au même titre que son bateau. Cette valeur dépend de l'état de la ressource, ce qui est de nature à inciter son détenteur à s'intéresser de près à cet état. La question du contrôle doit aussi être envisagée sous cet angle, comme le montre l'exemple néerlandais des « groupes de cogestion » institués pour améliorer la gestion des QIT. 
Lourdement chargée sur le plan idéologique, la question de la transférabilité des droits est souvent mal posée. Nonobstant son statut légal, un droit individuel ouvrant l'accès à une ressource rare fait généralement l'objet de transactions sous une forme ou une autre, car ces transactions répondent à des nécessités évidentes (que l'on imagine un instant, dans le monde d'aujourd'hui, une agriculture dans laquelle les agriculteurs ne seraient pas autorisés à acheter, vendre ou louer des terres). Dans le cas de la France, où la nontransférabilité des droits de pêche est inscrite dans la loi, une analyse économétrique des prix des navires d'occasion a mis en évidence le fait que, pour acquérir un navire muni d'un permis de mise en exploitation et, éventuellement, de licences de pêche (juridiquement strictement annuelles et non dissociables du couple navire-propriétaire), les pêcheurs paient un surcoût évalué à $50 \%$ de la valeur du navire en moyenne (Guyader et al., 2006). La véritable question ne porte donc pas sur l'opportunité de rendre les droits de pêche transférables (ils le sont), mais sur l'intérêt de conférer à ces transferts un caractère transparent et juridiquement sécurisé. Il existe des arguments sérieux en faveur d'une réponse positive à cette question, en termes d'équité comme d'efficacité. Par exemple, l'absence de distinction entre valeur du navire et valeur des droits de pêche, qui n'est ellemême que le corollaire de la non-reconnaissance de jure d'une valeur existant de facto, vient fortement compliquer l'évaluation de la rentabilité des projets d'investissement. L'incertitude pour le candidat à l'investissement est évidemment d'autant plus grande que le régime des droits d'accès à la ressource manque de clarté et de stabilité juridique.

Les instruments économiques de régulation de l'accès à la ressource, et plus spécifiquement les systèmes de quotas individuels transférables de capture (QIT), se voient généralement reconnaître une certaine efficacité en ce qui concerne la lutte contre la surcapacité et la tendance au derby fishing. Dans le même temps, ils sont souvent accusés de conséquences néfastes sur le plan social. Cette critique comporte elle-même deux volets: il est reproché aux QIT, d'une part de réduire l'emploi à la pêche et, d'autre part, de favoriser la concentration des droits de pêche (ces deux reproches ne se confondent pas).

En ce qui concerne l'emploi, il est assez clair que la réduction d'une situation de surcapacité ne constitue, dans aucun secteur, une circonstance favorable à l'emploi. Dans le cas de la pêche, le problème est aggravé par les limites strictes que la nature impose à l'expansion de la production. Une fois ces limites atteintes (ce qui tend à être la règle de nos jours, comme le montrent les statistiques de la FAO), les gains de productivité, qui concernent la pêche comme tout autre secteur productif, se concilient difficilement avec le maintien de l'emploi dans le secteur, quel que soit le système de régulation adopté. La question de la transférabilité des droits est assez latérale par rapport à ces considérations, et l'expérience indique que le refus de reconnaître explicitement cette transférabilité ne contribue pas nécessairement à protéger l'emploi à la pêche. Ainsi, alors que l'Islande a connu un recul de l'emploi à la pêche de $26 \%$ sur la période 1984-1997 pendant laquelle fut introduit puis généralisé le système des QIT dans ce pays (soit 2,2\% par an en moyenne), l'emploi à la pêche au sein de l'UE a diminué de $21 \%$ entre 1990 et 1998 (soit 2,9\% \% par an en moyenne), sans que cette diminution puisse être mise en relation, sauf de façon marginale, avec un système de droits explicitement transférables ${ }^{8}$.

\footnotetext{
8 À l'intérieur de l'UE, seuls les Pays-Bas se sont dotés, à ce jour, d'un système de QIT. La Grande-Bretagne dispose d'un système relativement complexe de droits de pêche partiellement transférables.
} 
La question de la concentration des droits de pêche est, quant à elle, largement politique $^{9}$. Dans un système productif où les droits de production sont librement transférables, ces droits tendent normalement à se retrouver entre les mains des producteurs dont l'efficacité économique est la plus grande, dans la mesure où ce sont eux qui ont le plus fort consentement à payer pour les acquérir. Le degré de concentration qui en résulte dépend principalement des économies d'échelle que ces producteurs sont susceptibles de réaliser. Il peut être jugé excessif par les pouvoirs publics, s'ils considèrent que le corps social est prêt à payer un certain prix, en termes d'efficacité économique, pour le maintien d'une structure productive plus conforme à certaines normes sociales. Cette question n'est pas spécifique à la pêche, et on la retrouve fréquemment posée dans des secteurs comme l'agriculture, le commerce de détail, les taxis... En ce qui concerne la pêche, dans le cadre d'un système de droits transférables, l'autorité de régulation a la faculté de limiter la transférabilité des droits : elle peut définir de façon plus ou moins restrictive les personnes (physiques ou morales) autorisées à détenir des droits de pêche et plafonner le nombre de droits qu'une personne peut détenir au sein d'une pêcherie. On constate que les pays ayant mis en place un système de QIT recourent systématiquement à ces deux leviers.

L'expérience des pays ayant adopté un mécanisme de droits individuels transférables pour gérer l'accès aux ressources halieutiques indique que, dans la majorité des cas, la question la plus délicate est celle de l'allocation initiale des droits. C'est, là aussi, une question d'ordre essentiellement politique, renvoyant en principe aux normes sociales en vigueur en matière d'équité. Même si, sur le plan technique, l'allocation initiale ne conditionne pas l'efficacité d'un mécanisme à base de droits transférables, un tel mécanisme est politiquement difficile à instaurer en l'absence d'un consensus minimum sur cette question. Cette considération conduit le plus souvent les gouvernements à allouer initialement les droits de façon gratuite, sur la base des antériorités de pêche (grandfathering).

Ce type d'opération est souvent assimilé à une " privatisation de la ressource ». Cette interprétation semble reposer sur une confusion entre la ressource et les usages qui en sont faits. Dans un système de QIT par exemple, les quotas individuels sont généralement définis comme des pourcentages permanents d'un TAC, lui-même révisé périodiquement par l'autorité en charge de l'aménagement de la pêcherie sur la base des avis scientifiques. Les exploitants ne sont donc pas propriétaires de la ressource mais titulaires du droit d'exploiter, dans des conditions définies, un certain pourcentage de la fraction de la ressource que le régulateur ouvre à la pêche. Ce droit d'usage transférable peut lui-même présenter un degré de liquidité et de sécurité juridique plus ou moins élevé, le rapprochant plus ou moins d'un droit de propriété.

\section{L'aménagement des pêcheries côtières}

La notion de « bande côtière » n'a pas de statut juridique explicite (Curtil, 2001). Dans cette étude, nous définirons celle-ci comme la zone maritime à l'intérieur des douze milles délimitant les eaux territoriales.

\footnotetext{
${ }^{9}$ Il peut aussi exister un motif proprement économique pour limiter la concentration de droits, qui est de prévenir les effets néfastes d'un pouvoir de monopole. Cette question est rarement pertinente à l'échelle d'une pêcherie, dans la mesure où la taille des marchés des produits de la mer est généralement très supérieure à celle des pêcheries.
} 
Les pêcheries côtières représentent une proportion significative de l'activité de pêche dans beaucoup de régions du monde. Au sein de l'Union européenne par exemple, les navires de longueur inférieure à 15 mètres, qui sont largement inféodés à la bande côtière, représentent $87 \%$ du nombre total de navires de pêche professionnelle (Berthou et al. 2005). En ce qui concerne la France, si l'on définit comme « côtiers » les navires consacrant plus de $75 \%$ de leur temps de pêche total à la bande côtière et comme « mixtes » ceux qui y consacrent entre $25 \%$ et $75 \%$, il ressort des statistiques disponibles pour l'année 2003 que ces deux ensembles de navires représentaient respectivement $72 \%$ et $16 \%$ de l'effectif total des navires de pêche professionnelle de la façade Mer du Nord/ Manche/Atlantique. En termes d'emploi (exprimé en équivalents temps plein), leurs parts respectives s'élevaient respectivement à $46 \%$ et $38 \%$ (Leblond et al., 2006).

Le cadre analytique général présenté dans les deux sections précédentes s'applique au cas des pêcheries côtières. Cependant, certaines spécificités de ces pêcheries donnent une coloration particulière au problème de leur aménagement. Dans cette section, nous recensons un ensemble de questions méritant une attention particulière dans le contexte des pêcheries côtières. Ces questions sont relatives au contexte institutionnel, aux caractéristiques biologiques des stocks, aux caractéristiques technico-économiques des flottilles et aux interactions entre usagers de la bande côtière.

\section{Contexte institutionnel}

Le contexte institutionnel de l'aménagement des pêcheries côtières diffère de celui des pêcheries hauturières. La convention de Montego Bay sur le droit de la mer (1982) reconnaît aux États côtiers des droits exclusifs sur les ressources naturelles (halieutiques et autres) au sein d'une bande de 200 milles définie comme leur « zone économique exclusive » (ZEE). Plus près des côtes, les activités se déroulant à l'intérieur des eaux territoriales font généralement l'objet de régulations spécifiques. Ainsi, dans l'UE, alors que la PCP a consacré le principe de la communautarisation des zones de pêche constituées par les ZEE des pays membres, la bande des 12 milles fait l'objet d'une exception confirmée par la réforme de 2002: les États côtiers peuvent y réserver l'exercice de la pêche aux navires nationaux, sous réserve de respecter les antériorités historiques dûment répertoriées. L'aménagement des activités halieutiques au sein de la bande côtière est fréquemment exercé par des services déconcentrés de l'État (en France, au niveau des préfectures de région), des collectivités locales, des organisations professionnelles ou des combinaisons de ces différentes institutions.

\section{Ressources}

Étant donné les caractéristiques de leur cycle de vie, de nombreuses espèces exploitées par la pêche peuvent être considérées comme essentiellement côtières. Cette caractéristique, associée à la précédente, offre des possibilités intéressantes pour l'aménagement sur une base locale des pêcheries côtières. Cependant, on relève également l'existence de stocks " chevauchants » ou " migrateurs », dont l'exploitation ne peut être gérée sur une base strictement locale. De nombreuses espèces qui sont exploitées principalement au large dépendent fortement de la zone côtière pour une partie de leur cycle vital, ce qui crée des situations similaires à celles que l'on rencontre dans des pêcheries séquentielles. Les conséquences des décisions d'aménagement prises au sein de la zone côtière peuvent 
alors déborder très largement cette zone. Elles doivent donc être cohérentes avec celles qui concernent la pêche au large, ce qui est de nature à compliquer les schémas d'aménagement.

\section{Flottilles}

À l'instar des stocks, les navires de pêche sont plus ou moins dépendants de la bande côtière. Les plus petits d'entre eux sont en général totalement inféodés à cette zone, étant donné leur faible rayon d'action. Ils compensent fréquemment cette faiblesse par une grande polyvalence, pratiquant une diversité de métiers. Au-delà de l'interaction classique entre pêcheurs résultant du caractère commun de la ressource exploitée, la diversité des métiers pratiqués au sein d'un espace relativement restreint suscite des interactions techniques plus ou moins complexes entre flottilles (incompatibilité de la pratique simultanée des arts traînants et des arts dormants dans une même zone par exemple), qui nécessitent des mesures d'aménagement spécifiques. Une autre difficulté tient au fait que les débarquements des flottilles côtières sont souvent mal connus, du fait des modes de commercialisation diffus qu'elles adoptent (faible taux de passage en criée notamment). Enfin, il faut tenir compte du fait que l'accès aux ressources de la bande côtière n'est généralement pas réservé aux navires strictement côtiers : des navires qui, par leurs caractéristiques physiques, sont aptes à pêcher au large, viennent souvent y exercer une partie de leur activité. Même si cette partie est minime par rapport à leur effort de pêche total, elle peut avoir des conséquences importantes pour les pêcheries côtières compte tenu de la puissance de pêche relativement importante de ces navires « chevauchants ».

\section{Interactions entre usages}

À l'intérieur de la bande côtière, les interactions entre usages impliquant la pêche sont multiples. Une première catégorie concerne les interactions au sein de la pêche professionnelle, qui présentent fréquemment, comme on l'a vu, un degré de complexité plus élevé à la côte qu'au large. Une seconde catégorie concerne les interactions entre pêcheurs professionnels et pêcheurs récréatifs, concentrés pour l'essentiel dans la bande côtière. Enfin, la pêche (professionnelle ou récréative) interagit avec de multiples usages non halieutiques de la bande côtière, mais aussi des bassins versants auxquels cette dernière sert d'exutoire. Ces interactions peuvent avoir un caractère spatial (compétition spatiale avec les éoliennes en mer par exemple) ou biologique (impact des effluents des activités terrestres sur les écosystèmes côtiers par exemple). Dans ce dernier cas, les interactions entre pêche et autres usages ont souvent un caractère diffus, et leur vecteur matériel n'est pas toujours connu avec une grande précision. En outre, les poids économiques des activités concernées, exprimés en termes de valeur ajoutée ou d'emploi, sont souvent très disparates, et leur comparaison tourne rarement à l'avantage de la pêche (même en tenant compte des activités « amont» et « aval » de la filière). Cependant, les interactions entre pêche côtière et autres usages ne sont pas uniquement négatives. En premier lieu, cette activité a souvent une image identitaire forte, qui participe à la conscience de soi des populations habitant le littoral, et cela même lorsque les liens économiques de ces populations avec la pêche sont devenus assez ténus. En outre, la pêche côtière joue souvent un rôle attractif important vis-à-vis de la fréquentation touristique (cf. fréquentation des ports de pêche). Enfin, par sa dépendance vis-à-vis de l'état des écosystèmes côtiers, la pêche côtière peut jouer un rôle d'activité témoin vis-à-vis des efforts déployés par la collectivité pour la conservation 
de ces écosystèmes. Cela suppose néanmoins que les techniques et l'effort de pêche mis en œuvre soient eux-mêmes compatibles avec cette conservation, ce qui peut nécessiter certaines adaptations.

\section{Le cas breton}

Cette dernière section introduit le cas d'étude faisant l'objet de ce rapport ${ }^{10}$. Après une brève présentation de la pêche professionnelle en Bretagne et des principales tendances de son évolution, elle aborde les problèmes posés par la régulation de l'accès à la ressource, particulièrement dans le cas de la bande côtière.

\section{Une activité très diversifiée}

Le profil de la pêche professionnelle bretonne peut être esquissé à l'aide de quelques chiffres : 1537 navires en activité, $315000 \mathrm{~kW}$ de puissance motrice nominale, 7053 marins embarqués, 302000 tonnes de captures estimées (hors algues), environ 466 millions d'euros de chiffre d'affaires en 2004 (MAP/Ofimer, 2005). Avec environ $44 \%$ du chiffre d'affaires de la pêche maritime française, la Bretagne est, de loin, la première région halieutique du pays. À l'échelle européenne, elle représente environ $6 \%$ de la valeur de l'ensemble des débarquements des pêcheries communautaires. Rapporté au contexte régional, le poids économique de cette activité apparaît beaucoup plus modeste: en 2002, l'emploi à la pêche représentait moins de $0,7 \%$ de l'emploi total en Bretagne, contre $5 \%$ dans l'agriculture par exemple. Selon une étude réalisée pour la Commission européenne, en 1997, le taux de dépendance de l'emploi local à l'égard de la filière pêche-aquaculture (incluant les activités amont et aval) était de $2 \%$ à l'échelle de la région, et de 3,4\% à l'échelle des zones d'emploi du littoral breton (Cofrepêche, 1999). Parmi celles-ci, il ne dépassait $5 \%$ que dans la zone d'emploi de Quimper, où il atteignait le niveau record de 8,5\%.

Les données globales qui viennent d'être présentées ne rendent pas compte d'une caractéristique essentielle de la pêche bretonne, qui est sa très grande diversité. Celle-ci peut se décliner selon plusieurs critères.

Le critère le plus immédiat, celui de la taille des navires, est fortement corrélé à celui de la localisation de l'activité. La classe des moins de 12 mètres, numériquement dominante avec un peu plus d'un millier de navires, exerce l'essentiel de son activité dans la bande côtière. Au nombre de 260 environ, les navires de 12 à 20 mètres ont une activité mixte côte-large, alors que les navires de plus de 20 mètres, au nombre de 200 environ, opèrent essentiellement au large.

La diversité de la pêche bretonne est également très importante en matière d'engins utilisés et d'espèces ciblées.

Les engins les plus utilisés sont le chalut et le filet, suivis du casier, de la drague, de la palangre et de la ligne.

Selon les estimations administratives, le chiffre d'affaires 2004 de la pêche professionnelle bretonne se répartissait approximativement ainsi : $24 \%$ pour le thon tropical, $52 \%$

\footnotetext{
${ }^{10}$ Cette section reprend pour l'essentiel le texte d'un article écrit par les auteurs du présent rapport et publié en 2006 dans la revue Bretagne[s] (Boncœur et al., 2006). Sauf exceptions dûment mentionnées, les données utilisées dans cette section sont reprises de Anon, 2006 ; Berthou, 2003 ; Boncœur, 2005 ; Boude, 2005 ; Talidec, 2005. Les données sur les flottilles sont issues du SIH de l'Ifremer.
} 
pour les autres poissons, $14 \%$ pour les crustacés, $10 \%$ pour les mollusques et moins de $1 \%$ pour les algues.

Le regroupement des navires en fonction des stratégies d'activité conduit à segmenter la flotte de pêche en flottilles. On peut distinguer quatre flottilles principales en Bretagne: les chalutiers (exclusifs ou mixtes), les dragueurs (combinant éventuellement la pratique de la drague avec celles d'autres engins, à l'exclusion du chalut), les " dormants » (pratiquant exclusivement les arts dormants tels que filet, casier et ligne) et les senneurs. Les dormants sont les plus nombreux, suivis de près par les chalutiers et, à bonne distance, par les dragueurs.

La diversité de la pêche bretonne a également une dimension géographique. Au-delà des particularismes locaux, on observe des différences importantes entre les façades nord et sud de la région. En 2003, plus de $80 \%$ des navires de Bretagne Nord avaient une longueur inférieure à douze mètres, alors qu'en Bretagne Sud la proportion ne dépassait pas $59 \%$. Les différences concernent également les engins pratiqués et les espèces ciblées. En Manche ce sont la drague et le casier qui dominent, alors qu'en Atlantique ce rôle est tenu par le chalut. Parallèlement, les grands crustacés et les coquillages occupent une place plus importante dans les débarquements des navires de Bretagne Nord que dans ceux de Bretagne Sud, où prédominent les poissons et la langoustine.

La forme juridique des armements est une autre dimension de la diversité de la pêche bretonne. Certes, près de $90 \%$ des navires sont détenus par des entrepreneurs individuels qui en sont en même temps les patrons. Mais la part des sociétés de capitaux prédomine chez les navires de grande taille, de sorte que la moitié de la puissance motrice de la flotte est détenue par ce type d'entreprises. Toutefois, la distinction juridique entre sociétés de capitaux et entreprises individuelles ne recouvre pas la césure traditionnelle (et quelque peu obscurcie aujourd'hui) entre « pêche industrielle » et « pêche artisanale ». En 2003, plus de la moitié des navires armés par des sociétés de capitaux avaient une longueur inférieure à 24 mètres, et plus d'une société sur deux n'armait qu'un seul navire.

\section{Des navires moins nombreux mais plus puissants, un emploi en recul}

Durant les deux décennies qui ont suivi la mise en place du volet « conservation » de la PCP en 1983, le nombre de navires de pêche immatriculés en Bretagne a diminué de plus de moitié. La puissance motrice totale de la flotte n'a diminué que d'un peu moins d'un tiers, ce qui témoigne d'une augmentation de la puissance motrice moyenne par navire d'environ $60 \%$. Deux classes de longueur ont été relativement épargnées par la réduction des effectifs: les navires de 9 à 12 mètres et, surtout, les navires de 20 à 24 mètres. La baisse de la puissance motrice globale ne reflète pas la réalité de l'évolution des capacités de captures, en raison de l'importance du progrès technique au cours de la période (généralisation du GPS, de l'électronique embarquée pour la détection, efficacité accrue des engins de pêche...).

La réduction du nombre de navires s'est accompagnée d'une diminution de l'emploi à la pêche. Après avoir augmenté de $6 \%$ entre 1985 et 1988, le nombre de marins embarqués a reculé en Bretagne de $47 \%$ sur les onze années suivantes. Cette baisse est plus forte que celle de la puissance motrice totale, ce qui témoigne d'un processus de substitution capital-travail. Le recul de l'emploi à la pêche s'est poursuivi pendant les années 20002004 (2,9\% par an en moyenne). Au-delà de la pêche stricto sensu, c'est l'ensemble de la filière régionale pêche-aquaculture qui est affecté par le recul de l'emploi. Selon l'étude 
précitée, entre 1991 et 1997 l'emploi total dans la filière a baissé de $38 \%$ dans les zones d'emploi de Saint-Malo et de Lorient, de $31 \%$ dans celle de Quimper et de $11 \%$ dans celle de Vannes. Quel que soit l'indicateur retenu, le taux de dépendance de l'économie locale vis-à-vis de la filière est partout en recul.

\section{La question du renouvellement de l'outil de production}

La flotte de pêche bretonne vieillit régulièrement depuis une quinzaine d'années et son âge moyen atteignait 22 ans en 2004. Ce phénomène est largement imputable aux politiques publiques. Après avoir stimulé par des aides massives l'entrée en flotte de nouvelles unités techniquement performantes dans les années quatre-vingt (en particulier des chalutiers de 20-25 mètres), ces politiques ont par la suite limité administrativement le renouvellement des capacités, suscitant dans la deuxième moitié des années quatre-vingtdix une envolée des prix des navires d'occasion (+65\%, en moyenne, de 1996 à 2001). Dénoncée par les professionnels de la pêche comme une entrave à l'installation des jeunes, cette hausse a conduit les collectivités territoriales à accorder des subventions à l'acquisition de navires d'occasion (à la fin des années quatre-vingt-dix, plus de la moitié de ces transactions sont subventionnées, le taux de subvention atteignant en moyenne $20 \% \mathrm{du}$ prix de la transaction). Dans ce contexte, le renouvellement de l'outil de production est considéré par les professionnels comme une priorité. Mais celle-ci doit affronter quelques incertitudes majeures et relever plusieurs défis.

Un premier facteur d'inquiétude concerne l'état de la ressource et son évolution future. Selon une analyse portant sur 53 stocks de Manche Ouest et du golfe de Gascogne, 33 stocks se trouvent aujourd'hui dans un état biologique considéré comme «à risques » et 10 dans un état jugé « critique ». La contribution aux débarquements des stocks jugés « en bon état» ne dépasse pas $20 \%$. Les limites imposées par la ressource apparaissent également à travers l'évolution à long terme des débarquements: en ce qui concerne les pêcheries du golfe de Gascogne, on observe sur la période 1973-2002, à la fois une érosion du tonnage total et une diminution de la part relative des espèces « nobles ».

L'évolution du marché constitue une autre source d'incertitude. Même s'il existe en Bretagne une forte consommation de proximité, le marché des produits de la pêche bretonne ne peut être considéré comme régional. Qu'il s'agisse de la pêche industrielle ou de la petite pêche côtière, ces produits sont vendus dans la France entière et, souvent, à l'étranger (en premier lieu en Espagne). En sens inverse, ils subissent les effets d'une concurrence qui, de plus en plus, revêt un caractère mondial et s'élargit aux produits d'aquaculture. L'épisode d'effondrement des prix en 1993-1994 illustre de façon spectaculaire l'impact que peuvent exercer ces facteurs sur le marché français. Aujourd'hui dominé par les grandes et moyennes surfaces, ce marché se présente comme porteur lorsqu'on le considère globalement: la consommation par habitant est passée en France de 27,5 kg en 1990 à 34,4 kg en 2004 (Ofimer, 2005) alors que, dans le même temps, la consommation de viande stagnait. Mais cette progression a bénéficié essentiellement aux producteurs extérieurs, dont le taux de pénétration sur le marché français est passé de $46 \%$ en 1990 à $62 \%$ en 2004. Pour une bonne part, ce phénomène s'explique par l'évolution de la structure de la consommation, vis-à-vis de laquelle l'offre intérieure manifeste une inadaptation croissante. L'évolution du marché français des produits de la mer est en effet très différenciée selon les segments: de 1999 à 2004, les ventes aux particuliers de produits traiteurs réfrigérés ont progressé de $26 \%$ en euros constants, alors que les ventes de poissons frais entiers ont reculé de $27 \%$. 
Les professionnels de la pêche tentent, avec des résultats divers, d'améliorer la valorisation de leurs produits par des opérations d'auto-labellisation. La validation par le marché d'une opération comme la marque « ligneurs de la pointe de Bretagne » résulte non seulement de la qualité intrinsèque du produit, mais aussi de son image de produit d'une pêche sélective et « écologiquement responsable 》. Dans les prochaines années, le marché français pourrait voir se développer des pratiques d'écolabellisation de produits halieutiques, à l'instar de ce qui existe déjà dans d'autres pays développés. L'expérience indique que le succès de ce type de démarche dépend, dans une large mesure, de la notoriété et de la crédibilité de l'organisme qui accorde le label.

Si le marché des produits de la mer est source d'importantes incertitudes, la question des coûts et celle du financement représentent de véritables défis. De janvier 1999 à janvier 2006, le prix du gazole payé par les pêcheurs est passé de 0,11 euros à plus de 0,40 euros par litre. Cette hausse spectaculaire a affecté de façon importante la rentabilité des flottilles chalutières, dont l'intensité énergétique est trois à quatre fois plus élevée que celles des autres flottilles bretonnes. Diverses mesures compensatoires ont été prises par le gouvernement. Cependant, compte tenu des tendances lourdes du marché mondial de l'énergie, il paraît peu probable que la hausse récente du coût du carburant se ramène à une péripétie susceptible d'être lissée par des mesures conjoncturelles. Dans la perspective du renouvellement de la flotte de pêche, il semble au contraire raisonnable d'anticiper un coût du carburant durablement et significativement plus élevé que le niveau moyen enregistré entre le contre-choc pétrolier de 1985 et la fin des années quatre-vingt-dix. Préparer l'avenir en adaptant l'appareil productif à ce contexte constitue sans doute un enjeu majeur. La question n'est pas simplifiée par le fait que les techniques de pêche ayant jusqu'ici le plus symbolisé la modernité sont aussi, généralement, les plus gourmandes en énergie.

Dans le cadre de la réforme de la PCP intervenue en 2002, la décision a été prise de mettre fin aux aides publiques ayant pour effet d'accroître les capacités de capture (ces aides étaient jusqu'ici conditionnées au respect des engagements de réduction de capacité pris par les États membres dans le cadre des programmes d'orientation pluriannuels). Cette décision est difficilement contestable sur le fond, tant du point de vue de l'état des pêcheries communautaires que de celui de l'utilisation de l'argent public. Cependant, la sortie du système des aides publiques n'est pas nécessairement simple, ces aides étant souvent devenues, au fil du temps, un adjuvant obligé des plans de financement des investissements. Dans ces conditions, la tentation peut être forte d'exploiter les failles du nouveau système pour faire perdurer l'ancien sous des formes adaptées, dites « eurocompatibles ». On peut ici rappeler que les navires les plus touchés par la crise de 19931994 étaient généralement issus de la vague de construction subventionnée des années quatre-vingt. Le rapport d'audit commandé par le gouvernement à la suite de cet épisode (rapport Mettling et Hénaff, 1995) avait mis en évidence le caractère souvent hasardeux des plans de financement de ces navires, dans lesquels les aides publiques avaient joué un rôle de levier décisif.

\section{Clarifier le régime des droits d'accès à la ressource}

La réforme de la PCP contribue à faire évoluer le cadre institutionnel dans lequel s'inscrit la pêche bretonne. Il subsiste des interrogations quant à la portée réelle de certaines innovations introduites par cette réforme, par exemple la création des « conseils consultatifs régionaux ». À ces incertitudes communautaires viennent s'ajouter les ambiguïtés 
et les non-dits qui caractérisent, à l'échelle nationale, la régulation des droits individuels d'accès à la ressource.

En ce domaine, le durcissement des mesures de conservation décidé pour répondre à l'état alarmant de nombreux stocks communautaires pourrait jouer le rôle d'un révélateur. Outre les difficultés immédiates qui en résultent pour les professionnels de la pêche, ce durcissement pourrait être le vecteur d'une évolution institutionnelle importante au sein de la pêche française. En ce qui concerne les stocks soumis au régime communautaire des TAC, les quotas attribués chaque année à la France sont eux-mêmes, pour l'essentiel, répartis par l'État entre des organisations de producteurs (OP). Toutefois, jusqu'à une époque récente, ces sous-quotas revêtaient rarement, dans les faits, un caractère contraignant. La situation évolue rapidement sur ce point, ce qui devrait amener les OP à préciser de plus en plus nettement la répartition des sous-quotas qui leur ont été attribués entre leurs adhérents. En ce qui concerne les stocks sous TAC, la logique de l'évolution en cours semble donc pousser au développement d'une gestion par quotas individuels, pour le moment assez étrangère aux habitudes nationales. Alternative aux quotas individuels de capture, les quotas individuels d'effort n'échappent pas plus que ces derniers au problème de l'allocation initiale des droits. Par ailleurs, si le système des quotas de capture pose le problème du contrôle des débarquements, celui des quotas d'effort se heurte à la quasiimpossibilité de contrôler dans la durée l'effort de pêche effectif.

Deux circonstances particulières participent à la complexité du problème. En premier lieu, tous les navires ne sont pas membres d'une OP et la question, jamais clairement réglée jusqu'à présent, de la gestion des droits réservés aux navires hors OP pourrait prendre un tour plus difficile dans le contexte qui vient d'être décrit. En second lieu, l'expérience internationale indique sans ambiguïté que l'individualisation des quotas (de capture ou d'effort) suscite, pour des raisons tout à fait pratiques, l'apparition de mécanismes d'échanges entre détenteurs (supra, section 2). Contrairement à la situation qui prévaut depuis 1987 dans la conchyliculture en matière de transmission des concessions sur le domaine public maritime (sous un habillage formel destiné à sauver les apparences), en pêche de tels échanges de droits à produire sont prohibés par la loi française. Dans les faits cependant, l'analyse économétrique évoquée précédemment (Guyader et al., 2006) a clairement établi que les prix des navires sur le marché de l'occasion intègrent implicitement une valeur en relation avec les droits de pêche. Utilisant les navires comme supports, cette transférabilité implicite ne contribue guère à la transparence du marché des droits, et peut difficilement être considérée comme un mécanisme recommandable en termes d'efficacité aussi bien que d'équité.

\section{La gestion de la pêche dans la bande côtière}

Le régime communautaire des TAC ne couvre qu'une partie des stocks exploités par la pêche bretonne (en Bretagne Nord, cette partie correspond à moins de $20 \%$ du total des débarquements). Pour la plupart, les stocks qui ont un caractère côtier échappent à ce régime. Objet d'un atelier international organisé à Brest en janvier 2007 dans le cadre de ce projet de recherche (Anon., 2006), la gestion des activités de pêche dans la bande côtière présente une grande importance pour l'avenir de la pêche bretonne: près des troisquarts de la flotte régionale exercent l'essentiel de leur activité dans cette zone, et la valeur ajoutée que génèrent ces navires approche la moitié de celle de l'ensemble des navires 
de moins de 24 mètres immatriculés en Bretagne. C'est aussi une question particulièrement compliquée, car la bande côtière est un lieu de multiples interactions entre usages de l'espace et des ressources. Sans doute faut-il voir là une raison majeure pour laquelle la réforme de la PCP en 2002 a renforcé le régime particulier de cette zone. Ce régime accorde à l'État riverain une grande latitude pour gérer les activités halieutiques dans ses eaux intérieures et territoriales. Dans le cas de la France, cette gestion s'exerce essentiellement au niveau régional. En pratique, c'est le Comité régional des pêches maritimes et des élevages marins (CRPMEM) qui, sur la base de la loi du 2 mai 1991, prend la plupart des mesures de gestion concernant la pêche côtière, sous la forme de « délibérations » auxquelles le préfet de région confère un caractère obligatoire.

Le CRPMEM de Bretagne a, dans ce cadre, développé depuis une quinzaine d'années une véritable politique de gestion de la pêche dans la bande côtière bretonne, reposant pour l'essentiel sur l'instrument cadre qu'est la licence de pêche. Il existe aujourd'hui en Bretagne une cinquantaine de licences différentes, relevant de la catégorie " ressource » ou de la catégorie « métier » et couvrant des espaces de plus en plus étendus aux limites de la mer territoriale. La politique de licences du CRPMEM de Bretagne vise à conserver les ressources, mais aussi à réserver l'accès aux ressources de la bande côtière à des navires de taille et de puissance limitées, à répartir l'espace et à prévenir les conflits de pêche. Innovante en France, cette politique porte à son actif un certain nombre de réalisations significatives. Elle ne peut cependant rester figée sur ses acquis. Évoquée plus haut dans le contexte des stocks soumis au régime communautaire des TAC, la question de la clarification du régime des droits d'accès à la ressource se pose aussi pour les stocks à caractère côtier qui échappent à ce régime. Les mécanismes de régulation de l'accès mis en place par le CRPMEM n'apportent pour le moment qu'une réponse incomplète à cette question, ce dont témoigne le fait que les licences, réputées annuelles et non-transférables, font en réalité l'objet de transferts marchands en même temps que les navires auxquels elles sont attachées. Une autre question qu'auront à résoudre les professionnels de la pêche bretonne est celle de la relation existant entre les droits de pêche gérés par le CRPM (licences) et ceux que gèrent les OP (quotas). L'évolution en cours indique en effet que la zone d'interférence est en voie d'élargissement. 


\section{Chapitre 2}

\section{Production de connaissances à l'échelle régionale}

\section{Les espèces majeures de la bande côtière bretonne}

Répartition des stocks

Les ressources halieutiques décrites ici, du point de vue de leur état d'exploitation, sont celles du golfe de Gascogne et de la Manche Ouest, qui correspondent respectivement aux zones du CIEM ${ }^{11}$ VIII $(a, b)$ et VIIe (figure 1).

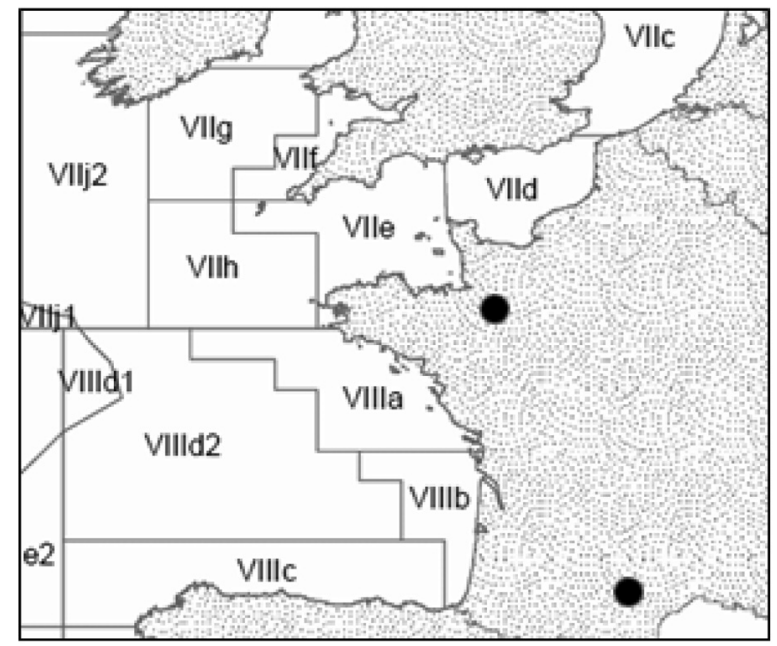

Figure 1. Carte des divisions CIEM.

${ }_{11}$ Conseil international pour l'exploration de la mer. 
Bien que $75 \%$ de l'activité de pêche française se situe dans la bande des 12 milles, les espèces exploitées par les flottilles bretonnes ne sont pas toutes inféodées à cette zone. Les aires de répartition de nombreux stocks ${ }^{12}$ peuvent en effet être très vastes : le stock dit « nord » du merlu par exemple a une aire de distribution qui s'étend du sud du golfe de Gascogne jusqu'au nord de l'Écosse.

Les distributions des principales espèces exploitées sont schématisées dans le tableau 1. Les pavés gris clair indiquent une moindre abondance dans la zone correspondante. Certains stocks ont une aire de répartition côtière dans les estuaires et à l'intérieur des 12 milles (bar, araignée, seiche, crevettes...), cependant, la majorité des espèces ont des aires de distribution qui dépassent les eaux territoriales (tableau 1).

Tableau 1. Distribution des stocks (Source: A. Forest, Atelier Amure, 2006)

\begin{tabular}{|l|l|l|l|l|}
\hline \multicolumn{1}{|c|}{ Espèces } & $\begin{array}{c}\text { Baies et } \\
\text { estuaires }\end{array}$ & $\begin{array}{c}\text { Douze } \\
\text { milles }\end{array}$ & $\begin{array}{c}\text { Au } \\
\text { large }\end{array}$ & $\begin{array}{c}\text { Hors zones } \\
\text { VII VIII }\end{array}$ \\
\hline Anguille & & & & \\
\hline Anchois & & & & \\
\hline Maquereau & & & & \\
\hline Chinchard & & & & \\
\hline Merlan & & & & \\
\hline Merlu & & & & \\
\hline Lieu jaune & & & & \\
\hline Tacaud & & & & \\
\hline Bar & & & & \\
\hline Baudroies & & & & \\
\hline Sole & & & & \\
\hline Cardine & & & & \\
\hline Langoustine & & & & \\
\hline Congre & & & & \\
\hline Sardine & & & & \\
\hline Rouget barbet & & & & \\
\hline Céteau & & & & \\
\hline Divers amphihalins & & & & \\
\hline Coquille Saint-Jacques & & & & \\
\hline Autres bivalves & & & & \\
\hline Ormeau, Buccin & & & & \\
\hline Seiche & & & & \\
\hline Encornets & & & & \\
\hline Crevettes & & & & \\
\hline Araignée & & & & \\
\hline Tourteau & & & & \\
\hline Algues & & & & \\
\hline & & & & \\
\hline & & & & \\
\hline
\end{tabular}

\footnotetext{
${ }^{12}$ Fraction exploitée d'une population.
} 
Ainsi, la distribution des stocks a pour conséquence de nombreuses interactions entre les flottilles qui doivent être prises en compte par la gestion de la zone côtière:

- compétition pour les ressources et les espaces;

- exploitations séquentielles: il s'agit de l'exploitation des différents stades de vie; par exemple les juvéniles de merlus sont capturés par les chalutiers côtiers de la grande vasière et les individus adultes par les fileyeurs du large;

- reports d'effort entre zones;

- interactions: côte/large, golfe de Gascogne/hors golfe, Manche Ouest/hors Manche Ouest.

Les débarquements français provenant du golfe de Gascogne sont d'environ 100000 tonnes, dont la majeure partie est pêchée dans la partie nord (8a), et ceux de la Manche Ouest (hors algues) sont d'environ 60000 tonnes (figures 2 et 3).

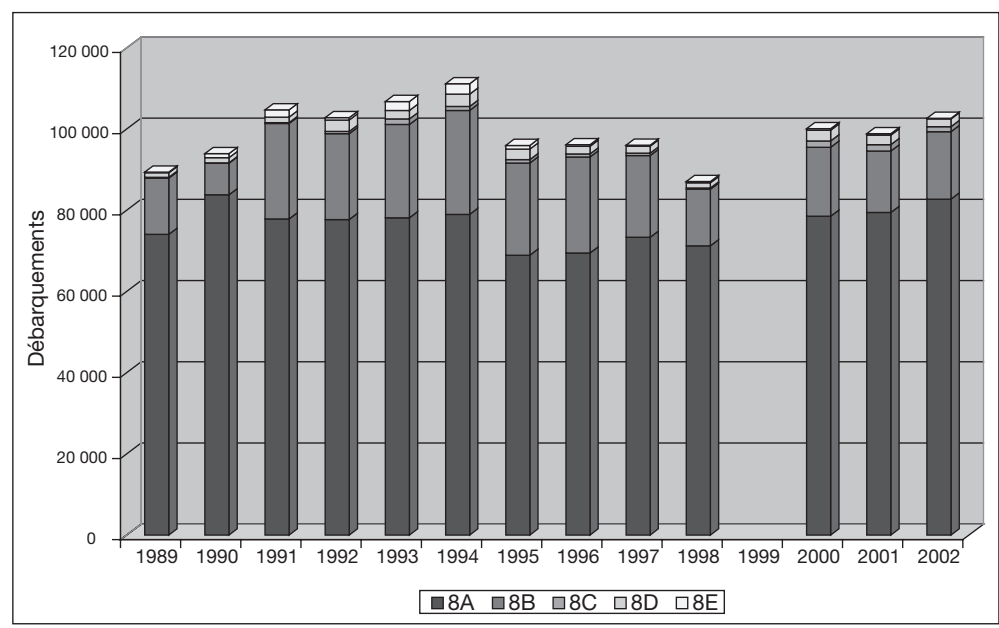

Figure 2. Débarquements français officiels (tonnes) provenant du golfe de Gascogne (source : DPMA).

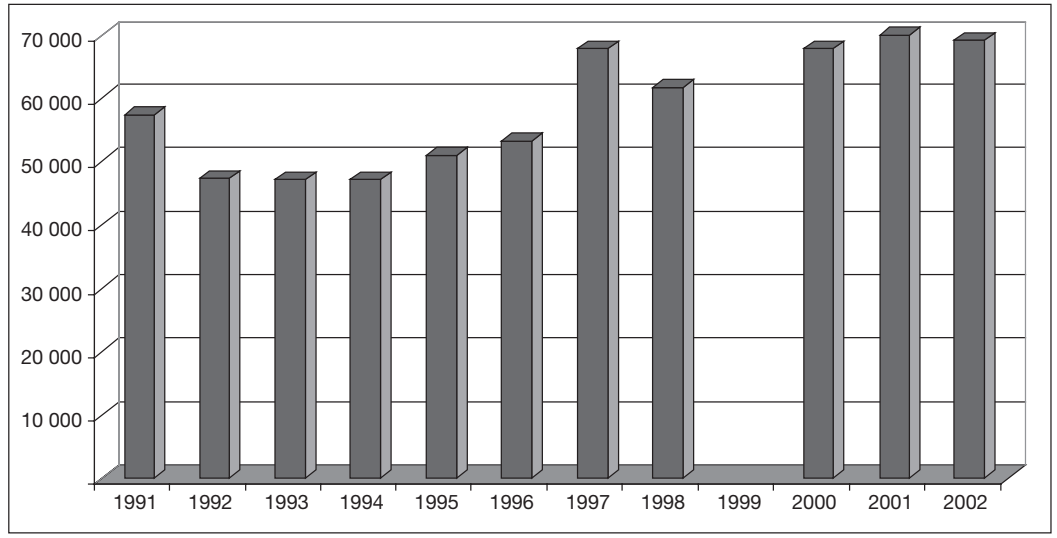

Figure 3. Débarquements français officiels (tonnes) provenant de la Manche Ouest (source : DPMA). 


\section{Fiabilité des diagnostics}

Le niveau de connaissance de l'état d'exploitation des ressources est très dépendant du système de gestion en vigueur: les stocks gérés au niveau communautaire soumis à $\mathrm{TAC}^{13}$ font chaque année l'objet d'évaluations menées dans le cadre international du CIEM pour répondre aux questions de la Commission européenne. En conséquence, ces stocks sont suivis de façon détaillée à travers notamment de séries chronologiques des structures démographiques des captures. Par ailleurs, de nombreuses ressources sédentaires comme les coquillages, dont l'exploitation est encadrée par des licences et pour lesquels des niveaux de prélèvements sont recommandés, font également l'objet d'estimations de biomasse et de suivis réguliers: on peut citer les gisements de coquilles Saint-Jacques, de palourdes, etc.

A contrario, les connaissances de l'état d'exploitation de nombreuses ressources peuvent être assez limitées, souvent en raison de l'absence d'encadrement de l'exploitation, ce qui a pour conséquence un suivi moins précis.

Ainsi les moyens scientifiques sont prioritairement alloués aux stocks pour lesquels des avis sont attendus chaque année, soit de la commission européenne, soit de commissions locales ou régionales dépendant des structures professionnelles $\left(\mathrm{CLPMEM}^{14}\right.$, CRPMEM).

La situation est contrastée, en matière d'encadrement de l'exploitation entre le golfe de Gascogne, où environ $50 \%$ des débarquements concernent des stocks soumis à TAC (figure 4), et la Manche Ouest où cette proportion est d'environ $15 \%$ (figure 5).

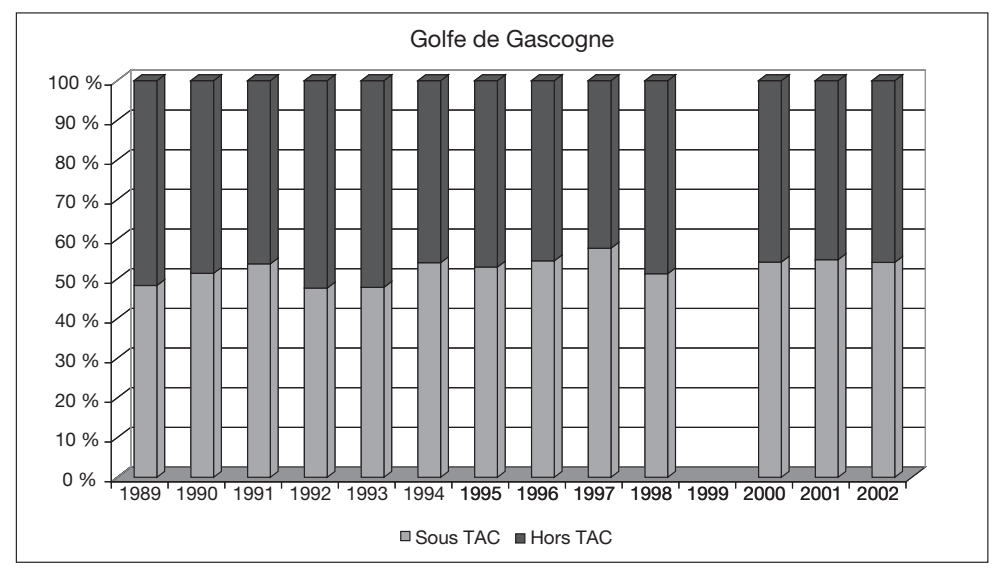

Figure 4. Proportions des débarquements sous TAC et hors TAC provenant du golfe de Gascogne (source : A. Forest, Atelier Amure, 2006).

\footnotetext{
13 Total autorisé de captures.

${ }^{14}$ Comité local (régional) des pêches maritimes et des élevages marins.
} 


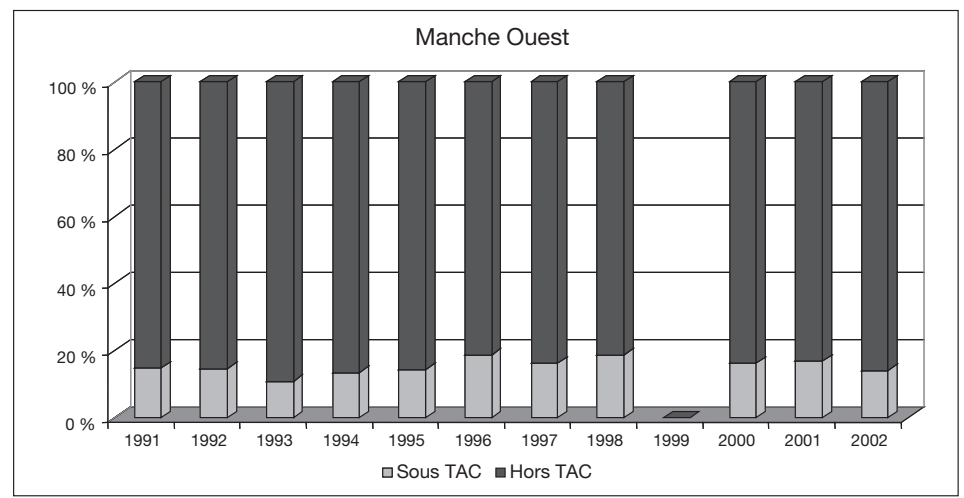

Figure 5. Proportions des débarquements sous TAC et hors TAC provenant de la Manche Ouest (source : A. Forest, Atelier Amure, 2006).

Ainsi, sur 53 stocks pris en compte, listés dans le tableau 2, la fiabilité du diagnostic est jugée (tableau 2 et figure 6 , page I):

- bonne pour 18 d'entre eux (34\%);

- moyenne pour $20(38 \%)$;

- faible pour $15(28 \%)$.

Tableau 2. Les stocks pris en compte (ceux entre parenthèses ne concernent pas la bande côtière).

\begin{tabular}{|l|l|}
\hline \multicolumn{2}{|c|}{ Stocks pris en compte } \\
\hline Gérés par TAC & Sans TAC associé \\
\hline Langoustine & Seiche \\
\hline Anchois & Calmars \\
\hline Maquereau & Coquille Saint-Jacques \\
\hline Chinchard & Homard \\
\hline (Merlan bleu) & Tourteau \\
\hline Germon & Araignée \\
\hline Dorade rose & Sardine \\
\hline Lieu jaune & Dorade grise \\
\hline Merlan & Requin taupe \\
\hline Merlu & Petite roussette \\
\hline (Morue) & Raie bouclée \\
\hline (Empereur) & Raie fleurie \\
\hline Baudroie blanche & Anguille \\
\hline Baudroie noire & Bar \\
\hline Sole & Grondin rouge \\
\hline Cardine & Rouget barbet \\
\hline Plie & Tacaud \\
\hline & \\
\hline
\end{tabular}




\section{État des stocks}

Sur les 53 stocks dont les espèces sont listées dans le tableau 2, 10 sont en bon état (ex. sardine du golfe de Gascogne, tourteau, bar, calmars, seiche...), 10 sont dans un état critique (ex. daurade rose, anguille, sole, plie, maquereau...). Les 33 autres sont dans une situation intermédiaire ou à risques: leur biomasse peut être faible (anchois, merlu, langoustine...) ou leur taux d'exploitation trop élevé, caractéristiques de conditions d'exploitation non optimales (figure 7 p. I).

Dans le golfe de Gascogne, $22 \%$ des captures proviennent de stocks en bon état, $62 \%$ de stocks en situation intermédiaire ou à risques, $16 \%$ de stocks en situation critique. En Manche Ouest, ces valeurs sont respectivement de $18 \%$, $79 \%$ et $3 \%$ (figure 8 p. I).

Les états des stocks sont qualifiés par rapport à des seuils de précaution, en matière de biomasse et de pression de pêche, c'est-à-dire des limites à ne pas franchir pour éviter des risques biologiques d'exploitation.

On définit des valeurs de biomasse limite et de précaution:

$\operatorname{Blim}^{15}$ : valeur de la biomasse de géniteurs au-dessous de laquelle la dynamique du stock n'est pas connue où le risque d'effondrement est très grand. Ainsi un stock dont la biomasse de géniteurs est inférieure à Blim a une capacité reproductrice réduite.

$\mathrm{Bpa}^{16}$ : valeur de la biomasse de géniteurs en dessous de laquelle il ne faut pas tomber afin que, compte tenu des incertitudes sur le diagnostic, il existe de bonnes chances de ne pas tomber au-dessous de Blim. Un stock dont la biomasse de géniteurs se situe au-dessus de Bpa a une bonne capacité reproductrice.

Si la biomasse de géniteurs d'un stock se trouve entre les deux valeurs Blim et Bpa, sa capacité reproductrice risque de se réduire.

Parallèlement, on définit des seuils en matière de mortalité par pêche, grandeur qui correspond à la proportion de poissons qui meurent chaque année du fait de la pêche:

Flim: mortalité par pêche limite, au-delà de laquelle il y a une très forte probabilité que le stock soit réduit et ne puisse pas assurer une exploitation durable. Un stock dont la mortalité par pêche est supérieure à Flim n'est pas exploité de manière durable.

Fpa: mortalité par pêche qu'il ne faut pas dépasser pour, en prenant en compte les diverses incertitudes, éviter tout risque de dépasser Flim. Si la mortalité par pêche d'un stock se situe en dessous de Fpa, l'exploitation de ce stock peut être qualifiée de durable.

Si la mortalité par pêche d'un stock se situe entre les deux valeurs Fpa et Flim, il y a un risque que l'exploitation du stock ne soit pas durable.

Sur la figure $9 \mathrm{p}$. II, les stocks sont placés selon leur situation par rapport aux seuils de précaution de mortalité par pêche et de biomasse de géniteurs. Les stocks situés dans la partie verte sont dans une situation d'exploitation satisfaisante, ceux dans la partie orange sont dans une situation à risque, ceux dans la partie rouge sont dans une situation critique.

Les scénarios de gestion des stocks doivent non seulement éviter que les seuils limites soient atteints, mais surtout doivent viser à rétablir les niveaux de biomasse de reproducteurs qui permettent une production maximale durable, dans une logique de développement durable en conformité avec les engagements des états pris au sommet mondial du développement durable de Johannesbourg en 2002.

\footnotetext{
15 Blim pour biomasse limite.

${ }^{16}$ Bpa pour biomasse de l'approche de précaution (Precautionary Approach).
} 
La figure 10 p. II indique que les stocks dont la situation par rapport aux points de précaution a été décrite sur la figure $9 \mathrm{p}$. II sont tous exploités au-delà du maximum de production durable, c'est-à-dire qu'ils sont dans une situation où toute augmentation d'exploitation conduira à une baisse de production globale.

Plus ils sont situés en haut à gauche, plus ils sont éloignés de l'optimum biologique: la mortalité par pêche qu'ils subissent est très au-delà de celle qui permet à une production maximale, par conséquent le gain de biomasse des reproducteurs à attendre d'une réduction de mortalité par pêche ramenée au niveau optimal est important.

Exemple: la mortalité par pêche subie par le merlu est d'environ $50 \%$ plus élevée que celle qui permettrait une production maximale, laquelle serait de $30 \%$ supérieure au niveau actuel.

\section{Focus sur les stocks intéressant particulièrement la pêche côtière bretonne}

\section{La sole (Solea vulgaris) de Manche Ouest}

Ce stock est situé sur la figure 9 parmi les stocks en situation critique. La figure 11 p. III montre clairement que la mortalité par pêche est très supérieure à Flim et que la biomasse des reproducteurs est inférieure à Bpa et proche de Blim. La recommandation de gestion du CIEM est de restaurer la biomasse des reproducteurs.

\section{Les baudroies de mer Celtique et du golfe de Gascogne}

- Baudroie blanche (Lophius piscatorius)

Le CIEM n'a pas émis de diagnostic sur ce stock en 2007 en raison des trop fortes incertitudes relatives aux débarquements déclarés, aux rejets en mer, et aux connaissances sur la croissance.

Les campagnes scientifiques de chalutage montrent que la biomasse augmente depuis 2000 et que les recrutements 2001, 2002 et 2004 ont été au-dessus de la moyenne (figure 12). La situation de ce stock est jugée stable. Il faut toutefois noter que les captures d'immatures sont importantes, et que leur réduction serait favorable à l'évolution du stock.

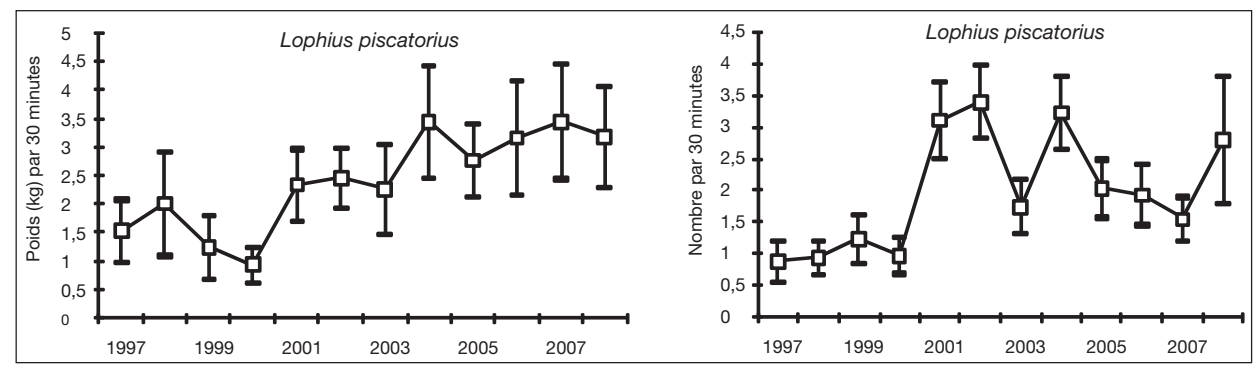

Figure 12. Indices d'abondance du stock de baudroies blanches de la mer Celtique et du golfe de Gascogne obtenus par les campagnes scientifiques (source : CIEM, 2007).

- Baudroie noire (Lophius budegassa)

Le CIEMn'a pas émis de diagnostic sur ce stock en 2007 en raison des trop fortes incertitudes relatives aux débarquements déclarés, aux rejets en mer, et aux connaissances sur la croissance.

Les campagnes scientifiques de chalutage montrent que la biomasse est stable et que les recrutements récents (2004-2006) ont été au-dessus de la moyenne (figure 13). La situation de ce stock est jugée stable. 


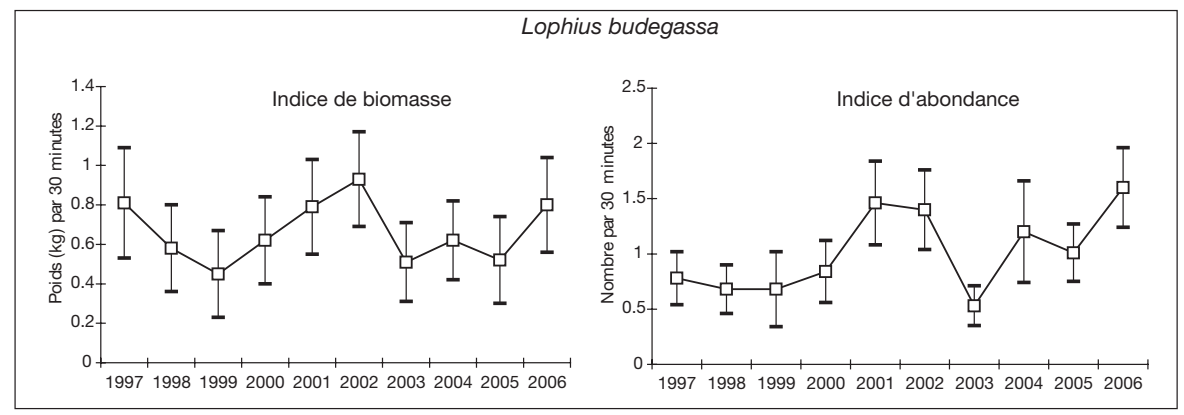

Figure 13. Indices d'abondance du stock de baudroies noires de la mer Celtique et du golfe de Gascogne obtenus par les campagnes scientifiques (source : CIEM, 2007).

Comme pour la baudroie blanche, on observe de fortes quantités d'immatures dans les captures. Leur préservation contribuerait à accroître la taille du stock dans les années à venir, suite aux bons recrutements récemment observés.

\section{La langoustine (Nephrops norvegicus) du golfe de Gascogne}

Il n'y a pas de point de référence biologique pour ce stock. La biomasse des reproducteurs est en augmentation, la mortalité par pêche est en légère baisse (figure 14). Les recrutements 2004 et 2005 ont été très bons. De très nombreuses captures non débarquées caractérisent l'exploitation de ce stock: les observations à bord des navires de pêche indiquent que $50 \%$ des captures sont rejetées, voire plus certaines années. La principale recommandation de gestion porte sur l'augmentation de la taille de première capture, qui peut être augmentée par un dispositif sélectif sur les chaluts.

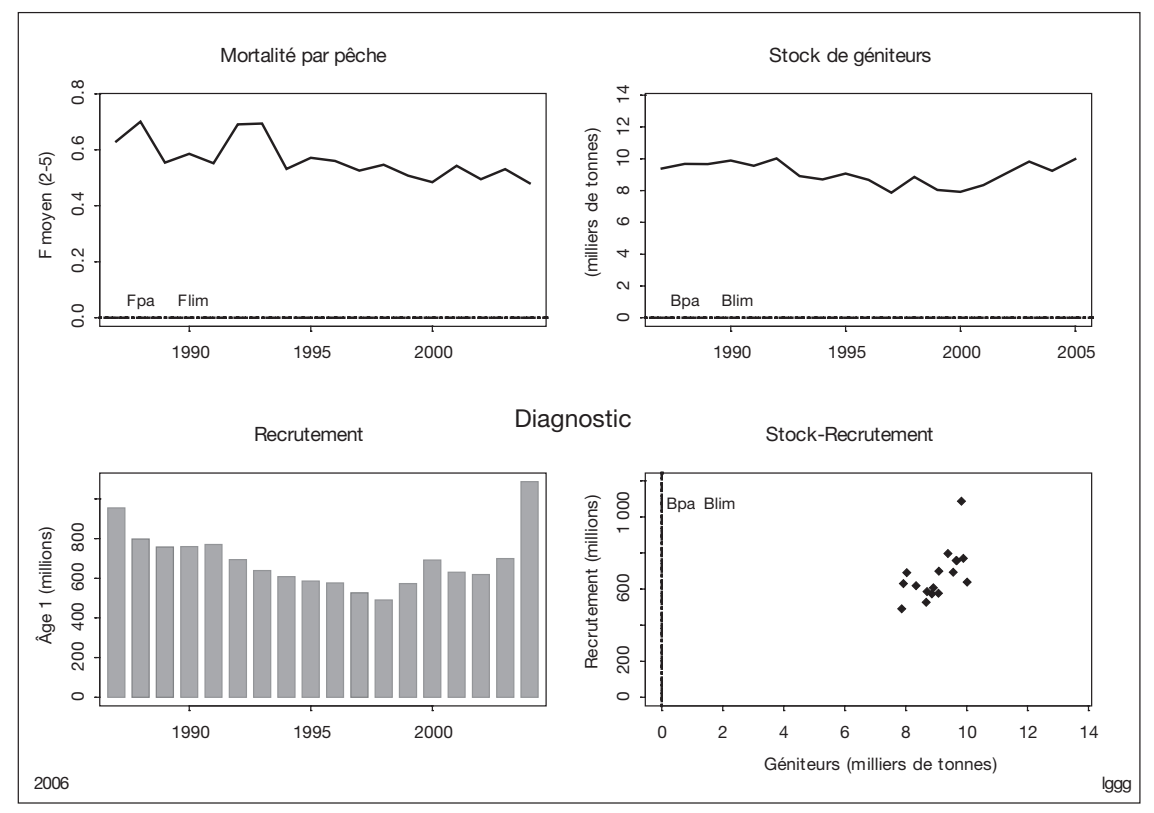

Figure 14. Indicateurs de l'état d'exploitation du stock de langoustines du golfe de Gascogne (source : CIEM, 2006). 


\section{Le merlu (Merluccius merluccius)}

Ce stock montre des signes de rétablissement après avoir été dans une situation à risque au cours des années quatre-vingt-dix. La mortalité par pêche a beaucoup baissé, ce qui a eu pour conséquence logique une augmentation de la biomasse (figure 15 p. III).

Les connaissances biologiques sur ce stock, en particulier la croissance, sont en cours de révision, suite à un programme de marquage qui a révélé une croissance deux fois plus rapide que celle estimée d'après les interprétations (erronées) des coupes d'otolithes. Les conséquences des incertitudes sur la croissance ne remettent pas en cause les tendances observées, elles ont cependant un impact sur les valeurs absolues des indicateurs (biomasse, mortalité par pêche etc.)

En termes de recommandation de gestion, il convient de maintenir la mortalité par pêche en dessous de Fpa, et d'éviter les captures de juvéniles de merlu qui ont un impact négatif sur le potentiel productif du stock et donc sur la durabilité de l'exploitation.

\section{La sole (Solea vulgaris) du golfe de Gascogne}

Ce stock est en dehors des limites de sécurité biologique: la mortalité par pêche est supérieure à Fpa et la biomasse des reproducteurs est inférieure à Bpa depuis 1999 (figure 16, p. IV). La capacité reproductrice est donc susceptible de se réduire. De plus les niveaux de recrutements récents sont plutôt bas. L'avis scientifique en terme de gestion préconise une réduction de la mortalité par pêche afin de restaurer la quantité de reproducteurs, puis d'évoluer vers une situation de production maximale. La situation de la pêcherie justifie un encadrement renforcé, en plus du TAC.

\section{La Coquille Saint-Jacques (Pecten maximus) de la baie de Saint-Brieuc}

Cette ressource fait l'objet d'évaluations annuelles directes au moyen d'un navire océanographique de l'Ifremer depuis une vingtaine d'années. Différents indicateurs sont obtenus: - la biomasse adulte constituée de coquilles de 2 ans et plus;

- la biomasse exploitable (coquilles de taille supérieure ou égale à $102 \mathrm{~mm}$ ) ;

- le recrutement (coquilles de deux ans) et le pré-recrutement (coquilles d'un an);

- la caractérisation de la croissance individuelle annuelle.

L'évaluation réalisée en 2007 est résumée ci-après.

Les travaux menés lors de la campagne d'évaluation ont consisté à réaliser des coups de drague sur 115 stations d'échantillonnage sur des distances constantes de 200 mètres à l'aide d'une drague expérimentale de 2 mètres d'ouverture, équipée de dents de $8,5 \mathrm{~cm}$ et d'un sac de maillage $50 \mathrm{~mm}$ (figure $17 \mathrm{p}$. IV). L'efficacité de l'engin de pêche est connue car elle a été étalonnée par des plongées sous-marines sur la trace de la drague pendant une longue période afin de permettre l'estimation de la structure réelle de la population à partir des captures expérimentales. Toutes les coquilles récoltées ont été âgées et mesurées.

Avant le démarrage de la saison de pêche 2007-2008, on peut considérer la situation du gisement coquillier comme une des meilleures enregistrées depuis trente ans en raison de la succession de bonnes reproductions entre 1998 et 2003. À titre indicatif, les biomasses adulte et exploitable actuelles sont 7 à 8 fois supérieures aux valeurs respectives de la fin des années quatre-vingt quand le stock s'est trouvé à son niveau le plus bas. Cependant, la diminution significative du reliquat de pêche entre 2006 et 2007 témoigne d'une pression de pêche élevée. En outre, la faible abondance de la classe née en 2004 a un impact 
négatif sur l'abondance globale et ce déficit sera encore plus perceptible dès que les plus anciennes classes ne contribueront plus au potentiel du stock.

La saison 2006-2007 a été marquée par une pression de pêche très élevée comme lors des deux saisons de pêche précédentes : ouverture la plus longue depuis trente ans accompagnée d'une augmentation continue de la capacité individuelle de capture, avec un dépassement de $15 \%$ du quota préconisé par l'Ifremer. On s'attend, de ce fait, à une réduction de la biomasse du stock malgré la forte abondance des animaux nés en 2005, par ailleurs caractérisés par une croissance individuelle rapide. Cependant, le nombre de classes d'âge participant significativement au potentiel du stock diminue. On peut s'attendre à terme à un stock plus fragile, constitué d'une seule classe majeure (celle de 2005), situation non observée depuis de nombreuses années.

Les ressources naturelles sont par nature fluctuantes et on ne peut exclure qu'une succession de reproductions induisant des abondances inférieures à la moyenne ne conduise à une période de récession relative. La pauvreté de la classe née en 2004 (qui risque d'être suivie par une classe squelettique issue de la reproduction 2007 compte tenu du profil thermique anormalement bas de l'été 2007) rappelle le rôle essentiel du facteur climatique dans les variations du recrutement et souligne la nécessité d'étaler dans le temps le prélèvement sur les « bonnes » classes d'âge pour amortir les inévitables « années creuses » en terme de recrutement.

Un meilleur encadrement de la production a été progressivement réalisé au sein de la pêcherie à travers l'évolution des dispositifs réglementaires et en particulier l'augmentation du maillage, de 72 à 85 (en 1985) puis à 92 mm (en 1996), ce qui a contribué à accroître le reliquat de pêche pour la saison de pêche suivante. Depuis longtemps, le problème de sélectivité a été globalement résolu sur ce gisement.

En 1990, face à l'appauvrissement alarmant du stock, les structures de gestion ont décidé d'appliquer de nouvelles mesures de limitation des puissances motrices des navires accédant à la ressource. Il convient toutefois de noter que l'amélioration de la capacité individuelle de capture ne tient plus tellement à la puissance motrice nominale: à puissance motrice donnée et à état de ressource constant, le navire «moyen » de la baie pêcherait aujourd'hui 40 à $45 \%$ de plus qu'il y a quinze ans. Ce fait explique en grande partie les difficultés actuelles d'ajustement de l'effort de pêche au prélèvement journalier et global souhaitable.

\section{Le bar (Dicentrarchus labrax)}

Le bar est exploité surtout en Manche et dans le golfe de Gascogne par divers engins (chalut pélagique, chalut de fond, filets calés, lignes et palangres). Il est aussi exploité par les pêches de loisir (du bord, en bateau; fusil sous-marin, traînes, lignes, palangres, filets). Les prélèvements de la pêche récréative ont été récemment évalués en France pour les années 2003 et 2006 par sondage téléphonique. Les prélèvements des pêcheurs amateurs seraient de l'ordre de 5000 tonnes, ce qui correspond à la production de la pêche professionnelle française.

Plusieurs populations peuvent être distinguées. Le CIEM considère qu'il existe plusieurs stocks de bar. Des considérations qui reposent sur des études de marquage, sur une analyse des variations observées dans le recrutement et sur les variations spatiotemporelles des indices d'abondance permettent d'avancer l'hypothèse de 6 stocks. La délimitation de ces stocks est telle qu'ils se chevauchent. Ainsi, en Manche occidentale, 3 stocks se mélangeraient en saison hivernale. 
En 2004, le CIEM $^{17}$ a réalisé des évaluations sur les composantes de Manche-mer Celtique. Il ressort que ces stocks sont exploités à un niveau proche du rendement maximal durable, mais la taille moyenne de capture est un peu basse, sans cependant altérer la capacité de reproduction. En effet, le CIEM souligne aussi que le réchauffement des eaux en Manche et en mer du Nord crée depuis 1989 des conditions environnementales favorables à la croissance et au recrutement du bar. La biomasse adulte est stable en mer Celtique, voire en augmentation en Manche et mer du Nord. Il n'existe pas suffisamment d'informations pour procéder à des évaluations similaires dans le golfe de Gascogne. Toutefois les indices d'abondance obtenus à partir des campagnes océanographiques mettent en évidence une situation stable dans le golfe de Gascogne.

\section{Le crabe tourteau (Cancer pagurus)}

Le tourteau est une espèce dont l'exploitation est conséquente puisque les débarquements annuels en France varient entre 6000 et 8000 tonnes.

Le principal engin de pêche du tourteau est le casier. D'autres engins, comme le filet ou le chalut, permettent sa capture mais les rendements sont beaucoup plus faibles, le tourteau est alors une prise accessoire.

Les débarquements français sont réalisés à plus de $75 \%$ par la flottille des caseyeurs exclusifs et par la flottille des caseyeurs-fileyeurs. Pour la première flottille, on peut distinguer la sous-flottille des navires de plus de 18 mètres ciblant exclusivement le tourteau et qui, aujourd'hui, débarque près de $40 \%$ de la production nationale. Le reste de cette flottille (composée de navires de plus faible taille) est constitué de navires qui ne ciblent pas le tourteau, ou uniquement au cours d'une saison, le homard et l'araignée étant les autres espèces ciblées. Pour la flottille des caseyeurs-fileyeurs, le tourteau peut être une espèce cible lorsque l'activité principale du navire au cours d'une saison est le casier, l'activité de fileyage n'entraînant qu'une capture accessoire du tourteau.

Le reste des débarquements en tourteaux est réalisé par la flottille des fileyeurs exclusifs à hauteur de $15 \%$ de la production nationale et par la flottille des chalutiers exclusifs pour moins de $10 \%$.

\section{Évolution de la production nationale}

Le tourteau est pêché sur l'ensemble du littoral de la Manche et de l'Atlantique. Néanmoins, certains secteurs sont exploités plus que d'autres en raison des différences d'abondance. On distingue deux périodes dans les débarquements (figure $18 \mathrm{p}$. V):

- de 1984 à 1992 on observe une diminution des débarquements de près de 8000 tonnes à 5500 tonnes ;

- ensuite les débarquements augmentent légèrement et se stabilisent autour de 6500 tonnes. L'année 2004 est caractérisée par des débarquements nettement plus importants avec près de 7800 tonnes.

Cette évolution des débarquements est consécutive à des changements dans les flottilles qui débarquent le tourteau. Ainsi, la diminution des débarquements jusqu'en 1992 s'explique par un nombre décroissant de navires de moins de 15 mètres dans la flottille des caseyeurs. Cette diminution est par la suite en partie compensée par deux facteurs:

17 http://www.ices.dk/committe/acfm/comwork/report/2004/oct/seabass.pdf 
- l'augmentation du nombre de fileyeurs qui débarquent du tourteau en prise accessoire. Selon l'espèce cible (notamment la lotte), les débarquements de tourteaux ne sont pas négligeables;

- l'augmentation des débarquements de la sous-flottille des caseyeurs hauturiers.

On peut mesurer l'importance de la sous-flottille des caseyeurs hauturiers dans l'exploitation de l'espèce puisque depuis 1984, ces derniers débarquent au minimum $35 \%$ des apports totaux et certaines années ce chiffre avoisine les $50 \%$.

\section{État du stock}

Les particularités de la biologie des crustacés entraînent des difficultés dans l'évaluation de l'état des stocks par les méthodes habituellement utilisées. Le tourteau ne déroge pas à cette règle, aussi le travail mené jusqu'à présent a consisté à un suivi de son indice d'abondance par secteur. Ce suivi se base sur les données de la sous-flottille des caseyeurs hauturiers.

L'estimation de cet indice est effectuée par une modélisation de type GLM des captures par unité d'effort (CPUE). Cette approche permet de tenir compte de multiples paramètres qui peuvent influencer l'indice d'abondance. Les estimations qui ont été réalisées permettent de mettre en évidence que globalement (figure 19 p. V) on n'observe pas de tendance de l'indice d'abondance du tourteau. Néanmoins, des variations annuelles sont notées. L'indice d'abondance de la Manche Ouest se situe autour de $1500 \mathrm{~kg}$ pour 1000 casiers et celui du golfe de Gascogne autour de $2000 \mathrm{~kg}$. Par ailleurs, on observe que l'évolution des indices de la Manche Ouest et du golfe Gascogne est synchrone ce qui traduirait l'existence d'un même stock sur l'ensemble de ces zones (figure 20).

L'ensemble de ces éléments laisse présager d'un état satisfaisant du stock de tourteaux. Ce diagnostic doit être étayé par des travaux en cours de réalisation suite à l'application de nouveaux modèles d'évaluation.

Le tourteau est une espèce dont le stock ne semble pas susciter d'inquiétude particulière. Néanmoins, il est nécessaire d'améliorer les approches de suivi afin de limiter au maximum les incertitudes qui subsistent sur l'état de ce stock.

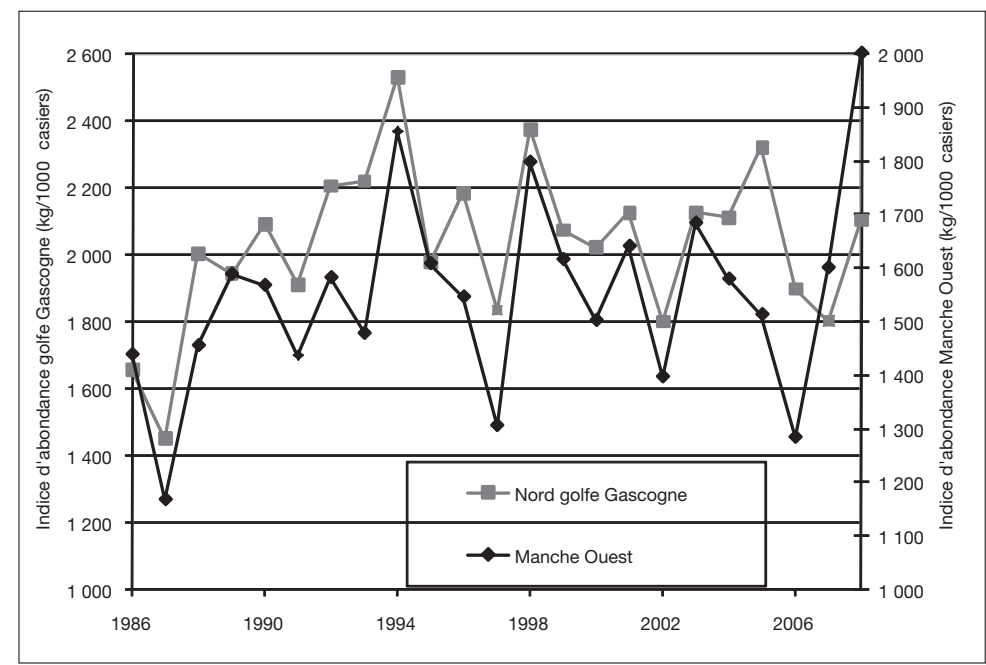

Figure 20. Représentation en double axe de l'indice d'abondance (kg pour 1000 casiers) de tourteaux pour la zone de la Manche Ouest et du golfe de Gascogne. 
Globalement le mode d'exploitation du tourteau est satisfaisant. En effet, le casier étant le principal engin de capture, ce mode de pêche assure une sélectivité complète, élément très positif pour le stock. Néanmoins, les autres modes de pêche entraînent une mortalité importante des rejets.

\section{La palourde (Ruditapes phillipinarum) du golfe du Morbihan}

Depuis 1997, le gisement de palourdes japonaises (Ruditapes phillipinarum) du golfe du Morbihan fait l'objet d'une évaluation directe annuelle. Il n'existe pas de point de référence biologique pour ce stock. La biomasse estimée fluctue environ d'un facteur 2 entre les années extrêmes soit entre 870 et 1800 tonnes, ce qui correspond à des effectifs de 150 à 250 millions de palourdes (figure 21). Ce stock a connu trois années de fort recrutement 1998, 2005 et 2006. Les biomasses totale et exploitable sont en augmentation régulière depuis 2004.
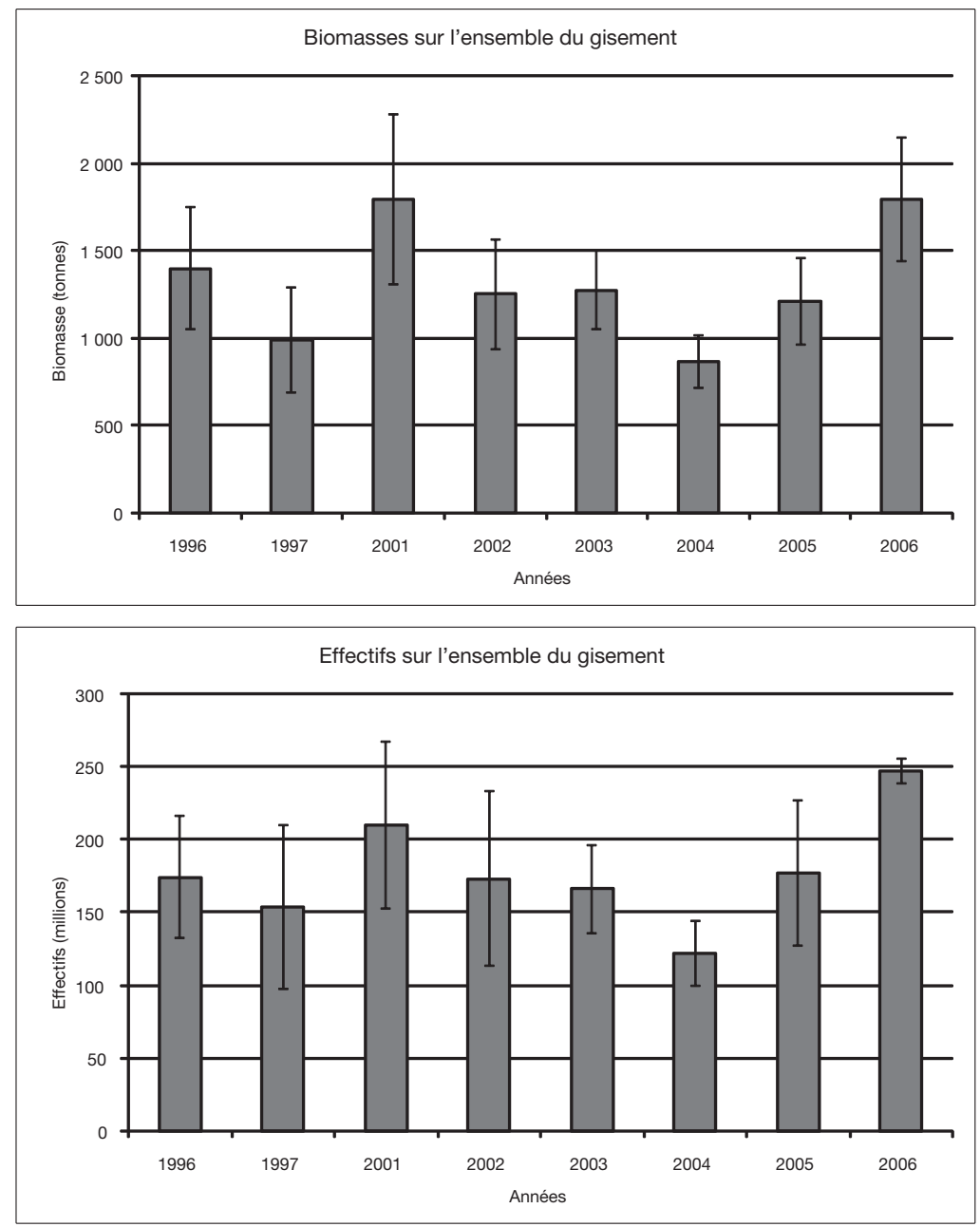

Figure 21. Évolution des effectifs et des biomasses totaux sur l'ensemble du gisement. 
Après un épisode de surcapacité de pêche en 2000 et 2001, des mesures de gestion restrictives ont été mises en place. Elles visent essentiellement à réduire l'effort de pêche par: - la diminution sensible du nombre de licences accordées (- $25 \%$ ) qui était de 285 en 2000 pour 214 aujourd'hui;

- la réduction adaptative de la période d'ouverture à la pêche en fonction de la biomasse disponible. La fermeture se situant entre fin août et la fin octobre alors qu'elle avait lieu fin décembre dans les années 1995-2000.

Cette mesure va également dans le sens d'une plus grande protection des zones d'hivernage des bernaches gravant;

- l'interdiction de la pêche les week-ends et jours fériés et celle d'effectuer deux marées au cours d'une même journée;

- la surveillance du secteur par un garde juré financé par les structures professionnelles ;

- la palourde atteint la taille de première commercialisation en moins de trois ans, le niveau des captures est en relation avec le recrutement de l'année n-2.

La politique dite des reliquats de pêche, qui consiste à ne pas exploiter la totalité de la biomasse exploitable, menée ces dernières années permet d'une part, de palier la variabilité du recrutement, et d'autre part, d'augmenter progressivement la taille moyenne des palourdes commercialisables et la biomasse exploitable présentes sur le site et d'améliorer ainsi l'état de ce gisement.

Les recommandations de gestions sont adaptatives sur le court terme en fonction du potentiel du gisement évalué annuellement et tendent à maîtriser l'effort de pêche pour pratiquer une politique de reliquat de pêche et insistent sur le strict respect de la taille de première capture.

\section{Flottilles et revenus}

\section{Présentation de la flotte de pêche bretonne, ses caractéristiques, son activité et son évolution récente}

La flotte de pêche bretonne regroupe les navires inscrits au fichier « flotte de pêche national » au 31 décembre de l'année $n$, immatriculés dans les quartiers maritimes de Saint-Malo à Vannes. Les navires armés en cultures marines petite pêche (CMPP) ne sont pas pris en compte.

Les informations utilisées sont les suivantes:

- données déclaratives (journaux de bords, fiches de pêche) du ministère de l'Agriculture et de la Pêche;

- données de débarquements des criées;

- données collectées par les enquêteurs de l'Ifremer sur l'activité et l'économie des entreprises de pêche (cf. chapitre 4. Méthodologies développées).

\section{Chiffres clés de la flotte de pêche bretonne en 2003}

Sur la base des informations du fichier « flotte nationale » au 31 décembre 2003, la flotte de pêche bretonne regroupe 1619 navires pour $337096 \mathrm{~kW}, 75259$ Tjb et 4769 marins embarqués. 
Le poids de la Bretagne est très important au niveau des façades mer du Nord - Manche - Atlantique (MNMA) et représente autour de $45 \%$ des capacités de production globales, mesurées à partir du nombre des navires, des marins ou de la puissance et jauge totales.

Tableau 3. Chiffres clés de la flotte bretonne en 2003 (source: Ifremer/SIH - DPMA).

\begin{tabular}{|c|c|c|c|c|}
\hline & 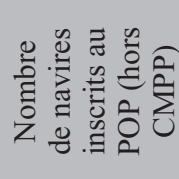 & 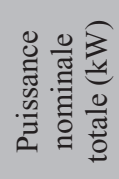 & 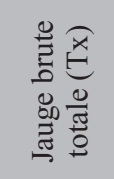 & 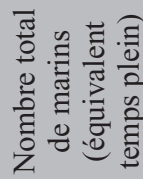 \\
\hline Région Bretagne & 1619 & 337096 & 75259 & 4769 \\
\hline Région Bretagne Nord & 666 & 89114 & 14223 & 1705 \\
\hline Région Bretagne Sud & 953 & 247982 & 61036 & 3064 \\
\hline $\begin{array}{l}\text { Façade mer du Nord - Manche - Atlantique } \\
\text { (MNMA) }\end{array}$ & 3803 & 721587 & 135239 & 11486 \\
\hline Façade mer du Nord - Manche (MNM) & 1600 & 290372 & 47291 & 5083 \\
\hline Façade Atlantique (A) & 2203 & 431215 & 87948 & 6403 \\
\hline Part de la Bretagne dans façade MNMA (\%) & 43 & 47 & 56 & 42 \\
\hline Part Bretagne Nord dans façade MNM (\%) & 42 & 31 & 30 & 34 \\
\hline Part Bretagne Sud dans façade Atlantique (\%) & 43 & 58 & 69 & 48 \\
\hline
\end{tabular}

Une première analyse de la répartition de la flotte par quartier maritime met en lumière trois groupes de quartiers selon le nombre de navires qui y sont immatriculés (figure 22 p. VI):

- les quartiers de plus de 200 navires : en 2003 Le Guilvinec est le seul quartier appartenant à cette catégorie, alors qu'en 1993 Concarneau et Lorient en faisaient encore partie avec respectivement 250 et 220 navires;

- les quartiers de 100 à 200 navires : du nord au sud, on y trouve Saint-Brieuc, Paimpol, Morlaix et Brest pour la Bretagne Nord; Concarneau, Lorient et Auray pour la Bretagne Sud. À l'exception de Concarneau et de Lorient, les autres quartiers relevaient de cette même catégorie en 1993 ;

- les quartiers de moins de 100 navires : Saint-Malo, Camaret, Douarnenez, Audierne et Vannes.

\section{Évolution de la flotte de pêche de 1983 à 2003}

Les données disponibles à l'échelle de la Bretagne nous permettent d'examiner l'évolution de la flotte avec un recul de 20 ans. Il apparaît donc qu'en 20 ans, la flotte bretonne a diminué de $54 \%$, passant de près de 3500 navires en 1983 à près de 1600 en 2003. Parallèlement, la puissance nominale moyenne des navires a augmenté de $60 \%$ (figure 23). 


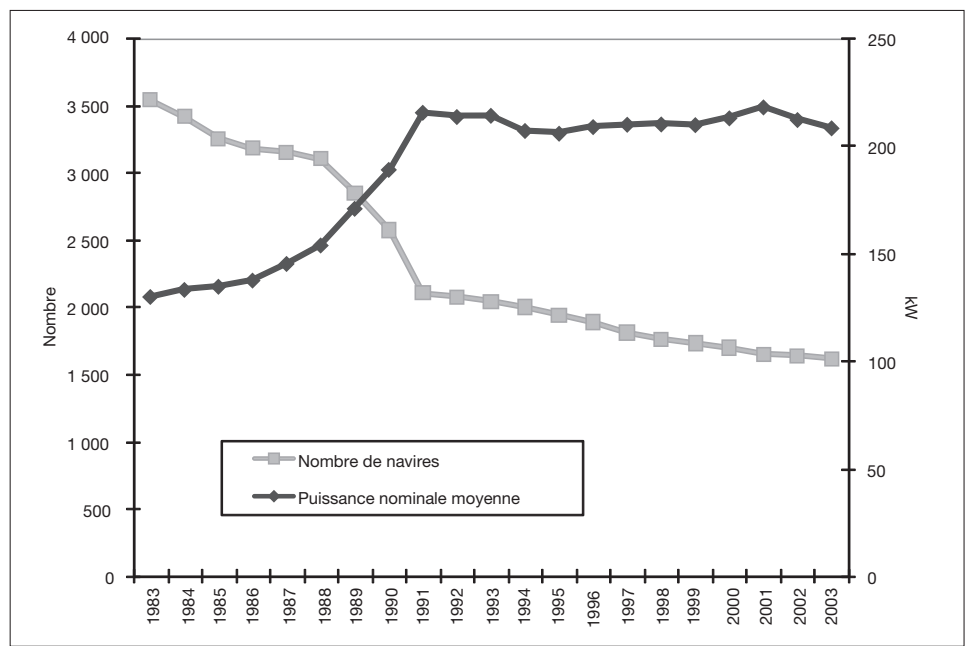

Figure 23. Évolution des effectifs de navires et de la puissance nominale moyenne de 1983 à 2003 en Bretagne.

Un élément marquant de l'évolution de la flotte bretonne sur 20 ans est la diminution drastique de la flotte de moins de 12 mètres, qui passe de 2629 navires en 1983 à 1094 navires en 2003 (figure 24). Cela n'a cependant pas contribué à modifier la structure en taille de la flotte bretonne qui reste majoritairement composée de navires de moins de 12 mètres. En effet, près de $70 \%$ des navires en 2003 appartiennent à cette catégorie de taille contre $74 \%$ en 1983, avec une diminution assez forte de cette proportion au début des années quatre-vingt-dix suite aux choix publics d'incitations aux investissements dans les flottilles du large à la fin des années quatre-vingt.

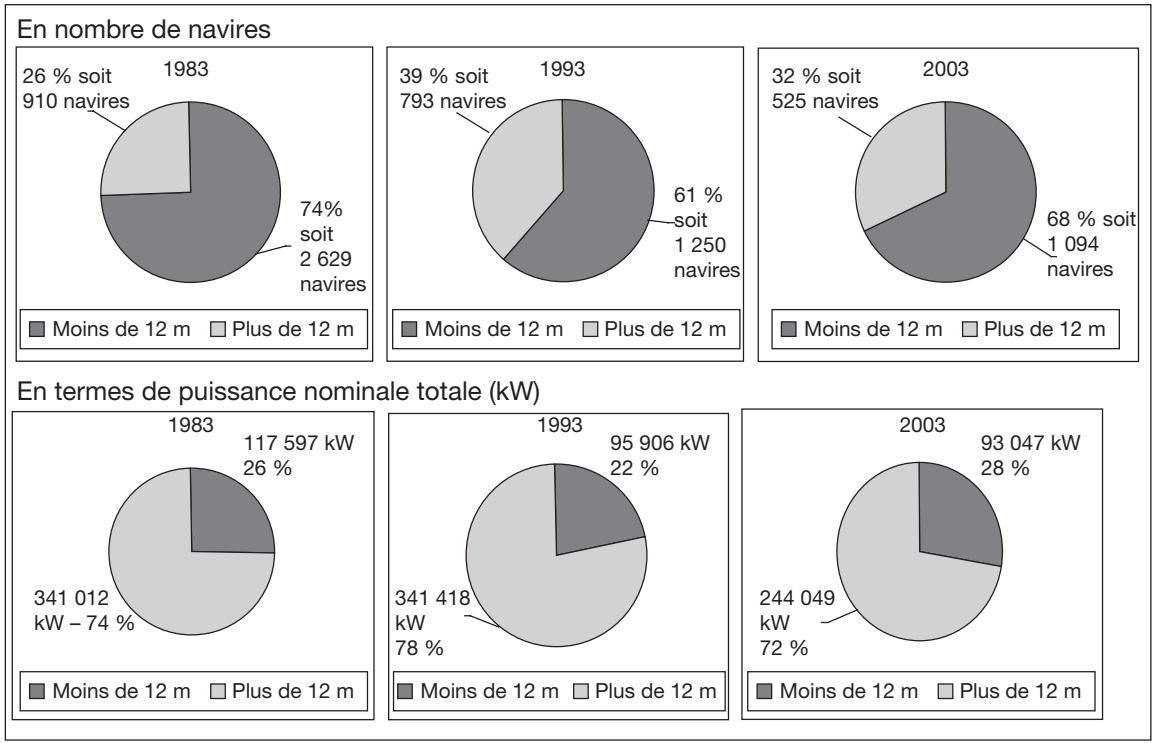

Figure 24. Flotte bretonne. Évolution du poids respectif par catégorie de taille. 


\section{Structure de la flotte de pêche bretonne en 2003}

En 2003, le navire moyen en Bretagne a une longueur de 12,80 mètres pour $208 \mathrm{~kW}$, $46 \mathrm{Tjb}$ et près de 3 hommes embarqués. Toutes les catégories de taille de navires sont représentées au sein de la flotte de pêche bretonne (figure 25).

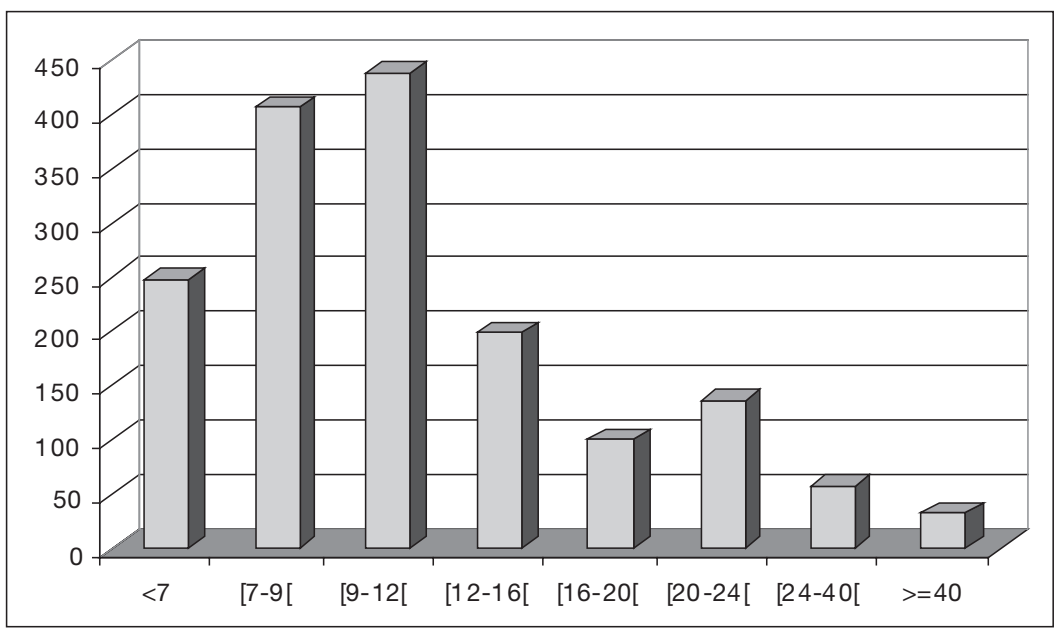

Figure 25. Bretagne. Nombre de navires par catégorie de longueur (en mètres) en 2003.

Les structures de flotte en termes de taille de navires sont très différentes selon les façades (figure 26 et tableau 4).

En Bretagne Nord, le navire moyen a une longueur de près de 11 mètres pour $134 \mathrm{~kW}$, $21 \mathrm{Tjb}$ et un peu moins de 3 hommes embarqués;

Le navire moyen de Bretagne Sud a une longueur d'environ 14 mètres pour $260 \mathrm{~kW}$, $64 \mathrm{Tjb}$ et un peu plus de 3 hommes embarqués.

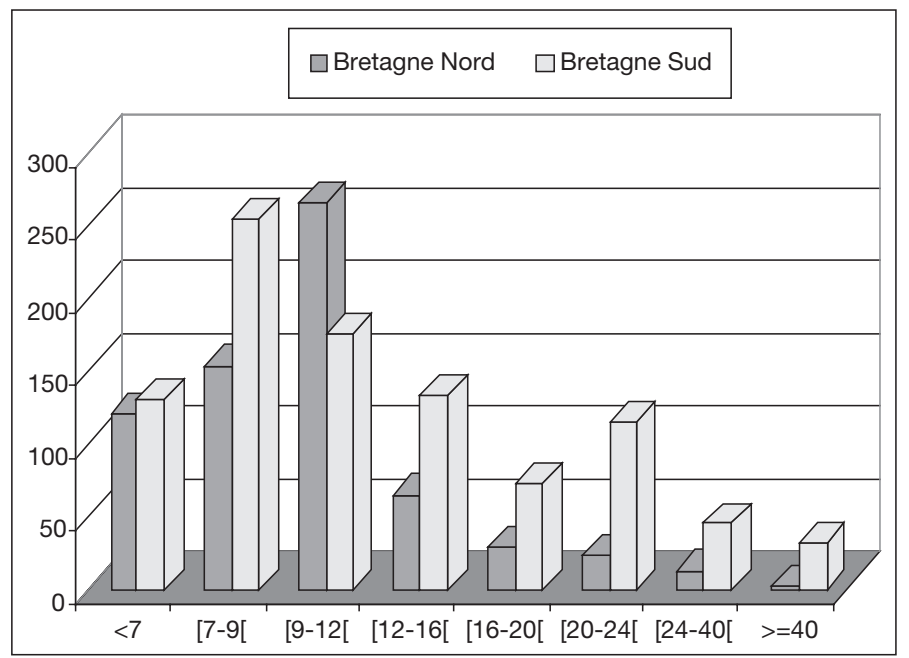

Figure 26. Bretagne. Nombre de navires par catégorie de longueur et par façade en 2003 (source : Ifremer/SIH). 
Ainsi, alors que la flotte de pêche commerciale de Bretagne Nord est concentrée sur les navires de moins de 12 mètres, la flotte de Bretagne Sud est représentée au sein de toutes les catégories de taille y compris les plus de 40 mètres.

Tableau 4. Caractéristiques du navire moyen par classe de taille au total et par façade en 2003 (source: Ifremer/SIH).

\begin{tabular}{|c|c|c|c|c|c|c|c|c|}
\hline BRETAGNE & $<7 \mathrm{~m}$ & {$[7-9[\mathrm{~m}$} & {$[9-12[\mathrm{~m}$} & {$[12-16[\mathrm{~m}$} & {$[16-20[\mathrm{~m}$} & {$[20-24[\mathrm{~m}$} & {$[24-40[\mathrm{~m}$} & $>=40 \mathrm{~m}$ \\
\hline Nombre de navires & 249 & 406 & 439 & 198 & 101 & 136 & 57 & 33 \\
\hline Puissance totale $(\mathrm{kW})$ & 9145 & 30245 & 53657 & 41267 & 29649 & 55219 & 33850 & 84064 \\
\hline Jauge (Tjb) & 441 & 1661 & 4719 & 7024 & 6911 & 17557 & 13548 & 49383 \\
\hline Nombre de marins & 274 & 530 & 1002 & 741 & 520 & 835 & 479 & 388 \\
\hline Âge moyen des navires & 21 & 25 & 22 & 21 & 20 & 15 & 16 & 19 \\
\hline Âge moyen des patrons propriétaires embarqués & 46 & 43 & 42 & 42 & 43 & 42 & 48 & \\
\hline Bretagne Nord & $<7 \mathrm{~m}$ & {$[7-9[\mathrm{~m}$} & {$[9-12[\mathrm{~m}$} & {$[12-16[\mathrm{~m}$} & {$[16-20[\mathrm{~m}$} & {$[20-24[\mathrm{~m}$} & {$[24-40[\mathrm{~m}$} & $>=40 \mathrm{~m}$ \\
\hline Nombre de navires & 119 & 153 & 264 & 65 & 29 & 22 & 12 & 2 \\
\hline Puissance totale $(\mathrm{kW})$ & 4038 & 10813 & 32518 & 12955 & 8703 & 9233 & 5923 & 4931 \\
\hline Jauge (Tjb) & 217 & 678 & 2994 & 2120 & 2132 & 3102 & 2066 & 4783 \\
\hline Nombre de marins & 132 & 222 & 621 & 235 & 171 & 150 & 81 & 94 \\
\hline Âge moyen des navires & 22 & 26 & 22 & 19 & 22 & 14 & 12 & 25 \\
\hline Âge moyen des patrons propriétaires embarqués & 48 & 43 & 41 & 42 & 44 & 41 & 53 & \\
\hline Bretagne Sud & $<7 \mathrm{~m}$ & {$[7-9[\mathrm{~m}$} & {$[9-12[\mathrm{~m}$} & {$[12-16[\mathrm{~m}$} & {$[16-20[\mathrm{~m}$} & {$[20-24[\mathrm{~m}$} & {$[24-40[\mathrm{~m}$} & $>=40 \mathrm{~m}$ \\
\hline Nombre de navires & 130 & 253 & 175 & 133 & 72 & 114 & 45 & 31 \\
\hline Puissance totale $(\mathrm{kW})$ & 5107 & 19432 & 21139 & 28312 & 20946 & 45986 & 27927 & 79133 \\
\hline Jauge (Tjb) & 224 & 983 & 1726 & 4904 & 4779 & 14455 & 11481 & 44600 \\
\hline Nombre de marins & 142 & 308 & 381 & 506 & 349 & 685 & 398 & 294 \\
\hline Âge moyen des navires & 20 & 24 & 21 & 22 & 20 & 15 & 17 & 19 \\
\hline Âge moyen des patrons propriétaires embarqués & 44 & 43 & 43 & 42 & 43 & 42 & 46 & \\
\hline
\end{tabular}

En Bretagne Nord, à l'intérieur de la catégorie, le segment de flotte des 9 à 12 mètres concentre l'essentiel des moyens de production y compris en termes d'emplois (621 marins pour un total Bretagne Nord de 1700 marins environ). En Bretagne Sud, si l'on s'appuie sur le nombre d'effectifs embarqués, deux catégories de taille prédominent: la flotte des 20-24 mètres et celle des 12-16 mètres avec respectivement 685 et 506 marins embarqués. Les autres catégories de taille génèrent autour de 300 et 400 emplois directs à la mer.

L'âge moyen relativement plus faible des navires de plus de 20 mètres témoigne des choix d'investissement à l'échelle de la façade, à la fin des années quatre-vingt, qui se sont portés vers des navires du large par rapport aux côtiers.

Si l'on fait abstraction des plus de 24 mètres, qui sont généralement propriété de sociétés, l'âge moyen des propriétaires embarqués ne diffère pas sensiblement d'une catégorie de taille de navires à une autre, ni d'une façade à une autre, à l'exception des navires de moins de 7 mètres de Bretagne Nord où la moyenne d'âge est sensiblement plus élevée.

\section{Évolution de la structure de la flotte bretonne entre 1990 et 2003}

\section{Caractéristiques moyennes}

Les évolutions des caractéristiques du navire moyen témoignent d'une relative stabilité de la longueur moyenne dans les deux façades sur la totalité de la période, d'une tendance à l'augmentation des puissances et jauges moyennes jusqu'en 2001 avec néanmoins une inflexion de cette tendance à partir de 2001. Cette dernière s'accompagne par ailleurs d'une tendance à l'augmentation de la taille moyenne des équipages ce qui semble témoigner d'une légère baisse de l'intensité capitalistique (rapport capital - travail) sur les années récentes, particulièrement en Bretagne Sud (figures 27 et 28). 


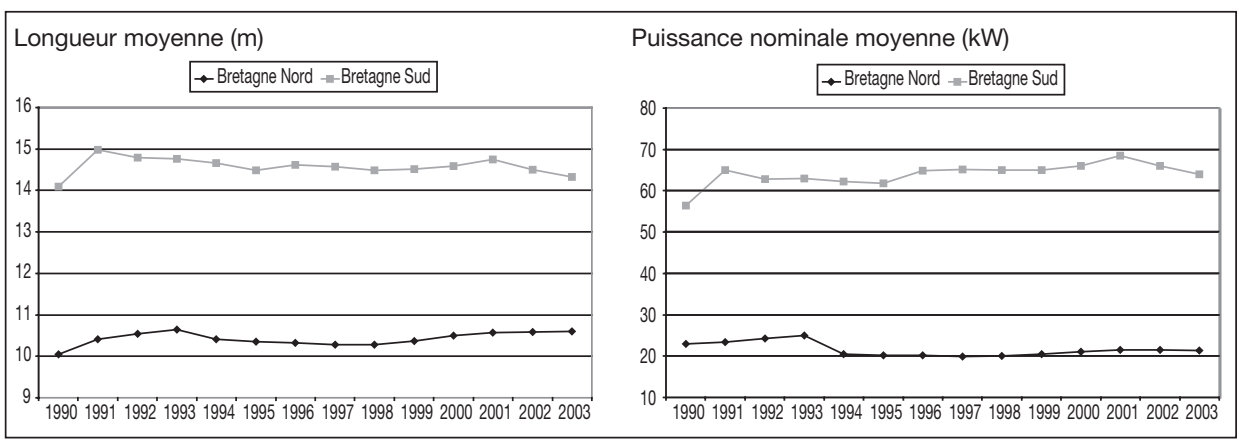

Figure 27. Évolution des caractéristiques du navire moyen par façade entre 1990 et 2003 (1/2).

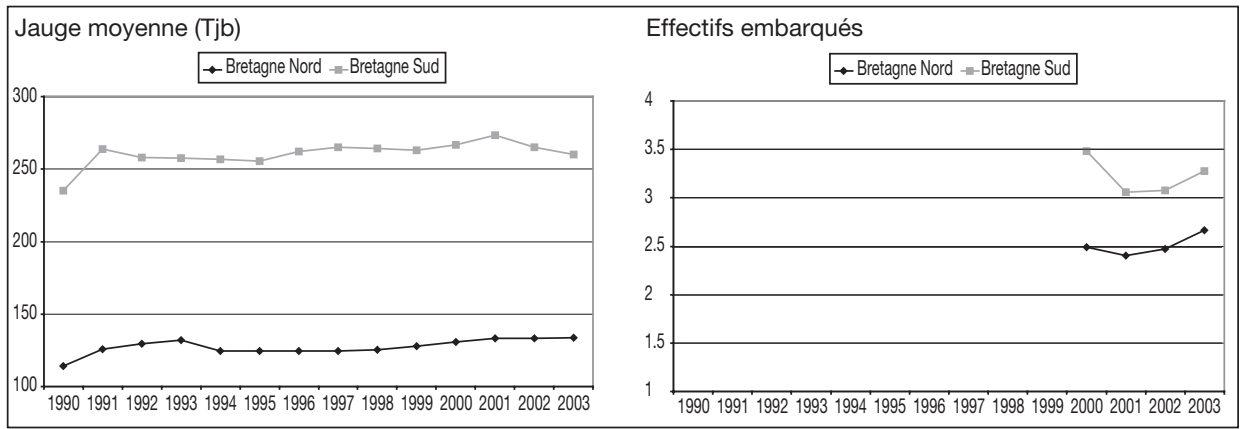

Figure 28. Évolution des caractéristiques du navire moyen par façade entre 1990 et $2003(2 / 2)$. (source : Ifremer/SIH).

\section{Une tendance au vieillissement de la flotte}

La structure de la flotte de pêche par classe d'âge a sensiblement évolué depuis 1990 avec une tendance au vieillissement des unités de production qui s'accentue depuis 1995. En 1990, un peu plus de $50 \%$ de la flotte des navires bretons avaient moins de 15 ans et près de $90 \%$ avaient moins de 25 ans. En 2003, seulement $20 \%$ des navires ont moins de 15 ans et la proportion de navires de moins de 25 ans est passée à moins de $70 \%$ (figure 29 p. VI).

\section{Évolution des structures de propriété}

La structure de propriété des navires dans le secteur des pêches est largement dominée par l'entreprise individuelle. Le propriétaire du navire est ainsi une personne physique qui fait l'objet d'une codification spécifique dans les fichiers sur la flotte de pêche nationale. Par ailleurs, la date de naissance de chaque propriétaire physique (généralement le patron de pêche) est disponible dans les fichiers, ce qui permet d'analyser les structures d'âge des propriétaires embarqués et leurs évolutions. Chaque société (personne morale propriétaire du navire) a également une codification propre. Une société ou une entreprise individuelle peuvent être propriétaires d'un ou plusieurs navires.

En première analyse, on n'examinera que le cas des entreprises individuelles (personnes physiques propriétaires des navires). 
Depuis 1994, la proportion des patrons embarqués par classe d'âge reste sensiblement la même : $25 \%$ ont moins de 35 ans, $55 \%$ ont entre 35 et 50 ans et $20 \%$ ont plus de 50 ans. Entre 1990 et 1994, la crise de la pêche avait conduit à une diminution de la part des patrons propriétaires de moins de 35 ans qui s'est stabilisée depuis autour de $25 \%$ (figure 30 p. VII).

Depuis une quinzaine d'années, le contexte est marqué par une restriction de l'accès au secteur des pêches maritimes (par le biais de permis de mise en exploitation ou PME notamment) et une diminution des aides publiques à la construction neuve, générant comme on a pu l'observer ces dernières années une augmentation très nette du prix des navires sur le marché de l'occasion (Guyader O. et al., 2003). L'évaluation de l'impact de ce contexte sur l'évolution de la propriété des navires est effectuée en comparant les années 1995 et 2003. Le choix de ne pas retenir l'année 1993 est motivé par la volonté de ne pas biaiser l'analyse par les effets immédiats de la crise du début de la décennie 1990.

Une première approche consiste à examiner pour chaque structure de propriété le type de capital ou bateau possédé (taille et âge).

\section{Remarques}

- Par structure de propriété, on distinguera la société de capitaux, l'entreprise individuelle où le patron propriétaire embarqué a moins de 35 ans, l'entreprise individuelle où le patron propriétaire embarqué a entre 35 et 50 ans, l'entreprise individuelle où le patron propriétaire embarqué a plus de 50 ans.

- Le type de bateau résulte d'un croisement entre des classes de taille et des classes d'âge, chacun de ces deux éléments ayant un impact important dans le prix du navire sur le marché de l'occasion.

Il y a une très nette différence entre le capital possédé par les sociétés et celui que possèdent les entreprises individuelles, quelle que soit la classe d'âge des patrons propriétaires embarqués. La nature du capital possédé par les patrons entrepreneurs individuels est presque exclusivement constituée de navires de moins de 24 mètres, avec une très large proportion de moins de 12 mètres (autour de 70 à $80 \%$ ). Les sociétés de capitaux en revanche concentrent leurs investissements dans les navires de plus de 12 mètres (figure 31 p. VII).

Des évolutions très marquées sont observées par rapport à 1995 (figure 31 p. VII). D'abord en ce qui concerne les entreprises individuelles, les investissements continuent à ne concerner que les navires de moins de 24 mètres, mais avec une diminution nette de la proportion des 12-24 mètres particulièrement au sein de la catégorie des patrons de 35 à 50 ans. Pour ce qui concerne les sociétés, les investissements continuent à ne concerner que les navires de plus de 12 mètres, mais alors que les navires appartenaient généralement aux classes d'âge de moins de 15 ans (au moins pour les moins de 40 mètres) en 1995, la nature du capital s'étend aux navires de plus de 15 ans en 2003. On peut y voir deux facteurs explicatifs: le vieillissement naturel de la flotte détenue par les sociétés, d'une part, et l'incitation à une structuration en sociétés de capitaux, fiscalement plus avantageuse pour l'acquisition de navires, y compris sur le marché de l'occasion, d'autre part.

\section{L'activité de la flotte de pêche commerciale bretonne: rayon d'action et polyvalence}

En 2003, sur la totalité des navires inscrits au registre de la flotte nationale, on recense seulement 44 bateaux inactifs à l'échelle de la Bretagne soit $3 \%$ de la flotte bretonne inscrite au fichier « flotte nationale ». L'activité annuelle de ces navires peut s'exercer 
exclusivement ou très largement dans les 12 milles (navire côtier), en dehors des 12 milles (navire du large) ou une partie du temps dans et hors des 12 milles (navire mixte). De même, l'activité annuelle d'un navire sera plus ou moins polyvalente selon qu'il utilisera au cours de l'année un ou plusieurs engins.

\section{Rayon d'action des navires}

La flotte bretonne est très largement côtière avec $75 \%$ des navires dont l'essentiel de l'activité en 2003 s'exerce dans la bande côtière, contre $77 \%$ en 1994 (figure 32).
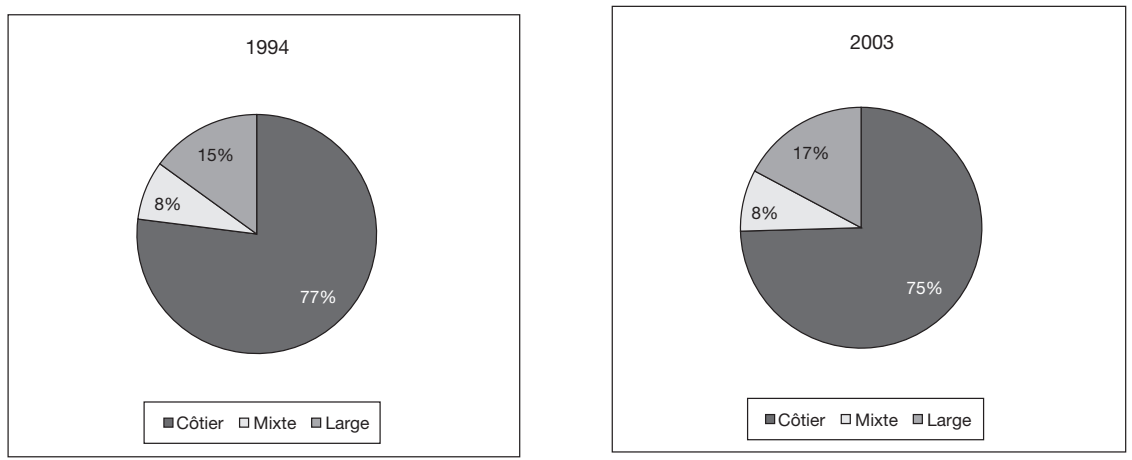

Figure 32. Rayon d'action des navires de la flotte bretonne en 1994 et 2003.

\section{Polyvalence des navires}

En 2003, un navire breton exerce en moyenne deux métiers par an (mesurés ici en termes d'engins utilisés au cours de l'année simultanément ou non). Cette moyenne reste inchangée par rapport à 1994.
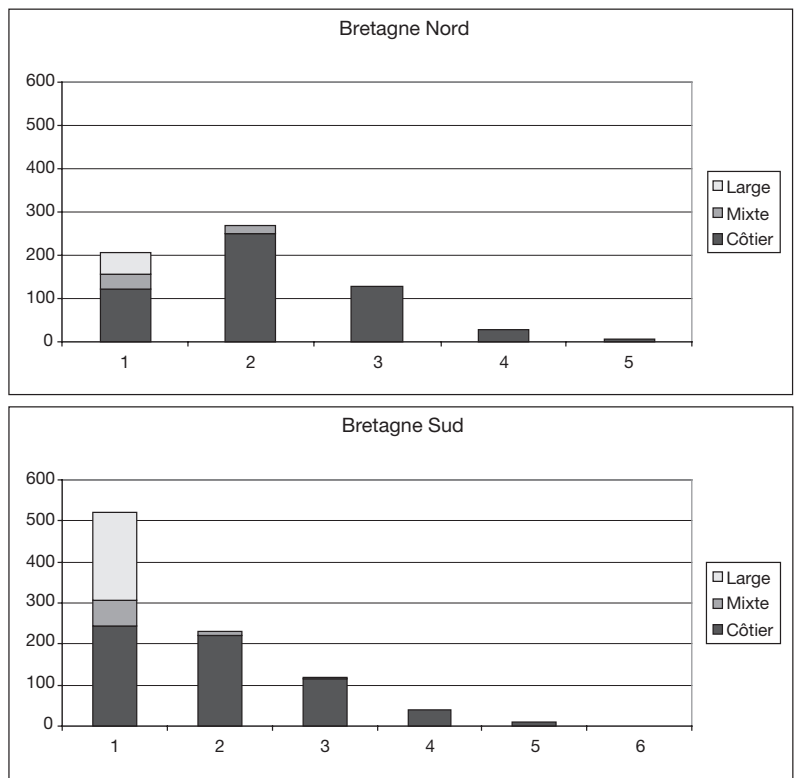

Figure 33. Polyvalence des navires par façade (source : Ifremer/SIH). 
La flotte de Bretagne Sud apparaît moins polyvalente que celle de Bretagne Nord (figure 33): on observe que la proportion de navires exerçant deux ou trois métiers dans l'année y est moins importante. Quelle que soit la façade, les flottilles du large et une très grande majorité des flottilles mixtes sont exclusivement « mono-métier ».

\section{L'activité de la flotte de pêche commerciale bretonne: les métiers structurants}

Le métier est défini par la combinaison d'un engin et d'une espèce cible (ou d'un groupe d'espèces cibles).

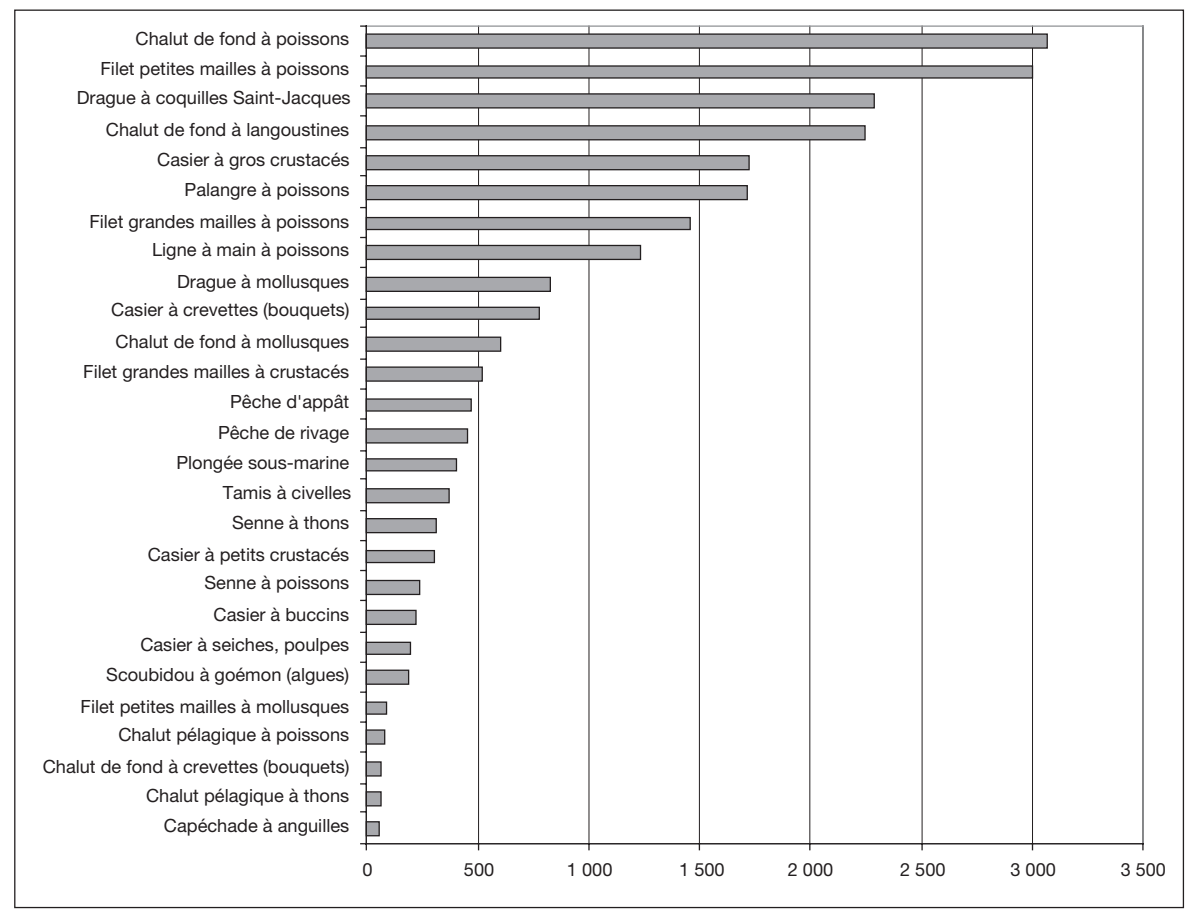

Figure 34. Bretagne. Nombre de mois d'activité par métier en 2003 (source : Ifremer/SIH).

Si l'on s'appuie sur les calendriers d'activité des navires, huit métiers peuvent être considérés comme structurant l'activité de la flotte de pêche commerciale bretonne (figure 34):

- le chalut de fond à poissons ;

- le filet petites mailles à poissons;

- la drague à coquilles Saint-Jacques;

- le chalut de fond à langoustines;

- le casier à gros crustacés;

- la palangre à poissons ;

- le filet grandes mailles à poissons;

- la ligne à main à poissons.

Chacun de ces métiers totalise au minimum 1000 mois d'activités et est exercé par 200 navires et plus en 2003. La hiérarchie de ces métiers n'est pas la même selon que l'on considère le nombre de mois d'activités total ou le nombre de navires actifs sur ce métier. 
L'importance des métiers est variable selon les façades.

En Bretagne Nord, l'activité est incontestablement dominée par le métier de la drague à coquilles Saint-Jacques. Viennent ensuite le casier à gros crustacés et les métiers du filet (grandes mailles et petites mailles à poissons). Chacun de ces métiers totalise au moins 1000 mois d'activité et 150 navires à l'échelle de la façade.

En Bretagne Sud, les quatre métiers dominants (au moins 1000 mois d'activité et 150 navires) sont les métiers du chalut (chalut de fond à poissons et chalut de fond à langoustines), le filet petites mailles à poissons et de la palangre à poissons. Les deux premiers sont majoritairement le fait de navires du large, notamment le chalut de fond à poissons.

Entre 1994 et 2003, malgré les évolutions notables de la flotte de pêche observées précédemment, les métiers qui dominent l'activité de la flotte de pêche commerciale bretonne n’ont pas changé (tableau 5).

Tableau 5. Importance hiérarchique en Bretagne des métiers selon le critère de mois d'activité total en 1994, 2000 et 2003 (source: Ifremer/SIH).

\begin{tabular}{|l|c|c|c|}
\hline \multicolumn{1}{|c|}{ Métiers - Nombre total de mois d'activité } & Rang 2003 & Rang 2000 & Rang 1994 \\
\hline Chalut de fond à poissons & 1 & 1 & 1 \\
\hline Filet petites mailles à poissons & 2 & 2 & 3 \\
\hline Drague à coquille Saint-Jacques & 3 & 5 & 5 \\
\hline Chalut de fond à langoustines & 4 & 3 & 2 \\
\hline Casier à gros crustacés & 5 & 6 & 4 \\
\hline Palangre à poissons & 6 & 4 & 6 \\
\hline Filet grandes mailles à poissons & 7 & 8 & 7 \\
\hline Ligne à main à poissons & 8 & 7 & 8 \\
\hline
\end{tabular}

Les huit principaux métiers recensés en 2003 étaient exactement les mêmes en 2000 et en 1994 avec quelques modifications dans leur importance hiérarchique. Si le métier de chalut de fond à poissons reste le métier dominant sur la période, en revanche le chalut de fond à langoustines passe de la deuxième place en 1994, à la troisième en 2000 et à la quatrième en 2003. Les évolutions différenciées de flotte entre la Bretagne Nord et la Bretagne Sud expliquent très largement ces changements de hiérarchie et la place de plus en plus importante occupée par les métiers de Bretagne Nord à l'échelle de la Bretagne.

\section{Les flottilles de pêche bretonne: définition, description et évolution}

Sur la base des calendriers d'activité des navires de pêche collectés en routine depuis 2000 (et ponctuellement les années précédentes), l'Ifremer a développé différentes méthodes de segmentation de la flotte en flottilles (Berthou et al, 2003). Cette segmentation vise à « individualiser des groupes de patrons pêcheurs ou d'unités d'exploitation, ayant des stratégies d'exploitation relativement homogènes. Ces stratégies s'expriment par le choix d'une combinaison particulière de métiers pratiqués. C'est la notion de type d'exploitation ou de flottille. Ainsi, la flotte de pêche d'une région donnée est structurée en différentes flottilles ». La méthode retenue est décrite au chapitre 4 (Méthodologies développées).

On distingue finalement 6 flottilles (figure 35 p. VIII) :

- les chalutiers : navires pratiquant le chalut en combinaison avec ou sans autres engins;

- les dragueurs : navires pratiquant la drague en combinaison avec ou sans autres engins à l'exception du chalut; 
- les dormants : navires pratiquant exclusivement des engins dormants (ligne, palangre, casier, filet);

- les divers : petits métiers de plongée ou de tamis;

- les bolincheurs;

- les senneurs tropicaux.

Un groupe d'inactifs à la pêche est par ailleurs identifié. Il s'agit des navires enregistrés au fichier national de la flotte de pêche au 31 décembre de l'année 2003 mais n'ayant pas pratiqué d'activités de pêche au cours de l'année.

Deux flottilles prédominent au sein de la flotte de pêche bretonne : la flottille des chalutiers et la flottille des dormants totalisant chacune plus de 500 navires. La flottille des dragueurs arrive en troisième position en nombre de navires.

L'analyse typologique a été également effectuée dans une perspective historique (tableau 6). Ainsi, sur la base des différentes sources d'information existantes (calendriers d'activité des navires, fiches de pêche, logbooks et données de vente), l'Ifremer a entrepris l'exercice de segmentation de la flotte de pêche en flottilles depuis 1990. Chaque navire appartenant à la flotte de pêche nationale est affecté à une flottille sur la base de la méthodologie systématisée présentée ultérieurement.

Tableau 6. Composition des flottilles par classe de taille et évolution entre 1990 et 2003 (source: Ifremer/SIH).

\begin{tabular}{|c|c|c|c|c|c|c|c|c|c|}
\hline 1990 & $<7 \mathrm{~m}$ & {$[7-9[\mathrm{~m}$} & {$[9-12[\mathrm{~m}$} & {$[12-16[\mathrm{~m}$} & {$[16-20[\mathrm{~m}$} & {$[20-24[\mathrm{~m}$} & {$[24-40[\mathrm{~m}$} & $>=40 \mathrm{~m}$ & TOTAL \\
\hline Chalutiers & 3 & 35 & 177 & 266 & 153 & 129 & 104 & 23 & 890 \\
\hline Bolincheurs & & & & 14 & 1 & & & 1 & 16 \\
\hline Dragueurs & 34 & 130 & 113 & 17 & 1 & & & & 295 \\
\hline Senneurs tropicaux & & & & & & & & 34 & 34 \\
\hline Dormants + Divers & 491 & 469 & 239 & 61 & 59 & 5 & 10 & 0 & 1334 \\
\hline Total & 528 & 634 & 529 & 358 & 214 & 134 & 114 & 58 & 2569 \\
\hline \multicolumn{10}{|c|}{ Variations entre 1990 et $2003(\%)$} \\
\hline & $<7 \mathrm{~m}$ & {$[7-9[\mathrm{~m}$} & {$[9-12[\mathrm{~m}$} & {$[12-16[\mathrm{~m}$} & {$[16-20[\mathrm{~m}$} & {$[20-24[\mathrm{~m}$} & {$[24-40[\mathrm{~m}$} & $>=40 \mathrm{~m}$ & TOTAL \\
\hline Chalutiers & -100 & -51 & -20 & -55 & -54 & -2 & -47 & -74 & -40 \\
\hline Bolincheurs & & & & 21 & & & & & 31 \\
\hline Dragueurs & -65 & -18 & 39 & -18 & & & & & -1 \\
\hline Senneurs tropicaux & -56 & & & & & & & -24 & -24 \\
\hline Dormants + Divers & & -43 & -43 & -25 & -58 & 60 & -90 & & -48 \\
\hline Total & -53 & -36 & -17 & -45 & -53 & 1 & -50 & -43 & -37 \\
\hline
\end{tabular}

Les évolutions témoignent de tendances différentes selon les flottilles et les classes de taille des navires. Les flottilles de chalutiers, des dormants et des senneurs tropicaux ont subi une forte diminution d'effectifs au cours de la période. En revanche, les flottilles des bolincheurs et des dragueurs ont connu respectivement une augmentation et une stabilité des effectifs de navires.

À l'intérieur des flottilles, les évolutions sont également contrastées en termes de classe de longueurs des navires. Au sein de la flottille des chalutiers, les diminutions ont été partout importantes, à l'exception du segment des 20 à 24 mètres où les effectifs sont restés relativement stables sur la période. Pour ce qui concerne les dragueurs, la stabilité est en fait le résultat d'une forte diminution des effectifs de moins de 9 mètres compensée par une augmentation des effectifs de 9 à 12 mètres. 


\section{Conclusion}

Cette étude a permis de réactualiser les travaux antérieurs de caractérisation de la flotte de pêche bretonne avec des données récentes. Elle met en évidence un certain nombre de résultats dont quelques-uns sont recensés ci-après :

- la Bretagne est une région incontournable dans le secteur des pêches maritimes nationales puisqu'elle contribue à près de $45 \%$ des capacités de production globales, mesurées à partir du nombre des navires, des marins ou de la puissance et jauge totale ;

- la flotte bretonne a subi une diminution drastique de ses effectifs entre 1983 et 2003 qui a majoritairement concerné les navires de moins de 12 mètres ;

- la flotte bretonne est encore avant tout une flotte côtière: $70 \%$ des navires font moins de 12 mètres et $75 \%$ des navires ont une activité essentiellement côtière ;

- on observe un vieillissement des unités de production: $75 \%$ des navires ont plus de 15 ans ;

- la diminution de la flotte n'a pas modifié fondamentalement la hiérarchie des activités de pêche puisque le chalut et le filet restent les engins de pêche prédominants. Par ailleurs, les huit principaux métiers exercés par les navires en 2003 sont les mêmes que ceux observés en 2000.

$\mathrm{Au}$ total, cette réactualisation permet de réaffirmer la nécessité de disposer de séries historiques et de bases de données pérennes de l'activité des navires, de leur production et de leur situation économique. Ces données sont indispensables à l'élaboration de diagnostics sur les pêcheries et les flottilles.

\section{Les revenus à la pêche dans la bande côtière bretonne}

\section{Vue synthétique relative à l'année 2003 et analyse rétrospective de trois segments de flottille}

Les résultats présentés sont issus de la base de données comptable de l'Observatoire économique régional des pêches. Ils ne couvrent donc pas la totalité de la pêche bretonne: les navires de plus de 24 mètres, en particulier, ne sont pas pris en compte. Par rapport aux documents annuels de l'Observatoire, la présente étude innove principalement sur trois points:

- Elle s'appuie sur le gradient du rayon d'action des navires de pêche, renseigné dans le Système d'informations halieutiques $(\mathrm{SIH})$ de l'Ifremer. Les résultats économiques sont présentés en distinguant les navires « côtiers », les navires « mixtes » et les navires du « large ».

- Elle utilise une segmentation de la flotte de pêche bretonne élaborée conjointement avec le SIH de l'Ifremer. Cette segmentation est plus cohérente que la nomenclature traditionnelle de l'observatoire, elle-même issue d'ajustements empiriques successifs plutôt que d'une réflexion taxonomique globale ${ }^{18}$.

- Elle inclut une étude rétrospective, menée à partir de trois échantillons constants de navires présents dans la base depuis 1994. Pour ces navires, on dispose (sur la période 1996-2004 pour les chalutiers-dragueurs et 1994-2003 pour les chalutiers exclusifs), de

\footnotetext{
${ }^{18}$ Dans le rapport 2003 de l'Observatoire, cette nomenclature a évolué dans le sens d'un rapprochement avec celle qui est utilisée ici (Observatoire économique régional des pêches, Résultats des flottilles artisanales 20022003. Fédération bretonne de la coopération maritime, Quimper, septembre 2004). Outre ses effets sur la lisibilité du document, cette évolution améliore la cohérence entre les différentes sources d'information statistique sur la pêche en Bretagne.
} 
séries continues de données comptables. On peut ainsi construire une image rétrospective de meilleure qualité que celle qui est obtenue à partir de l'exploitation des données brutes de la base. En effet, la composition de celle-ci varie d'une année sur l'autre, de sorte qu'il est difficile, à partir des données brutes, de faire la distinction entre les évolutions enregistrées par les navires et les changements dans la composition de la base ${ }^{19}$.

\section{Résultats économiques des navires par classe de longueur en 2003}

La population de la flotte de pêche bretonne se compose de 1619 navires en 2003, dont 1575 ont pu être identifiés selon le rayon d'action (tableau 7).

- Navires « côtiers »: navires passant plus de $75 \%$ de leur temps d'activité (mesuré en mois-métier) à l'intérieur de la bande des 12 milles;

- Navires « mixtes » : navires passant entre $25 \%$ et $75 \%$ de leur temps d'activité dans la bande des 12 milles;

- Navires du « large »: navires passant plus de $75 \%$ de leur temps d'activité au-delà de la bande des 12 milles.

Tableau 7. Population de la flotte de pêche bretonne par classe de longueur et de gradient (2003) (source : Ifremer).

\begin{tabular}{|l|c|c|c|c|c|c|c|c|c|}
\cline { 2 - 10 } \multicolumn{1}{c|}{} & $<7 \mathrm{~m}$ & {$[7-9[\mathrm{~m}$} & {$[9-12[\mathrm{~m}$} & {$[12-16[\mathrm{~m}$} & {$[16-20[\mathrm{~m}$} & {$[20-24[\mathrm{~m}$} & {$[24-40[\mathrm{~m}$} & $>=40 \mathrm{~m}$ & Total \\
\hline Côtier & 228 & 388 & 402 & 110 & 25 & 1 & & & 1154 \\
\hline Mixte & & 2 & 32 & 65 & 20 & 8 & 1 & & 128 \\
\hline Large & & 2 & & 23 & 56 & 125 & 55 & 32 & 293 \\
\hline Total & 228 & 392 & 434 & 198 & 101 & 134 & 56 & 32 & 1575 \\
\hline
\end{tabular}

Tous les navires présents dans la base de l'Observatoire et actifs en 2003 ont été pris en compte, soit un total de 547 navires identifiés selon le gradient et représentant un peu plus du tiers de l'effectif de la flotte de pêche bretonne segmentée par le temps d'activité en bande côtière (tableau 8). Le taux d'échantillonnage est de $30 \%$ pour les navires « côtiers », atteint $60 \%$ pour les unités « mixtes », et $41 \%$ pour les bateaux du « large ». Cependant, la représentativité varie selon la classe de longueur. Il s'élève à plus de $56 \%$ pour les navires de 12 à 24 mètres, mais n'atteint que $5 \%$ pour les bateaux de moins de 7 mètres. Seul ce dernier segment est sous-représenté.

Tableau 8. Taux d'échantillonnage (en \%) par classes de longueur et de gradient (2003) (source: Observatoire économique régional des pêches - Ifremer).

\begin{tabular}{|l|c|c|c|c|c|c|c|c|c|}
\cline { 2 - 10 } \multicolumn{1}{c|}{} & $<7 \mathrm{~m}$ & {$[7-9[\mathrm{~m}$} & {$[9-12[\mathrm{~m}$} & {$[12-16[\mathrm{~m}$} & {$[16-20[\mathrm{~m}$} & {$[20-24[\mathrm{~m}$} & {$[24-40[\mathrm{~m}$} & $>=40 \mathrm{~m}$ & Total \\
\hline Côtier & 5 & 28 & 36 & 59 & 76 & 0 & 0 & 0 & 30 \\
\hline Mixte & & 0 & 56 & 65 & 60 & 63 & 0 & & 60 \\
\hline Large & & 0 & & 91 & 54 & 56 & 0 & 0 & 41 \\
\hline Total & 5 & 28 & 38 & 65 & 60 & 56 & 0 & 0 & 35 \\
\hline
\end{tabular}

\footnotetext{
${ }^{19}$ La technique de l'échantillon constant est utilisée dans le rapport annuel de l'Observatoire, mais uniquement sur le court terme. Chaque année, on construit un échantillon de navires présents dans la base également l'année précédente, pour analyser les évolutions d'une année à l'autre. L'opération dont les résultats sont présentés ici a consisté à transposer cette méthode au long terme.
} 
Le tableau 9 renseigne sur les caractéristiques techniques des segments de longueur. Les navires de 9 à 12 mètres localisés majoritairement en bande côtière sont plus âgés (21,5 ans) que les unités « mixtes » (15,9 ans). Les critères de longueur, de tonnage et de puissance motrice permettent de distinguer, au sein d'une même classe, les bateaux « côtiers », des « mixtes » et ceux du « large ». En effet, le rayon d'action est positivement corrélé à la taille du navire selon ces trois caractéristiques. Ceci est vérifié pour les quatre classes de longueur pour lesquelles deux ou trois gradients sont identifiés ([9-12[ $\mathrm{m}$, [12-16[ m, [16-20[ m, [20-24[ m).

Tableau 9. Caractéristiques techniques selon la classe de longueur et le gradient (2003) (source: Observatoire économique régional des pêches/Ifremer).

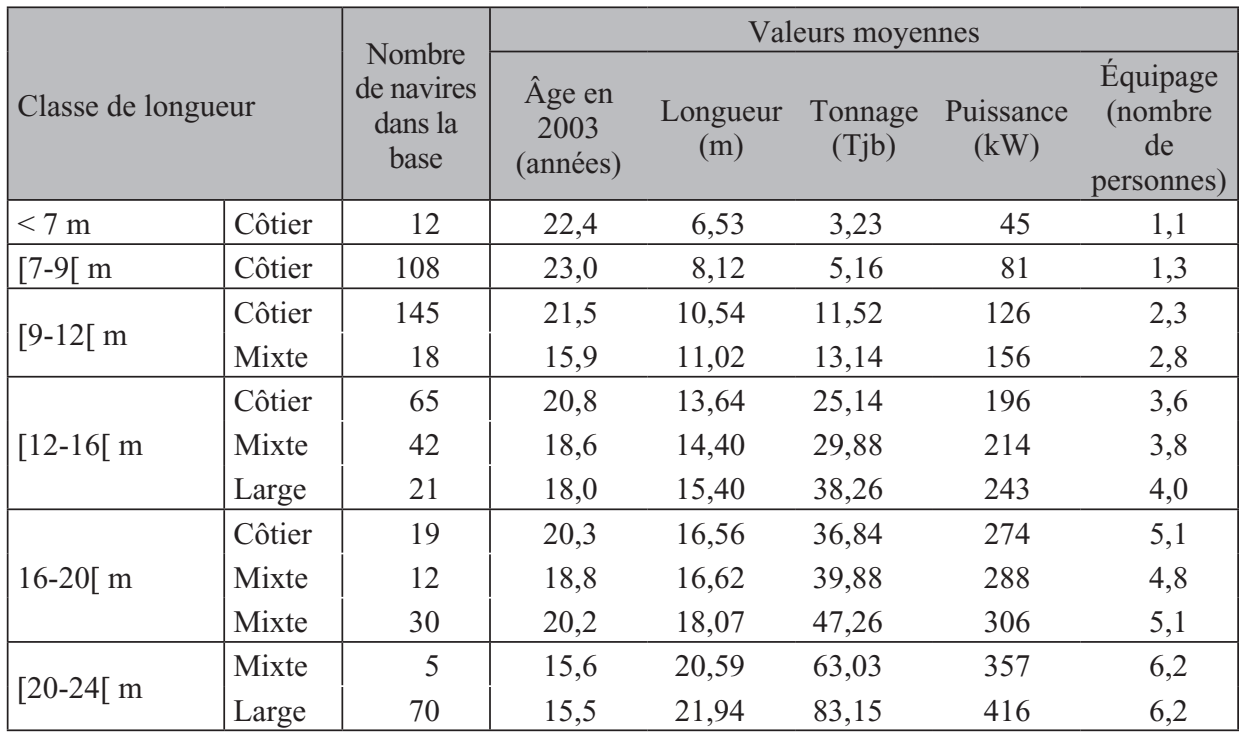

La mesure des performances économiques des unités de pêche s'appuie sur la valeur ajoutée brute unitaire ${ }^{20}$. Considérée comme un indicateur du niveau de production, au même titre que le chiffre d'affaires, la valeur ajoutée brute est rapportée à deux indicateurs d'utilisation des facteurs de production. Le facteur travail est appréhendé selon le nombre d'hommes embarqués. Le facteur capital est approché à partir de la puissance motrice (exprimée en kilowatts, $\mathrm{kW}$ ). Ainsi, le rapport de la valeur ajoutée brute à un facteur de production physique fournit une mesure de la productivité apparente. À la différence de la productivité appréhendée à partir de la production brute (ou chiffres d'affaire), la

\footnotetext{
${ }^{20}$ La valeur ajoutée est égale à la différence entre la valeur de la production (assimilable au chiffre d'affaires en l'absence de variation de stocks) et celle des consommations intermédiaires (biens non durables et services extérieurs consommés dans le processus productif). Elle représente, en première approximation, l'excédent de la valeur créée sur la valeur détruite dans le cadre du processus productif. Cependant, elle ne tient pas compte de la perte de valeur subie par le capital fixe au cours de ce processus, du fait de l'usure ou de l'obsolescence (perte de valeur que la pratique comptable appréhende à travers la notion d'amortissement). C'est pourquoi, il s'agit d'une valeur ajoutée brute.
} 
mesure de performance obtenue en fonction de la valeur ajoutée brute exclut les facteurs intermédiaires ${ }^{21}$ (détruits au cours du cycle de production).

La figure 36 p. VIII présente un indicateur de productivité apparente du facteur travail en comparant la valeur ajoutée brute (VAB) à la taille de l'équipage. Les valeurs extrêmes vont de $22 \mathrm{k} €$ à $78 \mathrm{k} €$ par an et par homme embarqué. Le fait le plus significatif concerne les classes de longueur de plus de 12 mètres. En effet, les navires opérant essentiellement en bande côtière obtiennent des résultats supérieurs aux navires « mixtes » et « larges ». La VAB par tête atteint $64 \mathrm{k} €$ pour les côtiers de 12 à 16 mètres contre 58 à $61 \mathrm{k} €$ pour les unités appartenant aux mêmes classes de longueur mais affectant moins de $75 \%$ de leur temps d'activité dans la bande des 12 milles. Une situation similaire apparait pour les bateaux de 16 à 24 mètres. La productivité apparente du travail est proportionnelle au temps passé en bande côtière. Enfin, les unités de 9 à 12 mètres travaillant majoritairement en bande côtière affichent un résultat proche de celui des navires mixtes de même classe de longueur.

Les résultats économiques sont présentés dans la figure $37 \mathrm{p}$. IX à travers deux indicateurs de productivité apparente du capital. La valeur ajoutée brute est rapportée à la puissance motrice $(\mathrm{kW})$ d'une part, et d'autre part, à la jauge $(\mathrm{Tjb})$.

Les résultats s'échelonnent de 540 euros (côtiers $<7$ mètres) à 1350 euros (mixtes de 20 à 24 mètres) par unité de puissance motrice. La productivité apparente du capital des navires côtiers est supérieure à celle des bateaux mixtes, elle-même plus élevée que celle des unités du large. Cette relation s'explique en partie par une motorisation croissante selon la classe de longueur et en fonction du gradient d'activité (cf. tableau 9). La productivité par unité de jauge brute atteint un seuil maximal de 10500 euros (les mixtes de 9-12 mètres) et un niveau minimal de 5000 euros (20-24 mètres du large). Ce dernier indicateur révèle les moindres performances des unités du large (moins de 6500 euros par $\mathrm{Tjb}$ ), tandis que les côtiers réalisent plus de 7500 euros de VAB par unité de jauge brute.

La répartition de la $\mathrm{VAB}$ totale des navires bretons de moins de 24 mètres en fonction du rayon d'action montre le poids dominant de la pêche pratiquée en bande côtière (figure 38 p. IX). L'extrapolation des résultats moyens, obtenus sur la base de l'échantillon, à la population de navires actifs immatriculés en Bretagne en 2003 indique une part relative de $49 \%$ en faveur des navires côtiers, tandis que la part des navires du large atteint $37 \%$ de la VAB totale.

\section{Résultats économiques par segment de flottille en 2003}

Cette section utilise la base de données comptables de l'observatoire, croisée d'une part avec le gradient d'activité des navires (large, mixte, côtier), et d'autre part, selon le segment de flottille. Finalement, 544 navires ont été identifiés selon ce double croisement.

Les navires de la base ont été répartis en 8 segments (tableau 10):

- fileyeurs exclusifs : navires pratiquant exclusivement les métiers du filet;

- chalutiers exclusifs : navires pratiquement exclusivement les métiers du chalut ;

- dormants polyvalents : navires associant différent métiers relevant des arts dormants (filet, casier, ligne);

\footnotetext{
${ }^{21}$ Les consommations intermédiaires incluent notamment les dépenses en carburant-lubrifiant, les vivres, la glace, le matériel de pêche, les frais d'entretien-réparations, l'assurance. Ces facteurs associent donc des biens et des services.
} 
- dragueurs: navires pratiquant les métiers de la drague, éventuellement en association avec d'autres métiers (sauf le chalut);

- ligneurs exclusifs : navires pratiquant exclusivement les métiers de l'hameçon (ligne, palangre);

- caseyeurs exclusifs : navires pratiquant exclusivement les métiers du casier ;

- chalutiers non exclusifs : navires pratiquant le chalut et d'autres métiers (principalement la drague);

- bolincheurs : navires pratiquant le métier de la bolinche (ou senne tournante).

Tableau 10. Caractéristiques techniques selon le gradient et le segment de flottille (2003) (source: Observatoire économique régional des pêches/Ifremer).

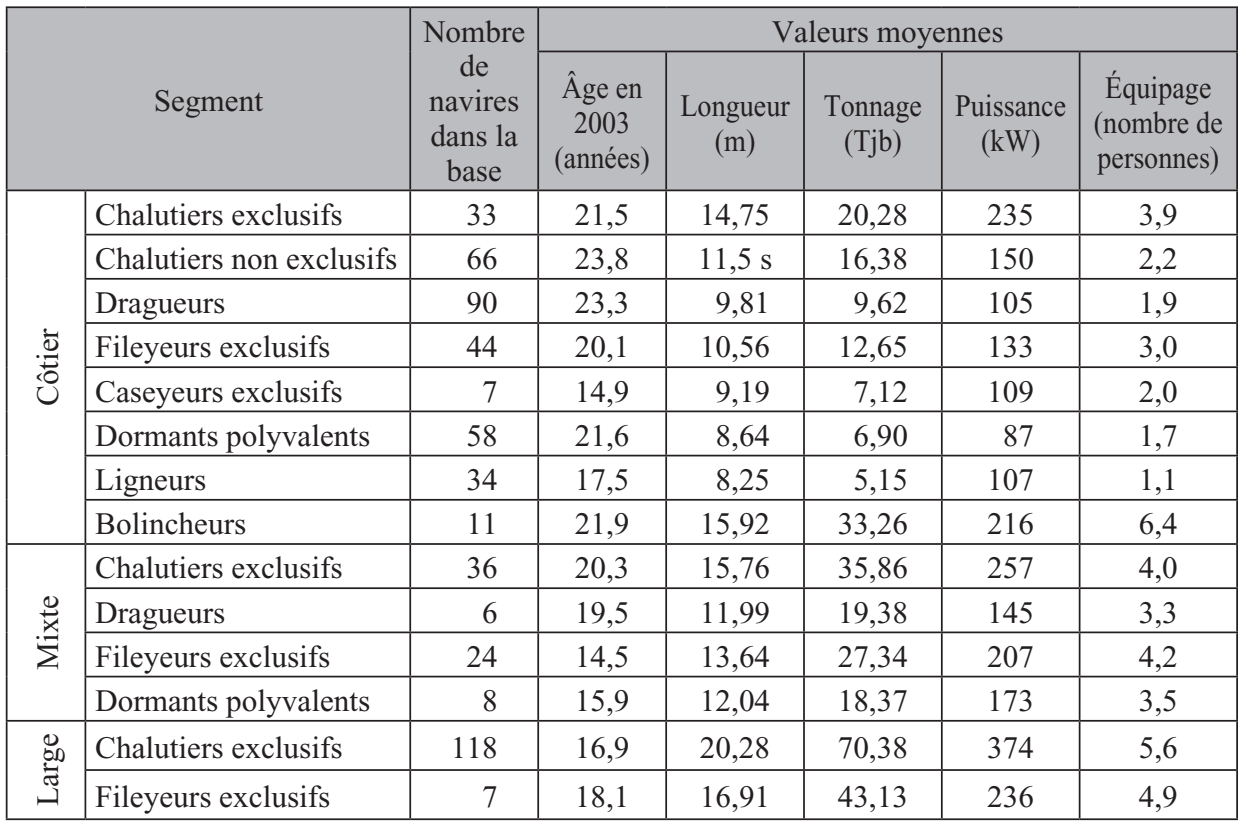

La productivité apparente du travail fluctue de $42 \mathrm{k} €$ (dragueurs côtiers) à $85 \mathrm{k} €$ (bolincheurs) (figure 39 p. IX). La flottille des bolincheurs, opérant essentiellement en bande côtière, devance nettement l'ensemble des autres segments, et notamment les deux flottilles du large (chalutiers exclusifs et fileyeurs exclusifs). Deux flottilles côtières obtiennent un résultat proche des unités du large. Il s'agit des ligneurs et des chalutiers exclusifs.

La productivité du capital approchée par la puissance motrice fait apparaître un résultat modeste pour les ligneurs en regard de la productivité apparente du travail (figure 40 p. X). En revanche, les fileyeurs exclusifs côtiers enregistrent une productivité du capital supérieure à celle de tous les autres segments côtiers (exception faite des bolincheurs), devançant même deux segments mixtes (dormants polyvalents et chalutiers exclusifs). Décrite en fonction de la jauge brute, la productivité du capital des côtiers est supérieure à celle des deux flottilles du large. Elle s'élève à plus de 10000 euros par Tjb pour les côtiers pratiquant les arts dormants, soit un score supérieur à celui de toutes les flottilles mixtes et du large. 


\section{Conclusion}

Les revenus à la pêche dans la bande côtière bretonne ont été appréhendés à partir des résultats économiques issus de la base de données comptables de l'Observatoire économique régional des pêches. L'identification des navires, provenant du Système d'informations halieutiques de l'Ifremer, selon leur répartition spatiale (côtiers, mixtes et large) constitue une innovation majeure dans la production d'informations économiques à la pêche, dont l'étape précédente fut la comparaison méthodologique des indicateurs économiques mesurés à partir des données de l'Observatoire économique régional des pêches de Bretagne et d'enquêtes de l'Ifremer (Boncœur et al., 2004).

L'étude met en évidence des écarts de performances, selon les indicateurs de productivité apparente du travail et du capital, pour l'année 2003. Notamment, les navires d'une même classe de longueur opérant essentiellement en bande côtière obtiennent des productivités apparentes du travail par tête supérieures à celles des navires « mixtes » et du « large ». Certains navires côtiers obtiennent même des niveaux de productivité du travail comparables ou supérieurs à ceux de groupes de navires de plus grande taille. En termes de productivité du capital, la comparaison des mêmes classes de longueur pour les bateaux de plus de 12 mètres indique des résultats supérieurs pour les unités d'avantage présentes en bande côtière. Ainsi, la productivité apparente du capital des navires côtiers est supérieure à celle des bateaux mixtes, elle-même plus élevée que celle des unités du large. Ici encore, certains navires côtiers obtiennent même des niveaux de productivité du capital comparables ou supérieurs à ceux de groupes de navires de plus grande taille.

L'extrapolation des résultats obtenus sur l'échantillon montre que les navires de moins de 24 mètres opérant essentiellement en bande côtière représentent la moitié de la VAB pour l'année 2003. La contribution des unités du large, consacrant plus de $75 \%$ de leur temps de pêche hors de la bande côtière, est de $37 \%$.

La présentation des résultats par segment de flottille a permis d'isoler le cas des bolincheurs mais également celui des ligneurs. Ces deux segments côtiers affichent des résultats supérieurs ou proches de flottilles mixtes et celle du large en terme de productivité apparente du travail. La situation est différente si l'indicateur utilisé est celui de la productivité du capital. Trois flottilles côtières dégagent des performances supérieures à des segments mixtes et larges. Il s'agit des bolincheurs, des fileyeurs exclusifs et des chalutiers exclusifs.

\section{Situation en 2005 et évolution depuis 1998}

L'analyse des revenus à la pêche est menée à partir des données comptables de l'Observatoire économique régional des pêches de Bretagne. La première partie décrit la méthodologie et la composition de l'échantillon constant portant sur les années 2004 et 2005. Les résultats économiques de l'année 2005 sont présentés dans la seconde partie. La ventilation des produits (approchés par le montant des ventes brutes) et des charges d'exploitation y est précisée. La troisième partie est consacrée à une étude rétrospective de trois flottilles importantes, sur la période 1998-2005. Enfin, la dernière partie traite de l'impact du coût du carburant sur les revenus.

\section{Méthodologie et échantillon}

La population de référence est la flottille de pêche bretonne constituée de 1530 navires au 31 décembre 2005, dont 47 unités inactives (Ifremer-DPMA). Les navires de 24 mètres 
et plus sont peu représentés au sein de la base de l'observatoire breton ( 5 unités pratiquant le chalutage et dont la longueur n'excède pas 25 mètres).

Parmi les navires de l'échantillon (base de l'observatoire en 2005) pratiquant les arts traînants (chalutiers exclusifs, chalutiers-dragueurs et goémoniers-coquilliers), 150 ont une longueur inférieure à 12 mètres, représentant $38 \%$ des navires de cette catégorie immatriculés en Bretagne en 2005. 189 unités ont une longueur comprise entre 12 et 24 mètres, soit $63 \%$ de la population de référence.

Les arts dormants regroupent les fileyeurs, les caseyeurs, les bolincheurs et les canots. 170 navires ont une longueur de moins de 12 mètres, soit $28 \%$ des unités bretonnes. Cinquante-six navires pratiquant les arts dormants sont classés dans le segment des 12-24 mètres, représentant $62 \%$ de l'effectif total en Bretagne.

Le taux d'échantillonnage global atteint $37 \%$ de la population de référence sur l'échantillon 2005 et $31 \%$ sur l'échantillon constant 2004-2005 (tableau 11).

Tableau 11. Population mère et échantillon en 2005

(Sources: Ifremer/SIH-DPMA BCS-Observatoire économique régional des pêches de Bretagne).

\begin{tabular}{|c|r|r|r|r|r|r|}
\hline & $\begin{array}{c}\text { Classe } \\
\text { de longueur }\end{array}$ & $\begin{array}{c}\text { Échantillon } \\
2005\end{array}$ & $\begin{array}{c}\text { Taux (\%) } \\
\text { d'échan- } \\
\text { tillonnage } \\
2005\end{array}$ & $\begin{array}{c}\text { Échantillon } \\
\text { constant } \\
2004-2005\end{array}$ & $\begin{array}{c}\text { Taux (\%) } \\
\text { d'échan- } \\
\text { tillonnage } \\
\text { 2004-2005 }\end{array}$ & $\begin{array}{c}\text { Flottille } \\
\text { bretonne au } \\
31-12-2005\end{array}$ \\
\hline \multirow{3}{*}{ Arts traînants } & $<12 \mathrm{~m}$ & 150 & 38 & 113 & 28 & 400 \\
\cline { 2 - 7 } & {$[12-24 \mathrm{~m}[$} & 189 & 63 & 175 & 58 & 301 \\
\cline { 2 - 7 } & $>=24 \mathrm{~m}$ & 5 & 9 & 5 & 9 & 54 \\
\hline \multirow{3}{*}{ Arts dormants } & $<12 \mathrm{~m}$ & 170 & 28 & 149 & 24 & 615 \\
\cline { 2 - 7 } & {$[12-24 \mathrm{~m}[$} & 56 & 62 & 35 & 39 & 90 \\
\hline Inactifs & $>=24 \mathrm{~m}$ & 0 & 0 & 0 & 0 & 23 \\
\hline Total & & 0 & 0 & 0 & 0 & 47 \\
\hline
\end{tabular}

Le Cedem et le département d'économie maritime de l'Ifremer ont entrepris en 2004 un programme de recherche sur la typologie des flottilles bretonnes (Boncœur et al., 2004; Le Floc'h et al., 2006). Les travaux engagés dans ce programme ont abouti à une redéfinition des flottilles représentées dans le rapport annuel de l'Observatoire économique régional des pêches de Bretagne (Observatoire économique régional des pêches de Bretagne, 2006).

Les navires sont affectés à l'une des 7 flottilles identifiées selon le principal engin utilisé :

- les chalutiers exclusifs: les chalutiers exclusifs pratiquent en exclusivité les métiers du chalut;

- les chalutiers-dragueurs : les engins utilisés sont le chalut de fond et/ou la drague et à titre complémentaire des engins dormants;

- les goémoniers-coquilliers : les engins utilisés sont le scoubidou pour l'exploitation des algues (principalement les laminaires) et dans de nombreux cas, la drague (principalement pour la coquille Saint-Jacques);

- les fileyeurs : les engins utilisés sont le filet (filet à poissons et filet à crustacés), et à titre complémentaire d'autres engins dormants (casier et ligne); 
- les caseyeurs : les engins utilisés sont le casier (casier à crustacés, à crevettes), et à titre complémentaire d'autres engins dormants (filet et ligne);

- les bolincheurs: l'engin utilisé est la bolinche ou senne tournante;

- les canots: les engins utilisés sont la ligne ou la palangre (ligne ou palangre à bars notamment) et à titre complémentaire d'autres engins dormants (filet et casier, ou divers métiers).

Le tableau 12 présente les caractéristiques techniques moyennes des navires sur la base de l'échantillon constant 2004-2005. Les navires pratiquant les arts traînants sont identifiés au sein de quatre segments (trois classes de longueur pour les chalutiers exclusifs et un segment pour les chalutiers-dragueurs intégrant également les goémoniers-coquilliers). Les unités relevant principalement des arts dormants (filet, casier, bolinche, métiers de la ligne) sont dissociées en deux classes de longueur (plus ou moins de 12 mètres).

Tableau 12. Caractéristiques techniques des échantillons constants 2004-2005 (source: Observatoire économique régional des pêches).

\begin{tabular}{|c|c|c|c|c|c|c|}
\hline \multirow[b]{2}{*}{ Échantillon } & \multirow[b]{2}{*}{$\begin{array}{c}\text { Nombre } \\
\text { de navires } \\
\text { dans la } \\
\text { base }\end{array}$} & \multicolumn{5}{|c|}{ Valeurs moyennes } \\
\hline & & $\begin{array}{c}\text { Âge en } \\
2005 \\
\text { (années) }\end{array}$ & $\begin{array}{l}\text { Longueur } \\
\text { (m) }\end{array}$ & $\begin{array}{c}\text { Tonnage } \\
(\mathrm{Tjb})\end{array}$ & $\begin{array}{c}\text { Puissance } \\
(\mathrm{kW})\end{array}$ & $\begin{array}{l}\text { Équipage } \\
\text { (nombre } \\
\text { de per- } \\
\text { sonnes) }\end{array}$ \\
\hline Chalutiers exclusifs $<16 \mathrm{~m}$ & 44 & 22,1 & 14,9 & 33,7 & 225 & 3,5 \\
\hline Chalutiers exclusifs $16-20 \mathrm{~m}$ & 39 & 20,8 & 17,2 & 42,6 & 303 & 4,9 \\
\hline Chalutiers exclusifs $>20 \mathrm{~m}$ & 68 & 16,7 & 22,3 & 88,2 & 430 & 6,1 \\
\hline Chalutiers dragueurs & 142 & 24,7 & 10,6 & 12,4 & 131 & 2,2 \\
\hline Dormants $<12 \mathrm{~m}$ & 149 & 21,3 & 9,6 & 7,9 & 107 & 1,8 \\
\hline Dormants $\geq 12 \mathrm{~m}$ & 35 & 16,5 & 14,4 & 31,2 & 220 & 4,5 \\
\hline Ensemble des navires & 477 & 21,3 & 13,2 & 27,6 & 195 & 3,2 \\
\hline
\end{tabular}

\section{Résultats économiques en 2005}

La situation économique des armements artisans bretons en 2005 est décrite à travers la ventilation des ventes brutes, sur la base de l'échantillon 2005 (570 navires). La segmentation de l'échantillon répond au double critère de la flottille d'appartenance (ellemême définie selon l'engin de pêche utilisé) et de la classe de longueur. Au sein de cet échantillon, seize segments sont identifiés. Le montant moyen des ventes brutes s'étale de $250 \mathrm{k} €$ à $750 \mathrm{k} €$ pour les unités de plus de 12 mètres (les bolincheurs ont tous des longueurs supérieures à 12 mètres) (figure 41). La production débarquée des navires de moins de 12 mètres est comprise entre 60 et $210 \mathrm{k} €$ (figure 41). La part des consommations intermédiaires et des taxes dans le montant des ventes brutes varie de $28 \%$ pour les bolincheurs à $57 \%$ pour les chalutiers exclusifs de plus de 20 mètres. Si la rémunération brute de l'équipage atteint généralement plus de $30 \%$ des ventes brutes (avec un seuil maximal de $47 \%$ chez les bolincheurs), elle reste en deçà de $30 \%$ chez les navires de taille inférieure à 9 mètres (seulement $7 \%$ pour les canots). Le mode de rémunération de l'équipage, traditionnellement fondé sur le système à la part, n'est pas toujours appliqué sur les unités de moins de 12 mètres. Sur les navires ayant un seul homme à bord, l'excédent brut d'exploitation (EBE) englobe généralement le revenu du travail. Cette particularité traduit en partie la proportion plus élevée de l'EBE chez les dormants de moins de 12 mètres. 


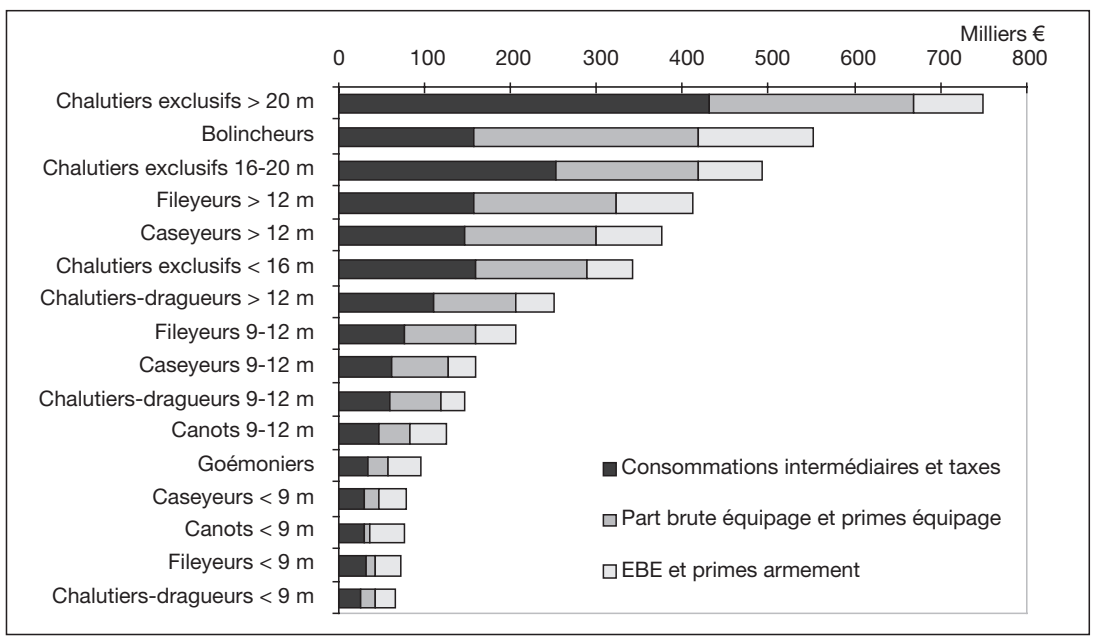

Figure 41. Ventes brutes, rémunération brute de l'équipage et de l'armement en 2005 (source : Observatoire économique régional des pêches).

La comparaison des indicateurs économiques en 2004 et 2005 est menée à partir de l'échantillon constant constitué de 477 navires (figure 42). La situation des navires pratiquant les arts traînants s'est dans l'ensemble détériorée en 2005, en particulier en termes d'EBE. Cet indicateur a reculé de $7 \%$ à $13 \%$. Seuls les chalutiers exclusifs de 16-20 mètres et les chalutiers-dragueurs de moins de 9 mètres ont enregistré une amélioration de l'EBE (avec cependant un léger recul des ventes brutes pour les chalutiers de 16-20 mètres). Une situation globalement inversée apparaît dans le cas des unités pratiquant les arts dormants. À l'exception des caseyeurs de plus de 12 mètres, tous les segments bénéficient en 2005 d'une progression des ventes brutes (de $2 \%$ à $10 \%$ ). Cette amélioration se traduit généralement par une augmentation de l'EBE (notamment pour les goémoniers).

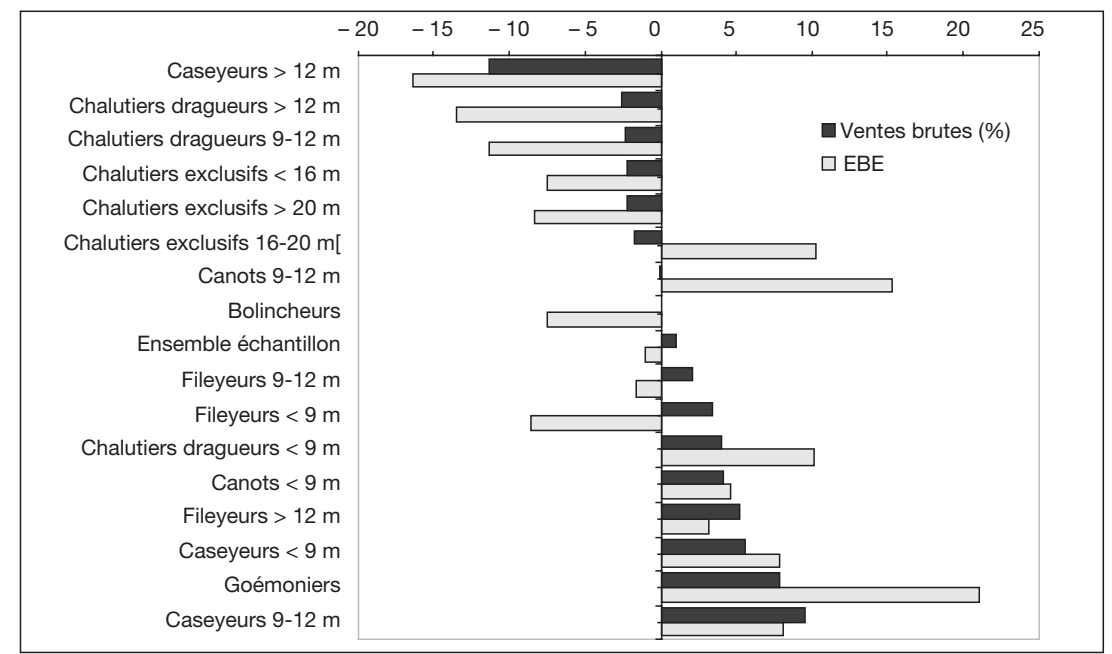

Figure 42. Évolution (en \%) des ventes brutes et de l'EBE entre 2004 et 2005 (en euros constants 2005) (source : Observatoire économique régional des pêches). 
L'évolution des ventes brutes est le résultat d'un effet prix et d'un effet quantité des espèces débarquées. Les éléments permettant l'étude de la structure des débarquements proviennent d'une base de données distincte de la base d'information de type comptable, ce qui pose certains problèmes.

La source d'information sur les débarquements ne reflète pas obligatoirement l'élément comptable des ventes brutes et ceci pour deux raisons essentielles. La première est liée à la disponibilité des données. Un navire renseigné dans la base comptable de l'Observatoire n'est pas obligatoirement présent dans la base de débarquement, et réciproquement. La seconde explication relève du mode de commercialisation des espèces. Les informations sur les débarquements sont collectées au sein des criées. Par conséquent, l'ensemble des produits de la pêche commercialisés hors du circuit des criées n'est pas comptabilisé dans la base gérée par l'Observatoire. Il peut donc persister, en particulier pour les navires de moins de 12 mètres, un écart important entre le montant des ventes brutes inscrit en tant qu'élément comptable et celui calculé en fonction des débarquements en criée.

Compte tenu de ces limites, les informations retenues sur l'évolution des prix et des quantités ne concernent que 7 flottilles (les bolincheurs et caseyeurs ne sont pas représentés), pour un échantillon total de 185 navires. L'ensemble de l'échantillon subit un léger recul des prix et une stabilité des quantités débarquées (figure 43). Les chalutiers exclusifs de moins de 20 mètres accusent une baisse importante des quantités ( $-7 \%$ à $-17 \%$ ) tandis que les chalutiers de plus de 20 mètres augmentent de $6 \%$ les volumes débarqués, accompagnés d'un recul des prix de même ampleur.

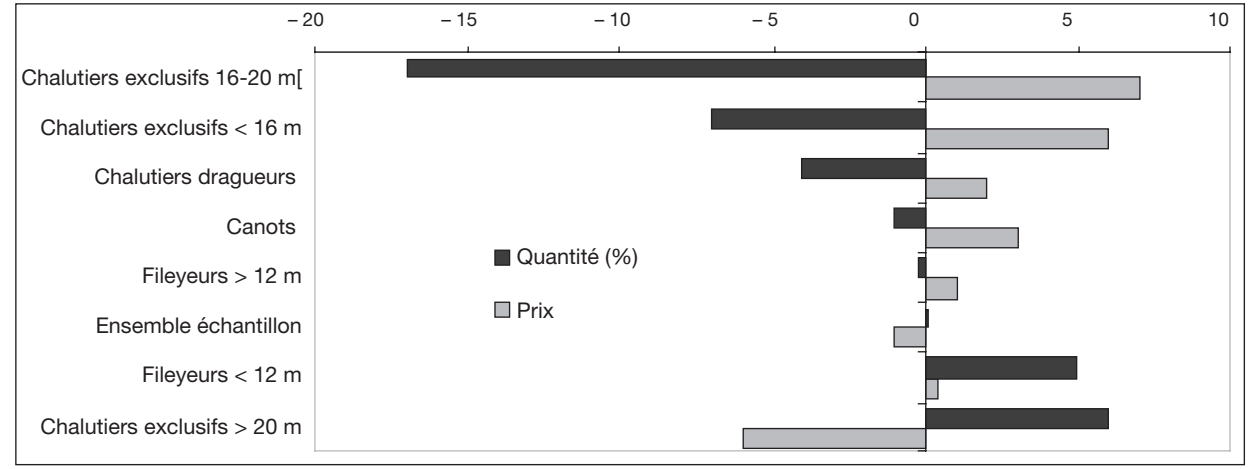

Figure 43. Évolution (en \%) des prix et des quantités entre 2004 et 2005 (en euros constants 2005, ventes en criée) (source : Observatoire économique régional des pêches).

La valeur des trois premières espèces débarquées en 2005 représente au minimum $43 \%$ des ventes brutes moyennes chez les chalutiers exclusifs de plus de 20 mètres, et au plus $81 \%$ chez les canots (figure 44 p. X). Si la baudroie, la langoustine et la sole figurent historiquement parmi les principales productions ciblées par les chalutiers exclusifs, l'apparition du saint-Pierre en troisième position chez les plus de 20 mètres est une situation inédite depuis une décennie.

La ventilation du chiffre d'affaires moyen met en avant le poids du composant énergétique chez les chalutiers, et à l'inverse, la part plus faible de la rémunération de l'armement (EBE et primes armement) chez ces mêmes navires (figure 45 p. X). À l'opposé, les dormants de moins de 12 mètres sont faiblement sensibles à la dépense de carburant mais 
consacrent plus de $30 \%$ du chiffre d'affaires à la rémunération de l'armement (la règle du système à la part est en effet peu adaptée aux équipages constitués d'un seul homme à bord). La situation en 2005 a été profondément modifiée en raison de la hausse importante du prix du carburant pour les flottilles de chalutiers exclusifs. Ainsi, la part des dépenses énergétiques s'est élevée à $26 \%$ du chiffre d'affaires chez les unités de plus de 20 mètres, soit 8 points de plus qu'en 2004. Si cette augmentation est moins marquée chez les chalutiers de moins de 20 mètres, elle reste cependant significative (plus de 7 points pour les 16-20 mètres et plus 6 points pour les 12-16 mètres). Les rémunérations de l'équipage et de l'armement n'ont pas été affectées. Les autres charges d'exploitation (hors salaires et carburant) ont compensé, par une baisse d'ampleur similaire à la hausse des dépenses de carburant, cette élévation du poste énergétique dans la ventilation du chiffre d'affaires. Les autres flottilles (chalutiers dragueurs et dormants) ont peu souffert de cette évolution (moins de $3 \%$ d'augmentation du poste carburant dans le chiffre d'affaires en 2005).

\section{Analyse rétrospective sur la période 1998-2005}

Une analyse rétrospective a été entreprise pour trois flottilles, sans distinction de taille, sur la période 1998-2005 (tableau 13). Cette analyse requiert la constitution d'un échantillon constant de navires présents dans la base comptable de l'Observatoire ${ }^{22}$.

Tableau 13. Caractéristiques techniques des échantillons constants 1998-2005 (source: Observatoire économique régional des pêches)

\begin{tabular}{|c|c|c|c|c|c|c|}
\hline \multirow[b]{2}{*}{ Échantillon } & \multirow{2}{*}{$\begin{array}{l}\text { Nombre } \\
\text { de navires } \\
\text { dans la } \\
\text { base }\end{array}$} & \multicolumn{5}{|c|}{ Valeurs moyennes } \\
\hline & & $\begin{array}{c}\text { Âge en } \\
2005 \\
\text { (années) }\end{array}$ & $\begin{array}{l}\text { Longueur } \\
\text { (m) }\end{array}$ & $\begin{array}{l}\text { Tonnage } \\
(\mathrm{Tjb})\end{array}$ & $\begin{array}{l}\text { Puissance } \\
(\mathrm{kW})\end{array}$ & $\begin{array}{l}\text { Équipage } \\
\text { (nombre de } \\
\text { personnes) }\end{array}$ \\
\hline Chalutiers exclusifs & 95 & 20,7 & 18,4 & 56,8 & 326 & 5,0 \\
\hline Chalutiers dragueurs & 32 & 26,5 & 10,9 & 13,9 & 135 & 2,2 \\
\hline Dormants & 64 & 20,0 & 10,2 & 11,6 & 140 & 2,3 \\
\hline Ensemble des navires & 191 & 21,5 & 14,4 & 34,2 & 231 & 3,2 \\
\hline
\end{tabular}

La figure 46 p. XI retrace, d'une part, l'évolution du chiffre d'affaires (CA) et des rémunérations de l'équipage et de l'armement, et d'autre part, l'évolution des charges moyennes hors salaires, pour l'ensemble de l'échantillon constitué de 191 unités. Si la période étudiée est marquée par une relative stabilité du chiffre d'affaires et des salaires bruts, on observe une nette dégradation de la rémunération de l'armement (EBE et primes armement) en 2004 et 2005. Le taux de croissance instantanée de l'EBE est de - $4 \%$ sur la période 1998-2005 ${ }^{23}$. Cette évolution reflète en partie la forte hausse des dépenses de carburant. Le coût énergétique a en effet plus que doublé entre 1998 et 2005 pour l'ensemble des navires. Une évolution à la baisse du chiffre d'affaires, même marginale, accompagnée d'une élévation du poste carburant conduit mécaniquement à une érosion importante de la rémunération du capital. Pour compenser ce phénomène, les armements ont comprimé les autres charges (hors salaires) en 2005, au sein desquels figurent notamment les dépenses

\footnotetext{
${ }_{22}$ Un degré de liberté d'une année a été conservé: un navire peut faire partie de l'échantillon s'il a été renseigné sur la période au moins 7 années sur 8.

${ }^{23}$ La fonction log-linéaire est de la forme suivante: $\ln (\mathrm{EBE}+$ primes $)=-0,0401 \times$ Année $+91,395$, avec un $\mathrm{r}^{2}$ égal à $63 \%$.
} 
de matériel de pêche, d'entretien et de réparation. En 2004, ces dépenses représentaient $15,8 \%$ du chiffre d'affaires, et $14,7 \%$ en 2005.

Une forte proportion de la flottille de chalutiers dragueurs est localisée dans les ports riverains ou voisins de la baie de Saint-Brieuc (Erquy, Saint-Quay-Portrieux, Paimpol), à proximité de la pêcherie de coquilles Saint-Jacques. La situation économique de ces navires, inféodés aux pêcheries situées dans la bande côtière des 12 milles nautiques, s'est particulièrement dégradée en 2005 (figure 47 p. XI et XII). L'érosion de la rémunération de l'armement (EBE et primes armement) est nette sur la dernière année. La stabilité du chiffre d'affaires en 2004, et ce depuis 2001, a permis d'amortir l'augmentation des dépenses de carburant et des autres charges (hors salaires), survenue en 2004. Au contraire, chiffre d'affaires et rémunération de l'équipage ont évolué à la baisse en 2005 et retrouvent un niveau proche de celui atteint en 1999, au moins pour le chiffre d'affaires. Le poste carburant a progressé en 2001, tandis que l'ensemble de l'échantillon enregistrait une baisse.

Les chalutiers exclusifs, principalement localisés en Bretagne Sud (quartiers maritimes du Guilvinec, de Concarneau et de Lorient) sont les unités les plus affectées par la hausse du prix du carburant. Les dépenses énergétiques ont été multipliées par 2,5 en 2005 par rapport à 1998 (figure 48 p. XII). Sur la même période, les rémunérations des équipages ont peu évolué, à l'exception d'une baisse en 2000 (reflétant déjà l'impact du prix du carburant). La baisse de la rémunération de l'armement atteint en moyenne annuelle $6,7 \%{ }^{24}$. À chiffre d'affaires quasi constant (stabilité sur la période d'étude), les charges d'exploitation (hors salaires, taxes et cotisations) accusent un recul significatif en 2005, de l'ordre de - $22 \%$ par rapport à l'année 1998. Les dépenses d'entretien-réparation et de matériel de pêche représentent la moitié de ces charges et sont en partie assimilées à un coût fixe.

Les dormants sont les seules unités à éviter une érosion de la rémunération de l'armement en fin de période (figure 49 p. XIII). La situation économique de ces navires se maintient en 2005 à un niveau comparable à celui qui a été constaté en 2003 et 2004. Les salaires bruts ont même atteint en 2005 le niveau le plus élevé sur la période. Si l'augmentation du coût énergétique est près de deux fois supérieure en 2005 à ce qu'il était en 1998, cette évolution n'affecte pas sensiblement les revenus.

\section{Impact du prix du carburant}

Les analyses précédentes sur l'évolution des revenus à la pêche ont clairement mis en évidence le poids stratégique du carburant chez les chalutiers exclusifs. Si la forte hausse du prix de ce facteur en 2005 n'est pas en soi un fait surprenant (une situation similaire mais de plus faible ampleur était apparue en 2000), elle a provoqué au sein du secteur une prise de conscience de la nécessité d'intégrer ce phénomène.

\section{Évolution du prix du carburant}

L'évolution du prix courant du gazole est retracée sur la figure 50 p. XIII. Les statistiques disponibles depuis 1985 proviennent de la Direction des ressources énergétiques et minérales (Direm) du ministère de l'Économie et des Finances. Elles traduisent les prix hors taxes (TVA, TIPP et autres taxes) des sociétés déclarant leur prix hebdomadaire

\footnotetext{
${ }^{24}$ Ce taux de croissance instantané est très proche de la hausse annuelle du prix du carburant sur la période 19942005 qui s'élève à 6,9\%. La fonction log-linéaire de l'évolution de la rémunération de l'armement des chalutiers exclusifs est du type : $\ln (\mathrm{EBE}+$ primes $)=-0,0674 \times$ Année $+146,18$, avec un $\mathrm{r}^{2}$ égal à $77 \%$.
} 
auprès de la Direm. Les prix annuels du gazole pêche (fournis par la Coopérative maritime du Pays bigouden), inférieurs de 10 à $16 \%$ aux prix nationaux, sont décrits sur la même figure depuis 1994.

La hausse intervenue en 2000 avait été compensée par un allégement des charges sociales et une exonération de la taxe criée et de la redevance d'équipement des ports de pêche (Observatoire économique régional des pêches, 2002). Si cette augmentation a laissé place à un recul du prix du gazole jusqu'en 2002, l'augmentation actuelle s'inscrit davantage dans une perspective de long terme, amenant les acteurs à explorer de nouvelles pistes visant à réduire la consommation de combustible.

La montée spectaculaire des cours du pétrole depuis 2003 est donc à relativiser à l'échelle des deux dernières décennies. En effet, le cours du pétrole en euro courant pour l'année 1985 était à mi-chemin des cours constatés en 2004 et 2005. En euro constant sur la base de l'année 2005, le prix au litre du carburant à la pêche a augmenté de 6,9\% par an depuis 1994 (figure 51 p. XIV).

\section{Application d'un mécanisme de compensation}

La sensibilité des armements à la pêche au coût du facteur énergétique, en particulier les navires utilisant les arts traînants, a conduit les acteurs à intégrer cette contrainte durable dans la gestion de leur outil de production. Cela s'est traduit par la création d'un nouvel outil de compensation en 2004, baptisé FPAP (fonds de prévention des aléas à la pêche). Ce nouvel outil a été institué en novembre 2004. Ce nouveau mécanisme a pour vocation principale de réduire l'impact du prix du carburant ${ }^{25}$.

Le FPAP, constitué sous forme de syndicat professionnel, a été créé le 9 avril 2004, sous l'impulsion de la Confédération de la coopération, de la mutualité et du crédit maritimes. Le FPAP intervient sur les marchés à terme du pétrole pour acquérir des options financières auprès d'établissements financiers spécialisés. Selon les options d'achats (Call) souscrites par le FPAP auprès d'établissements financiers, ceux-ci reversent au FPAP la différence entre la cotation moyenne mensuelle de l'indice de référence pour les produits pétroliers et le seuil maximal (dans le seul cas où la cotation mensuelle excède le seuil maximal de l'option choisie). Cette différence est ensuite reversée aux adhérents du FPAP ${ }^{26}$.

La mise en place de cet outil à l'échelle nationale a eu deux effets sur la structure des charges d'exploitation. Le premier, sans conséquence notable sur les revenus à la pêche, concerne la perception par le FPAP de la cotisation des pêcheurs adhérents ${ }^{27}$. Le second effet porte sur le montant de l'indemnisation perçue au titre du FPAP. Ce produit vient en

\footnotetext{
${ }^{25}$ Le montant de l'indemnisation est intégré aux frais communs dans le compte d'exploitation des entreprises de pêche artisanale (Observatoire économique régional des pêches, 2006).

${ }^{26}$ L'état français a participé au financement du FPAP. Cette intervention financière a fait l'objet, le 19 avril 2006, de l'ouverture de la procédure formelle d'examen de la part de la Commission européenne (Union Européenne, 2006). La Commission européenne considère en effet que la participation de l'État français à hauteur de 65 millions d'euros au 19 avril 2006 au FPAP relève d'une mesure discriminatoire à l'égard des autres secteurs d'activité économique à l'échelle européenne. Une enveloppe supplémentaire de 22 millions d'euros a été versée par l'État au FPAP au second semestre 2006. La contribution financière des pêcheurs adhérents s'élève à 4 millions d'euros.

${ }^{27}$ Il est d'ailleurs difficile de distinguer dans les comptes d'exploitation, le montant annuel pour chaque entreprise de pêche de la cotisation au FPAP. Celui-ci est compris dans les « diverses charges externes de l'armement ». Le montant de la cotisation implique un droit d'adhésion de 150 euros et une cotisation annuelle pour la couverture de risques d'un montant minimum de 200 euros. Cette cotisation annuelle pour l'année 2005 est calculée pour un volume de carburant sur une base de 0,00 euros par litre.
} 
déduction des charges supportées par l'équipage ${ }^{28}$ et a nécessité la construction d'un nouvel agrégat dans les comptes d'exploitation. Dans la mesure où cet encaissement répond directement à la forte hausse du prix du carburant pour l'année 2005, il convient de comparer le coût brut (sans indemnité FPAP) du carburant en 2005 et le coût net (après déduction de cette indemnité) supporté par les navires. La figure 52 p. XIV met en rapport la dépense énergétique par flottille (sur la base de l'échantillon 2004-2005 de 477 navires) en pourcentage des ventes brutes pour les années 2004 et 2005. La hausse réelle du poste gazole a été limitée à $2 \%$ chez les chalutiers dragueurs et à $1 \%$ chez les chalutiers exclusifs.

$\mathrm{Si}$ la tendance à une élévation du prix des énergies fossiles sur la longue période est désormais une certitude (Brook et al., 2004), l'ajustement des comportements en matière de consommation physique des produits pétroliers (que ce soit à usage final ou intermédiaire) obéit essentiellement à un horizon de courte période. Sur la période 1998-2005, on observe une baisse de la consommation physique de carburant pour l'ensemble des flottilles (base de 191 unités). Cette baisse atteint $20 \%$ en 2005, comparée au niveau de consommation en 1998 (pour un montant de chiffre d'affaires identique) (figure 53 p. XIV). Cette tendance concerne essentiellement les flottilles des chalutiers dragueurs et des dormants pour lesquelles une réduction annuelle de $2 \%$ est constatée. Plus sensibles au coût du carburant, les chalutiers exclusifs ont réduit plus faiblement leur consommation physique $(-0,4 \%$ par an) sur la période 1998-2005 et maintiennent leur niveau d'utilisation énergétique depuis 2000.

Le comportement des unités les plus dépendantes du facteur gazole ne semble donc pas avoir été modifié au cours de cette période affectée par deux mouvements de hausse du prix du pétrole ${ }^{29}$.

Une analyse centrée sur les chalutiers exclusifs montre que la hausse du prix du carburant entre 2004 et $2005^{30}$ n'a pas entraîné une modification des comportements en termes de consommation physique au sein des trois segments (tableau 14). L'ensemble de ces navires a utilisé environ 340 tonnes de carburant en 2004 comme en 2005. Le mécanisme de compensation créé en 2004 (FPAP) a neutralisé totalement l'effet prix.

Tableau 14. Consommation moyenne (en tonnes) de carburant des chalutiers exclusifs en 2004 et 2005 (source: Observatoire économique régional des pêches).

\begin{tabular}{|l|c|c|c|}
\cline { 2 - 4 } \multicolumn{1}{c|}{} & Échantillon & $\begin{array}{c}\text { Consommation } \\
\text { moyenne en 2004 }\end{array}$ & $\begin{array}{c}\text { Consommation } \\
\text { moyenne en 2005 }\end{array}$ \\
\hline Chalutiers exclusifs $<16 \mathrm{~m}$ & 44 & 163 & 163 \\
\hline Chalutiers exclusifs $12-16 \mathrm{~m}$ & 38 & 288 & 291 \\
\hline Chalutiers exclusifs $>20 \mathrm{~m}$ & 66 & 489 & 483 \\
\hline Ensemble des chalutiers exclusifs & 148 & 340 & 339 \\
\hline
\end{tabular}

\footnotetext{
${ }^{28}$ La structure des comptes à la pêche artisanale (Le Pichon, 1978) distingue en effet les charges d'équipage et les charges d'armement après déduction de frais communs à ces deux éléments. Il est d'usage de considérer dans ces frais communs les dépenses de carburant.

${ }^{29}$ Du point de vue de l'analyse conjoncturelle, traitant des faits économiques du présent et d'un passé proche, un évènement extérieur (prévisible ou aléatoire) assimilé à un choc exogène devrait entraîner une modification du comportement des acteurs. Or, la consommation physique de carburant des chalutiers exclusifs n'apparaît pas modifiée à la suite des « deux chocs pétroliers » de l'année 2000 et 2005, comme si aucun mouvement conjoncturel n'était survenu.

${ }^{30}$ Le prix du gazole pêche a augmenté de $35 \%$ entre ces deux années, alors que l'évolution annuelle depuis 1994 atteint $6,9 \%$.
} 
L'examen des comportements individuels (figure 54) montre une dispersion symétrique des comportements en matière de consommation énergétique. La marge de fluctuation des comportements individuels se situe entre $-20 \%$ et $+20 \%$, une fois écartées les valeurs extrêmes (lesquelles atteignent $-67 \%$ et $+58 \%$ pour le segment des 20-25 mètres). La valeur du premier quartile est comprise entre $-5 \%$ et $-7 \%$ et celle du troisième quartile se situe entre $5 \%$ et $8 \%$ selon le segment de chalutiers.

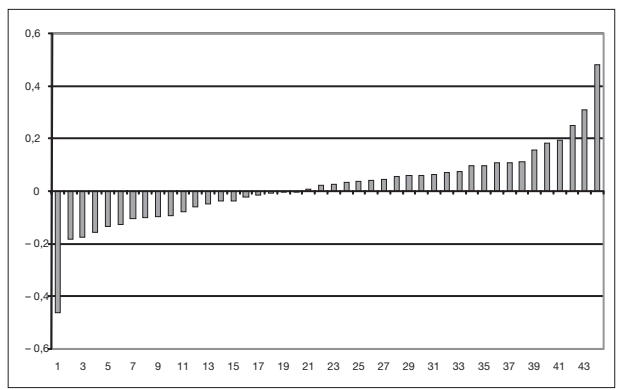

(a) Chalutiers exclusifs $12-16 \mathrm{~m}$

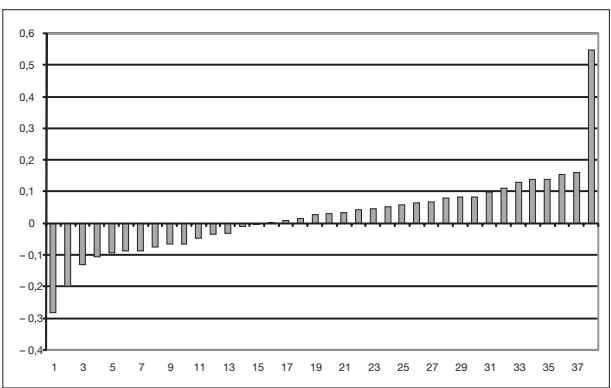

(b) Chalutiers exclusifs $16-20 \mathrm{~m}$

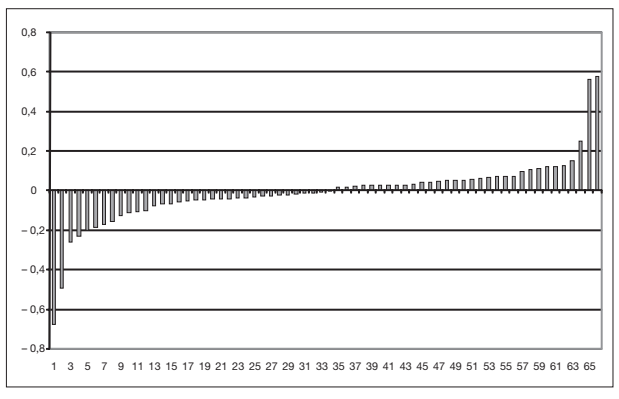

(c) Chalutiers exclusifs $20-25 \mathrm{~m}$

Figure 54. Coût du carburant (brut et net en \%) des ventes en 2004 et 2005 (source : Observatoire économique régional des pêches).

\section{Conclusion}

L'examen des revenus à la pêche en 2005 met en avant l'impact du coût du carburant. Si les navires pratiquant les arts dormants semblent peu affectés par l'augmentation du gazole, les chalutiers exclusifs accusent un recul net de la rémunération du capital (EBE et primes armement), déjà entamé lors des années antérieures. La mise en place d'un outil compensatoire a permis de neutraliser l'impact du coût énergétique, en particulier sur les revenus du travail, en 2005. Se pose cependant le problème du financement d'un tel mécanisme, notamment dans une perspective de hausse du coût énergétique sur le long terme. En effet, les analyses de prospective sur l'évolution du prix du pétrole concluent généralement, dans le scénario le plus favorable, à un maintien des cours de l'année 2005 (Maurice, 2001; Brook et al., 2004). Dans ce contexte, des mesures d'adaptation des flottes de pêche de type structurel devraient être privilégiées afin d'anticiper les hausses futures du coût du composant énergétique. 


\section{Cadre juridique: réflexions sur les spécificités de la pêche dans la bande côtière}

Le régime communautaire de conservation et d'exploitation durable des ressources halieutiques ${ }^{31}$ repose sur les principes essentiels de la compétence exclusive de la Communauté européenne et de l'égal accès des navires de pêche communautaires ${ }^{32}$ aux eaux et aux ressources de l'ensemble des eaux communautaires.

Toutefois, dans une bande côtière de 12 milles à compter des lignes de base des États membres, il est institué, d'une part, un régime dérogatoire au principe de l'égal accès reconnaissant de facto aux États le droit de limiter la pêche à leurs propres ressortissants sous quelques réserves ${ }^{33}$. Il est permis, d'autre part, aux États d'exercer un pouvoir général de conservation et de gestion des ressources susceptible désormais de s'appliquer aux navires d'autres États membres ${ }^{34}$.

Ces particularités construisent dans le cadre du régime communautaire général un régime juridique spécifique de la pêche à l'intérieur des 12 milles $^{35}$.

En matière d'accès, on peut rappeler que le régime dérogatoire avait théoriquement pour justification première la réservation de la pêche dans la bande côtière aux navires pratiquant la petite pêche côtière ${ }^{36}$. Le règlement du 20 décembre 2002 confirme cette position en rappelant que ce régime joue « au bénéfice de la conservation par la limitation de l'effort de pêche dans les eaux communautaires les plus sensibles et (permet) de préserver les activités de pêche traditionnelles dont est extrêmement dépendant le développement économique et social de certaines populations du littoral ${ }^{37}$. En dépit de ces déclarations d'intention, il est clair que le régime dérogatoire s'est effectivement traduit dans tous les États membres par l'instauration d'un privilège d'accès fondé sur la nationalité. Ce constat n'impose cependant pas que soit remis en cause l'objectif initial.

Quant à la délégation de pouvoirs faite aux États en matière de gestion et de conservation des stocks d'espèces inféodés aux fonds côtiers ${ }^{38}$, elle est pertinente dans la mesure où l'échelle nationale (régionale ou locale) parait la mieux adaptée. Par contre, la réintroduction d'une gestion de zone (la bande des 12 milles) dans le cadre d'une approche de la gestion désormais fondée sur les écosystèmes ${ }^{39}$ est moins convaincante.

L'instauration d'un régime communautaire spécifique de conservation et de gestion des ressources de la bande côtière suit logiquement les recommandations des instruments

\footnotetext{
${ }^{31}$ Règlement $n^{\circ} 2371 / 02$ du 20/12/2002, JOCE du 31/12/2002, p. 59

32 Tout navire battant pavillon d'un EM.

33 Sans préjudice de régimes applicables aux navires de pêche communautaires battant pavillon d'autres états membres au titre des « relations de voisinage » (R. 2371/02, article 17).

34 Sous réserve qu'aucune mesure de conservation et de gestion n'ait été adoptée par la Communauté spécifiquement pour cette zone (R. 2371/02, article 9).

${ }^{35}$ Cette spécificité n'a rien en soi de surprenant. La pêche littorale et côtière a depuis tout temps fait l'objet d'un traitement particulier tant sur le plan du droit interne des états que sur celui du droit international.

36 « Le régime applicable à la zone côtière comprise entre la limite des 6 milles et celle des 12 milles avait fondamentalement pour objet d'en réserver l'accès aux navires pratiquant la petite pêche côtière », COM (2001) 135 final (livre vert de 2001), point 5.1.4.2.

${ }^{37} \mathrm{La}$ CJCE a eu l'occasion de confirmer - implicitement - la légalité du régime dérogatoire en écartant l'hypothèse d'une violation du principe de non-discrimination (CJCE du 17 mars 2005, aff. C-91/03, Espagne/Conseil). 38 C'était le sens du système en vigueur avant la réforme de 2002 qui faisait référence à des « stocks strictement locaux».

${ }^{39}$ R. 2371/02, article 2
} 
internationaux relatifs à la gestion des pêches, particulièrement celles du « code de conduite pour une pêche responsable » de la $\mathrm{FAO}^{40}$.

La France a tiré les conséquences des larges délégations de pouvoirs qui lui sont faites $^{41}$. Le dispositif interne constitue un ensemble assez complet de mesures qui juxtapose des interdits (chalutage dans les 3 milles, ...), des instruments de gestion (licences, quotas, limitation de l'effort de pêche, mesures d'urgence...), des mesures d'ordre et de précaution (caractéristiques et modalités de pose des engins de pêche...) sans parvenir, toutefois, à définir une «politique » française de la pêche dans la bande côtière. L'État semble, sur ce point, s'en remettre largement aux autorités locales et notamment aux comités régionaux de l'organisation interprofessionnelle des pêches.

En vertu de la part prépondérante qu'elle représente dans l'économie nationale des pêches et de l'implication forte de ses comités interprofessionnels en matière de gestion, la Bretagne constitue un observatoire particulièrement convaincant de l'évolution des modes de gestion de la bande côtière française. Dans la circonscription du Comité régional des pêches et des élevages marins de Bretagne, la politique de conservation et de gestion des ressources se traduit essentiellement par l'instauration d'un régime de «licences » de pêche.

La spécificité de la pêche de la bande côtière dépend de facteurs qui sont, selon le cas, internes et externes à l'activité elle-même.

Sur un plan interne, un premier facteur est institutionnel. La décentralisation du pouvoir de gestion facilite une implication directe des professionnels dans la prise de décision. De fait, les comités interprofessionnels définissent, dans le cadre d'un système de codécision, d'authentiques politiques régionales de gestion et de conservation des ressources. En l'absence d'une ligne politique nationale, cette délégation de pouvoirs est cependant susceptible de poser un problème de cohérence: cohérence des mesures prises dans des circonscriptions régionales voisines, des mesures imposées de part et d'autre de la limite de la mer territoriale.

Le second facteur est matériel. La spécificité des pêches côtières découle de la possibilité de mettre en place une gestion ciblée de chaque stock d'une même espèce mais encore une gestion sélective basée sur une meilleure adéquation entre le stock exploité et le métier (engin) pratiqué. Elle se caractérise par une restriction progressive de l'accès aux stocks locaux au profit d'entreprises artisanales et de navires d'une taille et/ou d'une puissance limitée et plus généralement dans un contrôle - a priori - strict de l'effort de pêche déployé. Cette spécificité se traduit également par la place croissante que joue l'occupation de l'espace dans la politique de gestion.

Les facteurs externes concourant à la détermination d'une spécificité de la pêche dans la bande côtière prennent une importance croissante en matière de gestion. À vrai dire,

\footnotetext{
40 Point 10.1.1 « Les états devraient veiller à ce que, compte tenu de la fragilité des écosystèmes côtiers, du caractère limité de leurs ressources naturelles et des besoins des communautés côtières, un cadre juridique, institutionnel et de définition des politiques approprié soit adopté pour permettre l'utilisation durable et intégrée de ces ressources »; code adopté par la conférence de la FAO (28e session) le 31 octobre 1995.

${ }^{41}$ Décret $n^{\circ}$ 90-94 du 25 janvier 1990 « pris pour l'application des articles 3 et 13 du décret du 9 janvier 1852 modifié sur l'exercice de la pêche maritime » (JORF 27/01/1990) modifié par décret n 2006-738 du 27 juin 2006 (JORF du 28 juin 2006). Il a notamment pour objet de fixer des mesures de conservation et de gestion dans les eaux intérieures et les eaux territoriales. Voir également le décret n 90-95 du 25 janvier 1990 modifié pris pour l'application de l'article 3 du décret du 9 janvier 1852 modifié « fixant les conditions générales d'exercice de la pêche maritime dans les zones de pêche non couvertes par la réglementation communautaire de conservation et de gestion » (JORF 27/01/90).
} 
cette spécificité résulte souvent moins d'une application particulière de ces facteurs au domaine des pêches côtières que de l'importance qu'ils sont susceptibles de prendre dans cet espace. Ils consistent pour l'essentiel dans les effets produits sur l'activité de pêche par des instruments juridiques de régulation non ciblés sur la pêche et relatifs notamment à la mise en œuvre au niveau international, communautaire et national d'une politique environnementale ${ }^{42}$.

\section{Facteurs internes (la gestion régionale de la bande côtière)}

La France développe un modèle particulier de cogestion associant étroitement administration et professionnels ${ }^{43}$. L'essentiel des pouvoirs de gestion est décentralisé au niveau des autorités régionales. Les nouvelles dispositions communautaires relatives à la gestion des bandes côtières nationales sont censées amplifier le phénomène ${ }^{44}$.

Si les conditions générales d'exercice de la pêche dans la bande côtière fixées par les décrets de $1990^{45}$ reconnaissent d'importants pouvoirs aux autorités administratives compétentes $^{46}$, en pratique les comités régionaux des pêches maritimes assurent l'essentiel de la gestion quotidienne des activités professionnelles de la bande côtière ${ }^{47}$. Ce pouvoir s'exerce à travers la mise en œuvre de délibérations rendues exécutoires par l'autorité administrative ${ }^{48}$.

Le système en vigueur a l'avantage d'impliquer directement les acteurs de la pêche dans la prise de décisions. Cependant, il comporte des faiblesses qui soulignent l'absence d'une authentique politique nationale de la pêche côtière. Une telle politique dont l'objet serait principalement de fixer le principe d'une limitation générale de l'accès à la bande côtière aux navires inférieurs à une taille et/ou à une puissance définie (s), y compris les modalités de réservation à ces navires des espèces sous quotas accessibles dans ces eaux, serait susceptible d'aborder notamment les questions relatives:

- aux règles de gestion des stocks chevauchant les limites des circonscriptions des comités régionaux ${ }^{49}$;

- au cadrage des instruments de gestion de l'accès qui sont à la disposition des comités régionaux, en ce qui concerne notamment l'usage des licences de pêche mais aussi

\footnotetext{
${ }^{42}$ D'autres politiques (urbanisme, loisirs, aquaculture, transports, etc.) interfèrent avec la politique de la pêche mais il s'agit pour l'essentiel de tenter de régler des compétitions dans l'espace ou pour des ressources. L'interférence entre politique environnementale et politique de la pêche est plus insidieuse dans la mesure où elle concerne directement le système de gestion et de conservation.

43 Il s'agit toutefois d'une conception très particulière de la cogestion dans la mesure où l'organisation interprofessionnelle entièrement placée sous la tutelle de l'autorité administrative ne peut être considérée comme un partenaire à part entière.

${ }^{44}$ Cf. « Scénarios d'aménagement des pêches dans la bande côtière bretonne. étude juridique et économique », rapport d'exécution, 2003

45 Décret n 90-94 et 90-95 du 25 janvier 1990 modifiés, JORF du 27 janvier 1990.

46 Respectivement, articles 1 et 5 des décrets précités.

47 Les comités régionaux ont une compétence ratione loci. Exerçant leurs pouvoirs dans un « ressort territorial », les mesures qu'ils prennent s'appliquent à tous les pêcheurs qui exercent leur activité dans le cadre de cette circonscription.

48 Décret $n^{\circ}$ 92-335 du 30-03-92, art. 22 (JORF du $1^{\text {er }}$ avril 1992).

49 Le comité national, par une délibération de 1998 ( $n^{\circ}$ 4/98), a déjà eu l'occasion de préciser qu'en cas de gestion d'un stock partagé, la délivrance de droits de pêche devait se faire dans le cadre de délibérations uniformes entre les deux comités régionaux concernés.
} 
l'interprétation des modalités d'application des critères relatifs à l'attribution des droits d'accès - antériorité, orientations du marché, équilibres socio-économiques - définis par le décret du 9 janvier $1852^{50}$.

- à l'usage de méthodes de pêche respectueuses de l'environnement et limitant l'impact sur les écosystèmes conformément aux objectifs de la PCP.

En outre, de façon très générale, l'échelon national doit s'assurer de la compatibilité des mesures locales au droit communautaire, afin d'écarter les risques de litiges.

\section{Les limites d'une gestion régionalisée des ressources (gestion des stocks partagés et chevauchants)}

Une trop grande disparité entre les systèmes de gestion régionaux ${ }^{51}$ est une source de conflits $^{52}$; une harmonisation est souhaitable ${ }^{53}$.

Plus précisément, la constitution de systèmes de gestion régionaux fait naître des difficultés à la marge des circonscriptions. Les comités voisins doivent gérer en commun les stocks chevauchant leurs « frontières » communes ou qui migrent d'une circonscription à l'autre ${ }^{54}$. Ils peuvent avoir recours à la commission nationale ${ }^{55}$ mais ils n'y sont pas contraints.

\footnotetext{
${ }^{50}$ Critères des antériorités des producteurs, des " orientations du marché » et des "équilibres socioéconomiques » (cf. article $3, \S 1$ du décret du 9 janvier 1852). Si les éclaircissements apportés par l'arrêté du 26 décembre 2006 « établissant les modalités de répartition et de gestion collective des possibilités de pêche (quotas de captures et quotas d'effort de pêche) des navires français immatriculés dans la Communauté européenne » (JORF du 29 décembre) quant à la nature et à la portée de ces critères sont les bienvenus, ils ne concernent pas directement l'attribution des licences de pêche.

${ }^{51}$ Disparité qui n'est pas nouvelle (voir par exemple les applications particulières de la réglementation du chalutage dans les différentes bandes côtières régionales).

${ }^{52}$ Le conflit ouvert entre haut et bas normands à propos des navires autorisés à pêcher la coquille Saint-Jacques sur des stocks qu'ils partagent en baie de Seine (les deux comités régionaux ont adopté des spécifications différentes en ce qui concerne les navires autorisés à pêcher) en est une illustration (cf. TA Rouen du 5 décembre 2001, CRPMEM de Basse-Normandie/Préfet de la région Haute-Normandie et CRPMEM de Haute-Normandie, requête $\mathrm{n}^{\circ}$ 99359).

${ }^{53}$ En dépit de l'existence au sein du comité national d'une commission «bande côtière ». Les commissions " poissons migrateurs et des estuaires », « crustacés », « coquillages de pêche », dans la mesure où leur compétence s'étend à des espèces dont une part essentielle est exploitée dans la bande côtière sont également concernées par la gestion de la bande côtière.

${ }^{54}$ Lorsqu'il s'agit de 2 comités placés sous une même autorité, il peut être prévu une procédure de délibérations uniformes ou, le cas échéant, d'un renvoi au comité national. Il faut tenir compte toutefois d'une difficulté particulière qui concerne l'autorité chargée de rendre les délibérations obligatoires lorsqu'il s'agit de comités n'ayant pas la même autorité de tutelle. Selon l'article 22 du décret n 92-335, l'autorité compétente pour rendre obligatoire les délibérations d'un comité régional est le préfet de région territorialement compétent aux termes du décret n ${ }^{\circ} 90-94$ - notamment -. Il n'est pas prévu le cas où des comités régionaux relevant de deux autorités compétentes distinctes prendraient des mesures conjointes. Cependant, l'article $1^{\text {er }}$ du décret n ${ }^{\circ} 90-94$ qui désigne ces autorités compétentes stipule (5, dernier alinéa), que lorsque la zone géographique dans laquelle s'appliquent les mesures de gestion prévues par le décret relèvent de plusieurs autorités administratives locales, celles-ci sont prises par arrêté du ministre chargé des pêches. On peut donc supposer qu'une mesure prise conjointement par deux comités régionaux relevant de deux autorités distinctes devra être également rendue obligatoire par le ministre chargé des pêches.

${ }^{55}$ Le comité national est chargé de coordonner l'action des comités régionaux et locaux (décret $\mathrm{n}^{\circ}$ 92-335; article 2, point h).
} 
D'une portée plus déterminante est la question de la gestion des stocks chevauchant la limite des 12 milles ou migrant de part et d'autre de cette limite. Elle constitue une lacune évidente de la PCP. Il s'agit de stocks non soumis au système des quotas communautaires ${ }^{56}$ dont l'aire de répartition déborde les bandes côtières pour s'étendre à une sous-région ou une région maritime (Manche Est, Manche Ouest, ...). La gestion de ces stocks libres d'accès qui peuvent représenter une source d'activité importante à l'échelle régionale n'est pas prise en compte par la législation communautaire. Quant aux États membres, leur pouvoir de gestion reste confiné à leurs bandes côtières ${ }^{57}$. Dans ces circonstances, la création des conseils consultatifs régionaux (CCR) offre une opportunité intéressante ${ }^{58}$. La compétence très générale des CCR inclut sans doute les stocks hors quotas au sujet desquels ces organismes sont en mesure de faire des propositions de mesures de gestion concertées. Il est ainsi possible d'harmoniser la gestion de ce type de stocks au niveau d'une région maritime, bandes côtières comprises. Il y aurait avantage à défendre dans ces enceintes une ligne politique nationale dans la mesure où le cadre d'un CCR comme celui des « eaux occidentales septentrionales » recouvre les eaux de la zone communautaire de pêche de l'Atlantique Nord-Est et donc les circonscriptions de sept des dix comités régionaux métropolitains.

\section{Le système de gestion des ressources de la bande côtière bretonne}

La pêche dans la bande côtière, en Bretagne particulièrement, se caractérise par une gestion extrêmement ciblée et détaillée des ressources disponibles. Un mode de gestion dans lequel l'espace tient une place importante. La politique de gestion du comité régional de Bretagne est mise en œuvre essentiellement à travers un système de « licence de pêche », instrument auquel le comité a donné un caractère très polyvalent. La gestion des activités dans l'espace qui n'échappe pas non plus au régime de licence, repose généralement sur la mise en œuvre d' « accords de cohabitation » qui tendent à prévenir les conflits entre métiers.

\section{La gestion de l'effort de pêche}

Par nature, la licence est un instrument de contrôle de l'effort de pêche qui implique le plus souvent l'idée d'un «numerus clausus ». En fait, le comité régional des pêches de Bretagne en use comme d'un cadre qui permet d'instaurer d'authentiques « régimes » d'exploitation d'une ressource ou d'un métier particulier ${ }^{59}$. La plupart des activités de

\footnotetext{
${ }^{56}$ La pêche des stocks sous quotas obéit en principe à des mesures de gestion particulières fixées dans le cadre de la PCP.

57 Sauf à prendre, dans leurs eaux sous juridiction, des mesures de gestion applicables uniquement à leurs ressortissants (R. 2371/02, article 10).

${ }^{58}$ Il existe d'ores et déjà des « rencontres » informelles de gestion concertée qui visent notamment à instaurer un dialogue entre des instances professionnelles provenant d'une même zone géographique ou pratiquant la pêche d'une même espèce de poisson. Les professionnels français participent ainsi à des rencontres bilatérales avec l'Espagne (COFEP), l'Irlande (COFIR) ou régionale pour la Manche Ouest (CO-Manche). La Commission encourage ces réunions « dans la mesure où elles permettent de régler des problèmes d'importance locale pour autant qu'elles respectent les processus décisionnels », COM (99) 747 final.

${ }^{59}$ Le comité fait une interprétation extensive de la définition de l'autorisation de pêche telle qu'elle est formulée par l'article 3 § I point a du décret du 9 janvier 1852.
} 
pêche de la bande côtière bretonne sont tributaires de la délivrance d'une licence de pêche et sont ainsi soumises à des régimes spécifiques d'exploitation ${ }^{60}$.

On distingue sommairement trois types de licences: les licences « ressources», « métier» et « zone ».

La licence « ressources » est historique. Ce type de licences s'attache à régler la gestion et l'exploitation de diverses espèces de coquillages, des algues, des oursins, des crustacés.

La licence «métier » ne diffère de la précédente que par son objet ${ }^{61}$. Les licences « métiers » instaurées par le comité régional (palangre, filet, chalut, bolinche) ont pour vocation première de gérer l'accès aux pêcheries plurispécifiques et notamment aux pêcheries de poissons.

Enfin, il peut exister des licences « zone » ayant pour vocation la gestion d'un espace particulier.

Le régime de licences mis en place par le CRPMEM de Bretagne permet, entre autres choses, de limiter progressivement l'effort de pêche et de le réguler en fonction des zones d'accès ${ }^{62}$.

La poursuite de cet objectif induit d'une part une limitation de l'effort de pêche global par le contingentement des autorisations - qui n'est cependant pas systématique -, d'autre part par la restriction progressive de l'accès à la bande côtière à des navires d'une taille et d'une puissance limitées. Enfin, le système tend à répartir de façon optimale dans l'espace, les efforts déployés.

L'exclusion des navires hauturiers de la bande côtière permet de protéger les métiers côtiers traditionnels. Sur ce point, les licences en vigueur formalisent progressivement deux ordres de grandeur:

- une longueur maximale de 12 à 13 mètres à l'exception notable des licences « métiers » (bolinche, 17 mètres; le chalut de fond en mer d'Iroise, 18 mètres; le filet, 16 mètres au-delà des lignes de base; palangre, 16 mètres);

- une puissance maximum de 200/250 $\mathrm{CV}^{63}$.

Cependant, la plupart des licences prévoient des dérogations afin de ne pas exclure de la pêcherie des unités de pêche excédant ces limites et disposant d'antériorités. Outre que la dérogation ne vaut que pour la zone pour laquelle la licence a été délivrée, elle ne perdure que tant que le couple navire/propriétaire demeure identique et à certaines conditions ${ }^{64}$ :

Une typologie rapide de l'existant montre que les licences sont susceptibles de contenir l'éventail des mesures de gestion normalement à la disposition des gestionnaires des pêches. L'objectif du comité est de parvenir progressivement à encadrer l'exploitation de la plupart des ressources de la bande côtière quel que soit par ailleurs leur état de conservation. Voir notre précédente étude sur les « Les instruments concourants à la gestion spatiale des activités de pêche dans la bande côtière bretonne » in "Scénarios d'aménagement des activités de pêche dans la bande côtière bretonne. Étude juridique et économique », rapport d'activité 2004.

${ }^{60}$ En 2007, le Comité régional en créé une soixantaine de licence différentes (en fonction d'une espèce ou d'un métier donné, dans une zone donnée). Il en attribue environ 4000.

${ }^{61}$ La distinction peut apparaître assez artificielle dans la mesure où la pratique de certains métiers est indissolublement liée à une ressource donnée.

${ }^{62}$ Les délibérations des comités régionaux peuvent notamment porter sur « l'ajustement de l'effort de pêche concernant la taille et la puissance des navires » (Décret 92-335 du 30/03/92, article 22; JORF du $1^{\text {er }}$ avril 1992).

${ }^{63}$ Les licences « bolinche », « filets », « palangre » et « chalut de fond - Iroise » ne prévoient pas de limitation de puissance.

${ }^{64}$ Ces conditions concernent la plupart des licences « coquille Saint-Jacques », les licences « bolinche », « filets », « palangre » et « chalut de fond - Iroise »; cf. délibération du CRPMEM Bretagne « derogataires- 
- que le navire ne subisse pas de modifications entraînant une augmentation de la taille ou de la puissance;

- que le navire conserve un lien économique réel avec le quartier d'origine d'immatriculation du navire ${ }^{65}$;

- que le navire continue à pêcher régulièrement dans la zone définie par la licence.

Les navires bénéficiant de dérogations sont appelés à disparaître progressivement de la bande côtière.

La modulation de l'accès en fonction des caractéristiques des navires s'affine encore dans le cadre de certaines licences. Ainsi, la licence « filets » prévoit différentes zones d'activités définies à compter du rivage (terre/lignes de base droite; $0 / 6$ milles ; 0/12 milles ; 6/12 milles) où l'accès est modulé en fonction de la taille du navire. Celle-ci diminue à mesure que l'activité s'exerce dans une zone plus proche de la côte.

Cette politique de régulation de l'activité dans l'espace en fonction des caractéristiques des navires devrait être systématisée à l'échelle nationale. Il s'agit sans aucun doute d'un défi majeur à relever dans le cadre de la gestion de la bande côtière ${ }^{66}$.

La réservation de la bande côtière aux navires d'une taille et d'une puissance limitées interfère directement avec le système de répartition des quotas au niveau national dans la mesure où la fraction de certains quotas attribués à la France ne sera accessible qu'aux navires côtiers. De ce point de vue, l'arrêté du 26 décembre $2006^{67}$ laisse au ministre des pêches une marge de manœuvre.

En effet, le principe de la répartition des quotas repose sur la prise en compte de trois composantes parmi lesquelles se trouve celle des « équilibres économiques ». L'article 7 de l'arrêté prévoit qu' « en fonction des équilibres socio-économiques, le ministre chargé des pêches maritimes peut répartir le quota en imposant des critères d'accès à la pêcherie, le cas échéant au moyen du régime d'autorisations de pêche spécifiques en vertu des décrets $\mathrm{n}^{\circ}$ 90-94 et $\mathrm{n}^{\circ}$ 90-95.

Les critères d'accès peuvent être notamment les différents métiers et engins de pêche, les façades maritimes d'immatriculation des navires des producteurs, les zones de pêche, les lieux de débarquements ».

crpm-2006-A » du 1er décembre 2006. Ces dispositions se justifient par le fait qu'un assouplissement même mineur des règles mises en place par les professionnels ne doit pas conduire au maintien artificiel d'unités de pêche (cf. $3^{\mathrm{e}}$ considérant).

${ }^{65}$ Ce point est intéressant dans la mesure où il reproduit à l'échelle locale une condition fixée au niveau national en vue d'éviter la captation des quotas français par des navires d'autres États membres. Faut-il comprendre l'expression de lien économique réel dans le sens qui lui est donné par la circulaire ministérielle du 31/08/98? Pour que ce lien existe entre le navire et son quartier d'origine, il faudrait: soit que $50 \%$ au moins des prises soient débarquées dans un port du quartier et une part substantielle de ces prises mises en vente localement; soit que $50 \%$ au moins de l'équipage employé à bord réside dans une zone à partir de laquelle s'exerce l'activité de pêche; soit que la moitié au moins des expéditions de pêche partent d'un port du quartier. Cette interprétation aurait pour effet de « régionaliser » ces droits de pêche.

${ }^{66}$ Cf. par exemple, "Rapport sur l'exercice de la pêche dans la zone côtière de la France ", J. Bolopion, A. Forest, J. Sourd, Janvier 2000. Cf. $1^{\text {re }}$ des 10 recommandations; rapport du Parlement européen sur la pêche côtière et les problèmes rencontrés par les pêcheurs côtiers du 26 avril 2006, doc. A6-0141/2006 final.

${ }^{67}$ Arrêté du 26 décembre 2006 établissant les modalités de répartition et de gestion collective des possibilités de pêche (quotas de captures et quotas d'effort de pêche) des navires français immatriculés dans la Communauté européenne; JORF du 29/12/2006 
La bande des 12 milles est-elle susceptible de constituer par elle-même une zone de pêche au sens de ces critères? Juridiquement, rien ne paraît s'y opposer.

Enfin, la réservation de la bande côtière et des avantages qui y sont liés à une certaine catégorie de navires doit-elle interdire à ces derniers d'exercer leurs activités au-delà des limites de la mer territoriale?

La dimension spatiale de la gestion des activités de pêche dans la bande côtière bretonne n'est pas réservée exclusivement à la régulation de l'effort de pêche, elle permet également d'ajuster les mesures de gestion en fonction des zones de pêche. Ainsi les licences « filets» et « palangre » prévoient un ajustement des mesures de gestion par zones ou par secteur ${ }^{68}$.

La gestion des activités de pêche dans l'espace côtier doit également tenir compte des contraintes relatives à la cohabitation des métiers dans un espace restreint. Exceptionnellement, cet objectif peut être poursuivi dans le cadre d'une licence. Le plus souvent, il est l'objet d' « accords de cohabitation ».

\section{La gestion des activités dans l'espace}

Les accords de cohabitation ${ }^{69}$ en vigueur dans la bande côtière bretonne sont un mode de gestion le plus souvent non réglementaire - et souvent informel-des activités de pêche dans l'espace, spécialement dédié à la prévention des conflits entre métiers. Ces accords sont conclus et mis en œuvre par l'organisation interprofessionnelle.

La réglementation interne comporte des dispositions vouées à la résolution des problèmes de cohabitation entre les métiers. La mise en œuvre des dispositifs est du ressort des autorités administratives et professionnelles.

Le préfet de région est compétent pour prendre les mesures relatives à la cohabitation pour les eaux sous souveraineté et juridiction française où s'exerce sa compétence au terme du décret $n^{\circ} 90-94$ sous réserve des mesures concernant une zone géographique qui relève de plusieurs autorités administratives locales et sans préjudice des compétences de police générale exercées par le préfet maritime ${ }^{70}$.

Plus clairement, les comités régionaux peuvent prendre des délibérations qui portent notamment sur « les mesures d'ordre de précaution destinées à organiser la compatibilité entre les métiers » et plus précisément qui sont relatives à « la définition de zonages ou de carroyages particuliers, et la fixation de règles de cohabitation entre les différents métiers ».

La gestion spatiale des activités de pêche dans la bande côtière est certainement un important axe de réflexion des années à venir. Hormis l'organisation de la cohabitation des métiers entre eux qui peut fort bien être réglée par les pêcheurs eux-mêmes, c'est l'ensemble de l'organisation de multiples activités s'exerçant dans l'espace côtier qui doit être envisagé.

\footnotetext{
${ }^{68}$ La licence « filets » divise la côte bretonne en 4 zones et la licence " palangre » en 10 secteurs; cf. délibération filets-CRPM-2006-A du 29/09/2006 portant création et fixant les conditions d'attribution de la licence de pêche du poisson aux filets et délibération palangre-CRPM-2007-A du 30/03/2007 portant création et fixant les conditions d'attribution de la licence de pêche du poisson à la palangre dans les eaux de la circonscription du CRPMEM.

${ }^{69}$ Pour une brève typologie des accords bretons, voir notre précédente étude sur les « Les instruments concourant à la gestion spatiale des activités de pêche dans la bande côtière bretonne » in " Scénarios d'aménagement des activités de pêche dans la bande côtière bretonne. Étude juridique et économique », rapport d'activité 2004. ${ }^{70}$ L'article 23 dispose, « afin de permettre le bon ordre des activités de pêche, ...l'Autorité administrative prend, en tant que de besoin, les mesures relatives aux modalités d'utilisation (ou de pose) des engins de pêche... ».
} 


\section{Facteurs externes (contraintes des instruments de gestion de l'environnement sur les activités de pêche)}

Toute réflexion sur l'interaction et les conflits d'usage entre les diverses activités s'exerçant sur le littoral et dans la zone côtière française doit désormais être replacée dans le cadre de la politique de la gestion intégrée des zones côtières (GIZC) dont les lignes directrices sont fixées au niveau communautaire.

Prenant en considération l'article $17^{71}$ du plan d'action 21 (« agenda $\left.21 »\right)$ adopté au Sommet de la CNUED à Rio en juin 1992, la Commission européenne a défini en 2000 une stratégie sur l'aménagement intégré des zones côtières en Europe ${ }^{72}$. En 2002, le Parlement européen et le Conseil ont fait une recommandation relative à « la mise en œuvre d'une stratégie de gestion intégrée des zones côtières $\rangle^{73}$ définissant les éléments et principes sur lesquels seront fondées les approches stratégiques nationales et demandent aux États de leur faire un rapport sur l'application de cette recommandation. L'ensemble de ces contributions fait l'objet, en août 2006, d'une évaluation pour le compte de la Commission ${ }^{74}$. La France s'exécute en juin $2006^{75}$. C'est pour elle l'occasion de définir sa propre stratégie ${ }^{76}$. Celle-ci est fondée sur « la concertation et la coordination de la vision des acteurs et de leurs actions » et non « sur l'organisation ou la réglementation ». L'approche «s'appuie sur les instruments réglementaires sectoriels existants » et « il n’y a donc pas de modification du rôle institutionnel des acteurs; le processus engagé est celui de la territorialisation, chacun conservant ses responsabilités ». En définitive, il «n'existe pas d'action intégrée; mais des actions - sectorielles ou transversales - qui doivent être conduites de manière coordonnée pour concourir à un objectif global à l'échelle du périmètre (géographique et thématique) considéré » ainsi, « chaque acteur conserve son rôle et le plein exercice de ses compétences »" Il faut comprendre que les démarches entreprises dans le cadre de la GIZC, en France, n'auront pas de caractère normatif et que continueront de prévaloir les politiques sectorielles.

La question de l'intégration des pêches dans l'aménagement intégré des zones côtières a été spécifiquement envisagée dans le code de conduite pour une pêche responsable ${ }^{78}$. Sur le plan communautaire, le livre vert sur l'avenir de la PCP rappelle que la stratégie

\footnotetext{
${ }^{71}$ Relatif à la « protection des océans et de toutes les mers et des zones côtières et protection, utilisation rationnelle et mise en valeur de leurs ressources biologiques ».

${ }_{72}$ COM (2000) 547 final du 27/09/2000.

${ }_{73}$ Recommandation 2002/413/CE du 30 mai 2002.

${ }^{74}$ Evaluation of Integrated coastal zones management (ICZM) in Europe, final report, 18/08/2006:(http:// ec.europa.eu/environment/iczm/pdf/evaluation_iczm_report.pdf).

${ }^{75}$ Rapport français d'application de la recommandation du Parlement européen et du Conseil du 30 mai 2002 relative à la mise en œuvre d'une stratégie de gestion intégrée des zones côtières en Europe :(http://ec.europa. eu/environment/iczm/evaluation/iczm_national_reporting_france.htm).

76 « dans le respect de l'environnement et avec le souci d'éviter les conflits entre usagers anciens (pêcheurs) et nouveaux (exploitants d'aquaculture, de granulats, d'éoliennes) », cf. communiqué du Premier ministre à l'issue du CIMer du 29 avril 2003.

${ }^{77}$ La loi du 23 février 2005 sur le développement des territoires ruraux crée une instance nationale de concertation pour la politique du littoral, le Conseil national du littoral (CNL) chargé de définir les objectifs et de préciser les actions qu'il juge nécessaires pour l'aménagement, la protection et la mise en valeur du littoral, dans une perspective de gestion intégrée des zones côtières; JORF du 24 février 2005.

${ }_{78}$ Point 10.1.3. « Les États devraient favoriser l'adoption de pratiques de pêche qui permettent d'éviter les conflits entre les utilisateurs des ressources halieutiques, ainsi qu'entre ces derniers et d'autres usagers de la zone côtière ». L'article 10 a notamment donné lieu à l'élaboration d'une directive technique pour une pêche responsable «l'intégration des pêches dans l'aménagement des zones côtières », FAO, 1999.
} 
européenne pour l'aménagement intégré des zones côtières (AIZC) vise à améliorer la compatibilité des politiques européennes - dont la PCP - qui ont des retombées sur les zones côtières ainsi que d'assurer la cohérence entre la mise en œuvre de la PCP et les mesures nationales relatives à l'utilisation de la zone côtière ${ }^{79}$. La communication de la commission sur l'AIZC de 2000 à propos de la compatibilité des politiques sectorielles avec l'AIZC se réfère à la pêche surtout au regard du maintien du régime dérogatoire des 12 milles afin « de pouvoir planifier et aménager la pêche côtière dans le cadre d'un processus d'AIZC à long terme ».

Aux termes du règlement de 2002, il ressort que l'intégration de la politique de la pêche dans le cadre de la politique européenne de la GIZC opère selon deux axes : la PCP doit permettre par l'application du principe de précaution, de minimiser les répercussions des activités de pêche sur les écosystèmes marins - en ce sens, elle rejoint les objectifs généraux de la stratégie européenne pour le milieu marin ${ }^{80}$; elle doit être compatible avec les autres politiques communautaires et notamment celle de l'environnement ${ }^{81}$.

En attendant d'en connaître un peu plus sur la programmation française dans le cadre de la GIZC, il est possible d'évaluer les impacts - actuels ou potentiels - sur la gestion des pêches de la bande côtière ${ }^{82}$ de la mise en œuvre d'instruments juridiques divers. Cette évaluation concerne au premier chef les instruments relatifs à la protection de l'environnement marin et plus particulièrement à la protection de la biodiversité.

\section{Protection du milieu marin et de la diversité biologique}

Les instruments relatifs à la protection de l'environnement sont-ils susceptibles de créer des contraintes sur la gestion des activités de pêche? Bien que leur domaine d'application ne se limite pas à la bande côtière, il importe ici d'évaluer les impacts qu'ils pourraient produire dans cet espace.

\section{La stratégie européenne pour la protection du milieu marin}

Elle doit constituer le pilier environnemental de la future politique maritime de l' $\mathrm{UE}^{83}$. Elle servira de guide au développement de la GIZC ${ }^{84}$ en Europe. La protection de la biodiversité marine ${ }^{85}$ est un élément de cette stratégie. Elle se traduit en partie par la mise en place du réseau Natura $2000^{86}$.

\footnotetext{
79 COM (2001)135 final, point 5.5.4.

80 Cf. infra.

81 Règlement $n^{\circ} 2371 / 02$, article 2 ; précité.

${ }^{82}$ Les notions de bande côtière et de zone côtière ne se confondent pas. La communication initiale de la Commission sur l'aménagement intégré des zones côtières (COM (95)511 final/2 du 29/11/1995) définissait la zone côtière comme « une bande terrestre et marine dont la largeur varie en fonction de la configuration du milieu et des besoins d'aménagement » en précisant que si pour « le domaine halieutique, il est courant de limiter la zone côtière aux eaux territoriales », cette limite « ne correspond pas à une unité biologique ni à une unité de gestion bien individualisée ». La zone côtière peut donc «s'étendre bien au-delà de ces eaux territoriales ». Quant à la communication suivante sur ce même sujet du 4/10/2000 (COM (2000)547 final/2), elle note qu'il « serait inopportun de donner une définition géographique a priori de la "zone côtière" ").

83 COM (2006)275 final.

${ }^{84}$ COM (2007)308 final.

85 COM (2006)216 final.

${ }^{86}$ Cf. directive 92/43/CEE du Conseil du 21 mai 1992 « concernant la conservation des habitats naturels ainsi que de la faune et de la flore sauvage » (JOCE L 206, p. 7). Cette directive prévoit la constitution d'un réseau écologique européen cohérent de zones spéciales de conservation, dénommé « Natura 2000 ». Ce réseau, formé
} 
La stratégie européenne pour le milieu marin est une politique intégrée de protection et de restauration du milieu marin. Elle tend à obtenir un «bon état écologique » du milieu marin en Europe à l'horizon de 2021. Il s'agit de donner une vision d'ensemble du milieu marin et de fixer des objectifs et des principes communs à l'échelle communautaire dans le cadre d'une directive ${ }^{87}$. Sur la base de stratégies qu'ils auront définies dans le cadre de 3 grandes régions marines (ou sous-régions), les États devront élaborer des programmes de mesures afin de parvenir à l'objectif fixé. Pour les questions relevant de la compétence communautaire, la Commission pourra indiquer les solutions applicables ou le cas échéant adapter la réglementation communautaire (notamment en ce qui concerne la politique commune de la pêche).

La stratégie repose notamment sur « une approche axée sur les écosystèmes, en vertu de laquelle les activités humaines affectant le milieu marin seront gérées d'une façon intégrée favorisant la conservation et l'utilisation durable et équitable de la diversité biologique $»$; elle détermine la marche à suivre pour protéger les écosystèmes marins. La pêche commerciale étant perçue comme une des principales menaces du milieu marin, la recherche d'une limitation des répercussions de la pêche sur les écosystèmes constitue depuis la réforme de 2002 l'un des objectifs majeurs de la politique de la pêche; des mesures ont d'ores et déjà été prises dans ce sens ${ }^{88}$.

Le domaine d'application de la directive concerne l'ensemble des eaux sous souveraineté ou juridiction des États (y compris, dans une certaine mesure, les eaux intérieures) ${ }^{89}$.

Selon les termes de la directive, les États membres devront évaluer leurs eaux marines sur la base notamment d'une analyse de l'état écologique de ces eaux couvrant les types d'habitats (notamment ceux recensés au titre du réseau Natura 2000) et les composantes biologiques (dynamique des populations de mammifères et d'oiseaux marins mais également de toutes espèces couvertes par la législation communautaire ou des accords internationaux) ainsi que sur une analyse des principaux impacts et pressions, et notamment l'activité humaine, qui influencent les caractéristiques et l'état écologique de ces eaux (cela inclut naturellement les activités de pêche).

Sur cette base, les États définiront un «bon état écologique »-en tenant compte de ces mêmes éléments -, ainsi que des objectifs environnementaux mesurables et opérationnels (comprenant le cas échéant des points de référence limites et cibles). Pour chaque région (ou sous-région) concernée, les États élaboreront des mesures par référence aux objectifs définis qui peuvent comprendre notamment des mesures de régulation à l'entrée (intensité autorisée d'une activité humaine), de régulation spatiale et temporelle (lieu et moment où une activité est autorisée) ou d'incitation économique (incitant les usagers de l'écosystème à agir de manière à contribuer à la réalisation des objectifs fixés).

\footnotetext{
par des sites abritant des types d'habitats naturels et des habitats des espèces répertoriés, doit « assurer le maintien ou, le cas échéant, le rétablissement, dans un état de conservation favorable (de ces habitats) dans leur aire de répartition naturelle ». Le réseau Natura 2000 comprend également les zones de protection spéciale classées par les États membres en vertu des dispositions de la directive 79/409/CEE du Conseil du 2 avril 1979 « concernant la conservation des oiseaux sauvages » (JOCE L 103, p. 1).

${ }_{87}$ Une directive constitue un cadre juridique contraignant à l'intérieur duquel les États disposent de la compétence et des moyens pour parvenir au résultat fixé. Les mesures de gestion spécifiques n'ont pas à être définies au niveau communautaire.

88 Cf. infra.

${ }^{89}$ Le domaine d'application de la directive fait l'objet de débats, notamment au regard de la nécessaire cohérence entre celui-ci et celui de la directive « eau » (directive 2000/60/CE).
} 
Le texte spécifie que les mesures de gestion des pêches sont exclues de l'application de la directive et ne peuvent être prises que dans le cadre de la $\mathrm{PCP}^{90}$. À cet effet, il est prévu que les objectifs de la directive soient pris en compte au titre de la prochaine réforme de la PCP. La PCP doit devenir l'instrument d'application des objectifs de la directive à la pêche. Il s'ensuit que l'État, en vertu des pouvoirs dont il dispose en matière de gestion dans la bande côtière ou au titre des mesures d'urgence ${ }^{91}$, sera tenu de prendre toutes mesures afin de minimiser les incidences de la pêche sur les écosystèmes ${ }^{92}$.

L'État membre sera logiquement amené à assurer la cohérence des mesures de gestion et de conservation des ressources de pêche de la bande côtière avec les mesures de régulation qu'il prendra afin de réaliser les objectifs environnementaux qu'il se sera fixés au titre de la directive ${ }^{93}$.

Par ailleurs, la stratégie européenne de protection du milieu marin doit renforcer encore la ferme position tenue par la Communauté dans la préservation de la biodiversité à la fois par sa participation à diverses conventions internationales et par la poursuite des objectifs généraux fixés par la législation environnementale - notamment à travers la constitution du réseau Natura 2000.

\section{Le maintien de la diversité biologique}

La Communauté s'est clairement engagée dans le cadre de diverses conventions internationales et particulièrement dans celui de la convention sur la diversité biologique (CDB) en faveur du maintien de la diversité biologique ${ }^{94}$, de l'utilisation viable et durable de la biodiversité marine et de la création, d'ici à 2012, d'un réseau mondial de zones marines protégées ${ }^{95}$.

L'article 8 de la CDB demande aux parties contractantes de créer un système de zones protégées $^{96}$. À l'échelle de la Communauté, la mise en œuvre de cette recommandation se traduit essentiellement par la constitution du réseau Natura 2000 bien que cet instrument n'ait pas une vocation exclusivement maritime. L'obligation pour les États membres de désigner, en application de la directive « habitats ${ }^{97}$, des sites Natura 2000 situés partiellement ou entièrement en mer doit contribuer de façon déterminante à ce processus.

90 En vertu de la compétence exclusive communautaire.

91 Règlement 2371/02, article 8.

92 Règlement 2371/02, article 9 . La mise en œuvre de la directive aura pour effet de renforcer une obligation à laquelle l'État est déjà contraint.

93 L'État sera tenu à une obligation de résultat quant à l'obtention d'un bon état écologique de ses eaux en 2021.

94 La communauté s'est également engagée à mettre en place avant 2010 un réseau de zones protégées dans le cadre des conventions Ospar (protection du milieu marin de l'atlantique N-E) et Helcom (protection du milieu marin de la Baltique). Ces 2 conventions reconnaissent que les sites marins Natura 2000 répondent aux critères d'inclusion dans le réseau Ospar/Helcom. Elle adhère également à la convention de Barcelone ainsi qu'à son protocole de 1995 relatif aux aires spécialement protégées et à la diversité biologique en Méditerranée.

95 Cf. Convention sur la diversité biologique, $2^{\mathrm{e}}$ conférence des parties (COP), décision II-10 (dit « mandat de Jakarta »); 4 e COP, décision IV-5 (programme de travail découlant du mandat de Jakarta); 7e COP, décision VII-5 (actualisation du programme de travail). Cet objectif rejoint celui fixé par le Plan d'application du Sommet mondial pour le développement durable adopté à Johannesbourg le 4-09-2002; À/conf.199.20, annexe, chap. IV, § 32).

${ }^{6}$ Comprise comme « toute zone géographiquement délimitée qui est désignée, ou réglementée, et gérée en vue d'atteindre des objectifs spécifiques de conservation ».

${ }^{97}$ précité. 
Par ailleurs, la France qui est également partie à certaines de ces conventions (CDB, Ospar, convention de Barcelone - et protocole de 1995 -) crée et organise son propre réseau d'aires marines protégées.

\section{Constitution d'un réseau Natura 2000 en mer}

La directive communautaire « habitats » prévoit la constitution d'un réseau écologique européen cohérent de zones spéciales de conservation (ZSC), dénommé « Natura 2000 ». Ce réseau est formé par des sites abritant des types d'habitats naturels et des habitats des espèces dont il doit « assurer le maintien ou, le cas échéant, le rétablissement, dans un état de conservation favorable». Il comprend également les zones de protection spéciale (ZPS) classées par les États membres en vertu des dispositions de la directive 79/409/CEE ${ }^{98}$. Chaque État membre contribue à la constitution de Natura 2000 en désignant des sites en tant que zones spéciales de conservation puis en établissant à l'intérieur de ces zones les mesures de conservation nécessaires.

Le domaine d'application des directives « habitats » couvre le territoire européen ce qui en l'espèce comprend comme l'admet le Conseil ${ }^{99}$ les eaux sous souveraineté (eaux intérieures et mer territoriale), la zone économique de 200 milles ainsi que le plateau continental $^{100}$.

La France crée de nombreux sites Natura 2000 qui s'appliquent partiellement en mer - rarement au-delà de quelques milles marins -, sans qu'aucun pour l'instant ne soit exclusivement dédié à une zone marine du milieu de haute mer ${ }^{101}$.

Afin de respecter ses engagements internationaux, la Communauté est cependant tenue de développer la composante marine du réseau Natura 2000 et les États membres de prendre les dispositions utiles ${ }^{102}$. L'achèvement du réseau Natura 2000 en mer est prévu pour 2008; les objectifs de gestion devant être agréés et mis en œuvre en 2010. La France doit s'atteler à cette tâche de façon urgente ${ }^{103}$.

La pêche commerciale étant une activité d'une importance déterminante pour la préservation du milieu marin et la conservation de la biodiversité marine, comment figurer la problématique de l'interaction entre les politiques de la pêche et de la préservation de la

\footnotetext{
98 précité.

99 Conseil «pêches », Luxembourg, 23/04/2001 (annexe aux conclusions du Conseil sur l'intégration des exigences environnementales et du développement durable dans la PCP, § 15) http://ue.eu.int/ueDocs/cms_Data/ docs/pressData/en/agricult/ACF20DE.html; confirmation par la CJCE (aff. C-6/04 du 20/10/2005, Commission/ Royaume-Uni, § 117).

${ }^{100}$ Le plateau continental qui peut s'étendre jusqu'à 350 milles des lignes de base de l'État, comprend les fonds marins et leur sous-sol au-delà de la mer territoriale (convention sur le droit de la mer, article 76). L'État exerce des droits souverains et exclusifs sur les ressources naturelles du plateau continental (qui comprennent les organismes vivants appartenant aux espèces sédentaires).

${ }_{101}$ Au 30 juin 2007, la Communauté répertoriait 90 sites français ayant une partie maritime pour une surface totale de $5593 \mathrm{~km} 2$; http://ec.europa.eu/environment/nature/Natura2000/barometer/docs/sci.pdf.

${ }^{102}$ L'obligation pour les États de désigner des sites ne dépend pas de l'environnement dans lequel il se situe mais de critères écologiques relatifs à la protection d'un habitat ou d'une espèce telles que désignés par les annexes de la directive. De même, leurs obligations de parvenir à un état de conservation favorable ne se distinguent pas selon que le site soit terrestre ou marin.

${ }^{103}$ Message de Malahide du 27 mai 2004 http://ec.europa.eu/environment/nature/biodiversity/policy/pdf/ malahide_message_final.pdf. En France, un « comité national des aires marines » s'est réuni le 2 juillet 2007 pour définir une stratégie nationale pour la création des aires marines protégées et des sites Natura 2000 en mer.
} 
biodiversité ? Il importe de mesurer plus précisément, l'incidence de la création des sites Natura 2000 en mer - et des mesures de gestion proposées - sur les activités de pêche.

\section{Incidence de la désignation des sites Natura 2000 sur la gestion des activités de pêche}

La Commission ${ }^{104}$ note que l'incidence des activités de pêche sur l'environnement marin ${ }^{105}$ s'exprime, notamment:

- directement par le prélèvement indifférencié d'espèces recherchées et non recherchées, mettant en cause leur conservation;

- indirectement, par la modification des flux énergétiques dans le réseau alimentaire, ce qui peut avoir un effet sur l'état de conservation d'autres espèces de l'écosystème;

- directement, par la modification de l'environnement physique et la menace qu'elle représente pour la diversité des habitats, ayant des incidences sur leur capacité d'abriter à la fois des espèces commerciales et non commerciales (par exemple, le chalutage de fond) ${ }^{106}$.

Afin de corriger une relative incapacité de la politique communautaire de gestion et de conservation des ressources à résoudre ces difficultés, un des objectifs de la réforme de 2002 était d'adopter une démarche de gestion de la pêche fondée sur les écosystèmes. Cela suppose d'intégrer a priori les considérations d'environnement dans la définition de la politique de gestion - et directement dans les mesures d'application - et non de façon réactive lorsqu'il est constaté que l'environnement est déjà fortement dégradé.

La politique de la pêche doit concourir à la préservation de l'environnement même si cet objectif peut sembler paradoxal. Ce faisant, chaque acte de gestion doit être accompli en tenant compte de ses effets potentiels sur l'environnement. Cette exigence semble récemment prendre une tournure plus radicale puisque la Communauté entend observer scrupuleusement l'obligation faite aux États parties par l'assemblée générale des Nations unies de procéder à une évaluation d'impact sur l'environnement comme une condition de l'autorisation des activités de pêche ${ }^{107}$.

Dans ce contexte, la désignation des zones de protection Natura 2000 doit permettre de concilier l'exigence de conservation des espèces menacées avec les impératifs de la pêche commerciale au-delà des effets attendus de l'application du système général de gestion et de conservation (limitation des captures et de l'effort de pêche, plans de reconstitution, mesures techniques...). Dans ces zones de protection, la Communauté de sa propre initiative ou sur la suggestion des États, devra mettre en œuvre les mesures

\footnotetext{
104 Communication de la Commission concernant les éléments d'une stratégie d'intégration des exigences de l'environnement dans la PCP, COM (2001)143 final.

105 Il existe évidemment une incidence inverse des modifications de l'environnement sur la pêche.

106 Un « plan d'action en faveur de la diversité biologique dans le domaine de la pêche » retient également que « la réduction de la pression de pêche engendrée par une diminution de la fréquence de passage des engins de pêche remorqués sur les fonds marins sera bénéfique pour les habitats ainsi que pour la faune et la flore benthiques. Certains de ces habitats et espèces sont toutefois extrêmement fragiles, rares ou représentatifs et pourraient être fortement affectés en dépit de la réduction générale de l'effort de pêche. Il convient donc d'identifier les zones concernées afin d'en protéger les habitats et certaines espèces conformément aux exigences de la directive « Habitats », et le plan d'action s'inspirera du processus Natura 2000 pour atteindre cet objectif », COM (2001) 162 final.

107 Communication de la Commission relative aux pratiques de pêche destructrices en haute mer et à la protection des écosystèmes vulnérables d'eaux profondes du 17/10/2007, COM (2007)604 final et une résolution de l'assemblée générale des Nations unies du 6 mars 2007, A/RES/61/105.
} 
nécessaires (par priorité de nature réglementaire) à la conservation des habitats et des espèces menacées $^{108}$.

Dans le cadre de la PCP, la Communauté a pris, surtout depuis 2002, diverses mesures de gestion qui visent directement la préservation des écosystèmes ${ }^{109}$. Ces mesures de protection des habitats ou des espèces qui peuvent le cas échéant poursuivre des objectifs fixés dans le cadre de sites Natura 2000, ne sont pas nécessairement limitées à ces espaces. En effet, la PCP constitue un instrument de protection de la biodiversité dont le domaine d'application est sensiblement plus large que celui de la directive « habitats ». Ainsi, des mesures prises sous couvert de la PCP peuvent-elles avoir pour effet de $\operatorname{protéger~}^{110}$ :

- des éléments naturels non visés dans les annexes de la directive « Habitats »;

- des éléments visés dans les annexes de la directive, mais présents dans des zones ne relevant pas de la juridiction des États membres;

- des éléments inscrits aux annexes et situés dans des zones marines sous la juridiction des États membres, mais qui ne sont pas inclus dans une zone spéciale de conservation ou site d'intérêt communautaire (du fait qu'ils sont situés en dehors d'un SIC ou en attente de proposition/désignation).

La politique de la pêche peut ainsi édicter des mesures de conservation dans le cadre de ZSC ou de SIC qui visent à réaliser les objectifs des directives « Habitats », de même qu'elle peut anticiper la désignation de sites communautaires par les États en imposant des restrictions aux activités pêche dans un but de préservation de la biodiversité ${ }^{11}$.

108 Le Plan d'action communautaire pour l'intégration des exigences de la protection de l'environnement dans la PCP, COM (2002)186 final, s'il indique comme étant une priorité absolue l'amélioration des méthodes de pêche en vue notamment de réduire les prises accessoires et l'impact sur les habitats, demande à la Commission d'étendre les mesures de gestion à la protection des habitats et espèces non commerciales contre les effets des activités de pêche.

109 - interdiction de l'enlèvement des nageoires de requin à bord des navires (afin de prévenir les prises de requins visant uniquement à vendre les nageoires), règlement $(\mathrm{CE}) \mathrm{n}^{\circ} 1185 / 2003$;

- fermeture temporaire de la pêche au lançon en vigueur au large du Firth of Forth (Écosse) compte tenu des répercussions de la pêche sur la survie des populations de prédateurs (oiseaux, mammifères marins, gros poissons), règlement $\mathrm{n}^{\circ} 850 / 98$, art. 29 bis ;

- dispositifs de dissuasion acoustiques pour prévenir les captures accidentelles de cétacés dans les pêcheries, règlement $(\mathrm{CE}) \mathrm{n}^{\circ} 812 / 2004$;

- mesures de protection des habitats sensibles tels que les prairies de posidonies et les colonies coralliennes en Méditerranée; cf. règlement (CE) 1967/2006] ;

- réglementation de l'utilisation des filets dérivants sur les navires de pêche; cf. règlement (CE) n 894/97 ;

- institutions de zones de protection des habitats vulnérables situés en eau profonde (notamment des récifs coralliens), règlement $(\mathrm{CE}) \mathrm{n}^{\circ} 41 / 2007$ (art. 27 et 35); voir les considérations suivantes sur les règlements $(\mathrm{CE})$ $\mathrm{n}^{\circ} 602 / 2004$ et $\mathrm{n}^{\circ} 1568 / 2005$.

110 Voir « lignes directrices pour l'établissement du réseau Natura 2000 dans le milieu marin », p. 124 (Commission, mai 2007); http://ec.europa.eu/environment/nature/Natura2000/marine/index_en.htm

111 Voir par exemple le règlement $(\mathrm{CE}) \mathrm{n}^{\circ}$ 602/2004 du 22 mars 2004 modifiant le règlement 850/98 et concernant la protection des récifs coralliens en eau profonde contre les effets du chalutage dans une zone située au nord-ouest de l'Écosse (JOUE L 211 du 21/08/2003, p. 14) au motif que « la directive 92/43/CEE du Conseil du 21 mai 1992 concernant la conservation des habitats naturels ainsi que de la faune et de la flore sauvages (...) inclut les récifs dans les habitats naturels d'intérêt communautaire dont la conservation requiert la désignation de zones spéciales de conservation. Le Royaume-Uni a formellement exprimé son intention de désigner les « Darwin Mounds » comme zone spéciale de conservation afin de protéger ce type d'habitat, en réponse aux obligations qui lui incombent en vertu de la directive ». (article $1^{\mathrm{er}}$ ). Cette mesure qui concerne une zone de 
La désignation de sites Natura 2000 par les États peut incidemment constituer pour ces derniers - lorsque c'est leur intérêt - ou pour la Communauté, un moyen de limiter radicalement les activités de pêche dans certaines zones en dehors de toute considération directement liée à la gestion des pêches proprement dite. À cet égard, il suffit de rappeler qu'il est fait obligation à l'État par la directive « habitats » de désigner des sites abritant des types d'habitats naturels ou des habitats des espèces figurant à ses annexes et d'en assurer le maintien ou, le cas échéant, le rétablissement, dans un état de conservation favorable ${ }^{112}$. En outre, il semble que la Communauté fasse une interprétation assez large des types d'habitats naturels d'intérêt communautaire de la catégorie des « eaux marines et milieux à marées » (annexe I, § 1.1) dont la conservation nécessite la désignation de $\mathrm{ZSC}^{113}$.

La mise en cohérence entre la politique de la pêche et la politique environnementale s'étend à la haute mer dans les zones qui échappent à la juridiction des États membres avec la mise en chantier d'une proposition de règlement « relatif à la protection des écosystèmes marins vulnérables de haute mer contre les effets néfastes de l'utilisation des engins de pêche de fond ${ }^{114}$. La Communauté se donne ainsi les moyens de respecter l'objectif fixé par l'assemblée générale des Nations unies aux États parties de prendre les mesures nécessaires dans les eaux internationales - en agissant notamment dans le cadre des $\mathrm{ORP}^{115}$ et d'arrangements internationaux - afin d'éliminer les pratiques de pêche destructrices en mer et protéger les écosystèmes vulnérables d'eaux profondes ${ }^{116}$.

$1560 \mathrm{~km}^{2}$ est extrêmement pénalisante pour les navires pratiquant traditionnellement le chalutage de fond dans cette région.

Également en ce qui concerne des mesures de protection d'habitats d'intérêt communautaire au sens de la directive « Habitats », le règlement $(\mathrm{CE}) \mathrm{n}^{\circ}$ 1568/2005 du Conseil du 20 septembre 2005 « modifiant le règlement (CE) $n^{\circ} 850 / 98$ en ce qui concerne la protection des récifs coralliens en eau profonde contre les effets de la pêche dans certaines zones de l'océan Atlantique » interdisant aux bateaux « d'utiliser tout filet maillant, filet emmêlant ou trémail à des profondeurs supérieures à 200 mètres ainsi que tout chalut de fond ou engin traînant similaire opérant en contact avec le fond de la mer » dans les eaux baignant les Açores, Madère et les îles Canaries, JOUE L 252 du 28/09/2005, p. 2.

Récemment, la Commission a proposé d'interdire toute activité de pêche dans certaines zones de l'ouest et sud-ouest de l'Irlande en vue de protéger des habitats fragiles (classés habitats naturels d'intérêt communautaire par la directive 92/43/CEE) dans la mesure où « la réparation des dommages causés à ces habitats par le passage d'engins de pêche actifs ou dormants est soit impossible, soit très lente et très difficile; cf. COM (2007) 570.

112 L'État est tenu à une obligation de résultat.

113 Voir le « Manuel d'interprétation des habitats de l'Union européenne » : (http://ec.europa.eu/environment/ nature/legislation/habitatsdirective/docs/2007_07_im.pdf) et les précisions apportées sur 3 types d'habitats particuliers (bancs de sables à faible couverture permanente d'eau marine; récifs; structures sous-marines) par le manuel « lignes directrices pour l'établissement du réseau Natura 2000 dans le milieu marin », chapitre III.

114 COM (2007) 605 du 17 octobre 2007. Le règlement concerne les navires battant pavillon communautaire menant des activités de pêche avec des engins de fond en haute mer (hors zones placées sous la responsabilité d'une ORP pour ce type d'activités ou lorsque des mesures provisoires sont prises dans le cadre de la constitution d'une nouvelle ORP). La pêche est soumise à la délivrance par les autorités nationales d'un permis de pêche spécial après avoir réalisé une évaluation des impacts potentiels des activités de pêche prévues par le navire. L'utilisation d'engins de fond au-delà de 1000 mètres est interdite.

115 Organisation régionale de pêche.

116 La Communauté est en grande partie à l'origine de cette proposition. L'assemblée demande que des mesures soient prises au plus tard fin 2008; cf. résolution du 8 décembre 2006, A/RES/61/105 et communication de la commission du 17 octobre 2007, COM (2007) 604, précités. 
Dans leurs eaux sous souveraineté et juridiction ${ }^{117}$, les États membres doivent proposer les sites nécessaires pour achever la composante marine du réseau Natura 2000. Ils doivent prendre les mesures de gestion nécessaires dont la responsabilité leur incombe étant en cela limités par leurs obligations internationales.

S'agissant de la pêche, le principe général est que les mesures de gestion doivent être prises par la Communauté dans le cadre de sa compétence exclusive sur la base de l'article 37 du traité, conformément aux principes de la PCP. En effet, dans les zones désignées $^{118}$, outre des mesures spécifiques, la protection des habitats ou des espèces nécessitera souvent de réglementer les activités de pêche elles-mêmes ${ }^{119}$. Lorsque la conservation des habitats et des espèces des sites Natura 2000 nécessitera des mesures de gestion des pêches, l'État devra fournir à la Commission les éléments nécessaires pour que la Communauté puisse fixer les règles. En outre, le caractère réglementaire des mesures de gestion et de conservation de la pêche, le système de contrôle, la définition des infractions et le régime de sanctions offrent de nombreux avantages à l'utilisation du cadre réglementaire de la PCP pour la conservation de la biodiversité sur celui moins contraignant de la directive « Habitats ».

Si en matière de pêche, le principe est celui de la compétence communautaire, rappelons que l'État dispose de pouvoirs importants dans la bande côtière. S'agissant des mesures de conservation prises dans le cadre des sites Natura 2000 compris dans les limites de sa mer territoriale, il pourra exercer pleinement ses pouvoirs de gestion de pêche. Ainsi, la définition des régimes de gestion des aires Natura 2000 comprises dans les limites de la mer territoriale ressort pour l'essentiel de l'autorité de l'État.

En outre, les devoirs de l'État en matière de conservation des habitats dans les ZSC sont étroitement contrôlés. Ainsi, les «États membres prennent des mesures pour éviter la détérioration des habitats naturels et des habitats d'espèces ainsi que les perturbations touchant les espèces pour lesquelles les zones ont été désignées $(\ldots){ }^{120}$. Cette « obligation de portée générale » est complétée par une disposition stipulant que l’État, sous réserve d'impératifs d'intérêt public majeur, ne doit donner son accord à tout «plan » ou « projet » non directement lié ou nécessaire à la gestion du site mais « susceptible d'affecter ce site de manière significative », qu'après s'être assuré (selon une « évaluation appropriée de ses incidences sur le site eu égard aux objectifs de conservation de ce site ») qu'il ne portera pas atteinte à l'intégrité du site concerné. La Cour de justice a jugé que cette disposition avait des conséquences directes sur la mise en œuvre par l'État de ses pouvoirs ${ }^{121}$.

\footnotetext{
117 À l'exception des eaux des DOM-TOM (cf. directive 79/409/CEE, art. 1er et 92/43/CE, art. 2).

118 ZSC mais des mesures de conservation doivent être prises dès qu'un site est sélectionné comme site d'importance communautaire (dir. 92/43/CE, art. $4 \S 5$ ).

119 En consultation avec les parties intéressées et notamment les conseils consultatifs régionaux.

120 Directive 92/43/CE, article $6 \S 2$.

121 Ibid. (§ 3). La CJCE voit dans cette disposition l'instauration d'une procédure « visant à garantir, à l'aide d'un contrôle préalable, qu'un plan ou un projet non directement lié ou nécessaire à la gestion du site concerné, mais susceptible d'affecter ce dernier de manière significative, n'est autorisé que pour autant qu'il ne porte pas atteinte à l'intégrité de ce site » (selon la Cour, un «plan » ou « projet » au sens de la directive doit être entendu comme couvrant, notamment, des interventions destinées à l'exploitation des ressources du sol; c'est-à-dire, en l'espèce, de modes de pêche susceptibles d'affecter les habitats naturels et les habitats d'espèces se situant sur le sol de la mer). En outre, la Cour précise que cette disposition doit être interprétée en ce sens que « lorsqu'un tel plan ou projet risque de compromettre les objectifs de conservation du site concerné, il doit nécessairement être considéré comme susceptible d'affecter ce dernier de manière significative ». Plus clairement encore, « lorsque
} 
S'agissant de la protection des espèces animales nécessitant une protection stricte, seul, en principe, le caractère intentionnel de la capture, de la mise à mort ou de la perturbation de ces espèces durant certaines périodes particulières de leur vie est prohibé. Toutefois, la détérioration ou la destruction des sites de reproduction ou des aires de repos sont prohibées en toutes circonstances. En outre, les États membres doivent instaurer un système de contrôle des captures et mises à mort accidentelles de ces espèces et, le cas échéant, «prendre les mesures de conservation nécessaires pour faire en sorte que les captures ou mises à mort involontaires n'aient pas une incidence négative importante sur les espèces en question ${ }^{122}$.

En définitive, les conséquences des activités de pêche - des méthodes pratiquées doivent instamment être prises en compte dans le cadre de la mise en œuvre du réseau Natura 2000.

En ce qui concerne le type de mesures de conservation que l'État doit prendre, l'article 6 de la directive « Habitats » précise qu'elles peuvent être de nature réglementaire, administrative ou contractuelle. Toutefois, si un État membre choisit les mesures contractuelles, il lui incombe toujours l'obligation d'établir à titre permanent les mesures de conservation nécessaires qui répondent « aux exigences écologiques des types d'habitats naturels de l'annexe I et des espèces de l'annexe II présents sur les sites » et de respecter l'objectif général de la directive, tel qu'il est défini à l'article $2 \S 1^{123}$.

La démarche contractuelle adoptée par la France pour la mise en œuvre de l'article 6 (§1 et 2) de la directive « Habitats » qui concerne les titulaires de droits réels et personnels sur des espaces terrestres ne peut manifestement être transposée en mer dans des espaces pour partie inclus dans le domaine public de l'État (DPM) ou pour des ressources considérées comme un patrimoine collectif. On peut douter, par ailleurs, que la simple adhésion par les professionnels ou les utilisateurs des espaces marins à la charte Natura $2000^{124}$ suffise à la France pour assurer le respect des objectifs communautaires.

\section{Le système français des aires marines protégées}

Les sites Natura 2000 ayant une partie maritime sont compris au titre des aires marines protégées à laquelle l' « agence des aires marines protégées » apporte son concours ${ }^{125}$.

\footnotetext{
subsiste une incertitude quant à l'absence d'effets préjudiciables pour l'intégrité dudit site liés au plan ou au projet considéré, l'autorité compétente devra refuser l'autorisation de celui-ci ». Cf. CJCE (Grande chambre) du 7 septembre 2004, aff. C-127/02 « Landelijke Vereniging et autres », sp. § 31 à 61 . Il va de soi que cette précision de la Cour de Luxembourg ne manquera pas d'avoir d'importantes répercussions sur les diverses pêches pratiquées à l'aide d'engins traînants ou grattant le fond de la mer dans le périmètre des zones spéciales de conservation.

${ }_{122}$ Ibid., article $12, \S 4$.

${ }^{123}$ Cf. Manuel « Gérer les sites Natura 2000. Les dispositions de l'article 6 de la directives Habitats », p. 21.

124 Code de l'Environnement (article L 414-3 § II): « Les titulaires de droits réels et personnels portant sur les terrains inclus dans le site ainsi que les professionnels et utilisateurs des espaces marins situés dans le site peuvent adhérer à une charte Natura 2000. La charte Natura 2000 comporte un ensemble d'engagements définis par le document d'objectifs et pour lesquels le document d'objectifs ne prévoit aucune disposition financière d'accompagnement. ».

${ }^{125}$ Code de l'Environnement (art. L 334-1).
} 
Lorsque le site comprend majoritairement des espaces marins, l'établissement du « document d'objectifs $»^{126}$ et le suivi de sa mise en œuvre sont confiés à l'autorité administrative - en association avec le « comité de pilotage » qu'elle préside ${ }^{127}$ - Toutefois, la loi précise ${ }^{128}$ que les mesures de conservation ou de rétablissement des habitats naturels et des espèces menacés ou les mesures de prévention destinées à prévenir leur détérioration «ne conduisent pas à interdire les activités humaines dès lors qu'elles n'ont pas d'effets significatifs sur le maintien ou le rétablissement dans un état de conservation favorable de ces habitats naturels et de ces espèces » et que, notamment, la pêche et les activités aquacoles, pratiquées dans les conditions et sur les territoires autorisés par les lois et règlement en vigueur, « ne constituent pas des activités perturbantes ou ayant de tels effets ». Autrement dit, le législateur considère que pour autant que les activités de pêche puissent être concernées par les objectifs de préservation des sites Natura 2000, la réglementation de la pêche constitue un cadre et une garantie suffisante au respect de ces objectifs. Cet a priori particulièrement optimiste des Autorités françaises contraste fortement avec les analyses suivies d'effets ${ }^{129}$ des Autorités communautaires sur le sujet; pour qu'il soit justifié, encore faudrait-il admettre que ces mêmes autorités, dans le cadre des pouvoirs qu'elles exercent et des mesures qu'elles prennent, aient effectivement pris la mesure des enjeux d'une gestion écosystémique des pêches et de la nécessité de minimiser les impacts de la pêche sur les écosystèmes.

La question se pose différemment lorsque la plus grande partie maritime d'un site Natura 2000 se trouve incluse dans le périmètre d'un parc naturel marin. Dans ce cas, le plan de gestion du parc a valeur de document d'objectifs du $\operatorname{site}^{130}$; il convient donc d'appliquer le régime juridique des parcs naturels marins (PNM).

À l'intérieur des PNM, le « conseil de gestion » sous l'autorité de l'Agence des aires marines protégées élabore un plan de gestion et définit un programme d'actions. Le conseil peut proposer toute mesure nécessaire à la protection et à la gestion durable du parc - notamment en matière d'occupation du domaine public maritime et de pêche - et peut, le cas échéant ${ }^{131}$, donner un avis conforme à l'autorisation de toute activité susceptible d'altérer de façon notable le milieu marin du parc ${ }^{132}$. Cela concerne notamment les licences de pêche prévues par les articles 4 et 22 du décret $n^{\circ} 92-335$ du 30 mars $1992^{133}$. À défaut de détenir un pouvoir réglementaire dans le domaine de la gestion de la pêche, il s'agit pour le Conseil, en l'espèce, d'un pouvoir - théorique? - d'empêcher ${ }^{134}$. Rappelons que ces licences constituent l'instrument emblématique de la gestion de la pêche dans la bande côtière bretonne ${ }^{135}$.

\footnotetext{
${ }^{126}$ Ce document comprend les objectifs de développement durable du site permettant d'assurer la conservation et, éventuellement, la restauration des habitats naturels et des espèces qui justifient la désignation du site ainsi que des propositions de mesures permettant d'atteindre ces objectifs (code de l'Environnement, art. R 414-11).

127 Code de l'Environnement (art. L 414-2 § 8).

128 Code de l'Environnement (art L 414-1 § 5).

129 Cf. supra.

${ }_{130}$ Code de l'Environnement (art. R 414-8).

131 La compétence de droit commun appartient à l'Agence des aires marines protégées.

132 Code de l'Environnement (art. L 334-5 et R 334-33).

${ }^{133}$ Décret fixant les règles d'organisation et de fonctionnement du Comité national des pêches maritimes et des élevages marins ainsi que des comités régionaux et locaux des pêches maritimes et des élevages marins.

134 Mais aussi est-ce bien la voie de la concertation qui a été choisie pour la définition et la mise en œuvre du plan de gestion.

135 Cf. supra.
} 
La multiplicité des actions envisagées dans le cadre de la gestion du premier parc naturel marin ${ }^{136}$ afin d'organiser les activités de pêche et en prévenir les effets témoigne de la place essentielle qu'occupera la pêche dans la réalisation des objectifs du parc.

Les intéressantes propositions du document d'orientation ${ }^{137}$ qui permet de fixer les orientations de gestion ${ }^{138}$ soulignent cependant le caractère essentiellement incitatif et « recommandatoire » des mesures préconisées notamment pour la poursuite des objectifs d' « exploitation durable des ressources halieutiques » et de « soutien à la pêche côtière professionnelle $»^{139}$.

Sans préjuger du résultat final, il faudra attendre trois ans afin de mesurer de la portée réelle des mesures proposées par le futur plan de gestion.

Enfin, on ne manquera pas de s'interroger sur les raisons qui ont incité le législateur à ne pas accorder au Conseil de gestion des parcs naturels marins les pouvoirs dévolus à l'établissement public d'un parc national dans les espaces maritimes qu'il couvre et qui consistent en la possibilité de proposer aux autorités administratives compétentes de soumettre la pêche à un régime particulier ${ }^{140}$.

S'agissant de la prochaine désignation des sites Natura 2000 marins en France, notons que la liste des aires marines protégées (AMP) prévue à l'article 334-1 du Code de l'Environnement qui n'en prévoit pas l'existence, n'est pas exhaustive. L'article R 334-2 du Code de l'Environnement définit la procédure à suivre pour identifier d'autres aires marines protégées concernées par l'agence.

Le moment est venu d'inclure des sites marins dans les aires marines protégées placées sous contrôle de l'agence. À ce sujet, se posera nécessairement la question de l'application de l'article L 414-1 ${ }^{141}$ dont le libellé est en manifeste contradiction avec l'objet même que la Communauté fixe à ces espaces.

D'autres instruments de gestion de l'environnement sont susceptibles d'exercer des contraintes sur les activités de pêche.

\footnotetext{
136 Parc naturel marin d'Iroise créé par décret 2007-1406 du 28-09-2007 (JORF du 2-10-2007).

$137 \mathrm{http}$ ///iroise-parcnational.gouv.fr/medias/documents/www/contenu//enquete_publique/odg_version_7.pdf.

138 Ces orientations préfigurent le plan de gestion qui doit être élaboré par le conseil de gestion du parc.

139 Il n'est ainsi question que:

- d'encourager les mesures techniques allant dans le sens d'une plus grande sélectivité ;

- de conserver les potentialités des écosystèmes marins en terme de production de ressources ;

- d'assurer la protection d'habitats considérés comme critique pour la pérennité et la survie des espèces et des ressources (nourriceries, frayère) ;

- de définir une stratégie de restauration des stocks et des populations surexploitées ;

- de proposer aux structures socioprofessionnelles les mesures de gestion adaptées à la reconquête des stocks les plus stratégiques pour les professionnels tout en garantissant la pérennité des entreprises ;

- de faire de l'espace du parc, une zone pilote en matière de gestion des ressources halieutiques de haute qualité environnementale, sociale et de sécurité au travail ;

- d'encourager la prise en compte de la protection de l'environnement marin dans la gestion des pêches ;

- en étudiant notamment leur impact potentiel sur les milieux, d'évaluer les prélèvements possibles, proposer des mesures de régulation ;

- de valoriser les produits issus d'une pêche responsable et respectueuse de la ressource (labellisation);

- de participer à la prévention des conflits d'usages entre les divers types de pêcheries.

140 Code de 1'Environnement (art. L 331-14).

141 Cf. supra.
} 


\section{La protection de ces espèces par le contrôle du commerce international}

Elle fait précisément l'objet de la Convention internationale sur le commerce des espèces de la faune et de la flore sauvage menacées d'extinction (dite convention CITES) ${ }^{142}$ mise en vigueur dans le cadre communautaire depuis $1984^{143}$.

Il importe de noter l'inscription récente ${ }^{144}$ d'espèces de poissons à haute valeur commerciale destinées à la consommation humaine à l'annexe II de la convention CITES $^{145}$. Ce mouvement pourrait s'étendre à d'autres espèces directement concernées par la pêche maritime commerciale et notamment à certaines espèces dont l'état de conservation est préoccupant ${ }^{146}$. Cette éventualité pose cependant un certain nombre de difficultés quant à l'application des critères d'inscription sur la liste de l'annexe II de la CITES des espèces exploitées commercialement et gérées dans le cadre des politiques de gestion des pêches. Cette difficulté porte notamment sur l'évaluation du risque d'extinction d'une espèce qui doit être pris en compte dans le cadre de ces critères d'inscription ${ }^{147}$. L'inscription d'espèces commerciales à l'annexe II de la convention pose également des problèmes de nature juridique dans la mesure où elle est susceptible de faire surgir des conflits entre la mise en œuvre de la convention et le droit international de la pêche ${ }^{148}$.

\section{Focus régionaux}

\section{Golfe du Morbihan/Mor-Braz}

L'analyse de la pêche professionnelle a été effectuée sur le site du Mor-Braz, situé en Bretagne Sud. Cette zone correspond en mer aux limites des quartiers maritimes d'Auray et de Vannes et à terre aux zones d'emploi d'Auray et de Vannes. Ce site a été choisi en raison de la très forte présence des activités de la pêche côtière.

\footnotetext{
${ }^{142}$ Convention signée à Washington le 3 mars 1973, O.N.U., vol. 993, p. 243.

143 Règlement $n^{\circ}$ 338/97 du Conseil du 9 décembre 1996 relatif à la protection des espèces de flore et de faune sauvage par le contrôle du commerce (JOCE L 61 du 3/3/97, p. 1), modifié en dernier lieu par un règlement $n^{\circ}$ 834/204 de la Commission du 28 avril 2004 (JOUE L 127 du 29/04/04, p. 40).

${ }^{144}$ Napoléon et Datte de mer à la $13^{\mathrm{e}}$ session de la conférence des parties, Bangkok, 2-14 octobre 2004 et anguille européenne à la 14 session de la conférence des parties, La Haye, 3-15 juin 2007 (pour cette espèce, l'entrée en vigueur a été repoussée au 13 mars 2009).

${ }^{145}$ Inscription à l'Annexe II (qui comprend les espèces qui ne sont pas nécessairement menacées d'extinction, mais dont le commerce doit être contrôlé pour éviter une utilisation incompatible avec leur survie), avec un quota d'exportation annuel nul du napoléon, de la « datte de mer » en Méditerranée.

146 Voir notamment, la menace qui pèserait sur le thon rouge; Le marin, 23/11/2007.

${ }^{147}$ Sur cette question, cf., notamment, une circulaire de la FAO n ${ }^{\circ} 954$ « Évaluation de la validité des critères d'inscription des espèces aquatiques commercialement exploitées sur les listes de la CITES », FAO, Rome 2000. Notons que la CITES et la FAO ont en octobre 2006 formalisé leur rapport de collaboration dans un protocole d'accord. En vertu de ce dernier, la FAO et la CITES examineront conjointement l'évaluation scientifique, juridique et technique des espèces aquatiques faisant l'objet d'une exploitation commerciale, inscrites ou proposées dans les Annexes de la Convention. Ce type de collaboration devrait s'étendre aux Organisations régionales de pêche.

${ }^{148}$ Cf. FAO, rapport sur les pêches n $746,2004$.
} 


\section{Les activités de pêche des navires d'Auray et Vannes et le système halieutique}

\section{Les activités de pêche professionnelle : présentation générale des navires des quartiers maritimes d'Auray et de Vannes}

Les navires de pêche professionnelle immatriculés dans les quartiers maritimes d'Auray et de Vannes (AY et VA) n'appartiennent pas tous à la même catégorie administrative. Les navires inscrits au fichier national de la flotte française sont titulaires d'un PME (Permis de mise en exploitation). Ces derniers étaient inscrits jusqu'en 2002 dans les Programmes d'orientation pluriannuels (POP), pour simplifier on les nommera « navires inscrits aux POP ». Cependant, dans la zone d'étude, certains navires exercent la pêche sans être inscrits au fichier national de la flotte, par la suite ils seront désignés « navires hors POP ». Il s'agit de navires armés en culture marine petite pêche (CMPP) ou ayant un rôle « bivalves ». En plus des pêcheurs embarqués, une vingtaine de pêcheurs à pied professionnels (non inscrits maritimes) exercent leur activité sur la zone d'étude, mais elle reste encore mal connue.

La flotte étudiée comprend 257 navires inscrits au fichier national de la flotte en 2002 et environ 200 navires hors POP.

Les navires de cette flotte:

- sont généralement de petite taille et de faible puissance;

- exercent leur activité essentiellement dans la bande côtière;

- ont une activité régulière sur l'année;

- sont très polyvalents (le degré de polyvalence est moins élevé pour les navires hors POP).

Les navires d'Auray et de Vannes inscrits aux POP ont exercé 93 métiers au cours de l'année 2002. Vingt-cinq métiers contribuent à $80 \%$ de l'activité totale. Le métier le plus exercé est le tamis à civelles que ce soit en termes de mois d'activité ou de nombre de navires. Ensuite, viennent la drague à coquilles Saint-Jacques, le casier à bouquets, le tramail à soles, la pêche à la main des palourdes, la palangre de fond à bars, le chalut de fond à divers poissons, la ligne à main à bars... Les navires hors POP ont pratiqué 19 métiers et les 4 métiers principaux représentent presque $90 \%$ de l'activité totale de ces navires. Il s'agit de la pêche à pied à palourdes (61 \% de l'activité totale), de la pêche à pied des moules, de la plongée en apnée des palourdes et de la pêche à pied des oursins.

Les arts dormants prédominent, ils ont représenté la moitié de l'activité de toute la flotte en 2002. Ces engins sont diversifiés : palangres, pièges, filets maillants, filets soulevés, lignes. Les arts traînants, essentiellement la drague à coquillages, le chalut de fond et le tamis à civelles sont pratiqués par les navires POP uniquement et représentent un quart de l'activité totale. L'activité de pêche dans ce secteur est caractérisée par l'importance de la pêche à la main (à pied ou en apnée), elle représente un quart de l'activité totale et est très pratiquée par les navires hors POP.

Concernant la gestion des pêches, on notera l'importance des licences de pêche dans ces deux quartiers maritimes; 17 types de licences ont été recensés:

- une licence nationale : la licence pour la pêche dans les estuaires et la pêche des poissons migrateurs, dite licence $\mathrm{CIPE}^{149}$;

- deux licences régionales: la licence de pêche aux filets et la licence de pêche aux crustacés $^{150}$;

\footnotetext{
149 Commission interprofessionnelle des poissons migrateurs et des estuaires.

150 À l'exception des langoustines et des pouce-pied.
} 
- 14 licences locales : il s'agit surtout de pêches à la main ou à la drague de coquillages sur des gisements particuliers.

S'ajoute à ces licences, des autorisations de pêche, délivrées par les Affaires maritimes, pour la pêche des pétoncles dans le golfe du Morbihan, des coques et des palourdes dans l'estuaire de la Vilaine.

Le système de production ne peut exister indépendamment d'autres activités en amont qui lui fournissent ses approvisionnements et d'autres activités en aval qui lui permettent de commercialiser ses captures. L'ensemble constitue ce que l'on peut appeler le système halieutique.

Focus sur le comportement économique des flottilles de pêche du golfe du Morbihan

Une étude particulière a été menée sur les flottilles de pêche du golfe du Morbihan. Elle va au-delà de la description des caractéristiques techniques des bateaux en prenant en compte :

- la diversité des métiers pratiqués au cours de l'année;

- les interactions entre métiers qui peuvent générer des conflits pour l'espace et/ou pour la ressource;

- les stratégies de pêche qui s'expriment par le choix des métiers pratiqués au cours de l'année;

- les situations économiques des différentes flottilles de pêche professionnelle dans le golfe du Morbihan.

La population concernée a été identifiée à partir du traitement des bases de données sur l'activité des navires en 2001 qui permet de recenser 175 navires ayant pêché au moins une fois dans le golfe du Morbihan.

Cette flotte comprend 92 navires inscrits au $\mathrm{POP}^{151}$ : 67 navires ont fait l'objet d'une enquête détaillée sur leurs métiers et zones de pêche dans le golfe du Morbihan et 25 navires n'étaient pas inclus dans le plan d'échantillonnage initial.

Ces informations ont été utilisées pour décrire les caractéristiques physiques des navires, l'intensité de l'activité dans le golfe du Morbihan, les métiers pratiqués, la saisonnalité des métiers et pour cartographier le nombre de mois d'activité et le nombre de navires par secteur. De plus, la pêche professionnelle des palourdes et des oursins a fait l'objet d'une étude détaillée.

Si les métiers pratiqués au sein du golfe du Morbihan sont divers, la prédominance des activités de pêche à la main a particulièrement été mise en en évidence, particulièrement en apnée, pour l'exploitation des gisements de palourdes, d'un point de vue de la population impliquée et du poids économique de cette activité.

L'analyse des modalités d'exploitation des gisements de palourde a révélé l'hétérogénéité des flottilles en matière d'engins utilisés, de stratégies d'allocation de leur effort de pêche entre les zones du golfe, d'une part, et les zones hors du golfe, d'autre part, et enfin, de la structuration de leurs recettes et de leurs coûts annuels d'exploitation.

La situation économique de la flotte de pêche du golfe de Morbihan a été appréhendée à partir des enquêtes directes effectuées en 2002 auprès d'un échantillon de 65 patrons pêcheurs sur leurs résultats en 2001. Une flottille regroupe des navires proches d'un point de vue des combinaisons de métiers qu'ils exercent et si un navire peut exercer plusieurs métiers, il ne peut appartenir qu'à une seule flottille. Par ailleurs, un navire peut exercer

\footnotetext{
${ }^{151}$ En réalité, 94 navires inscrits au POP ont déclaré une activité de pêche dans le golfe du Morbihan en 2001 mais deux d'entre eux exercent une activité exclusivement conchylicole.
} 
un métier caractéristique d'une flottille et ne pas appartenir à cette flottille. Ainsi pour illustration, 26 navires pratiquent la drague à palourdes dans le golfe du Morbihan en 2001 alors que la flottille des dragueurs à palourdes et oursins n'est finalement composée que de 22 navires. Les différentes combinaisons de métiers pratiqués par les navires dans le golfe du Morbihan ont permis d'identifier 6 flottilles significatives et de répartir les navires du golfe du Morbihan selon cette typologie:

- plongeurs en apnée à palourdes et oursins (activité fortement exercée par les navires hors POP);

- pêcheurs à pied à palourdes (activité fortement exercée par les navires hors POP);

- dragueurs à palourdes et oursins;

- verveux à anguilles et casiers à seiches et bouquets;

- palangres et filets à bar et divers poissons;

- casiers à seiches et petits crustacés.

Les flottilles prédominantes sont les «plongeurs en apnée à palourdes » d'une part et les « pêcheurs à pied à palourdes », d'autre part.

Le tableau 15 récapitule certains éléments essentiels de l'activité et de la situation économique des flottilles de pêches dans le golfe du Morbihan en 2001 (hors activité pêche à la main).

Tableau 15. Synthèse de l'activité et de la situation économique en 2001 des flottilles de pêche professionnelle du golfe du Morbihan, hors pêche à la main (source: Ifremer/SIH).

\begin{tabular}{|c|c|c|c|c|}
\hline & $\begin{array}{l}\text { Dragueurs } \\
\text { à palourdes } \\
\text { et oursins }\end{array}$ & $\begin{array}{l}\text { Verveux à } \\
\text { anguilles } \\
\text { et casiers } \\
\text { à seiches/ } \\
\text { bouquets }\end{array}$ & $\begin{array}{c}\text { Palangres } \\
\text { et filets } \\
\text { à bars/ } \\
\text { divers } \\
\text { poissons }\end{array}$ & $\begin{array}{l}\text { Casiers } \\
\text { à seiches } \\
\text { et petits } \\
\text { crustacés }\end{array}$ \\
\hline Nombre de navires dans la flottille & 22 & 6 & 12 & 10 \\
\hline Longueur moyenne $(\mathrm{m})$ & 9,25 & 8,77 & 7,88 & 8,38 \\
\hline Puissance moyenne (kW) & 91 & 67 & 69 & 78,5 \\
\hline Âge moyen (ans) & 22 & 29 & 24 & 20 \\
\hline Équipage moyen (homme) & 1,9 & 1,3 & 1 & 1,2 \\
\hline $\begin{array}{l}\text { Nombre de mois de présence dans } \\
\text { le golfe du Morbihan }\end{array}$ & 5 & 8 & 8 & 7 \\
\hline $\begin{array}{l}\text { Poids effectif du golfe du Morbihan par } \\
\text { rapport à l'activité totale dans toutes les zones } \\
\text { de pêche }(\%)\end{array}$ & 29 & 69 & 62 & 60 \\
\hline Part du carburant dans le CA (\%) & 4 & 6 & 6 & 5 \\
\hline Part des engins dans le CA (\%) & 5 & 10 & 7 & 5 \\
\hline Part des coûts personnels dans le CA (\%) & 51 & 56 & 51 & 50 \\
\hline $\begin{array}{l}\text { Chiffre d'affaires annuel d'un navire moyen en } \\
2001(€)\end{array}$ & 103000 & 49000 & 53500 & 74500 \\
\hline $\begin{array}{l}\text { Salaire annuel brut moyen en } 2001 \text { y compris } \\
\text { cotisations salariales }(€)\end{array}$ & 25268 & 16361 & 24396 & 22862 \\
\hline $\begin{array}{l}\text { Estimation des recettes brutes totales } \\
\text { provenant du golfe du Morbihan en } 2001(\mathrm{k} €)\end{array}$ & {$[265-1055]$} & [133-271] & [302-494] & [110-739] \\
\hline Taux de valeur ajoutée $(\%)$ & 82 & 74 & 79 & 82 \\
\hline
\end{tabular}


On peut remarquer une assez forte disparité des résultats, en particulier pour ce qui concerne les estimations de recettes brutes totales, avec de très forts écarts à l'intérieur d'une même flottille, ainsi que pour ce qui concerne les salaires bruts annuels moyens.

Plus précisément les structures de coût de production des diverses flottilles présentées sur la figure 55 p. XV illustrent les caractéristiques de chaque flottille.

Pour ce qui concerne les indicateurs de performance à savoir le taux de valeur ajoutée (valeur ajoutée/chiffre d'affaires) et le taux de profit (excédent net exploitation/valeur du capital investi), les disparités entre flottilles sont de l'ordre d'un à quatre (figure 56).

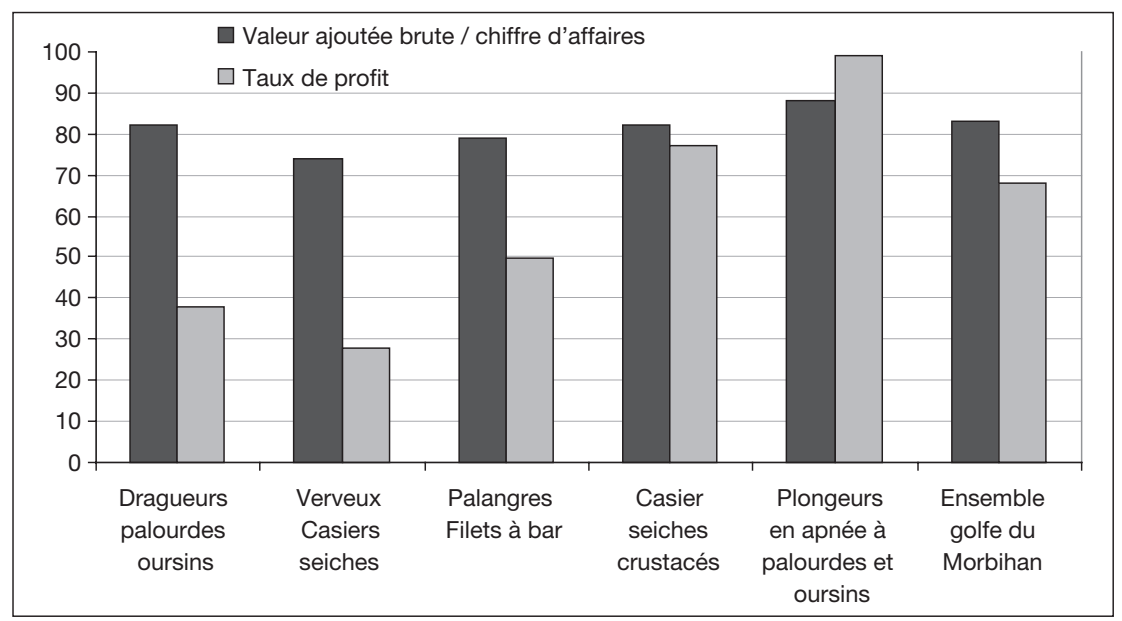

Figure 56. Taux de valeur ajoutée et taux de profit (\%) des flottilles du golfe du Morbihan (source : Ifremer/SIH).

\section{Le système halieutique}

Le système halieutique se définit comme l'ensemble des acteurs qui interviennent pour faire vivre les activités de pêche ainsi que les relations qu'ils ont établies entre eux et avec leurs partenaires extérieurs. La figure 57 schématise le système pêche côtière de la zone étudiée; pour simplifier, les structures situées hors de la zone d'étude n'ont pas été représentées.

Les secteurs situés en amont de la pêche sont divers et très difficiles à cerner. Les établissements de ces secteurs n'ont généralement pas une activité uniquement en relation avec la pêche. La part de la pêche est même souvent accessoire par rapport à celles de la plaisance et de la conchyliculture.

Le commerce de gros des produits de la pêche côtière est essentiellement réalisé par une vingtaine de mareyeurs et des grossistes de la zone mais aussi par des mareyeurs et des grossistes de Lorient ou de Nantes. Des industries (conserveries et ateliers de fumaison) sont très présentes surtout sur la presqu'île de Quiberon mais transforment peu ou pas de produits de la pêche côtière locale. Quant au commerce de détail, une centaine d'étals de poissonniers sédentaires et ambulants et de grandes surfaces proposent des produits de la mer. Ces établissements commercialisent une partie des produits de la pêche côtière locale en les vendant en frais essentiellement. On peut noter la diversité des circuits de commercialisation des produits de la pêche côtière où interviennent plusieurs intermédiaires avant le stade de consommation finale: mareyeurs, grossistes, supermarchés et hypermarchés, poissonniers, restaurateurs. 


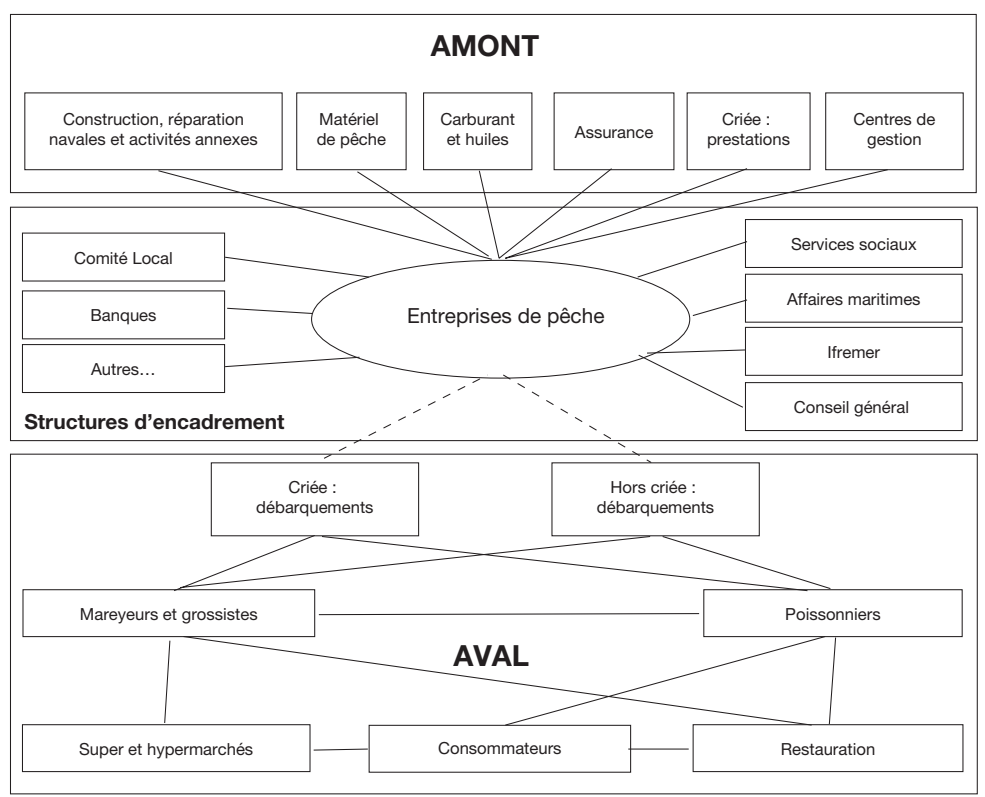

Figure 57. Système halieutique du Mor-Braz.

On rencontre quatre types de circuit de commercialisation:

- le circuit direct: producteur $\rightarrow$ consommateur;

- le circuit court: producteur $\rightarrow$ détaillant, restaurateur $\rightarrow$ consommateur;

- le circuit raccourci: producteur $\rightarrow$ mareyeur $\rightarrow$ détaillant, restaurateur $\rightarrow$ consommateur;

- le circuit long: producteur $\rightarrow$ mareyeur $\rightarrow$ grossiste $\rightarrow$ détaillant, restaurateur $\rightarrow$ consommateur.

Les trois premiers circuits sont les plus fréquents pour les produits de la pêche locale, le nombre d'intermédiaires y reste limité.

Plus d'une dizaine de structures d'encadrement participent au fonctionnement du secteur de la pêche dans la zone d'étude. Leurs missions sont variées : gestion, contrôle, surveillance de la pêche, aides financières et techniques, recherche, formation... Cependant, ces structures ont généralement un champ d'action plus large que les deux zones d'emploi étudiées : il s'agit souvent de structures départementales voire même régionales. De plus, à part quelques organismes propres à la pêche, les structures d'encadrement sont souvent communes à toutes les activités maritimes comme la pêche, l'aquaculture, la conchyliculture, voire même la plaisance et la marine marchande. Dans certains cas, elles s'occupent même d'autres secteurs d'activité : c'est le cas des banques et des assurances par exemple. Pour ces raisons, il est très difficile de déterminer la part de la pêche côtière des navires d'Auray et de Vannes dans l'activité de ces structures.

\section{Les effets économiques induits}

En s'appuyant sur cette description des agents qui constituent le système halieutique de la pêche côtière, il est possible d'identifier et de faire une première évaluation des effets économiques induits dans les zones d'emploi par les activités de pêche côtière en termes monétaires et d'emploi. 


\section{Les effets monétaires}

Les flux monétaires considérés sont ceux qui sont directement générés par les unités de production (achats et ventes des entreprises de pêche) et ceux qui sont induits par ces flux directs tout au long de la filière.

\section{Les flux économiques monétaires directs}

Il s'agit de reconstituer les différents flux monétaires créés par les entreprises de pêche côtière de la zone étudiée. Cela concerne aussi bien les flux d'inputs (approvisionnement, entretien, réparation...) que ceux d'outputs (ventes de la production débarquée). À cette étape, il n'est pas possible de faire la distinction entre la part de ces flux qui proviennent ou entrent dans la zone étudiée et la part des flux qui concernent l'extérieur. Il s'agit donc de flux globaux sans considération quant à leur origine ou à leur destination géographique, non compris ceux liés à l'investissement.

Les dépenses en carburant et en lubrifiants représentent $6 \%$ du chiffre d'affaires total de la flotte. Cependant, cette moyenne ne reflète pas les grandes variations entre les flottilles: ce poste représente $12 \%$ pour la flottille des chalutiers de plus de 9 mètres, à l'opposé il représente $2 \%$ pour la flottille des divers métiers côtiers. Il existe une corrélation positive entre la pratique du chalut et les frais de carburant. Les postes de réparation et d'entretien équivalent à $8 \%$ du chiffre d'affaires.

Par contre, les frais en vivres, glace et appâts sont très faibles : les trois postes regroupés comptent pour un peu plus de $1 \%$ du chiffre d'affaires. Les frais en vivres ne sont pas toujours pris en charge par l'armement. De plus, les navires étudiés sont des navires côtiers, la durée des marées peut être très courte (quelques heures). La plupart d'entre eux rentrent tous les soirs à terre. Il y a donc peu, voire pas de vivres embarqués. Les frais de vivres sont les plus importants pour la flottille des chalutiers de plus de 12 mètres qui partent pour des marées plus longues ( 24 heures en moyenne) et où l'équipage est plus nombreux. La durée des marées ne nécessite généralement pas l'embarquement de glace. Néanmoins l'été, certains navires s'approvisionnent en glace à la criée. L'achat de glace est plus important pour la flottille des arts dormants de 7 à 9 mètres et pour celle des chalutiers de 12 mètres, mais reste marginal: moins de $1 \%$ du chiffre d'affaires. Les dépenses en appâts ne concernent pas toute la flotte: les chalutiers n'utilisent pas d'appât (encore appelé boëtte), par contre les entreprises de pêche qui utilisent des casiers, des palangres ou des lignes, appâtent leurs engins. On retrouve logiquement les dépenses les plus élevées dans les strates des arts dormants ( 1 à $3 \%$ du chiffre d'affaires suivant la longueur des navires).

Presque un quart (23\%) du chiffre d'affaires réalisé par la flotte Auray Vannes serait utilisé en consommations intermédiaires. La valeur ajoutée brute dégagée par cette activité constituerait en moyenne plus des trois quarts du chiffre d'affaires (77\%). Suivant les flottilles, cette répartition varie : le taux de valeur ajoutée serait de $61 \%$ pour la flottille des chalutiers de plus de 12 mètres et de $93 \%$ pour les divers métiers côtiers. Les consommations intermédiaires sont très élevées pour la flottille des chalutiers de plus de 12 mètres, la consommation en carburant et en services représente une part importante du chiffre d'affaires : respectivement 12 et $11 \%$. À l'opposé, les consommations intermédiaires sont réduites à leur minimum pour la flottille des divers métiers côtiers, elles ne correspondent qu'à $7 \%$ du chiffre d'affaires. En général, plus la longueur du navire augmente, plus le taux de valeur ajoutée diminue.

Les débarquements des navires d'Auray et de Vannes représenteraient 21,4 millions d'euros $(\mathrm{M} €)(+/-1,85 \mathrm{M} €)$. Une partie de ces débarquements est réalisée hors de la 
zone pour environ 3,2 M€. Sur la zone, presque les trois quarts sont vendus par un autre circuit de commercialisation que la criée. Les principaux acheteurs des produits de la mer au débarquement sont de loin les mareyeurs et les grossistes : ils achètent environ les deux tiers des débarquements.

Les effets économiques directs de l'activité de pêche dans le secteur d'étude se traduiraient donc par la mobilisation d'environ $21 \mathrm{M} €$ dont les trois quarts (77\%) constituent une création de richesse. L'évaluation des flux monétaires induits par ces flux est beaucoup plus délicate.

\section{Les flux économiques monétaires indirects}

Il s'agit de suivre la diffusion des différents effets induits directs décrits précédemment. Les agents du système ayant été identifiés, il reste à établir la grandeur des flux qui les relient entre eux.

Les flux vers l'amont représenteraient $20 \%$ du chiffre d'affaires pour une valeur estimée à 4,2 millions d'euros $(\mathrm{M} €)(+/-0,40 \mathrm{M} €)$. À cela, il faut ajouter le flux d'investissement destiné à l'acquisition de navires d'occasion, la modernisation ou la remotorisation des navires soit au minimum 2,28 M€. Certains flux n'ont pas pu être calculés à partir des données disponibles comme par exemple l'achat ou la location d'appareils de bord et de télécommunication, les frais d'expertises...

La création de valeur ajoutée est estimée à 16,5 M€ $(+/-1,7 \mathrm{M} €)$, elle se décompose en salaires et charges (62\% de la valeur ajoutée), impôts et taxes divers et en excédent brut d'exploitation.

L'approvisionnement en direct sur la zone (à la criée de Quiberon ou auprès de pêcheurs) peut être important pour certains maillons de la filière des produits de la mer : il représente près de la moitié des achats pour les mareyeurs et les grossistes et un peu moins du quart pour les poissonniers. Par contre, il est faible pour les supermarchés et pour les hypermarchés (environ $7 \%$ ).

\section{Les multiplicateurs de revenu}

Un des objectifs de l'étude est de déterminer les effets induits par la pêche côtière des navires d'Auray et de Vannes sur la zone côtière limitrophe (zones d'emploi d'Auray et de Vannes). Les effets induits en termes de revenu peuvent être approchés à partir de multiplicateurs. Deux multiplicateurs ont été construits :

- un multiplicateur de revenu pour la filière: il détermine la valeur de la marge commerciale induite dans la filière pour un euro débarqué sur la zone;

- un multiplicateur de revenu pour le système: il détermine la valeur induite dans le système halieutique pour un euro débarqué sur la zone.

D'après ces premières estimations, on peut retenir que :

- un euro débarqué sur la zone par les navires d'Auray et de Vannes induit au moins 0,50 euro en marge commerciale dans la filière pêche (hors restauration);

- un euro débarqué par les navires d'Auray et de Vannes induit au minimum 1,50 euro dans le système halieutique (sur la zone et hors zone). Sont comprises dans cette valeur: les dépenses en consommations intermédiaires, une part des investissements, la valeur ajoutée dégagée par l'activité de pêche, les marges commerciales en aval.

En l'absence de données supplémentaires et compte tenu des hypothèses retenues, ces estimations sont certainement très largement minimisées (tous les flux n'ont pas pu être pris en compte) et sont donc à utiliser avec précaution. De plus, elles reflètent l'impact de 
la pêche côtière des navires d'Auray et de Vannes sur une zone géographique limitée à la zone côtière limitrophe.

\section{Les emplois}

L'ensemble des analyses précédentes s'est attaché à appréhender les différents flux monétaires générés par la pêche côtière. Afin de compléter l'analyse, il faut aussi s'intéresser au facteur travail et essayer de calculer un multiplicateur d'emploi généré par la pêche côtière.

\section{Emplois indirects et emplois induits par la pêche côtière sur la zone d'étude.}

L'objectif poursuivi est d'estimer l'impact en termes d'emploi de la pêche côtière c'està-dire de connaître quel est le volume d'emplois générés par l'activité des navires d'Auray et de Vannes dans la zone d'étude. Outre les personnes directement employées dans les secteurs couverts, c'est-à-dire les emplois en mer (emplois directs), deux types d'emplois, appelés emplois indirects et emplois induits, ont été pris en compte. Les emplois indirects sont techniquement liés aux emplois directs, en amont, en aval ou dans les structures d'encadrement de la pêche. Les emplois induits sont suscités par la consommation finale des ménages percevant des revenus dans le cadre d'emplois directs ou indirects.

La pêche côtière des quartiers maritimes d'Auray et de Vannes génère environ 700 emplois en mer. L'ensemble des structures des zones d'emplois d'Auray et de Vannes génère au total 165 emplois à terre directement liés à l'activité de pêche côtière, ce chiffre constituant un minimum : 35 emplois se situent à l'amont, 90 à l'aval et 40 dans les structures d'encadrement du secteur. Les emplois induits, issus de la consommation finale des ménages tirants leurs revenus dans le cadre d'emplois directs ou indirects de la pêche côtière sur la zone étudiée représentent entre 280 et 400 emplois sur les deux zones d'emplois (le coefficient d'induction se situant entre 0,34 et 0,47 ). Par rapport à la population active ayant un emploi, le total de ces emplois représente entre $1,20 \%$ et $1,31 \%$ du total des emplois de la zone d'étude.

\section{Multiplicateur d'emploi}

À partir de ces données, il est possible de calculer le nombre d'emplois à terre généré par l'activité d'un pêcheur côtier. Il faut rappeler que le nombre d'emplois directs, ne correspondant pas à un nombre en équivalent temps plein, est surestimé. Le nombre d'emplois indirects dénombré est quant à lui sûrement sous-estimé. Le calcul de cet indicateur est donc délicat et tend à être sous-estimé.

D'après les données récoltées, un emploi en mer (sur les navires d'Auray ou de Vannes) générerait au moins 0,7 emploi à terre (en emplois indirects et induits) dans les zones d'emplois d'Auray et de Vannes. L'ordre de grandeur peut apparaître comme relativement faible. Il faut garder à l'esprit qu'il s'agit d'un multiplicateur qui ne comprend que les emplois directement liés à l'activité de pêche côtière d'Auray et de Vannes sur une petite zone et qu'il représente un minimum. Il reflète les spécificités locales et ne peut être généralisable à l'ensemble de la pêche côtière.

\section{Mer d'Iroise}

Dans le cadre du Système d'informations halieutiques de l'Ifremer, une enquête exhaustive sur l'activité des navires de pêche opérant à partir des ports de la Manche et de l'Atlantique a été mise en place à partir de l'année 2000. L'enquête vise en particulier 
à reconstituer pour chaque navire, un calendrier d'activité précisant, à l'échelle mensuelle, le ou les métiers de pêche pratiqués, c'est-à-dire les engins de pêche mis en œuvre, les espèces ciblées, ainsi que les zones principales de pratique.

À partir de l'information collectée dans le cadre de cette enquête, et des données déclaratives disponibles concernant les débarquements et l'activité des navires, il a été possible d'identifier un ensemble de navires ayant travaillé au moins une fois en 2001 dans l'un des quatre rectangles statistiques de la mer d'Iroise (rectangles 25E4, 25E5, 26E4 et $26 \mathrm{E} 5$ figure 58, p. XV). Le groupe de navires ainsi constitué et son activité ont ensuite été analysés dans le but de mieux décrire la nature des activités de pêche professionnelle pratiquées dans la zone.

\section{Caractéristiques techniques, effectifs embarqués, et origine de la flotte de pêche ayant travaillé en mer d'Iroise en 2001}

La flotte des navires ayant travaillé au moins une fois en mer d'Iroise en 2001 comprend 412 navires, pour un effectif total d'environ 1329 marins. Les caractéristiques des navires sont les suivantes (tableau 16):

Tableau 16. Caractéristiques techniques moyennes de la flotte de navires (année 2001).

\begin{tabular}{lcc}
\hline & Moyenne & Coefficient de variation (\%) \\
\hline Effectif (marins) & 3,2 & 113,4 \\
Puissance (kW) & 199 & 138,2 \\
Jauge (Tjb) & 31 & 215,7 \\
Longueur (m) & 13 & 65,3 \\
Âge du navire (ans) & 19 & 43,5 \\
\hline
\end{tabular}

La plupart des navires (95\%) est immatriculée dans les quartiers maritimes de la région Bretagne, avec 236 navires (57\%) immatriculés dans les quartiers de Bretagne Nord (Saint-Malo à Camaret) et 155 navires (38\%) immatriculés dans les quartiers de Bretagne Sud (Douarnenez à Vannes, figure 59).

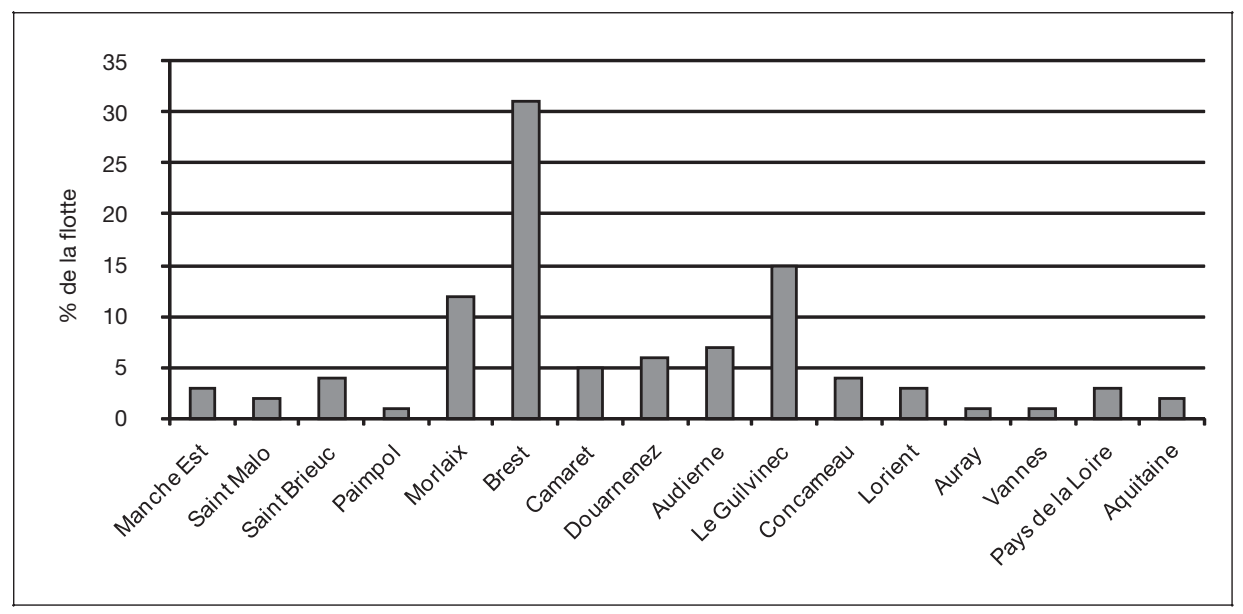

Figure 59. Distribution de la flotte de navires par quartier maritime d'immatriculation. 


\section{Activité de la flotte de pêche ayant travaillé en mer d'Iroise en 2001}

Au total en 2001, l'ensemble des navires ayant travaillé au moins une fois en mer d'Iroise a déployé 4400 mois d'activité de pêche, soit une moyenne de 10,7 mois d'activité par navire.

L'analyse des calendriers de pêche permet d'identifier des espèces cibles et les engins utilisés pour les capturer. Les six principaux métiers cités sont le filet grandes mailles à lottes, qui représente $8,4 \%$ de l'activité totale des navires, suivi des métiers de la ligne à bars $(7,4 \%)$, du casier à gros crustacés $(7,2 \%)$, du chalut de fond à lottes $(7,1 \%)$, de la drague à coquilles Saint-Jacques $(5,8 \%)$, et du filet petites mailles à poissons $(5,7 \%)$ (figure 60).

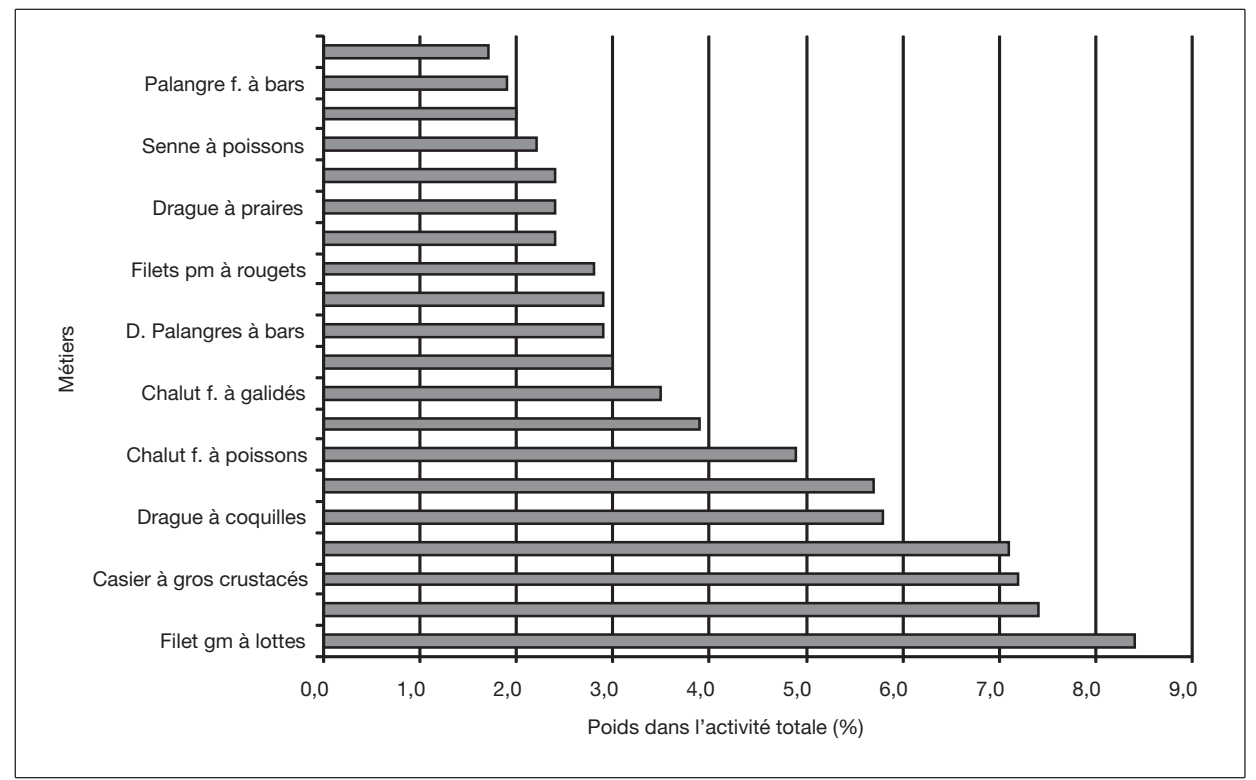

Figure 60. Principaux métiers de pêche pratiqués (poids dans l'activité globale en \%). ${ }^{152}$

La polyvalence de la flotte de navires étudiée justifie la mise en œuvre de typologies visant à identifier les combinaisons de métiers les plus fréquemment observées au niveau de sous-ensembles de la flotte, appelés « flottilles ». Le tableau 17 présente la structure de la flotte du point de vue de cette classification.

L'origine par région des navires constituant ces flottilles est décrite dans la figure 61 p. XVI.

\footnotetext{
${ }^{152}$ Filet $\mathrm{pm}$ : filet petites mailles ; Filet gm : filet grandes mailles ; Chalut $\mathrm{f}$ : : chalut de fond à panneaux (1 nav.).; Chalut $\mathrm{p}$. : chalut pélagique à panneaux ; D. palangres : diverses Palangres ; Palangres $\mathrm{f}$. : palangre de fond ; Palangre fl. : palangre flottante.
} 
Tableau 17. Flottilles bretonnes. Appartenance des navires ayant travaillé en mer d'Iroise en 2001.

\begin{tabular}{lcrrcc}
\hline \multicolumn{1}{c}{ Flottilles } & $\begin{array}{c}\text { Nombre } \\
\text { de navires }\end{array}$ & $\begin{array}{c}\text { Jauge } \\
\text { totale } \\
(\mathrm{Tjb})\end{array}$ & $\begin{array}{c}\text { Puissance } \\
\text { totale } \\
(\mathrm{kW})\end{array}$ & $\begin{array}{c}\text { Effectif total } \\
\text { (hommes) }\end{array}$ & $\begin{array}{c}\text { Longueur } \\
\text { moyenne } \\
(\mathrm{m})\end{array}$ \\
\hline Chalutiers exclusifs & 106 & 9323 & 49903 & 696 & 23 \\
Dragueurs & 90 & 790 & 7900 & 148 & 9,3 \\
Dormants polyvalents & 82 & 608 & 6887 & 131 & 8,2 \\
Fileyeurs exclusifs & 49 & 804 & 6783 & 133 & 10,8 \\
Métiers de l'hameçon exclusifs & 45 & 409 & 4689 & 68 & 8,5 \\
Bolincheurs & 13 & 453 & 2757 & 88 & 15,8 \\
Divers & 11 & 62 & 520 & 12 & 7,7 \\
Caseyeurs exclusifs & 10 & 358 & 1684 & 38 & 13 \\
Chalutiers non exclusifs & 6 & 96 & 874 & 15 & 11,2 \\
\hline
\end{tabular}

\section{Distribution spatiale de l'activité de pêche}

La distribution spatiale de l'activité de la flotte peut être appréhendée par le biais de plusieurs indicateurs complémentaires.

\section{Gradient côte-large}

Le premier concerne le degré d'éloignement des navires par rapport à la côte, mesuré de manière qualitative en termes de « gradient côte-large » dans l'enquête activité ${ }^{153}$. Le caractère avant tout côtier de cette flotte apparaît nettement (figure 62 p. XVI).

Les navires dont la pratique de pêche au large est dominante sont principalement des chalutiers exclusifs (90\% des navires « large »). Ceux dont la pratique de pêche alterne entre la côte et le large sont essentiellement des chalutiers exclusifs (38\% des navires «mixtes »), des fileyeurs exclusifs $(27 \%)$ et des dormants polyvalents $(19 \%)$.

\section{Zones de pêche}

Le second type d'information permettant d'analyser la distribution spatiale de l'activité des navires a trait aux zones principales de pratique des métiers cités dans l'enquête activité.

\section{Zones principales de pratique des activités de pêche}

Les rectangles statistiques de la mer d'Iroise (cf. carte supra) sont cités comme l'une des trois principales zones de pratique du métier de pêche, pour près des deux tiers de l'activité de pêche mesurée (2836 mois). Le rectangle statistique $25 \mathrm{E} 5$ est cité comme zone principale pour $37 \%$ de cette activité, contre $19 \%$ pour le $26 \mathrm{E} 5,11 \%$ pour le $25 \mathrm{E} 4$ et $5 \%$ pour le $26 \mathrm{E} 4$.

\footnotetext{
153 Pour chaque métier renseigné dans le calendrier d'activité d'un navire, une information concernant la localisation de la pratique de ce métier est recueillie. Les trois modalités possibles sont: " côte » (métier pratiqué à moins de 12 milles nautiques du rivage); « large » (métier pratiqué à plus de 12 milles nautiques du rivage) et « mixte » (métier pratiqué à la fois à la côte et au large).
} 


\section{Poids de la mer d'Iroise dans l'activité globale des flottilles}

Il est également possible de représenter la répartition de l'activité globale par flottille d'appartenance des navires, entre la zone Iroise et l'extérieur. Ainsi, en 2001, ce sont les navires de la flottille bretonne des chalutiers exclusifs qui apparaissent les moins dépendants de la zone « Iroise » (14\% de leur activité dans la zone, cf. figure 63). Les bolincheurs, chalutiers non-exclusifs et caseyeurs exclusifs dépendent de manière significative, mais non exclusive de la zone pour leurs activités de pêche. Les autres flottilles apparaissent nettement plus fortement dépendantes de la zone, puisqu'au moins $80 \%$ de leur activité s'y déroule en 2001 (plus de $90 \%$ pour les dragueurs et les métiers de l'hameçon exclusifs).

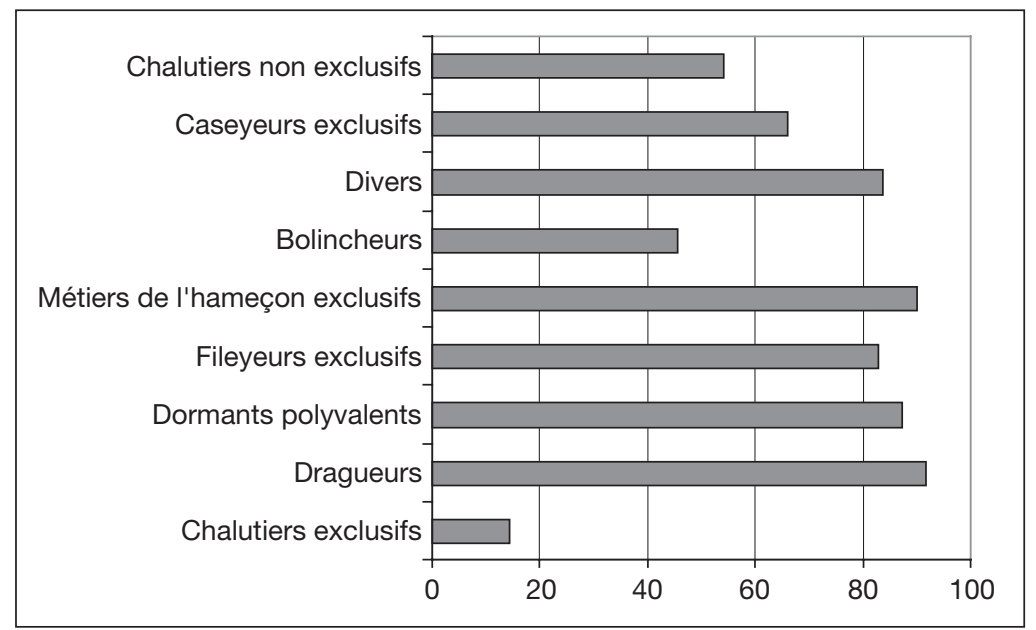

Figure 63. Degré de dépendance (\%) en mer d'Iroise (année 2001).

\section{Résultats de l'enquête économique menée auprès des navires de pêche de la mer d'Iroise}

Les enquêtes économiques auprès de la flotte de pêche opérant en mer d'Iroise ont été conduites en 2000 et 2001. Les exemples de résultats présentés ci-après ont trait à l'année 2000.

\section{Intensité capitalistique de l'activité de pêche}

L'intensité capitalistique mesure le rapport entre les facteurs capital et travail mobilisés dans l'activité de production. Ce rapport peut être mesuré par exemple par le ratio de la valeur du capital investi (mesurée par la valeur assurée du navire) sur l'effectif moyen (mesuré en équivalent temps plein) employé sur l'unité de pêche. La figure 64 présente la valeur de ce ratio pour les différents groupes de navires, classés par ordre croissant de la variable mesurée.

La valeur moyenne de ce ratio pour l'ensemble de l'échantillon est de $66,5 \mathrm{k} €$ par homme. Elle varie beaucoup, entre un minimum de 40,4 $\mathrm{k} €$ par homme pour les palangriers dragueurs et un maximum de 131,4 k€ par homme pour les chalutiers de plus de 16 mètres. Hormis les flottilles des petits chalutiers, des ligneurs et des goémoniers, qui sont composées de navires relativement plus petits, on observe une corrélation positive entre la taille des navires et leur intensité capitalistique. 


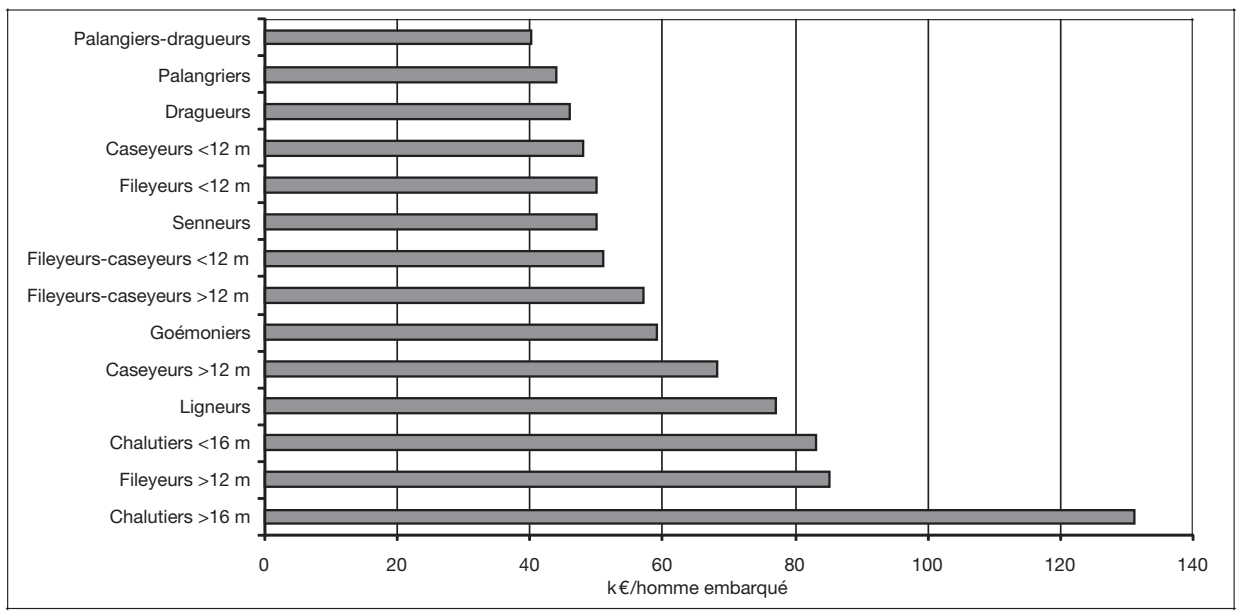

Figure 64. Capital investi $(\mathrm{k} €)$ par homme embarqué.

\section{Productivité apparente des facteurs}

La productivité simple est calculée comme le ratio du chiffre d'affaires (en euros) sur le nombre moyen d'hommes à bord en équivalent temps plein pour le facteur travail, et le ratio du chiffre d'affaires (en euros) sur la valeur assurée du navire (en milliers d'euros) pour le facteur capital. La productivité journalière est ensuite calculée en rapportant la productivité simple au nombre de jours de mer effectués par les navires; tandis que la productivité horaire du travail est calculée en rapportant la productivité simple au nombre annuel d'heures moteur effectuées.

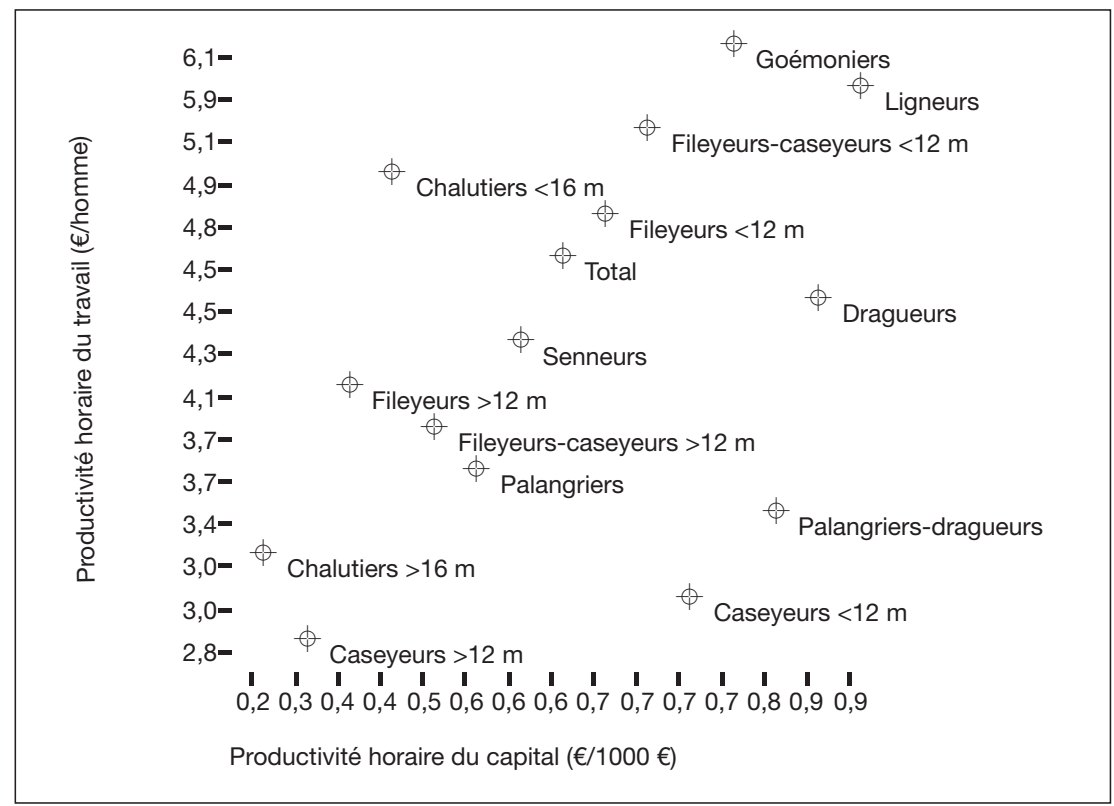

Figure 65. Relation entre productivité horaire du travail et productivité horaire du capital par flottille. 
L'enquête fait apparaître des niveaux de productivité très variables suivant les flottilles. La figure 65 permet de synthétiser les résultats par flottille. Elle fait apparaître différentes catégories de flottilles:

- les flottilles présentant des niveaux de productivité élevés pour les deux facteurs de production (principalement les goémoniers, ligneurs et fileyeurs-caseyeurs de moins de 12 mètres); - les flottilles présentant des niveaux de productivité faibles pour les deux facteurs (grands chalutiers et grands caseyeurs);

- les flottilles présentant des niveaux de productivité élevés pour le capital mais faibles (caseyeurs de moins de 12 mètres, palangriers-dragueurs) ou moyennes (dragueurs) pour le travail;

- les flottilles présentant des niveaux de productivité proches ou inférieurs à la moyenne observée pour l'ensemble de l'échantillon, pour les deux facteurs (chalutiers de moins de 16 mètres, fileyeurs, fileyeurs-caseyeurs de plus de 12 mètres et palangriers).

\section{Taux de profit}

Le taux de profit se définit comme le rapport entre le résultat d'exploitation et la valeur du capital, estimée à partir de la valeur assurée des navires. Il est globalement de $18,9 \%$ et varie entre 3,3\% pour les petits caseyeurs et 31,8\% pour les grands caseyeurs (tableau 18). L'analyse des taux de profit montre que la rentabilité des navires pratiquant des arts dormants est en général meilleure que celle des navires pratiquant les arts traînants. Les caseyeurs de moins de 12 mètres représentent le segment de flotte le moins rentable, tandis que les fileyeurs de moins de 12 mètres et les caseyeurs de plus de 12 mètres ont les taux de profit les plus élevés.

Tableau 18. Taux de profit (\%) des flottilles

(Source: Ifremer/SIH, résultats de l'enquête économique; année de référence 2000).

\begin{tabular}{l|c}
\multicolumn{1}{c|}{ Flotilles } & Moy. pondérée (\%) \\
\hline Caseyeurs $<12 \mathrm{~m}$ & 3,3 \\
Goémoniers & 9,4 \\
Ligneurs & 10,3 \\
Fileyeurs $>12 \mathrm{~m}$ & 16,6 \\
Chalutiers $>16 \mathrm{~m}$ & 17,0 \\
Senneurs & 18,0 \\
Palangriers & 19,1 \\
Palangriers-dragueurs & 19,7 \\
Chalutiers $<16 \mathrm{~m}$ & 20,5 \\
Fileyeurs-caseyeurs $<12 \mathrm{~m}$ & 21,1 \\
Dragueurs & 23,3 \\
Fileyeurs-caseyeurs $>12 \mathrm{~m}$ & 26,6 \\
Fileyeurs $<12 \mathrm{~m}$ & 28,1 \\
Caseyeurs $>12 \mathrm{~m}$ & 31,8 \\
\hline Total & 18,9 \\
\hline
\end{tabular}

La différence de métiers pratiqués n'est toutefois pas le seul facteur explicatif des écarts de rentabilité entre les navires. En effet, les classes de longueurs semblent être un facteur de différenciation important mais la corrélation ne joue pas dans le même sens 
selon le métier pratiqué. Ainsi, le taux de profit des chalutiers de plus de 16 mètres est inférieur à celui des chalutiers de moins de 16 mètres (17\% contre 20,5\%). Il en est de même chez les fileyeurs $(16,6 \%$ contre $28,1 \%)$. Par contre, les taux de profit des caseyeurs et des fileyeurs caseyeurs de plus de 12 mètres sont supérieurs à ceux des moins de 12 mètres, respectivement $31,8 \%$ contre $3,3 \%$, et $26,6 \%$ contre $21,1 \%$.

On constate alors que les navires pratiquant les arts dormants sont en général les navires les plus rentables, exception faite des petits caseyeurs, des goémoniers et des ligneurs. Les différences de rentabilité entre les flottilles pratiquant des arts dormants et celles pratiquant des arts traînants s'expliquent peut-être par l'augmentation du coût du gazole observée en 2000.

Si l'on met en perspective le taux de profit avec le degré de dépendance à la mer d'Iroise comme zone de pêche (figure 63), on constate que les flottilles fortement dépendantes présentent pour la plupart des niveaux de rentabilité plus élevés. Exception faite des grands caseyeurs (groupe ayant le taux de profit le plus élevé), les flottilles les plus rentables sont généralement celles dont les navires sont fortement inféodés à la mer côtière (fileyeurs-caseyeurs, dragueurs et petits fileyeurs).

\section{Pêche récréative: résultats de l'enquête nationale sur la pêche de loisir concernant la Bretagne}

L'Ifremer, en tant que maître d'œuvre pour la direction des pêches maritimes et de l'aquaculture (DPMA), a été chargé de réaliser une enquête nationale pilote sur la pêche de loisir en mer. L'objectif de l'étude était de quantifier l'impact de la pêche de loisir (récréative et sportive) en mer sur la ressource halieutique, et les retombées économiques de cette activité.

La technique retenue est celle de l'enquête téléphonique en ayant recours à un institut de sondage spécialisé, (Institut BVA). La méthode repose sur des interviews téléphoniques réalisées, à partir d'un questionnaire ad hoc, sur la population cible métropolitaine des plus de 15 ans. Ainsi, 15000 ménages ont été interrogés en 5 vagues réparties au cours de l'année 2006 (tableau 19). L'unité de sondage utilisée est le ménage ce qui permet d'améliorer le taux apparent de pénétration (pourcentage de foyers avec au moins un pêcheur récréatif), la maîtrise de la représentativité et le coût de construction des échantillons. Au sein d'un ménage (contenant 1 ou plusieurs pêcheurs de loisir), on interroge un seul pêcheur choisi de façon aléatoire.

Tableau 19. Répartition des vagues d'enquête au cours de l'année 2006.

\begin{tabular}{|l|l|l|l|}
\cline { 2 - 5 } \multicolumn{1}{c|}{} & \multicolumn{3}{c|}{ France métropolitaine } \\
\cline { 2 - 5 } \multicolumn{1}{c|}{} & Période d'enquête & Nombre d'interviews & Période de référence \\
\cline { 2 - 5 } \multicolumn{1}{c|}{} & \multicolumn{3}{c|}{ Phase test } \\
\hline VAGUE 1 & 5 au 11 avril 2006 & 2061 ménages interrogés & Janvier, février et mars 2006 \\
\hline & \multicolumn{3}{|c}{ Phase d'étude } \\
\hline VAGUE 2 & 15 au 24 Juin 2006 & 3003 ménages interrogés & Avril et mai 2006 \\
\hline VAGUE 3 & Septembre 2006 & 5012 ménages interrogés & Juin, juillet et août 2006 \\
\hline VAGUE 4 & Novembre 2006 & 3003 ménages interrogés & Septembre et octobre 2006 \\
\hline
\end{tabular}


Les échantillons des ménages sont construits en tenant compte du lieu du domicile (région, taille de la commune) et de critères sociodémographiques. Compte tenu des précédentes expériences, il a été décidé de suréchantillonner la zone littorale, où le taux de pénétration des enquêtes, les fréquences des pratiques et les prélèvements se sont révélés plus importants qu'à l'intérieur du pays (figure 66).

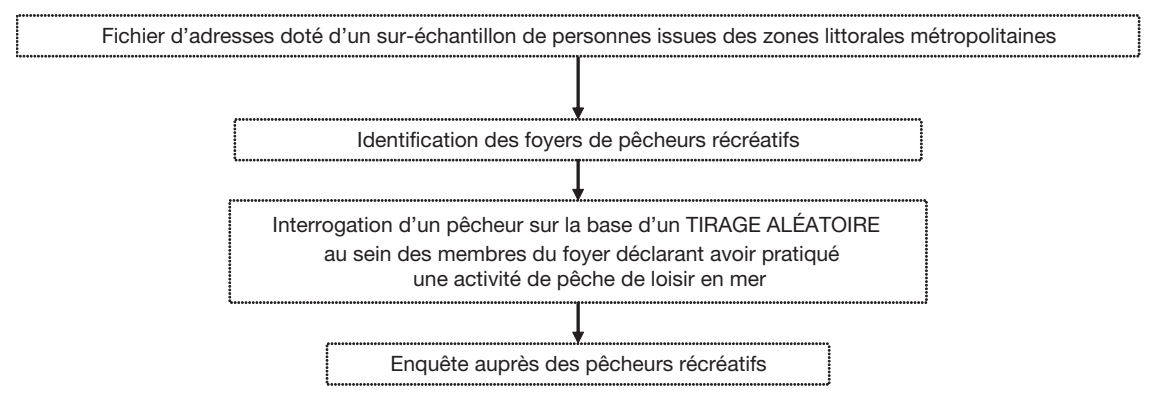

Figure 66. Schéma de la stratégie et du plan de sondage.

Les données sont ensuite redressées afin que l'échantillon interviewé ait les mêmes propriétés que la population française des plus de 15 ans. Le redressement consiste à corriger le poids de chaque ménage de telle sorte que le cumul des poids pour les 62 items définis corresponde aux 62 valeurs recherchées correspondant à la situation de référence nationale. La fourchette de variation maximale considérée comme acceptable pour la variation des poids est de 0,25 à 4 .

Le questionnaire comporte cinq parties (cf. annexe):

- les volets A et B portent sur d'une part l'activité de pêche récréative des trois derniers mois de l'année en cours (2006) et d'autre part sur la dernière sortie de pêche, pour laquelle des informations plus détaillées et quantitatives, notamment économiques et sur la composition des captures, peuvent être collectées ;

- le volet C a pour but de cerner l'activité annuelle du pêcheur en 2005 ;

- le volet D permet d'approfondir les caractéristiques des embarcations possédées et les coûts liés à leur possession et à leur utilisation ;

- le volet E se rapporte à l'expérience du pêcheur et sa perception de la situation actuelle de la pêche de loisir en mer (évolution de la ressource, encadrement de l'activité de pêche de loisir...).

Tous ces volets visent à caractériser les pratiques des points de vue sociologique (profil et comportement du pêcheur, origine géographique, lieux de pêche), technique (types de pêche, engins utilisés, fréquences et saisonnalité des sorties, volume et composition des captures), et économique (les dépenses générées par ce loisir, coûts directs de l'activité et impact pour l'économie locale).

À la demande de l'Ifremer, BVA à partir de cette étude nationale, a mené une analyse spécifique auprès des Français ayant pratiqué la pêche de loisir (récréative et sportive) en mer en 2005 et/ou 2006 en Bretagne dans au moins l'un des quatre départements bretons. Pour analyser les particularités de la Région Bretagne une sélection des questionnaires a été réalisée, en fonction des lieux de pêche déclarés. Les résultats présentés ci-après concernent les interviewés ayant effectué au moins une sortie de pêche de loisir (récréative et sportive) en mer en 2005 et/ou 2006 dans au moins l'un des départements suivants : Côtes-d'Armor (22), Finistère (29), Ille-et-Vilaine (35) et Morbihan (56). Au titre de l'année 2005 (partie $\mathrm{C}$ du questionnaire), on dénombre 255 répondants. Au titre 
de l'année 2006 (partie A et B du questionnaire), on dénombre 114 répondants. La taille de l'échantillon de base des pêcheurs ayant réalisé au moins une sortie en 2005 et/ou 2006 est faible, il faut donc évaluer les résultats avec précaution.

Au niveau métropolitain, le nombre de pêcheurs de loisir en mer a été estimé à 2,45 millions $(+/-0,15)$ en 2005 tous modes de pêche confondus. $71 \%$ déclarent avoir fait au moins une sortie de pêche à pied en 2005. En 2005, un pêcheur réalise en moyenne $12,8(+/-22,5)$ sorties par an mais l'intervalle de confiance est très grand. Les captures ont été estimées en 2005 à: 15000 tonnes de poissons, 12000 à 15000 tonnes de coquillages, 1500 tonnes de crustacés, 500 tonnes de céphalopodes ${ }^{154}$.

\section{Pêche de loisir en mer en Bretagne en 2005}

Cette partie concerne les pêcheurs de loisir en mer interrogés au titre de l'année 2005. Elle correspond aux résultats des données dans la partie $\mathrm{C}$ du questionnaire. Après redressement, l'extraction concerne 255 foyers qui représentent un total de 383 pêcheurs de loisir.

Par extrapolation, on estime à $600000(+/$-36000) le nombre de personnes ayant réalisé au moins une sortie de pêche en Bretagne en 2005. Cela représente environ $25 \%$ des 2,45 millions de pêcheurs de loisir estimés en 2005. Cela fait de la Bretagne une des régions les plus fréquentées par les pêcheurs récréatifs. Le Finistère est le département le plus fréquenté à l'échelle métropolitaine: $9 \%$ des pêcheurs y ont réalisé au moins une sortie.

\section{Caractéristiques socio-démographiques}

Les caractéristiques socio-démographiques des pêcheurs en Bretagne sont proches de celles de l'ensemble des pêcheurs de loisir en mer métropolitains (tableau 20).

Tableau 20. Caractéristiques socio-démographiques (\%) des pêcheurs de loisir en mer déclarant au moins une sortie de pêche en Bretagne en 2005 sur la base de 291 interrogés.

\begin{tabular}{|c|c|c|}
\hline & Bretagne & Métropole \\
\hline \multicolumn{3}{|l|}{ Sexe } \\
\hline Homme & 57 & 58 \\
\hline Femme & 43 & 42 \\
\hline \multicolumn{3}{|l|}{ Âge } \\
\hline $15-24$ ans & 7 & 6 \\
\hline $25-34$ ans & 17 & 15 \\
\hline $35-49$ ans & 28 & 30 \\
\hline $50-64$ ans & 30 & 29 \\
\hline 65 ans et plus & 18 & 20 \\
\hline \multicolumn{3}{|l|}{ Profession } \\
\hline Agriculteur & - & 1 \\
\hline Artisan, commerçant, chef d'entreprise, prof. libérale & 10 & 12 \\
\hline Cadre, profession intermédaire & 23 & 21 \\
\hline Employé, personnel de service & 16 & 16 \\
\hline Ouvrier & 15 & 16 \\
\hline Retraité / autres inactifs & 36 & 33 \\
\hline
\end{tabular}

154 Cf. Anonyme, 2007. Enquête relative à la pêche de loisir (récréative et sportive) en mer en métropole et dans les DOM: synthèse des résultats intermédiaires. Ifremer/DPMA. 
On constate que les personnes pêchant en Bretagne sont surtout originaires des départements bretons ou de la région parisienne (figure 67, p. XVII), $42 \%$ des pêcheurs en Bretagne résident dans la région.

\section{Particularité des pêcheurs ayant réalisé au moins une sortie en Bretagne et résidant en Bretagne}

Sur le nombre estimé de 2,45 millions de pêcheurs de loisir en mer en France, environ 316000 pêcheurs résident en Bretagne, soit $13 \%$.

\section{Modes de pêche pratiqués}

Comme dans les résultats nationaux, la pêche à pied est le mode de pêche le plus représenté, $76 \%$ des personnes interrogées ont déclaré pratiquer ce mode de pêche. Au cours de l'année 2005, un pêcheur a pratiqué en moyenne 1,4 mode de pêche. Il y a peu de chasse sous-marine (figure 68 p. XVII). Au total, $88 \%$ des pratiques ne nécessitent pas d'embarcation.

\section{Répartition des sorties sur l'année}

La majorité des sorties en Bretagne est réalisée en été. Quatre-vingt cinq pour cent des personnes ayant pêché en Bretagne en 2005 déclarent au moins une sortie de pêche entre juin et août (figure 69). Le nombre moyen de sorties en Bretagne durant cette période est d'environ sept par pêcheur. Aux autres périodes, la fréquentation diminue nettement.

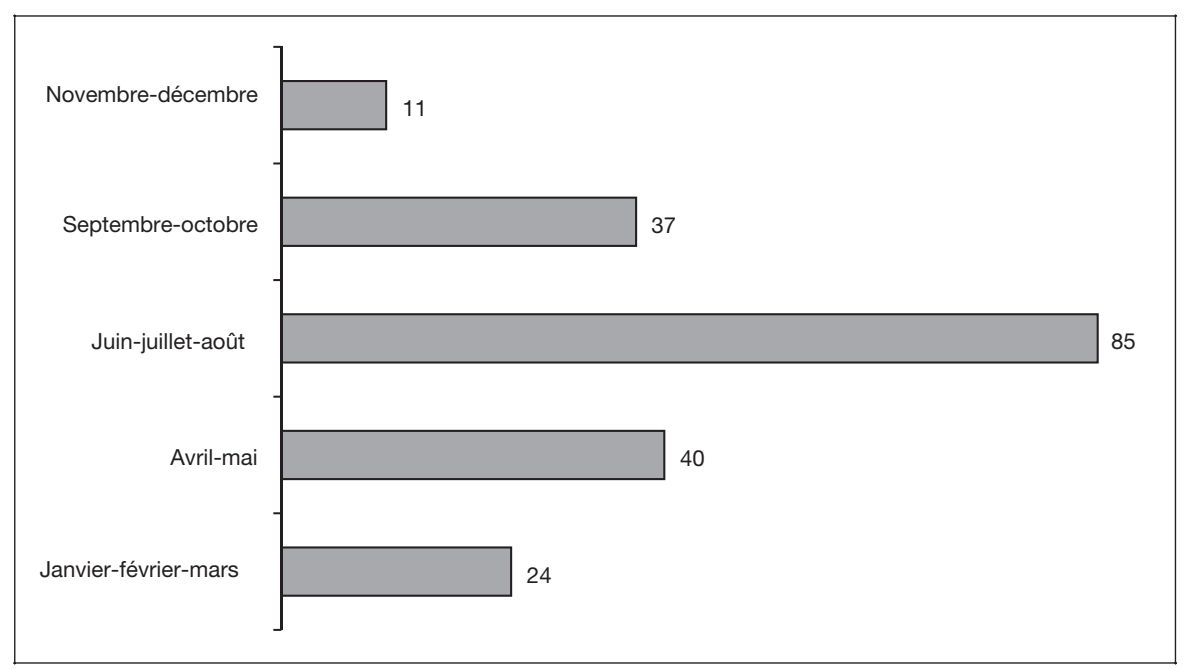

Figure 69. Répartition des sorties de pêche (\%) des personnes ayant fait au moins une sortie de pêche en Bretagne en 2005.

\section{Sorties par département}

Parmi les départements, celui du Finistère est le plus fréquenté. Il regroupe $37 \%$ des sorties de pêche réalisées dans la région (tableau 21). L'Ille-et-Vilaine ne totalise que $13 \%$ bien qu'elle ne possède que $5 \%$ du linéaire de côte breton.

Les pêcheurs ayant réalisé au moins une sortie en 2005 en Bretagne ont réalisé 98,6 \% de leur sortie en Bretagne, soit la quasi-totalité. 
Tableau 21. Proportion des pêcheurs ayant réalisé au moins une sortie dans le département.

\begin{tabular}{|l|c|c|c|c|}
\hline \multicolumn{1}{|c|}{ Départements } & Effectif & $\%$ & $\begin{array}{c}\text { Linéaire } \\
\text { de côte } \\
\text { en km }\end{array}$ & $\begin{array}{c}\text { Ratio nombre } \\
\text { de sorties par } \\
\text { rapport au } \\
\text { linéaire de } \\
\text { côte }\end{array}$ \\
\hline 29 Finistère & 94 & 37 & $\begin{array}{c}1019,0 \\
(46 \%)\end{array}$ & 0,9 \\
\hline 56 Morbihan & 76 & 30 & $\begin{array}{c}603,8 \\
(27 \%)\end{array}$ & 1,5 \\
\hline 22 Côtes-d'Armor & 73 & 29 & $\begin{array}{c}483,2 \\
(22 \%)\end{array}$ & 1,4 \\
\hline 35 Ille-et-Vilaine & 32 & 13 & $\begin{array}{c}113,9 \\
(5 \%)\end{array}$ & 2,5 \\
\hline
\end{tabular}

\begin{tabular}{|c|}
\hline $\begin{array}{c}\text { Nombre de sorties } \\
\text { moyen (sur la base des } \\
\text { pêcheurs ayant effectué } \\
\text { au moins une sortie dans } \\
\text { le département concerné) }\end{array}$ \\
\hline 10 \\
\hline 12 \\
\hline 9 \\
\hline 9
\end{tabular}

\section{Groupes d'espèces pêchées - quantités pêchées}

Si l'essentiel des sorties à lieu en Bretagne, les captures déclarées par les enquêtés au titre de l'année 2005 ont également pu être réalisées à l'occasion d'activités de pêche pratiquées dans d'autres régions littorales. $75 \%$ des pêcheurs ayant fréquenté la Bretagne en 2005 ont fait au moins une prise de coquillage, contre $67 \%$ sur l'ensemble de la France. $56 \%$ des pêcheurs ayant fréquenté la Bretagne en 2005 ont fait au moins une prise de crustacés, contre $61 \%$ sur l'ensemble de la France (figure 70 p. XVII). En Bretagne, contrairement à la métropole, la proportion de pêcheurs ayant réalisé au moins deux prises de crustacées est supérieure à celle des pêcheurs ayant réalisé au moins deux prises de coquillages.

La plupart des pêcheurs ne pêchent pas plus de $2 \mathrm{~kg}$ par an quel que soit le type de capture (figures 71 p. XVIII ; 72 p. XVIII et XIX ; 73 p. XIX et 74 p. XX).

Pour les coquillages, le rendement moyen des pêcheurs ayant réalisé au moins une prise est supérieur en Bretagne à celui observé au niveau métropolitain: 6,5 kg contre $5,2 \mathrm{~kg}$ (figure $71 \mathrm{p}$. XVIII).

En revanche pour les crustacés, le rendement moyen des pêcheurs ayant réalisé au moins une prise est inférieur en Bretagne à celui observé au niveau métropolitain: 4,8 kg contre $5,5 \mathrm{~kg}$ (figure $72 \mathrm{p}$. XVIII et XIX).

Pour les poissons, le rendement moyen des pêcheurs ayant réalisé au moins une prise est supérieur en Bretagne à celui observé au niveau métropolitain: 12,6 kg contre $11 \mathrm{~kg}$ (figure 73 p. XIX).

Pour les céphalopodes, le rendement moyen des pêcheurs ayant réalisé au moins une prise est inférieur en Bretagne à celui observé au niveau métropolitain: 3,6 kg contre $5,5 \mathrm{~kg}$ (figure $74 \mathrm{p} . \mathrm{XX}$ ).

\section{Détail des espèces recherchées}

Les captures des personnes ayant pêché en Bretagne en 2005, sont similaires aux déclarations obtenues sur l'ensemble de la France. Pour les poissons, on retrouve en tête des espèces citées : le bar et le maquereau. En revanche, la daurade n'est qu'au quatrième rang derrière le lieu, alors qu'elle apparaît en troisième position dans les résultats nationaux. Pour les coquillages, les coques arrivent au premier rang des espèces citées alors qu'elles sont au deuxième rang sur l'ensemble de la France (figure 75). 

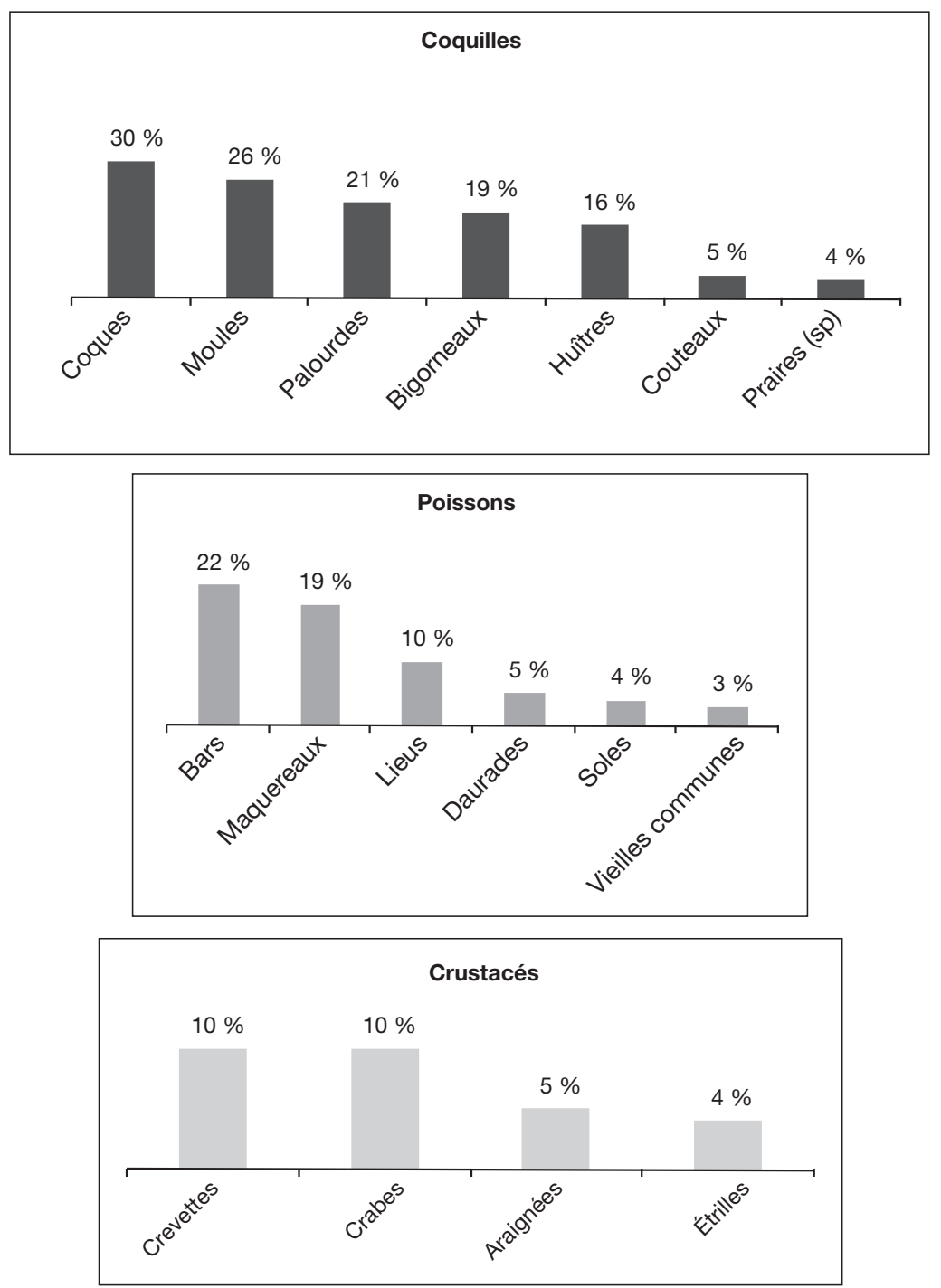

Figure 75. Principales espèces recherchées ou pêchées par les pêcheurs ayant réalisé au moins une sortie en Bretagne en 2005.

\section{Estimation du poids pêché par type de prise}

Par extrapolation, une première estimation des captures réalisées par les pêcheurs ayant effectué au moins une sortie en Bretagne en 2005 a été établie. Ces captures sont d'environ : 3500 tonnes de poissons, 2100 tonnes de coquillages, 2100 tonnes de crustacés et 200 tonnes de céphalopodes.

Rappelons que l'ensemble de ces captures n'a pas été totalement réalisé en Bretagne. Cependant, comme 98,6 \% du nombre total des sorties réalisées par les pêcheurs ayant fait au moins une sortie en Bretagne en 2005 se sont déroulés en Bretagne, on peut considérer que l'estimation donne une bonne approximation des prélèvements effectués sur les côtes bretonnes. 


\section{Dépenses moyennes}

La figure 76 présente l'estimation des dépenses moyennes réalisées par les pêcheurs de loisirs ayant pêché en Bretagne. Dans la catégorie « Autres », les dépenses les plus fréquemment citées sont l'alimentation (pique-nique ou restaurant) et les frais de transports (carburant pour la voiture ou le bateau).

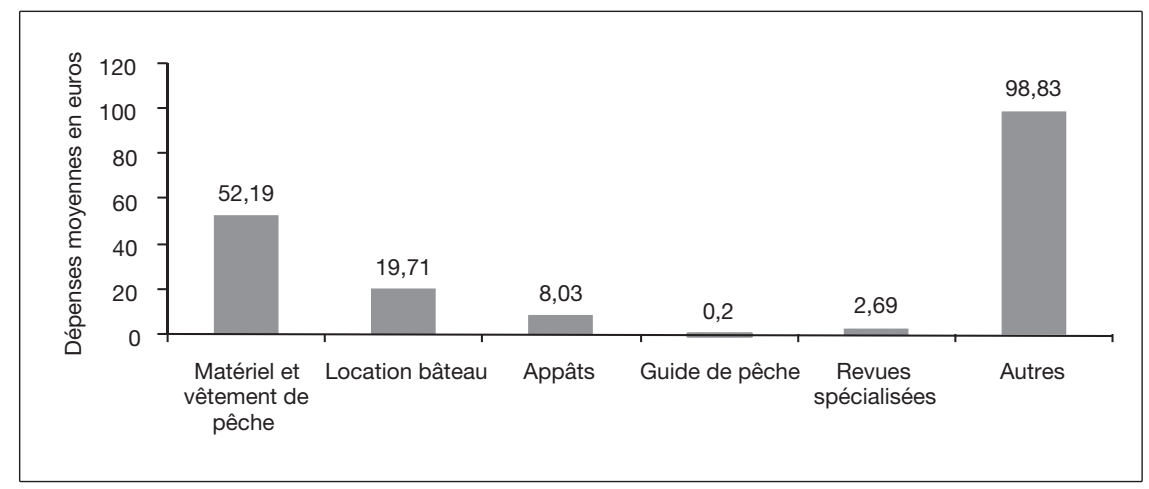

Figure 76. Répartition de dépenses consacrées à la pêche de loisir en 2005 par les pêcheurs ayant réalisé au moins une sortie en Bretagne en 2005.

\section{Pêche de loisir en mer en Bretagne en 2006}

Cette partie concerne les pêcheurs de loisir en mer interrogés au titre de leur dernière sortie de pêche en 2006. Elle correspond aux résultats des réponses données dans les parties A et B du questionnaire. Après redressement, l'extraction concerne 114 pêcheurs ayant réalisé leur dernière sortie de pêche dans un des quatre départements de la région Bretagne.

\section{Mode de pêche utilisé lors de la dernière sortie}

La pêche à pied apparaît ici encore comme le mode de pêche le plus pratiqué (figure 77), avec $62 \%$ des dernières sorties de pêche qui y sont consacrées. La pêche en bateau est également bien représentée, comparée aux résultats métropolitains.

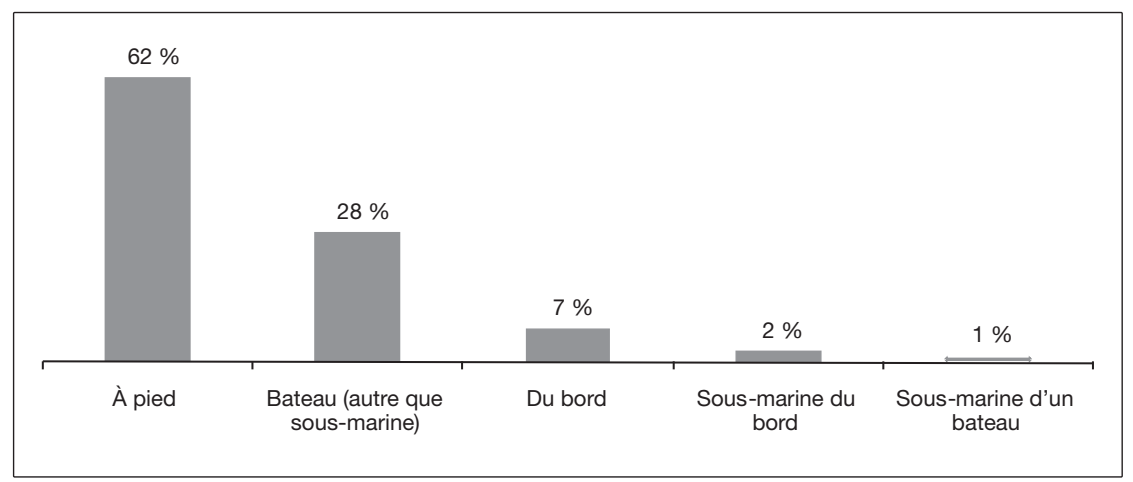

Figure 77. Principal mode de pêche pratiqué lors de la dernière sortie en 2006 (base 114 pêcheurs interrogés). 


\section{Principaux engins}

La pêche à pied avec outils est l'engin de pêche le plus utilisé pour la dernière sortie en Bretagne. Trente-neuf pour cent des pêcheurs en Bretagne déclarent l'avoir pratiquée contre $28 \%$ au niveau métropolitain. La pêche à pied totalise $56 \%$ des pratiques contre $48 \%$ au niveau métropolitain. Le casier est également plus utilisé en Bretagne. La pêche à la canne est moins représentée avec $25 \%$ des pratiques contre $36 \%$ au niveau métropolitain (figure 78).

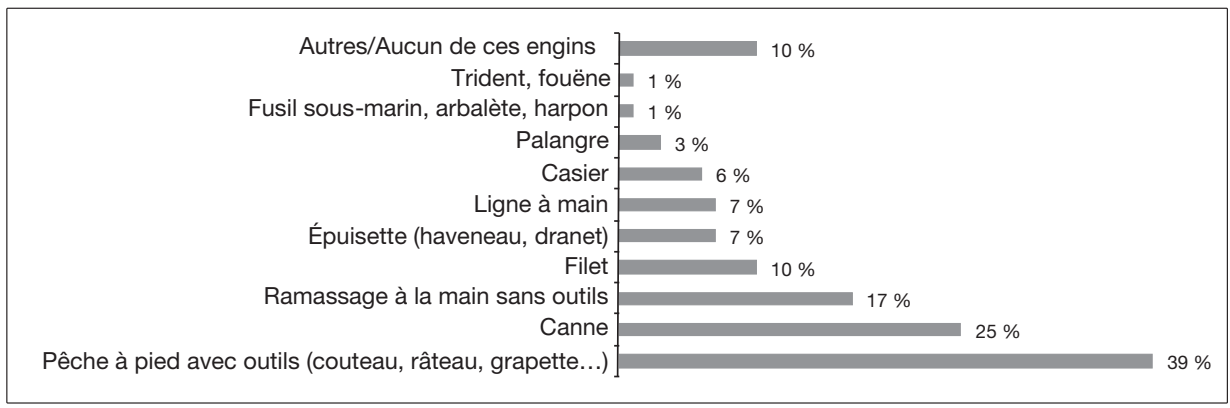

Figure 78. Les principaux engins de pêche utilisés dans le cadre de la dernière sortie de pêche en 2006 (base : 114 pêcheurs interrogés).

\section{Lieu de pêche}

Pour 2006, c'est le Morbihan qui compte le plus de dernières sorties de pêche avec $35 \%$ des sorties (figure 79 p. XX). Comme en 2005, ce sont les départements du Finistère et du Morbihan dans lesquels il y a le plus de sorties de pêche. Ceci est à rapporter au linéaire de côte des différents départements bretons.

La figure $80 \mathrm{p}$. XX permet d'avoir une idée de la fréquentation des différents départements bretons au cours de l'année 2006.

Plages, grève et rochers sont les lieux les plus fréquentés ce qui est à mettre en rapport avec l'importance de la pratique de la pêche à pied (figure 81). Les résultats sont ici similaires à ceux observés au niveau métropolitain.

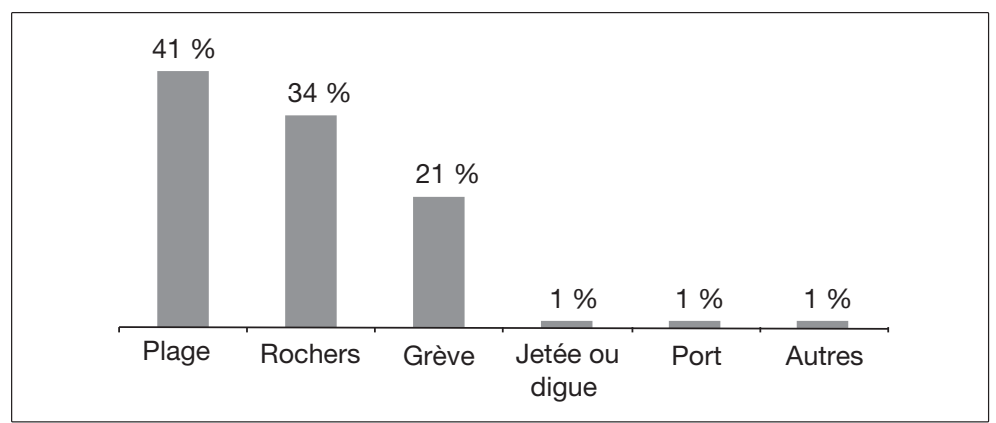

Figure 81. Type de lieu de pêche fréquenté lors la dernière sortie en Bretagne en 2006 pour les pêcheurs à pied et du bord (base : 74 pêcheurs interrogés). 


\section{Profil des sorties embarquées}

Les bateaux s'éloignent peu des côtes. Soixante et un pour cent des dernières sorties réalisées en Bretagne ont été effectuées à moins de 3 milles nautiques (figure 82). Les embarcations contiennent de $2(40 \%$ des sorties) à 3 personnes (42\% des sorties) à bord. Pour les sorties en bateau une majorité de personnes (environ $55 \%$ ) était invitée à bord, seules $38 \%$ des personnes interrogées étaient propriétaires de leur embarcation (figure 82).
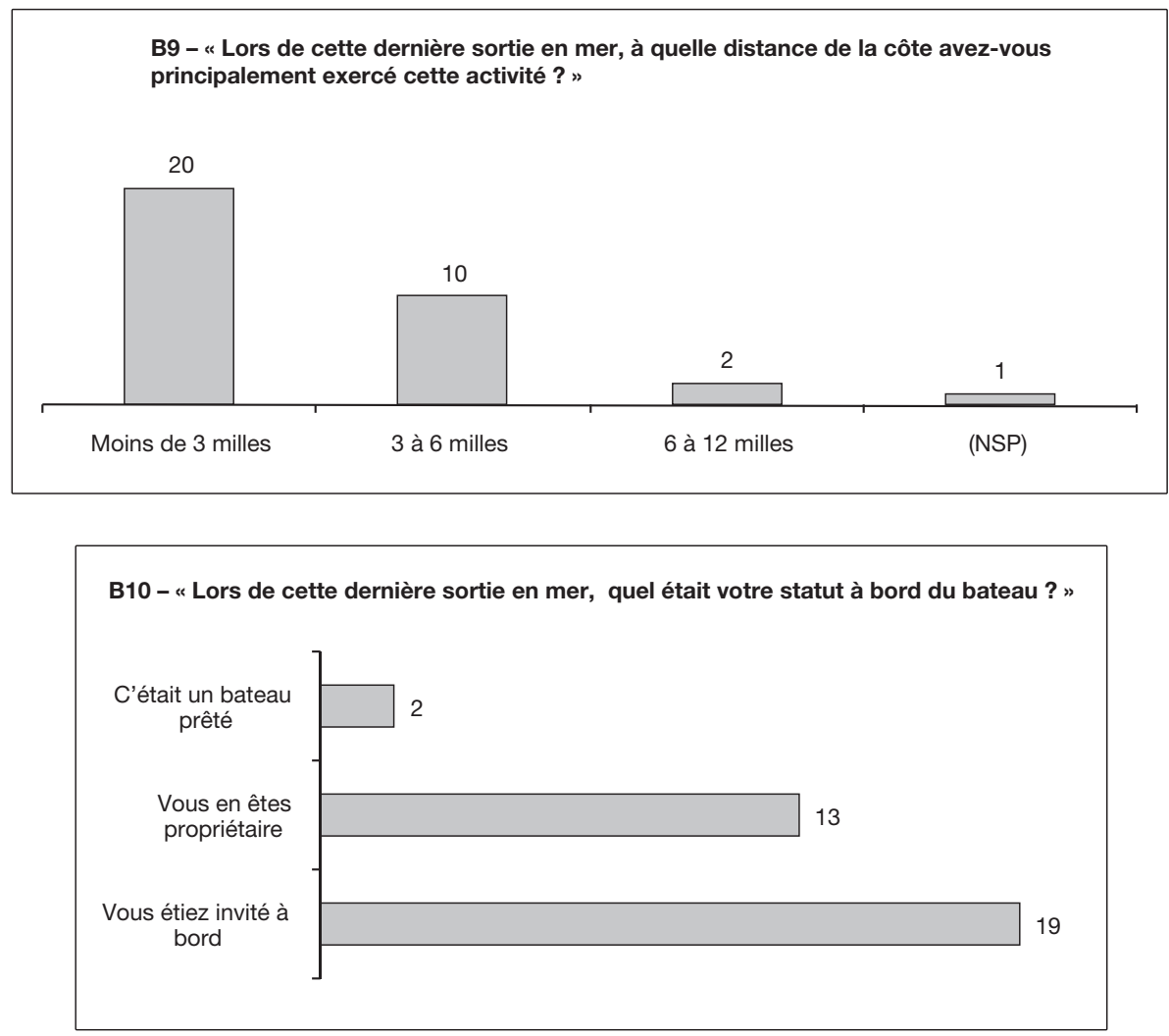

Figure 82. Distance par rapport à la côte et statut du pêcheur à bord lors de la dernière sortie en Bretagne en 2006 (résultats présentés en effectifs).

\section{Informations relatives aux bateaux de pêche plaisance}

Cette partie concerne les pêcheurs qui ont réalisé au moins une sortie en 2005 ou leur dernière sortie en 2006 en Bretagne. Elle correspond aux résultats de la partie D qui regroupe les questions relatives aux caractéristiques des bateaux et des dépenses pour le bateau. La base de référence est de 291 pêcheurs ayant réalisé au moins une sortie en Bretagne en 2005 ou leur dernière sortie de pêche en Bretagne en 2006. Parmi ceux-ci, $16 \%$ sont propriétaires d'une embarcation (figure 83). Au niveau métropolitain, ils sont $14 \%$. 


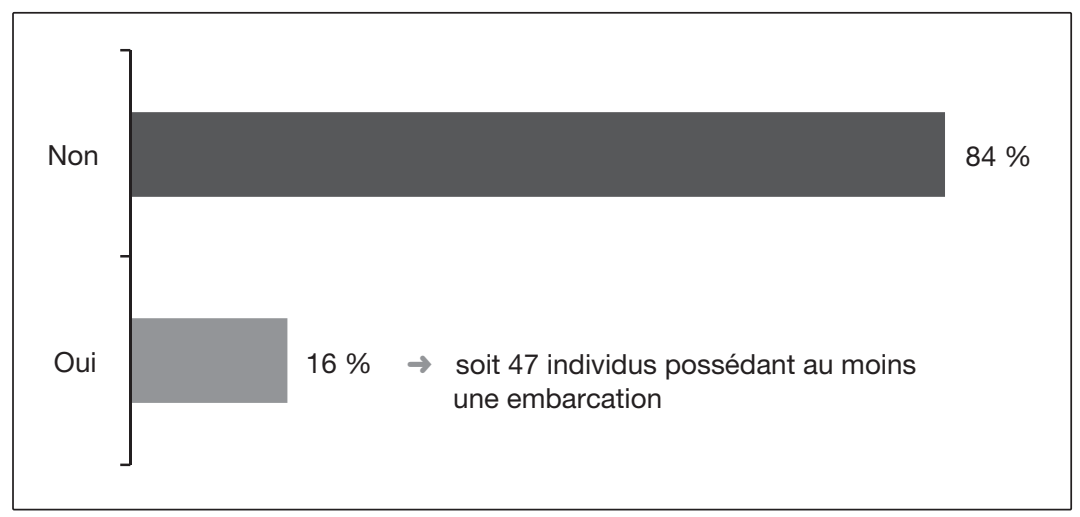

Figure 83. Proportion (\%) de personnes possédant un bateau parmi les pêcheurs ayant réalisé au moins une sortie en Bretagne en 2005 ou leur dernière sortie de pêche en Bretagne en 2006 (base : 291 pêcheurs interrogés).

L'échantillon des possesseurs de bateau est faible (47 répondants), les résultats doivent donc être évalués avec précaution. Les trois principaux types d'embarcations possédées et utilisées pour la pêche sont les bateaux de pêche promenade, les voiliers et les barques/ canots (figure 84). Les pneumatiques n'arrivent qu'en quatrième position alors qu'ils sont au deuxième rang au niveau métropolitain. De même les coques open sont un peu moins représentées que dans l'échantillon métropolitain.

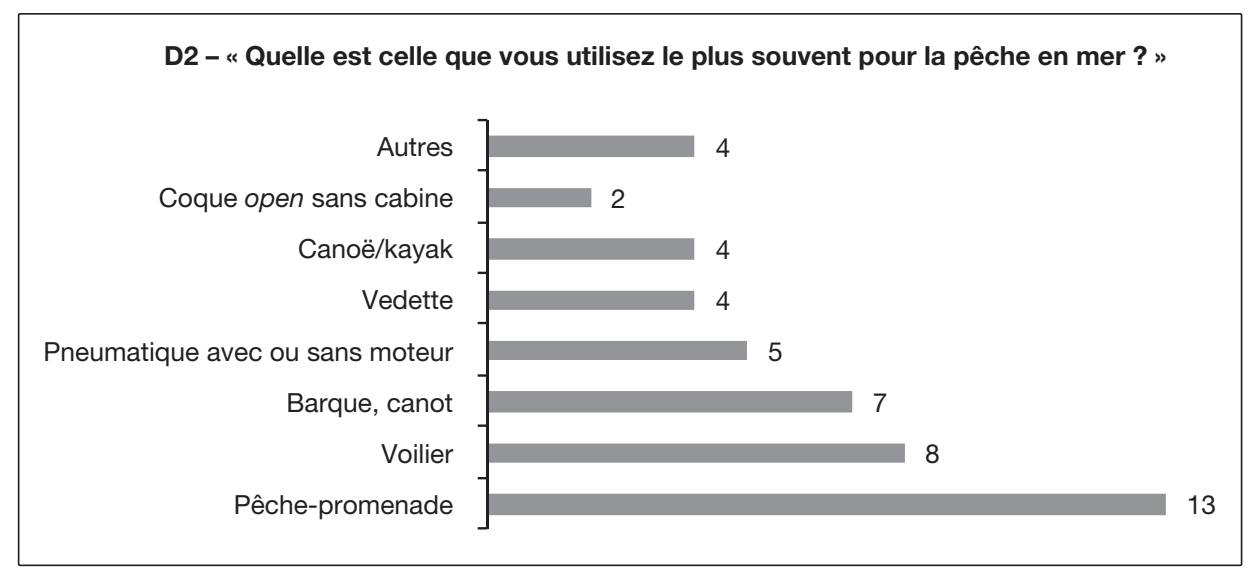

Figure 84. Types d'embarcations les plus fréquemment utilisés pour la pêche (résultats présentés en effectifs).

Les embarcations sont de petite taille: la majorité d'entre elles mesurent entre 5 et $7,49$ mètres (figure 85 ), pour une longueur moyenne de 5,1 mètres (+/- 0,5$)$. La puissance moyenne du moteur est de $41 \mathrm{CV}(+/-15)$. Elle est inférieure à la moyenne métropolitaine de $61 \mathrm{CV}$. Quarante-trois pour cent des bateaux ont moins de 10 ans. 

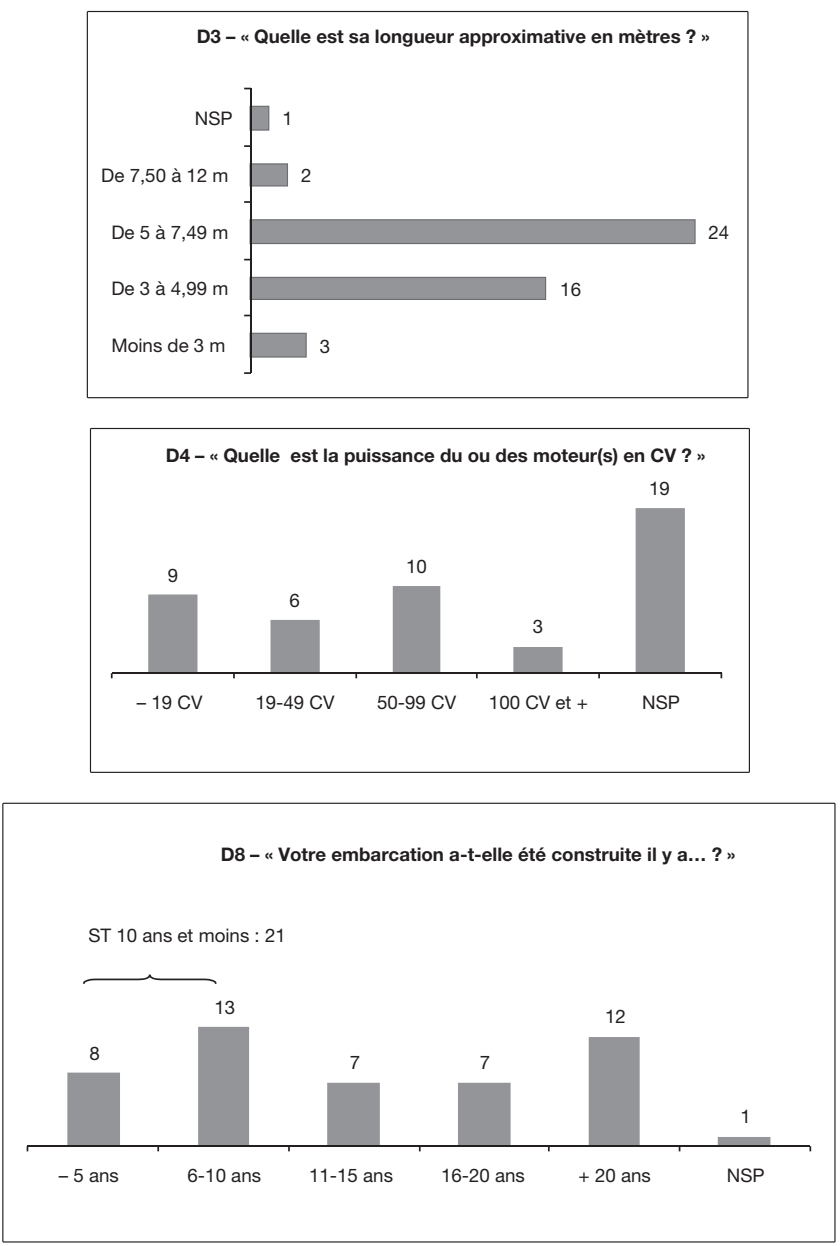

Figure 85. Caractéristiques des embarcations utilisées pour la pêche de loisir par les pêcheurs fréquentant la Bretagne (résultats présentés en effectifs).

Une faible partie des embarcations est amarrée à un ponton ou une bouée, la majorité des bateaux utilisés pour la pêche est gardée à terre ou sur une remorque (figure 86) : 53 \% des bateaux sont à terre contre $45 \%$ dans l'échantillon métropolitain.

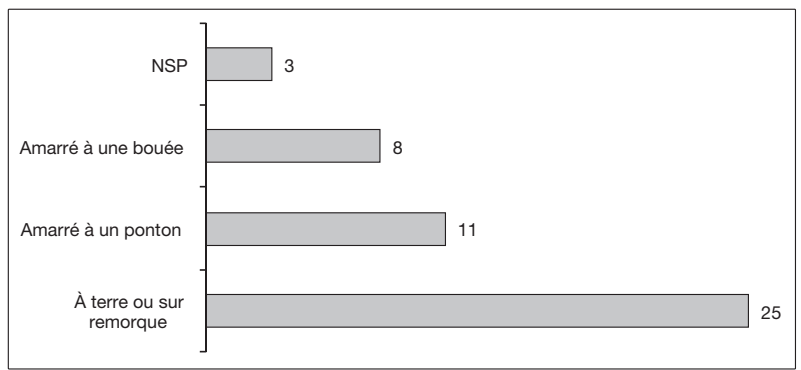

Figure 86. Emplacement du bateau la majeure partie du temps (résultats présentés en effectifs). 


\section{Informations relatives aux pêcheurs de loisirs}

Cette partie concerne les pêcheurs qui ont réalisé au moins une sortie en 2005 ou leur dernière sortie en 2006 en Bretagne. Elle correspond aux résultats des réponses données dans la partie E, qui regroupe les questions se rapportant à l'expérience du pêcheur et sa perception de la situation actuelle de la pêche de loisir en mer. La base de référence est de 291 pêcheurs ayant réalisé au moins une sortie en Bretagne en 2005 ou leur dernière sortie de pêche en Bretagne en 2006.

Le taux d'appartenance des pêcheurs enquêtés à une association ou d'un club de pêche en mer est très faible : $1 \%$ (figure 87 ).

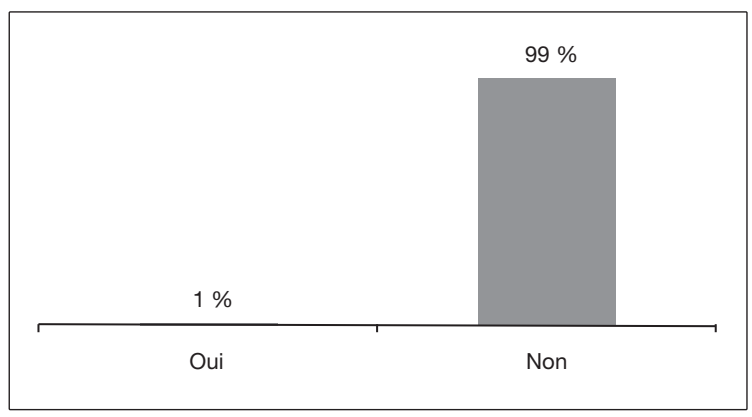

Figure 87. Appartenance à une association de pêche en mer (291 pêcheurs interrogés).

Quarante-deux pour cent des pêcheurs ayant pêché en Bretagne en 2005 ou en 2006 ont le sentiment d'avoir consacré toujours autant de temps à la pêche au cours de ces 5 dernières années (figure 88 p. XXI), contre $39 \%$ au niveau métropolitain. Ils sont $40 \%$ à penser qu'ils y ont consacré moins de temps, contre $42 \%$ au niveau métropolitain.

Soixante-quatre pour cent des pêcheurs ayant pêché en Bretagne en 2005 ou en 2006 ont le sentiment que la ressource est en diminution; contre $59 \%$ au niveau métropolitain (figure 89 p. XXI).

Comme sur l'ensemble de la métropole, les pêcheurs ayant fréquenté la Bretagne en 2005 ou en 2006 se déclarent majoritairement bien informés sur les réglementations relatives aux fermetures de la pêche pour des raisons sanitaires, sur la taille minimale autorisée des espèces pêchées et sur les réglementations en vigueur. En revanche, une plus faible part (40\% des pêcheurs) se considère bien informée sur l'évolution de la ressource (figure 90 p. XXI).

Les pêcheurs ayant fréquenté la Bretagne en 2005 ou en 2006 se déclarent majoritairement favorables aux mesures suivantes de protection et de gestion de la ressource: mise en place de repos biologique, limitation de prises par sortie, renforcement des contrôles à l'encontre des contrevenants et mise en place d'un permis pour certaines espèces. En revanche une minorité (34\%) se déclare favorable à la mise en place d'un permis.

\section{Typologie}

Sur la base des données collectées pour l'année 2005, une typologie par classe de pêcheurs de loisir en mer a été réalisée au niveau métropolitain d'après les variables explicatives suivantes:

- nombre de sorties par période;

- mode de pêche; 
- zone de pêche;

- zone de résidence (littoral/non littoral);

- groupes d'espèces pêchées et possession d'un bateau.

Elle permet de faire ressortir les grands groupes de pêcheurs, de quantifier leur proportion au sein de la population des pêcheurs de loisir et de les resituer par rapport à leur niveau d'équipement, leur période et leur fréquence de pratique. La figure $91 \mathrm{p}$. XXII présente la répartition des pêcheurs ayant fréquenté la Bretagne en 2005 ou en 2006 dans cette typologie.

Plus de la moitié des pêcheurs de loisir sont des pêcheurs à pied avec un faible niveau d'équipement. Trois catégories de pêcheurs pratiquent toute l'année, avec une fréquence élevée et un fort niveau d'équipement : les pêcheurs confirmés (pêche du bord), les pêcheurs réguliers (pêche à pied et du bord), les chasseurs sous-marins. Ces pratiquants représentent au total $18 \%$ des pêcheurs en Bretagne.

Deux catégories de pêcheurs ne pratiquent que l'été: les pêcheurs d'été du bord, les pêcheurs plaisanciers. Ils représentent $20 \%$ des pêcheurs en Bretagne. 



\section{Chapitre 3 \\ Scénarios de gestion}

\section{Régulation de l'accès de la pêcherie de coquilles Saint-Jacques de la baie de Saint-Brieuc (1962-2006). Évolution et éléments de diagnostic}

\section{Le contexte historique}

L'évolution historique du stock de coquilles Saint-Jacques de la baie de Saint-Brieuc au cours des dernières décennies est présentée dans les travaux de Piboubès (1973) enrichis par les travaux de Faure (1965, 1966), Dupouy (1978), Dupouy et al. (1983), Fifas (1991), Fifas (1996), Fifas et al. (2003).

Le stock de la baie de Saint-Brieuc a une vie relativement courte. Les traditions de pêche dans les ports de la baie de Saint-Brieuc sont relativement récentes et peu marquées. Les ports de Paimpol étaient orientés pendant longtemps vers la marine marchande alors que les rapports des agglomérations côtières de Saint-Brieuc avec la mer étaient pratiquement inexistants exception faite des pêches à la morue en Terre-Neuve et en Islande avantguerre. Après la guerre, la pêche est estivale, voire épisodique (goémon, pêche à pied, oursins). L'exploitation des bancs de praires à partir de 1953 permet de prolonger les saisons.

La coquille Saint-Jacques semble avoir déjà fait des apparitions et disparitions successives depuis le XVIII ${ }^{\mathrm{e}}$ siècle. Avant-guerre, elle est apparue aux alentours de 1925-1929 et elle a fait l'objet d'une petite pêche artisanale à voile; après la guerre, on la rencontre vers 19471949. La ressource semble disparaître dans les années cinquante par l'invasion des pieuvres, puis des étoiles de mer. La découverte du stock actuel a eu lieu en 1961 et la campagne de la saison 1962-1963 ${ }^{155}$ a marqué le début réel de l'exploitation. Le développement de cette

\footnotetext{
${ }^{155}$ L'hypothèse qui lie le développement des stocks coquilliers en Manche (baie de Saint-Brieuc, baie de Seine) à la disparition des pieuvres est plausible. Elle met l'accent sur le fait qu'à quelques dizaines ou centaines de kilomètre de distance, l'équilibre des populations d'une même espèce peut évoluer vers des directions totalement opposées (en termes d'abondance des coquilles Saint-Jacques, la Manche entre dans la période faste comparée aux gisements de la rade de Brest et de Bretagne Sud qui ont connu un effondrement).
} 
pêche était initialement dû à l'arrivée massive, à partir de 1963, des navires étrangers à la baie; il s'agissait notamment des navires brestois dont les équipages étaient déjà spécialisés dans la pêche à la coquille Saint-Jacques. Leur arrivée a été provoquée par l'effondrement du stock de la rade de Brest à la suite de l'hiver très rigoureux de 1962-1963. L'évolution des principaux paramètres de l'exploitation du stock est illustrée par la figure $92 \mathrm{p}$. XXII.

La montée spectaculaire des débarquements entre 1965 et 1975 n'est pas seulement imputable à l'abondance du recrutement à la pêcherie, mais également à l'accroissement de la capacité de capture des navires. Quant à l'évolution de l'effort de pêche, on est à présent à des valeurs divisées par 7 ou 9 par rapport aux années soixante. Les rendements horaires autrefois étroitement liés au recrutement (e.g. impact des cohortes 1970 et 1973 aux saisons de pêche 1972-1973 et 1975-1976) à l'instar de la rade de Brest dans les années cinquante, se sont stabilisés notamment depuis le début des années quatre-vingt-dix à des niveaux comparables à l' «âge d'or » alors que les recrutements sont souvent inférieurs. La puissance motrice moyenne a évolué progressivement selon trois stades distincts (ajustement d'un modèle à deux arcs exponentiels; figure 92 p. XXII). Lors de la première phase (jusqu'au milieu des années soixante-dix), la puissance motrice a augmenté (près de $15 \%$ par an en moyenne) en raison de l'apport massif de nouvelles constructions liées à la " ruée vers l'or » (nouveaux pêcheurs à origines professionnelles très diversifiées) tandis que les constructions neuves de la deuxième phase (augmentation de la puissance de $4 \%$ /an stoppée vers 1990 en raison des limitations plus drastiques en terme de puissance motrice) ont vu le jour notamment grâce au système des subventions à la flottille. Lors de la troisième phase, les limitations aux caractéristiques des navires ont conduit à une stabilisation: depuis une quinzaine d'années, le rôle de l'assimilation du progrès technique lié à l'informatique à l'augmentation de la capacité de capture devient de plus en plus déterminant (Guyader et Fifas, 1999; Guyader et al. 2004; Fifas et Arzel, 2005).

Pendant cette période, l'arsenal réglementaire a évolué en fonction de la raréfaction de la ressource et de la volonté des exploitants riverains d'assurer l'exclusivité de la pêche. Les premières limitations (sur la jauge) ont vu le jour en $1966{ }^{156}$ pour freiner l'arrivée des Camaretois (possédant de grosses unités à 7 hommes à bord), mais ces derniers ont eu gain de cause auprès de la direction des Pêches. Vers la fin des années soixante et début des années soixantedix, on a procédé à des tirages au sort des navires extérieurs. Le système a été modifié en 1972 avec la création d'une instance spécifique au sein du comité interprofessionnel chargé de la gestion des stocks de coquillages et de crustacés (CRUSCO) qui a instauré un système de licence de pêche attribuée au couple patron/navire ${ }^{157}$ en 1973 avec des limitations relatives aux caractéristiques des navires (400 ch/294 kW et 16 mètres en 1974), à l'effort de pêche à partir de la saison $1975-1976^{158}$, puis à l'instauration des TAC depuis 1976. Depuis 1990, de nouvelles limitations ont été fixées ( $250 \mathrm{ch} / 184 \mathrm{~kW}$ et 13 mètres).

\footnotetext{
${ }^{156}$ La saison de pêche 1965/1966 fut particulièrement mauvaise (820 tonnes de production avec un rendement horaire ramené aux deux tiers de la saison précédente). Étant donné qu'on assistait à l'époque à l'exploitation d'une pêcherie de recrutement, on peut déduire une mauvaise reproduction en 1963 très probablement liée aux conditions climatiques extrêmes de l'année («l'hiver du siècle »; cf. rade de Brest où la surexploitation a conduit à des effets beaucoup plus néfastes).

157 Le premier système des licences de pêche en France.

${ }^{158}$ La cause de cette première limitation (l'effort de pêche a été divisé par deux entre deux saisons consécutives) est d'ordre biologique (suite à une intervention de l'ISTPM): la cohorte pléthorique de 1973, la plus riche observée sur la série temporelle des 40 ans, a été suivie de la classe née en 1974 de très faible abondance.
} 


\section{Caractéristiques biologiques}

Les gisements coquilliers en Bretagne, plus particulièrement en baie de Saint-Brieuc, ont fait l'objet d'études et d'analyses approfondies en matière de connaissance et de modélisation des paramètres biologiques et d'exploitation (Dao et al., 1975; Véron, 1979; Dao, 1985; Fifas, 1991). Pour le stock briochin, les acquis dans le cadre des programmes nationaux de recherche tels le Programme national de déterminisme du recrutement (PNDR) et le Programme de biodiversité ont permis également de mieux cerner les mécanismes de fonctionnement à l'échelle de l'individu et de la population (Boucher et al., 1985; Boucher, 1987; Fifas et al., 1990). Des modèles bio-économiques développant une approche structurale de leur composante biologique afin d'analyser des scénarios d'évolution de la pêcherie ont vu le jour (Meuriot et al., 1987; Guyader et Fifas, 1999; Guyader et al., 2000; Guyader et Fifas, 2000).

Le recrutement de la coquille Saint-Jacques en baie de Saint-Brieuc est caractérisé par une forte variabilité interannuelle (facteur de 1 à 15) qui constitue un inconvénient majeur pour la proposition des mesures de gestion de la ressource sur la base d'un «état moyen » et rend indispensables les méthodes d'évaluation directe par dragages expérimentaux sur une base annuelle. La relation entre le stock de géniteurs et le recrutement est masquée par des causes fluctuantes externes intervenant principalement au début du cycle de développement lors de la maturation sexuelle et des étapes larvaires (Boucher, 1985 ; Boucher et al., 1985 ; Fifas et al., 1990). Depuis la restauration de la ressource en début des années quatrevingt-dix, on observe un lissage des variations de la production (facteur de 1 à 2) comparativement à la variabilité du recrutement (facteur de 1 à 7 sur la même plage temporelle). Cette relative stabilité de la production résulte d'une gouvernance devenue plus efficace au fil des années même s'il existe toujours des marges d'amélioration (cf. tableau 22).

Tableau 22. Biomasse adulte, biomasse exploitable, quota proposé et quota officiellement réalisé.

\begin{tabular}{cccrcrrrr}
\hline $\begin{array}{c}\text { Année/ } \\
\text { mois }\end{array}$ & $\begin{array}{c}\text { Coquilles } \\
\text { de } 2 \text { ans }\end{array}$ & $\begin{array}{c}\text { Coquilles } \\
\text { de } 3 \text { ans } \\
\text { et }+\end{array}$ & $\begin{array}{c}\text { Bio- } \\
\text { masse } \\
\text { adulte }\end{array}$ & $\begin{array}{c}\text { Biomasse } \\
\text { exploi- } \\
\text { table }\end{array}$ & $\begin{array}{c}\text { Quota } \\
\text { proposé }\end{array}$ & $\begin{array}{c}\text { Quota } \\
\text { officiel } \\
\text { réalisé }\end{array}$ & Excès & $\begin{array}{c}\text { Excès } \\
(\%)\end{array}$ \\
\hline Sept 1991 & 5700 & 2960 & 8660 & 4650 & 2500 & 3320 & 820 & 33 \\
Sept 1992 & 6470 & 5800 & 12270 & 6770 & 3000 & 4700 & 1700 & 57 \\
Sept 1993 & 3780 & 10910 & 14690 & 10080 & 3500 & 5300 & 1800 & 51 \\
Sept 1994 & 3810 & 14070 & 17880 & 10850 & 5000 & 5530 & 530 & 11 \\
Sept 1995 & 1820 & 10740 & 12560 & 9430 & 3500 & 4550 & 1050 & 30 \\
Sept 1996 & 2960 & 7840 & 10800 & 7390 & 3000 & 3730 & 730 & 24 \\
Sept 1997 & 4340 & 9200 & 13540 & 8560 & 3000 & 3710 & 710 & 24 \\
Sept 1998 & 1190 & 7600 & 8790 & 6710 & 2000 & 2830 & 830 & 42 \\
Sept 1999 & 2280 & 6140 & 8420 & 5780 & 2000 & 2710 & 710 & 36 \\
Sept 2000 & 5500 & 9240 & 14740 & 9900 & 3700 & 3570 & -130 & -4 \\
Sept 2001 & 8240 & 7580 & 15820 & 7990 & 4500 & 5600 & 1100 & 24 \\
Août 2002 & 5350 & 19070 & 24420 & 16260 & 6600 & 6190 & -410 & -6 \\
Sept 2003 & 6670 & 17250 & 23920 & 15720 & 5700 & 6510 & 810 & 14 \\
Sept 2004 & 9640 & 21350 & 31000 & 20730 & 6000 & 7365 & 1365 & 23 \\
Sept 2005 & 7890 & 22210 & 30100 & 20480 & 5800 & 6980 & 1180 & 20 \\
Août 2006 & 3800 & 29050 & 32850 & 27350 & 6500 & 7505 & 1005 & 15 \\
Sept 2007 & 8920 & 22310 & 31230 & 23220 & & & & \\
\hline
\end{tabular}




\section{La capacité de capture en fonction de la puissance motrice et du progrès technique}

\section{La puissance motrice}

Le rôle de la puissance motrice sur l'augmentation de la capacité de capture a été modélisé sur les groupes d'âge principaux (2 et 3) ciblés lors des opérations de pêche. La capturabilité est fonction croissante de l'abondance du groupe en question et de la puissance motrice moyenne (Fifas, 1991; Fifas, 1993 ; figure 93 p. XXIII) :

$$
q=\exp \left[\frac{\mathrm{N}^{\mathrm{CV}}}{\alpha+\beta \cdot \mathrm{N}^{\mathrm{CV}}}\right]
$$

avec:

$\mathrm{N}=$ abondance $; \mathrm{cv}=$ puissance motrice moyenne de la flottille; $\beta<0 ; \alpha>0$.

Le modèle ajusté sur les cohortes nées entre 1972 et 1981 n'est valide qu'à la plage temporelle stricte de référence. D'un côté, dès les premières années de l'exploitation mécanisée du stock, il y avait vraisemblablement peu d'impact de la puissance motrice sur la capacité de capture, exception faite de la faculté des déplacements rapides en cas de puissance élevée ${ }^{159}$. De l'autre côté, les années récentes caractérisées par de nouvelles limitations des puissances motrices et par l'avènement du développement informatique amenuisent l'effet de la puissance motrice : comme on l'observe sur la figure 93 p. XXIII, de nos jours la puissance motrice moyenne en tant que facteur explicatif de la capturabilité du groupe d'âge 2 n'intervient qu'à près de $25 \%$. De plus, la pêcherie du recrutement des années soixante et soixante-dix n'est plus valable du fait de l'augmentation du diamètre des anneaux métalliques des dragues : par conséquent, un modèle actualisé doit se référer sur l'ensemble des âges.

\section{Le progrès technique}

Nous avons utilisé les résultats des analyses de cohortes données de 1972 à 1998 acquises par échantillonnage des captures commerciales et nous avons calibré par la suite les résultats par les campagnes annuelles d'évaluation directe de la ressource afin d'intégrer au calcul des vecteurs de mortalité les décès indirectement induits par la pêche (fraude hivernale et estivale, mortalité par « casse », mortalité par rejet) (Fifas, 1991; Guyader et Fifas, 1999). Un modèle de capturabilité tous groupes d'âge confondus a ainsi été bâti. Le modèle est composite, il tient compte de la puissance motrice moyenne comme variable explicative corrigée par un coefficient d'assimilation du progrès technique au-delà d'une année limite. La capturabilité qi de l'année i s'écrit comme suit (tableau 23, figure 94 p. XXIV):

$$
\begin{array}{cccr}
\mathrm{q}_{\mathrm{i}}=\mathrm{b} \times \mathrm{CV} \times \mathrm{i}^{\mathrm{c}} & \text { si } & \text { i } & \operatorname{Lim} \\
\mathrm{q}_{\mathrm{i}}=\mathrm{b} \times \mathrm{CV} \times \mathrm{i}^{\mathrm{c} \cdot[1+\alpha \cdot(i-\operatorname{Lim})]} & \text { si } & \text { i }>\operatorname{Lim}
\end{array}
$$

\footnotetext{
159 La drague à volet dépresseur introduite en 1968 et le remplacement des treuils mécaniques par des treuils hydrauliques à partir de 1970-1972 ont constitué deux étapes déterminantes au rôle explicatif de la puissance motrice dans la capacité de capture.
} 
Tableau 23. Résultats de l'ajustement du modèle de capturabilité tous groupes d'âge confondus en baie de Saint-Brieuc. Données de 1974 à 2000.

\begin{tabular}{|c|c|c|c|c|c|r|}
\hline \multirow{2}{*}{ Paramètre } & \multirow{2}{*}{ Valeur } & \multirow{2}{*}{ Écart type } & \multirow{2}{*}{ CV } & \multicolumn{3}{|c|}{ Matrice des corrélations } \\
\cline { 5 - 7 } & & & & $\mathrm{C}$ & $\alpha$ & \multicolumn{1}{c|}{ Lim } \\
\hline $\mathrm{b}$ & 2,1384 & 0,3633 & 0,1699 & $-0,917$ & 0,044 & $-0,220$ \\
$\mathrm{C}$ & 0,4428 & 0,2064 & 0,4462 & & $-0,103$ & 0,466 \\
$\alpha$ & 0,0253 & 0,0296 & 1,1708 & & & 0,513 \\
Lim & 1989 & 11,0827 & 0,6927 & & & \\
\hline \multicolumn{6}{c}{ nombre d'années : $\mathrm{n}=27$} \\
\hline
\end{tabular}

où : $b, C, \alpha$, Lim coefficients du modèle.

À la lumière de ces résultats significatifs $\left(\mathrm{R}^{2}\right.$ près de 0,88$)$, on constate une augmentation annuelle moyenne de la capacité de capture de 12 à $14 \%$ avec un renforcement notamment au milieu des années quatre-vingt provoqué par l'apport de navires neufs grâce au système de subventions à la pêche de l'époque. Actuellement, sans augmentation de la puissance motrice moyenne (stabilisée à $170 \mathrm{ch} / 125 \mathrm{~kW}$ ) depuis une quinzaine d'années, le progrès technique apporte un accroissement de la capacité de capture de $2,5 \%$ par an (coefficient a).

La capturabilité ou capacité de capture est la mortalité par pêche « vue par le pêcheur». Afin d'examiner la mortalité « vue par la coquille Saint-Jacques », nous devons intégrer dans les calculs la sélectivité liée aux engins de pêche (figure 95 p. XXIV). On peut déduire que la sélectivité masque l'évolution de la capacité de capture: les augmentations successives des maillages ont réduit les coefficients instantanés de mortalité par pêche $\mathrm{F}$ aux alentours de 0,4 par an contre 0,7 il y a 25 ans. Avant l'adoption des TAC, la pêcherie de recrutement, il y a une trentaine d'années, était caractérisée par de fortes fluctuations de F.

L'analyse porte par la suite sur la série antérieure au système des licences de pêche. Le profil de l'exploitation de l'époque (absence des dragues à volet, limitations du poids des dragues jusqu'à 1966-68, treuils mécaniques de pêche) nous incite à considérer une capturabilité constante de 0,40 environ. L'étude nous permet de constituer la série complète de l'abondance du recrutement depuis la cohorte 1963. Une cinétique positive du recrutement est observée de 1966 à 1970 alors qu'en 1963 et 1965 les abondances sont faibles (figure 96 p. XXIV). Au regard de cette cinétique, on peut discerner une périodicité avec un cycle de dix ans environ (abondances de recrutement à la hausse vers la fin des décennies) qui n'est pas sans rappeler l'évolution cyclique de l'énergie solaire (Meunier, 2004) et de la température (Fifas et Arzel, 2005).

\section{Impact bioéconomique de l'accroissement de la capacité de capture}

L'augmentation de la capacité de capture malgré le contrôle de la variable puissance motrice induit un gaspillage économique et une menace permanente du système de conservation qui est basé sur l'ajustement permanent de l'effort de pêche nominal comme variable principale de contrôle. Actuellement, en raisonnant sur la base d'une flottille de pêche de 250 navires environ et sous l'hypothèse d'un progrès technique de l'ordre de 
2,5\% par an, on se situe au-delà de l'optimum économique. Néanmoins, ces surcapacités doivent être relativisées en raison de la polyvalence des navires (figure 97 p. XXV). Sur ce dernier point, l'analyse mérite d'être reconduite à la lumière des contraintes actuelles croissantes liées à l'augmentation du prix du carburant particulièrement pénalisant dans le cas des engins traînants (cf. rôle prépondérant des navires dragueurs chalutiers en baie de Saint-Brieuc). Ces contraintes associées à la richesse actuelle du gisement coquillier conduiront vraisemblablement à une diminution de la polyvalence de la flottille de pêche.

\section{Conclusion}

La coquille Saint-Jacques, actuellement quatrième espèce en valeur de débarquement en France, a une histoire récente conditionnée par le réchauffement climatique malgré les fluctuations périodiques de la température sur des cycles de 7 à 8 ans. Après l'accident climatique de l'hiver 1963 concernant les gisements de la façade atlantique, la baie de Saint-Brieuc est devenue le gisement prépondérant à l'échelle régionale. On y observe, par ailleurs, les densités en coquilles Saint-Jacques les plus élevées en Europe. On doit y chercher principalement l'influence des conditions environnementales, mais également l'impact positif de quelques mesures d'encadrement réglementaire notamment en termes de sélectivité des engins de pêche. Cependant, la capacité de capture se développe continuellement et le stock a failli connaître un déclin irréversible après une chute alarmante vers la fin des années quatre-vingt. De nos jours, avec le développement de l'informatique embarquée, l'encadrement des pêches par des moyens traditionnels a épuisé ses moyens. Seule, la limitation draconienne du nombre d'heures de pêche ne sera pas suffisante à terme tandis qu'en matière de surexploitation biologique, on possède peu de marges de progression, la surcapacité économique de la flottille est effective depuis longtemps.

\section{Langoustine du golfe de Gascogne}

\section{Impacts des scénarios de pêche sélective}

La pêcherie de langoustines du golfe de Gascogne a fait l'objet de nombreux travaux sur la période 2000-2006, tant sur la mise au point d'un protocole d'observation des captures en mer, que sur les simulations d'amélioration de la sélectivité des chaluts. L'impact de l'amélioration de la sélectivité a été évalué de façon intégrée: les analyses ont permis de montrer les effets de scénarios de gestion à la fois en termes d'évolution du stock et de performances économiques des chalutiers langoustiniers. Un exemple non actualisé (2002) de développement d'une approche bioéconomique est donné ci-dessous. Le modèle de simulation peut évidemment être appliqué à des séries de données intégrant les années récentes. Il s'agit ici d'en montrer l'intérêt et le type de résultats qu'il produit.

\section{Caractéristiques de la pêcherie de langoustines du golfe de Gascogne}

Les informations collectées par l'Ifremer sur l'activité halieutique et économique des navires en 2000 permettent de caractériser cette flottille exploitant cette pêcherie (Berthou et al., 2002). La langoustine est pêchée au chalut de fond par une flottille d'environ 230 navires français. Le navire moyen mesure 15 mètres pour $235 \mathrm{~kW}$, possède un équipage de 3 hommes et est âgé de 19 ans. Le capital investi par homme employé est 
estimé en moyenne à $105 \mathrm{k} €$ révélant une forte intensité capitalistique comparée à une moyenne nationale dans le secteur de la pêche évaluée à $75 \mathrm{k} €$.

L'activité de ces navires a généré, en 2000, un chiffre d'affaires total estimé à $75 \mathrm{M} €$. La part de la langoustine dans le chiffre d'affaires de ces navires est estimée à $39 \%$ en moyenne, mais est très variable d'un navire à l'autre. La spécialisation plus ou moins forte de la flottille conditionne sa réactivité à des modifications de l'environnement économique, biologique ou réglementaire lié au stock de langoustine. Pour $31 \%$ des navires, plus de la moitié de leur chiffre d'affaires annuel repose essentiellement sur la langoustine, mais cette proportion est encore plus forte dans les quartiers du Guilvinec ou de Concarneau. La Bretagne Sud concentre ainsi $68 \%$ des chalutiers langoustiniers et $64 \%$ des emplois directs associés à cette activité. En plus d'un déterminant géographique, cette spécialisation semble être inversement liée à la taille des navires. L'importance de l'activité langoustine varie selon les navires: pour $52 \%$ des navires, elle constitue l'essentiel de l'activité annuelle, $36 \%$ de la flotte l'a exercée en 2000 à temps partiel dont $26 \%$ entre 3 et 6 mois, enfin pour $11 \%$ des navires, elle a constitué une activité très épisodique. Les activités connexes de ces navires sont essentiellement du chalutage (chalut de fond dirigé sur le poisson pour $22 \%$ et chalut pélagique pour $9 \%$ ). Des variations d'intensité d'activité s'observent également au cours de l'année: $70 \%$ des débarquements totaux de langoustines sont concentrés sur quatre mois de l'année, entre mai et août, le minimum de l'activité se situant en janvier.

La pêcherie la plus importante se situe autour des îles de Glénan. Elle y est exploitée à partir des ports bigoudens et de Concarneau. Selon les années entre 50 et $60 \%$ des débarquements proviennent de cette pêcherie pour laquelle la faible distance des ports d'attache permet de commercialiser la langoustine vivante pêchée le jour même. La pêcherie située au large de Groix et de Belle-Île-en-Mer est fréquentée par des navires de Concarneau, de Lorient et du quartier maritime de Saint-Nazaire. Celle des vasières de l'Île d'Yeu, de Rochebonne et de la Gironde, est exploitée par les bateaux des Sables-d'Olonne, de La Rochelle et de La Cotinière.

Les débarquements sont effectués quasi exclusivement par des navires français ${ }^{160}$. Après avoir culminé à 7000 tonnes/an dans le milieu des années 1970, les débarquements ont fortement chuté pour atteindre 3000 tonnes/an à la fin des années 1990 (figure 98 p. XXV). Ils ont été de 3800 tonnes en 2001. La diminution du nombre de navires ciblant cette espèce (400 en 1978, 300 en 1987, 230 en 2000), ne peut expliquer seule cette baisse des apports, d'autant plus qu'elle a été compensée au moins partiellement par des gains d'efficacité (chaluts jumeaux, gros bourrelets) et par une augmentation du temps de pêche individuel.

Sur le plan du marché de langoustines, la chute des débarquements de langoustine n'a été que partiellement compensée par l'augmentation des importations. La part de marché de la production nationale par rapport à la consommation apparente (production nationale + importations - exportations) n'est plus que de $46 \%$ contre $56 \%$ en 1992 , $75 \%$ en 1983. Le prix moyen au débarquement en francs constants, proche de 8 euros a retrouvé les niveaux des années quatre-vingt après avoir fortement chuté au début des années quatre-vingt-dix.

${ }^{160}$ On notera cependant que l'Espagne dispose d'un quota représentant $6 \%$ du TAC. 
La majeure partie des langoustines capturées mesure de 5 à $11 \mathrm{~cm}$ (longueur totale), (figure 99 p. XXVI). La taille de commercialisation étant de $8,5 \mathrm{~cm}$, les rejets sont donc importants : en 2001, ils représentent environ la moitié en nombre des langoustines capturées et $30 \%$ en poids. Des études ont montré qu'environ $30 \%$ des langoustines rejetées à la mer survivent (Guéguen et Charuau, 1975).

\section{Simulations de scénarios de gestion}

\section{Matériels et méthodes}

Les données de base sont celles que le groupe de travail du CIEM en charge de l'analyse de cette pêcherie a utilisées pour réaliser l'estimation du stock et les prévisions de captures à court terme (CIEM, 2002b). On rappellera ici simplement les grandes lignes de la méthode d'évaluation retenue. La base de données porte sur les années 1987 à 2001 ; les compositions en taille des captures françaises par sexe sont transposées en compositions en âge en utilisant les paramètres de croissance (technique du «slicing »); l'évaluation des stocks de mâles et de femelles est réalisée par la méthode classique d'analyse de population virtuelle (VPA); les nombres d'individus par groupe d'âge au premier janvier 2002 fournis par la VPA constituent la population initiale utilisée dans les simulations.

Les simulations présentées sont réalisées sexes combinés et les résultats comparés à la situation de référence (statu quo) correspondant à l'année 2002. Les mortalités par pêche aux âges en 2002 sont estimées comme moyenne de celle fournies par la VPA pour la période 1999-2001; celles des années suivantes sont calculées par application d'un multiplicateur $\mathrm{mF}$ en fonction du scénario analysé (cf. ci-dessous). Ces simulations intègrent deux sources d'incertitude. Faute de relation claire entre stock et recrutement, pour chaque année de la période de simulation, le recrutement est tiré aléatoirement parmi les recrutements estimés par la VPA au cours de la période 1990-1999. De plus, l'abondance par âge dans la population est supposée être estimée avec une erreur log-normale d'écart type (sa); les valeurs de sa étant également estimées par la VPA. Un tirage aléatoire de type « Monte-Carlo » permet d'estimer les quartiles des distributions des captures et de la biomasse pour les différents scénarios de gestion.

Les effets d'une protection accrue des jeunes langoustines ont été simulés en modulant la mortalité par pêche à laquelle sont soumis les premiers groupes d'âge (groupes d'âge 1 et 2) par rapport aux individus plus âgés (groupe d'âge $3+$ ). Les limites approximatives de taille (longueur de carapace Lc) pour chaque groupe d'âge sont les suivantes:

- groupe d'âge 1: moins de $19 \mathrm{~mm} \mathrm{Lc}$;

- groupe d'âge 2 : entre 19 et $26 \mathrm{~mm} \mathrm{Lc}$;

- groupe d'âge 3+ : plus de $26 \mathrm{~mm} \mathrm{Lc} \mathrm{(26} \mathrm{mm} \mathrm{correspond} \mathrm{approximativement} \mathrm{à} \mathrm{la} \mathrm{taille}$ minimale au débarquement retenue par les organisations françaises de producteurs).

Chaque scénario est identifié par les multiplicateurs de mortalité par pêche $(\mathrm{mF})$ appliqués à chaque groupe d'âge : à titre d'exemple, le scénario « (.0/.5/1.) » correspond à un scénario dans lequel, par rapport à la situation de référence, la mortalité par pêche est nulle sur le groupe 1, divisée par 2 pour le groupe 2, maintenue constante sur le groupe $3+$; dans un scénario «(.7/.7/.7)», le diagramme d'exploitation n'est pas modifié, mais la mortalité par pêche est diminuée de $30 \%$ pour tous les groupes d'âge.

Aucun point de référence biologique n'a, pour le moment, été adopté par le CIEM pour le stock de langoustines du golfe de Gascogne, ni en terme de biomasse, ni en terme de mortalité par pêche. Par ailleurs, il n'existe pas d'objectif de gestion explicite pour cette 
pêcherie. Aussi, en mai 2002, devant les signes de dégradation de l'état du stock (biomasse des reproducteurs et recrutement au plus bas), le CIEM a proposé divers scénarios devant permettre une « augmentation significative » de la biomasse afin d'augmenter les chances de bons recrutements pour les années à venir (ou de limiter les risques de mauvais recrutements). Au début des années 1990, biomasse et recrutement étaient élevés, et c'est le niveau de biomasse correspondant à cette période (soit environ 18000 tonnes) qui a été retenu comme objectif. Les scénarios proposent une restauration de la biomasse à court terme, qui suppose une réduction de $50 \%$ du taux d'exploitation en 2003 par rapport à 2001, ou à moyen terme (2006-2007), au travers de réductions progressives du taux d'exploitation ou d'améliorations de la sélectivité (CIEM, 2002a). Même si la biomasse de 18000 tonnes ne constitue pas un vrai point de référence biologique (dans le sens où ce n'est pas une valeur calculée selon les méthodes habituelles), implicitement, on peut considérer que ces objectifs sont conformes à une approche de précaution, puisque proposés par le CIEM.

Reprenant ces bases, nous avons examiné la capacité de différents scénarios de gestion à permettre une reconstitution de la biomasse au voisinage de 18000 tonnes soit à court terme (2004), soit à moyen terme (2007).

Les résultats des simulations biologiques en termes de débarquements (médians) ont été utilisés dans le modèle de simulation économique, avec un exemple d'utilisation des résultats des fractiles 25 et $75 \%$. Le paramétrage du modèle de simulation s'est appuyé sur les données économiques collectées par voie d'enquête auprès d'un échantillon représentant $31,5 \%$ de la flottille langoustinière en 2000. La méthodologie de sondage, les variables collectées ainsi que le processus de validation de données sont précisés par Daurès et al, (2002a), Daurès et al., (2002b) et Daurès et al. (2002c). La comparaison des caractéristiques de l'échantillon et de la population est présentée dans le tableau 24. Les résultats économiques sont analysés par catégories de nombre de personnes embarquées, critère de regroupement pertinent d'un point de vue de la réduction de la dispersion intraclasse du chiffre d'affaires. Pour une catégorie de taille d'équipage sont spécifiées les longueurs minimale et maximale des navires enquêtés sur un plan économique (tableau 25).

Tableau 24. Comparaison des caractéristiques de l'échantillon et de la population de navires langoustiniers.

\begin{tabular}{lcccc}
\hline Classe équipage & $\begin{array}{c}\text { Inf. ou égal } \\
\text { à 2 hommes }\end{array}$ & $\begin{array}{c}\text { Sup. 2 à } \\
3 \text { hommes }\end{array}$ & $\begin{array}{c}\text { Sup. 3 à } \\
5 \text { hommes }\end{array}$ & $\begin{array}{c}\text { Sup. } \\
5 \text { hommes }\end{array}$ \\
\hline Flotte langoustine VIII en 2000 & & & & \\
Nombre de navires & 50 & 74 & 96 & 9 \\
Part moyenne lang. VIII dans CA annuel (\%) & 44 & 47 & 31 & 24 \\
Débarquement annuel moyen lang. VIII & 9,6 & 15,3 & 17,5 & 19,8 \\
Longueur moyenne (m) & 12,03 & 14,24 & 16,05 & 19,88 \\
Chiffre d'affaires total moyen 2000 (k€) & 164,6 & 257,8 & 428,8 & 721,2 \\
Échantillon Données économiques 2001 & & & & \\
Nombre de navires & 18 & 17 & 31 & 6 \\
\% du total (taux d'échantillonnage) & 36 & 23 & 32 & 67 \\
Longueur moyenne (m) & 12,43 & 14,38 & 16,60 & 21,84 \\
Chiffre d'affaires total moyen 2000 (k€) & 154,6 & 233,9 & 423,4 & 651,0 \\
Longueur min et max. (m) & $10,6-14,3$ & $12,9-15,8$ & $14,1-19,1$ & $19,3-24,3$ \\
\hline
\end{tabular}


Tableau 25. Indicateurs économiques (année 2000) utilisés dans les simulations de gestion pour les sous-flottilles langoustinières du golfe de Gascogne.

\begin{tabular}{lcccc}
\hline Classe équipage & $\begin{array}{c}\text { Inf. ou égal } \\
\text { à 2 hommes }\end{array}$ & $\begin{array}{c}\text { Sup. 2 à } \\
\text { 3 hommes }\end{array}$ & $\begin{array}{c}\text { Sup. 3 à } \\
5 \text { hommes }\end{array}$ & $\begin{array}{c}\text { Sup. } \\
\text { 5 hommes }\end{array}$ \\
\hline Salaire annuel brut par homme embarqué $(\mathrm{k} €)$ & 27,8 & 27,1 & 30,8 & 31,1 \\
Excédent brut d'exploitation $(\mathrm{k} €)$ & 37,4 & 52,5 & 99,6 & 167,3 \\
$\begin{array}{l}\text { Résultat courant économique }(\mathrm{k} €) \\
\text { Valeur ajoutée brute }(\mathrm{k} €)\end{array}$ & 11,3 & 11,5 & 23,5 & 53,4 \\
Part carburant huile/chiffre d'affaires (\%) & 94,6 & 136,7 & 241,6 & 365,7 \\
Part autres frais communs/chiffre d'affaires & 15 & 17 & 17 & 17 \\
$\begin{array}{l}\%) \\
\text { Part frais armements/chiffre d'affaires (\%) }\end{array}$ & 6 & 8 & 10 & 10 \\
\hline
\end{tabular}

Le modèle de simulation développé est fortement inspiré de la méthodologie utilisée pour l'analyse économique des avis de l'ACFM (Salz et Frost, 2000) validée par le Comité scientifique technique et économique des pêches de la Commission européenne. Le principe général est que les entreprises ajustent leur effort nominal de pêche aux contraintes sur les débarquements totaux de la flottille considérée. Dans ce cas d'étude, le volume de langoustines débarquées chaque année est réparti entre les différentes sous-flottilles, puis par navire sur la base d'une clé de répartition fondée sur les débarquements historiques des trois dernières années. L'hypothèse ensuite retenue est que le nombre de jours de mer passés par navire dans la pêcherie s'ajuste, si les débarquements par navires sont limitants, en proportion de l'état du stock exprimé en termes de biomasse féconde. Les variables économiques endogènes sont les taxes de débarquements basées sur la valeur des débarquements et les coûts de carburants qui dépendent de l'activité des unités de pêche exprimée en jours de mer.

On suppose également que la production des autres espèces (principalement merlu, poissons plats, céphalopodes) est liée à celle de la langoustine. L'analyse des statistiques de débarquements montre une stabilité des profils de production au cours des dernières années. Cependant, cette hypothèse de production jointe devra être approfondie dans les travaux à venir, de manière à identifier plus précisément les possibilités de substitution entre espèces. Une autre composante de la simulation intègre la sensibilité des prix de la langoustine à la première vente au volume débarqué par cette flottille et aux prix des importations. Une première analyse économétrique des séries de prix depuis environ vingt ans, donne le résultat suivant:

$$
\mathrm{p}=52,19 \times \operatorname{pimp}^{0,635} \times \text { qnat }^{0,277}
$$

avec:

$\mathrm{p}$ : prix au débarquement (en euros)

pimp : prix des importations (en euros)

qnat: débarquements nationaux de langoustine (tonnes)

Les paramètres estimés sont significatifs au seuil de $1 \%$ et la qualité d'ajustement de la régression est satisfaisante $(\mathrm{R} 2=0,85)$. L'ensemble des simulations proposées par la suite suppose un prix d'importation constant de 6,55 euros. Le prix du gazole est paramétré à 0,27 euro/l sur la période. 


\section{Description des scénarios simulés}

Le tableau 26 fournit la liste des scénarios qui ont été simulés en termes de réduction de la mortalité par pêche et/ou d'amélioration du profil d'exploitation.

Tableau 26. Description des scénarios de gestion simulés pour la langoustine du golfe de Gascogne.

\begin{tabular}{|l|c|c|c|c|c|c|c|}
\hline \multicolumn{1}{|c|}{ Scénarios } & Statu quo & 1 & 2 & 3 & 4 & 5 & 6 \\
\hline $\mathrm{mF}-2003$ & $(1 . / 1 . / 1)$. & $(.9 / .9 / .9)$ & $(.8 / .8 / .8)$ & $(.7 / .7 / .7)$ & $(.5 / .5 / .5 /)$ & $(.0 / 1 . / 1)$. & $(0 . / 0.5 / 1)$. \\
$\mathrm{mF}-2004 \ldots$ & & $(.9 / .9 / .9)$ & $(.8 / .8 / .8)$ & $(.7 / .7 / .7)$ & $(.5 / .5 / .5)$ & $(.0 / 1 . / 1)$. & $(.0 / .5 / 1)$. \\
& & & & & & & \\
$\begin{array}{l}\text { Simulations } \\
\text { économiques }\end{array}$ & OUI & & & & & & \\
\hline
\end{tabular}

\begin{tabular}{|l|c|c|c|c|c|}
\hline \multicolumn{1}{|c|}{ Scénarios } & 7 & 8 & 9 & 10 & 11 \\
\hline $\mathrm{mF}-2003$ & $(.8 / .8 / .8)$ & $(1 . / 1 . / 1)$. & $(1 . / 1 . / 1)$. & $(1 . / 1 . / 1)$. & $(1 . / 1 . / 1)$. \\
$\mathrm{mF}-2004 \ldots$ & $(.0 / .5 / .8)$ & $\begin{array}{c}(0 . / .5 / 1 .) \\
+\mathrm{TAC} 3000 \mathrm{t}\end{array}$ & $\begin{array}{c}(0 . / .5 / 1 .) \\
+\mathrm{TAC} 3200 \mathrm{t}\end{array}$ & $\begin{array}{c}(0 . / .5 / 1 .) \\
+\mathrm{TAC} 3500 \mathrm{t}\end{array}$ & $(0 . / .5 / 1)$. \\
Simulations & & & & OUI & \\
économiques & & OUI & & \\
\hline
\end{tabular}

Les scénarios 1 à 4 correspondent respectivement à une réduction de la mortalité par pêche de $10 \%, 20 \%, 30 \%$ et $50 \%$ pour tous les groupes d'âges à partir de 2003 (pas d'amélioration du profil d'exploitation).

Le scénario 5 correspond à une protection totale du groupe $1(\mathrm{mF} 1=0)$ et à un maintien de la mortalité par pêche sur les autres groupes d'âge; ces nouveaux paramètres s'appliquent à partir de 2003.

Le scénario 6 simule une plus grande protection des petites langoustines à partir de 2003 : protection totale du groupe d'âge $1(\mathrm{mF} 1=0)$, diminution de $50 \%$ de la mortalité par pêche sur le groupe $2(\mathrm{mF} 2=0,5)$, maintien du taux d'exploitation sur le groupe d'âge $3+$.

Le scénario 7 tient compte du fait que la mise au point et l'adoption par la profession de chalut plus sélectif ne seront pas immédiates; il simule une réduction de $20 \%$ de la mortalité par pêche à partir de 2003, puis une amélioration du profil d'exploitation à partir de 2004.

Enfin, suite aux diverses réunions entre les organisations professionnelles, l'Administration et l'Ifremer, une autre option de gestion a été explorée, qui consiste à limiter les captures aux environs de 3000 à 3500 tonnes/an par un TAC fixé pour plusieurs années, avec une amélioration du profil d'exploitation à partir de 2004 (0./.5/1.) ; dans ce cas, la mortalité par pêche est adaptée dès 2003 de sorte que les captures ne dépassent pas le TAC objectif. Les scénarios 8,9 et 10 correspondent respectivement à des TAC annuels fixés à 3000 tonnes, 3200 tonnes, 3500 tonnes pendant plusieurs années; le scénario 11, donné pour comparaison, correspond à une amélioration du profil d'exploitation à partir de 2004 .

Les résultats de ces simulations ne doivent pas être interprétés comme des valeurs absolues. Ils sont en effet tributaires des hypothèses émises (notamment le recrutement aléatoire); ils indiquent cependant des tendances et permettent des comparaisons entre les divers scénarios. De même, compte tenu des incertitudes, des différences entre résultats ne sont significatives que dans la mesure où elles sont nettes. Enfin, il s'agit d'exemples permettant de mettre en évidence les effets de diverses mesures, mais ne constituent en aucun 
cas des propositions d'objectifs de gestion dont la définition relève des gestionnaires et des usagers.

\section{Évolution de la biomasse des reproducteurs et des débarquements}

\section{Biomasse des reproducteurs}

Les résultats sont fournis par le tableau 27 et la figure 100.

Tableau 27. Probabilités (exprimées en \%) que la biomasse féconde de langoustines du golfe de Gascogne atteigne le seuil de 18000 tonnes selon divers scénarios de gestion.

\begin{tabular}{|c|r|r|r|r|r|r|r|r|r|r|r|r|}
\hline Scénario & $\mathrm{Sq}$ & 1 & 2 & 3 & 4 & 5 & 6 & 7 & 8 & 9 & 10 & 11 \\
\hline 2002 & 0 & 0 & 0 & 0 & 0 & 0 & 0 & 0 & 0 & 0 & 0 & 0 \\
\hline 2003 & 3 & 3 & 3 & 3 & 3 & 3 & 3 & 3 & 3 & 3 & 3 & 3 \\
\hline 2004 & 8 & 12 & 14 & 17 & 30 & 9 & 14 & 14 & 17 & 14 & 11 & 8 \\
\hline 2005 & 12 & 19 & 27 & 34 & 59 & 12 & 25 & 31 & 38 & 32 & 26 & 19 \\
\hline 2006 & 13 & 21 & 31 & 46 & 75 & 14 & 28 & 41 & 56 & 48 & 38 & 26 \\
\hline 2007 & 15 & 22 & 36 & 49 & 82 & 16 & 32 & 46 & 68 & 62 & 49 & 29 \\
\hline 2008 & 14 & 25 & 36 & 52 & 86 & 15 & 32 & 50 & 76 & 69 & 56 & 32 \\
\hline 2009 & 16 & 24 & 38 & 52 & 87 & 18 & 33 & 50 & 84 & 76 & 62 & 33 \\
\hline 2010 & 13 & 21 & 36 & 54 & 90 & 15 & 31 & 52 & 88 & 83 & 70 & 31 \\
\hline 2011 & 15 & 23 & 37 & 56 & 90 & 16 & 31 & 54 & 91 & 86 & 75 & 31 \\
\hline
\end{tabular}

En l'absence de toute mesure (statu quo) la probabilité que la biomasse atteigne le seuil de 18000 tonnes est faible; en fait, cette dernière reste stable et très proche de son plus bas niveau, y compris à long terme.

Les scénarios 1 à 3 montrent que sans amélioration du profil d'exploitation, une réduction immédiate de la mortalité par pêche d'au moins $30 \%$ (scénarios 1 à 3 ) est nécessaire pour que la biomasse atteigne 18000 tonnes à moyen terme (2007-2008) avec une probabilité supérieure à $50 \%$.

Une réduction immédiate plus forte $(-50 \%$, scénario 4$)$ permet une restauration beaucoup plus rapide puisque la biomasse dépasse 18000 tonnes dès 2005 avec une probabilité de plus de $50 \%$ puis continue d'augmenter pour atteindre environ 23000 tonnes à long terme.

Une amélioration du diagramme d'exploitation permettant dès 2003 de ne capturer aucune petite langoustine d'âge 1 (scénario 5) ne conduit pas à une restauration de la biomasse au niveau requis. Ce résultat s'explique par le fait que les langoustines du groupe 1 (moins de $19 \mathrm{~mm} \mathrm{Lc)} \mathrm{sont} \mathrm{peu} \mathrm{abondantes} \mathrm{(ou} \mathrm{sous-estimées)} \mathrm{dans} \mathrm{les} \mathrm{captures.}$

L'augmentation de la biomasse est plus nette lorsque la mortalité par pêche est nulle sur le groupe d'âge 1 et réduite de $50 \%$ sur les individus d'âge 2 (scénario 6).

Les résultats des scénarios 5 et 6 mettent donc en évidence que pour être efficace en termes de reconstitution de la biomasse, l'amélioration du diagramme d'exploitation doit être importante et permettre la sauvegarde accrue d'une grande partie des langoustines de moins de $26 \mathrm{~mm}$. Cependant la probabilité d'atteindre le seuil de 18000 tonnes reste faible, inférieure à $50 \%$, même à long terme.

Le scénario 7 prend en compte le fait que l'amélioration du diagramme d'exploitation ne sera pas immédiate; il intègre également une réduction de la mortalité par pêche de 
$20 \%$ à partir de 2003. La biomasse féconde atteint 18000 tonnes en 2008 (probabilité $50 \%$ ) et se stabilise à ce niveau à long terme.

Les derniers scénarios (8 à 10) simulent l'impact d'une amélioration sensible du diagramme d'exploitation en 2004 et des limitations de captures (TAC) à divers niveaux. Ils montrent que la biomasse féconde atteint 18000 tonnes (probabilité de $50 \%$ ) en 2006, 2007 ou 2008 selon que le TAC est de 3000,3200 ou 3500 tonnes.

Statu quo F.

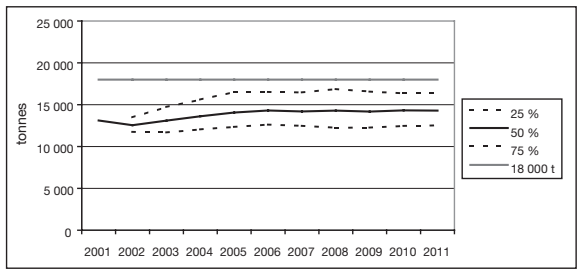

Scénario 2 - Réduction uniforme de F de $20 \%$ $(.8 / .8 / .8)$.

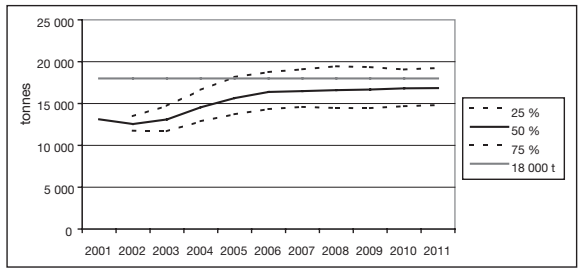

Scénario 4 - Réduction uniforme de F de $50 \%$ $(.5 / .5 / .5)$.

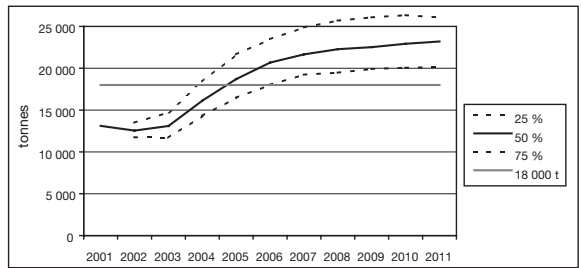

Scénario 6 - Pas de capture âge 1 , réduction $50 \%$ âge $2(0 . / .5 / 1$.).

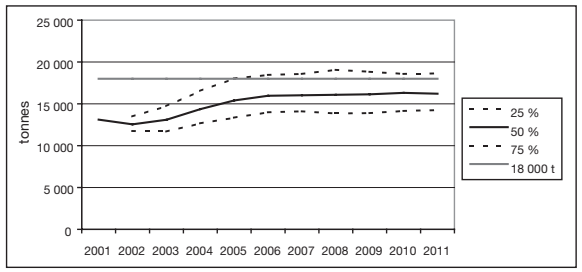

Scénario 1 - Réduction uniforme de F de $10 \%$ (.9/.9/.9).

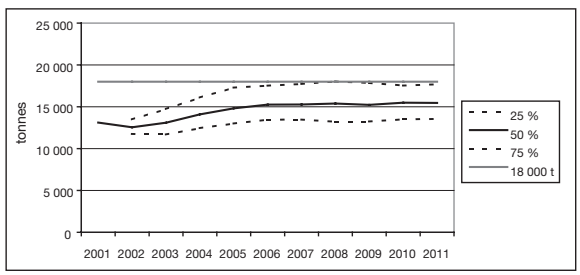

Scénario 3 - Réduction uniforme de F de $30 \%$ $(.7 / .7 / .7)$.

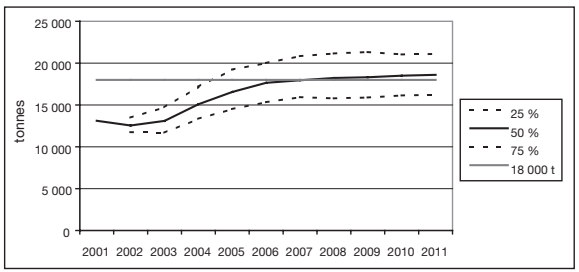

Scénario 5 - Pas de capture âge 1 (0./1./1.).

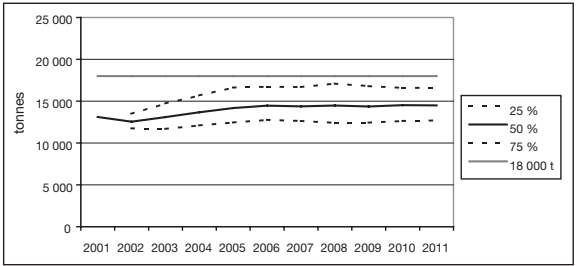

Scénario $7-(.8 / .8 / .8)$ puis $(0 . / .5 / .8)$.

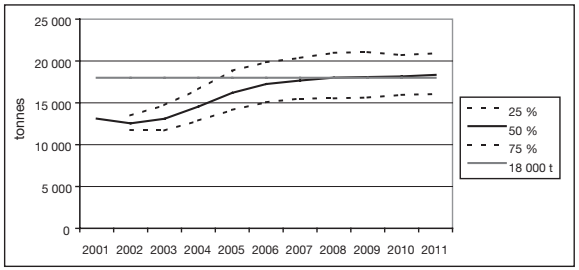


Scénario $8-(0 . / .5 / 1$.) puis TAC 3000 tonnes.

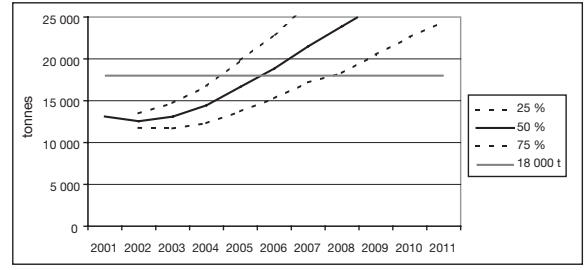

Scénario $10-(0 . / .5 / 1$.) puis TAC 3500 tonnes.

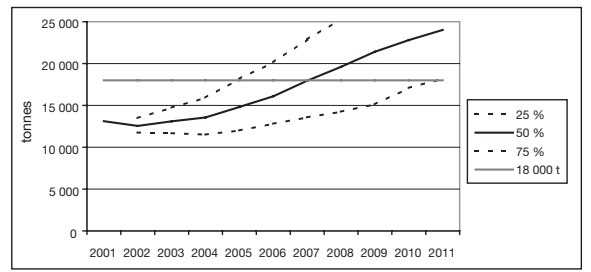

Scénario $9-(0 . / .5 / 1$.) puis TAC 3200 tonnes.

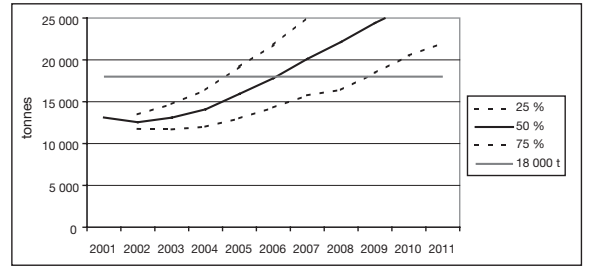

Scénario $11-(0 . / .5 / 1$.$) .$

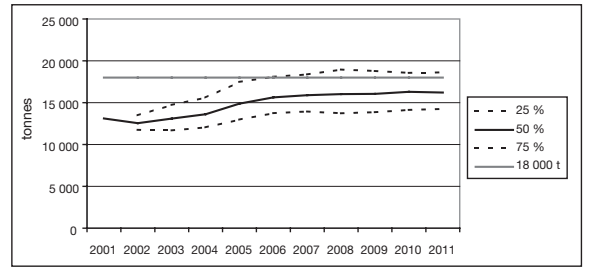

Figure 100. Évolution de la biomasse féconde de la langoustine du golfe de Gascogne selon divers scénarios de gestion.

Statu quo F.

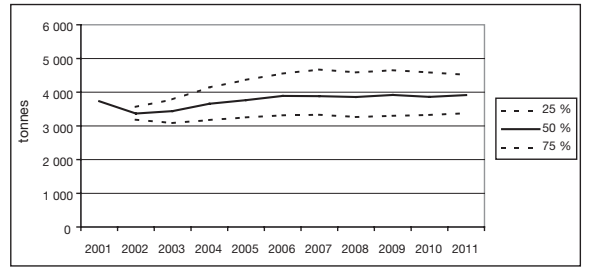

Scénario 2 - Réduction uniforme de F de $20 \%$ $(.8 / .8 / .8)$.

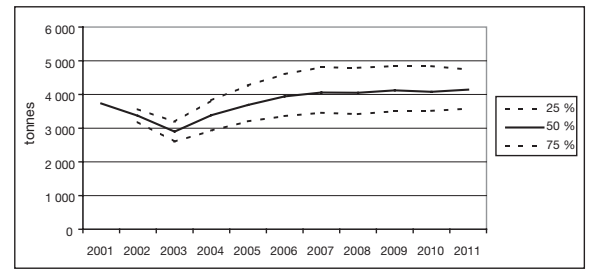

Scénario 4 - Réduction uniforme de F de $50 \%$ $(.5 / .5 / .5)$.

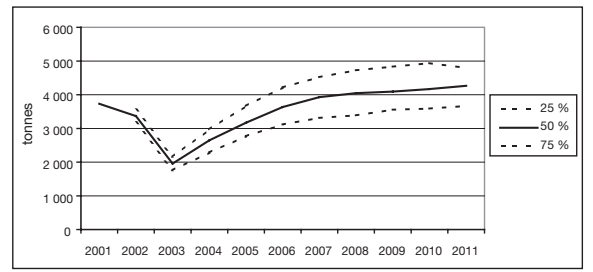

Scénario 1 - Réduction uniforme de F de $10 \%$ (.9/.9/.9).

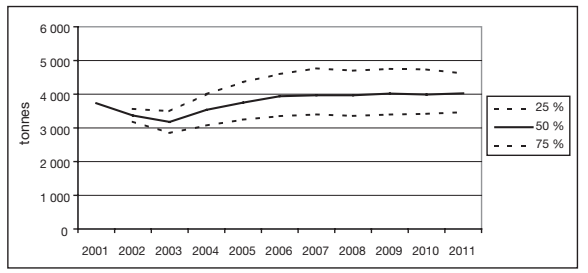

Scénario 3 - Réduction uniforme de F de $30 \%$ (.7/.7/.7).

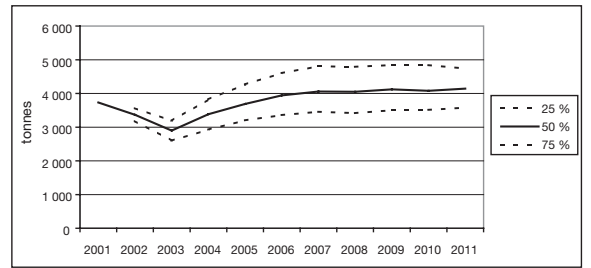

Scénario 5 - Pas de capture âge 1 (0./1./1.).

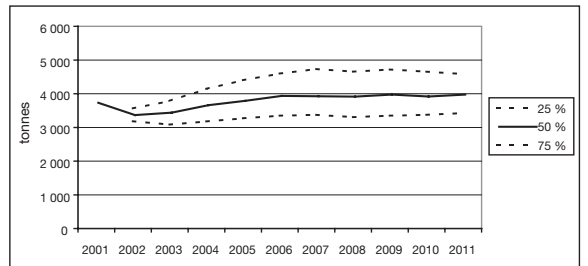


Scénario 6 - Pas de capture âge 1 , réduction $50 \%$ âge $2(0 . / .5 / 1$.$) .$

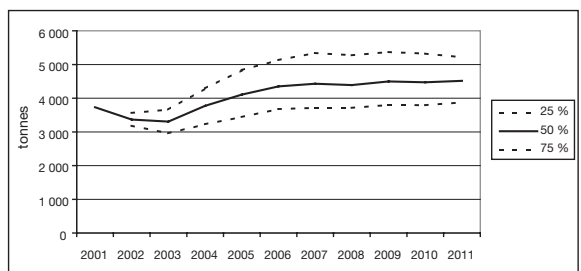

Scénario $8-(0 . / .5 / 1$.$) puis TAC 3000$ tonnes.

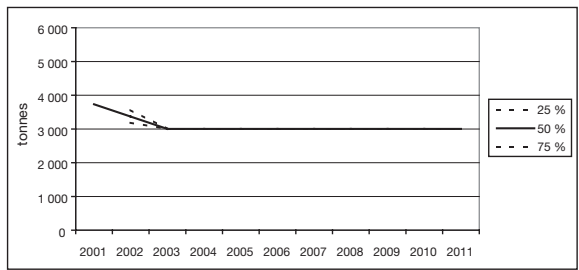

Scénario $10-(0 . / .5 / 1$.$) puis TAC 3500$ tonnes.

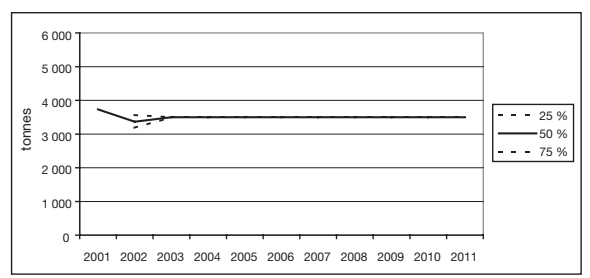

Scénario $9-(0 . / .5 / 1$.$) puis TAC 3200$ tonnes.

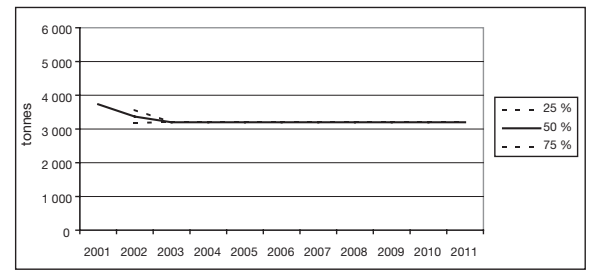

Scénario $11-(0 . / .5 / 1$.$) .$

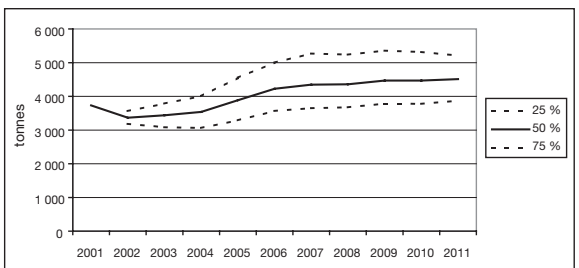

Figure 101. Évolution des débarquements de langoustines du golfe de Gascogne selon divers scénarios de gestion.

\section{Débarquements}

Les résultats sont fournis par le tableau 28 et la figure 101.

Toute mesure de limitation du taux d'exploitation ou d'amélioration du diagramme d'exploitation ne peut que se traduire que par une baisse immédiate des captures. Aussi, pour tous les scénarios qui simulent ce type de mesure, les débarquements à court terme sont inférieurs à ceux du statu quo (sauf le scénario 5 dont on a vu qu'il était très peu efficace).

En cas de diminution du taux d'exploitation seul, le retour des débarquements au niveau statu quo est d'autant plus long et les pertes cumulées d'autant plus fortes que la réduction est importante. Toutefois, seule une diminution d'au moins $30 \%$ permet à la biomasse féconde d'atteindre 18000 tonnes.

Le scénario 6 montre que l'effet de la seule amélioration du profil d'exploitation en vue de protéger les langoustines de moins de $26 \mathrm{~mm}$ Lc entraîne des pertes à court terme relativement faibles, un retour rapide aux débarquements statu quo et des gains à moyen terme importants; toutefois, ce scénario ne permet pas de revenir à une biomasse de 18000 tonnes. 
Tableau 28. Impact (en \% par rapport au statu quo) sur les débarquements annuels et cumulés de différents scénarios de gestion (les débarquements annuels retenus sont les valeurs médianes de la distribution des résultats fournis par les simulations).

\begin{tabular}{|l|c|c|c|c|c|c|c|c|c|c|c|}
\hline Scénarios & 1 & 2 & 3 & 4 & 5 & 6 & 7 & 8 & 9 & 10 & 11 \\
\hline 2003 & -8 & -16 & -24 & -43 & 0 & -4 & -16 & -13 & -7 & 2 & 0 \\
\hline 2004 & -3 & -7 & -13 & -28 & 0 & +3 & -9 & -18 & -13 & -4 & -3 \\
\hline 2005 & 0 & -2 & -5 & -16 & +1 & +9 & -1 & -20 & -15 & -7 & +3 \\
\hline 2006 & +1 & +1 & +1 & -7 & +1 & +12 & +6 & -23 & -18 & -10 & +9 \\
\hline 2007 & +2 & +5 & +5 & +1 & +1 & +14 & +11 & -23 & -18 & -10 & +12 \\
\hline & & & & & & & & & & & \\
\hline $2003-2007$ & -1 & -4 & -7 & -18 & 1 & 7 & -1 & -20 & -14 & -6 & 4 \\
\hline & & & & & & & & & & & \\
\hline Année où Bf > 18000 t. & - & - & 2008 & 2005 & - & - & 2008 & 2006 & 2007 & 2008 & - \\
\hline
\end{tabular}

Le scénario 7, qui couple une diminution de mortalité par pêche et une amélioration du profil d'exploitation, se traduit par des pertes à court terme importantes, des gains à long terme moins élevés que dans le cas du scénario 6, mais il permet une reconstitution de la biomasse aux environs de 18000 tonnes.

Les scénarios qui prévoient une amélioration du diagramme d'exploitation et des TAC constants procèdent d'une autre logique (volonté de limiter les captures pour des raisons commerciales), et entraînent dans tous les cas des pertes à long terme dans la mesure où les TAC simulés sont inférieurs aux débarquements correspondant au statu quo. Il faut rappeler ici que, comme le montre la figure 102, cet objectif implique à terme une diminution du taux d'exploitation (réduction de la capacité de capture et/ou de l'activité des flottilles).

TAC de $3000 \mathrm{t}$

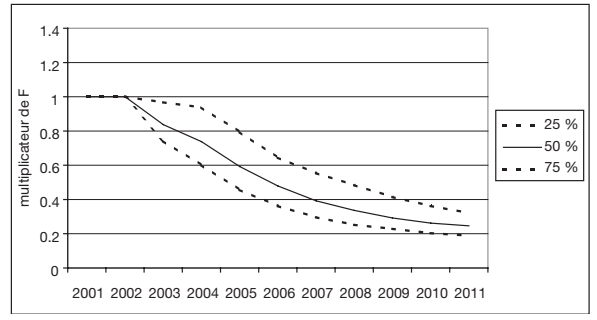

TAC de $3200 t$

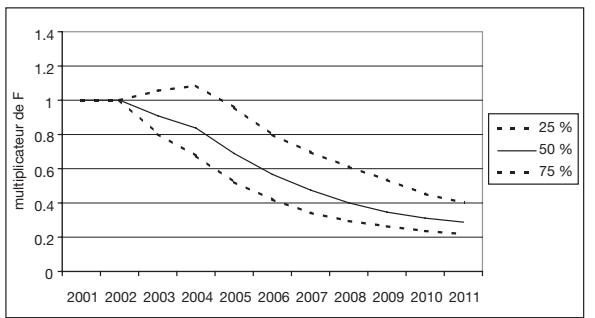

TAC de $3500 t$

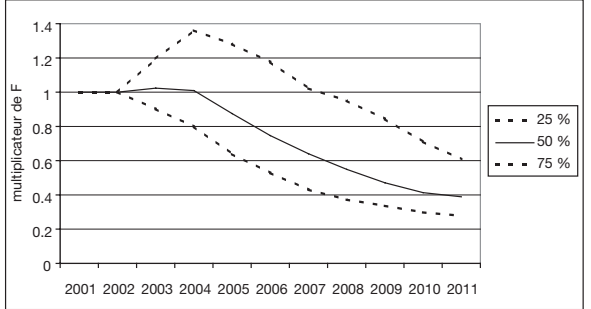

Figure 102. Évolution du multiplicateur global de la mortalité par pêche $(\mathrm{mF})$ pour des scénarios de TAC constant (en plus de l'amélioration du profil de capture). 
En résumé, les scénarios les plus efficaces en termes de reconstitution de la biomasse (scénarios $4,8,9,10,3,7$ ) sont aussi ceux pour lesquels les pertes immédiates et/ou cumulées sont les plus fortes. À l'inverse, ceux qui n'impliquent pas une baisse des débarquements à court terme (scénarios $11,1,2)$ ne permettent pas d'augmentation sensible de la biomasse.

Il s'agit donc de trouver un compromis entre ce qui est souhaitable biologiquement et acceptable économiquement. Les simulations économiques apportent donc un éclairage indispensable avant toute prise de décision politique.

\section{Évolution des indicateurs économiques}

Outre les indicateurs économiques, l'exercice de modélisation développé permet de caractériser l'évolution du nombre de jours de mer déployés par les unités de pêche pour les quatre sous-flottilles mentionnées ci-dessus. Ne sont présentés ici que les résultats pour un navire moyen de la flottille langoustinière. Pour chacun des scénarios, l'activité de la flottille décline du fait du caractère contraignant du TAC. Elle est notamment fortement réduite $(-35 \%)$ dans le cas de l'application du scénario $4(\mathrm{mF}=0,5)$ puis elle se stabilise ensuite à environ 140 jours de mer par navire en moyenne (figure 103 p. XXVI). Les autres scénarios supposent une réduction non négligeable de l'effort nominal de pêche (entre 8 et $17 \%$ en 2003 par rapport à 2002), l'amélioration de l'état du stock impliquant par la suite de réduire le nombre de jours de mer pour stabiliser les débarquements.

La traduction monétaire de cet ajustement est caractérisée par une réduction mécanique des coûts dits variables, comme le carburant ainsi que les taxes de débarquement, évoluant en fonction de la valeur débarquée. Les effets sur les prix liés à une réduction des prises sont limités en raison d'un coefficient d'élasticité prix de la demande relativement faible. Alors que le prix moyen atteint 8,10 euros $/ \mathrm{kg}$, l'écart maximum entre deux scénarios atteint 0,40 euros/ $\mathrm{kg}$ en 2003 pour une différence de production de 1500 tonnes. L'augmentation des prix et la diminution des coûts ne compensent que partiellement la réduction du volume offert comme l'indique la figure 104 p. XXVI.

Les différentes variables suivent des tendances relativement similaires à scénario donné. Le déclin des indicateurs de référence est particulièrement prononcé dans le cas du scénario $4(\mathrm{mF}=0,5)$; il atteint par exemple $42 \%$ pour le salaire brut en 2003 qui cependant se rétablit rapidement pour dépasser les valeurs associées aux autres scénarios en fin de période.

Au cours des dernières années, la situation économique a été relativement satisfaisante pour les navires composant cette flottille à la fois en termes de revenu du travail ou de rémunération du capital investi par l'armement. Le scénario de statu quo ou de TAC égal à 3500 tonnes (scénario 10) permet respectivement d'augmenter et de stabiliser la rémunération des facteurs mais avec une restauration de la biomasse très limitée, particulièrement dans le cas de l'option statu quo. La restriction du TAC à 3000 tonnes (scénario 8) réduit sensiblement le salaire annuel brut moyen pour les équipages de cette flottille ( $27 \mathrm{k} €$ ) ainsi que l'excédent brut d'exploitation. En moyenne, ce scénario permet toujours de financer les coûts financiers ainsi que l'amortissement économique des navires. Cependant, certaines entreprises ayant investi récemment peuvent se trouver fragilisées dans ce contexte et une analyse complémentaire devrait permettre d'affiner cette question. La situation des navires se détériore de manière significative si le scénario 4 est adopté. L'excédent brut d'exploitation reste en moyenne positif, mais la profitabilité des entreprises mesurée par le résultat économique courant devient négative ( $-30 \mathrm{k} €$ en 2003) et le reste durant une période deux années (figures 105 et 106 p. XXVII). Les conditions de viabilité des entreprises ne sont 
donc plus systématiquement assurées pour un grand nombre d'entreprises sauf à ce qu'elles puisent dans leur propre trésorerie.

Au terme de quatre années, pour les scénarios 8 (TAC de 3000 tonnes) et 4 ( $\mathrm{mF}=0,5)$ les situations du stock exprimé en termes de biomasse féconde sont relativement proches. Pour autant, les conditions d'exploitation des entreprises sont très différentes durant cette période de transition. La situation économique des entreprises se détériore fortement dans le cas du scénario 4, ce qui risque d'engendrer la cessation de paiement, puis la sortie de l'activité de pêche d'un grand nombre d'entre elles. Les conditions de transition vers une pêche durable doivent donc être étudiées avec attention de manière à évaluer les conditions de viabilité des entreprises durant ces phases d'ajustement. Cet aspect a notamment été développé dans le cadre des travaux de l'OCDE (OCDE, 2000) sur la transition vers une pêche responsable et en particulier dans le cas de pêcheries de coquilles Saint-Jacques de la baie de Saint-Brieuc (Guyader et Fifas, 2000).

Ces résultats soulignent également qu'il est nécessaire de mener des analyses de risque de manière à identifier l'impact de facteurs aléatoires sur les conditions d'exploitation de la pêcherie. À ce stade, seules les incertitudes sur la taille de la population initiale et sur le recrutement prévu au cours de la période étudiée ont été simulées. Les aléas touchant certains paramètres économiques devront être intégrés ultérieurement. Ils permettraient par exemple d'identifier l'impact de mauvais recrutements successifs qui ralentiraient la restauration du stock et donc le rétablissement de la situation économique des entreprises.

Il est également utile du point de vue de l'analyse économique d'évaluer les résultats économiques cumulés sur la période retenue. À cette fin, un exercice d'actualisation, qui revient à transformer une valeur future en valeur présente équivalente par le biais d'actualisation traduisant la préférence d'une collectivité pour le présent, a été mené. Les différents scénarios peuvent alors être comparés du point de vue d'indicateurs de performance et sur une période donnée (tableau 29).

Tableau 29. Résultats de l'actualisation sur quelques indicateurs clés en fonction de différents scénarios de gestion du stock de langoustines du golfe de Gascogne.

\begin{tabular}{|l|c|c|c|c|c|c|c|c|c|}
\hline $\begin{array}{l}\text { Taux } \\
\text { d'actualisation }\end{array}$ & \multicolumn{3}{|c|}{$2 \%$} & \multicolumn{3}{c|}{$10 \%$} & \multicolumn{3}{c|}{$15 \%$} \\
\hline $\begin{array}{l}\text { En millions } \\
\text { d'euros }\end{array}$ & EBE & $\begin{array}{c}\text { Salaires } \\
\text { bruts }\end{array}$ & Total & EBE & $\begin{array}{c}\text { Salaires } \\
\text { bruts }\end{array}$ & Total & EBE & $\begin{array}{c}\text { Salaires } \\
\text { bruts }\end{array}$ & Total \\
\hline Statu quo & 103 & 40 & 143 & 83 & 32 & 115 & 73 & 28 & 102 \\
\hline TAC 3 500 tonnes & 87 & 36 & 123 & 69 & 29 & 99 & 61 & 26 & 87 \\
\hline TAC 3 3000 tonnes & 69 & 32 & 101 & 55 & 26 & 81 & 49 & 23 & 72 \\
\hline mF $=0,5$ & 74 & 33 & 107 & 57 & 26 & 83 & 49 & 23 & 71 \\
\hline
\end{tabular}

Quel que soit le niveau de préférence, le scénario de statu quo produit les résultats économiques les plus élevés en termes de rémunération des moyens de production mobilisés sur la période 2003-2007. Le rationnement du TAC à 3500 tonnes limite les bénéfices attendus par rapport à la situation de statu quo, puis le classement entre les deux derniers scénarios évolue en fonction du taux d'actualisation retenu. Si la préférence pour le présent est faible (taux d'actualisation de $2 \%$ ), les gestionnaires devraient alors privilégier le scénario $4(\mathrm{mF}=0,5)$ par rapport à un TAC de 3000 tonnes. Le choix devrait s'inverser pour un taux d'actualisation proche de $15 \%$. 


\section{Discussion - conclusion}

La problématique des conditions d'exploitation de la langoustine dans le golfe de Gascogne n'est pas nouvelle, et toutes les études précédentes ont montré que l'amélioration de la sélectivité des chaluts des langoustiniers aurait à terme un impact positif non seulement sur le stock de langoustines lui-même, mais aussi sur celui du merlu qui constitue une capture accessoire importante. L'approche bioéconomique développée ici a permis de mieux évaluer l'impact de différents scénarios de gestion de la pêcherie de langoustines du golfe de Gascogne à la fois en terme d'évolution du stock et d'indicateurs de performance des entreprises. Le modèle de simulation reste très simple au moins du point de vue de la composante économique. Il peut être qualifié de statique dans la mesure où il n'intègre pas ou peu les possibilités de réactions des pêcheurs à leur environnement économique, réglementaire, etc. Cet aspect devra être développé, notamment de manière à intégrer les possibilités de substitution entre pêcheries.

Dans les conditions actuelles de l'exploitation, les rejets de langoustines sont importants, et leur impact sur les potentialités de production du stock est fort. Les simulations réalisées montrent que ce sont les langoustines de moins de $26 \mathrm{~mm}$ de longueur céphalothoracique qui doivent être épargnées, alors qu'elles sont actuellement largement capturées puis rejetées. Pour reprendre un slogan de la profession elle-même, le tri doit se faire sur le fond et non sur le pont. Les travaux en cours sur la mise au point d'une grille sélective permettent de penser qu'un tel objectif est à portée de main. On notera aussi que les nombreuses observations réalisées à bord des langoustiniers montrent une forte variabilité spatio-temporelle de la distribution des rejets de langoustines. L'amélioration du diagramme d'exploitation de la langoustine n'est donc pas qu'une question technique (amélioration de la sélectivité) mais peut aussi relever de choix de stratégies de pêche visant à éviter les périodes et les zones où la petite langoustine est abondante.

À ce problème de captures de jeunes langoustines (surexploitation de croissance), est venue s'ajouter une mortalité par pêche excessive, non soutenable à long terme dans les conditions actuelles d'exploitation et qui a entraîné une baisse progressive de la biomasse des reproducteurs. Cette situation peut surprendre, dans la mesure où le nombre de langoustiniers a eu tendance à diminuer; elle est en fait le résultat de l'amélioration de l'efficacité de la flottille au travers du progrès technique tant au niveau du navire lui-même que du train de pêche et qui compense au moins pour partie la diminution du nombre de navires. Ainsi, selon des informations préliminaires, les gains d'efficacité d'un langoustinier par adoption de chaluts jumeaux seraient de l'ordre de 30 à $50 \%$. Faute de disposer des données nécessaires, ces gains ne sont pas pris en compte dans la plupart des évaluations de stocks, ce qui est actuellement reconnu comme une des causes majeures de surestimation de l'abondance des stocks et de sous-estimation de la mortalité par pêche: contrairement à une idée largement répandue, les diagnostics scientifiques sur l'état des ressources et des pêcheries sont souvent plutôt « optimistes » par rapport à la réalité.

Sous l'effet conjugué de ces deux causes, depuis plusieurs années, le stock de langoustines du golfe de Gascogne montre des signes de surexploitation. En l'absence d'objectif de gestion explicite, le CIEM a proposé des scénarios permettant la reconstitution de la biomasse. Suite aux rencontres entre gestionnaires, professionnels et scientifiques, d'autres scénarios ont été simulés, et leurs conséquences biologiques et économiques ont été présentées ici.

Si le seul objectif est une restauration de la biomasse au niveau élevé des années quatre-vingt-dix, plusieurs voies sont possibles: réduction de la mortalité par pêche (ce 
qui suppose la maîtrise du développement du progrès technique), amélioration du diagramme d'exploitation, combinaison des deux types de mesures. Les simulations réalisées montrent qu'une restauration de la biomasse aux environs de 18000 tonnes à moyen terme suppose à la fois une protection accrue des petites langoustines et une diminution du taux d'exploitation; cette dernière peut être obtenue au travers d'une diminution des capacités de captures (réduction de la flottille) et d'une réduction de leur activité (nombre de jours de pêche).

En complément d'une amélioration du diagramme d'exploitation de cette pêcherie, d'autres objectifs ont également été évoqués par les gestionnaires et les professionnels, en particulier la limitation des débarquements, au travers d'un TAC relativement bas par rapport aux captures historiques. Les résultats obtenus ici montrent que, conjugués avec une amélioration du diagramme d'exploitation de la langoustine, des TAC compris entre 3000 tonnes et 3500 tonnes assurent une reconstitution de la biomasse des reproducteurs à moyen terme.

Ces résultats permettent aussi de mettre en évidence que des objectifs qui viseraient au maintien d'un TAC fixe et d'une mortalité par pêche constante ne seraient pas compatibles : à terme, le maintien des débarquements aux environs de 3200 tonnes/an nécessiterait un ajustement de l'effort de pêche, au travers de la limitation des capacités de capture des flottilles ou de leur activité.

Enfin, ces analyses montrent également que la réduction de $50 \%$ de la mortalité par pêche n'est pas viable économiquement pour les unités de pêche ciblant la langoustine. Certains scénarios identifiés comme répondant aux objectifs de gestion durable des stocks ne permettent donc pas une transition soutenable pour les entreprises impliquées dans la pêcherie. Cela ne signifie pas pour autant que la restauration du stock soit incompatible à moyen terme avec des objectifs d'amélioration de la performance économique des flottilles, mais les gestionnaires devront privilégier des scénarios permettant à la fois de maintenir la viabilité économique des entreprises de pêche et de reconstitution la biomasse.

Il convient également de mettre l'accent, sur les modalités de mise en œuvre éventuelle de ces scénarios dans cette pêcherie plurispécifique et en particulier sur les possibilités de réaction stratégique des pêcheurs. En effet, sans une gestion adéquate, les scénarios visant à fixer un $\mathrm{TAC}$ annuel à un faible niveau pourraient conduire à une fermeture précoce de la pêcherie dans la saison et être accompagnées, en l'absence d'encadrement, d'un certain nombre d'effets pervers bien identifiés (OCDE, 1997). Premièrement, dans ce type de situation, chaque pêcheur cherche à réaliser le maximum de captures avant que la pêcherie ne ferme. Cela se traduit par un renforcement de l'activité des flottilles, un accroissement de l'efficacité des unités de pêche au travers de l'innovation technique, et donc un développement de l'effort de pêche. Deuxièmement, la concentration des débarquements sur une période limitée peut générer des phénomènes de goulots d'étranglements associés à une chute des prix ou encore déstabiliser certains circuits de commercialisation des produits pêchés. Enfin, il a été souligné que les pêcheries langoustinières sont plurispécifiques et que les captures accessoires d'autres espèces constituent un élément important du chiffre d'affaire. Si les possibilités de redéploiement vers d'autres zones de pêche sont limitées, l'interdiction de débarquement de langoustines après que le quota soit atteint se traduira par des rejets importants de cette espèce, et ne contribuera pas à l'objectif de réduction de la mortalité par pêche réelle sur ce stock. 
Quel que soit l'objectif de gestion retenu (limitation directe du taux d'exploitation ou fixation d'un TAC à un niveau relativement bas), sa réalisation suppose donc la mise en œuvre d'instruments de régulation complémentaires à l'échelle des unités de production visant à contrôler soit leurs moyens de production soit leur production. Dans le premier cas, cela suppose de mettre en place un système de numerus clausus associé à une régulation du nombre de jours de pêche autorisés par navire, éventuellement associé à un calendrier de pêche. Les simulations réalisées ici montrent que l'adoption de ce système devrait conduire à diminuer le nombre de jours de pêche autorisés pour les prochaines années. La seconde option est d'allouer des quotas individuels aux unités de pêche, chaque quota étant une part du TAC de langoustines défini suivant une clé et des modalités d'allocation qui resteraient à préciser. Il reste à évaluer les avantages et inconvénients de ces options pour en faire une analyse comparative. Toutefois, la mise en œuvre de tels instruments est nécessaire pour limiter les effets négatifs, à la fois sur un plan biologique et économique, de la seule application d'un TAC.

\section{Comparaison entre la pêcherie chalutière langoustinière du golfe de Gascogne et celle au casier à langoustines de Loch Torridon en Écosse}

\section{Introduction}

Le coût social élevé des rejets et les limites des mesures d'amélioration de la sélectivité proposées depuis plusieurs décennies (notamment faible effectivité et résolution imparfaite des problèmes de productions jointes), nous amènent à analyser le cas d'une technique de pêche alternative, le casier, comme solution permettant de diminuer les productions jointes et donc les effets externes négatifs qu'elles peuvent engendrer. Selon l'utilisation qui en est faite, le casier peut être un engin parfaitement sélectif permettant de cibler les catégories voulues, donc d'éviter les productions jointes. La gestion est dès lors facilitée. Les solutions théoriques de gestion des externalités négatives basées sur des droits de propriété deviennent applicables.

L'analyse des conditions de rentabilité et des incitations au changement technique, à l'adoption du casier, s'appuie sur l'exemple de la pêcherie langoustinière au casier de Loch Torridon (Écosse), pêcherie éco-certifiée par le Marine StewardShip Council (MSC) que nous présentons dans cette section. Nous comparons la pêcherie langoustinière au casier de Loch Torridon et la pêcherie langoustinière au chalut du golfe de Gascogne du point de vue de leur système institutionnel et de gestion, de leurs captures par unité d'effort et prix par catégorie et de leurs performances économiques.

\section{Description de la flottille langoustinière au casier de Loch Torridon}

La langoustine est la principale espèce capturée en valeur en Écosse où elle représente un chiffre d'affaires de 56 millions d'euros en 2005 (32 millions d'euros pour l'églefin, deuxième espèce en valeur). Les principales pêcheries écossaises sont situées dans les zones de Firth of Forth, Moray Firth, nord et sud de Minch, l'estuaire de Clyde, le Fladen Ground et le centre nord de la mer du Nord. La plupart des langoustines sont capturées par des chalutiers $(90 \%$ des captures totales en poids effectuées par des chalutiers hauturiers 
opérant dans la zone centrale de mer du Nord et des chalutiers côtiers) mais la pêche au casier à langoustines s'est développée ces dernières années en particulier sur la côte ouest où la pêche au casier représente dans certaines zones jusqu'à $20 \%$ des captures de langoustines en poids et $40 \%$ en valeur. Dans l'ouest de l'Éosse, on observe une tendance au passage du chalut au casier, les casiers permettant la capture de langoustines de plus forte valeur et engendrant des consommations de carburant moindres ${ }^{161}$.

Les caseyeurs exploitent essentiellement les stocks de langoustines côtiers généralement inaccessibles aux chalutiers (soit pour des raisons de restrictions réglementaires soit parce que les fonds ne s'y prêtent pas). Des propriétés sélectives des engins et du changement de comportement des langoustines en présence de casiers résultent des structures de captures différentes entre les deux engins de pêche, casier et chalut. Les chalutiers capturent les plus jeunes individus dont une proportion importante est sous la taille minimale de débarquement $(20 \mathrm{~mm}$ ) et les caseyeurs capturent des individus de plus grande taille et de valeur unitaire plus élevée destinés à l'exportation de langoustines vivantes.

La pêcherie langoustinière au casier de Loch Torridon est située sur la côte nordouest de l'Écosse, dans le Loch Torridon et la zone fermée du détroit intérieur de Rona (figure 107 p. XXVIII). La flottille d'origine comptait seulement 3 navires et s'est développée dans les années quatre-vingt-dix. Depuis 2003, environ 13 navires ciblent la langoustine dans la zone fermée au chalutage depuis 2001 de Loch Torridon. Ce sont des navires d'une taille moyenne de 9,5 mètres. La flottille de Loch Torridon compte huit navires de moins de 10 mètres et cinq navires de plus de 10 mètres. Cinq navires sont des catamarans d'une largeur d'environ 5 mètres et d'une puissance moyenne de $200 \mathrm{~kW}$. L'effectif par navire est en moyenne de deux personnes. La pêche au casier à langoustines implique donc 20 à 30 pêcheurs dans cette zone. Six navires sont basés à Ardeslaig où se trouve la coopérative chargée du conditionnement et de l'exportation des langoustines Shieldaig Export et 7 autres navires opèrent depuis le port d'Applecross et depuis les autres ports de Loch Torridon et livrent les langoustines à Ardeslaig par camion.

La méthode de pêche est traditionnelle, les langoustines sont pêchées à l'aide de casiers contenant des appâts et déployés sur des lignes d'une centaine de casiers. Les casiers reposent sur le fond à environ 200 mètres de profondeur. Les appâts sont du hareng congelé (fournis par les chalutiers pélagiques de façon irrégulière) puis trempés dans une saumure pour ne pas attirer d'autres prédateurs que les langoustines comme c'est le cas lorsque le poisson est frais. Le maquereau est également parfois utilisé. Les casiers sont laissés au moins un jour sur les fonds habituellement deux jours et plus longtemps s'ils sont mis en pêche avant le week-end.

L'essentiel de l'activité de ces navires est le casier à langoustines, pratiqué toute l'année, cinq jours sur sept avec cependant une limite de 200 jours de mer autorisés. Quelques navires pratiquent également le casier à crabes mais de façon très occasionnelle. Un navire capture de 8 à 14 tonnes de langoustines par an pour une valeur de 120000 euros. La flottille débarque 100 à 150 tonnes de langoustines chaque année. Outre la dimension traditionnelle de la pratique du métier de casier à langoustines, la fermeture au chalutage de la zone de Loch Torridon à partir de 2001 et l'écolabel MSC en 2003 ont favorisé le développement de ce métier.

${ }^{161}$ Kirkland, Atkinson and Smith, 2007. Nephrops behaviour and ecology in relation to the commercial creel fishery. 


\section{État du stock de Loch Torridon}

La zone de Loch Torridon fait l'objet de collectes de données spécifiques. Les campagnes conduites par le FRS d'Aberdeen (Fisheries Research Service) donnent des informations sur l'abondance de la langoustine dans le loch Torridon et dans la zone interdite au chalutage. Des données de fécondité, recrutement et facteurs responsables de la mortalité naturelle sont récoltées lors des campagnes conduites par le FRS d'Aberdeen qui échantillonne également les rejets chaque trimestre. L'abondance et la densité de la population du Loch Torridon sont estimées grâce à des données de vidéo sous-marine de comptage de terriers de langoustine. Les données de débarquements déclarées dans les logbooks par les navires de plus de dix mètres et les débarquements journaliers des navires de moins de dix mètres transmis chaque semaine aux Fishery Officers permettent d'analyser les débarquements par unité d'effort. Des données de débarquements par unité d'effort sont disponibles depuis 1990. Par ailleurs, Shieldaig Export Limited enregistre très précisément toutes les données de débarquement et dispose d'informations journalières par navire sur les débarquements par classe de taille sur lesquelles se base la rémunération des marins. Des échantillonnages des débarquements de caseyeurs du North Minch contenant le Loch Torridon permettent une analyse des données de composition en taille. La série de données n'est cependant pas suffisante pour mener une analyse de cohortes standard pour le Loch Torridon. Une collaboration entre le Scottish Natural Heritage et le FRS Aberdeen évalue les effets du plan de gestion de Torridon: les données récoltées dans ce cadre suggèrent une augmentation de l'abondance du stock en 2005-2006. Des analyses de cohortes en longueur menées dans le cadre de la thèse de Adley (2007) montrent que le stock de Loch Torridon présente moins de risques de surexploitation de croissance que lorsqu'il avait été évalué en 1996-1998. Les campagnes de surveillance vidéo suggèrent les mêmes tendances.

\section{Comparaison des systèmes institutionnels et de gestion des deux pêcheries}

\section{Organisations institutionnelles}

La gestion de la pêcherie de Loch Torridon relève en partie de l'Union européenne (UE) par l'intermédiaire de la Politique commune de pêche (PCP) qui détermine la gestion globale en fixant les mesures techniques et les quotas pour la pêcherie. Dans ce cadre-là, les mesures de conservation (EC) $n^{\circ} 850 / 98$ sur les mesures techniques, les mesures de contrôle (EC) $\mathrm{n}^{\circ} 2847 / 93$ sur les logbooks et déclarations de captures et d'effort et les mesures de TAC et quotas sont en vigueur dans la pêcherie. Le Parlement écossais, par le biais d'un concordat sur un sujet spécifique avec le ministère britannique de l'Environnement, de l'Agriculture et des Affaires rurales (DEFRA), fait appliquer les lois (allocations de quotas, attribution des licences) dans les eaux écossaises par l'intermédiaire du ministère écossais de l'Environnement et des Affaires rurales (SEERAD, Scottish Executive Environment and Rural Affairs Department). Le département des pêches du DEFRA (SEERAD) gère les quotas via les organisations de producteurs selon les règles de gestion des quotas de la PCP. Depuis janvier 1999, les quotas sont distribués selon une allocation fixe de quota (FQA). Le quota de langoustines est ainsi distribué entre les organisations de producteurs (WSFFPO, SFO), les navires de moins de dix mètres et les navires hors organisations de producteurs ou dont l'organisation de producteurs n'est pas bénéficiaire de quotas. Les navires britanniques opérant dans les eaux territoriales écossaises s'exposent à la législation écossaise mise en place par le Parlement en vertu de la loi sur la pêche 
côtière en Écosse (Inshore Fishing (Scotland) Act 1984). Cette loi permet au Parlement écossais de réguler ou interdire certaines activités de pêche.

Les navires de moins de 10 mètres ont une licence délivrée par le Parlement écossais. Depuis le $1^{\text {er }}$ janvier 2000, tout navire de moins de 10 mètres pêchant plus de $12 \mathrm{~kg}$ de langoustines dans les zones IIa, IV, VI, VII doit déclarer ses débarquements. Des limites de captures mensuelles sont par ailleurs en vigueur. Les navires de plus de 10 mètres doivent remplir les logbooks en accord avec la mesure (EC) $n^{\circ} 2847 / 93$. Des mesures de maillage minimal et taille minimale sont également en vigueur.

En 2001, le Parlement écossais a confié la gestion à la communauté de Loch Torridon. Le plan de gestion définit trois zones de pêche. Les pêcheurs qui prennent part à la pêcherie de langoustines au casier du Loch Torridon pêchent conformément au plan de gestion volontaire mis en place à l'intérieur de la « zone fermée » par le Torridon Nephrops Management Group (TNMG) qui s'assure également que toutes les informations nécessaires à la certification sont récoltées efficacement et réfléchit à l'avenir de la pêcherie (figure 108). Le Scottish Natural Heritage contribue également à la gestion de la zone. La gestion de la pêcherie repose essentiellement sur une base responsable et volontaire. Les mesures dictées par le code n'ont aucune valeur légale. La désapprobation collective dans cette petite communauté sert de garantie de respect des règles. La coopérative Shieldaig Export Limited créée en 1996 par les pêcheurs de Loch Torridon joue également un rôle de contrôle et de taxation dans la gestion de la pêcherie. L'entreprise se réserve le droit de donner des avertissements aux navires qui ne respectent pas le code de conduite. Elle peut refuser leurs débarquements s'ils ne sont pas conformes aux engagements du code. Il s'agit d'une structure organisationnelle particulière chargée de l'exportation des langoustines vivantes. Elle fonctionne comme une coopérative, chaque pêcheur prenant des parts égales. L'entreprise s'occupe de la recherche de marchés, du contrôle du vivier et du transport des langoustines vers les marchés européens. Une meilleure stabilité des prix et des marchés sécurisés sont

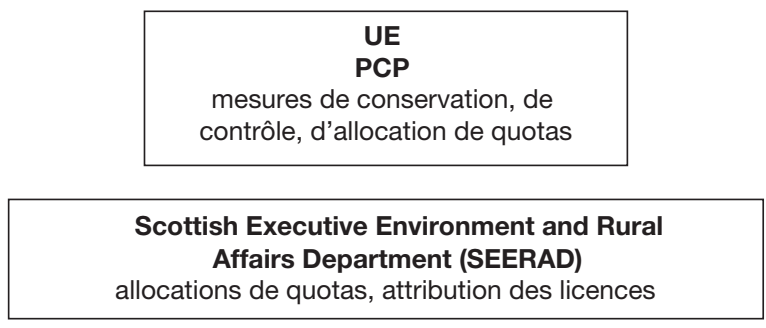

\section{Torridon Nephrops}

Management Group (TNMG)

plan de gestion volontaire mis en

place à l'intérieur de la « zone

fermée » gestion par le Scottish

Natural Heritage

Coopérative Shieldaig
Export Limited
rôle de contrôle et
taxation dans la gestion
de la pêcherie

Ecolabel Marine Stewardship Council

Moody Marine Limited, organisme certificateur

\begin{tabular}{l}
\hline $\begin{array}{l}\text { Organisations } \\
\text { de producteurs } \\
\text { WSFFPO, SFO }\end{array}$ \\
\hline $\begin{array}{l}\text { Highlands \& Islands } \\
\text { Fishermen's, rôle de } \\
\text { représentation des pêcheurs } \\
\text { et prospection technique }\end{array}$ \\
\hline
\end{tabular}

Figure 108. Organisation institutionnelle de la pêcherie langoustinière au casier de Loch Torridon (source - C. Macher). 


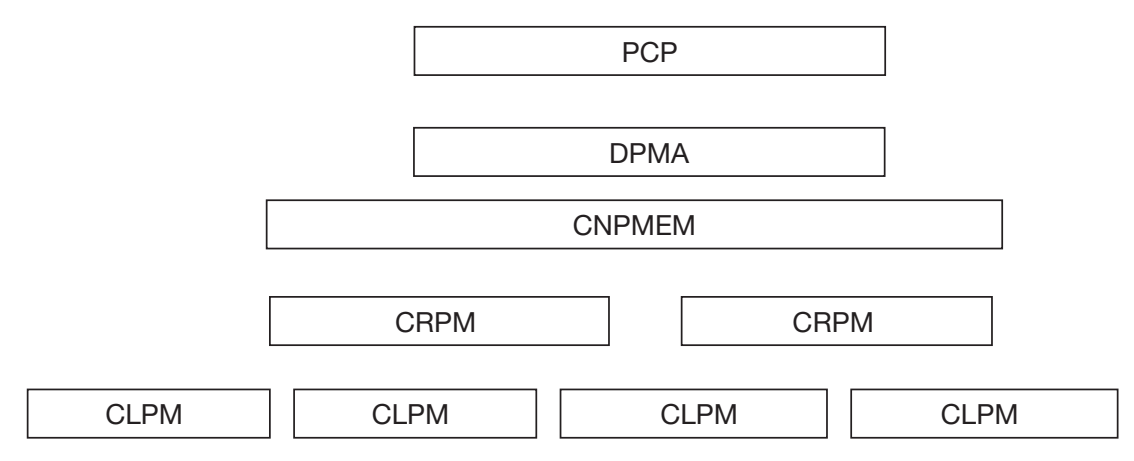

Organisations de producteurs

Figure 109. Organisation institutionnelle de la pêcherie chalutière langoustinière du golfe de Gascogne (source : C. Macher).

ainsi assurés. Highlands \& Islands Fishermen's Association joue le rôle de représentation des pêcheurs et de prospection technique (mise au point des fenêtres d'échappement). En janvier 2003, la pêcherie a obtenu une éco-certification en satisfaisant aux critères du Marine Stewardship Council qui portent essentiellement sur l'état d'exploitation du stock, les effets de la pêche sur l'écosystème, et le système de gestion de la pêcherie. Le processus d'évaluation mené par l'organisme certificateur Moody Marine Limited Centre for Marine and Coastal Studies avait débuté en janvier 2002. Malgré le coût élevé de la certification, celle-ci a apporté une reconnaissance internationale de bonne gestion et de durabilité.

Dans le cas de la pêcherie langoustinière du golfe de Gascogne comme dans le cas de la pêcherie au casier de Loch Torridon, un certain nombre de réglementations relève des règlements de la Commission européenne dont les mesures de conservation (EC) $\mathrm{n}^{\circ} 850 / 98$ sur les mesures techniques, les mesures de contrôle (EC) n ${ }^{\circ} 2847 / 93$ sur les logbooks et les mesures de TAC et quotas. La Commission européenne délivre également les permis de pêche spéciaux, licences d'accès aux stocks situés hors des eaux territoriales et dans la ZEE (entre les 12 et les 200 milles nautiques). La PCP est relayée au niveau national par la direction des pêches maritimes et de l'aquaculture (DPMA) du ministère de l'Agriculture et de la Pêche.

La gestion repose également sur des organisations professionnelles nationales (figure 109). Ainsi une commission " langoustine » rattachée au Comité national des pêches maritimes et des élevages marins (CNPMEM) décide avec les représentants professionnels des conditions techniques (longueur des bateaux, maillages, taille réglementaire, dispositifs sélectifs, etc.) pour l'attribution des permis de pêche spéciaux attribués chaque année. Deux cent vingt-huit permis de pêche spéciaux pour la langoustine du golfe de Gascogne ont été attribués en octobre 2004 aux chalutiers ayant pêché individuellement au moins 2 tonnes de langoustines par an. Au niveau régional et local, les comités régionaux et locaux des pêches (CRPMEM et CLPMEM) peuvent renforcer les conditions fixées par la commission « langoustine».

Au niveau national, cinq organisations de producteurs (OP) se partagent le quota français de langoustines du golfe de Gascogne (Opob, From Bretagne, Proma, Socosama, et l'OP de La Cotinière). Elles sont responsables de la part de quota qui leur est allouée et libres d'organiser l'étalement de la consommation du quota au long de l'année. 


\section{Mesures techniques de conservation}

Tableau 30. Comparaison des mesures techniques de conservation

\begin{tabular}{|c|c|c|}
\hline Mesures techniques & $\begin{array}{l}\text { Pêcherie langoustinière au } \\
\text { casier de Loch Torridon }\end{array}$ & $\begin{array}{c}\text { Pêcherie chalutière langoustinière } \\
\text { du golfe de Gascogne }\end{array}$ \\
\hline $\begin{array}{l}\text { Sélectivité, restriction } \\
\text { de maillage }\end{array}$ & & $\begin{array}{l}\text { Maillage de } 70 \mathrm{~mm} \text { dans le cul } \\
\text { de chalut, maillage de } 100 \mathrm{~mm} \\
\text { dans le box merlu }\end{array}$ \\
\hline Dispositif sélectif & $\begin{array}{l}\text { Sur une base volontaire: } \\
\text { fenêtre d'échappement dont } \\
\text { l'espacement est de } 22 \mathrm{~mm}\end{array}$ & $\begin{array}{l}\text { Obligatoire pour le PPS : panneau } \\
\text { à mailles carrées pour favoriser } \\
\text { l'échappement du merlu } \\
\text { Sur une base volontaire: grille } \\
\text { à langoustine d'espacement entre } \\
\text { barreaux } 13 \mathrm{~mm} \text { ou } 15 \mathrm{~mm}\end{array}$ \\
\hline $\begin{array}{l}\text { Restriction de pêche } \\
\text { de certaines espèces }\end{array}$ & Rejet des femelles grainées & \\
\hline $\begin{array}{l}\text { Pourcentages autorisés } \\
\text { de l'espèce cible } \\
\text { et des captures accessoires }\end{array}$ & & \\
\hline \multicolumn{3}{|l|}{ Restrictions sur les rejets } \\
\hline $\begin{array}{l}\text { Taille minimale } \\
\text { de débarquement }\end{array}$ & $\begin{array}{l}\text { Obligatoire: } 25 \mathrm{~mm} \\
\text { Appliquée: } 28 \mathrm{~mm} \\
\text { Mais la fenêtre } \\
\text { d'échappement permet } \\
\text { des captures }>40 \mathrm{~mm}\end{array}$ & $\begin{array}{l}\text { Obligatoire (CE) : } 20 \mathrm{~mm} \\
\text { Appliquée par les OP : } 28 \mathrm{~mm}\end{array}$ \\
\hline TAC et quotas & $\begin{array}{l}\text { Quotas (100 à } 150 \text { tonnes) } \\
\text { sur le quota global } \\
\text { de langoustines de la zone } \\
\text { de gestion C dans la sous- } \\
\text { zone CIEM VIa } \\
\end{array}$ & $\begin{array}{l}\text { Quota }=94 \% \text { TAC } \\
\text { zone CIEM VIIIab* }\end{array}$ \\
\hline $\begin{array}{l}\text { Limitation du nombre } \\
\text { de navires }\end{array}$ & Pas de numerus clausus & Numerus clausus : 250 navires \\
\hline $\begin{array}{l}\text { Limitation du nombre de jours } \\
\text { de mer, du temps de pêche }\end{array}$ & $\begin{array}{l}200 \text { jours de pêche par an } \\
\text { autorisés }\end{array}$ & \\
\hline $\begin{array}{l}\text { Limitation du nombre } \\
\text { d'engins de pêche }\end{array}$ & $\begin{array}{l}2 \text { jeux de } 800 \text { casiers } \\
\text { par navire de deux hommes }\end{array}$ & \\
\hline $\begin{array}{l}\text { Limitation du nombre } \\
\text { d'engins virés par jour }\end{array}$ & 1 jeu relevé par jour & \\
\hline Restriction sur les gréements & $\begin{array}{l}\text { Espacement obligatoire entre } \\
\text { les casiers: } 16 \text { mètres }\end{array}$ & \\
\hline Période de pêche & Toute l'année & Toute l'année \\
\hline $\begin{array}{l}\text { Restriction de pêche } \\
\text { dans certaines zones } \\
\text { ou à certaines périodes } \\
\text { de l'année et restriction } \\
\text { de l'utilisation de certains } \\
\text { engins }\end{array}$ & $\begin{array}{l}\text { Zone de pêche interdite } \\
\text { au chalutage toute l'année, } \\
\text { et autre zone de pêche } \\
\text { interdite au chalutage } \\
6 \text { mois par an, interdiction } \\
\text { de la drague toute l'année }\end{array}$ & $\begin{array}{l}\text { Chalutage interdit dans la zone } \\
\text { des } 3 \text { milles }\end{array}$ \\
\hline
\end{tabular}

* Le quota français de langoustines en 2004 était de 2961 tonnes pour la zone CIEM VIIIab. 


\section{Systèmes de gestion}

Les deux systèmes de gestion sont comparés en s'appuyant sur la typologie des mesures de gestion des pêcheries de Boncœur et al. (2003) et complétée par Guyader et al. (2005).

On distingue donc deux types de mesures selon l'objectif qu'elles poursuivent:

- les mesures techniques (lato sensu) visant à la conservation de la productivité du stock (tableau 30),

- les mesures de régulation de l'accès visant à allouer la capacité productive limitée du stock entre les entreprises de pêche (tableau 31).

Les mesures de régulation de l'accès sont classées suivant la variable de contrôle sur laquelle elles portent (effort ou captures) et la méthode de contrôle de laquelle elles relèvent, méthode économique basée sur les incitations ou méthode administrative (command and control).

Les mesures techniques relèvent essentiellement d'une gestion administrative mais peuvent être accompagnées d'incitations économiques à l'adoption d'engins sélectifs par exemple par éco-étiquettage des produits capturés par un engin plus sélectif ou taxation des captures de l'engin non sélectif.

Mesures de régulation de l'accès: allocation des capacités productives du stock entre les entreprises de pêche

Tableau 31. Comparaison des mesures de régulation de l'accès.

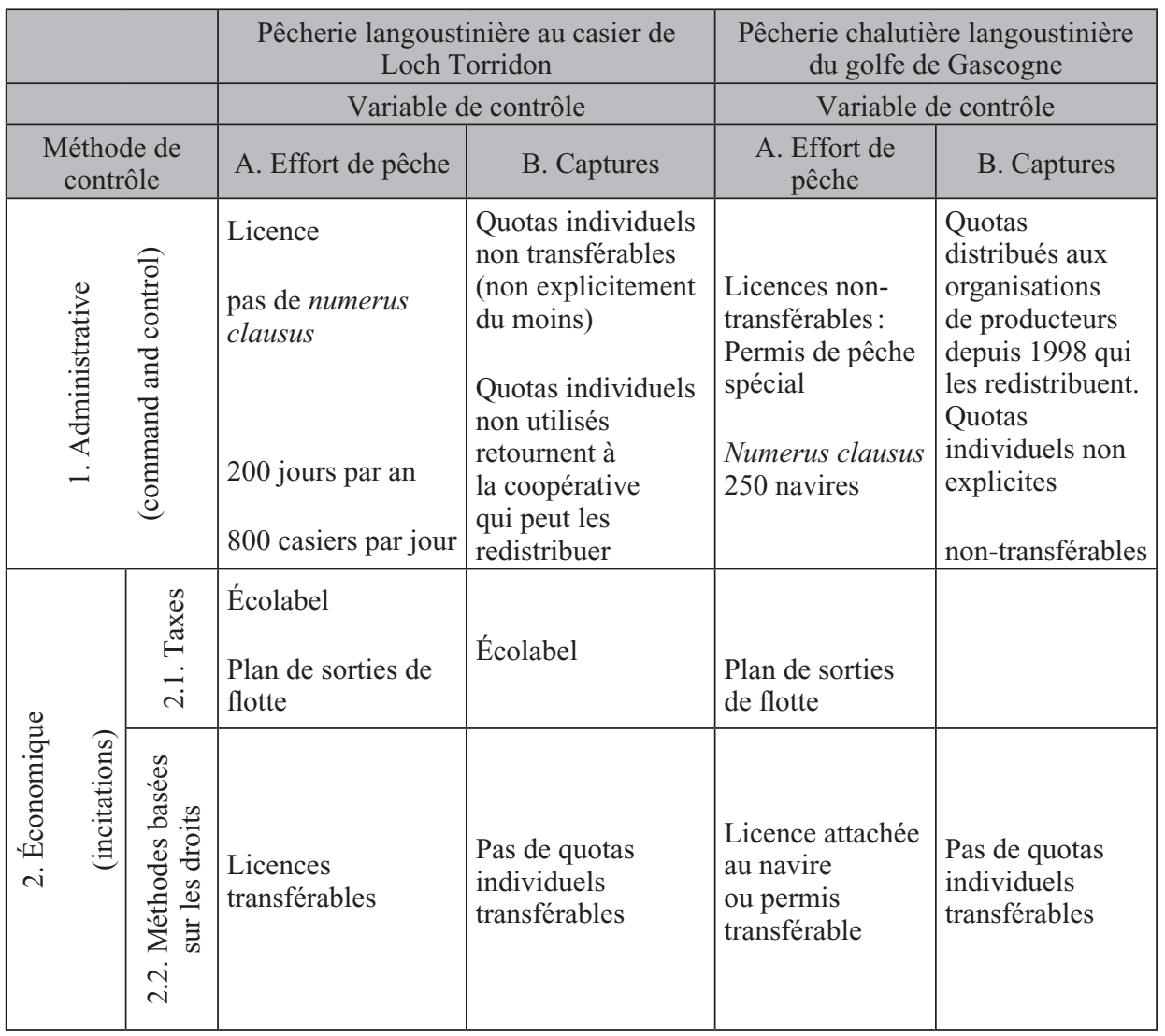


La pêcherie de Loch Torridon est encadrée essentiellement par des mesures techniques de conservation (tableau 30).

La pêcherie est soumise à un régime de quotas. Le TAC langoustine pour la zone CIEM V, la zone de gestion C (11300 tonnes en 2002), décidé au niveau européen, est distribué entre les différents États membres. Le quota anglais est ensuite alloué par le Department of the Environment Food and Rural Affairs (DEFRA) entre les organisations professionnelles, les autres organisations et les navires de moins de 10 mètres. En Écosse, le Scottish Executive Environment and Rural Affairs (SEERAD) est en charge de la réallocation des quotas. La pêcherie de Loch Torridon dispose ainsi d'un quota de 100 à 150 tonnes par an qui peut fluctuer en fonction des mesures sur les limites de captures, des variations de licences et de l'arrivée ou du départ de navires de la zone. Il existe des mécanismes de fermeture de la pêcherie lorsque les débarquements approchent le quota. Il existe des quotas individuels attribués sur une base de droits historiques. Dans la pêcherie de Loch Torridon, les patrons possédant un quota plus important que leur débarquement, donnent leur excès de quotas à Shieldaig Export Limited qui peut ensuite le redistribuer notamment à des patrons qui ne possèdent pas de droits historiques. Des limites de captures mensuelles ont été introduites en janvier 2000 pour les navires de moins de dix mètres capturant plus de $12 \mathrm{~kg}$ de langoustines dans les zones IIa, IV, VI, VII afin d'assurer la disponibilité du quota toute l'année.

Les mesures techniques encadrant la pêcherie relèvent du plan de gestion volontaire mis en place à l'intérieur de la « zone fermée » par le Torridon Nephrops Management Group (TNMG) et repris dans la charte de l'écolabel MSC qui prévoit les mesures suivantes:

- limitation du nombre de casiers : deux jeux de 800 casiers sont autorisés pour un navire et deux hommes et deux jeux de 400 casiers sont autorisés pour un homme et un navire. Deux jeux de 400 casiers à crabes sont autorisés pour deux hommes et un navire et 2 jeux de 200 casiers à crabes pour un homme et un navire;

- les casiers doivent être espacés de 16 mètres sur des lignes pouvant supporter 120 casiers;

- deux jeux de casiers peuvent être en pêche mais seul un jeu de casier peut être viré par jour;

- des dispositifs d'échappement doivent être installés sur les casiers;

- les femelles grainées doivent être rejetées à la mer;

- limitation du nombre de jours de mer à 200 jours de mer par an.

Tous les navires langoustiniers de la zone sont sujets aux règles de quotas et au plan de gestion de Torridon. Tout autre navire pénétrant dans la zone protégée doit se plier aux règles de gestion. Ces mesures sont appliquées sur une base volontaire. L'écolabel MSC joue cependant le rôle d'incitation économique à appliquer ces mesures. La charte porte à la fois sur la technique de pêche, le nombre d'engins, le respect des quotas et le respect des mesures dictées par le Code de gestion volontaire.

D'autres mesures techniques correspondant à l'application du règlement (EC) nº 850/98 sur les mesures techniques dans le cadre de la PCP sont en vigueur dans la pêcherie notamment la taille minimale de débarquement. La taille minimale de débarquement des langoustines imposée pour les stocks écossais de la côte ouest est de $20 \mathrm{~mm}$. Cependant, dans le cas des pêcheries au casier, les comportements et la taille des mailles signifient que très peu de langoustines en dessous de $25 \mathrm{~mm}$ sont capturées et les pêcheurs se sont imposés une taille minimale de débarquement de $28 \mathrm{~mm}$. Compte tenu de la grande différence de prix entre les langoustines de taille $\mathrm{S}$ et les catégories de plus grande taille, l'incitation à 
rejeter ou ne pas capturer les petites langoustines est grande. Des fenêtres d'échappement d'une largeur de $22 \mathrm{~mm}$ ont été récemment mises en place pour permettre aux petites langoustines de s'échapper. Elles sont maintenant installées sur environ 14000 casiers.

Le système permettant l'échappement de langoustines de moins de $40 \mathrm{~mm}$ de longueur de carapace a été mis au point. Les essais ont montré une augmentation de la taille minimum des langoustines capturées qui permet d'augmenter la valeur des captures et d'assurer la durabilité de la pêcherie. Les langoustines jugées sous la taille commerciale (c'est-à-dire en dessous de 30-40 mm de longueur de carapace) sont rejetées. Les pêcheurs appliquent donc une taille minimale de débarquement plus élevée que la réglementation et correspondant à la sélectivité des casiers et aux contraintes du marché.

Un régime de licences délivrées par le Parlement écossais est également en place mais ne prévoit pas pour l'instant de numerus clausus. Le coût de la licence sert de limitation à l'entrée dans la pêcherie.

Enfin, la flottille opère sur une zone fermée au chalutage. En 1984, la loi sur la pêche côtière en Écosse (Inshore Fishing (Scotland) Act) avait en effet levé l'interdit sur les engins mobiles de pêche dans une limite de 3 milles à partir des côtes, mettant ainsi les chalutiers et les caseyeurs en compétition directe pour la ressource d'une part et pour les zones de pêche d'autre part. À partir du début des années quatre-vingt-dix, les pêcheurs caseyeurs du Loch Torridon, craignant alors les dommages engendrés par les chalutiers sur les fonds, les engins de pêche et les captures accessoires qui donnent lieu à des captures réduites et des coûts supplémentaires ont commencé à militer pour la fermeture d'une zone aux engins mobiles. Le 1er novembre 2000, une zone fermée à tous les engins mobiles de pêche est établie. L'accès à cette «zone fermée » est initialement interdit pour une période de cinq ans. Cette zone s'étend de Red Point, y compris le Loch Torridon, à la partie sud de la zone Butec du détroit intérieur de Rona. La zone fermée au chalutage et réservée aux casiers représente environ $100 \mathrm{~km}^{2}$ dans les eaux du Loch Torridon. Les prises totales permises sont comprises entre 100 et 150 tonnes.

Le ministre écossais des pêches, Rhona Brankin, signe en 2001 une mesure interdisant le chalutage dans l'Inner Sound de Raasay et Loch Torridon. Trois zones de pêche sont créées pour permettre les comparaisons : une zone réservée aux chalutiers exclusivement, une zone mixte ouverte aux chalutiers et aux caseyeurs et une zone fermée à tous les engins mobiles de pêche. Le Scottish Statutory Instrument ${ }^{\circ} 174$ crée la zone réservée aux casiers.

Dans la zone fermée au chalutage en 2001, les caseyeurs capturaient environ $90 \%$ des langoustines capturées. Cette mesure a donc peu atteint les chalutiers qui ont reporté leur effort sur d'autres zones.

Les mesures de gestion encadrant la pêcherie langoustinière du golfe de Gascogne sont essentiellement des mesures de conservation. Il existe un TAC depuis 1987, régulièrement dépassé ces dernières années.

Des mesures techniques de maillage minimal et tailles minimales de débarquement réglementent également la pêcherie. Jusqu'en 1989, le maillage était de $50 \mathrm{~mm}$, puis il est passé à $55 \mathrm{~mm}$ de 1990 à 1999. Depuis 2000, il est de $70 \mathrm{~mm}$ dans le golfe de Gascogne (sauf dans le box merlu où il est de $100 \mathrm{~mm}$ ). La taille minimale de débarquement est fixée par les organisations de producteurs et est supérieure à la taille légale européenne: elle était de $26 \mathrm{~mm}$ de longueur céphalo-thoracique ( $8,5 \mathrm{~cm}$ de longueur totale) jusqu'en 2005 puis les organisations l'ont relevée à $9 \mathrm{~cm}$ de longueur totale. 
La flottille langoustinière est en outre soumise au plan de sortie de flotte qui explique la diminution du nombre de navires ciblant la langoustine (de 400 navires en 1978 à 300 en 1987 et autour de 230 depuis 2000).

Avant 2004, la pêcherie était en libre accès et soumise uniquement aux politiques publiques non spécifiques (permis de mise en exploitation national, plans de sortie).

Depuis 2004, des permis de pêche spéciaux ont été mis en place sur la base d'un numerus clausus de 250 navires. L'obtention du PPS est conditionnée par une limite en longueur des navires (21,5 mètres), et, pour l'année 2005, à l'adoption du dispositif sélectif pour le merlu (mailles carrées). Il n'existe pas en revanche de limitation de l'effort de pêche (en nombre de jours par exemple) ni de limite des captures individuelles, ni de contrainte sur les engins.

\section{Commentaires sur les systèmes de gestion}

La gestion des deux pêcheries repose essentiellement sur des mesures de conservation en particulier des mesures de maillage, taille minimale de débarquement, dispositif sélectif et un système de quotas. Dans le cas de la pêcherie au casier de Loch Torridon, une zone interdite au chalutage réserve l'accès aux seuls caseyeurs et évite ainsi l'encombrement, la compétition pour la ressource et les destructions d'engins observées lorsque casiers et chaluts cohabitent dans une même zone. La pêcherie au casier s'est par ailleurs entourée de mesures (applicables sur une base volontaire) notamment sur le nombre de casiers virés chaque jour et le nombre de jours de pêche. L'écolabel mentionne ces mesures et sert donc d'incitation économique à les appliquer. Le nombre réduit de navires opérant dans cette zone et le lieu unique de débarquement favorise également l'autosurveillance. Le système de quotas individuels associé à une limitation de l'effort en nombre de casiers permet de contenir la pression de pêche. Cependant, aucun numerus clausus n'est pour l'instant appliqué dans cette pêcherie, ce qui peut représenter un danger majeur. Il n'existe pas de limitation du nombre de licences. La valeur non-légale du code de conduite peut être également une limite à la bonne gestion.

En ce qui concerne les mesures de régulation de l'accès, celles-ci relèvent essentiellement d'une régulation administrative de l'effort et des captures souvent de façon informelle via la redistribution des quotas entre bateaux par les OP.

L'efficacité du système de quotas individuels (QI) dans la pêcherie au casier provient du fait que les droits historiques ont donné aux caseyeurs des quotas individuels non contraignants. D'autre part, en cas de QI contraignant, étant donné le peu de productions jointes, les navires ne seraient pas soumis à l'arbitrage entre rejeter ou débarquer.

La comparaison du système de gestion de la pêcherie au casier de Loch Torridon par rapport à celui de la pêcherie chalutière langoustinière du golfe de Gascogne met en évidence plusieurs points forts qui ont favorisé la rentabilité du casier à langoustines dans le Loch Torridon. La zone interdite au chalutage a permis aux caseyeurs d'exercer leur activité sans conflit avec les chalutiers. L'autogestion est rendue possible puisqu'il s'agit d'une petite communauté de pêcheurs opérant dans une zone définie par des éléments naturels et de faible surface. La forte sélectivité des engins de pêche et la limitation de l'effort (en nombre de casiers) garantissent la gestion durable du stock et limite les interactions avec d'autres pêcheries. Enfin, " l'écolabelling » a servi d'incitation à respecter les règles proposées sur une base volontaire mais n'ayant pas de valeur légale. 


\section{Captures par unité d'effort et structure des débarquements}

Les navires de la pêcherie langoustinière au casier de Loch Torridon débarquent en moyenne 9,5 tonnes de langoustines par an (jusqu'à 14 tonnes pour les navires les plus performants) et l'ensemble de la flottille des caseyeurs opérant dans cette zone débarque de 100 à 150 tonnes de langoustines par an. Les chalutiers langoustiniers du golfe de Gascogne débarquent, quant à eux, en moyenne 15.4 tonnes de langoustines par navire par an avec néanmoins une forte variabilité en fonction de la classe de longueur des navires ou du nombre d'hommes à bord. Les chalutiers langoustiniers ayant deux hommes à bord débarquent ainsi en moyenne 11,5 tonnes de langoustines par an.

Les deux flottilles présentent une saisonnalité quasi synchrone de l'activité et de la production de langoustines (figure 110 p. XXVIII). Les captures de langoustines par les caseyeurs de Loch Torridon et les chalutiers langoustiniers du golfe de Gascogne ont ainsi lieu essentiellement au printemps et en été, de février à août à Loch Torridon et d'avril à août dans le golfe de Gascogne. Entre les mois de février et août, 10 à 14 tonnes de langoustines sont débarquées par mois par la flottille des caseyeurs de Loch Torridon (2002-2004). De septembre à janvier, la flottille ne débarque plus que 6 à 7 tonnes par mois (2002-2004).

L'activité des langoustiniers (caseyeurs ou chalutiers) est concentrée sur la langoustine lors de ces mois de printemps-été. Dans le golfe de Gascogne, on observe ainsi un pic d'activité sur le métier de chalutage de fond à langoustine à cette époque de l'année. C'est à cette saison en effet que la langoustine est plus abondante et que les chalutiers du sud de la pêcherie notamment, moins spécialisés sur la langoustine, ciblent cette espèce.

\section{Captures par unité d'effort}

Les données disponibles pour Loch Torridon indiquent que 141774 casiers ont été levés par navire en 2003. Étant donné 200 jours de mer autorisés par an, en moyenne 709 casiers ont été virés par jour en 2003 avec néanmoins une variabilité saisonnière liée à l'activité des navires (figure 111).

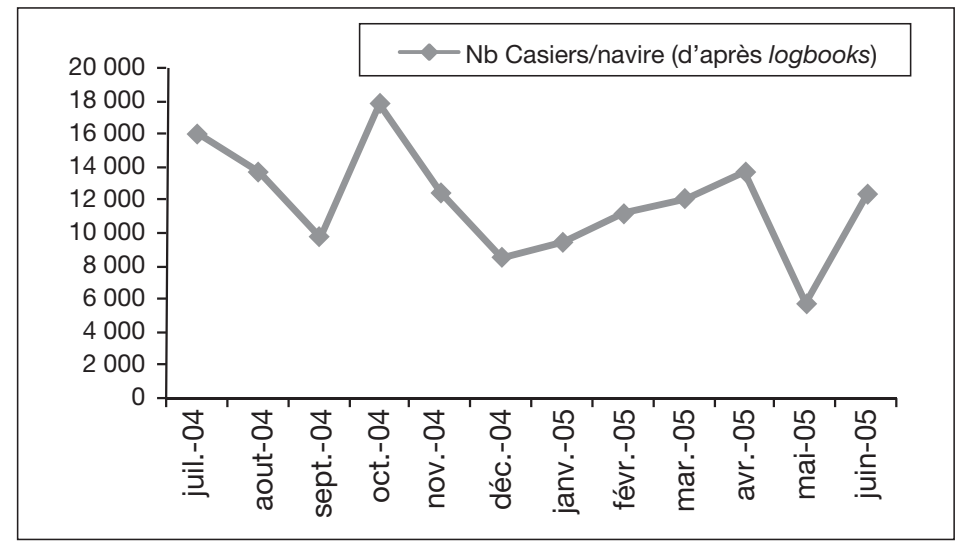

Figure 111. Nombre de casiers levés par mois par navire de la pêcherie de Loch Torridon.

Les données de captures de langoustines par navire et de nombre de casiers virés par an permettent de calculer que les captures par unité d'effort des caseyeurs de Loch Torridon sont en moyenne de $98 \mathrm{~g}$ par casier viré par jour de mer (par levée) avec néanmoins une variabilité saisonnière marquée (figure 112). 


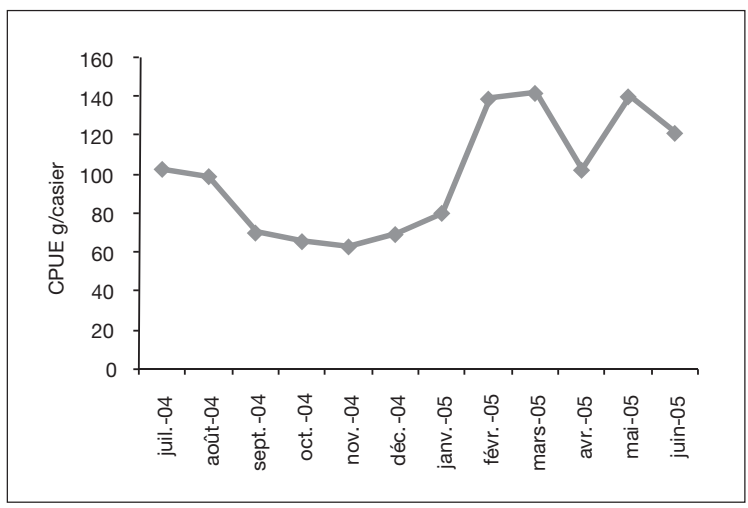

Figure 112. Captures par unité d'effort de langoustines en grammes par casier levé, par mois, dans la pêcherie de Loch Torridon.

Les marées durent dix à douze heures. Les navires partent généralement à $5 \mathrm{~h} 30$ et reviennent vers 17-18 heures Le temps de route est d'environ 2 heures aller-retour pour les navires d'Ardeslaig. Les navires totalisent donc environ 2000 heures de pêche par an pour 9.5 tonnes débarquées par navire (14 tonnes pour les plus performants). Les captures par unité d'effort en heure de pêche sont donc de $4,75 \mathrm{~kg} /$ heure de pêche en moyenne (jusqu'à $6,94 \mathrm{~kg} /$ heure de pêche pour les navires les plus performants de la pêcherie).

Du point de vue de la capturabilité des casiers, 200 jours de pêche sont autorisés par an mais les casiers sont refilés immédiatement après virage et restent donc en pêche toute l'année. D'autre part, deux jeux de 400 casiers sont autorisés par homme et par navire soit 1600 casiers pour un navire ayant deux hommes à bord. L'effort appliqué par navire ayant deux hommes à bord au stock correspond donc à 1600 casiers $\times 365 \mathrm{~J} \times 24 \mathrm{~h}=14$ millions de casiers. Étant donné 9,5 à 14 tonnes de langoustines débarquées par navire, la capture par unité d'effort est de 0,7-1 g par heure de pêche casier.

Dans le cas de la flottille langoustinière du golfe de Gascogne, les captures par unité d'effort utilisées par le groupe de travail d'évaluation du stock de langoustines (Anon., 2004) sont estimées pour les navires du quartier du Guilvinec au deuxième trimestre (figure 113). Elles indiquent pour 2003, que la capture par unité d'effort est de $13 \mathrm{~kg} / \mathrm{h}$ en moyenne en 2003.

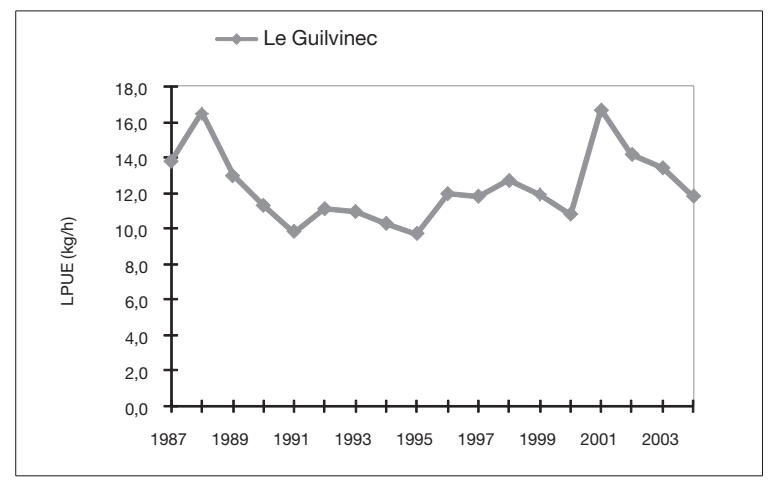

Figure 113. Débarquements de langoustines par unité d'effort en $\mathrm{kg} / \mathrm{h}$ des chalutiers langoustiniers du golfe de Gascogne du quartier du Guilvinec au deuxième trimestre. 
Les CPUE peuvent cependant varier entre $4,5 \mathrm{~kg} / \mathrm{h}$ et plus de $15 \mathrm{~kg} / \mathrm{h}$ selon la saison.

Les chalutiers de 12 mètres ayant deux hommes à bord capturent environ 11,5 tonnes de langoustines par an pour environ 2400 heures moteur par an, ce qui est comparable au tonnage réalisé par les navires de Loch Torridon.

\section{Catégories et structures des débarquements}

Si les captures par unité d'effort et tonnages totaux de langoustines débarquées sont du même ordre de grandeur entre le métier de casier à langoustines de Loch Torridon et le métier de chalut de fond à langoustines du golfe de Gascogne (pour des classes de longueur de navire ou de nombre d'hommes à bord comparables), les productions jointes diffèrent en revanche en fonction de la technique de production.

La flottille de caseyeurs de Loch Torridon débarque quatre catégories classées en catégorie $\mathrm{S}, \mathrm{M}, \mathrm{L}, \mathrm{XL}$ qui correspondent à la définition suivante (tableau 32):

Tableau 32. Définition des catégories de langoustines débarquées par la flottille des caseyeurs langoustiniers de Loch Torridon.

\begin{tabular}{ccccc} 
Catégories & Nb individus/kg & $\begin{array}{c}\text { Poids maximum } \\
(\mathrm{kg})\end{array}$ & $\begin{array}{c}\text { Correspondance } \\
\text { age }(\text { ans })\end{array}$ & $\begin{array}{c}\text { Distribution } \\
\text { débarquements }\end{array}$ \\
\hline $\mathrm{S}$ & $25-26$ & 0,040 & $5-6$ & 15 \\
$\mathrm{M}$ & $16-24$ & 0,063 & $7-8$ & 42 \\
$\mathrm{~L}$ & $9-15$ & 0,111 & $9+$ & 28 \\
$\mathrm{XL}$ & $<8$ & & $9+$ & 16 \\
\hline
\end{tabular}

La flottille débarque essentiellement des langoustines de taille $\mathrm{M}$ et $\mathrm{L}$ qui représentent environ $70 \%$ des débarquements (tableau 33).

Tableau 33. Débarquements totaux par catégorie en kilogramme. (Source: Shieldaig Export).

\begin{tabular}{crr} 
Catégorie & & \\
S 2002-2003 & \\
S & 19734 & 20396 \\
M & 42327 & 47132 \\
L & 39917 & 41489 \\
XL & 17387 & 22166 \\
\hline Total & 119365 & 131183 \\
\hline
\end{tabular}

Tableau 34. Captures par unité d'effort (en gramme) par casier levé par catégorie commerciale.

\begin{tabular}{cc}
\hline Catégories & CPUE (g/casier) \\
\hline S & 15 \\
M & 27 \\
L & 41 \\
XL & 14 \\
\hline Total & 98 \\
\hline
\end{tabular}

Les captures par unité d'effort se répartissent entre les catégories commerciales de la façon suivante (tableau 34).

Les tableaux 34 et 35 donnent la structure annuelle moyenne des débarquements. Les débarquements présentent d'autre part une saisonnalité marquée comme dans le cas de la pêcherie langoustinière du golfe de Gascogne.

Comparés aux débarquements de la flottille chalutière langoustinière du golfe de Gascogne, les débarquements de la flottille au casier sont constitués essentiellement d'individus de grande taille. 
Plus de $90 \%$ des débarquements de la flottille chalutière langoustinière du golfe de Gascogne sont constitués de langoustines d'âge inférieur ou égal à 5 (tableau 35), alors que plus de $80 \%$ des débarquements de langoustines par les caseyeurs de Loch Torridon sont constitués de langoustines d'âge supérieur à 6 (tableau 32).

Tableau 35. Définition des catégories de langoustines débarquées par la flottille chalutière langoustinière du golfe de Gascogne.

\begin{tabular}{ccccc}
\hline Catégories & Nb individus/kg & $\begin{array}{c}\text { Poids maximum } \\
(\mathrm{kg})\end{array}$ & $\begin{array}{c}\text { Corespondance } \\
\text { âge }(\text { ans })\end{array}$ & $\begin{array}{c}\text { Distribution } \\
\text { débarquements }(\%)\end{array}$ \\
\hline 40 & $>41$ & 0,024 & 1 à 4 & 27 \\
30 & $31-40$ & 0,032 & 4 et 5 & 71 \\
20 & $21-30$ & 0,048 & 5 et 6 & 1 \\
10 & $<20$ & & 6 et plus & 1 \\
\hline
\end{tabular}

Les catégories de langoustine sont définies par le règlement $(\mathrm{CE}) \mathrm{n}^{\mathrm{o}} 2406 / 96^{162}$.

Les catégories 10 et 20 débarquées par les chalutiers langoustiniers du golfe de Gascogne correspondent à la catégorie $\mathrm{M}$ de langoustines débarquées par les caseyeurs de Loch Torridon, la catégorie 30 correspond à la catégorie $\mathrm{S}$.

Les captures par casier sont donc des captures d'individus de plus de 5 ans.

\section{Rejets, prises accessoires et productions jointes environnementales}

Des campagnes à la mer ont montré des taux de rejet et prises accessoires limités dans le cas de la pêcherie au casier de Loch Torridon. Les campagnes d'échantillonnage menées par le Fisheries Research Service d'Aberdeen ont ainsi montré que sur 16,22 kg de langoustines capturées, $5,8 \mathrm{~kg}$ ont été rejetés. La taille moyenne des rejets de langoustine mâles était de 35,9 mm CL et la taille moyenne des rejets de langoustines femelles de 34,9 mm CL. Treize femelles grainées ont également été rejetées.

L'analyse des rejets d'autres espèces met en évidence la forte sélectivité du casier par rapport aux espèces. Sur 115 casiers relevés, 69 casiers ne contenaient aucune espèce accessoire (60\% des casiers ne contiennent aucune espèce accessoire).

Aucun casier ne contenait plus de deux espèces accessoires et au maximum 16 individus étaient présents. Une fenêtre d'échappement de $22 \mathrm{~mm}$ améliore encore davantage la sélectivité des casiers.

Les travaux menés sur la survie des rejets ont montré d'autre part que le taux de survie des rejets des chalutiers était d'environ $31 \%$ mais que le taux de survie des langoustines échappées du chalut était bien supérieur (81\%, Ulmestrand et al., 1998). La survie des rejets de langoustines capturées par des casiers peut être supposée proche de celle des langoustines qui s'échappent du chalut. Les études sur les dommages causés sur les langoustines par l'exposition à la lumière ont montré par ailleurs que ceux-ci n'affectaient pas leur survie (Chapman et al., 2000) ni leur comportement (Richardson, 1996). On peut donc supposer que la plupart des langoustines capturées par des casiers et rejetées survivent.

\footnotetext{
162 Règlement du Conseil du 26 novembre 1996 fixant des normes communes de commercialisation pour certains produits de la pêche Journal officiel $n^{\circ} L 334 d u$ 23/12/1996 p. 0001 à 0015 remplaçant les règlements (CEE) $n^{\circ} 103 / 76$ et (CEE) $n^{\circ}$ 104/76 sur les normes communes de commercialisation fixées, d'une part, pour certaines espèces de poissons par le règlement (CEE) $n^{\circ}$ 103/76 (2) et, d'autre part, pour certaines espèces de crustacés par le règlement (CEE) $n^{\circ} 104 / 76$ (3).
} 
Dans le cas de la pêcherie chalutière langoustinière en revanche, les taux de rejet sont très élevés $(60 \%$ des langoustines capturées sont rejetées en nombre et $30 \%$ en poids, Talidec et al., 2005) et la survie des rejets très faible (taux de mortalité de $70 \%$, Guéguen et Charuau, 1975).

Le casier, comme l'ensemble des engins dormants a peu d'impact sur l'écosystème. Dans le cas de la pêcherie chalutière langoustinière du golfe de Gascogne en revanche, une évaluation de l'effort déployé par les navires langoustiniers du golfe de Gascogne a mis en évidence que la Grande Vasière était chalutée sur toute sa surface de 3 à 6 fois par an avec les conséquences en terme d'impact sur les habitats que cela peut impliquer. (Bourillet et al., 2005)

Des études montrent cependant les risques liés à la pêche fantôme des casiers perdus (Ghost Fishing, Al-Masroori et al., 2004, Brown et Macfadyen, 2007). Dans le cas des casiers à langoustines, une étude du FRS d'Aberdeen a montré cependant que les casiers laissés plusieurs jours ne contenaient pas ou peu de captures.

\section{Prix et marchés}

Alors que le marché de la langoustine vivante française est un marché essentiellement local, la quasi-totalité des langoustines écossaises de Loch Torridon est exportée. La coopérative Shieldaig Export achète les langoustines aux caseyeurs et les exporte vivantes essentiellement vers l'Espagne ainsi que dans des restaurants français et londoniens où est acheminée également une partie des langoustines françaises de catégorie royale pêchées au chalut. Une partie des langoustines a été exportée vers l'Italie, mais le marché trop fluctuant a été abandonné.

Le fonctionnement en coopérative de Shieldaig Export garantit le même prix à tous les navires. La commercialisation vivante est rendue possible par une logistique appropriée $^{163}$. Un système de stockage en viviers géré par la coopérative Shieldaig Export permet d'adapter l'offre à la demande et ainsi de stabiliser les prix. Les grosses langoustines sont ainsi généralement exportées le jeudi et le vendredi pour être mises sur le marché avant le week-end. Les langoustines vivantes sont vendues 4 à 5 fois plus chères que les langoustines glacées.

La comparaison des prix par classe d'âge entre les langoustines du golfe de Gascogne pêchées au chalut et les langoustines pêchées au casier de Loch Torridon montre que pour des langoustines de classes de tailles à peu près équivalentes, la différence de prix est faible $^{164}$ (figure 114).

Compte tenu de la structure des débarquements, il existe en revanche une différence de prix moyen d'environ 4 euros/kg entre les langoustines pêchées au chalut et celles pêchées au casier (tableau 36). En 2003-2004, le prix moyen des langoustines de Loch Torridon était de 13 euros $/ \mathrm{kg}$ ce qui correspond à peu près au prix obtenu pour la catégorie royale vendue en France. Dans la pêcherie langoustinière chalutière du golfe de Gascogne, le prix moyen au débarquement en 2003 atteignait 9,10 euros/kg en 2003.

\footnotetext{
163 Le système de vivier et un conditionnement individuel permettent de conserver la qualité des langoustines qui sont ensuite exportées par avion.

164 Les langoustines de très grande taille pêchées au casier obtiennent en revanche des prix élevés sur les marchés de luxe et de façon croissante sur les marchés domestiques.
} 


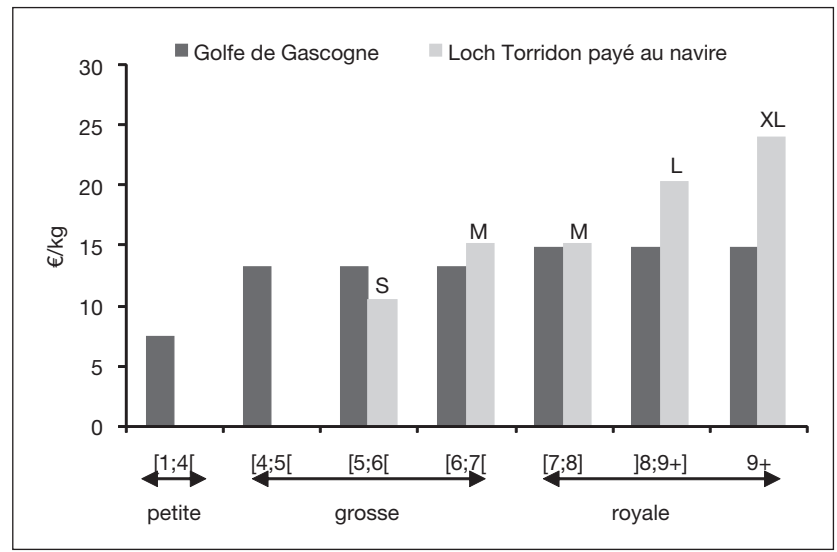

Figure 114. Comparaison des prix moyens en 2003-2004 en (euros/kg) des langoustines vivantes du golfe de Gascogne et de Loch Torridon (Source : Shieldaig Export Ifremer/SIH).

Tableau 36. Comparaison des prix moyens au kilogramme des langoustines pêchées au casier et des langoustines pêchées au chalut, des chiffres d'affaires moyens par navire et de la structure du chiffre d'affaires des deux pêcheries (source: d'après données Shieldaig Export et Ifremer/ $\mathrm{SIH})$.

\begin{tabular}{|l|c|c|}
\cline { 2 - 3 } \multicolumn{1}{c|}{} & $\begin{array}{c}\text { Loch Torridon } \\
\text { 2003-2004 }\end{array}$ & \begin{tabular}{c} 
Golfe de Gascogne \\
\multicolumn{1}{c|}{}
\end{tabular} \\
\hline Prix moyen de la langoustine $(€ / \mathrm{kg})$ & 13 & 9,10 \\
\hline $\begin{array}{l}\text { Chiffre d'affaires langoustine de la } \\
\text { pêcherie }(M €)\end{array}$ & 1,7 & 30 \\
\hline Chiffre d'affaires moyen par navire $(\mathrm{k} €)$ & 122 & 128 \\
\hline Structure du chiffre d'affaires $(\%)$ & $\begin{array}{c}\text { S }: 9 \\
\text { M et L }: 66 \\
\text { XL }: 25\end{array}$ & $40: 65$ \\
\hline
\end{tabular}

La majorité des débarquements de langoustines de Loch Torridon est en effet constituée de catégories M et L (catégories de taille intermédiaire) et non de catégories les plus petites comme dans le cas de la pêcherie chalutière langoustinière du golfe de Gascogne. L'effet de la structure en tailles des débarquements explique la différence de prix moyen observé $^{165}$, et la structure du chiffre d'affaires.

En 2003-2004, la pêcherie langoustinière de Loch Torridon a réalisé un chiffre d'affaires langoustine de 1,7 M€ soit en moyenne $122 \mathrm{k} €$ par navire qui provient pour plus de $90 \%$ de langoustines de catégories M, L et XL. Dans le golfe de Gascogne, la langoustine a rapporté aux chalutiers un chiffre d'affaires d'environ $30 \mathrm{M} €$ en 2003 dont $65 \%$ proviennent du débarquement des petites langoustines de catégorie 40 .

\footnotetext{
${ }^{165}$ La figure 114 laisse penser qu'il n'existe pas d'effet de l'écolabel MSC dans la formation des prix de la langoustine pêchée par les caseyeurs de Loch Torridon. Il n'existe cependant pas de données sur les préférences des consommateurs qui permettraient de mettre en évidence le rôle de l'écolabel MSC dans la formation des prix de la langoustine vivante pêchée au casier.
} 
L'analyse comparée de la structure des débarquements met en évidence que dans la pêcherie au casier, la majorité des débarquements est constituée de catégories $\mathrm{M}$ et $\mathrm{L}$ (catégories de taille intermédiaires) et non de catégories les plus petites comme dans le cas de la pêcherie chalutière langoustinière du golfe de Gascogne. Le stockage en vivier géré par la coopérative Shieldaig Export permet par ailleurs une certaine stabilité des prix. La commercialisation des grosses langoustines vivantes à des prix élevés (en Espagne notamment) dégage une forte valeur ajoutée. Cette commercialisation vivante est rendue possible par une logistique appropriée (viviers, conditionnements spécifiques...).

\section{Analyse des performances économiques des deux pêcheries}

L'analyse comparée des performances économiques du métier de caseyeurs à langoustine et du métier de chalutage de fond à langoustines s'appuie sur les données suivantes: - des données comptables récoltées pour les périodes 2002-2003 et 2003-2004 à Loch Torridon en 2006 sur les caseyeurs à langoustines;

- des données qualitatives issues d'embarquements et entretiens avec les pêcheurs de Loch Torridon;

- des données issues des enquêtes économiques menées par l'Ifremer chaque année auprès d'un échantillon représentatif de navires permettant d'estimer les coûts et revenus par métiers et flottilles (dans notre cas pour les caseyeurs et les chalutiers);

- des données qualitatives récoltées auprès des patrons des chalutiers langoustiniers du golfe de Gascogne;

- des données issues du rapport annuel sur les performances économiques des flottilles de pêches européennes 2005 et fournies par le Sea Fish Industry Authority.

Les performances économiques des navires de Loch Torridon sont comparées aux performances économiques de chalutiers langoustiniers de Bretagne Sud de caractéristiques techniques et d'effort de pêche nominal comparable (classe de longueur, nombre d'hommes à bord, nombre de jours de pêche et durée des marées comparables).

Tableau 37. Comparaison des caractéristiques entre les caseyeurs de Loch Torridon et la flottille des chalutiers langoustiniers du golfe de Gascogne de nombre d'hommes à bord et classe de longueur de navires proches de ceux des navires de Loch Torridon.

\begin{tabular}{|l|c|c|}
\cline { 2 - 3 } \multicolumn{1}{c|}{} & $\begin{array}{c}\text { Caseyeurs à langoustines } \\
\text { Loch Torridon }\end{array}$ & $\begin{array}{c}\text { Chalutiers langoustiniers } \\
\text { Bretagne Sud }\end{array}$ \\
\hline Nombre d'hommes à bord & 2 & 2 \\
Longueur moyenne $(\mathrm{m})$ & 10 & $12-14$ \\
Nombre de jours de mer & 200 & 199 \\
Durée marée (h) & 12 & 13 \\
Temps de pêche par jour (h) & 10 & 10 \\
Puissance moyenne (kW) & 200 & 165 \\
Âge moyen des navires (années) & 2 & 19 \\
\hline
\end{tabular}

Le tableau 37 met en évidence que les captures par unité d'effort de Loch Torridon sont du même ordre de grandeur que celles des chalutiers de mêmes caractéristiques techniques et que le tonnage annuel de langoustine est comparable. Dans le cas des caseyeurs, le tonnage moyen, un peu plus faible que celui des chalutiers, est compensé par un prix au 
kilogramme plus élevé (du fait de la structure des débarquements). Le chiffre d'affaires total est supérieur dans le cas des chalutiers langoustiniers car ces derniers tirent près de $40 \%$ de leur chiffre d'affaires des productions jointes. Les caseyeurs ont cependant un chiffre d'affaires langoustines plus important. De même, le chiffre d'affaires langoustine par heure de mer des caseyeurs est plus important que le chiffre d'affaires langoustine par heure des chalutiers (tableau 38).

Tableau 38. Comparaison de quelques indicateurs économiques entre les caseyeurs de Loch Torridon et la flottille des chalutiers langoustiniers du golfe de Gascogne de nombre d'hommes à bord et classe de longueur de navires proches de ceux des navires de Loch Torridon.

\begin{tabular}{|l|c|c|}
\cline { 2 - 3 } \multicolumn{1}{c|}{} & $\begin{array}{c}\text { Caseyeurs à langoustines } \\
\text { Loch Torridon }\end{array}$ & $\begin{array}{c}\text { Chalutiers langoustines } \\
\text { Bretagne Sud }\end{array}$ \\
\hline Prix moyen langoustine (euros/kg) & 13 & 9 \\
Tonnage moyen langoustine (t/navire) & 9,7 & 11,5 \\
Tonnage langoustine (min-max tonnes par & $9,5-14$ & $5,8-13$ \\
navire par an) & $4,75-6,94$ & 148000 \\
CPUE* (en kg par heure de pêche) & 124000 & 89000 \\
Chiffre d'affaires par navire (euros) & 124000 & 57 \\
Chiffre d'affaires de langoustines par navire & & 34 \\
(euros) & 52 & 26000 \\
Chiffre d'affaires par heure (euros/h) & 52 & \\
Chiffre d'affaires de langoustines par heure & & \\
(euros/h) & 30515 & \\
Salaire net par marin (euros/an) & & \\
\hline
\end{tabular}

On compare ensuite, à partir de données moyennes de structure en coût variable sur la période 2001-2003, les coûts par métier nécessaires pour générer 100 euros de langoustine dans le cas des caseyeurs de Loch Torridon et dans le cas des chalutiers langoustiniers de caractéristiques comparables (tableau 39). Les coûts nécessaires pour générer 100 euros de chiffre d'affaire toutes espèces confondues dans le cas des chalutiers langoustiniers figurent également. Le reste à partager moyen par heure de mer par métier est calculé pour la période $2001-2003$.

L'absence de frais de débarquements dans le cas des caseyeurs de Loch Torridon est spécifique au fonctionnement de cette pêcherie ${ }^{166}$. Il faut 8 euros de coûts variables pour générer 100 euros de langoustines dans le cas du métier casier contre 34 euros de frais pour générer 100 euros de langoustines et 21 euros de frais pour générer 100 euros de chiffre d'affaires toutes espèces confondues dans le cas du métier de chalutage de fond à langoustine. Ces résultats indiquent que le métier de caseyeur peut être un métier rentable dégageant un reste à partager par unité d'effort qui peut approcher celui du métier de chalutage de fond. La différence de reste à partager par heure de mer entre les métiers (48 euros par heure de mer pour les caseyeurs et 45 euros par heure de mer pour les chalutiers) provient

\footnotetext{
166 On pourrait donc prendre en compte les frais de débarquements obtenus par des caseyeurs français ( 3 euros pour 100 euros de chiffre d'affaires, flottille des caseyeurs de moins de 12 mètres de Manche - mer du Nord synthèse des flottilles 2003, 8,2 mètres de longueur moyenne, 2,2 hommes à bord, puissance $85 \mathrm{~kW}$ ).
} 
essentiellement des frais de carburant et huile et des frais d'engins ${ }^{167}$, de la différence de prix moyen des langoustines et des différences de captures par unité d'effort.

Tableau 39. Comparaison des frais par métier nécessaires pour générer 100 euros de chiffre d'affaires langoustine ou 100 euros de chiffre d'affaires toutes espèces confondues et comparaison des restes à partager par heure de mer par métier. Moyenne 2001-2003.

(Sources: Données comptables récoltées à Loch Torridon et données économiques de l'Ifremer/SIH).

\begin{tabular}{|l|c|c|c|}
\cline { 2 - 4 } \multicolumn{1}{c|}{} & \multicolumn{2}{|c|}{$\begin{array}{c}\text { Pour 100 euros de chiffre } \\
\text { d'affaires langoustines }\end{array}$} & $\begin{array}{c}\text { Pour 100 euros } \\
\text { de chiffre d'affaires } \\
\text { espèces confondues }\end{array}$ \\
\cline { 2 - 4 } \multicolumn{1}{c|}{} & $\begin{array}{c}\text { Caseyeurs } \\
\text { Loch Torridon }\end{array}$ & $\begin{array}{c}\text { Chalutiers } \\
\text { langoustiniers } \\
\text { Bretagne Sud }\end{array}$ & $\begin{array}{c}\text { Chalutiers } \\
\text { langoustiniers } \\
\text { Bretagne Sud }\end{array}$ \\
\hline Chiffre d'affaires total (euros) & 100 & 167 & 100 \\
Chiffre d'affaires langoustines (euros) & 100 & 100 & 100 \\
\hline Frais débarquement (euros) & 0 & 6,3 & 60 \\
Frais glace (euros) & 0 & 0,1 & 3,8 \\
Frais appât (euros) & 1,3 & 0 & 0,1 \\
Frais carburant et huile (euros) & 4,63 & 18,9 & 0 \\
Frais vivire (euros) & 0,5 & 0,8 & 11,3 \\
Frais engins gréément (euros) & 1,3 & 8,1 & 0,5 \\
Frais totaux (euros) & 8 & 34 & 21 \\
\hline Reste à partager (euros) & 92 & 133 & 79 \\
Reste à paratger (euros/ heure de mer) & 48 & 45 & 45 \\
\hline
\end{tabular}

${ }^{67}$ Les frais d'engin-gréement sont ici inclus dans les frais communs pour le calcul du reste à partager comme cela a pu être observé pour certains armements du golfe de Gascogne. 



\section{Chapitre 4 \\ Méthodologies développées}

\section{Enquêtes}

\section{Enquêtes sur l'activité des navires de pêche}

Les analyses de flottille sont classiquement fondées sur la connaissance par bateau des activités de pêche exprimées en nombre de mois d'activité globale et par métier, complétées par des données relatives aux zones de pêche fréquentées et à l'effort exprimé en jours de pêche ou en quantité de matériels mis en œuvre. Il est possible de réaliser des analyses plus fines reposant sur la composition par espèce des captures par opération de pêche ou marée pour les navires déclarant complètement leurs débarquements.

Le travail engagé par l'Ifremer en matière de suivi des activités part du constat qu'il est malheureusement exclu à terme prévisible de disposer d'une information déclarative complète en matière d'effort et de production par métier pour l'ensemble des navires français (établi sur la base des navires actifs au registre de la flotte, ou armés en conchyliculture mixte petite pêche (CMPP), source BCS-DPMA), comme l'illustre la figure ci-dessous (figure 115).

Un réseau pérenne d'observation des pêcheries a été mis en place à l'Ifremer en 2001, dans le cadre du $\mathrm{SIH}^{168}$ de l'Ifremer. L'objectif des enquêtes est de disposer pour chaque navire d'informations minimales mais exhaustives ${ }^{169}$ concernant: le calendrier d'activité mensuel de chaque navire; l'information attendue par mois concerne le nombre de jours de pêche, le port d'exploitation principal, le ou les métiers pratiqués dans le mois et,

\footnotetext{
168 Système d'informations halieutiques.

169 La collecte des données d'activité sur la façade atlantique est un des volets de la convention Statistiques de pêches DPMA-Ifremer. La collecte des données d'activité en Méditerranée est intégrée dans le volet contractualisation des données économiques confié à l'Ifremer (programmes 2003 et 2004).
} 
pour chaque métier, les deux zones de pêche principales et le gradient côte-large ${ }^{170}$. Des informations annuelles de complément sur l'activité du navire (nombre de jours de mer, nombre d'heures moteur, etc.) et sur l'exhaustivité ou non des déclarations du pêcheur (estimation du taux de suivi de l'effort et des productions via le système déclaratif) ${ }^{171}$.

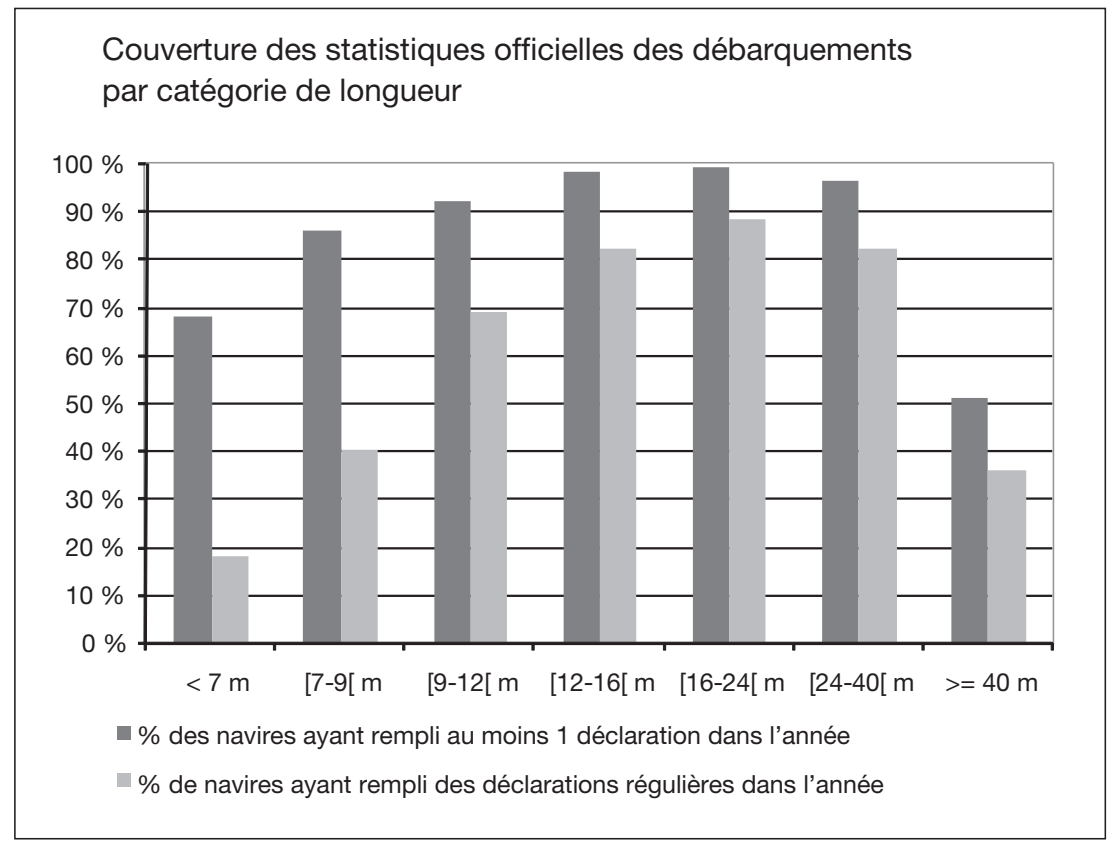

Figure 115. Couverture des statistiques officielles de débarquement en 2001.

Le questionnaire « activité » qui sert de base aux enquêtes est présenté page suivante.

Préalablement au travail d'enquête, les données disponibles à l'Ifremer sont transmises de manière synthétique aux enquêteurs sous forme de prédocumentation des questionnaires activités.

La prédocumentation résume l'ensemble des données d'origine administrative relatives aux caractéristiques des navires et des armements (fichier des navires de pêche commerciale, fichier armateur, fichier des couples navire - armateur), aux données d'activité potentielle (fichier des rôles d'armement), du flux déclaratif des efforts de pêche par engin et des débarquements (journaux de bord, fiches de pêche, données de ventes en

\footnotetext{
${ }^{170}$ La notion de gradient côte - large caractérise la zone de travail sous l'angle de l'éloignement par rapport à la côte. Il s'agit en particulier de déterminer si l'activité s'est développée ou non à l'intérieur de la bande côtière des 12 milles. Cette mer territoriale a juridiquement un statut particulier dans le contexte de la PCP; c'est un espace réservé aux flottilles nationales, sauf droits historiques dans la bande 6-12 milles de quelques navires européens. Elle constitue aussi la zone de compétence des comités régionaux des pêches et élevages marins en matière de gestion des pêcheries.

${ }^{171}$ Ce suivi exhaustif des activités de pêche, réalisé en routine par le réseau des enquêteurs de l'Ifremer est complété sur un large échantillon de navires par des enquêtes sur les caractéristiques de l'effort mensuel moyen par métier et les caractéristiques des engins mis en œuvre par métier.
} 


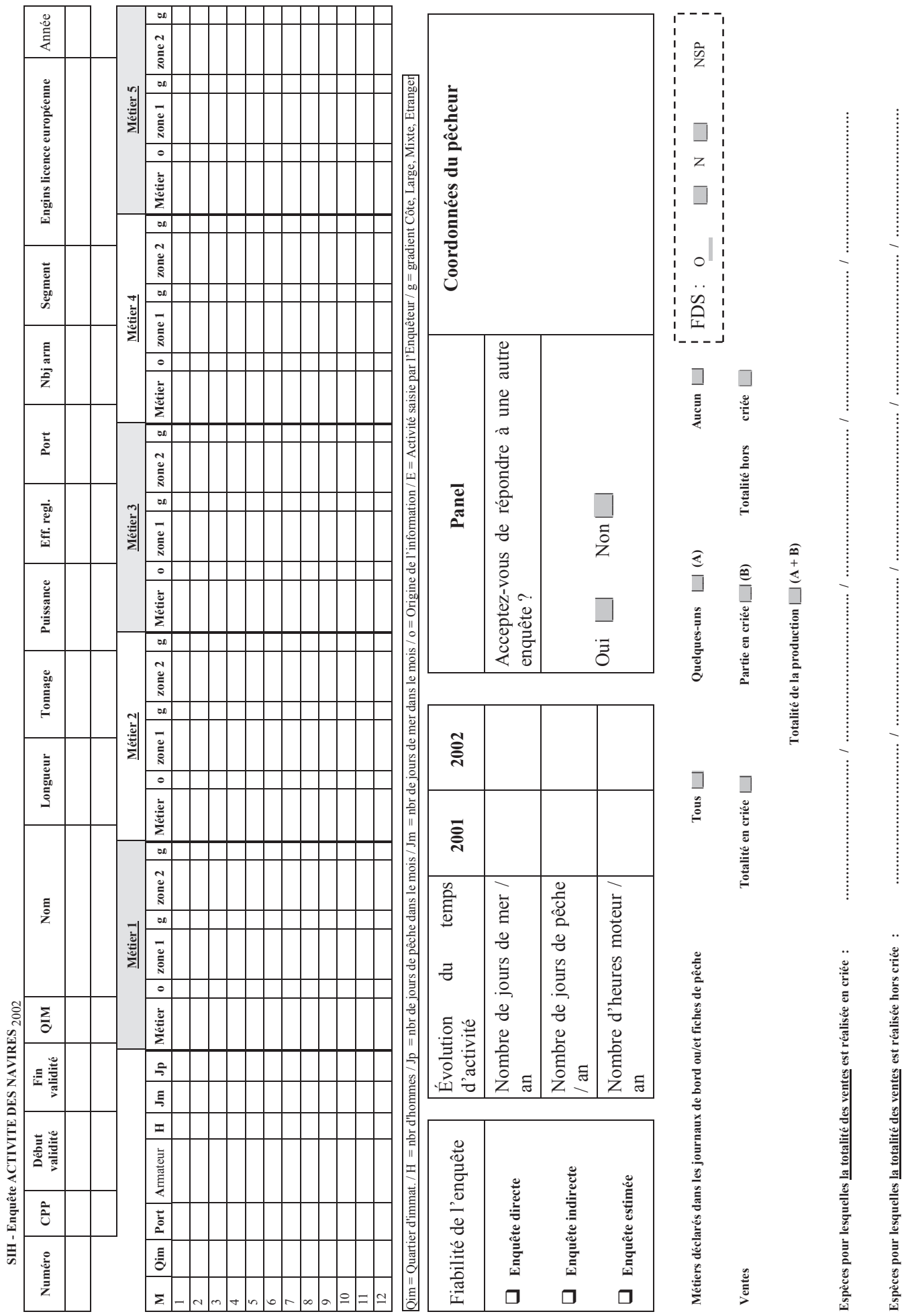


criées et hors criées). L'enquêteur s'appuie sur ces éléments, les confirme ou non et les complète par enquête sur le terrain.

Après transmission au site central de l'Ifremer, les données d'activité sont validées et stockées en base.

\section{Enquêtes économiques}

Les principaux objectifs de l'enquête économique sont d'améliorer la connaissance des pêcheries bretonnes dans leur diversité au travers d'une approche monétaire de l'activité des navires (production en valeur, moyens de production mobilisés), permettant une analyse et un suivi des performances économiques des flottilles et des indicateurs généraux du secteur des pêches comme par exemple la rémunération du capital investi et de l'emploi. Cette collecte a également pour finalité de renforcer les analyses scientifiques en matière de gestion durable des pêcheries, permettant de mesurer l'influence de l'environnement des entreprises (biologique, économique) et des politiques publiques sur la situation du secteur.

Les données collectées visent à restituer les indicateurs économiques par segment de flotte. La dispersion des résultats constatés l'année n-1 et le niveau de précision retenu comme cible permettent de définir le nombre de navires à sélectionner dans chaque strate. Les critères retenus sont la taille, le métier pratiqué et la région de rattachement du navire.

\section{Le plan d'échantillonnage}

Il est procédé à une structuration de la flotte de pêche métropolitaine qui s'appuie sur des variables disponibles à l'échelle de la population: taille du navire, appartenance à une flottille en terme de métiers pratiqués, localisation géographique du navire (Daurès et al., 2003). Ces informations sont en partie disponibles à partir du fichier des navires de pêche actifs. Par ailleurs, la totalité des navires de la flotte de pêche française métropolitaine fait l'objet d'une enquête annuelle sur les métiers exercés tout au long de l'année (type d'engins, espèces cibles et zones de pêche recensés mensuellement dans un calendrier d'activité) et de l'intensité de leur activité (nombre de jours de mer, d'heures moteur...).

Sur la base de ces informations individuelles et exhaustives, il est possible de mettre en place un plan d'échantillonnage stratifié pour la collecte des informations économiques permettant le calcul des indicateurs requis par le règlement. La stratification de la flotte de pêche est donc basée sur des critères d'activité (engins utilisés et combinaison de métiers au cours de l'année), de longueur du navire, de localisation géographique (région maritime d'appartenance).

On détermine un taux d'échantillonnage national à hauteur de près de $40 \%$ de la population totale. On calcule le nombre optimal de navires à enquêter pour chaque strate en fonction des contraintes du nombre total de navires à enquêter, de la taille de la strate et en tenant compte de la variabilité observée l'année précédente pour la variable Chiffre d'affaires. La formule suivante est ainsi appliquée:

$$
\mathrm{n}_{\mathrm{h}}=\operatorname{ENTIER}\left(\frac{\mathrm{n} \times \mathrm{N}_{\mathrm{h}} \times \mathrm{CV}_{\mathrm{yh}}}{\sum_{\mathrm{h}=1}^{\mathrm{H}} \mathrm{N}_{\mathrm{h}} \times \mathrm{CV}_{\mathrm{yh}}}\right)+1, \mathrm{~h}=1, \ldots, \mathrm{H}
$$

où $\mathrm{n}_{\mathrm{h}}$ est la taille de l'échantillon pour la strate $\mathrm{h}$. 
$\mathrm{N}_{\mathrm{h}}$ est la taille de la strate $\mathrm{h}$.

$\mathrm{CV}_{\mathrm{yh}}$ est le coefficient de variation du chiffre d'affaires (CA) pour la strate h et pour l'année y.

n est calculé de telle sorte que l'on obtienne au final le nombre total de navires à enquêter (soit environ 1900).

De plus, sachant qu'une strate est généralement représentée sur plusieurs quartiers, le nombre de navires à enquêter dans le quartier qi pour la strate h résultera d'un tirage systématique par quartier et taille.

L'information est collectée par enquête directe auprès des patrons pêcheurs pour un échantillon total d'environ 800 navires par an pour la France métropolitaine. Une partie de l'échantillon global est à nouveau enquêtée l'année suivante. L'objectif est de mesurer les variations interannuelles des indicateurs économiques qui ne soient pas affectées par des variations de structure de population d'une part et d'améliorer les procédures de validation des données collectées d'autre part.

Le panel représente au plus $75 \%$ du nombre de navires à enquêter dans une strate, le reste des navires étant choisi sur la base d'un tirage aléatoire. En d'autres termes, la part du tirage aléatoire dans le nombre d'enquêtes par strate doit toujours être au moins égale à $25 \%$ afin d'assurer le renouvellement annuel partiel du panel.

\section{Le questionnaire économique}

La collecte des données économiques répond à un objectif scientifique précis d'améliorer la connaissance de la flotte de pêche française dans sa diversité. Cela passe donc par: - un approfondissement de la connaissance en termes économiques de l'activité des navires et des moyens de production mis en œuvre (travail, capital investi);

- la mesure des recettes et des coûts générés annuellement par l'activité au niveau général et par métiers pratiqués.

Elle se réfère ici à des données individuelles détaillées relatives aux revenus et aux coûts des navires.

Le questionnaire est composé de 9 blocs de questions :

- bloc 1 : identité et situation de la personne enquêtée;

- bloc 2: activité du navire en termes de métiers pratiqués, engins utilisés, effort de pêche par métier et calendrier d'activité ;

- bloc 3 : coûts d'entretien et de renouvellement des apparaux (engins de pêche, gréements et auxiliaires de pont);

- bloc 4 : recettes et consommations intermédiaires au niveau général et par métier;

- bloc 5 : coûts d'exploitation du navire (taxes, cotisations sociales et diverses, coûts de maintenance et réparation...);

- bloc 6: évaluation physique et économique de l'investissement initial à la pêche;

- bloc 7: évaluation physique et économique de l'investissement dans les équipements autres que les engins de pêche (moteur, équipements de passerelle, équipements de stockage et de conditionnement des captures, équipements non embarqués);

- bloc 8: équipage et mode de rémunération;

- bloc 9: autres activités commerciales de l'armateur.

$\mathrm{Au}$ total, le questionnaire comprend près de 60 questions qui seront saisies au moyen d'un logiciel spécifique élaboré pour le transfert et l'organisation en base des données économiques collectées en site central. 
Ce questionnaire, qui a été élaboré dans le cadre d'un groupe de travail associant scientifiques et universitaires suite à des expériences d'enquêtes économiques plus locales déjà réalisées, s'est également beaucoup appuyé sur l'expérience de la statistique agricole, notamment dans le cadre du réseau d'information comptable agricole dans le passé.

\section{La méthode basée sur des enquêtes directes}

L'enquête directe porte sur un sous-échantillon d'environ 800 navires répartis le long des façades maritimes : mer du Nord, Manche, Atlantique et Méditerranée. Elle est réalisée à partir de moyens propres à l'Ifremer (réseau de techniciens enquêteurs) complétés par un appui externe en sous-traitance. Il convient de rappeler que le réseau de techniciens enquêteurs qui réalise une grande partie des enquêtes économiques sur la façade Atlantique connaît bien le monde de la pêche puisqu'il effectue par ailleurs l'enquête exhaustive annuelle sur l'activité des navires.

Un manuel d'enquête spécifie les méthodes de travail des enquêteurs pour limiter ainsi les biais possibles. Ce manuel fait l'objet de mises à jour régulières intégrant les évolutions méthodologiques nécessaires et les éventuels questionnements.

Les navires sont enquêtés sur l'année de référence n-1 sur la base d'un questionnaire harmonisé au niveau national. Par ailleurs, un questionnaire simplifié adapté aux navires déjà interrogés l'année précédente (panel) est utilisé.

Les données collectées par voie d'enquêtes directes permettent d'estimer des indicateurs de coûts et de revenus mais également des indicateurs relatifs au capital et à l'emploi. Les données de base font l'objet de procédures de validation, qualification, poststratification et redressement de l'information avant le calcul en routine des indicateurs et des estimateurs. Ces procédures s'appuient d'une part sur des contrôles de cohérence interne aux données collectées et d'autre part sur la confrontation de ces données avec des données externes: informations auxiliaires disponibles à travers des conventions avec les institutions publiques (réseau intercriées, logbooks, rôles d'équipage...), données économiques complémentaires collectées auprès des fournisseurs de la pêche et sociétés d'avitaillement (prix du gazole, des engins...). Par ailleurs, pour valider la structure des coûts d'exploitation, les données semi-agrégées de l'enquête économique sont confrontées autant que faire se peut aux données comptables collectées par ailleurs.

\section{Typologies de la flotte}

Avant d'aborder les méthodologies adoptées conduisant à l'élaboration de typologies de la flotte, il est utile de préciser quelques définitions et concepts.

\section{Quelques définitions: les opérations de pêche, les métiers et les flottilles}

L'opération unitaire de pêche est le trait de chalut, le coup de drague, la calée et la levée d'un filet. C'est le couple « technique (engin de pêche) - ressource ». Il détermine la notion de métier qui correspond à la mise en œuvre d'un engin de pêche sur une ou plusieurs espèces cibles sur une zone donnée et pendant une période donnée.

L'opération unitaire de pêche qui dure de quelques minutes à quelques heures sur une aire de quelques centaines de mètres à quelques kilomètres carrés est répétée par le pêcheur au cours de la sortie en mer, ou marée; cette dernière peut durer de quelques 
heures à quelques jours sur une aire de quelques kilomètres à quelques dizaines de kilomètres carrés. Ces marées sont elles-mêmes répétées durant la saison de pêche qui dure de quelques semaines à l'année, sur une aire de quelques kilomètres à quelques milliers de kilomètres carrés.

Dans son calendrier annuel d'activité, l'entreprise de pêche choisit de pratiquer un ou plusieurs métiers (en fonction de la ressource mais aussi de l'environnement économique et social): les navires spécialisés sur un métier toute l'année seront souvent mobiles, se déplaçant dans toute ou partie de l'aire de répartition de l'espèce recherchée (parfois très étendue), tandis que les navires sédentaires ou à faible rayon d'action seront souvent polyvalents, exerçant plusieurs métiers au cours de l'année et parfois au cours d'un même mois.

L'analyse des activités de pêche permet d'individualiser des groupes de patrons pêcheurs ou d'unités d'exploitation, ayant des stratégies d'exploitation relativement homogènes. Ces stratégies s'expriment par le choix d'une combinaison particulière de métiers pratiqués. C'est la notion de type d'exploitation ou de flottille. Ainsi, la flotte de pêche d'une région donnée est structurée en différentes flottilles.

Différentes flottilles peuvent pratiquer un même métier; selon les stratégies, il peut s'agir d'un métier de base, structurant ou au contraire un métier d'appoint voire d'opportunité.

En résumé, chaque unité de pêche alloue au cours de l'année un effort de pêche sur différentes ressources en pratiquant différents métiers selon la stratégie choisie, modulée par le contexte biologique et socio-économique.

\section{Traitements préliminaires des données d'activité}

\section{Tables de référence}

Les informations collectées par les enquêteurs décrivent l'activité du navire au niveau le plus détaillé. Ainsi, le(s) métier(s) pratiqué(s) chaque mois de l'année de référence sont renseignés à un niveau fin de couplage de l'engin et de l'espèce cible (ou groupe d'espèces). Il en va de même pour les zones de pêche (rectangles statistiques).

Dans l'objectif de pouvoir décrire l'activité des navires de manière plus ou moins détaillée selon les besoins des analyses, des procédures d'agrégation ont été définies pour l'ensemble des variables renseignées par les enquêteurs: métiers, zones de pêche, gradients (ou rayon d'action).

\section{Procédures d'agrégation des métiers}

La table de référence des métiers pratiqués propose actuellement aux enquêteurs 316 modalités (couples engin $\times$ espèce cible). Des regroupements de métiers sont ensuite opérés à plusieurs niveaux.

Les analyses typologiques qui suivent s'appuient sur les niveaux d'agrégation des métiers suivants (tableau 40):

- engin - groupe d'espèces (MET3);

- technique de pêche (MET 4);

- famille d'engins (MET5);

- catégories de grandes flottilles selon le règlement CE (MET8) 
Remarque : les procédures d'agrégation sont multiples et fonction des besoins propres à chaque analyse de flottille (problématiques régionales, zoom sur une pêcherie...).

Tableau 40. Table d'agrégation des métiers. Référentiel Ifremer.

\begin{tabular}{|c|c|c|c|c|c|c|c|}
\hline \multicolumn{2}{|c|}{$\begin{array}{c}\text { MET3 = Engin * Groupe } \\
\text { d'espèces }\end{array}$} & \multicolumn{2}{|c|}{$\begin{array}{l}\text { MET } 4 \text { = Technique } \\
\text { de pêche }\end{array}$} & \multicolumn{2}{|c|}{$\begin{array}{l}\text { MET } 5=\text { Famille } \\
\text { d'engins }\end{array}$} & \multicolumn{2}{|c|}{$\begin{array}{l}\text { MET } 8=\text { Flotille } \\
\text { RegCE }\end{array}$} \\
\hline M3_FPOAG & Casier à anguilles & \multirow{7}{*}{ M4_FPO } & \multirow{7}{*}{ Casier } & \multirow{7}{*}{ M5_C } & \multirow{7}{*}{ Casier } & & \\
\hline M3_FPOBU & Casier à buccins & & & & & & \\
\hline M3_FPOCE & $\begin{array}{l}\text { Casier à seiches, } \\
\text { poulpes }\end{array}$ & & & & & & \\
\hline M3_FPOCR & $\begin{array}{l}\text { Casier à crevettes } \\
\text { (bouquets) }\end{array}$ & & & & & & \\
\hline M3_FPODP & Casier à poissons & & & & & & \\
\hline M3_FPOGC & $\begin{array}{l}\text { Casier à gros } \\
\text { crustacés }\end{array}$ & & & & & & \\
\hline M3_FPOPC & $\begin{array}{l}\text { Casier à petits } \\
\text { crustacés }\end{array}$ & & & & & & \\
\hline M3_FVEAG & $\begin{array}{l}\text { Capéchade } \\
\text { à anguilles }\end{array}$ & \multirow{2}{*}{ M4_FVE } & \multirow{2}{*}{ Capéchade } & \multirow{2}{*}{ M5_V } & \multirow{2}{*}{ Capéchade } & & \\
\hline M3_FVEDP & $\begin{array}{l}\text { Capèchade } \\
\text { à poissons }\end{array}$ & & & & & & \\
\hline M3_GGMCC & \begin{tabular}{|l|} 
Filet grandes \\
mailles \\
à crustacés \\
\end{tabular} & \multirow{3}{*}{ M4_GGM } & \multirow{3}{*}{$\begin{array}{l}\text { Filet grandes } \\
\text { mailles }\end{array}$} & \multirow{6}{*}{ M5_F } & \multirow{6}{*}{ Filet } & & \\
\hline M3_GGMDP & \begin{tabular}{|l} 
Filet grandes \\
mailles \\
à poissons
\end{tabular} & & & & & & \\
\hline M3_GGMTH & $\begin{array}{l}\text { Filets à grandes } \\
\text { mailles à thons }\end{array}$ & & & & & M8_DO & Dormants \\
\hline M3_GPMCC & \begin{tabular}{|l|} 
Filet petites \\
mailles \\
à crustacés
\end{tabular} & \multirow{3}{*}{ M4_GPM } & \multirow{3}{*}{$\begin{array}{l}\text { Filet petites } \\
\text { mailles }\end{array}$} & & & & \\
\hline M3_GPMDP & \begin{tabular}{|l} 
Filet petites \\
mailles \\
à poissons \\
\end{tabular} & & & & & & \\
\hline M3_GPMML & \begin{tabular}{|l} 
Filet petites \\
mailles \\
à mollusques \\
\end{tabular} & & & & & & \\
\hline M3_LHDP & $\begin{array}{l}\text { Ligne à main } \\
\text { à poissons }\end{array}$ & \multirow{3}{*}{ M4_LH } & \multirow{3}{*}{ Ligne à main } & \multirow{3}{*}{ M5_LH } & \multirow{3}{*}{$\begin{array}{l}\text { Ligne à } \\
\text { main }\end{array}$} & & \\
\hline M3_LHML & $\begin{array}{l}\text { Ligne à main } \\
\text { à mollusques }\end{array}$ & & & & & & \\
\hline M3_LHTH & $\begin{array}{l}\text { Lignes à main } \\
\text { à thons }\end{array}$ & & & & & & \\
\hline M3_LLDP & $\begin{array}{l}\text { Palangre } \\
\text { à poissons }\end{array}$ & \multirow[t]{2}{*}{ M4_LL } & \multirow[t]{2}{*}{ Palangre } & \multirow[t]{2}{*}{ M5_LL } & \multirow[t]{2}{*}{ Palangre } & & \\
\hline M3_LLTH & Palangre à thons & & & & & & \\
\hline M3_PSM & $\begin{array}{l}\text { Plongée sous- } \\
\text { marine }\end{array}$ & M4_PSM & $\begin{array}{l}\text { Plongée sous } \\
\text { marine }\end{array}$ & M5_PL & $\begin{array}{l}\text { Plongée } \\
\text { sous marine }\end{array}$ & & \\
\hline M3_PRI & Pêche de rivage & M4_PRI & $\begin{array}{l}\text { Pêche de } \\
\text { rivage }\end{array}$ & M5_R & $\begin{array}{l}\text { Pêche de } \\
\text { rivage }\end{array}$ & & \\
\hline
\end{tabular}




\begin{tabular}{|c|c|c|c|c|c|c|c|}
\hline \multicolumn{2}{|c|}{$\begin{array}{c}\text { MET3 }=\underset{\text { Engin * Groupe }}{\text { d'espèces }}\end{array}$} & \multicolumn{2}{|c|}{$\begin{array}{l}\text { MET } 4=\text { Technique } \\
\text { de pêche }\end{array}$} & \multicolumn{2}{|c|}{$\begin{array}{l}\text { MET } 5=\text { Famille } \\
\text { d'engins }\end{array}$} & \multicolumn{2}{|c|}{$\begin{array}{c}\text { MET } 8=\text { Flotille } \\
\text { RegCE }\end{array}$} \\
\hline M3_SCOGO & $\begin{array}{l}\text { Scoubidou à } \\
\text { goémon (algues) }\end{array}$ & M4_SCO & Scoubidou & M5_S & Scoubidou & \multirow{17}{*}{ M8_MO } & \multirow{16}{*}{ Mobiles } \\
\hline M3_PS_DP & Senne à poissons & \multirow{2}{*}{ M4_PS } & \multirow{2}{*}{ Senne } & \multirow{2}{*}{ M5_P } & \multirow{2}{*}{ Senne } & & \\
\hline M3_PS_TH & Senne à thons & & & & & & \\
\hline M3_DRBCJ & $\begin{array}{l}\text { Drague à coquille } \\
\text { saint-jacques }\end{array}$ & \multirow{6}{*}{ M4_DRB } & \multirow{6}{*}{ Drague } & \multirow{6}{*}{ M5_D } & \multirow{6}{*}{ Drague } & & \\
\hline M3_DRCR & $\begin{array}{l}\text { Drague } \\
\text { à crevettes } \\
\text { (bouquets) }\end{array}$ & & & & & & \\
\hline M3_DRBDP & $\begin{array}{l}\text { Drague } \\
\text { à poissons }\end{array}$ & & & & & & \\
\hline M3_DRBEC & $\begin{array}{l}\text { Drague } \\
\text { à échinodermes }\end{array}$ & & & & & & \\
\hline M3_DRBGO & $\begin{array}{l}\text { Drague } \\
\text { à gélidium }\end{array}$ & & & & & & \\
\hline M3_DRBML & $\begin{array}{l}\text { Drague } \\
\text { à mollusques }\end{array}$ & & & & & & \\
\hline M3_OTBCR & $\begin{array}{l}\text { Chalut de fond } \\
\text { à crevettes } \\
\text { (bouquets) }\end{array}$ & \multirow{4}{*}{ M4_OTB } & \multirow{4}{*}{$\begin{array}{l}\text { Chalut de } \\
\text { fond }\end{array}$} & \multirow{7}{*}{ M5_H } & \multirow{7}{*}{ Chalut } & & \\
\hline M3_OTBDP & $\begin{array}{l}\text { Chalut de fond } \\
\text { à poissons }\end{array}$ & & & & & & \\
\hline M3_OTBLN & $\begin{array}{l}\text { Chalut de fond } \\
\text { à langoustines }\end{array}$ & & & & & & \\
\hline M3_OTBML & $\begin{array}{l}\text { Chalut de fond } \\
\text { à mollusques }\end{array}$ & & & & & & \\
\hline M3_OTMDP & $\begin{array}{l}\text { Chalut pélagique } \\
\text { à poissons } \\
\end{array}$ & \multirow{3}{*}{ M4_OTM } & \multirow{3}{*}{$\begin{array}{l}\text { Chalut } \\
\text { pélagique }\end{array}$} & & & & \\
\hline M3_OTMML & $\begin{array}{l}\text { Chalut pélagique } \\
\text { à mollusques } \\
\end{array}$ & & & & & & \\
\hline M3_OTMTH & $\begin{array}{l}\text { Chalut pélagique } \\
\text { à thons }\end{array}$ & & & & & & \\
\hline M3_TAMAG & Tamis à civelles & M4_TAM & Tamis & M5_T & Tamis & & \\
\hline M3_ELE & Élevage & M4_ELE & Élevage & M5_E & Élevage & \multirow{5}{*}{ M8_AU } & \multirow{5}{*}{ Autres } \\
\hline M3_AP & Appât & M4_AP & Appât & M5_AP & Appât & & \\
\hline M3_AUT & $\begin{array}{l}\text { Activite autre } \\
\text { que la pêche }\end{array}$ & M4_AUT & \begin{tabular}{|l|}
$\begin{array}{l}\text { Autres } \\
\text { activités }\end{array}$ \\
\end{tabular} & M5_X & \begin{tabular}{|l|} 
Autres \\
activités
\end{tabular} & & \\
\hline M3_INA & Inactif & M4_INA & Inactif & M5_INA & \begin{tabular}{|l|} 
Inactif \\
\end{tabular} & & \\
\hline M3_INE & Inexistant & M4_INE & Inexistant & M5_INE & Inexistant & & \\
\hline
\end{tabular}

\section{Procédures d'agrégation des zones de pêche}

La table de référence des zones de pêche présente actuellement 3087 modalités allant du sous-rectangle statistique aux grandes zones internationales définies par la FAO.

Dans le cas des analyses typologiques nationales, nous avons synthétisé les résultats à l'échelle de la division CIEM pour les zones de pêches non bordières du littoral atlantique français et de la sous-division pour les divisions 7 (mer d'Irlande, Manche et mer Celtique...) et 8 (golfe de Gascogne), étant donné la concentration des navires français dans ces zones (tableau 41). 
Tableau 41. Table d'agrégation des zones de pêche - Référentiel international.

\begin{tabular}{|c|c|c|c|}
\hline \multicolumn{2}{|r|}{ Division CIEM } & \multicolumn{2}{|r|}{ Sous Division CIEM } \\
\hline 1 & Mer de Barents & & \\
\hline 2 & Mer de Norvège, Spitzberg, île aux Ours & & \\
\hline 3 & Skagerrak, Kattegat, Sund, Belts, Baltique & & \\
\hline 4 & Mer du Nord & & \\
\hline 5 & Fonds d'Islande et des Féroë & & \\
\hline 6 & Rockall, ouest de l'Écosse & & \\
\hline \multirow{10}{*}{7} & \multirow{10}{*}{ Mer Irlande, Manche, mer Celtique... } & $7 \mathrm{~A}$ & Mer d'Irlande \\
\hline & & 7B & Ouest de l'Irlande \\
\hline & & $7 \mathrm{C}$ & Banc de Porcupine \\
\hline & & $7 \mathrm{D}$ & Manche orientale \\
\hline & & $7 \mathrm{E}$ & Manche occidentale \\
\hline & & $7 \mathrm{~F}$ & Canal de Bristol \\
\hline & & $7 \mathrm{G}$ & Mer Celtique Nord \\
\hline & & $7 \mathrm{H}$ & Mer Celtique Sud \\
\hline & & $7 \mathrm{~J}$ & Sud-ouest de l'Irlande Est \\
\hline & & $7 \mathrm{~K}$ & Sud-ouest de l'Irlande Ouest \\
\hline \multirow{5}{*}{8} & \multirow{5}{*}{ Golfe de gascogne } & $8 \mathrm{~A}$ & Golfe de Gascogne Nord \\
\hline & & $8 \mathrm{~B}$ & Golfe de Gascogne central \\
\hline & & $8 \mathrm{C}$ & Golfe de Gascogne Sud \\
\hline & & $8 \mathrm{D}$ & Golfe de Gascogne large \\
\hline & & $8 \mathrm{E}$ & Ouest du Golfe de Gascogne \\
\hline 9 & Eaux portuaires & & \\
\hline
\end{tabular}

\section{Procédure d'agrégation des gradients et de détermination du rayon d'action des navires}

À chaque zone de pêche, il est demandé aux enquêteurs d'associer une information complémentaire relative au gradient côte-large (tableau 42).

Tableau 42. Table d'agrégation des gradients. Référentiel Ifremer.

\begin{tabular}{|l|c|c|c|c|}
\hline \multicolumn{1}{|c|}{ Distance à la côte } & \multicolumn{2}{c|}{ Gradient Niveau 1 } & \multicolumn{2}{c|}{ Gradient Niveau 2 } \\
\hline Eaux continentales & $\mathrm{F}$ & Fluvial & $\mathrm{C}$ & Côtier \\
\hline Lagune, Grau, Étang & $\mathrm{G}$ & GLE & $\mathrm{C}$ & Côtier \\
\hline Intérieur de 12 milles & $\mathrm{C}$ & Côtier & $\mathrm{C}$ & Côtier \\
\hline Zones anglo-normandes & $\mathrm{J}$ & JerseyGuer & $\mathrm{C}$ & Côtier \\
\hline Dans et hors des 12 milles & $\mathrm{M}$ & Mixte & $\mathrm{M}$ & Mixte \\
\hline Hors des 12 milles & $\mathrm{L}$ & Large & $\mathrm{L}$ & Large \\
\hline Hors de ZEE européennes & $\mathrm{E}$ & Étranger & $\mathrm{L}$ & Large \\
\hline
\end{tabular}

En cumulant sur l'année, pour chaque bateau, le nombre de mois d'activité exercé par gradient, on détermine le « rayon d'action » annuel moyen du navire selon la procédure suivante: 
- les bateaux qui ont exercé plus de $75 \%$ de leur activité dans la zone des 12 milles sont qualifiés de « côtiers »;

- les bateaux qui ont exercé moins de $75 \%$ de leur activité dans la zone des 12 milles sont qualifiés de « larges »;

- les autres sont qualifiés de « mixtes ».

\section{Construction d'un tableau de données préalable à l'analyse typologique}

L'élaboration des typologies repose sur la construction d'un tableau annuel synthétique s'appuyant sur les informations contenues dans la base de données « Activité des navires » et les procédures d'agrégation des variables précédemment décrites (cf. tableau 43).

Outre les caractéristiques techniques et administratives individuelles, sont donc incluses les données résultant d'un traitement annuel:

- port d'exploitation principal;

- nombre de mois d'activité par sous-division CIEM ou division CIEM;

- caractérisation synthétique de l'activité selon le rayon d'action: côte, mixte ou large;

- cumul annuel du nombre de mois d'activité tous métiers confondus;

- cumul annuel du nombre de mois d'activité par métier selon différents niveaux d'agrégation.

Ces dernières données constituent les variables actives dans les analyses typologiques standards.

Tableau 43. Table synthétique. Informations sur l'activité annuelle par navire.

\begin{tabular}{|l|l|l|}
\hline \multicolumn{1}{|c|}{ Variables } & \multicolumn{1}{c|}{ Descriptif } & \multicolumn{1}{c|}{ Source } \\
\hline ocean & Atlantique/Méditerranée & POP \\
\hline Num_nav & Numéro d'immatriculation du navire & POP \\
\hline Nom_nav & Nom du navire & POP \\
\hline Q_immat & Quartier d'immatriculation du navire & POP \\
\hline Nom_quartier & Nom du quartier & POP \\
\hline RG & Région d'appartenance du navire & POP \\
\hline SRG & Sous-région d'appartenance du navire & POP \\
\hline Num_util & $\begin{array}{l}\text { Numéro d'immatriculation de l'armateur } \\
\text { du navire }\end{array}$ & POP \\
\hline An_nais & $\begin{array}{l}\text { Année de naissance de l'armateur } \\
\text { du navire }\end{array}$ & POP \\
\hline Cod_typ_nav & Type de navire (Type POP) & POP \\
\hline An_constr & Année de construction du navire & POP \\
\hline Long_cm & Longueur du navire en cm & POP \\
\hline Largeur & Largueur du navire & POP \\
\hline Jaugeb_100etx & Jauge du navire en 100ext & POP \\
\hline Jgt & Jauge jgt du navire & POP \\
\hline Puis_kw & Puissance du navire en kw & POP \\
\hline seg & Segmentation POP du anvire & POP \\
\hline eng1 & Engin POP 1 & POP \\
\hline eng2 & Engin POP 2 & POP \\
\hline
\end{tabular}




\begin{tabular}{|c|c|c|}
\hline Variables & Descriptif & Source \\
\hline eng3 & Engin POP 3 & POP \\
\hline Class_an_constr & $\begin{array}{l}\text { Classe des années de construction } \\
\text { des navires }\end{array}$ & REF Ifremer \\
\hline ClassL7 & Classe de longueur (7 classes) & REF Ifremer \\
\hline Libéllé_ClassL7 & Libéllé classe de longueur (7 classes) & REF Ifremer \\
\hline ClassL4 & Classe de longueur ( 4 classes) & REF Ifremer \\
\hline Libéllé_ClassL4 & Libéllé classe de longueur (4 classes) & REF Ifremer \\
\hline Port & Port d'exploitation principal du navire & Enquêtes activité SIH \\
\hline Nombre de mois renseigné & $\begin{array}{l}\text { Nombre de mois renseignés } \\
\text { dans les données Activité }\end{array}$ & Enquêtes activité SIH \\
\hline Nombre de mois actif & $\begin{array}{l}\text { Nombre de mois actifs dans les données } \\
\text { Activité }\end{array}$ & Enquêtes activité SIH \\
\hline $\begin{array}{l}\text { Nombre de mois actif en } \\
\text { pêche }\end{array}$ & Nombre de mois d'activité à la pêche & Enquêtes activité SIH \\
\hline \multicolumn{3}{|c|}{$\begin{array}{l}\text { Nombre de mois d'activité par 1) chaque métier 2) chaque gradient 3) chaque zone à différents } \\
\text { niveaux d'agrégation }\end{array}$} \\
\hline & $\begin{array}{c}\text { Niveau d'aggrégation } \\
\text { MET } 3 \text { / MET } 30 \text { / MET } 4 \text { / MET } 5 \\
\end{array}$ & REF Ifremer \\
\hline $\mathrm{Nb}$ met 3 & Nombre de MET3 pratiqué & Enquêtes activité SIH \\
\hline Cumul mois met 3 & Cumul des mois niveau MET3 & Enquêtes activité SIH \\
\hline M3_XX & $\begin{array}{l}\text { Nombre de mois d'activité par type } \\
\text { de métier (niveau d'agrégation) }\end{array}$ & Enquêtes activité SIH \\
\hline $\mathrm{Nb}$ met 30 & Nombre de MET30 pratiqué & Enquêtes activité SIH \\
\hline Cumul mois met 30 & Cumul des mois niveau MET30 & Enquêtes activité SIH \\
\hline M30_XX & $\begin{array}{l}\text { Nombre de mois d'activité par type } \\
\text { de métier (niveau d'agrégation) }\end{array}$ & Enquêtes activité SIH \\
\hline $\mathrm{Nb}$ met 4 & Nombre de MET4 pratiqué & Enquêtes activité SIH \\
\hline Cumul mois met 4 & Cumul des mois niveau MET4 & Enquêtes activité SIH \\
\hline M4_XX & $\begin{array}{l}\text { Nombre de mois d'activité par type } \\
\text { de métier (niveau d'agrégation) }\end{array}$ & Enquêtes activité SIH \\
\hline $\mathrm{Nb}$ met 5 & Nombre de MET5 pratiqué & Enquêtes activité SIH \\
\hline Cumul mois met 5 & Cumul des mois niveau MET5 & Enquêtes activité SIH \\
\hline M5_XX & $\begin{array}{l}\text { Nombre de mois d'activité par type } \\
\text { de métier (niveau d'agrégation) }\end{array}$ & Enquêtes activité SIH \\
\hline $\mathrm{Nb}$ met 7 & Nombre de MET7 pratiqué & Enquêtes activité SIH \\
\hline Cumul mois met 7 & Cumul des mois niveau MET7 & Enquêtes activité SIH \\
\hline M7_XX & $\begin{array}{l}\text { Nombre de mois d'activité par type } \\
\text { de métier (niveau d'agrégation) }\end{array}$ & Enquêtes activité SIH \\
\hline \multirow[t]{2}{*}{ M8_XX } & $\begin{array}{l}\text { Nombre de mois d'activité par type } \\
\text { de métier (niveau d'agrégation) }\end{array}$ & Enquêtes activité SIH \\
\hline & $\begin{array}{l}\text { Niveau d'aggrégation } \\
\text { Gradient } 1 / \text { Gradient } 2\end{array}$ & REF Ifremer \\
\hline nb grad niveau 1 & Nombre de gradient niv1 déclaré & Enquêtes activité SIH \\
\hline nb mois Gradient Niv 1 & $\begin{array}{l}\text { Nombre de mois d'activité par type de } \\
\text { gradient niv1 }\end{array}$ & Enquêtes activité SIH \\
\hline
\end{tabular}




\begin{tabular}{|l|l|l|}
\hline \multicolumn{1}{|c|}{ Variables } & \multicolumn{1}{|c|}{ Descriptif } & \multicolumn{1}{c|}{ Source } \\
\hline nb grad niveau 2 & Nombre de gradient niv2 déclaré & Enquêtes activité SIH \\
\hline nb mois Gradietn Niv 2 & $\begin{array}{l}\text { Nombre de mois d'activité par type de } \\
\text { gradient niv2 }\end{array}$ & Enquêtes activité SIH \\
\hline Rayon d'action & $\begin{array}{l}\text { Rayon d'action du navire (Côtier/Mixte/ } \\
\text { Large) }\end{array}$ & \multicolumn{1}{|c|}{ Zones de Pêche : } \\
Enquêtes activité SIH \\
\hline Nb zones & Nombre de zones déclarées & REF International \\
\hline Cumul mois zones & Cumul Mois_Zones & Enquêtes activité SIH \\
\hline Type Zone XX & $\begin{array}{l}\text { Nombre de mois d'activité Zone } \\
\text { Division }\end{array}$ & Enquêtes activité SIH \\
\hline ENQUETEêtes activité SIH \\
\hline ENQUETE_SEM_2001 & Pointeur Données économiques 2000 & $\begin{array}{l}\text { Enquêtes } \\
\text { économiques SIH }\end{array}$ \\
\hline
\end{tabular}

\section{Description de la flotte par métiers}

Une analyse préliminaire du tableau 44 synthétique met en évidence:

- l'importance de certaines familles d'engins d'un point de vue du nombre de navires concernés et de l'activité totale (en nombre de mois d'activité),

- la polyvalence de l'activité des navires.

Les résultats suivants sont issus du traitement des calendriers d'activité 2000 des navires de pêche de la façade Manche - mer du Nord - Atlantique (tableau 44).

Tableau 44. Description par famille d'engins de l'activité des navires en 2000.

\begin{tabular}{lccccc}
\hline Famille d'engins & Code engin & Nombre de navires & $\%$ navires & $\begin{array}{c}\text { Nombre de } \\
\text { mois d'activité }\end{array}$ & Activité \\
\hline Chalut & $\mathrm{H}$ & 1541 & 21,9 & 14749 & 29,8 \\
Filet & $\mathrm{F}$ & 1408 & 20 & 10959 & 22,2 \\
Casier & $\mathrm{C}$ & 1003 & 14,2 & 6829 & 13,8 \\
Drague & $\mathrm{D}$ & 960 & 13,6 & 5111 & 10,3 \\
Palangre & $\mathrm{LL}$ & 644 & 9,1 & 4210 & 8,5 \\
Tamis & $\mathrm{T}$ & 764 & 10,8 & 2987 & 6 \\
Ligne & $\mathrm{LH}$ & 340 & 4,8 & 2118 & 4,3 \\
Rivage & $\mathrm{R}$ & 124 & 1,8 & 657 & 1,3 \\
Senne & $\mathrm{P}$ & 66 & 0,9 & 651 & 1,3 \\
Appât & $\mathrm{AP}$ & 70 & 1 & 412 & 0,8 \\
Aquaculture & $\mathrm{A}$ & 37 & 0,5 & 330 & 0,7 \\
Scoubidou & $\mathrm{SC}$ & 50 & 0,7 & 239 & 0,5 \\
Plongée & $\mathrm{PL}$ & 32 & 0,5 & 160 & 0,3 \\
Autres métiers & $\mathrm{X}$ & 10 & 0,1 & 44 & 0,1 \\
\hline
\end{tabular}

Note: Les métiers d'appât, essentiellement au lançon pour la pêche à la palangre, l'aquaculture et les autres métiers que la pêche sont mentionnés pour mémoire. 
Deux familles d'engins, le chalut et le filet, dominent la flotte de Manche et Atlantique. Chacune représente plus de $20 \%$ des navires et plus de $22 \%$ de l'activité. Avec 1541 navires concernés et 14749 mois d'activité, les métiers du chalutage concernent près de $22 \%$ de la flotte et près de $30 \%$ des mois d'activité. Plus de 1400 navires de la zone d'étude pratiquent les métiers du filet, tout ou partie de l'année, soit près de 11000 mois.

Deux autres groupes, ceux du casier et de la drague, représentent chacun plus de $10 \%$ de la flotte totale (autour de 1000 navires) et plus de $10 \%$ de l'activité (respectivement 6800 mois et 5100 mois).

Un groupe de trois engins représente encore plus de $8 \%$ de l'activité globale: la palangre est le cinquième groupe d'engin en termes d'activité avec 4210 mois d'activité et le sixième en nombre de navires concernés. Le tamis à civelles est le cinquième engin en nombre de navires concernés (764) et représente près de 3000 mois d'activité.

Les autres engins ont une importance plus secondaire, le plus souvent inférieure à $1 \%$ de l'activité globale, il s'agit des métiers de rivage (essentiellement la pêche à pied), de la senne, du scoubidou ou de la plongée.

Il convient d'ajouter que 1650 navires ont eu, au cours de l'année 2000, une période d'inactivité évaluée en moyenne à 4,2 mois. Rapportée à la flotte totale des 4142 navires, l'inactivité moyenne est de 1,7 mois. On dénombre 120 navires considérés comme totalement inactifs à la pêche en 2000, dont 9 conchyliculteurs stricts.

Sur la population des 4022 navires ayant été actifs à la pêche en 2000, le nombre moyen d'engins mis en œuvre est de 1,7. Près de $50 \%$ des navires (1997 navires) ont utilisé un seul type d'engin en 2000, $32 \%$ en ont mis deux en œuvre. Seulement $14 \%$ de la flotte ont mis en œuvre 3 familles d'engins et $4 \%$ quatre. Les navires encore plus polyvalents pratiquant cinq ou six types d'engins représentent $1 \%$ de la flotte (figures 116 et 117 p. XXIX).

La polyvalence est maximale pour les navires de 7 à 12 mètres. Les navires de plus de 24 mètres pratiquent tous un seul type d'engin. La polyvalence est d'autant plus faible que le navire a un rayon d'action important.

Ce constat de la polyvalence d'une large partie de la flotte justifie la mise en œuvre de typologies permettant de dégager les stratégies d'exploitation principales (combinaisons de métiers les plus fréquentes).

\section{La méthode Ifremer de stratification de la flotte de pêche}

Les analyses typologiques ont pour objectif de dégager la structure d'un ensemble de navires de pêche d'une zone donnée en procédant à une classification des navires selon plusieurs critères permettant de distinguer des groupes homogènes; il existe donc plusieurs typologies possibles selon la question posée et les variables utilisées. Les analyses réalisées en standard au sein du SIH portent sur les bilans annuels de la flotte active au sens du registre de la flotte au 31 décembre de l'année de référence, d'une part, en mer du Nord - Manche - Atlantique et en Méditerranée, d'autre part; elles peuvent aussi porter sur un bassin donné ou une région particulière.

Les méthodes classiques pour l'élaboration des typologies font appel aux analyses multivariées ou à des classifications systématisées sur la base de critères définis. Ces différentes méthodes ont été mises en œuvre par l'Ifremer qui a retenu depuis 2003 l'option de la classification systématisée. 


\section{Systématisation de la typologie nationale 2002}

Un des objectifs du Système d'informations halieutiques de l'Ifremer est de fournir chaque année des indicateurs synthétiques de l'ensemble des flottilles françaises, incluant des tendances interannuelles. Pour cela, il est nécessaire de disposer d'une typologie des flottilles stabilisée par une systématisation de la procédure de segmentation de la flotte.

La systématisation de la procédure de classification s'appuie sur une hiérarchisation des engins prenant en compte notamment leur influence sur l'investissement et la structure des coûts. Les résultats des enquêtes économiques montrent le poids relativement plus élevé de l'investissement pour les navires ayant une stratégie d'exploitation fondée sur les engins traînants, à taille de navire équivalente. Ils montrent également une dichotomie dans la structure des coûts entre engins traînants et dormants : importance relative des frais de carburant dans les charges d'exploitation plus élevée pour les arts traînants et des frais d'engins pour les arts dormants.

Les étapes qui conduisent à la typologie systématisée 2002 de l'Ifremer sont précisées dans Berthou et al. (2003) et peuvent être résumées comme suit.

Une première étape consiste à distinguer les navires « inactifs à la pêche » que l'on peut scinder en trois sous-groupes : les « strictement inactifs », les « autres activités que la pêche » et enfin, les « éleveurs ». Pour le reste, on se situe au niveau d'agrégation des métiers suivants : casier/verveux - métiers de l'hameçon (ligne/palangre) - divers métiers côtiers (rivage/plongée) - filet - chalut - drague - tamis - senne - scoubidou. Un ordre de priorité des techniques de pêche a été établi dans le traitement : chalut - drague - tamis arts dormants (casier/verveux - métiers de l'hameçon - divers métiers côtiers - filet), la senne et le scoubidou faisant l'objet d'un traitement à part.

L'étape suivante consiste à traiter d'abord l'ensemble des navires qui pratiquent le chalut puis ceux qui pratiquent la drague, puis le tamis et enfin ceux qui pratiquent uniquement les arts dormants. À chaque étape, on distingue les « exclusifs ou purs » des «polyvalents » qui sont eux-mêmes différenciés en fonction du nombre de métiers pratiqués ( 2 métiers, 3 métiers, etc.). Tant que l'on travaille sur les navires pratiquant sur l'année un art traînant (chalut - drague - tamis), on ne différencie pas les arts dormants pratiqués par ailleurs par ces navires.

Il reste alors à classer les navires pratiquant seulement les arts « dormants ». Comme pour les « traînants », on commence par distinguer les « exclusifs ou purs ». Les règles de décision sont les suivantes:

Une hiérarchisation des engins est établie: filet - casier - métiers de l'hameçon divers métiers côtiers.

Les combinaisons d'engins dormants « filet/casier » - « filet/métiers de l'hameçon » et « casier/métiers de l'hameçon » sont considérées comme une stratégie de pêche à part entière.

Dans certains cas de navires très polyvalents, la notion de dominance a été intégrée.

Quelques cas particuliers doivent être traités dans une dernière phase.

Les navires pratiquant la senne constituent une flottille à part entière, exceptés ceux dont la pratique du chalut est dominante. Ces derniers sont alors affectés dans les différents groupes de « chalutiers ».

Les navires pratiquant le scoubidou sont eux soit affectés au groupe des « divers métiers côtiers » soit à d'autres groupes de « dormants » selon leur(s) activité(s) connexe(s).

On gère également le cas des navires utilisant la Ligne à thons, pratique associée au filet à thon ou au chalut pélagique. 
Au final, chaque navire de la flotte mer du Nord - Manche - Atlantique est affecté à une flottille et à une sous-flottille d'une manière totalement automatisée (succession de requêtes Access). Cela présente l'avantage de pouvoir être répété simplement d'une année sur l'autre.

L'analyse automatisée de la flotte mer du Nord - Manche - Atlantique en 2002 permet de distinguer 14 flottilles (dont une flottille de navires inactifs à la pêche) et 33 sousflottilles (dont trois sous-flottilles correspondant aux inactifs à la pêche ; tableau 45).

Tableau 45. Typologie nationale 2002.

\begin{tabular}{|c|c|c|}
\hline Flottille Ifremer & Sous-flottille Ifremer & Population 2002 \\
\hline \multirow{3}{*}{ Chalutiers exclusifs } & Chalutiers de fond purs & 585 \\
\hline & Chalutiers mixtes purs & 129 \\
\hline & Chalutiers pélagiques purs & 69 \\
\hline \multirow{3}{*}{ Chalutiers non exclusifs } & Chalutiers-dragueurs & 466 \\
\hline & Chalutiers-tamiseurs & 174 \\
\hline & Chalutiers arts dormants & 74 \\
\hline \multirow{2}{*}{ Senneurs } & Bolincheurs & 33 \\
\hline & Senneurs tropicaux & 28 \\
\hline \multirow{2}{*}{ Dragueurs } & Dragueurs purs & 63 \\
\hline & Dragueurs polyvalents & 337 \\
\hline \multirow{2}{*}{ Tamiseurs } & Tamiseurs purs & 135 \\
\hline & Tamiseurs arts dormants & 289 \\
\hline \multirow{2}{*}{ Fileyeurs } & Fileyeurs purs & 437 \\
\hline & Fileyeurs polyvalents & 13 \\
\hline \multirow{2}{*}{ Fileyeurs-caseyeurs } & Fileyeurs-caseyeurs purs & 232 \\
\hline & Fileyeurs-caseyeurs polyvalents & 88 \\
\hline \multirow{2}{*}{ Fileyeurs métiers de l'hameçon } & Fileyeurs métiers de l'hameçon purs & 107 \\
\hline & Fileyeurs métiers de l'hameçon polyvalents & 47 \\
\hline \multirow{2}{*}{ Caseyeurs } & Caseyeurs purs & 190 \\
\hline & Caseyeurs polyvalents & 5 \\
\hline \multirow{2}{*}{ Caseyeurs métiers de l'hameçon } & Caseyeurs métiers de l'hameçon purs & 78 \\
\hline & Caseyeurs métiers de l'hameçon polyvalents & 26 \\
\hline \multirow{6}{*}{ Métiers de l'hameçon } & Ligneurs purs & 71 \\
\hline & Ligneurs polyvalents & 4 \\
\hline & Palangriers purs & 74 \\
\hline & Palangriers polyvalents & 2 \\
\hline & Ligneurs-palangriers purs & 34 \\
\hline & Ligneurs-palangriers polyvalents & 1 \\
\hline Canneurs de Dakar & Canneurs de Dakar & 5 \\
\hline Divers métiers côtiers & Divers métiers côtiers & 79 \\
\hline \multirow{3}{*}{ Inactifs à la pêche } & Éleveurs & 11 \\
\hline & Autres activités que la pêche & 2 \\
\hline & Strictement inactifs & 97 \\
\hline TOTAL & & 3985 \\
\hline
\end{tabular}




\section{Structure de la flotte de pêche bretonne}

Cette structuration résulte de la méthode de stratification présentée ci-dessus sur laquelle ont été appliqués de nouveaux regroupements. Ces derniers s'appuient sur une volonté de mise en conformité avec les réalités régionales et d'harmonisation avec les structurations existantes notamment celle de l'Observatoire économique régional de Bretagne.

Le tableau 46 présente les regroupements permettant de passer de la typologie de la flotte mer du Nord - Manche - Atlantique à celle de la Bretagne.

Tableau 46. Typologies des flottes bretonne et nationale (2002).

\begin{tabular}{|c|c|c|c|}
\hline \multicolumn{2}{|c|}{ Typologie Bretagne } & \multicolumn{2}{|c|}{ Typologie de la flotte en mer du Nord - Manche - Atlantique } \\
\hline Flottille & Sous-flottille & Flottille & Sous-flottille \\
\hline \multirow{6}{*}{ Chalutiers } & \multirow{3}{*}{ Chalutiers exclusifs } & \multirow{3}{*}{ Chalutiers exclusifs } & Chalutiers de fond purs \\
\hline & & & Chalutiers mixtes purs \\
\hline & & & Chalutiers pélagiques purs \\
\hline & \multirow{3}{*}{$\begin{array}{l}\text { Chalutiers } \\
\text { non exclusifs }\end{array}$} & \multirow{3}{*}{$\begin{array}{l}\text { Chalutiers non } \\
\text { exclusifs }\end{array}$} & Chalutiers-dragueurs \\
\hline & & & Chalutiers arts dormants \\
\hline & & & Chalutiers-tamiseurs \\
\hline Bolincheurs & Bolincheurs & Senneurs & Bolincheurs \\
\hline \multirow{2}{*}{ Dragueurs } & \multirow{2}{*}{ Dragueurs } & \multirow{2}{*}{ Dragueurs } & Dragueurs purs \\
\hline & & & Dragueurs polyvalents \\
\hline \multirow{15}{*}{ Dormants } & Caseyeurs exclusifs & Caseyeurs & Caseyeurs purs \\
\hline & Fileyeurs exclusifs & Fileyeurs & Fileyeurs purs \\
\hline & \multirow{3}{*}{$\begin{array}{l}\text { Métiers de } \\
\text { l'hameçon exclusifs }\end{array}$} & \multirow{3}{*}{$\begin{array}{l}\text { Métiers de } \\
\text { l'hameçon }\end{array}$} & Ligneurs purs \\
\hline & & & Palangriers purs \\
\hline & & & Ligneurs-palangriers purs \\
\hline & \multirow{10}{*}{$\begin{array}{l}\text { Dormants } \\
\text { polyvalents }\end{array}$} & \multirow{2}{*}{ Fileyeurs-caseyeurs } & Fileyeurs-caseyeurs purs \\
\hline & & & Fileyeurs-caseyeurs polyvalents \\
\hline & & \multirow{2}{*}{$\begin{array}{l}\text { Fileyeurs métiers } \\
\text { de l'hameçon }\end{array}$} & Fileyeurs métiers de l'hameçon purs \\
\hline & & & Fileyeurs métiers de l'hameçon polyvalents \\
\hline & & \multirow{2}{*}{$\begin{array}{l}\text { Caseyeurs métiers } \\
\text { de l'hameçon }\end{array}$} & Caseyeurs métiers de l'hameçon purs \\
\hline & & & Caseyeurs métiers de l'hameçon polyvalents \\
\hline & & \multirow{2}{*}{$\begin{array}{l}\text { Métiers } \\
\text { de l'hameçon }\end{array}$} & Ligneurs polyvalent \\
\hline & & & Ligneurs-palangriers polyvalents \\
\hline & & Caseyeurs & Caseyeurs polyvalents \\
\hline & & Fileyeurs & Fileyeurs polyvalents \\
\hline \multirow{3}{*}{ Divers } & \multirow{3}{*}{ Divers } & Tamiseurs & Tamiseurs arts dormants \\
\hline & & $\begin{array}{l}\text { Divers métiers } \\
\text { côtiers }\end{array}$ & Divers métiers côtiers \\
\hline & & Tamiseurs & Tamiseurs purs \\
\hline $\begin{array}{l}\text { Senneurs } \\
\text { tropicaux }\end{array}$ & Senneurs tropicaux & Senneurs & Senneurs tropicaux \\
\hline \multirow{3}{*}{$\begin{array}{l}\text { Inactifs } \\
\text { à la pêche }\end{array}$} & \multirow{3}{*}{ Inactifs à la pêche } & \multirow{3}{*}{ Inactifs à la pêche } & Éleveurs \\
\hline & & & Autres activités que la pêche \\
\hline & & & Strictement Inactifs \\
\hline
\end{tabular}


Les regroupements précédents permettent donc d'aboutir à la typologie Bretagne, qui distingue 7 flottilles et 11 sous-flottilles. Le tableau 47 présente la répartition des navires de chacune des sous-flottilles par classe de longueur.

Tableau 47. Répartition des flottilles bretonnes par classe de longueur (2002).

\begin{tabular}{|c|c|c|c|c|c|c|c|c|c|}
\hline \multicolumn{10}{|c|}{ Typologie Bretagne } \\
\hline Flottille & Sous-flottille & $<7 \mathrm{~m}$ & $7-9 \mathrm{~m}$ & $\begin{array}{c}9-12 \\
\mathrm{~m}\end{array}$ & $\begin{array}{c}12-16 \\
\mathrm{~m}\end{array}$ & $\begin{array}{c}16-20 \\
\mathrm{~m}\end{array}$ & $\begin{array}{c}20-24 \\
\mathrm{~m}\end{array}$ & $\begin{array}{c}24-40 \\
\mathrm{~m}\end{array}$ & $\begin{array}{c}>=40 \\
\mathrm{~m}\end{array}$ \\
\hline \multirow[b]{2}{*}{ Chalutiers } & Chalutiers exclusifs & & & 6 & 92 & 68 & 131 & 58 & 9 \\
\hline & $\begin{array}{l}\text { Chalutiers non } \\
\text { exclusifs }\end{array}$ & & 16 & 134 & 35 & 3 & & & \\
\hline Bolincheurs & Bolincheurs & & & & 17 & 3 & 1 & & \\
\hline Dragueurs & Dragueurs & 18 & 107 & 153 & 14 & 1 & & & \\
\hline \multirow{4}{*}{ Dormants } & Caseyeurs exclusifs & 23 & 24 & 17 & & 11 & 4 & & \\
\hline & Fileyeurs exclusifs & 21 & 42 & 42 & 31 & 11 & 4 & & \\
\hline & \begin{tabular}{|l|} 
Métiers de \\
l'hameçon exclusifs \\
\end{tabular} & 21 & 72 & 12 & 2 & & & 1 & \\
\hline & \begin{tabular}{|l|} 
Dormants \\
polyvalents
\end{tabular} & 79 & 95 & 51 & 12 & 3 & & & \\
\hline Divers & Divers & 69 & 47 & 13 & & & & & \\
\hline $\begin{array}{l}\text { Senneurs } \\
\text { tropicaux }\end{array}$ & Senneurs tropicaux & & & & & & & & 28 \\
\hline $\begin{array}{l}\text { Inactifs à la } \\
\text { pêche }\end{array}$ & Inactifs à la pêche & 21 & 11 & 1 & & 2 & & & \\
\hline
\end{tabular}

\section{Conclusion}

L'Ifremer a développé une méthode et acquis un savoir-faire en matière de collecte de données sur l'activité des navires à l'échelle nationale. Cette méthode est basée sur une information minimale mais exhaustive : le calendrier d'activité mensuel par métier. Elle est appliquée ici au niveau d'un secteur géographique précis : la Bretagne.

Ce type d'information permet de produire, à différentes échelles, un certain nombre d'indicateurs robustes de l'activité des navires (métiers pratiqués, engins, zones de pêche...) en fonction des stratégies d'exploitation et de mesurer leur évolution au cours du temps.

Par ailleurs, la structuration en flottilles est un préalable pertinent pour l'organisation de plans d'échantillonnage pour la collecte de données plus spécifiques (effort de pêche, débarquements et rejets, données biologiques et économiques).

\section{Effets économiques induits de la pêche côtière}

Les recherches menées dans ce domaine avaient pour objectif de mesurer les effets économiques et sociaux de l'activité de pêche côtière (pêche pratiquée dans la limite des 12 milles) du Mor-Braz sur une zone terrestre littorale contiguë. Cela a été réalisé en quantifiant les flux monétaires existants entre les acteurs du système halieutique et en déterminant les emplois générés par leurs activités. En d'autres termes, l'objectif était de mesurer combien un euro de produits de la pêche commercialisés au débarquement dans 
la zone d'étude génère d'euros dans le système halieutique local, mais aussi combien un emploi de marin en mer génère d'emplois à terre.

La première étape consiste à définir les espaces géographiques sur lesquels portent les analyses. Pour ce qui concerne la zone littorale le cadre spatial de référence choisi est celui de la «zone d'emploi ». La définition qu'en donne l'Insee est la suivante: « une zone d'emploi est un espace géographique à l'intérieur duquel la plupart des actifs résident et travaillent. Effectué conjointement par l'Insee et les services statistiques du ministère du Travail, le découpage en zones d'emploi constitue une partition du territoire adaptée aux études locales sur l'emploi et son environnement. Les déplacements domicile-travail constituent la variable de base pour la détermination de ce zonage ». Les deux zones d'emploi retenues sont les zones d'emploi d'Auray et de Vannes. Elles couvrent plus de $90 \%$ de l'espace littoral concerné. Pour ce qui concerne les flottilles à prendre en considération, l'Ifremer a dénombré 945 navires qui exercent leur activité plus ou moins régulièrement dans cette zone. Ces navires proviennent de 21 quartiers maritimes qui s'étalent de Caen à Bayonne. La provenance des navires étant trop dispersée, l'étude se limitera aux navires immatriculés dans les quartiers maritimes d'Auray et de Vannes (AY et VA). Les limites physiques de ces deux quartiers sont le ruisseau de Loperet (Erdeven) et la pointe de Loscolo (Penestin).

La seconde étape consiste en une identification et en une description la plus précise possible des acteurs concernés, aussi bien en mer qu'à terre. Pour ce faire, la notion de système halieutique a été retenue. Il se définit comme l'ensemble des acteurs qui interviennent pour faire vivre les activités de pêche ainsi que les relations qu'ils ont établies entre eux et avec leurs partenaires extérieurs. Il est constitué de l'ensemble des entreprises de pêche concernées, des établissements d'amont (auprès desquels elles s'approvisionnent en biens et services), des établissements d'aval qui participent à la commercialisation et à la valorisation des produits et des structures d'encadrement du secteur: Administration, Enseignement, Recherche... L'unité de base retenue pour effectuer la récolte de données et l'analyse est l'établissement : c'est une unité de production localisée géographiquement, individualisée mais dépendant juridiquement d'une entreprise. L'établissement constitue le niveau le mieux adapté à une approche géographique de l'économie. Il est relativement homogène et son activité principale apparaît proche du produit.

Les recherches pour identifier les établissements du système se sont effectuées à partir soit du code d'activité principale exercée (APE), soit de l'activité de l'établissement. Onze secteurs d'activité ont été retenus comme ayant a priori un lien fort avec le secteur de la pêche côtière. Tous les secteurs d'activités liés à la pêche n'ont pu être distingués à partir des codes APE. Les établissements en amont et les structures d'encadrement ont un code APE non-spécifique à la pêche comme par exemple: le commerce de détail de carburant, les banques, les assurances, l'Administration. Les listes d'établissements et de structures d'encadrement ont été établies à partir des fichiers de la base de données Sirene de l'Insee, complétées par la consultation d'annuaires spécialisés, d'informations recueillies sur Internet et de contacts téléphoniques directs. Ce recensement permet d'avoir une liste d'établissements plus proche de la réalité que la liste de l'Insee mais doit être considérée comme encore incomplète. Le recueil d'informations plus précises a été réalisé grâce à de nombreuses enquêtes et entretiens. Cette méthode est coûteuse en temps et en moyens et dépend de la disponibilité des personnes enquêtées, mais elle est la seule possible pour obtenir les informations recherchées. 
Pour ce qui concerne les navires, la plupart des informations proviennent du SIH de l'Ifremer, complété par celles fournies par les pêcheurs et par les structures d'encadrement à partir d'entretiens.

La troisième étape consiste à reconstituer les différents flux monétaires créés par les entreprises de pêche côtière de la zone étudiée. Cela concerne aussi bien les flux d'inputs (approvisionnement, entretien, réparation...) que ceux d'outputs (ventes de la production débarquée). À cette étape, il n'est pas possible de faire la distinction entre la part de ces flux qui proviennent ou entrent dans la zone étudiée et la part des flux qui concernent l'extérieur. Il s'agit donc de flux globaux sans considération quant à leur origine ou à leur destination géographique.

Pour évaluer les effets économiques directs induits, les données utilisées proviennent du traitement d'enquêtes menées par l'Ifremer dans le cadre du SIH. Les enquêtes sont annuelles et non-exhaustives. La méthode consiste à obtenir des valeurs de certains paramètres caractérisant la population totale des navires d'Auray et de Vannes à partir d'un échantillon. Ces paramètres sont évalués à partir d'estimateurs, élaborés grâce à l'emploi de techniques statistiques simples. Pour cela, la flotte d'Auray et de Vannes a été scindée en 12 flottilles. Chaque flottille regroupe des navires ayant une homogénéité relative de leur structure d'exploitation du point de vue des caractéristiques des navires, des combinaisons de métiers et de la structure des coûts.

Le chiffre d'affaires (flux d'outputs), les consommations intermédiaires (entretien du navire, matériel de pêche, vivres, glace, appâts, carburant et lubrifiants, services) (flux d'inputs), la valeur ajoutée brute et les charges de personnels, ont été estimés pour chaque flottille et pour la flotte totale. Les données utilisées sont celles fournies par les enquêtes économiques de l'Ifremer. La population mère est composée de 252 navires dont 41 ont été enquêtés. Le niveau d'échantillonnage est donc finalement de $16 \%$. Une stratification en fonction de la typologie de l'Ifremer ne pouvait convenir car pour 3 flottilles le taux d'échantillonnage était nul. Une autre stratification a dû être envisagée avec comme contrainte de garder le concept de flottille de la typologie de l'Ifremer. Les objectifs étaient d'obtenir des strates où l'échantillon serait représentatif en termes de caractéristiques des navires et où les structures des coûts seraient homogènes. Pour être pertinente la classification obtenue doit être telle qu'elle permette d'obtenir une variabilité assez faible au sein de chacune des strates, mais aussi une variabilité suffisamment significative par rapport aux strates voisines.

Différentes options ont été explorées : 1'option la plus judicieuse est celle d'un regroupement par technique de pêche avec une stratification par longueur. C'est ainsi que douze strates ont été distinguées.

La part des débarquements réalisés en criée et hors criée ainsi que leur destination géographique (zone et hors zone) est déterminée à partir des données de ventes dans les criées des navires d'Auray et de Vannes (données du Réseau inter-criées, Ofimer) et des débarquements totaux. On en déduit la valeur des débarquements réalisés hors criée. Pour la destination des ventes hors criée, peu de données quantitatives sont disponibles. Les données du Comité local et la connaissance du terrain acquise pendant l'étude sont utilisées en complément.

Pour ce qui concerne les flux indirects induits et les investissements, les données récoltées ne permettent pas d'évaluer les flux monétaires indirects qui représentent les achats de fournitures des fournisseurs de la pêche. Les résultats présentés concernent donc la 
répartition des flux directs de consommations intermédiaires et le flux d'investissement, en se fondant pour ces derniers sur les informations fournies par le Conseil général.

Pour suivre les différents flux de la première vente à la consommation finale, on a besoin d'informations sur les différents maillons de la filière. Au niveau de la commercialisation des produits de la mer, on considère trois maillons : les commerces de gros (mareyeurs et grossistes), les rayons produits de la mer frais des supermarchés et des hypermarchés et les poissonniers. Pour chaque maillon, les estimations du chiffre d'affaires total et de la marge commerciale permettent une estimation des coûts d'achats de marchandises. Les résultats sont, chaque fois que cela est possible, comparés à d'autres sources de données. Dans un second temps, la répartition des achats et des ventes entre les différents fournisseurs et clients sert au calcul du multiplicateur de revenu. Ces estimations sont réalisées à partir du traitement des enquêtes menées auprès des mareyeurs, des poissonniers et des rayons marée des supermarchés et des hypermarchés des deux zones d'emploi. La part des achats réalisés à la criée ou en direct auprès des pêcheurs a pu être évaluée pour chaque maillon. Par contre, les données récoltées ne permettent pas de suivre la diffusion des flux induits par la pêche côtière d'Auray et de Vannes à chaque étape de la filière.

La plupart des structures d'encadrement du secteur ont un champ d'action bien plus large que la zone étudiée et il est très difficile de déterminer la part des flux à destination ou en provenance des entreprises de pêche d'Auray et de Vannes. Les informations récoltées ne permettent pas de quantifier tous les flux entre les structures qui jouent un rôle dans l'encadrement du secteur et les entreprises de pêche. Les estimations s'appuient d'une part sur une information de base constituée des enquêtes économiques de l'Ifremer et d'autre part, sur des informations obtenues lors d'une série d'entretiens. Ces informations ne sont pas suffisantes pour quantifier tous les flux existants, les estimations des flux présentées dans le rapport constituent donc un seuil minimum.

Le multiplicateur de revenus permet de calculer quels sont les revenus générés en marges et en valeur ajoutée par euro débarqué. Un multiplicateur de revenu pour la filière pêche (aval) et un multiplicateur de revenu pour le système halieutique ont été calculés.

Pour le multiplicateur "filière ", on tient compte des débarquements des navires d'Auray et de Vannes effectués sur la zone d'étude, répartis par type d'acheteur. Ces débarquements représentent les achats de marchandises de la première vente. Pour chaque maillon, on cherche à estimer les ventes de marchandises à partir des achats de marchandises et de la marge commerciale.

On part de la formule suivante: $\mathrm{V}=\mathrm{A}+\mathrm{M} \times \mathrm{CA}$ avec:

$\mathrm{V}$ : ventes de marchandises,

A : achats de marchandises (estimés à partir des débarquements),

M: marge commerciale en fonction du chiffre d'affaires (données d'enquêtes),

CA: chiffre d'affaires (donnée non connue).

N'ayant pas le chiffre d'affaires correspondant aux ventes des produits débarqués par les navires d'Auray et de Vannes, on l'exprime en fonction des ventes.

Alors, $\mathrm{V}=\frac{\mathrm{A}}{\left(1-\frac{\mathrm{M}}{\mathrm{C}}\right)}$ avec $\mathrm{C}$ qui représente la part de ventes de marchandises sur le chiffre d'affaires. Pour estimer ce paramètre, on utilise les données comptables nationales de l'Insee. 
Les ventes sont ensuite ventilées entre les différents clients à partir des données d'enquêtes. On réitère les calculs jusqu'aux achats des restaurateurs ou du consommateur final. La différence entre la somme des ventes aux restaurants et aux particuliers de chaque circuit de commercialisation et le montant des débarquements représente la marge réalisée par la filière pêche. Le multiplicateur de revenus pour la filière se calcule en faisant le rapport de la marge sur les débarquements.

Les marges commerciales utilisées sont la moyenne des données obtenues par enquête pour les mareyeurs et les grossistes, pour les supermarchés et pour les poissonniers. Pour les industries du poisson, on utilise la marge commerciale calculée directement en pourcentage des ventes de marchandises à partir des données comptables de l'Insee: elle est de $31 \%$. Pour la restauration, la marge n'a pas été calculée, les données comptables de l'Insee couvrant une variété trop importante de catégories de restauration. Par ailleurs, nous n'avions pas les moyens d'effectuer des enquêtes suffisamment précises pour ce secteur.

Le multiplicateur de revenu pour le système halieutique se calcule en faisant le rapport entre le chiffre d'affaires généré par l'activité de pêche des navires d'Auray et de Vannes et la somme des flux suivants :

- les flux directs vers l'amont, c'est-à-dire les consommations intermédiaires et les investissements destinés à l'acquisition de navires d'occasion, la modernisation ou la remotorisation des navires ;

- la valeur ajoutée générée par l'activité de pêche des navires d'Auray et de Vannes ;

- les marges commerciales dégagées par l'aval de la filière.

La dernière étape est d'estimer l'impact en termes d'emploi de la pêche côtière c'està-dire de connaître quel est le volume d'emplois générés par l'activité des navires d'Auray et de Vannes dans la zone d'étude. Outre les personnes directement employées dans les secteurs couverts (emplois directs), deux types d'emplois, appelés emplois indirects et emplois induits, ont été pris en compte. Les emplois indirects sont techniquement liés aux emplois directs, en amont, en aval ou dans les structures d'encadrement de la pêche. Les emplois induits sont suscités par la consommation finale des ménages percevant des revenus dans le cadre d'emplois directs ou indirects.

La méthode utilisée est fondée sur la théorie de la base, issue des travaux de Douglas North (1955). Sous certaines hypothèses, la méthode de la base permet de calculer le nombre d'emplois induits par les emplois que génère, de façon directe et indirecte, la présence dans une zone de certaines activités de base. Selon Boncœur (comm. pers.) dans sa version la plus simple, la méthode postule une homogénéité des effets d'induction de toutes les composantes de la base. Sous cette hypothèse, peut alors être calculé le nombre d'emplois induits par une composante quelconque de la population de base multipliant l'effectif de cette composante par un coefficient d'induction. Ce coefficient est obtenu en divisant le nombre total d'emplois induits dans la zone par la population de base de celle-ci (figure 118).

Pour calculer le coefficient d'induction, il est nécessaire de comptabiliser les emplois induits sur les deux zones d'emploi. L'Insee a fourni les emplois au lieu de travail selon l'activité économique en 200 postes pour les deux zones d'emplois concernées. Le dénombrement des emplois liés à ces activités est effectué à partir d'une exploitation complémentaire du recensement de la population de 1999.

Il faut d'abord identifier les activités au service des ménages locaux, les emplois liés à ces activités sont comptabilisés en emplois induits. La différence entre ces emplois induits 
et les emplois au lieu de travail des deux zones d'emploi est affectée à la population de la base avec les titulaires de revenus autonomes (chômeurs, militaires et retraités).

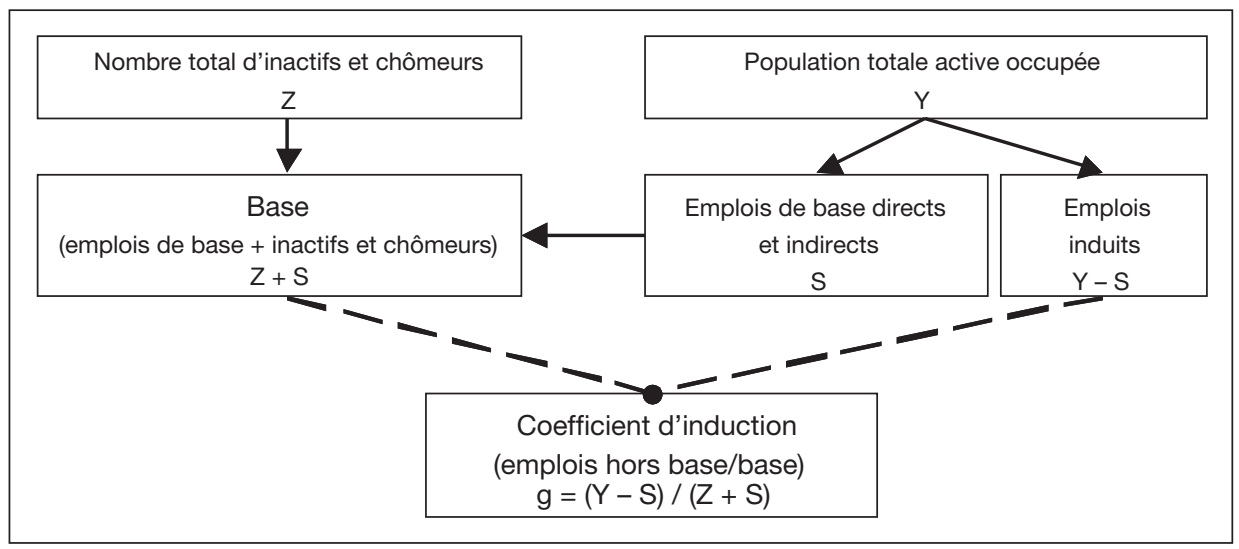

Figure 118. Détermination du coefficient d'induction (toutes activités confondues).

L'avantage de la méthode utilisée est qu'elle peut être mise en œuvre sous une forme relativement élémentaire et qu'elle procure des résultats relativement solides. Le nombre d'emplois directs est une donnée facilement accessible pour les navires. Cependant, les données ne correspondent pas à un nombre d'emplois en équivalent temps plein. Un premier dénombrement des emplois indirects est obtenu rapidement par les données fournies par la base de données de l'Insee et par des sites Internet spécialisés. Un complément a été apporté par le biais d'enquêtes. Ces enquêtes peuvent être téléphoniques, le nombre d'emploi étant donné facilement par les établissements. La difficulté réside dans la répartition de ces emplois entre les emplois liés à l'activité des navires d'Auray et de Vannes et ceux liés à d'autres activités ou à des activités de pêche hors zone.

Pour le calcul du coefficient d'induction, la distinction entre les emplois de base et les emplois induits n'est pas toujours évidente. Pour cette raison, on a utilisé un encadrement du coefficient en calculant un minimum et un maximum.

\section{Mesure des résultats économiques de la pêche professionnelle bretonne: comparaison des méthodes à base de données comptables et d'enquêtes de terrain ${ }^{172}$}

Disposer de données économiques fiables sur l'activité des flottes de pêche constitue une nécessité pour l'aménagement des pêcheries (FAO, 1995), comme pour la recherche scientifique. Cependant, l'accès à l'information est souvent un problème en ce domaine.

\footnotetext{
${ }^{172}$ Une version en langue anglaise de ce texte a été présentée à la $12^{\mathrm{e}}$ conférence biennale de l'IIFET, Tokyo, 20-30 juillet 2004 (Boncœur et al., 2004). L'analyse qui y est développée a été rendue possible grâce à l'aimable collaboration de l'Observatoire économique régional des pêches et du Système d'informations halieutiques de l'Ifremer. Les jugements qu'elle contient doivent être considérés comme propres à ses auteurs et n'engagent pas les deux organismes précités.
} 
Dans la plupart des cas, les statistiques accessibles publiquement se limitent aux débarquements, et leur qualité varie fortement selon les canaux de commercialisation utilisés.

C'est pourquoi la collecte de données économiques additionnelles concernant l'activité des navires de pêche est de plus en plus considérée comme une nécessité (Anon., 2001). Au niveau européen, le conseil des ministres a décidé en 2000 d'établir un programme communautaire pour la collecte des données (notamment économiques) nécessaires à l'évaluation de la situation du secteur des pêches ${ }^{173}$. Deux méthodes peuvent être utilisées à cet effet: le traitement statistique de bases de données préexistantes (généralement de nature comptable) et la réalisation d'enquêtes de terrain ad hoc. Chacune présente des avantages et des inconvénients et, pour des raisons pratiques, il peut être nécessaire de faire usage à la fois de l'une et de l'autre (Anon., 2001; Sabatella et Franquesa, 2003; Anon., 2003). Mais le traitement statistique de bases de données comptables et les enquêtes de terrain menées auprès des pêcheurs ne produisent pas nécessairement des résultats homogènes, ce qui pose le problème de la compatibilité de ces deux approches.

Pour étudier cette question, les deux méthodes ont été appliquées au même ensemble de navires sur la même période. La population étudiée est la flotte de pêche professionnelle bretonne, et la période d'observation est l'année 2001. La comparaison repose sur les deux sources suivantes: la base de données comptable de l'Observatoire économique régional des pêches maritimes et une enquête annuelle réalisée par l'Ifremer ${ }^{174}$. L'objectif poursuivi est d'élaborer une méthodologie harmonisée qui soit applicable aux données provenant des deux types de sources et qui maximise la fiabilité, la pertinence économique et l'homogénéité des résultats obtenus, quel que soit le type de source utilisé.

Après une brève présentation de la population mère, la représentativité des échantillons procurés par les deux sources est examinée. Un sous-échantillon commun est ensuite utilisé pour comparer les indicateurs économiques procurés par chaque méthode. La conclusion synthétise les résultats obtenus et suggère certaines solutions d'harmonisation.

\section{Cas d'étude et échantillons}

Cette section présente la population mère de l'étude et les deux sources d'information utilisées, puis examine la représentativité des deux échantillons qui en dérivent.

\section{Population mère}

L'étude repose sur le cas de la flotte de pêche professionnelle immatriculée en Bretagne, avec comme période de référence l'année 2001. La Bretagne est la première région halieutique française, représentant approximativement 30 à $40 \%$ de la pêche française, selon l'indicateur retenu (tableau 48).

\footnotetext{
${ }_{173}$ Règlement $\mathrm{CE} \mathrm{n}^{\circ} 1543 / 2000$, juin 2000, établissant un cadre communautaire pour la collecte et la gestion des données halieutiques nécessaires à la conduite de la politique commune de la pêche (OJ L176, 15.07.2000). 174 Afin de garantir la confidentialité des données individuelles, les deux bases de données ont fait l'objet de traitements séparés et n'ont donné lieu à aucune comparaison au plan individuel.
} 
Tableau 48. Pêche professionnelle en Bretagne et en France, année 2001 (Source: ministère de l'Agriculture et de la Pêche; Anon., 2002).

\begin{tabular}{lrrc} 
& {$[\mathrm{A}]$ Bretagne } & {$[\mathrm{B}]$ France } & {$[\mathrm{A}] /[\mathrm{B}](\%)$} \\
\hline Nombre de bateaux immatriculés & 1641 & 5686 & 29 \\
Nombre de pêcheurs & 8983 & 25548 & 35 \\
Puissance motrice de la flotte $(\mathrm{kW})$ & 359653 & 915021 & 39 \\
Valeur débarquée (106 euros) & 356 & 1066 & 33 \\
\hline
\end{tabular}

La flotte de pêche bretonne est fortement diversifiée, en termes de taille de navires comme de métiers pratiqués ${ }^{175}$. Basée sur une typologie élaborée par l'Ifremer, la figure 119 p. XXIX donne une vue synthétique de cette diversité :

En 2001, le nombre de navires de pêche professionnelle en activité s'élevait à 1609 . Plus d'un millier de ces navires avait une longueur inférieure à 12 mètres, alors que 105 navires dépassaient 24 mètres. En termes de métiers, l'essentiel de la flotte était composé des trois groupes suivants: 573 chalutiers (exclusifs ou mixtes), 416 navires utilisant d'autres types d'engins traînants (essentiellement des dragues) et 572 navires pratiquant exclusivement les arts dormants. En outre, la flotte de pêche bretonne comportait un groupe de 48 senneurs.

Les chalutiers se rencontrent dans toutes les classes de longueur au-dessus de 7 mètres, avec un pic dans la classe des 16-24 mètres (213 unités en 2001). En revanche, les navires appartenant aux deux autres groupes principaux ont, majoritairement, une longueur inférieure à 12 mètres. Le groupe des senneurs se décompose quant à lui en deux sous-groupes bien différenciés : 20 bolincheurs travaillant en Atlantique Nord et ayant le plus souvent une longueur comprise entre 12 et 16 mètres, et 28 thoniers-senneurs tropicaux dépassant tous 40 mètres de longueur.

\section{Sources d'information}

Une première source d'information économique concernant la flotte de pêche bretonne est la base de données de l'Observatoire économique régional des pêches. Créé en 1992 par la Fédération bretonne de la coopération maritime, cet observatoire fonctionne aujourd'hui en partenariat avec le Centre de droit et d'économie de la mer (Cedem, université de Bretagne occidentale) et le Comité régional des pêches maritimes et des élevages marins. Il collecte les données annuelles de 12 groupements comptables (couvrant 781 navires en 2001) et les données de débarquement mensuelles (limitées aux ventes en criées) de trois organisations de producteurs (couvrant 1140 bateaux en 2001). L'observatoire publie une étude annuelle sur la situation économique de la pêche artisanale bretonne (Observatoire économique régional des pêches, 2003) et réalise des études spécifiques pour les professionnels de la pêche et la recherche académique.

Une seconde source d'information est l'enquête réalisée chaque année, depuis 2000, par l'Ifremer auprès d'un échantillon de patrons et d'armateurs à l'échelle nationale. Cette enquête constitue la base de la composante économique du SIH développé par l'Ifremer

\footnotetext{
${ }^{175}$ Un métier se définit comme une combinaison espèce-zone-engin. Une flottille est un ensemble de navires pratiquant des métiers, ou des combinaisons de métiers similaires.
} 
(Berthou et al., 2003). Elle repose sur une analyse exhaustive des calendriers d'activité des navires, qui permet de stratifier la flotte de pêche française en une série de flottilles, au sein desquelles un tirage aléatoire est effectué pour constituer chaque année l'échantillon de l'enquête. Pour les besoins de la présente étude, un échantillon régional a été constitué à partir de l'échantillon national en extrayant de celui-ci les navires immatriculés en Bretagne.

\section{Représentativité des échantillons}

Lorsque l'on compare les deux sources d'information, la première question qui se pose est celle de la représentativité des échantillons sur lesquelles elles reposent (les deux échantillons seront dénommés ci-après " échantillon comptable » et " échantillon enquête »). Cette question est envisagée ici sous deux angles: la classe de longueur et le métier principal. Utilisant ces critères, les figures 120 et 121 p. XXX comparent la structure de la flotte de pêche bretonne (population mère) à celle des deux échantillons.

Il ressort de ces comparaisons que la structure de l'échantillon enquête donne une image plus fidèle de la population mère que celle de l'échantillon comptable, en termes de classe de longueur comme de métier principal. Dans les deux échantillons, les navires les plus grands et les plus petits sont sous-représentés, mais la distorsion est plus forte avec l'échantillon comptable, qui ne comporte qu'un nombre négligeable de navires de moins de 7 mètres ou de plus de 24 mètres. Symétriquement, la classe 16-24 mètres est fortement surreprésentée au sein de cet échantillon: elle constitue $48 \%$ de l'effectif de l'échantillon comptable, contre seulement $28 \%$ de l'effectif de la population mère. La surreprésentation des 16-24 mètres est moins forte au sein de l'échantillon de l'enquête (34\% de l'effectif de cet échantillon). Les distorsions en termes de métiers recoupent en partie les distorsions en termes de classe de longueur, ces deux caractères n'étant pas indépendants l'un de l'autre (cf. figure 121 p. XXX). En ce domaine, le résultat le plus net est la surreprésentation des chalutiers dans l'échantillon comptable (49\% de l'échantillon, contre seulement $36 \%$ dans la population mère), qui induit mécaniquement une sousreprésentation des autres flottilles. La composition par flottilles de l'échantillon enquête est plus proche de celle de la population mère.

L'explication des distorsions entre la structure de chaque échantillon et celle de la population mère réside dans la façon dont ces échantillons ont été constitués. Dans le cas de la base de données de l'Observatoire, aucun critère de représentativité n'est intervenu dans cette opération. L'échantillon est simplement le produit de l'adhésion des propriétaires de navires aux groupements comptables travaillant avec l'Observatoire. Pour des raisons historiques, la couverture des chalutiers 16-24 mètres est particulièrement forte. Parallèlement, peu de patrons de très petites unités adhèrent à des groupements comptables, et les bateaux relevant d'armements industriels n'en font pas partie non plus. La situation est différente dans le cas de l'échantillon de l'enquête Ifremer, qui a été conçu spécialement pour cet usage avec, comme il se doit, un souci de représentativité (Van Iseghem et al., 2004). Dans ce cas, la stratégie d'échantillonnage repose sur un taux-cible de $15 \%$ par groupe de navires (défini en termes de longueur et de métier), avec des possibilités de suréchantillonnage pour les groupes à faible effectif. Cependant, des difficultés pratiques s'opposent parfois à la réalisation des taux d'échantillonnage prévus. En effet, il n'est pas toujours possible de trouver en nombre suffisant des patrons ou armateurs qui soient disponibles et volontaires pour répondre à l'enquête. Pour des raisons diverses, 
cette condition est plus difficile à satisfaire dans les classes de longueur extrêmes que dans les tailles intermédiaires.

Cette contrainte, combinée au coût de la réalisation d'une enquête de terrain à large échelle, limite la taille de l'échantillon enquête. À l'échelle de la Bretagne, l'effectif de cet échantillon est de 258 navires pour l'année 2001, alors qu'il atteint 545 navires (en activité) pour l'échantillon comptable. En conséquence, le taux d'échantillonnage effectif par groupe de navires est, dans la majorité des cas, plus élevé avec l'échantillon comptable qu'avec l'échantillon enquête (figure 122). Les exceptions les plus marquantes se trouvent chez les bateaux de moins de 7 mètres, où la couverture réalisée par l'échantillon-enquête est sensiblement plus forte que celle qui est obtenue avec l'échantillon comptable.

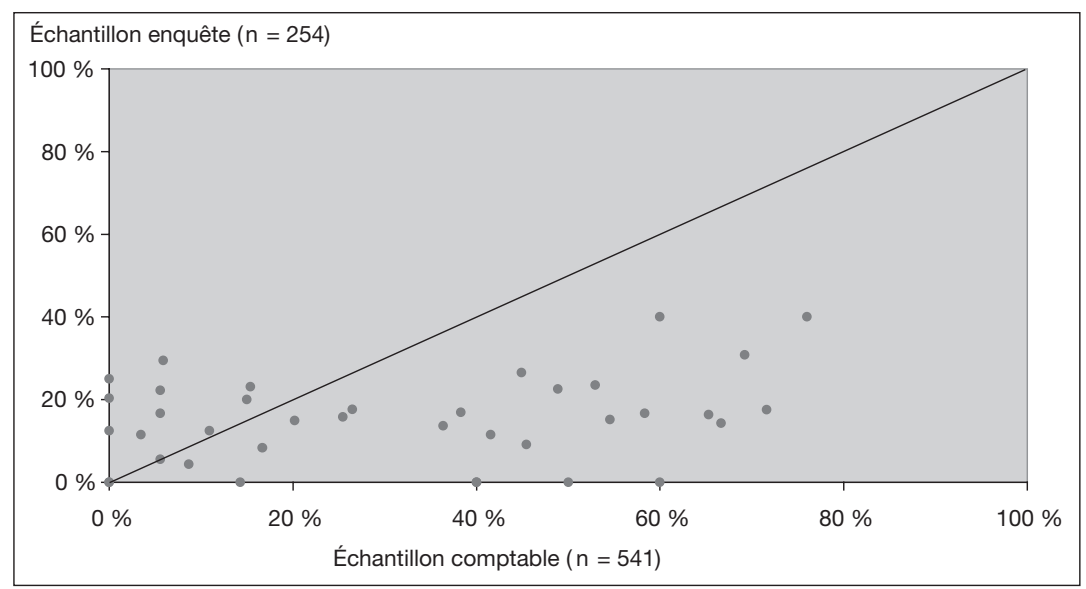

Figure 122. Taux d'échantillonnage comparés par groupe de navires (classe de longueur + métier).

\section{Comparaison des résultats économiques obtenus à l'aide des deux méthodes}

Dans cette section, les données économiques procurées par la base de données comptables et l'enquête de terrain sont comparées. L'analyse se concentre sur le chiffre d'affaires, les charges d'exploitation (hors amortissement) et l'excédent brut d'exploitation (EBE). Afin d'éliminer tout biais d'échantillonnage, un sous-échantillon commun, constitué par l'intersection des deux échantillons de base, a été constitué. Dénommé ci-après « échantillon commun », cet ensemble de navires comporte 127 unités. Sa composition par classe de longueur et par flottille est présentée dans le tableau 49 ci-dessous.

Tableau 49. Composition de l'échantillon commun.

\begin{tabular}{lcrrrrr}
\hline & $<7 \mathrm{~m}$ & $7-12 \mathrm{~m}$ & $12-16 \mathrm{~m}$ & $16-24 \mathrm{~m}$ & $>24 \mathrm{~m}$ & Total \\
\hline Senneurs & 0 & 0 & 3 & 0 & 0 & 3 \\
\hline Chalutiers & 0 & 10 & 10 & 33 & 3 & 56 \\
\hline Autres arts traînants & 1 & 21 & 4 & 1 & 0 & 27 \\
\hline Arts dormants & 2 & 28 & 10 & 1 & 0 & 41 \\
\hline Total & 3 & 59 & 27 & 35 & 3 & 127 \\
\hline
\end{tabular}


Étant donné le faible nombre de navires dans les deux classes de longueur extrêmes, seul le groupe des navires de 7 à 24 mètres sera considéré dans l'analyse comparative cidessous. Ce groupe comporte 121 navires, ce qui correspond à un taux d'échantillonnage de $9,5 \%$ de la population correspondante (1 276 bateaux), représentant elle-même $80 \%$ de l'effectif total de la flotte de pêche bretonne en 2001.

Tous les résultats économiques présentés dans cette section sont dérivés de l'échantillon commun. L'analyse est conduite dans un premier temps à l'échelle de l'ensemble de l'échantillon, puis par classe de longueur. En dernier lieu, sont présentés des tests de sensibilité des indicateurs de résultats à la méthode de mesure retenue.

\section{Comparaison globale}

À l'échelle de l'ensemble de l'échantillon (7-24 mètres), la figure 123 p. XXXI compare les résultats de chaque méthode en matière de valeur moyenne du chiffre d'affaires, des charges d'exploitation et de l'EBE.

Cette comparaison fait apparaître une bonne correspondance entre les deux méthodes pour ce qui est du chiffre d'affaires moyen: selon les réponses à l'enquête de terrain, le niveau de celui-ci est plus élevé que selon les données comptables, mais l'écart est très restreint ( $1 \%$ ). Il est plus important pour les charges d'exploitation et, par suite, pour l'EBE. L'excédent brut d'exploitation moyen issu de l'enquête dépasse celui que donne le traitement des données comptables d'un montant égal à $8 \%$ du chiffre d'affaires comptable.

Afin d'analyser l'écart en matière de charges d'exploitation, la figure 124 p. XXXI permet de comparer la décomposition des charges selon l'une et l'autre méthode. Il apparaît que, pour l'essentiel, l'écart est dû à trois postes: entretien et réparations du navire, charges salariales et charges diverses nettes. Considérés ensemble, ces trois postes représentent $95 \%$ de l'écart total entre les deux sources. En termes relatifs, l'écart le plus important concerne les charges diverses nettes, qui sont, en moyenne, $59 \%$ plus faibles selon les résultats de l'enquête que selon les données comptables. Mais ce taux s'applique à des montants modestes (pas plus de $2 \%$ du total des charges d'exploitation). En termes absolus, le principal facteur d'écart concerne les charges d'entretien et de réparation du navire: ce poste, qui est $41 \%$ plus faible selon l'enquête que selon la base de données comptable, représente $57 \%$ de l'écart total entre les deux sources. En termes relatifs, l'écart concernant les charges salariales est nettement plus limité : le coût moyen du travail selon les données d'enquête est seulement $3 \%$ plus faible que selon les données comptables. Mais ce taux s'applique à une base importante, les charges salariales représentant, en moyenne, environ $40 \%$ du total des charges d'exploitation (hors amortissement).

\section{Comparaison par classe de longueur}

La figure 125 p. XXXII présente, par classe de longueur, les écarts entre les deux sources d'information concernant les valeurs moyennes du chiffre d'affaires, des charges d'exploitation et de l'EBE. Afin de décrire ces écarts dans une échelle commune significative, le graphique les exprime en pourcentage du chiffre d'affaires comptable de la classe de longueur considérée.

La figure 125 fait apparaître une opposition entre les navires de plus de 12 mètres et ceux de moins de 12 mètres. Chez ces derniers, l'enquête de terrain donne des valeurs moyennes supérieures à la base de données comptable, pour le chiffre d'affaires $(+6 \%)$ 
mais aussi pour les charges d'exploitation (+ $4 \%$ du CA comptable). En conséquence, l'écart moyen concernant l'EBE est peu élevé chez les bateaux de moins de 12 mètres ( $1 \%$ du CA comptable environ). Il est plus important chez les bateaux de 12 mètres : dans la classe des 12-16 mètres, l'estimation moyenne de l'EBE issue de l'enquête de terrain excède celle que l'on tire de la base de données comptable d'un montant équivalent à $6 \%$ du CA comptable; chez les 16-24 mètres, l'écart, dans le même sens, atteint $11 \%$ du CA comptable. De plus, chez les navires de plus de 12 mètres, les deux sources donnent des estimations moyennes très proches l'une de l'autre pour le CA. L'écart constaté pour l'EBE est essentiellement imputable aux charges d'exploitation, pour lesquelles l'enquête de terrain fournit des estimations sensiblement plus faibles que la base de données comptables (écart atteignant $8 \%$ du CA comptable dans la classe des 12-16 mètres, et $10 \%$ du CA comptable chez les 16-24 mètres).

La figure 126 p. XXXII présente les écarts relatifs aux charges d'exploitation par classe de longueur et par type de charge. Comme il ressort de cette figure, trois types de charges peuvent être distingués :

- les charges salariales, qui sont significativement plus importantes selon l'enquête de terrain que selon la base de données comptables chez les bateaux de moins de 12 mètres, et inversement chez les navires de plus de 12 mètres ;

- les charges d'entretien/réparation du navire et les charges diverses nettes, qui sont plus faibles selon l'enquête de terrain que selon la base de données comptables dans toutes les classes de longueur;

- les autres types de charges, pour lesquels les écarts entre les deux sources sont faibles et n'ont pas de signe permanent.

\section{Analyse des écarts}

Les deux sections précédentes ont permis de dégager les résultats suivants.

La correspondance entre enquête de terrain et données comptables est généralement bonne pour le chiffre d'affaires, excepté chez les bateaux de moins de 12 mètres, où l'enquête de terrain produit des estimations généralement plus élevées que la base de données comptables.

Pour toutes les classes de longueur, la correspondance entre les deux sources est généralement bonne pour les catégories de charges suivantes: frais de débarquement, carburant et lubrifiant, glace, vivres, appâts, engins de pêche, primes d'assurance (considérées ensemble, ces charges représentent approximativement un cinquième du CA comptable chez les plus petites unités, et jusqu'à un tiers du CA comptable chez les plus grandes).

Pour toutes les classes de longueur, les estimations produites par l'enquête en matière de charges diverses nettes et d'entretien-réparation du navire sont plus faibles que celles de la base de données comptables (considérées ensemble, ces estimations représentent en moyenne $7 \%$ ou $10 \%$ du CA comptable, selon la source qui est utilisée pour leur estimation).

Pour ce qui concerne les charges salariales, les estimations issues de l'enquête de terrain sont plus fortes que celles de la base de données comptables pour les bateaux de moins de 12 mètres, et inversement pour les bateaux de plus de 12 mètres.

En matière de chiffre d'affaires, l'écart entre les deux sources qui a été constaté chez les bateaux de moins de 12 mètres doit être rapproché des modes de commercialisation des débarquements. Ces modes de commercialisation sont souvent plus informels dans le cas 
des petites unités, d'où il résulte que les données comptables donnent parfois une image approximative et incomplète des ventes réalisées par ces navires.

Concernant les charges diverses nettes et les charges d'entretien et de réparation du navire, l'écart que l'on constate dans toutes les classes de longueur provient de deux causes. La première est la difficulté d'obtenir par voie d'enquête une information précise et complète sur ces postes, qui sont par nature diversifiés (c'est particulièrement vrai pour les « charges diverses », catégorie hétérogène regroupant une collection de petites dépenses) et qui ont souvent un profil temporel irrégulier. Il est donc probable que les enquêtes de terrain sous-estiment la réalité concernant ces postes. Mais il existe une seconde cause d'écart, concernant plus spécialement le poste " entretien réparation du navire ». Un examen détaillé des éléments regroupés sous ce nom dans la base de données comptables permet de constater que certains d'entre eux doivent être considérés, d'un point de vue économique, plutôt comme des opérations d'investissement que comme des charges d'exploitation. Cette caractéristique, qui n'est sans doute pas étrangère à des considérations fiscales, reflète un certain flou dans les conventions en matière de classification des charges dans le secteur de la pêche ${ }^{176}$. Il en résulte vraisemblablement une surestimation des charges d'entretien et de réparation du navire dans la base de données comptables.

Les écarts symétriques constatés en matière de charges salariales reflètent des problèmes institutionnels. Il convient ici d'examiner séparément le cas des petites unités et celui des plus grandes unités.

Pour les bateaux d'une longueur inférieure à 12 mètres, l'enquête de terrain débouche généralement sur des estimations de charges salariales plus hautes que les données comptables. La rémunération de l'équipage étant en principe calculée selon le système du salaire à la part, une partie de cet écart résulte mécaniquement de celui qui a été constaté pour ces bateaux en matière de chiffre d'affaires (voir ci-dessus). Mais, paradoxalement, une cause majeure d'hétérogénéité entre les deux sources de données réside dans le fait que le système du salaire à la part n'est pas d'application générale pour les petites unités (et plus particulièrement pour celles qui ont moins de 9 mètres). Dans la version française du salaire à la part ${ }^{177}$, le patron propriétaire d'un bateau de pêche artisanale est normalement rémunéré à travers deux canaux, qui sont la part équipage (en tant que patron) et la part armement ${ }^{178}$ (en tant que propriétaire). Cependant, lorsque l'équipage se réduit au seul patron-propriétaire, comme c'est souvent le cas sur les plus petites unités, le système du salaire à la part n'est pas toujours appliqué. Dans ce cas, la rémunération du patronpropriétaire passe exclusivement par le canal de l'EBE, ce qui crée un biais par rapport aux bateaux où le salaire à la part est appliqué. Facteur d'hétérogénéité dans la manière dont la base de données comptables détermine l'EBE des navires, ce biais est corrigé dans les estimations issues de l'enquête de terrain, auxquelles une part-salariale virtuelle est appliquée lorsque le système du salaire à la part n'est pas pratiqué. Il résulte de cette correction une estimation en moyenne plus forte des coûts de main-d'œuvre sur les petites unités.

\footnotetext{
176 Sur ce point, la situation est plus claire dans le secteur agricole où existe un système normalisé qui, à ce jour, n'a pas d'équivalent dans le secteur halieutique.

177 À la différence, par exemple, de la pratique britannique, qui exclut toujours le patron de la part-équipage (Boncœur et al. 2000).

178 Nette de charges d'armement.
} 
Pour les navires de plus de 12 mètres, il a été signalé que la base comptable fournit des charges salariales en moyenne plus élevées que l'enquête de terrain. Dans ce cas, l'origine de l'écart ne réside pas dans le système du salaire à la part. En effet, celui-ci est appliqué sur tous les bateaux de l'échantillon ${ }^{179}$, et la part-équipage est déterminée à partir de ventes nettes et de charges communes dont les niveaux moyens diffèrent peu d'une source à l'autre ${ }^{180}$. L'examen des éléments enregistrés sous l'étiquette « charges salariales » dans la base de données comptables permet de constater que l'écart avec l'enquête de terrain provient ici essentiellement de certaines primes, qui sont comprises dans les charges salariales par la première source mais généralement pas par la seconde. Ces primes sont de deux types: primes-équipage, et primes-armement. Si la première catégorie peut à bon droit être considérée comme un élément du salaire, l'inclusion de primes-armement dans les charges salariales semble plus discutable. De ce fait, on peut estimer que la bonne estimation des charges salariales, dans le cas des navires de 12-24 mètres, se trouve à l'intérieur de la fourchette définie par les deux sources de données.

\section{Tests de sensibilité}

L'analyse des écarts suggère que les méthodes de comptabilisation des charges peuvent influencer de façon significative l'image des performances économiques des navires de pêche. Afin de tester cet effet, quatre scénarios ont été construits et appliqués à l'échantillon commun:

Le scénario 0 décrit simplement la situation actuelle (c'est-à-dire les données « brutes » issues de la base de données comptables et de l'enquête de terrain).

Le scénario 1 repose sur les hypothèses et conventions suivantes: les données comptables sont valides en ce qui concerne les charges diverses nettes (tous navires), alors que les données issues de l'enquête de terrain sont valides pour les charges salariales des navires de moins de 9 mètres; les primes-équipage sont incluses dans les charges salariales, mais pas les primes-armement.

Les scénarios $2 \mathrm{~A}$ et 2B modifient le scénario 1 en reprenant, respectivement, les données de l'enquête de terrain et les données comptables en matière d'entretien-réparation du navire.

Les conséquences de ces scénarios en matière d'EBE moyen sont présentées sur la figure 127 ci-dessous. Le passage du scénario 0 (situation actuelle) au scénario 1, puis au scénario 2A ou 2B se traduit par une diminution progressive de l'écart entre les estimations de performance économique issues des deux sources d'information ${ }^{181}$. Ce rapprochement est substantiel: exprimé en proportion du chiffre d'affaires moyen, l'écart passe de $7,5 \%$ dans le scénario 0 à $4,2 \%$ dans le scénario 2 , et tombe à moins de $0,5 \%$ dans le

\footnotetext{
179 Tous les bateaux de l'échantillon comptable, et donc tous ceux de l'échantillon commun, appartiennent au secteur dit « artisanal » de la pêche.

${ }^{180}$ Les charges dites communes, qui sont soustraites des ventes nettes avant de calculer la part-équipage, sont habituellement les suivantes : carburant, vivres, appâts, glace. L'examen de données issues des deux sources n'a pas fait apparaître d'écart majeur concernant ces charges. Il en va de même pour les frais de débarquement, qui interviennent dans le calcul des ventes nettes.

181 Représenté sur le graphique à l'échelle de l'ensemble de l'échantillon, ce phénomène est également constaté à l'échelle de chaque classe de longueur, à l'exception de la classe 9-12 mètres. Cette exception est due à l'écart concernant la valeur des débarquements qui affecte cette classe au sein de l'échantillon.
} 
scénario 2A ou le scénario 2B. Quelle que soit la source considérée, le niveau absolu de performance est plus élevé dans le scénario 2A que dans le scénario $2 \mathrm{~B}$. Les conventions retenues pour l'entretien et les réparations du navire rendent en effet les charges d'exploitation plus faibles dans le scénario 2A que dans le 2B. Pour les raisons exposées plus haut, la réalité se situe vraisemblablement quelque part entre ces deux scénarios.

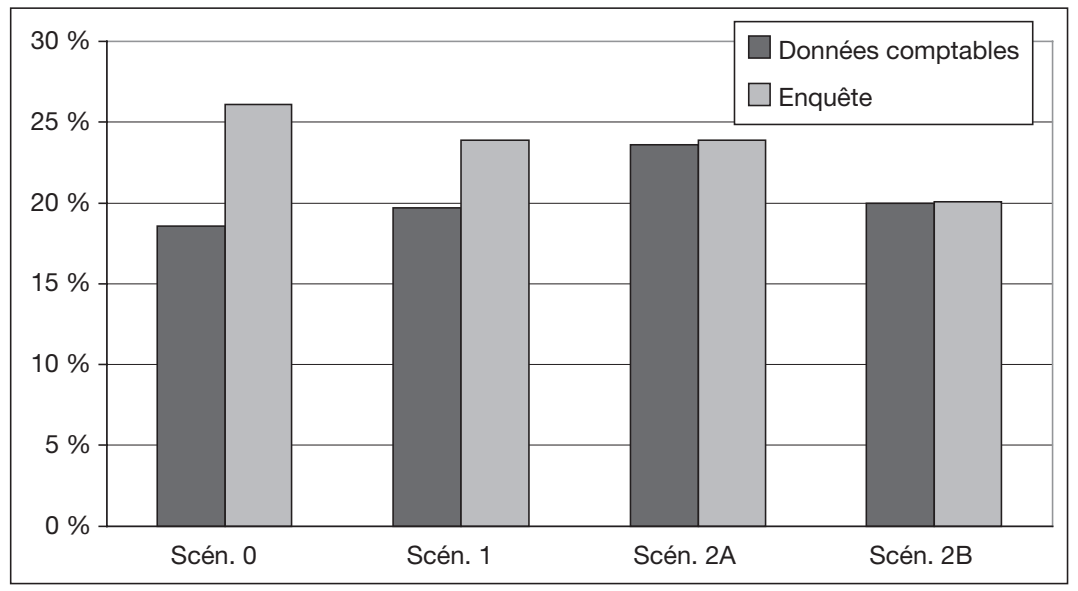

Figure 127. Ratio EBE/chiffre d'affaires. Impact des scénarios comportant différentes hypothèses et conventions de comptabilisation des charges d'exploitation.

\section{Conclusion}

Dans cette étude, deux méthodes de collecte et de traitement de l'information économique sur l'activité des navires de pêche ont été considérées : l'utilisation de données comptables préexistantes et l'enquête de terrain ad hoc. À cet effet, deux échantillons de la flotte de pêche bretonne ont été rapprochés, et un sous-échantillon commun a été construit afin de pouvoir comparer sans biais d'échantillonnage les conséquences des deux méthodes. Les principaux résultats de l'étude peuvent être résumés comme suit :

L'échantillon enquête donne une image de la population mère plus fidèle que l'échantillon comptable.

Cependant, cet avantage de l'enquête est contrebalancé, dans le cas étudié, par une taille d'échantillon sensiblement plus faible.

Il ressort de l'examen de l'échantillon commun que la correspondance statistique des deux méthodes est bonne en matière de chiffre d'affaires, à l'exception des petites unités pour lesquelles il est probable que les données comptables sous-estiment parfois la réalité.

La correspondance statistique des deux méthodes apparaît également bonne pour plusieurs catégories de charges d'exploitation : frais de débarquement, carburant et lubrifiant, glaces, appâts, vivres, engins de pêche et primes d'assurances.

En revanche, des écarts substantiels ont été relevés pour trois types de charges : charges salariales, entretien et réparations du navire, charges diverses nettes. Pour ces trois catégories, l'analyse des écarts a permis de dégager des explications qui, au vu des tests de sensibilité, rendent compte de l'essentiel des différences entre les EBE moyens issus des deux méthodes. 
Les résultats des tests de sensibilité suggèrent également certaines recommandations en vue d'harmoniser les méthodes. La liste de ces recommandations inclut:

- la fixation de règles homogènes pour la prise en compte du revenu du patron propriétaire, que le système du salaire à la part soit appliqué ou non (problème des petites unités embarquant une seule personne);

- un traitement homogène des primes, qui devrait sans doute différencier les primes équipage des primes armements (les premières étant à inclure dans les charges salariales, à la différence des secondes);

- la fixation de règles communes permettant de distinguer clairement les dépenses devant être incluses dans les charges d'exploitation au titre de l'entretien et des réparations du navire, de celles qui n'ont pas à y figurer car elles constituent des opérations d'investissement.

- le recours, autant que possible, aux estimations issues de données comptables pour les charges diverses, qui sont par nature difficiles à appréhender à travers une enquête de terrain.

Cette liste ne saurait être considérée comme complète. Pour des raisons pratiques, il n'a pas été possible, dans le cadre de l'étude, d'examiner les problèmes posés par charges d'amortissement et les charges financières. En ce qui concerne l'amortissement du capital fixe, d'autres études ont montré que l'écart entre les conventions comptables et la réalité économique pouvait être important (Alban et al., 2001), les premières étant influencées par des considérations fiscales qui ne sont pas nécessairement en harmonie avec la durée de vie économique réelle des immobilisations. Une complication supplémentaire vient du fait que, dans le système français de régulation de l'accès à la ressource, la valeur marchande d'un navire incorpore la valeur implicite des droits de pêche qui lui sont attachés et qui sont, de facto, vendus en même temps que lui (Guyader et al., 2003). 



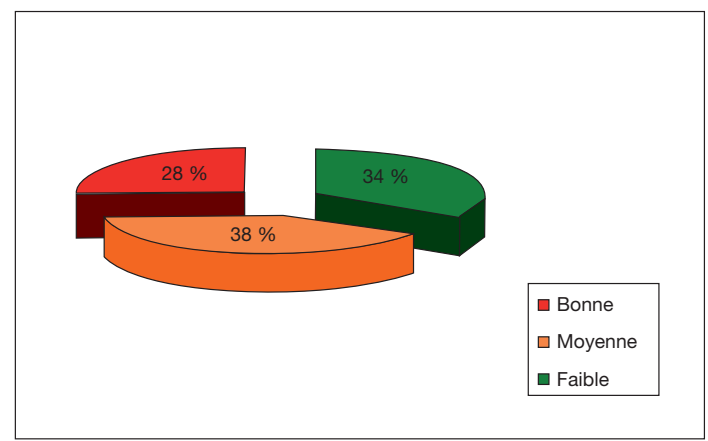

Figure 6. Fiabilité du diagnostic de l'état des ressources (source : A. Forest, Atelier Amure, 2006).

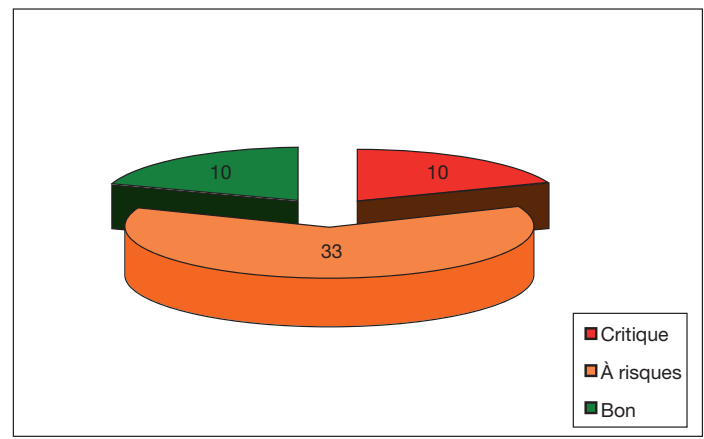

Figure 7. État des stocks et de l'exploitation (source : A. Forest, Atelier Amure, 2006).
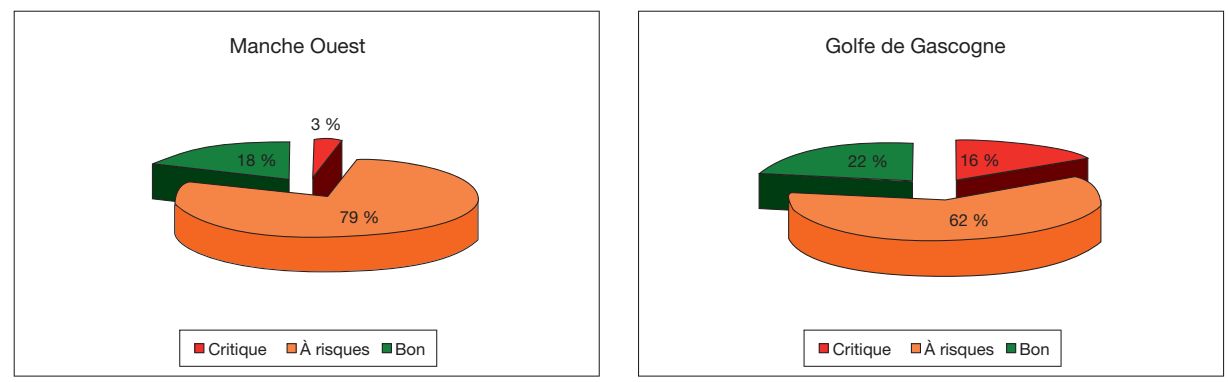

Figure 8. Répartition des productions en fonction de l'état des stocks (source: A. Forest, Atelier Amure, 2006). 


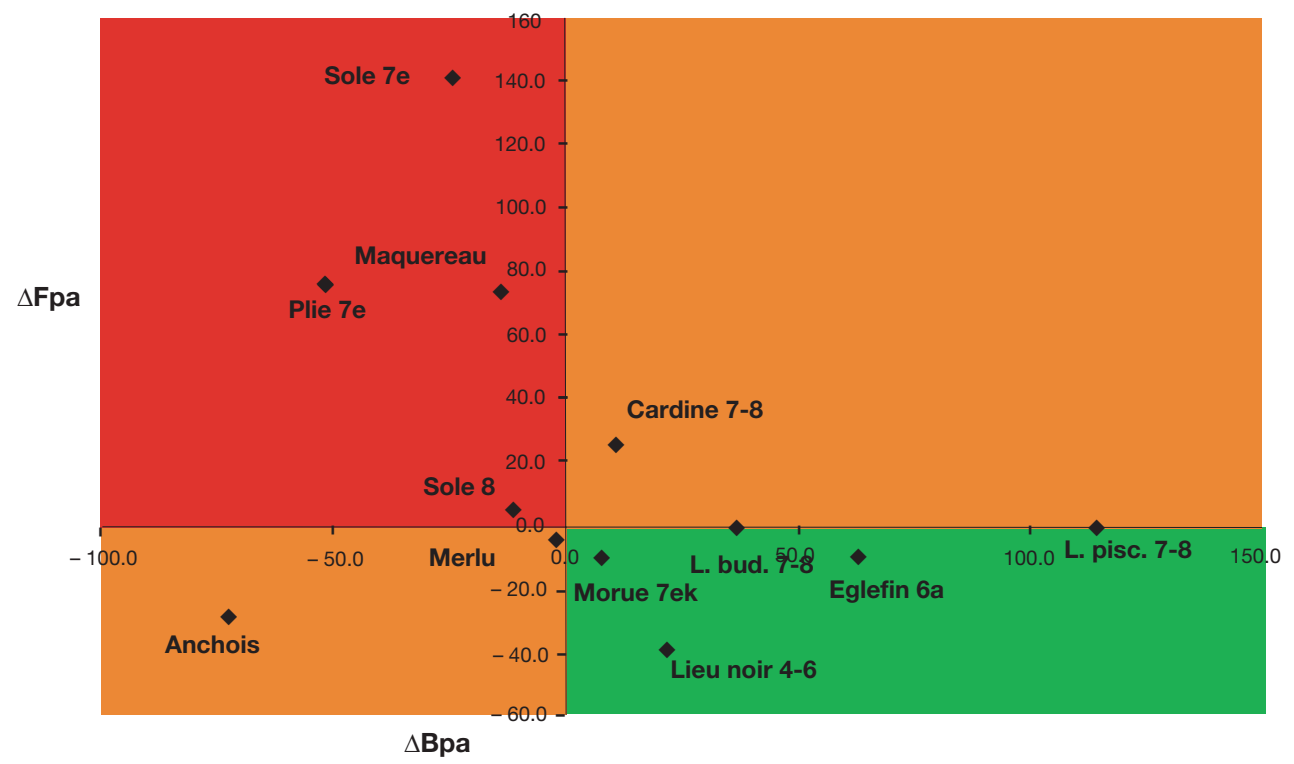

Figure 9. Situation des stocks par rapport aux points biologiques de précaution (source : A. Forest, Atelier Amure, 2006).

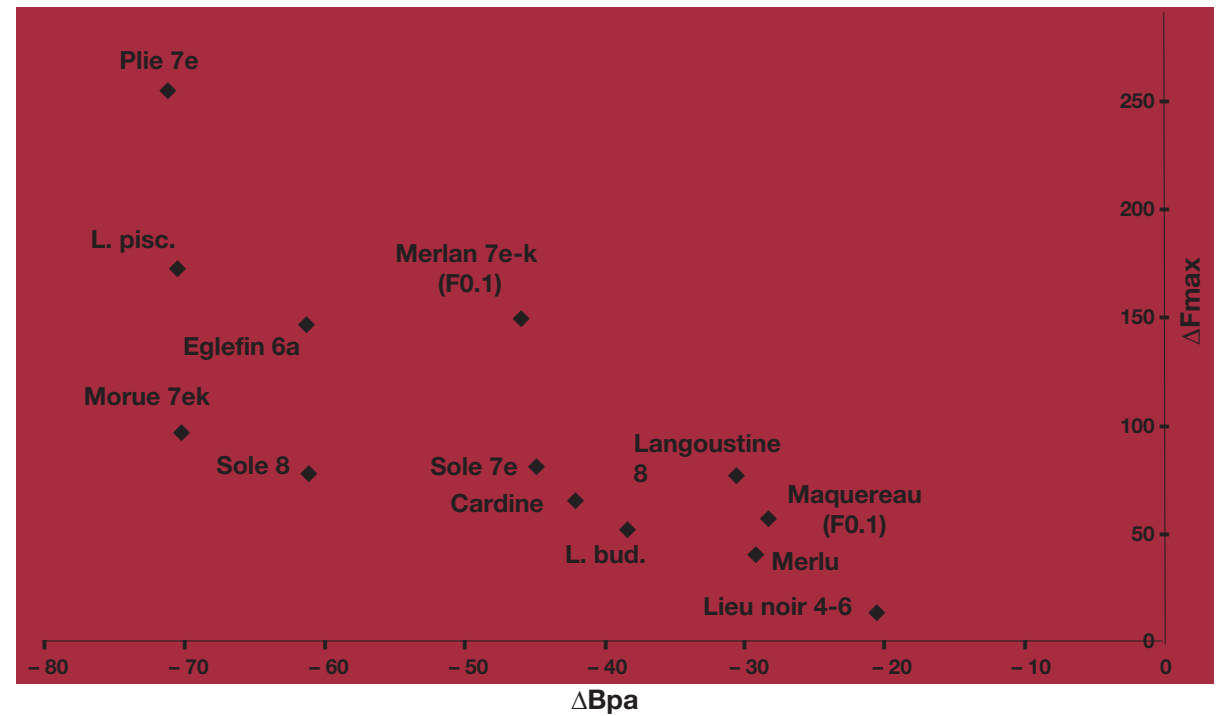

Figure 10. Situation des stocks au regard de la production maximale (source: A. Forest, Atelier Amure, 2006). 
Mortalité par pêche
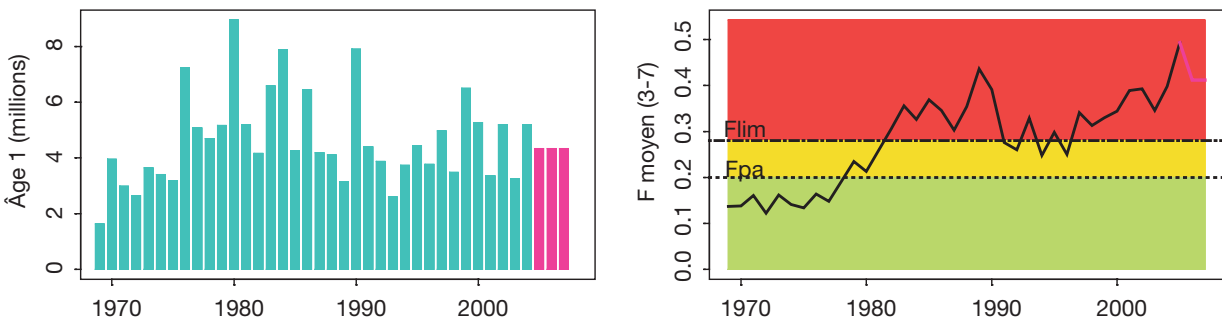

Prévisions (statu quo)

Stock de géniteurs

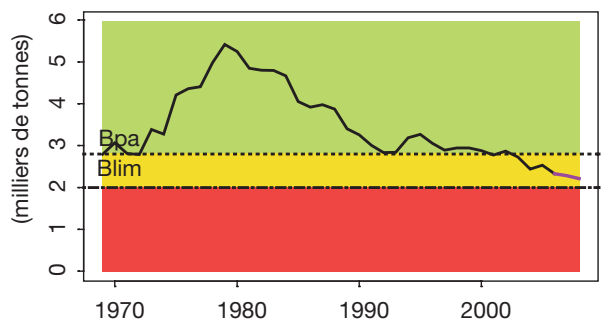

Débarquements

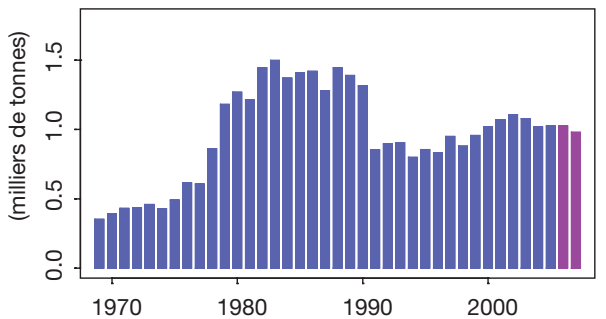

Figure 11. Indicateurs de l'état d'exploitation du stock de sole de Manche Ouest (source : CIEM, 2007).
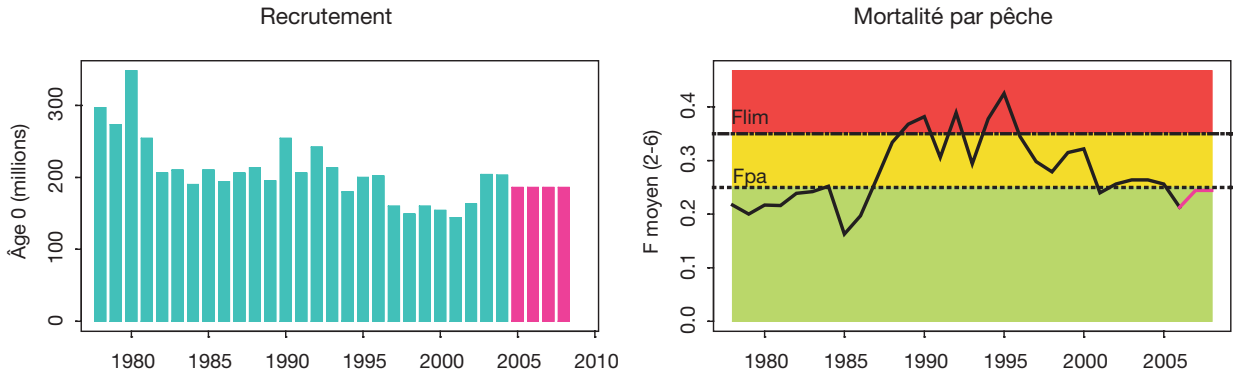

Prévisions (statu quo)

Stock de géniteurs

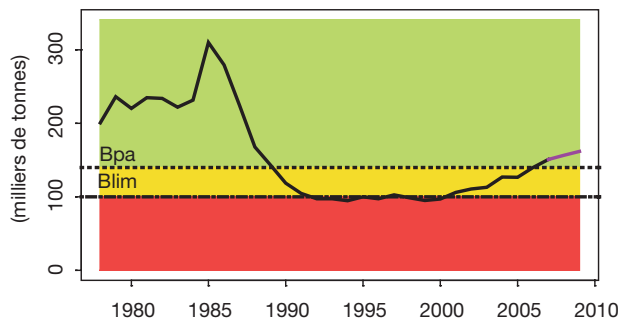

Débarquements

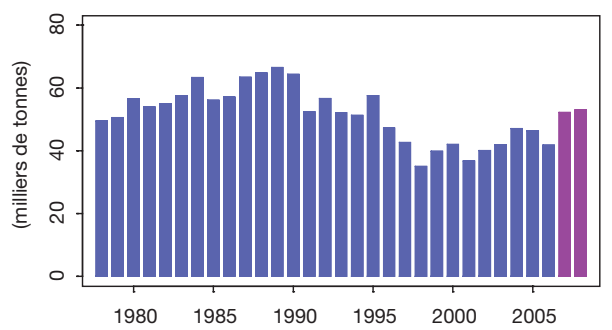

Figure 15. Indicateurs de l'état d'exploitation du stock nord de merlus (golfe de Gascogne jusqu'à la Norvège) (source : CIEM, 2007). 
Recrutement

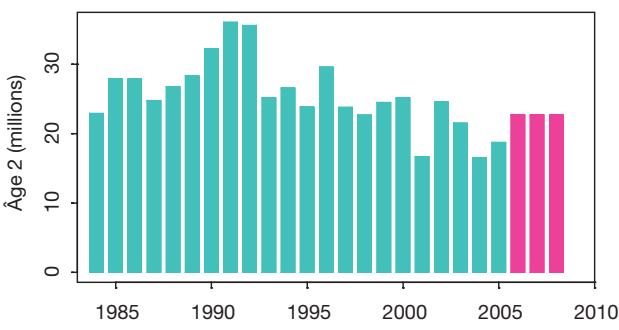

Mortalité par pêche

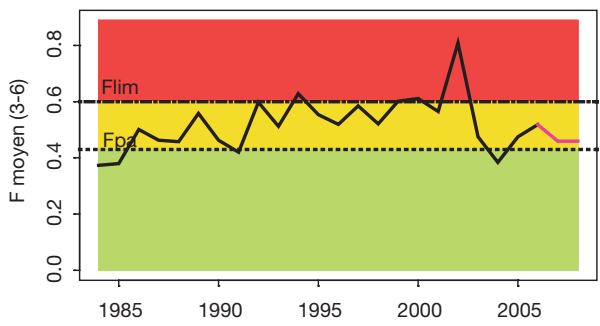

Débarquements

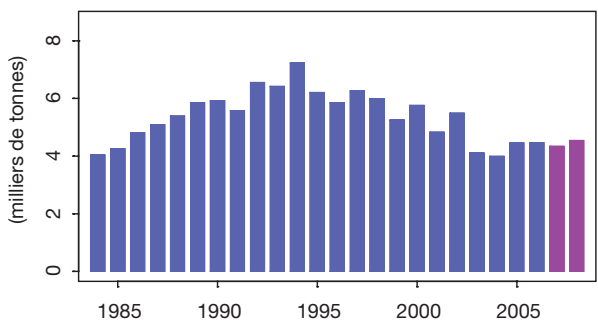

Figure 16. Indicateurs de l'état d'exploitation du stock de soles du golfe de Gascogne (source : CIEM, 2006).

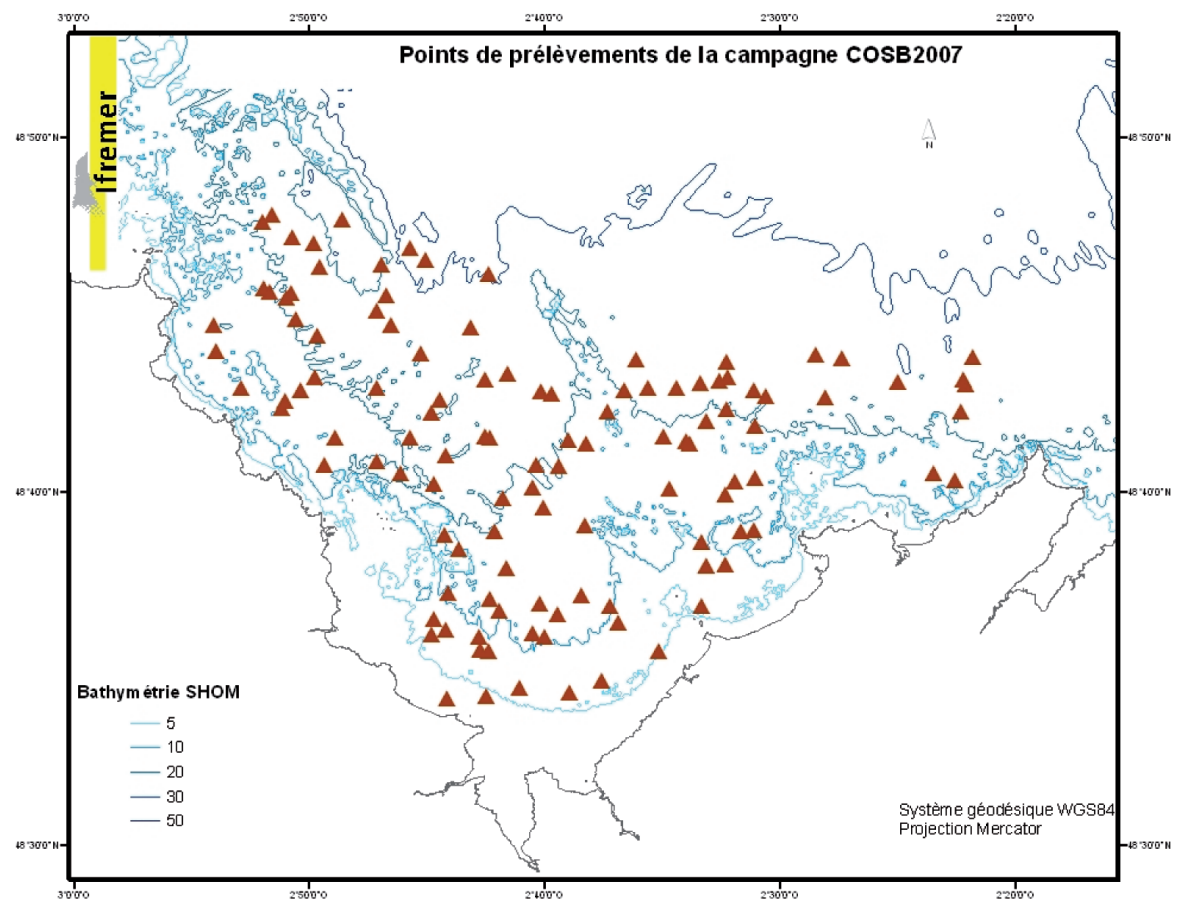

Figure 17. Campagne d'évaluation 2007. Répartition des 115 points d'échantillonnage en baie de Saint-Brieuc. 


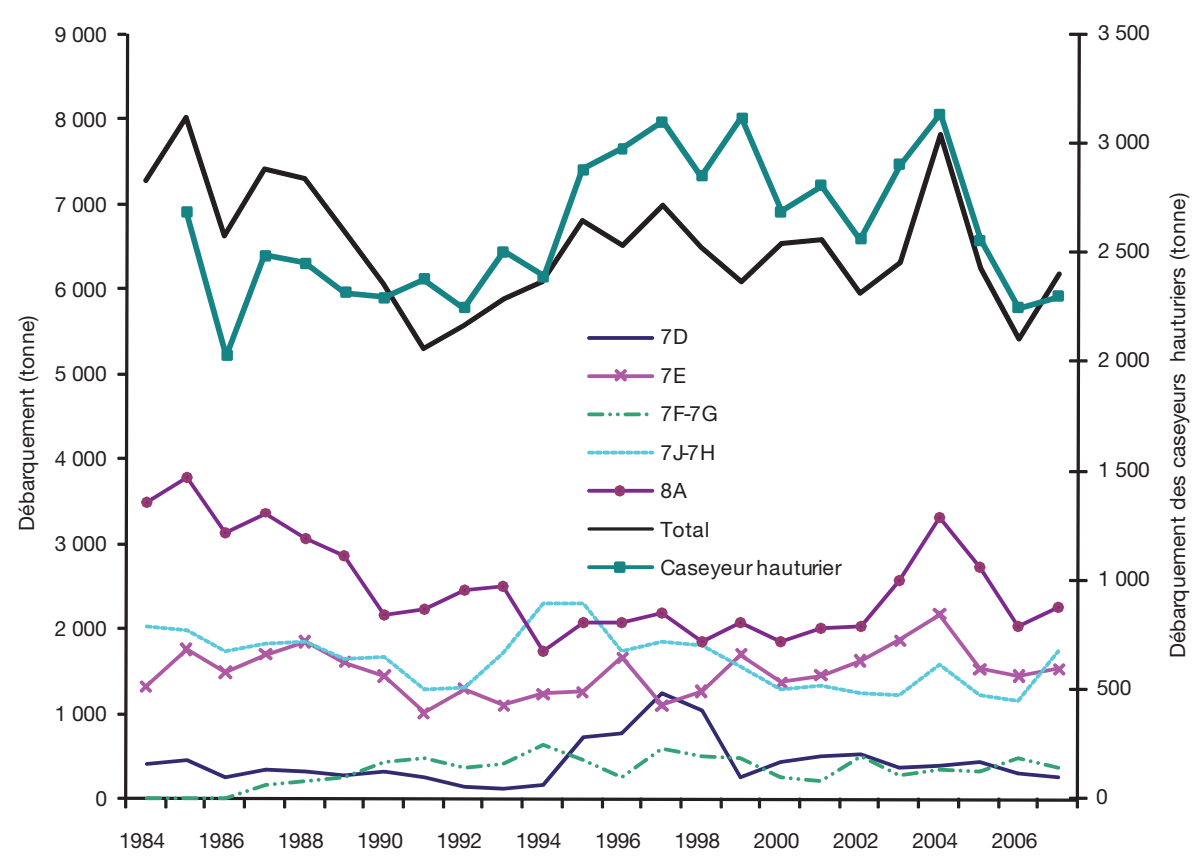

Figure 18. Débarquements de tourteaux selon le secteur de pêche et débarquements de la sousflottille des caseyeurs hauturiers, de 1984 à 2006.

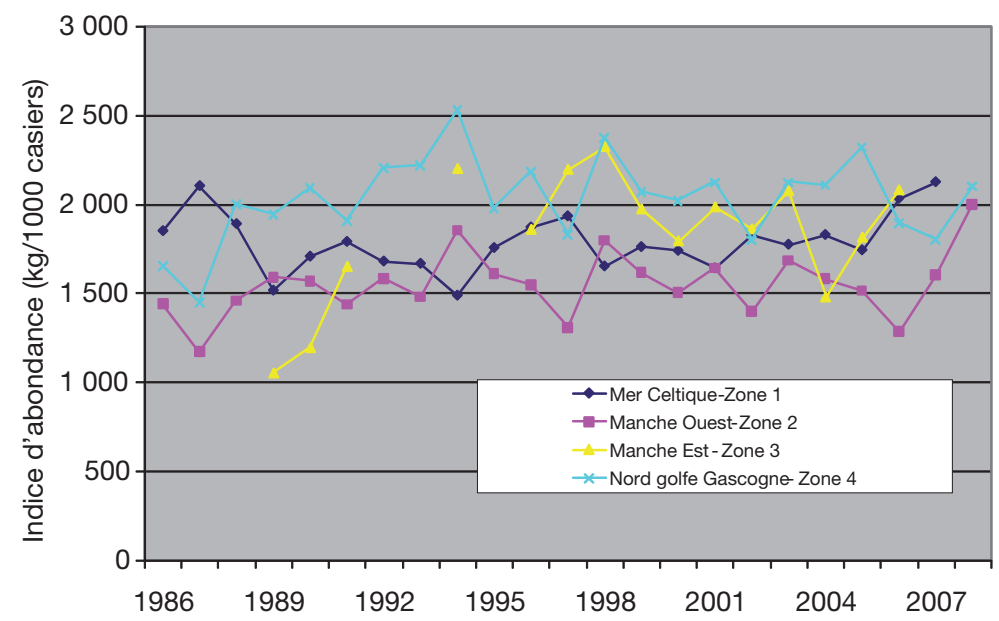

Figure 19. Indice d'abondance ( $\mathrm{kg}$ pour 1000 casiers) du tourteau de quatre zones fréquentées par les caseyeurs hauturiers. 


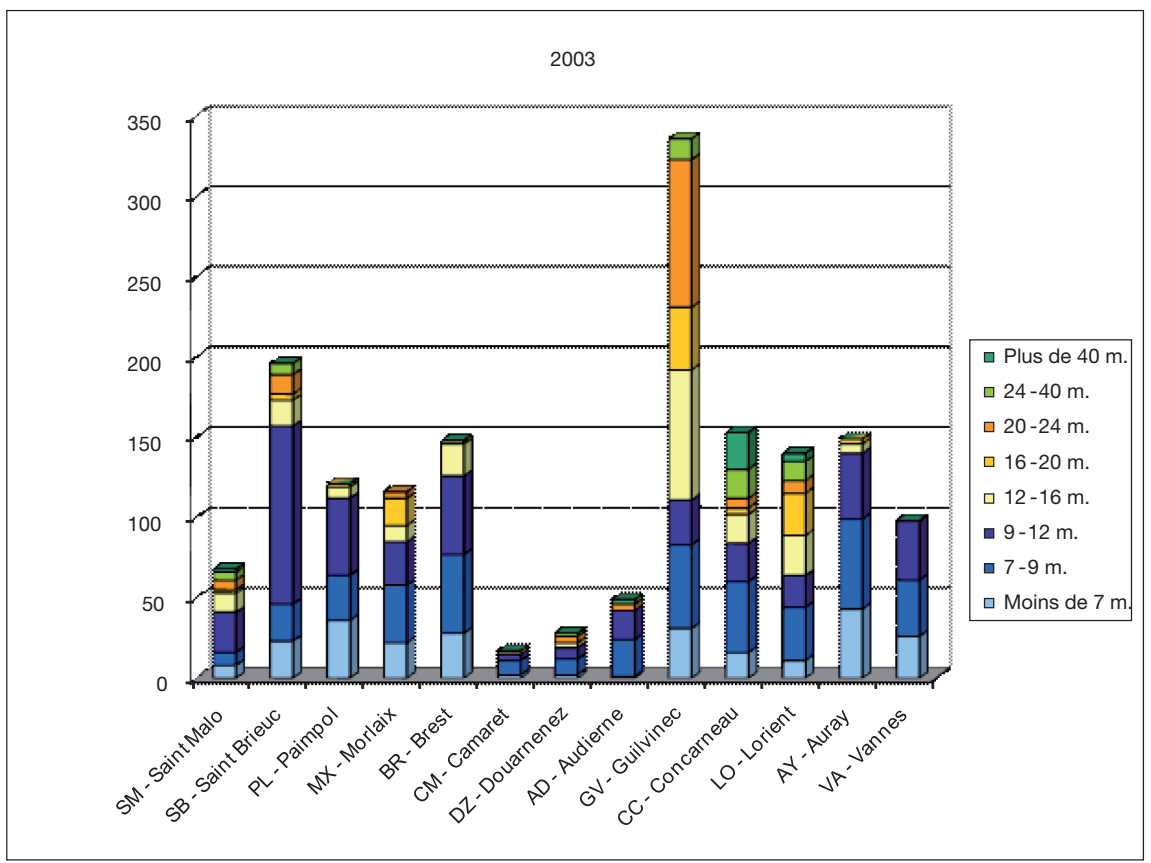

Figure 22. Répartition de la flotte bretonne par quartier maritime et classe de longueur des navires en 2003 (source : Ifremer/SIH-DPMA).

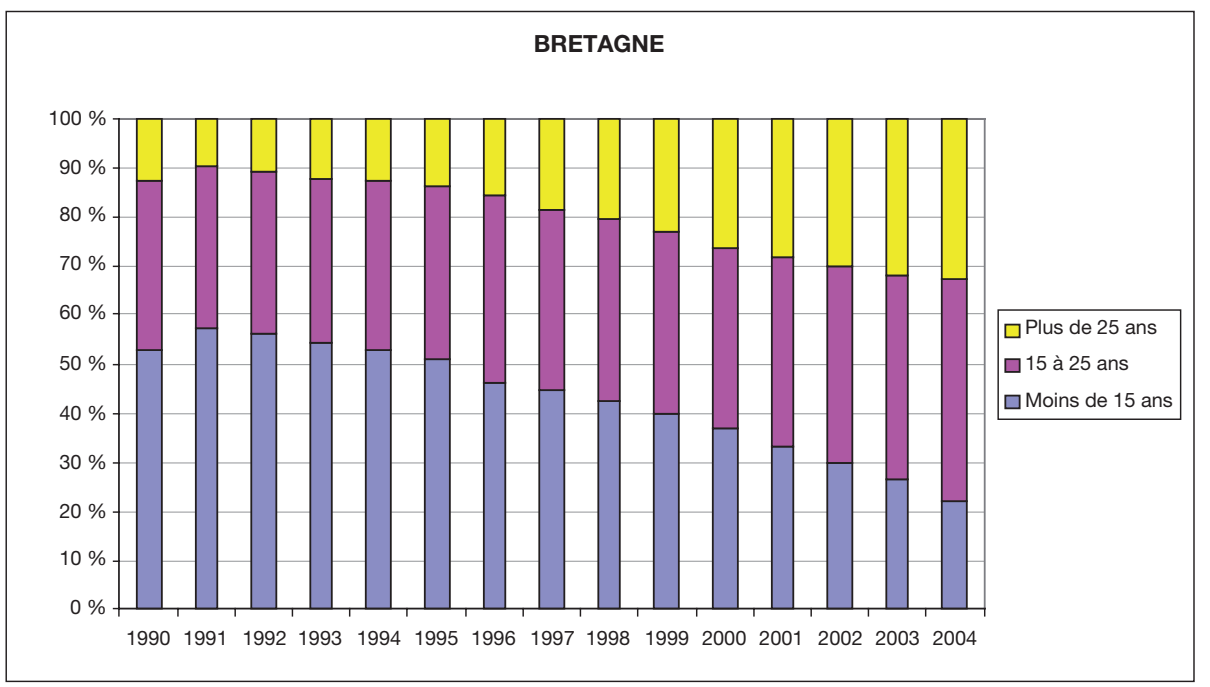

Figure 29. Évolution de la répartition de la flotte bretonne par classe d'âge 1990-2004. 


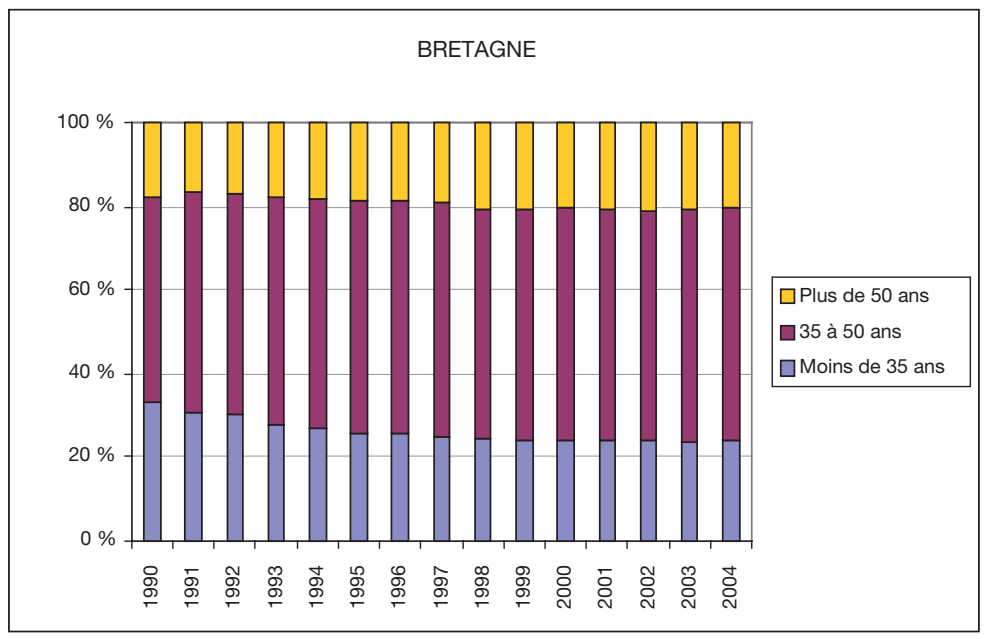

Figure 30. Évolution de la proportion de patrons propriétaires par classe d'âge sur la période 1990-2004 (source : Ifremer/SIH).
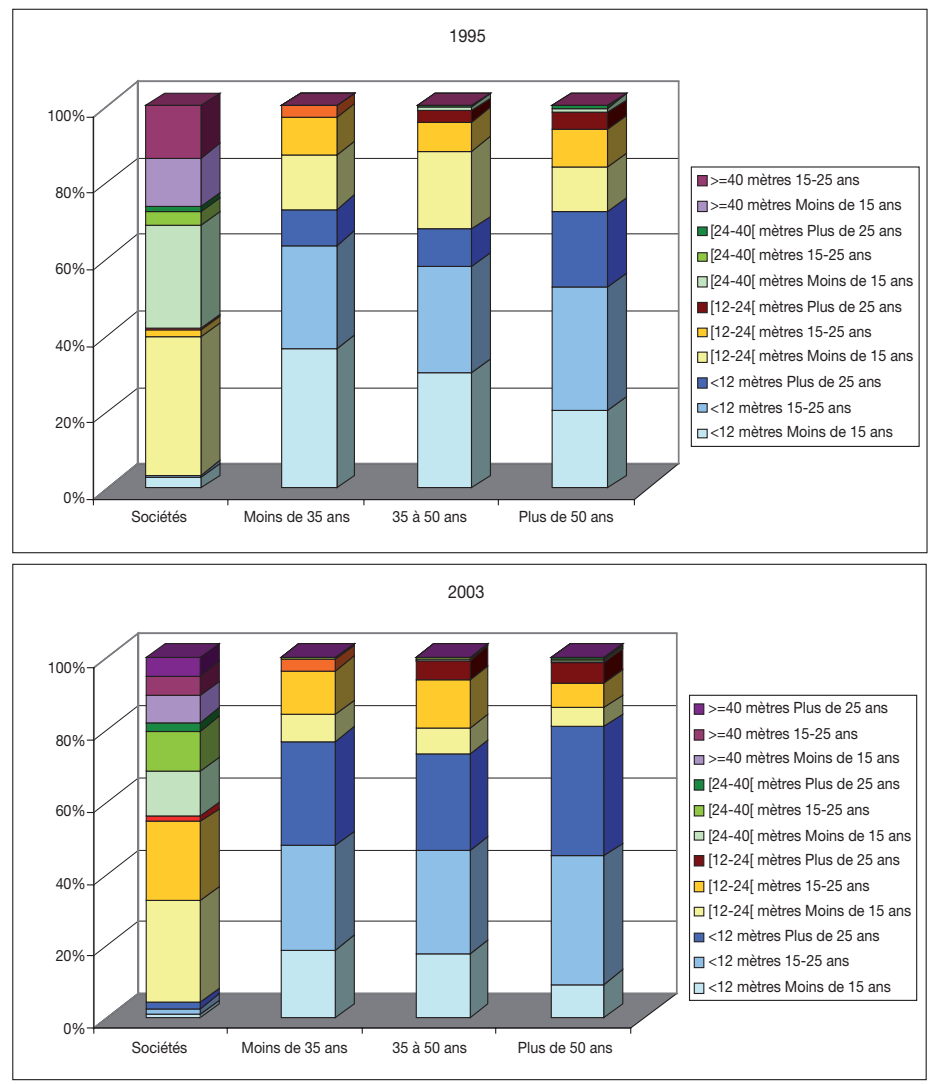

Figure 31. Nature du capital possédé (classe de taille * classe d'âge des navires) par type de propriétaires : 1995 et 2003 (source : Ifremer/SIH). 


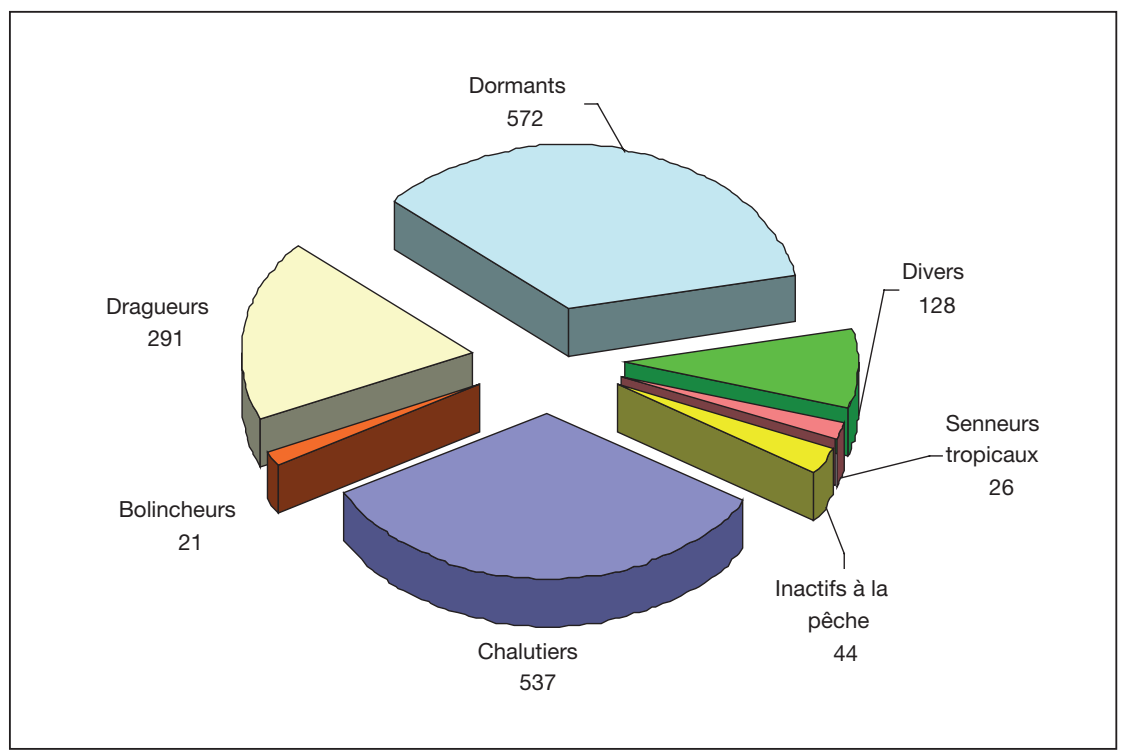

Figure 35. La composition en flottilles de la flotte de pêche bretonne en 2003. Nombre de navires (source : Ifremer/SIH).

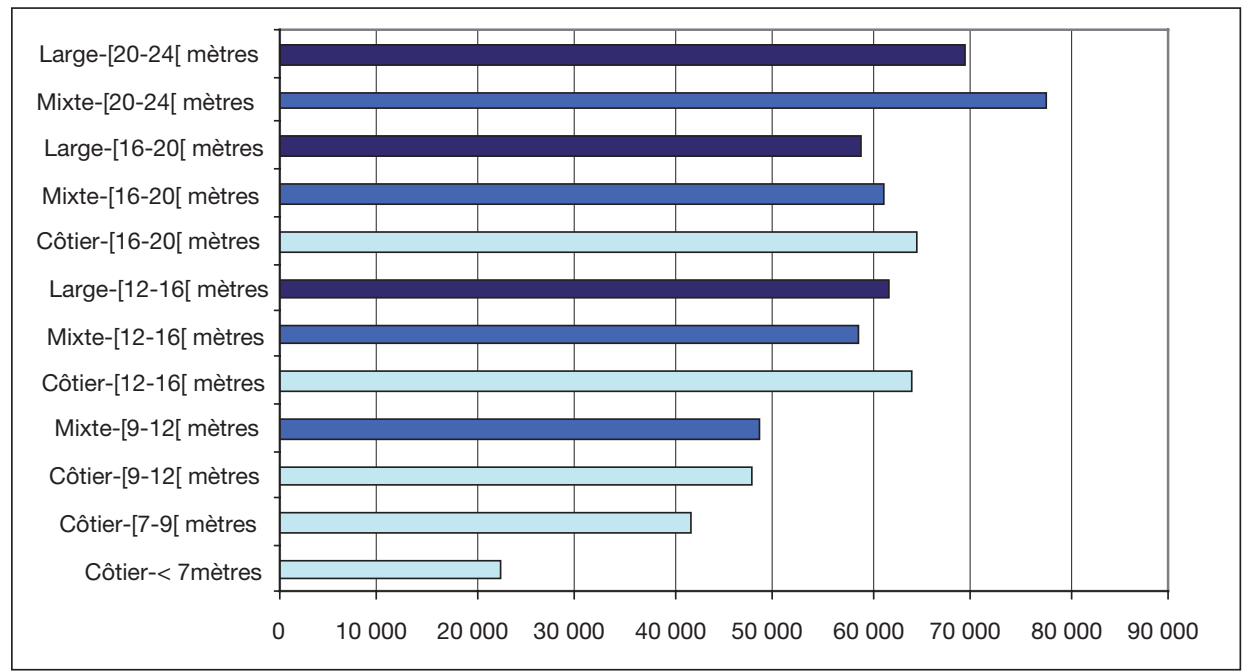

Figure 36. Productivité apparente du travail par classe de longueur (moyenne pondérée en euros par an, 2003). 


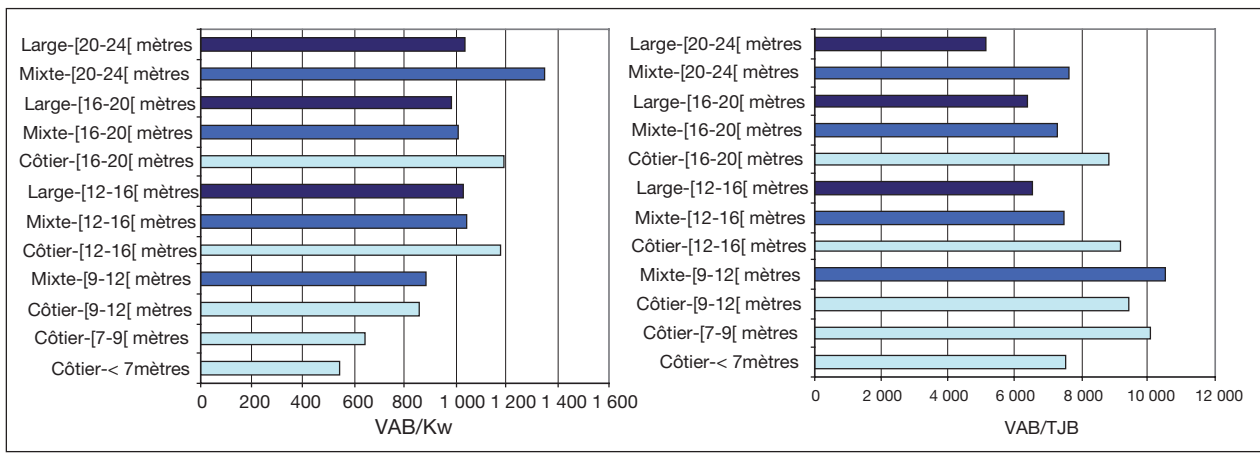

Figure 37. Productivité apparente du capital par classe de longueur (moyenne pondérée en euros par an, 2003).

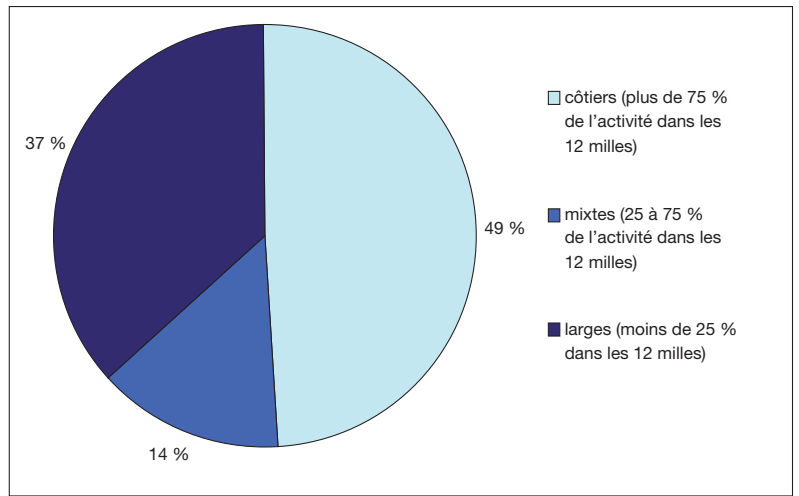

Figure 38. Répartition de la valeur ajoutée brute totale des navires bretons de moins de 24 mètres en 2003 .

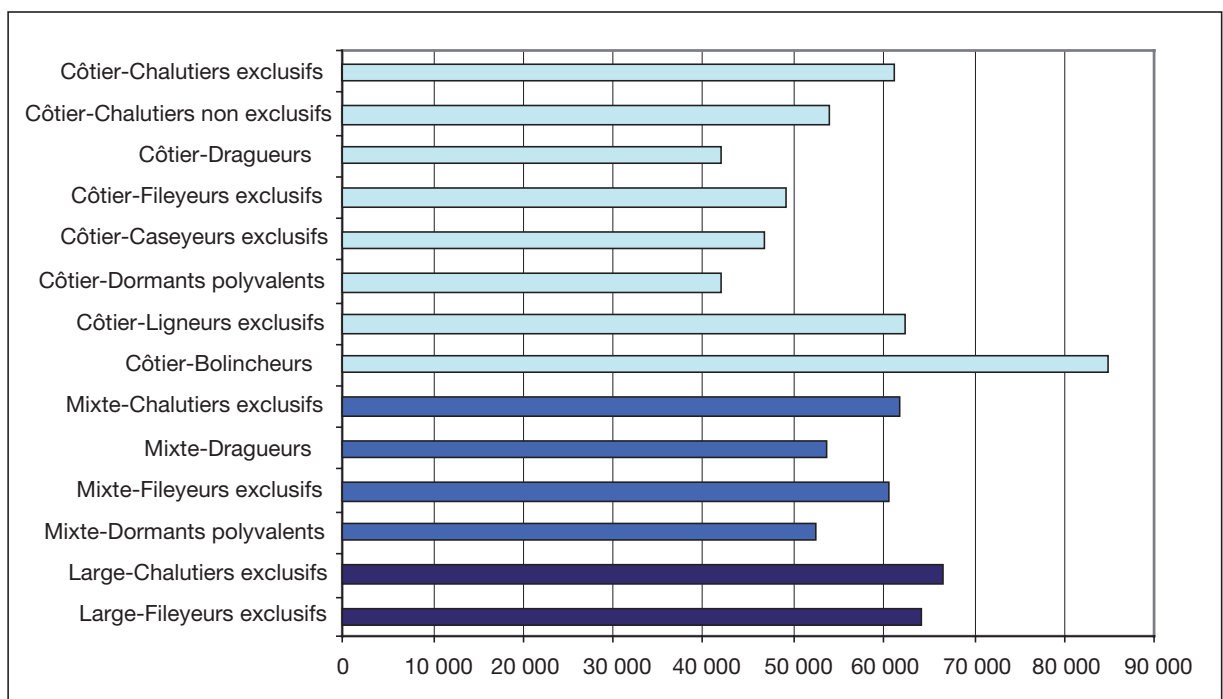

Figure 39. Productivité apparente du travail par flottille (moyenne pondérée en euros par an, 2003). 


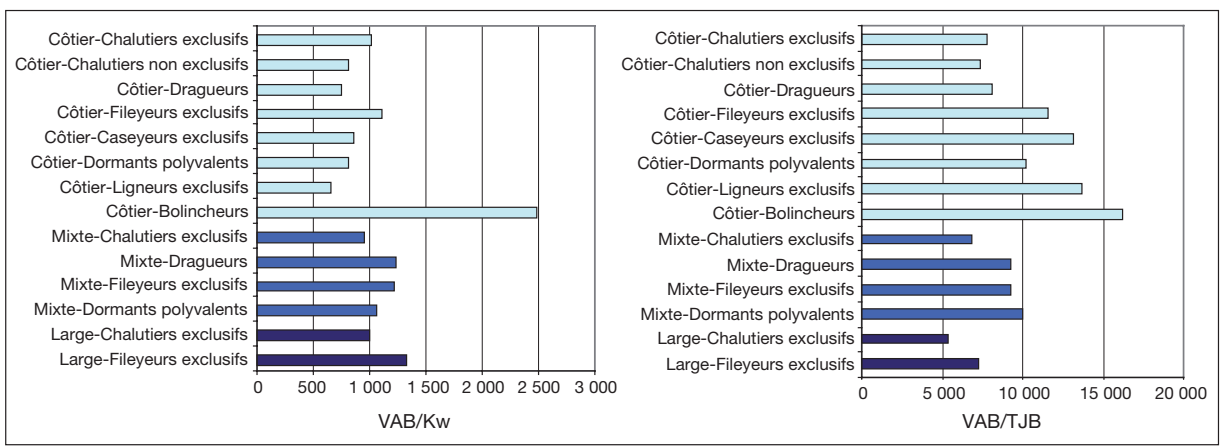

Figure 40. Productivité apparente du capital par flottille (moyenne pondérée en euros par an, 2003).

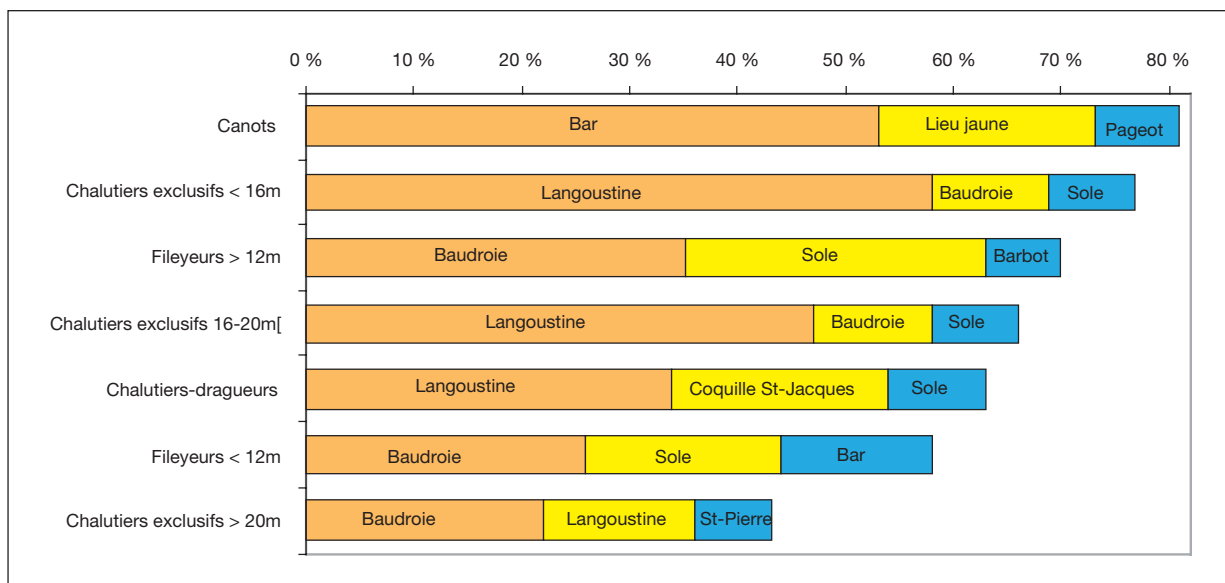

Figure 44. Les trois premières espèces débarquées en valeur, année 2005, en \% des ventes brutes (ventes en criées) (source : Observatoire économique régional des pêches).
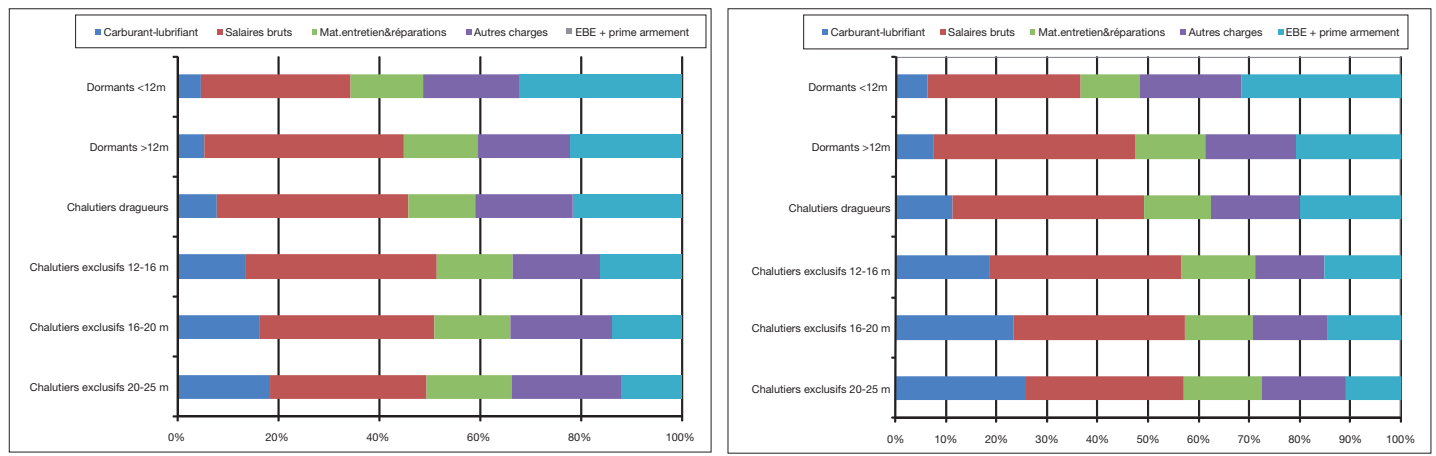

Figure 45. Ventilation du chiffre d'affaires moyen en 2004 et 2005

(source : Observatoire économique régional des pêches). 

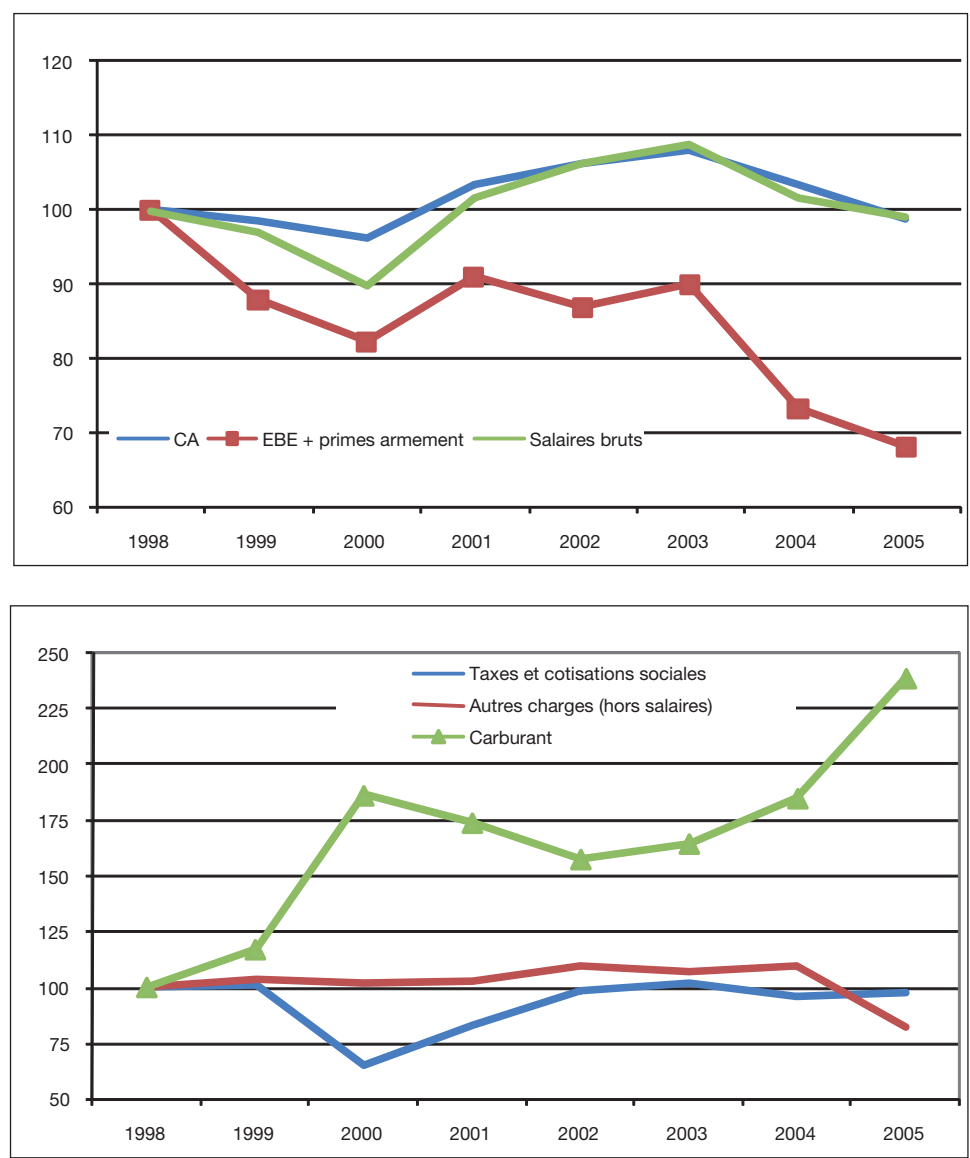

Figure 46. Ensemble de l'échantillon - évolution des agrégats comptables, 1998-2005 (euros constants, 2005) (source : Observatoire économique régional des pêches).

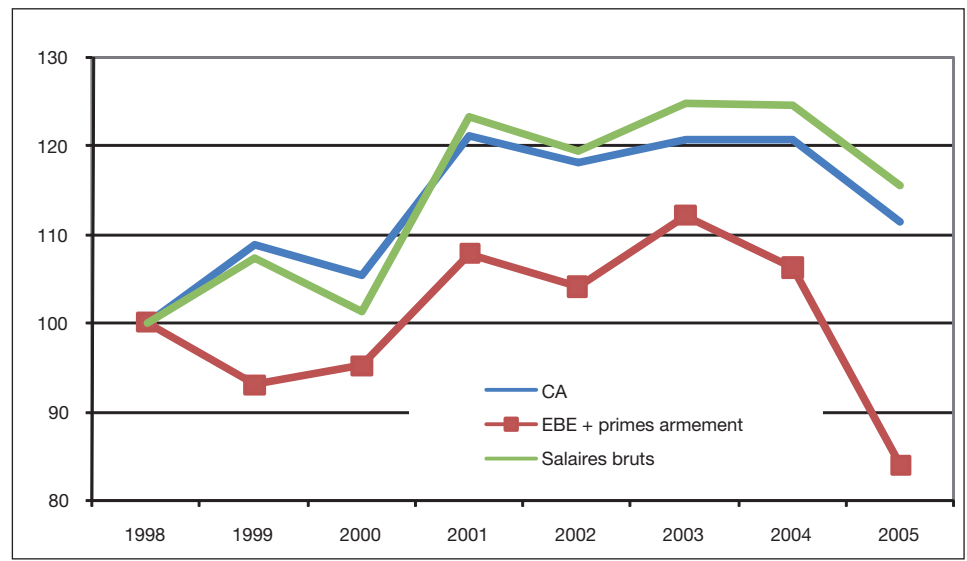

Figure 47. Chalutiers-dragueurs - évolution des agrégats comptables, 1998-2005 (euros constants 2005) (source : Observatoire économique régional des pêches). 


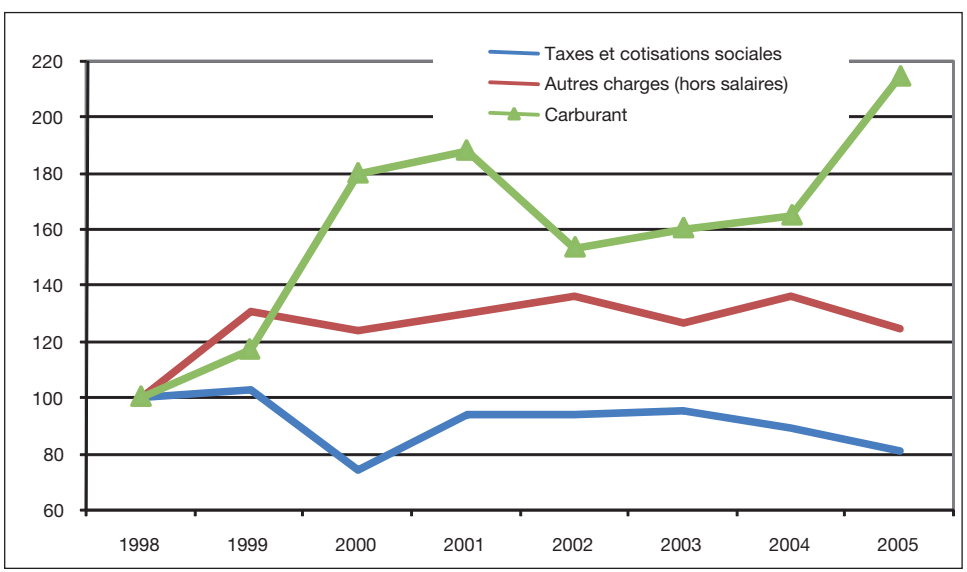

Figure 47 (suite). Chalutiers-dragueurs - évolution des agrégats comptables, 1998-2005 (euros constants 2005) (source : Observatoire économique régional des pêches).
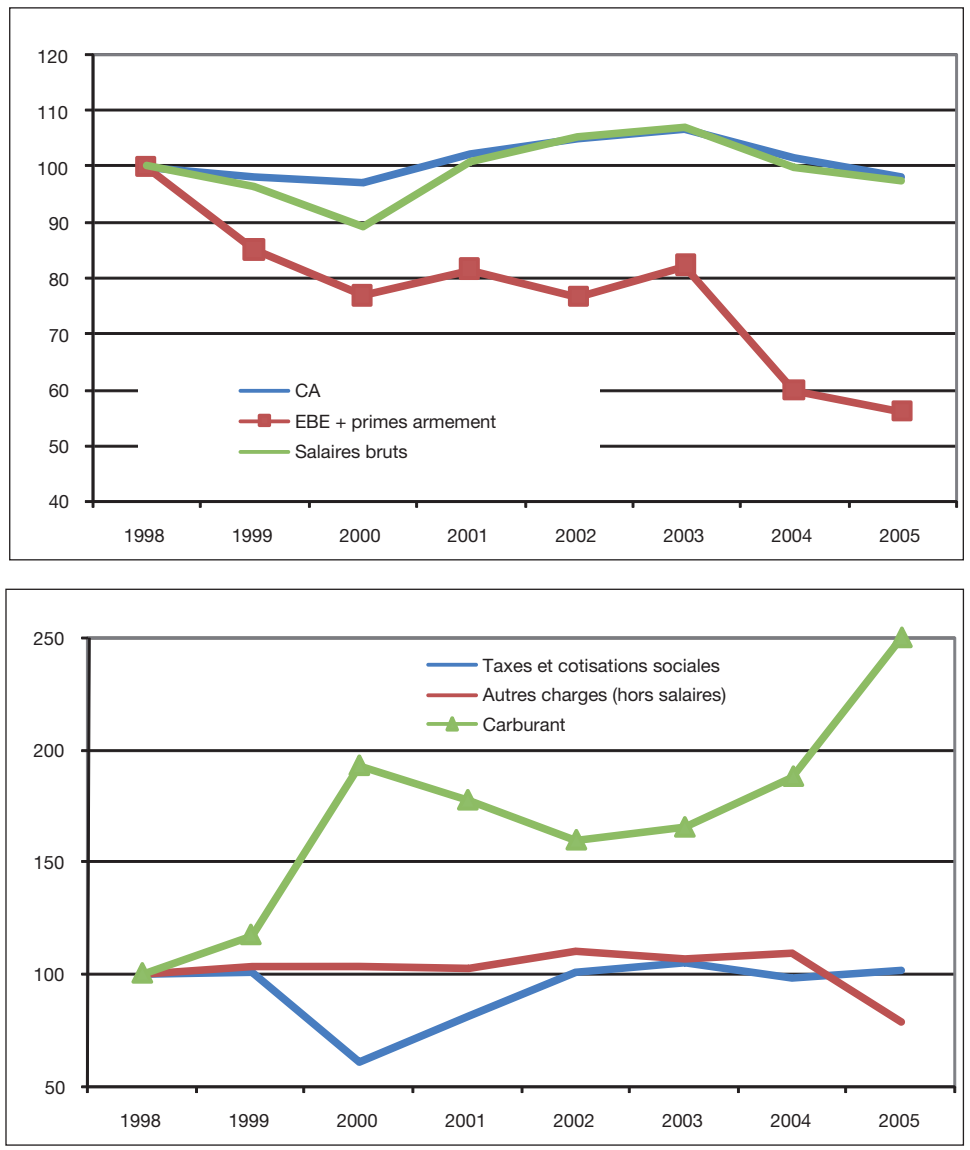

Figure 48. Chalutiers exclusifs - évolution des agrégats comptables, 1998-2005 (euros constants 2005) (source : Observatoire économique régional des pêches). 

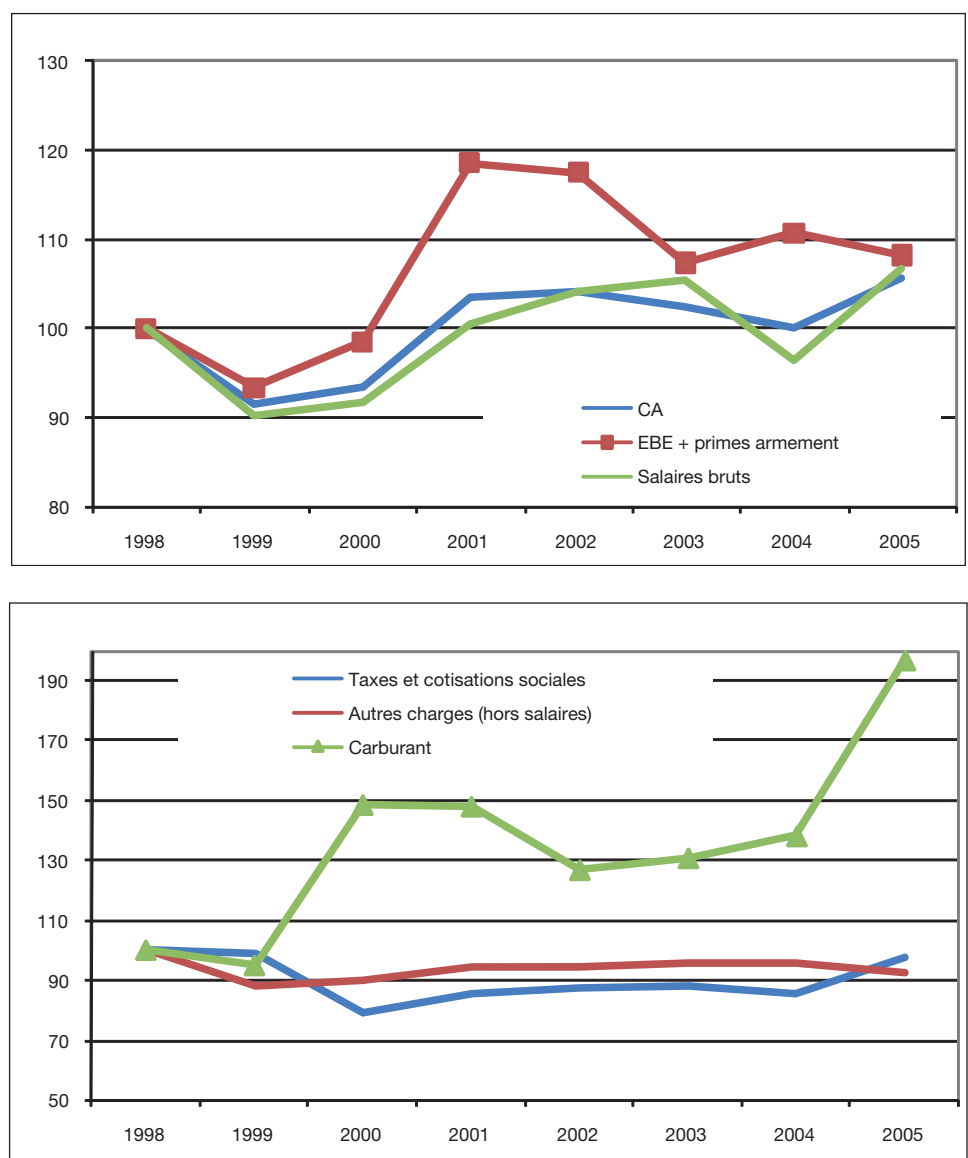

Figure 49. Dormants - Évolution des agrégats comptables, 1998-2005 (euros constants 2005) (source : Observatoire économique régional des pêches).

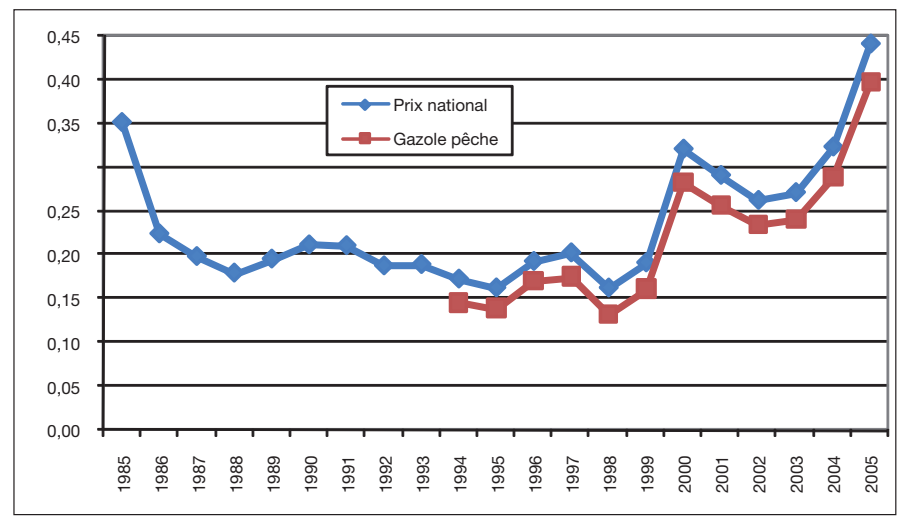

Figure 50. Évolution du prix annuel du gazole, en euros courants (source : ministère de l'Économie, des Finances et de l'Industrie (prix national) et coopérative maritime du Pays bigouden (prix du gazole pêche)). 


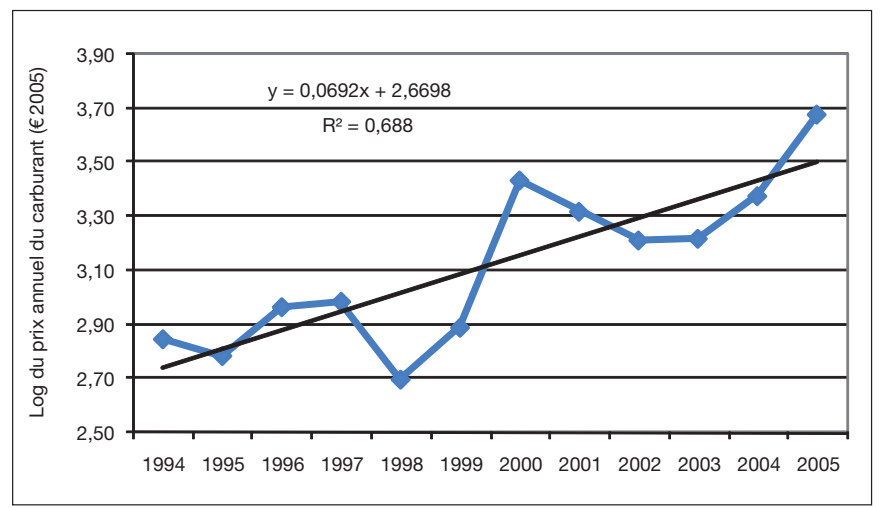

Figure 51. Évolution du prix annuel du carburant (en Log) par litre (euros constants, 2005) (source : coopérative maritime du Pays bigouden).

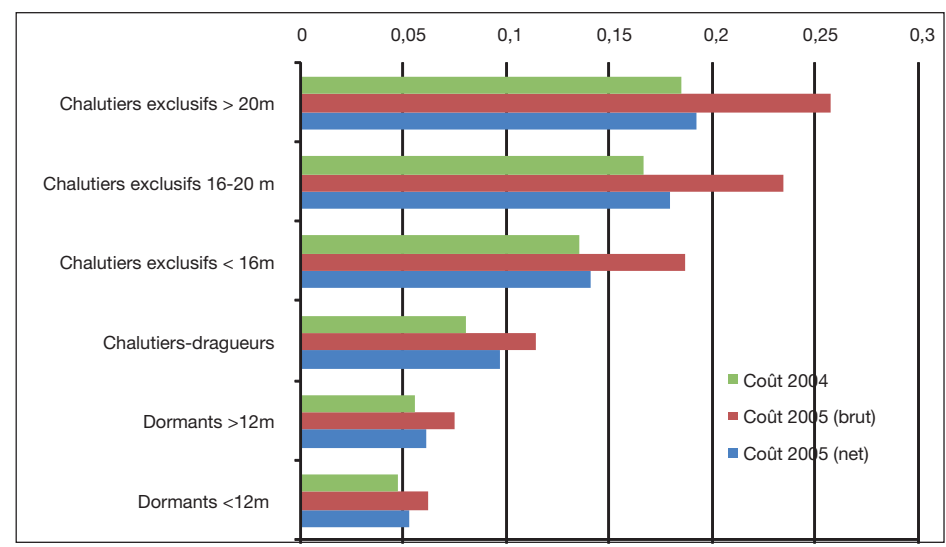

Figure 52. Coût du carburant (brut et net) en \% des ventes en 2004 et 2005 (source : Observatoire économique régional des pêches).

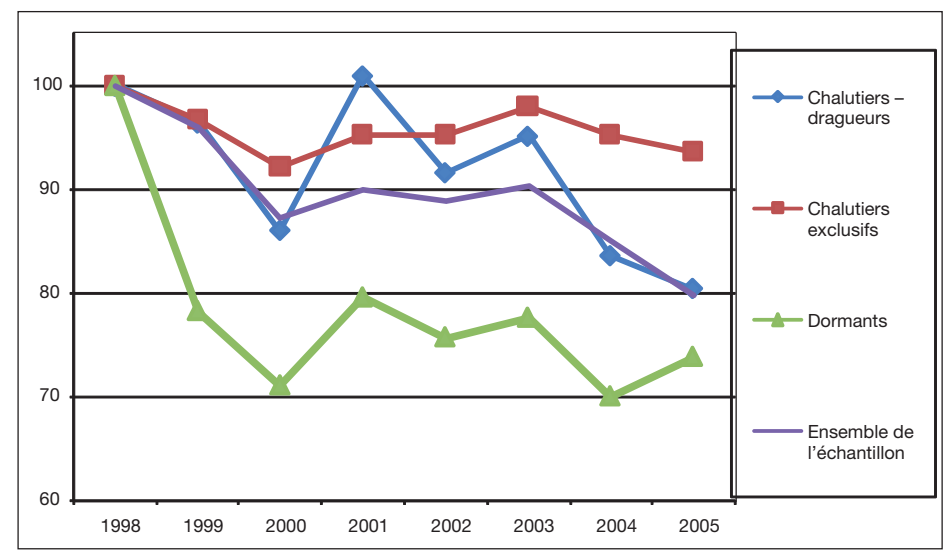

Figure 53. Évolution de la consommation physique de carburant par navire, évolution 1998-2005 (source : Observatoire économique régional des pêches). 


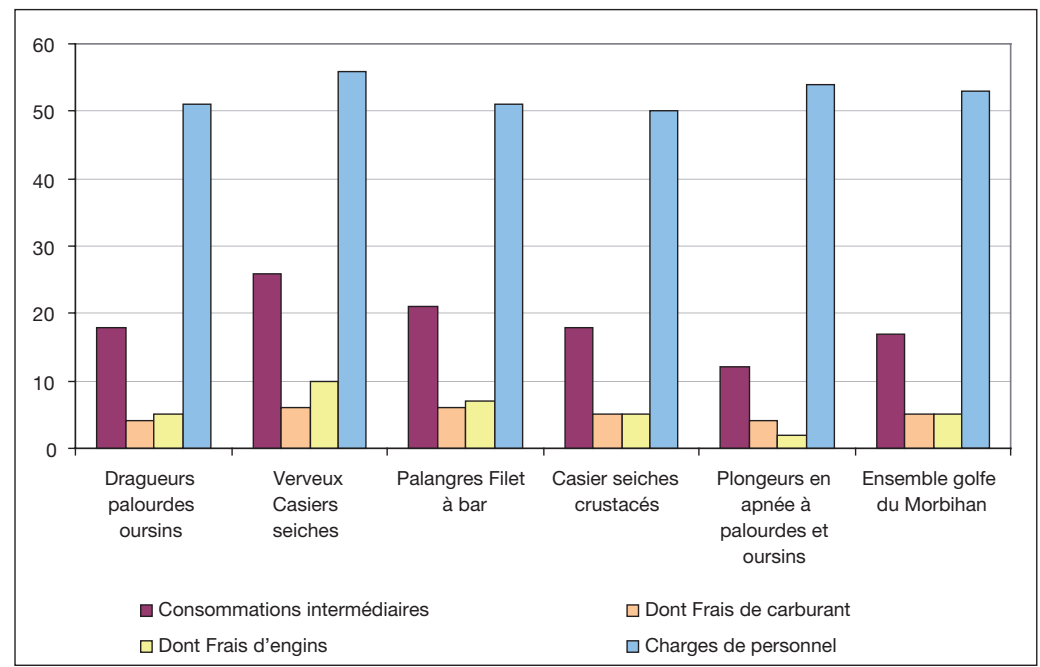

Figure 55. Structure des coûts des flottilles du golfe du Morbihan (source : Ifremer/SIH).

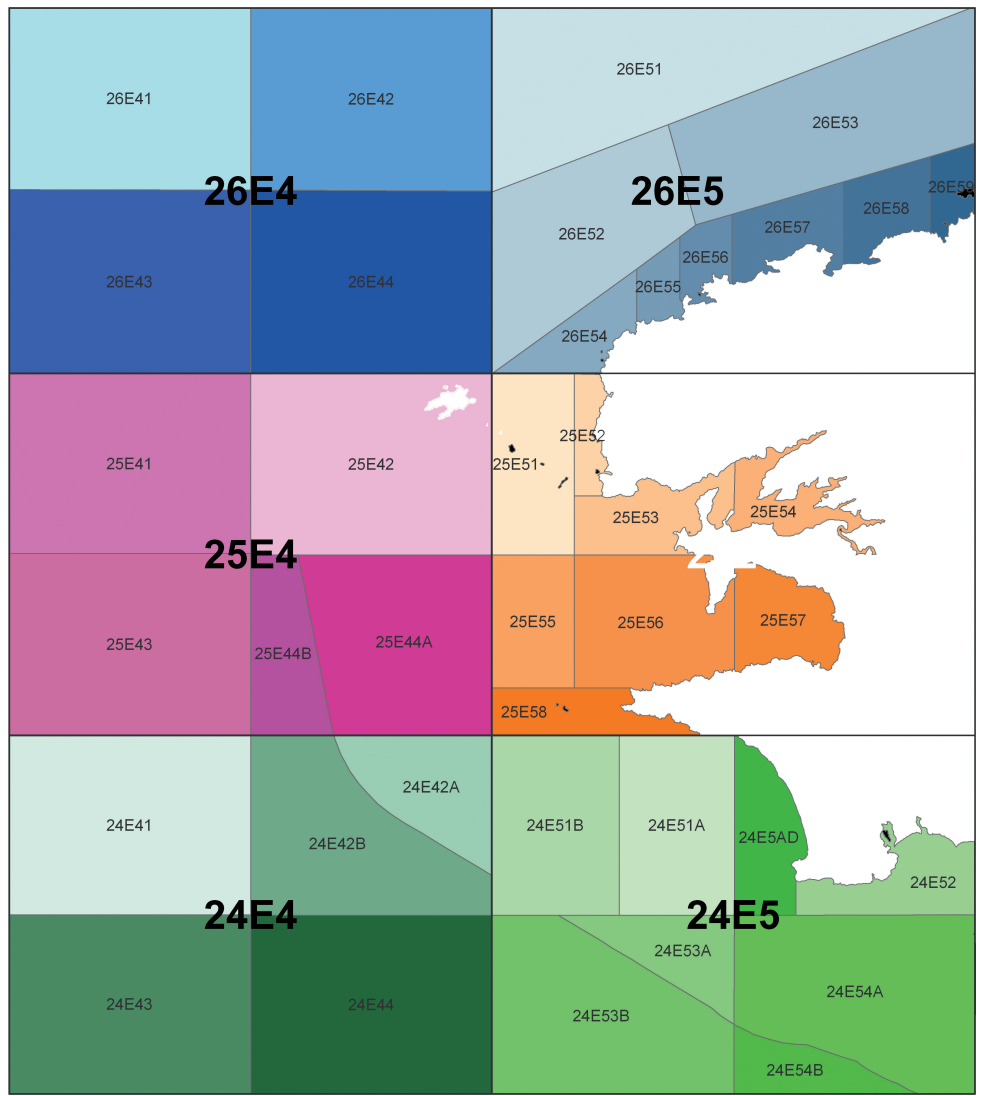

Figure 58. Zones statistiques de suivi des activités de pêche en mer d'Iroise. 


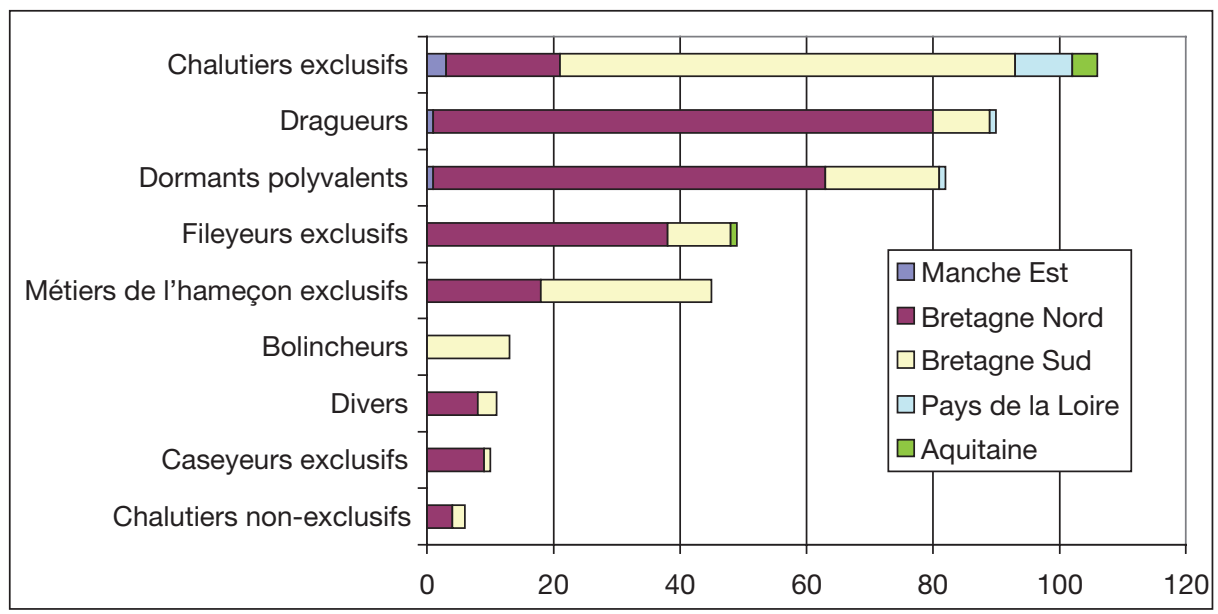

Figure 61. Régions d'origine des navires

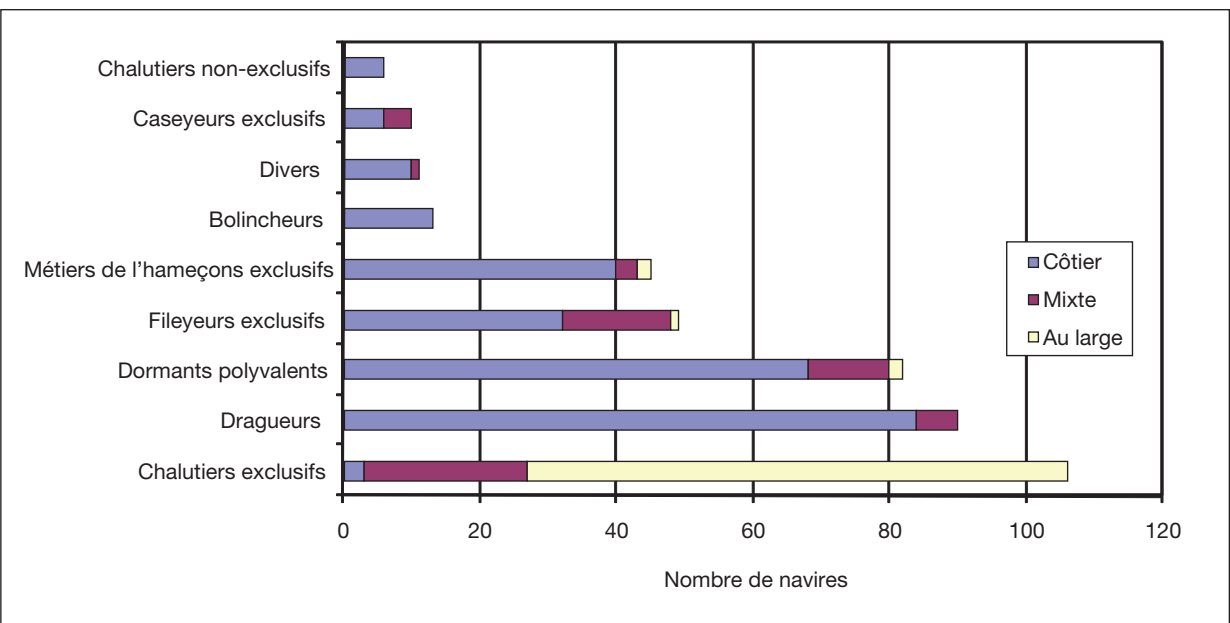

Figure 62. Répartition de la flotte selon le gradient dominant d'activité et la flottille bretonne d'appartenance. 


\section{Illustrations - Chapitre 2}
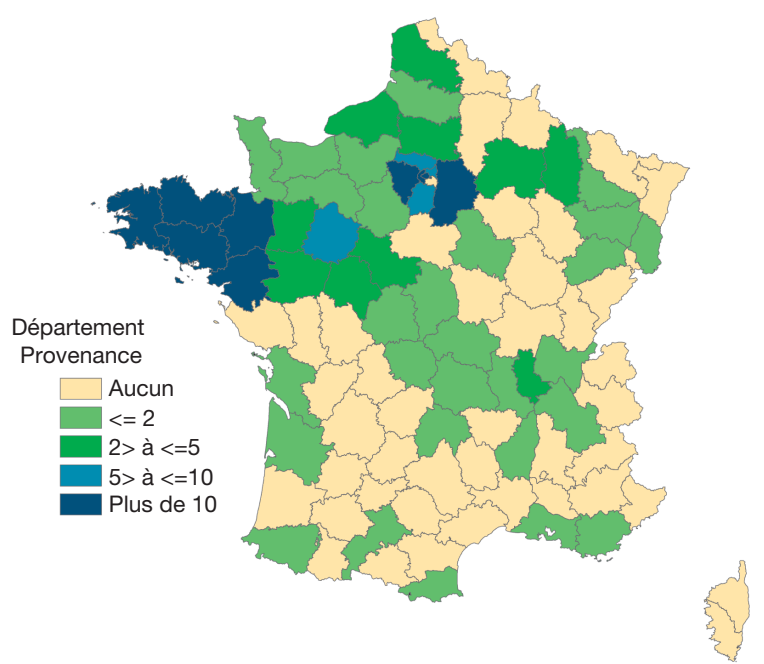

Figure 67. Département d'origine des personnes ayant fait au moins une sortie de pêche en Bretagne en 2005.

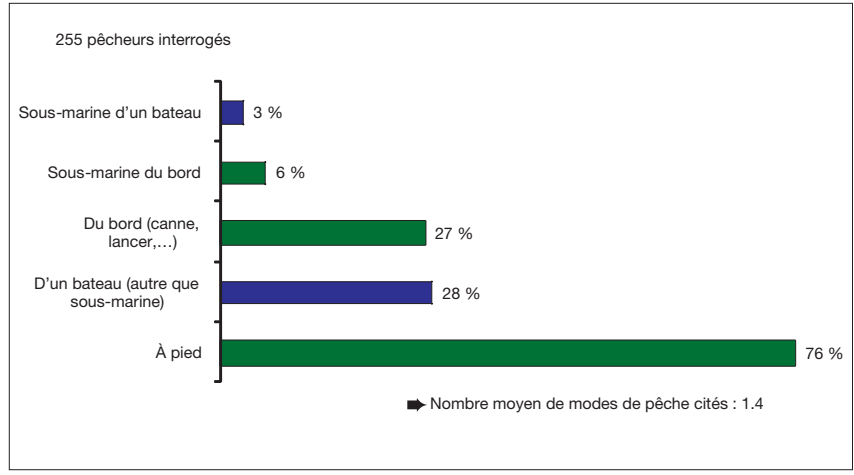

Figure 68. Modes de pêche cités par les personnes ayant fait au moins une sortie de pêche en Bretagne en 2005 (plusieurs réponses possibles par personne).

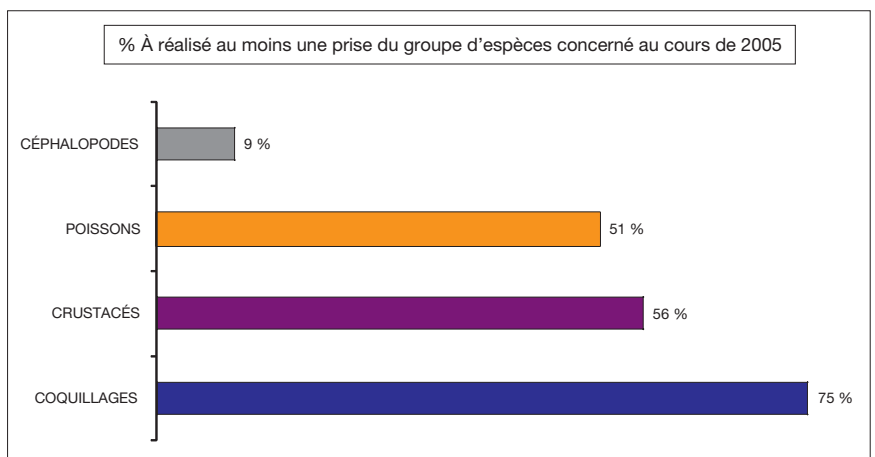

Figure 70. Groupes d'espèces pêchées en 2005 par les pêcheurs ayant réalisé au moins une sortie en Bretagne en 2005. 

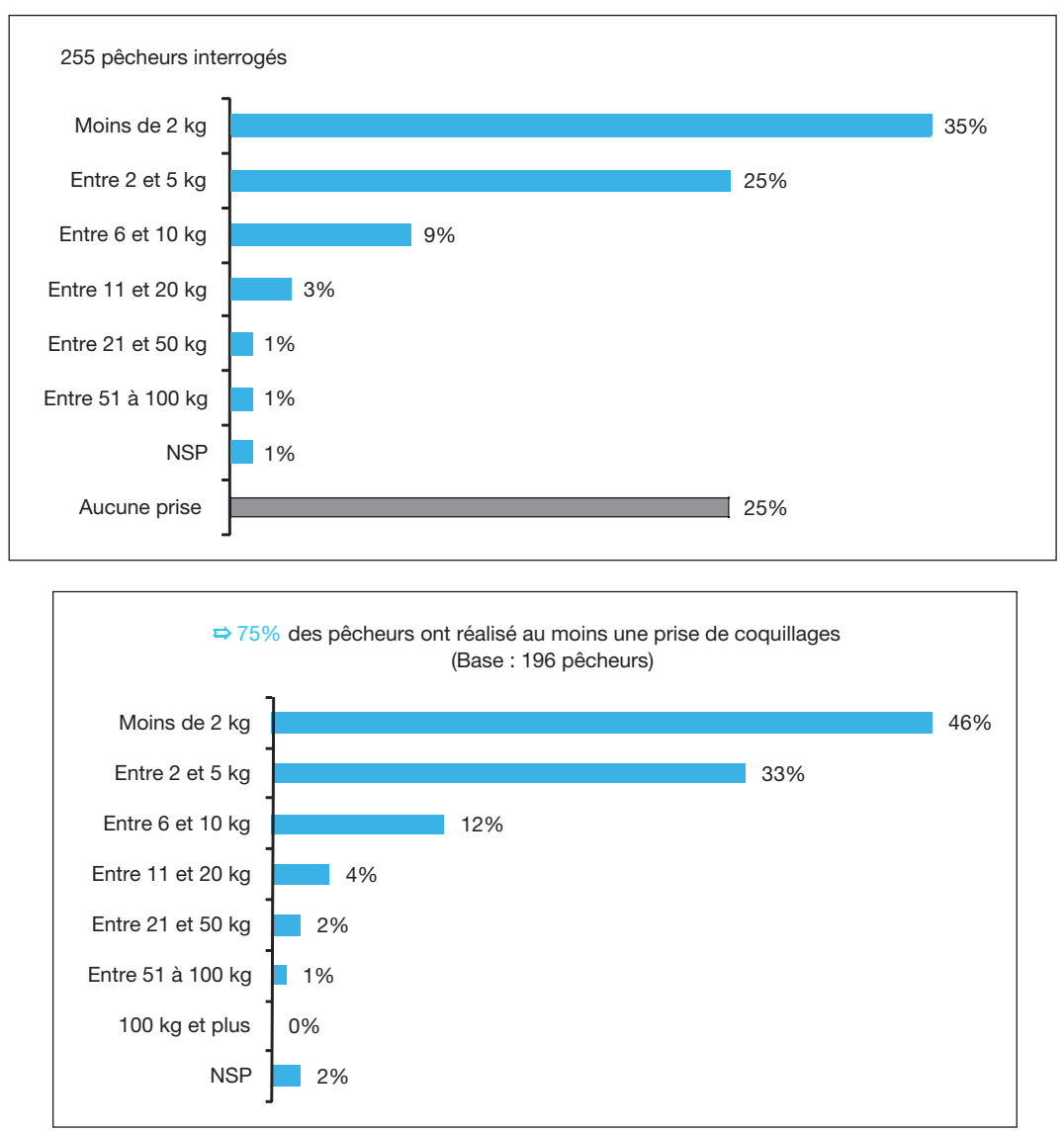

Poids moyen de coquillages pêchés sur :

$\Rightarrow$ L'ensemble des pêcheurs $2005: 3,6 \mathrm{~kg}$

$\Rightarrow$ Les pêcheurs 2005 ayant réalisé au moins une prise : $6,5 \mathrm{~kg}$

Figure 71. Rendements en coquillages par les pêcheurs ayant réalisé au moins une sortie en Bretagne en 2005.

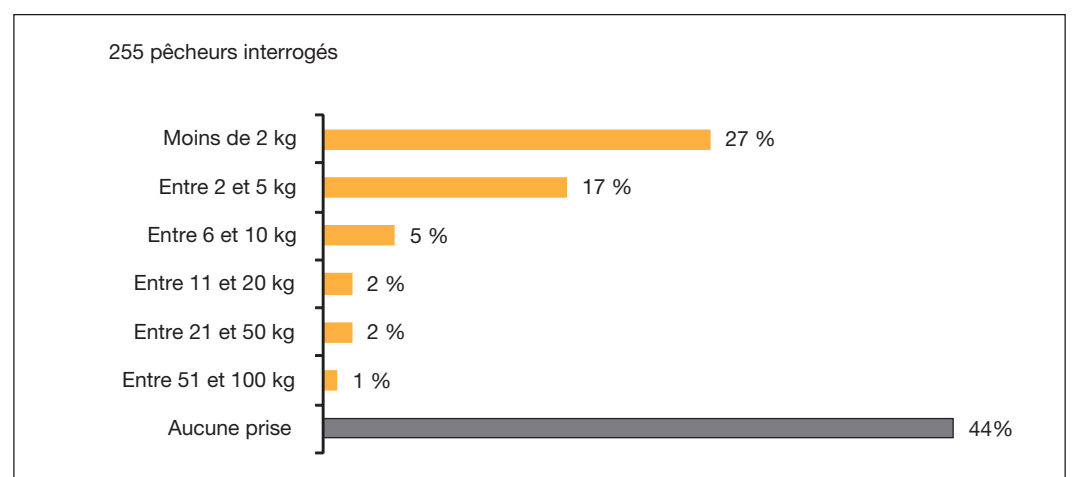

Figure 72. Rendements en crustacés par les pêcheurs ayant réalisé au moins une sortie en Bretagne en 2005. 


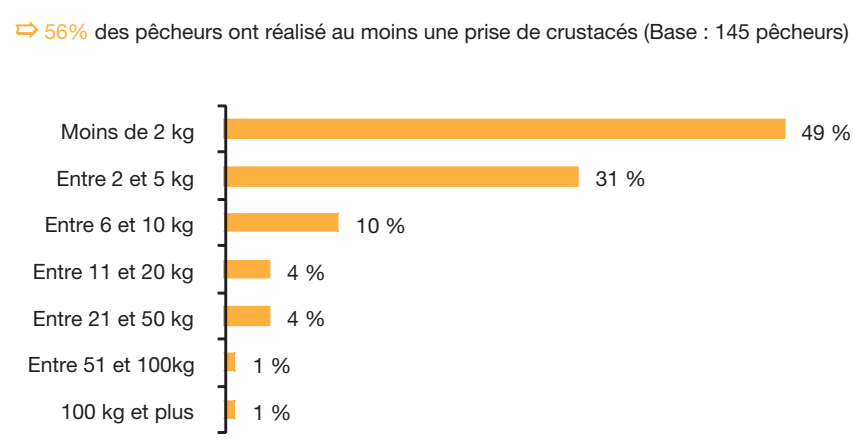

Poids moyen de crustacés pêchés sur :

$\Rightarrow$ L'ensemble des pêcheurs $2005: 3,6 \mathrm{~kg}$

$\Rightarrow$ Les pêcheurs 2005 ayant réalisé au moins une prise : 4,8 kg

Figure 72 (suite). Rendements en crustacés par les pêcheurs ayant réalisé au moins une sortie en Bretagne en 2005.

255 pêcheurs interrogés
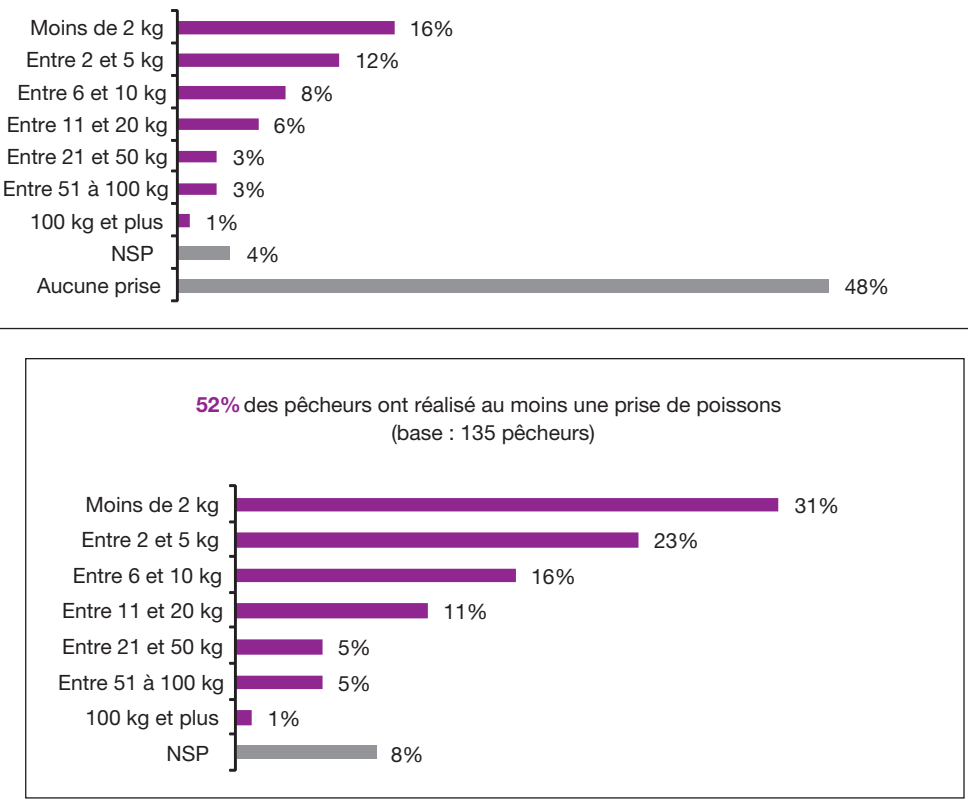

Poids moyen de poissons pêchés sur :

L'ensemble des pêcheurs 2005 : 6,2 kg

Les pêcheurs 2005 ayant réalisé au moins une prise : 12,6 kg

Figure 73. Rendements en poissons par les pêcheurs ayant réalisé au moins une sortie en Bretagne en 2005. 


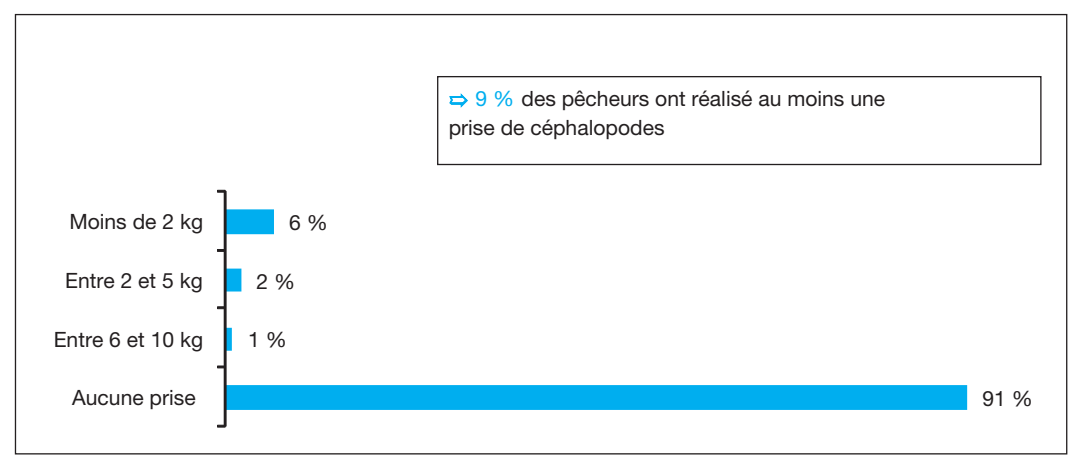

Poids moyen de céphalopodes pêchés sur :

$\Rightarrow$ L'ensemble des pêcheurs $2005: 0,3 \mathrm{~kg}$

$\Rightarrow$ Les pêcheurs 2005 ayant réalisé au moins une prise : $3,6 \mathrm{~kg}$

Figure 74. Rendements en céphalopodes par les pêcheurs ayant réalisé au moins une sortie en Bretagne en 2005.

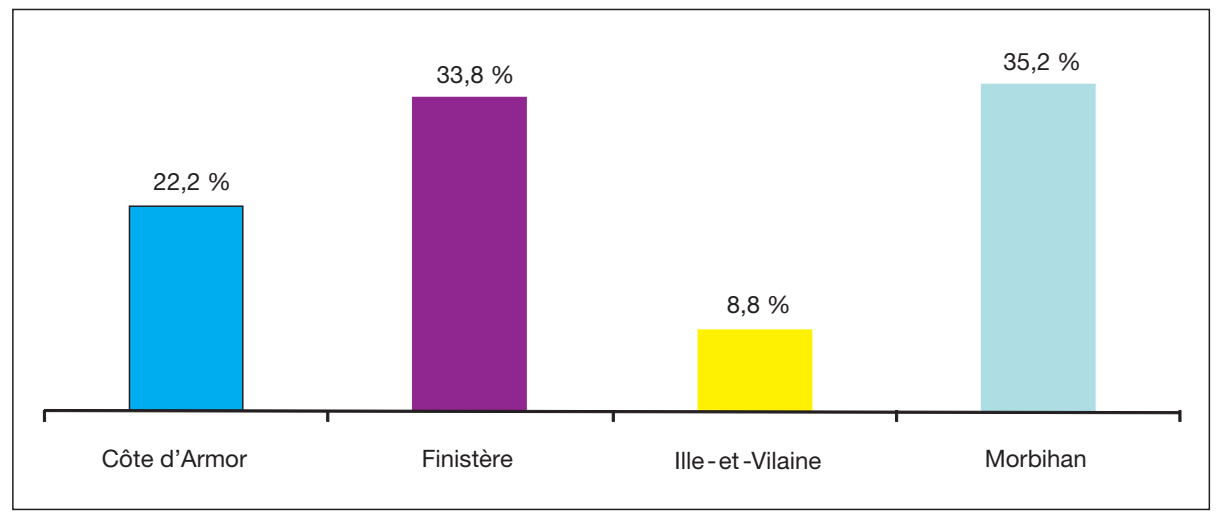

Figure 79. Fréquentation des différents départements bretons pour la dernière sortie de pêche en 2006.

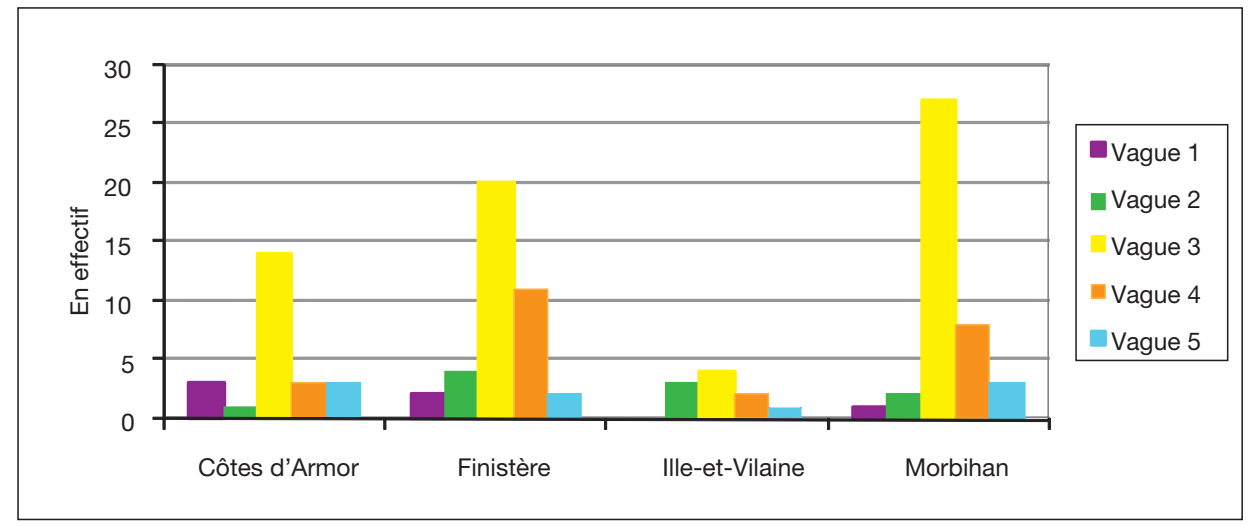

Figure 80. Fréquentation des différents départements bretons pour la dernière sortie de pêche par vague d'enquête en 2006. 


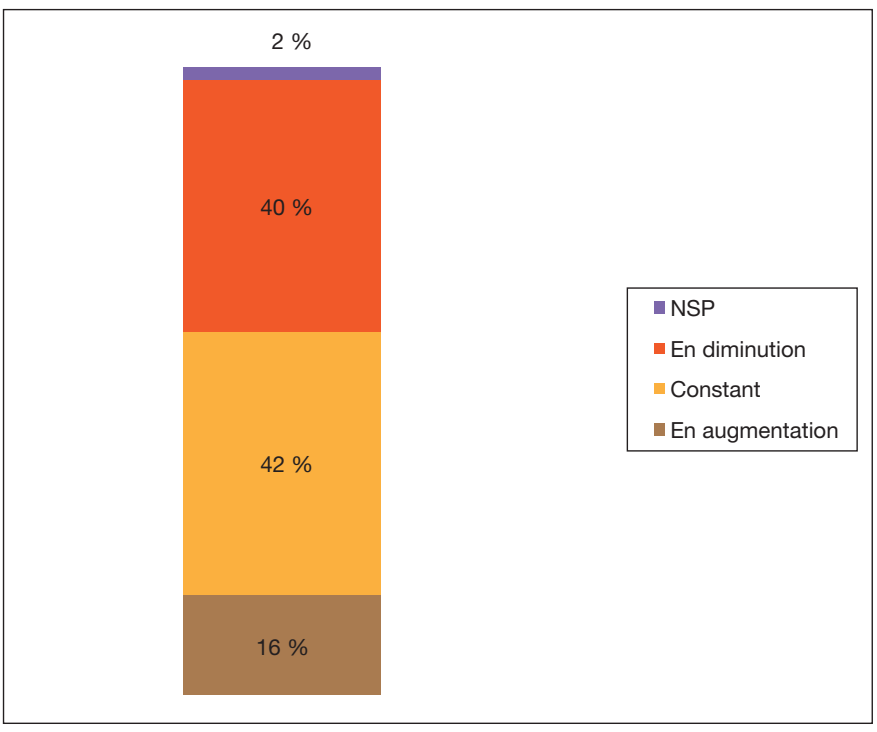

Figure 88. Évolution du temps consacré à la pratique de la pêche en mer au cours des 5 dernières années (base 291 pêcheurs interrogés).

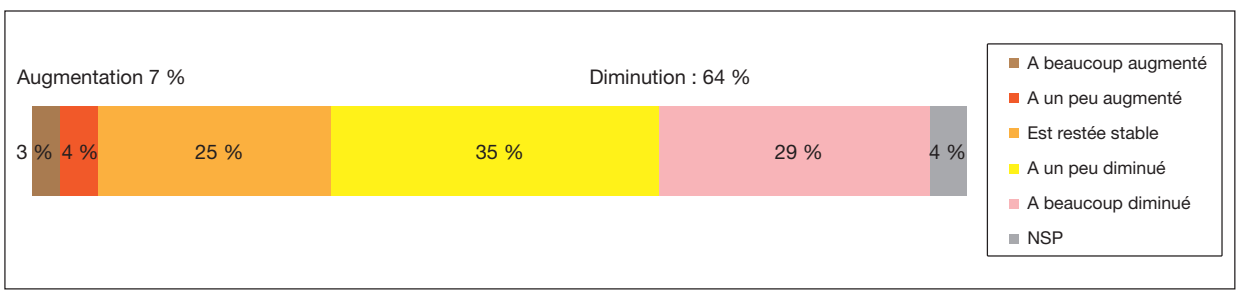

Figure 89. Sentiment des pêcheurs sur l'évolution de la ressource au sens large (poissons, coquillages, crustacés...) au cours de ces 5 dernières années (base 291 pêcheurs interrogés).

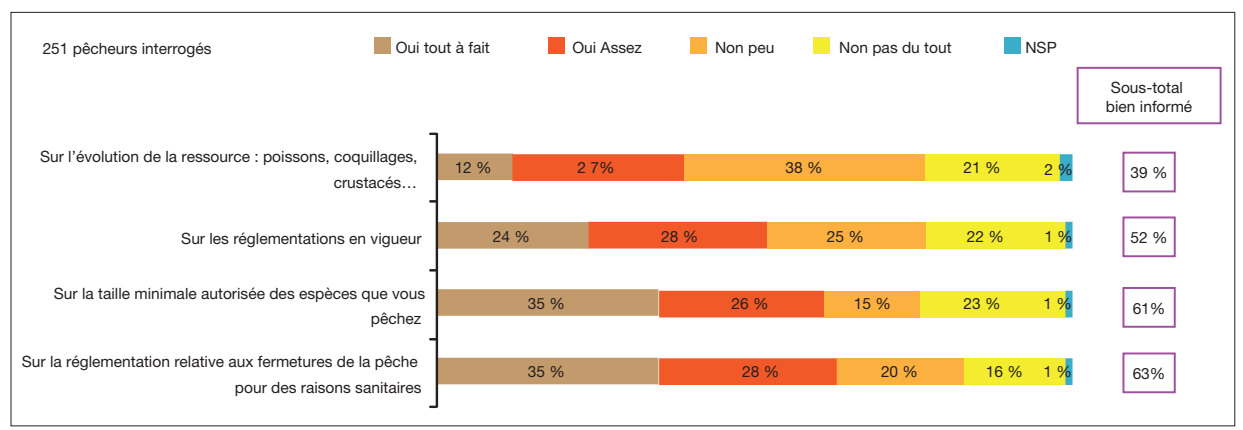

Figure 90. Sentiments généraux des pêcheurs ayant fréquenté la Bretagne en 2005 et/ou en 2006 vis-à-vis des réglementations et de l'évolution des ressources. 


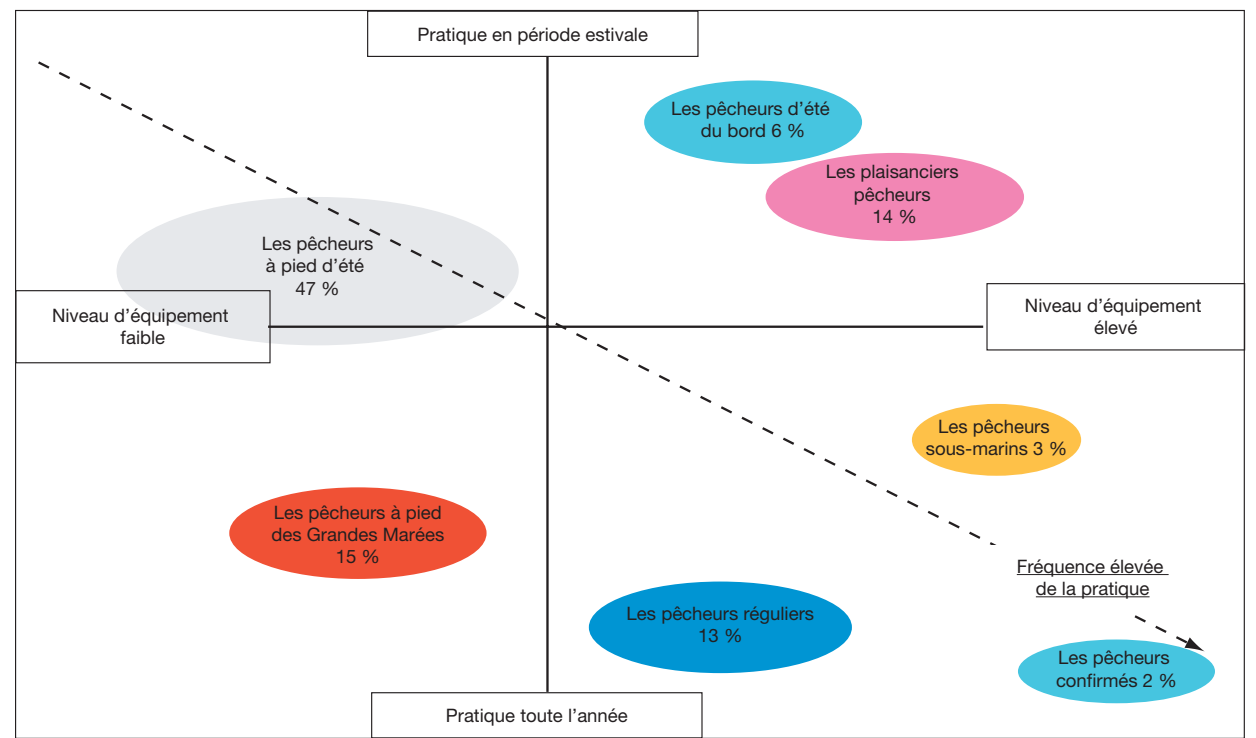

Figure 91. Répartition des pêcheurs ayant fréquenté la Bretagne en 2005 ou en 2006 en fonction de leur typologie par rapport aux principaux facteurs explicatifs.

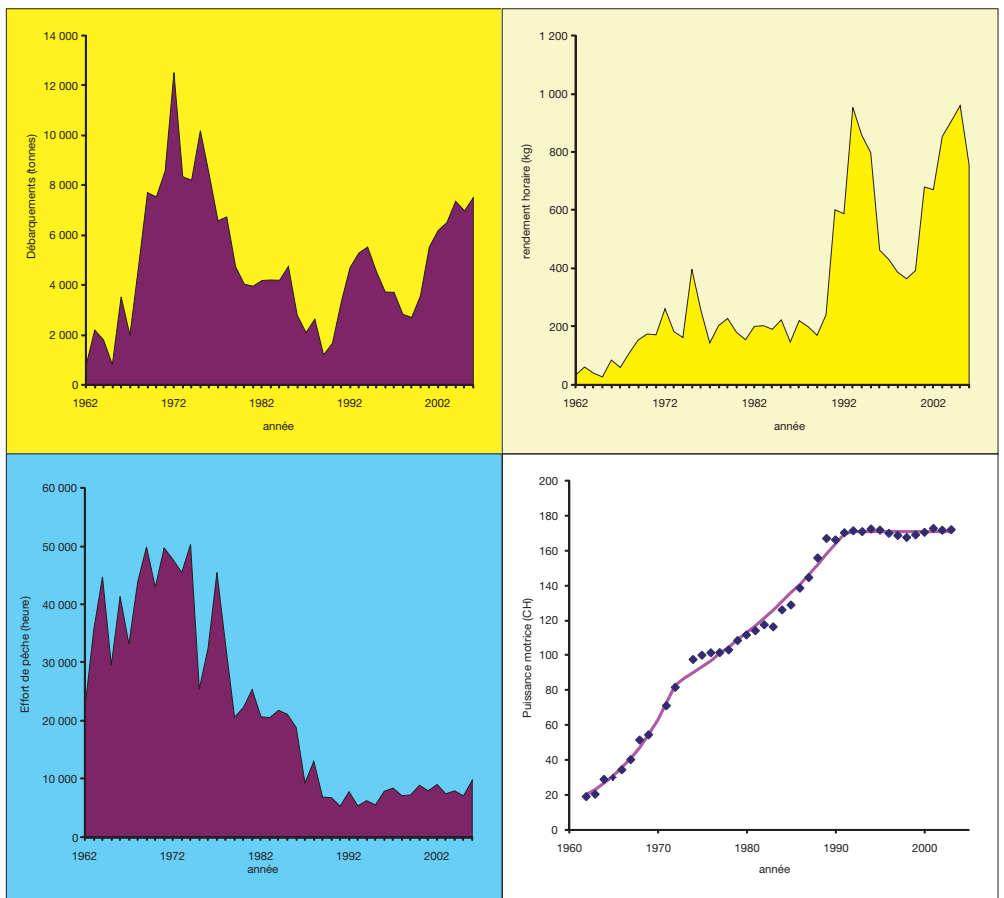

Figure 92. Évolution des paramètres principaux de l'exploitation du stock de la baie de SaintBrieuc depuis 1962. De gauche à droite et de haut en bas - (1) les débarquements officiels ; (2) le rendement horaire (CPUE) ; (3) l'effort de pêche et (4) la puissance motrice moyenne de la flottille de pêche. 


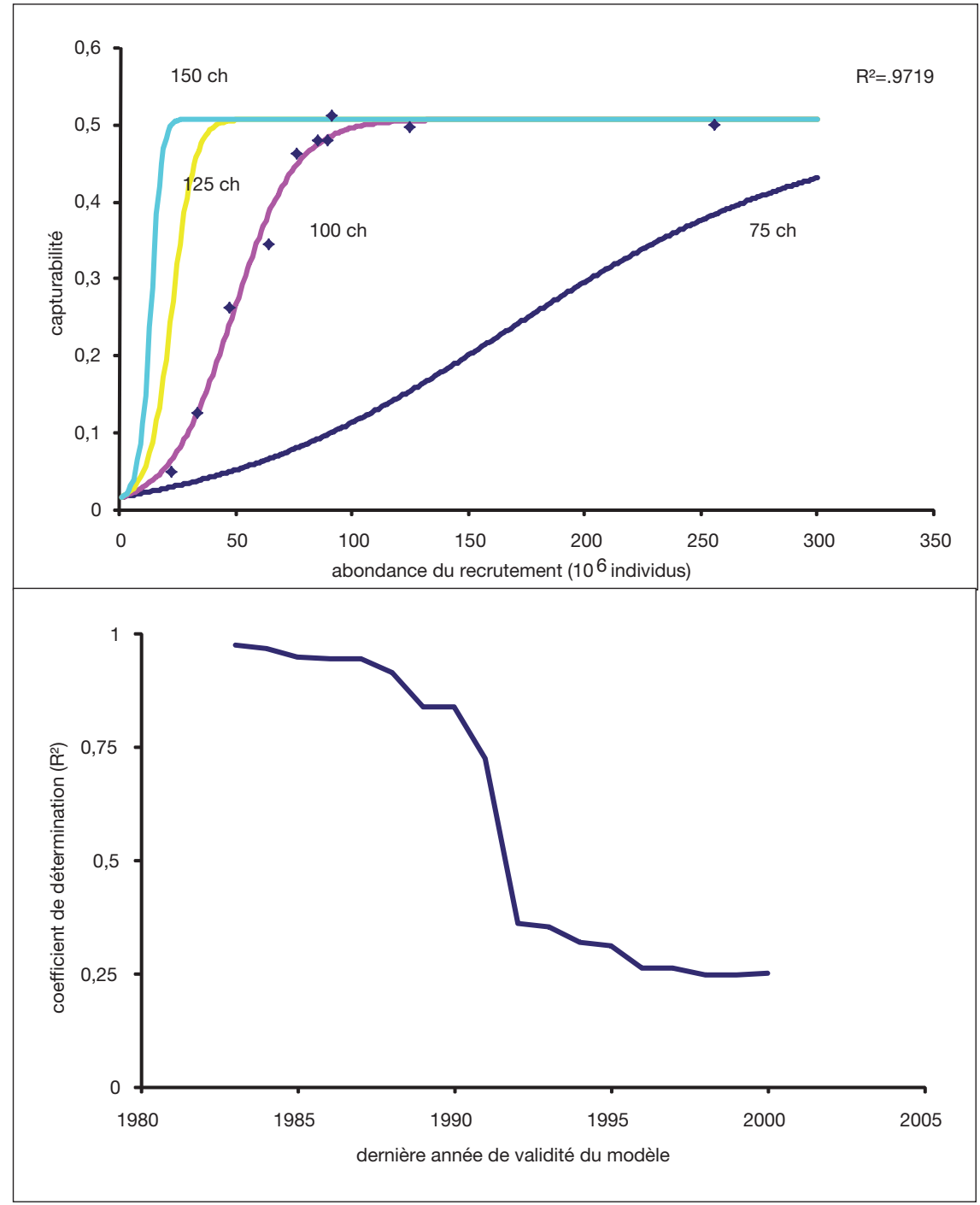

Figure 93. En haut : Modèle de capturabilité (exemple du groupe d'âge 2) en fonction de l'abondance du groupe (cohortes nées entre 1972 et 1981) et de la puissance motrice moyenne. En bas : Évolution du coefficient de détermination $\left(\mathrm{R}^{2}\right)$ du modèle de capturabilité (équation [1]) en fonction de la dernière année de validité du modèle. 


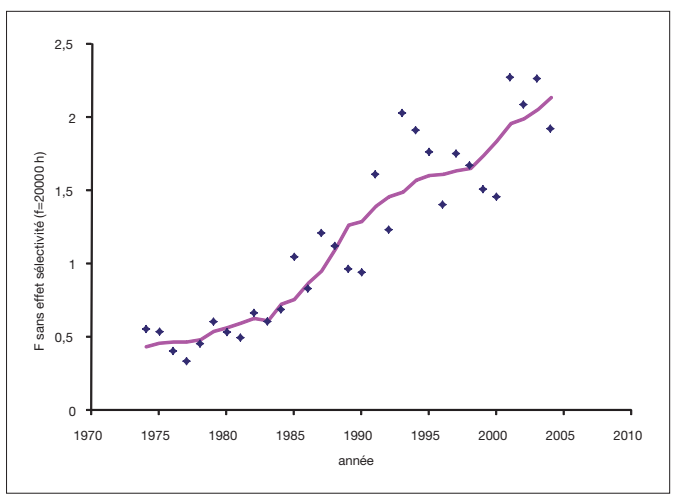

Figure 94. Modèle de capturabilité (exprimée comme mortalité par pêche à un effort de pêche de $20000 \mathrm{~h}$ après dissociation de la composante de sélectivité). Tous groupes d'âge confondus.

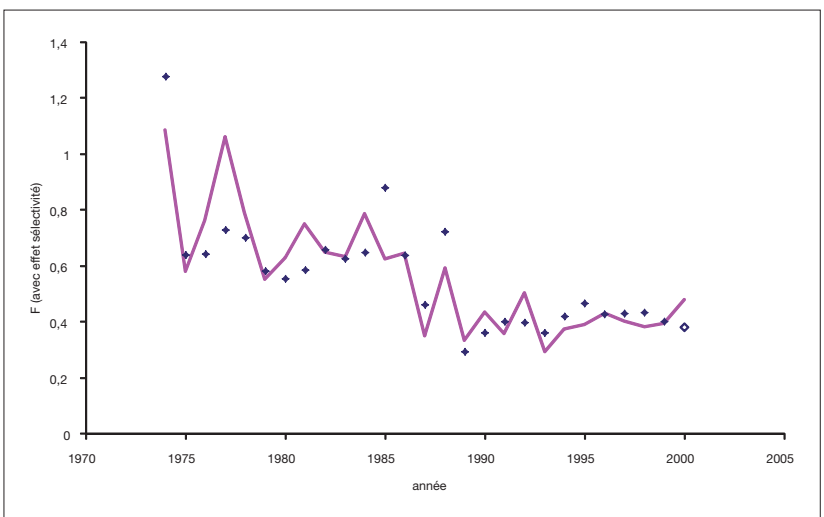

Figure 95. Baie de Saint-Brieuc. Évolution de la mortalité par pêche tous groupes d'âge confondus avec effet de sélectivité lié aux maillages.

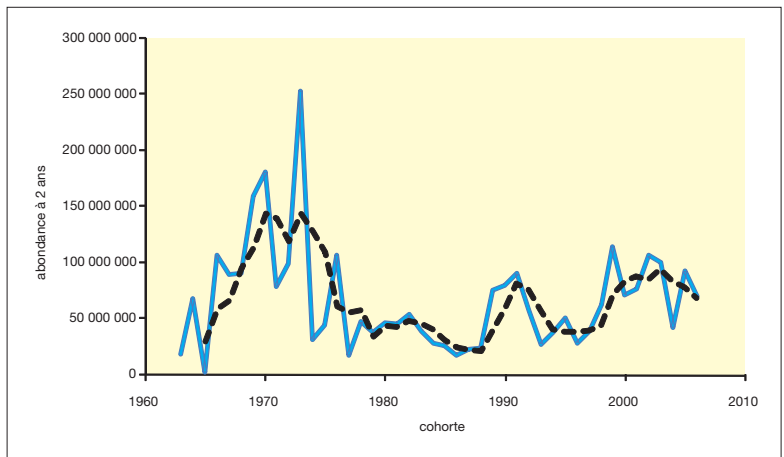

Figure 96. Cinétique du recrutement de coquilles Saint-Jacques (abondance à l'âge de deux ans) en baie de Saint-Brieuc. Note : L'abondance de la cohorte 2006 à l'âge 2 est obtenue par extrapolation à partir de l'abondance de la même cohorte à l'âge $1, C f$. Relation $\mathrm{N}(\mathrm{GR} 2) .=1,555^{*} \mathrm{~N}(\mathrm{GR} 1) .0,765$, les abondances $\mathrm{N}$ étant exprimées en milliers d'individus ; $\mathrm{r}^{2}=0,860$. 


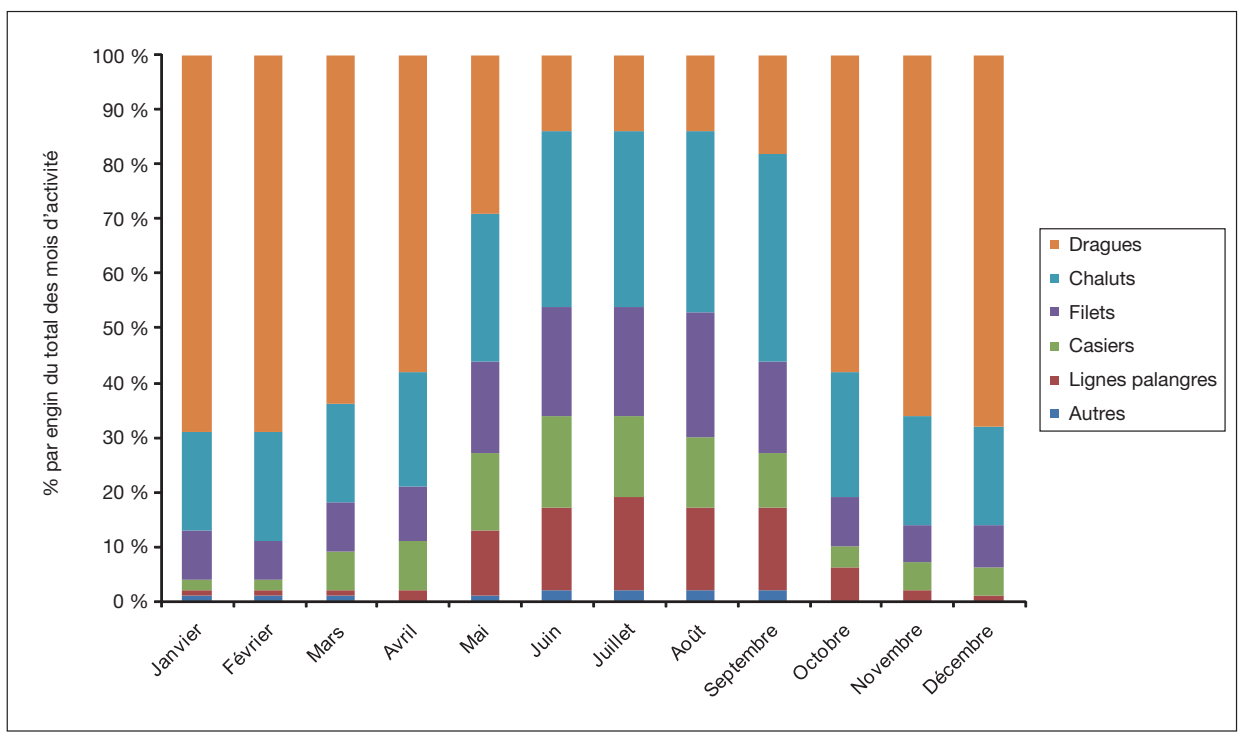

Figure 97. Indicateurs de polyvalence saisonnière de la flottille de dragueurs à coquilles Saint-Jacques de la baie de Saint-Brieuc.

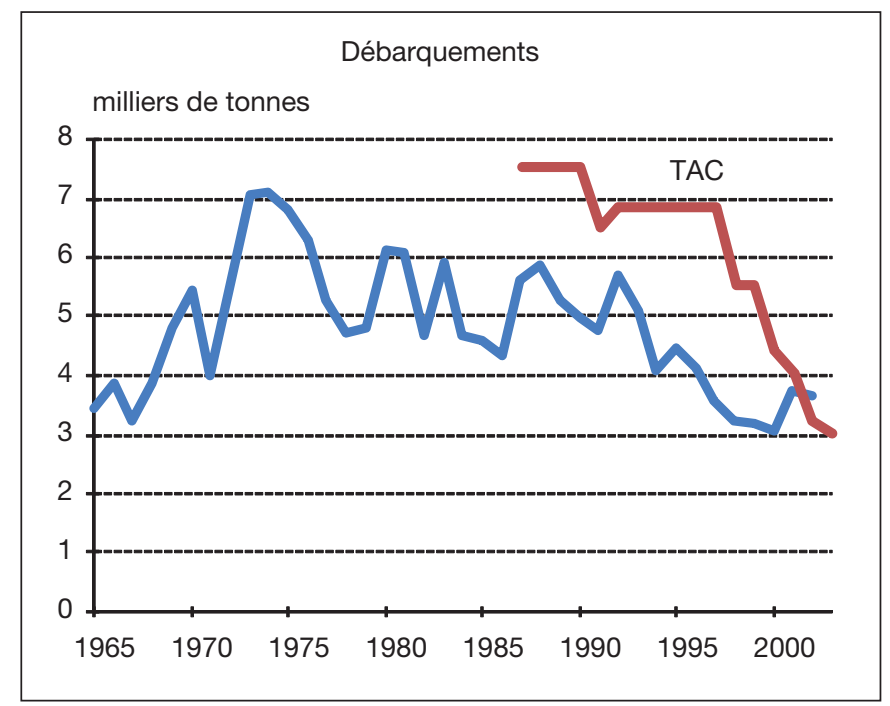

Figure 98. Évolution des débarquements de langoustines en provenance du golfe de Gascogne de 1965 à 2001. 


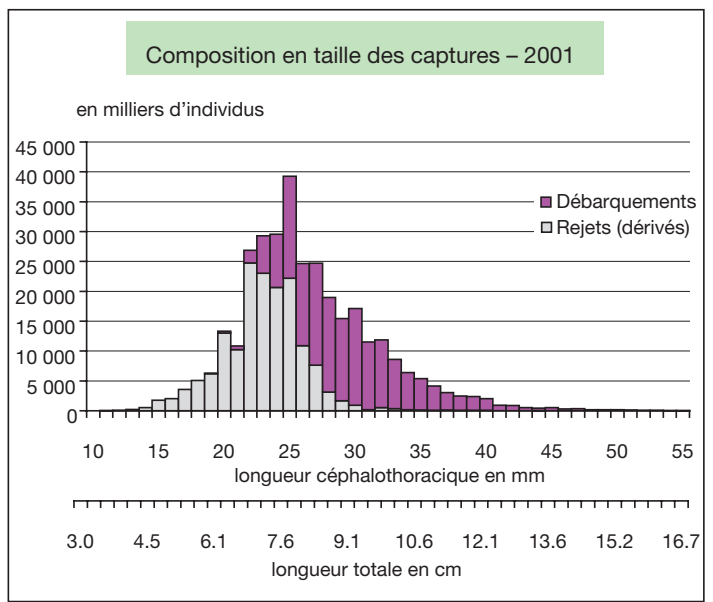

Figure 99. Composition en taille des débarquements et de rejets de langoustines en provenance du golfe de Gascogne pour l'année 2001.

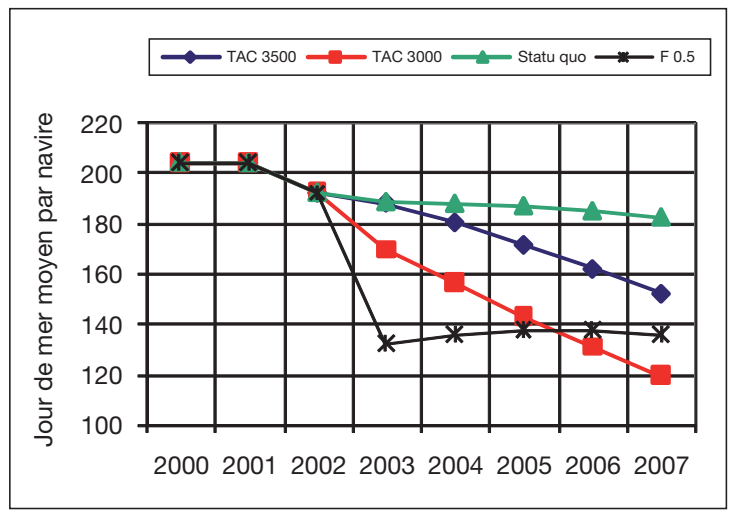

Figure 103. Évolution du nombre de jours de mer pour un navire langoustinier selon différents scénarios de gestion du stock de langoustines du golfe de Gascogne.

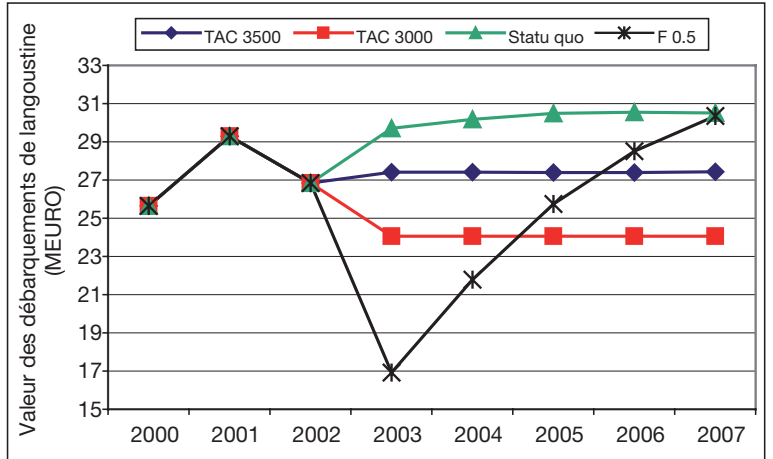

Figure 104. Évolution de la valeur totale des débarquements de langoustines selon différents scénarios de gestion du stock de langoustine du golfe de Gascogne. 


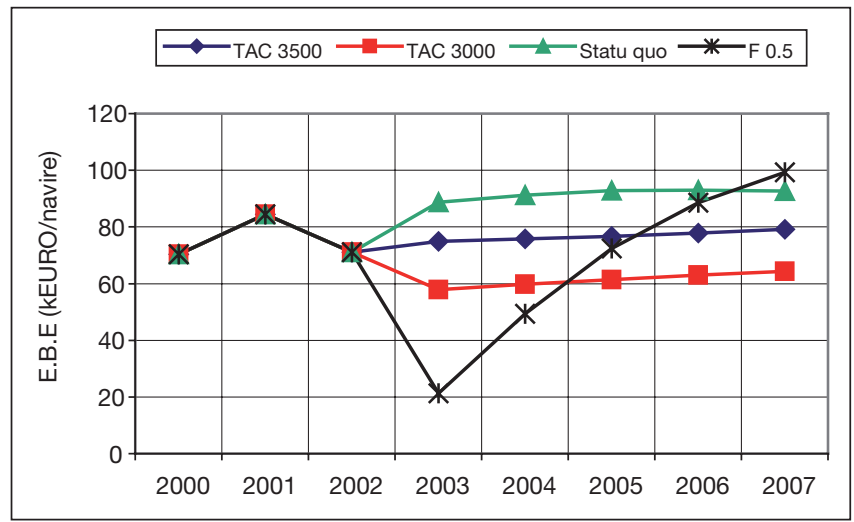

Figure 105. Évaluation de l'excédent brut d'exploitation d'un navire langoustinier selon différents scénarios de gestion du stock de langoustines du golfe de Gascogne.

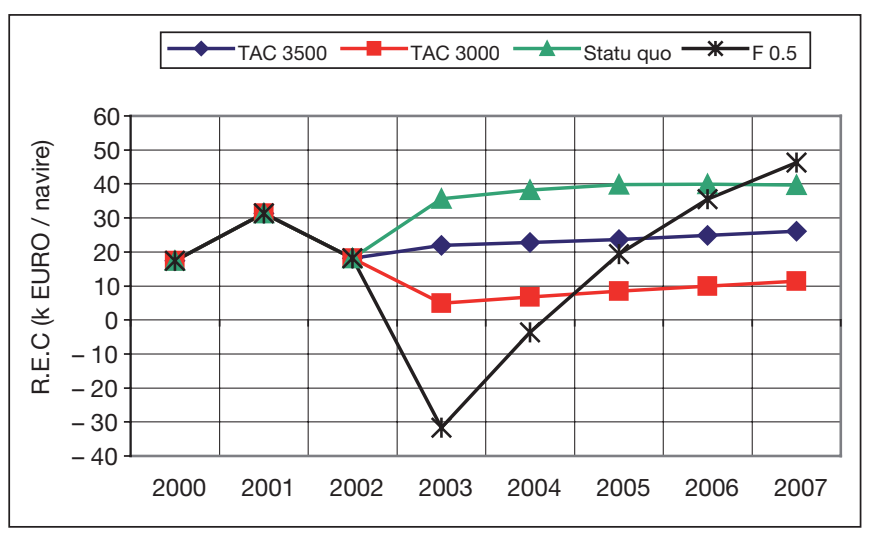

Figure 106. Évolution du résultat économique courant d'un navire langoustinier selon différents scénarios de gestion du stock de langoustines du golfe de Gascogne. 


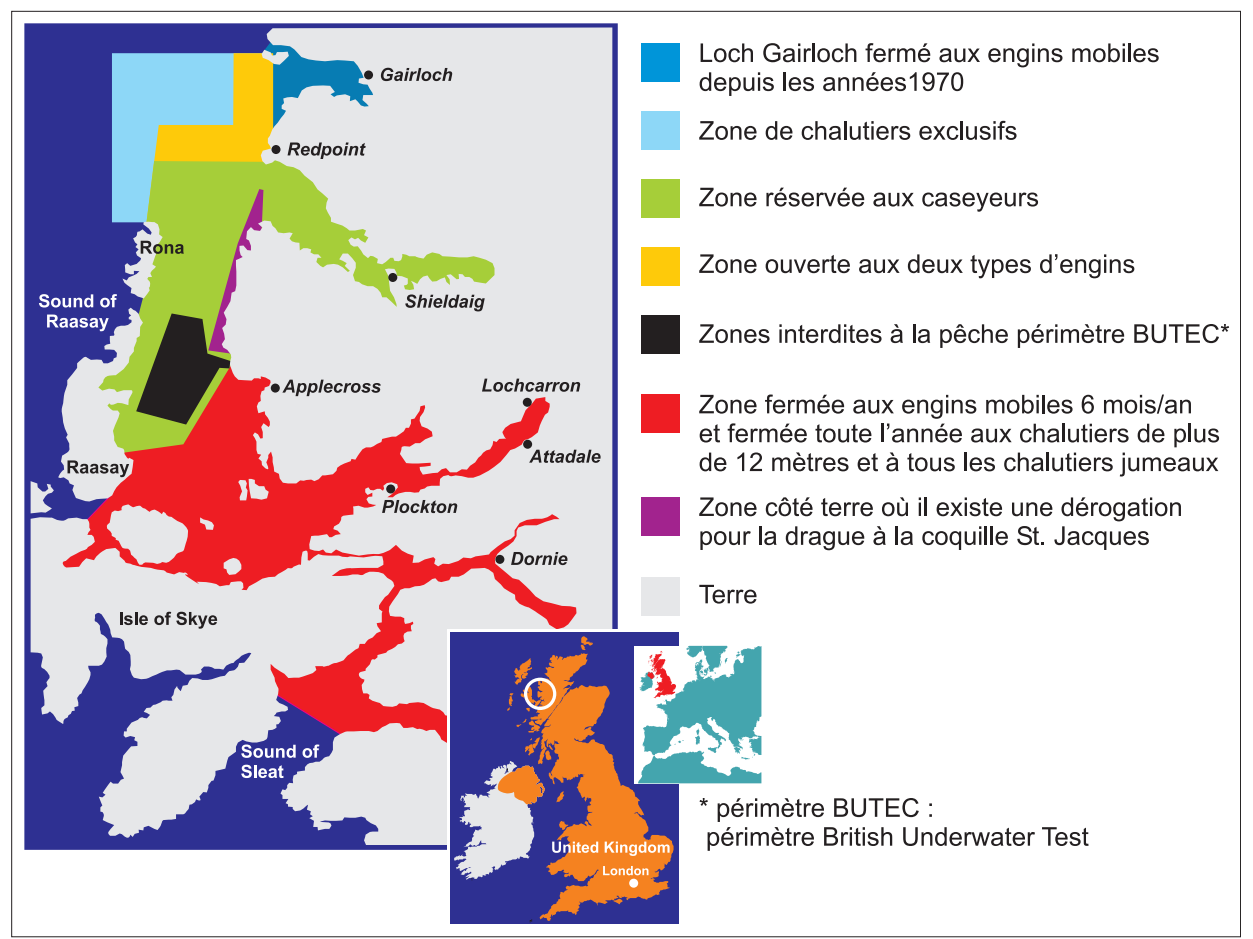

Figure 107. Zones de pêche des caseyeurs à langoustines de Loch Torridon (sources - Carte de localisation WWF et carte des différentes zones Shieldaig Export).

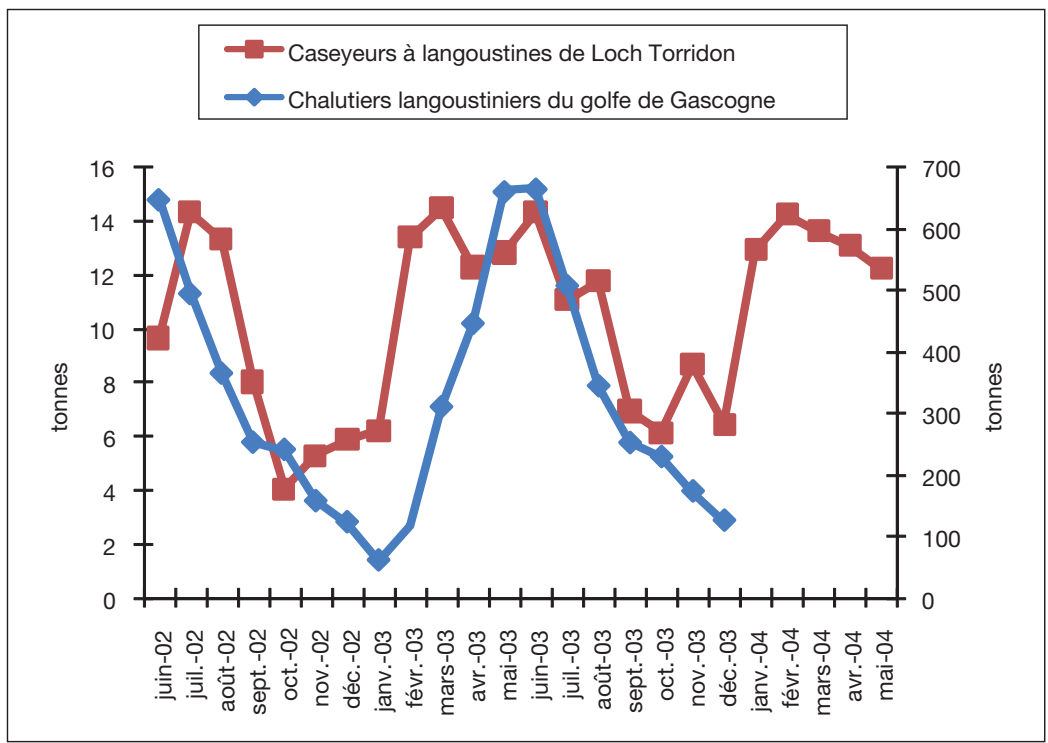

Figure 110. Débarquements totaux de langoustines des navires de la flottille des caseyeurs à langoustines de Loch Torridon et des chalutiers langoustiniers du golfe de Gascogne, en tonnes par mois (sources : Shieldaig Export Ltd - Ifremer/SIH). 

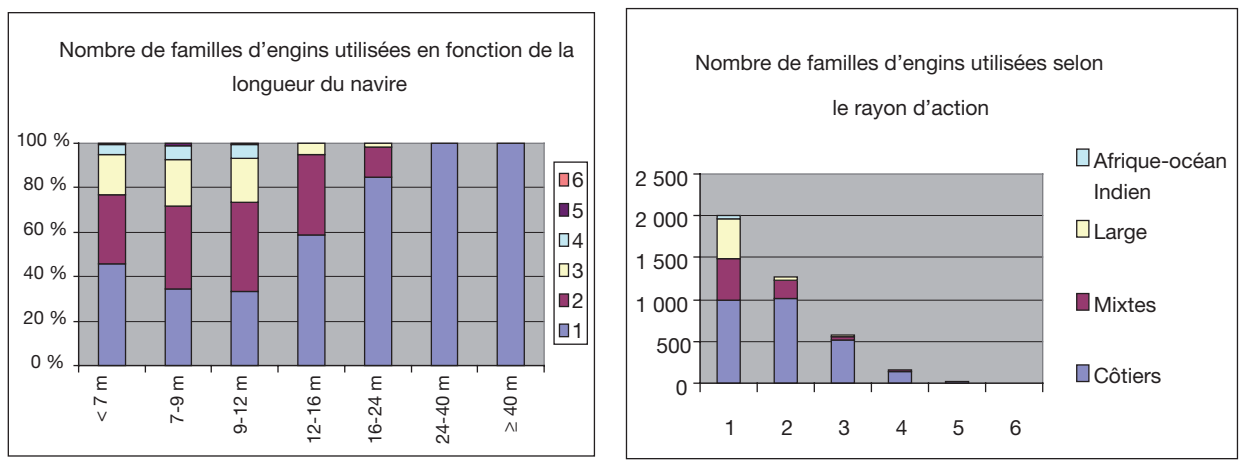

Figures 116 et 117. Polyvalence de l'activité des navires en 2000.

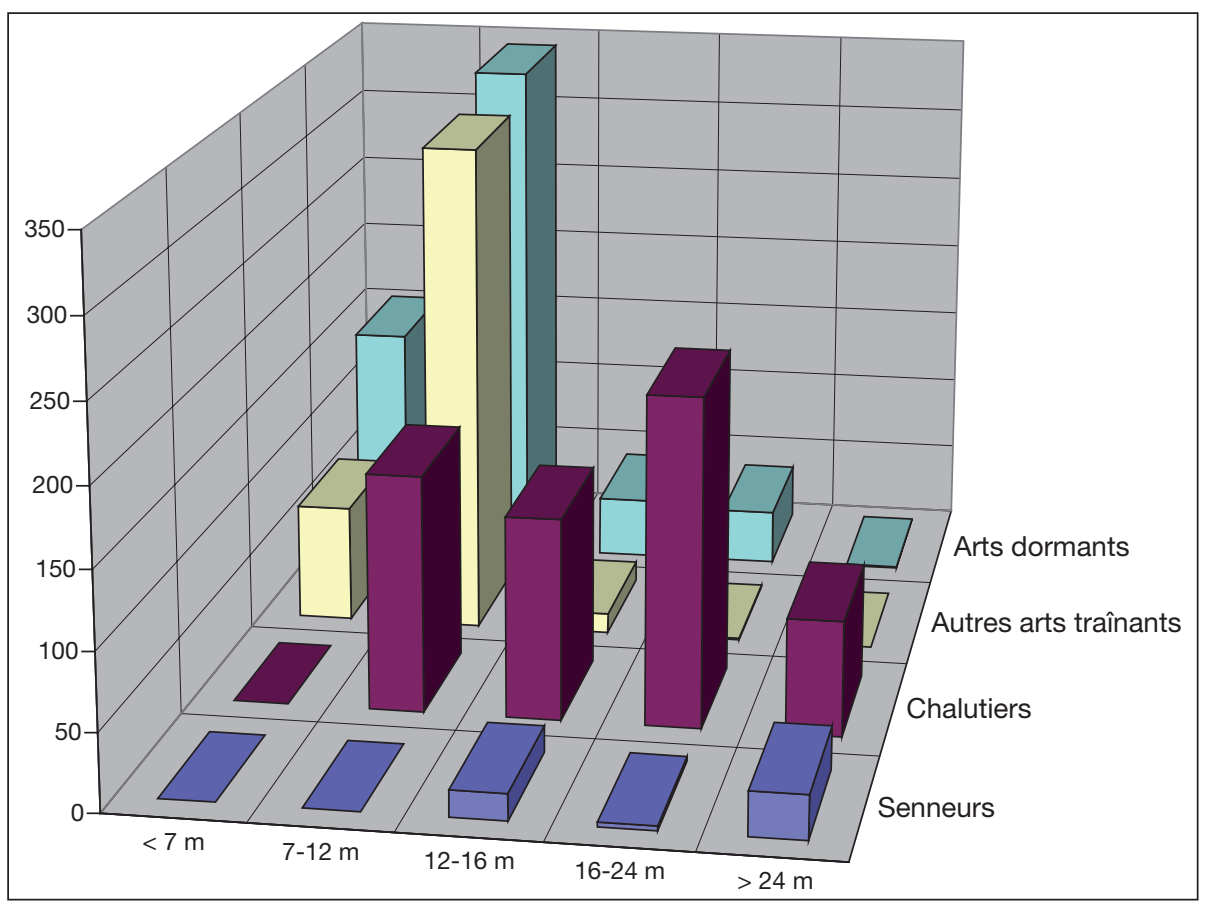

Figure 119. La flotte de pêche bretonne en 2001. Nombre de navires par classe de longueur et par flottille. 


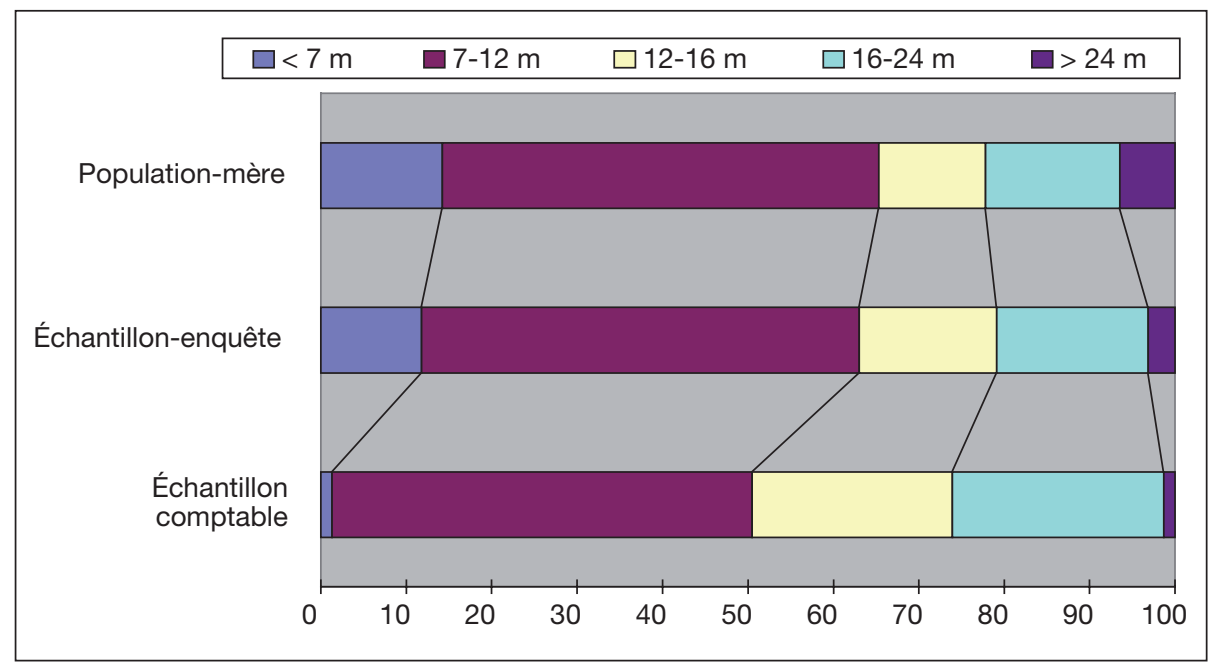

Figure 120. Structure comparée de la population mère et des échantillons par classe de longueur (en \%).

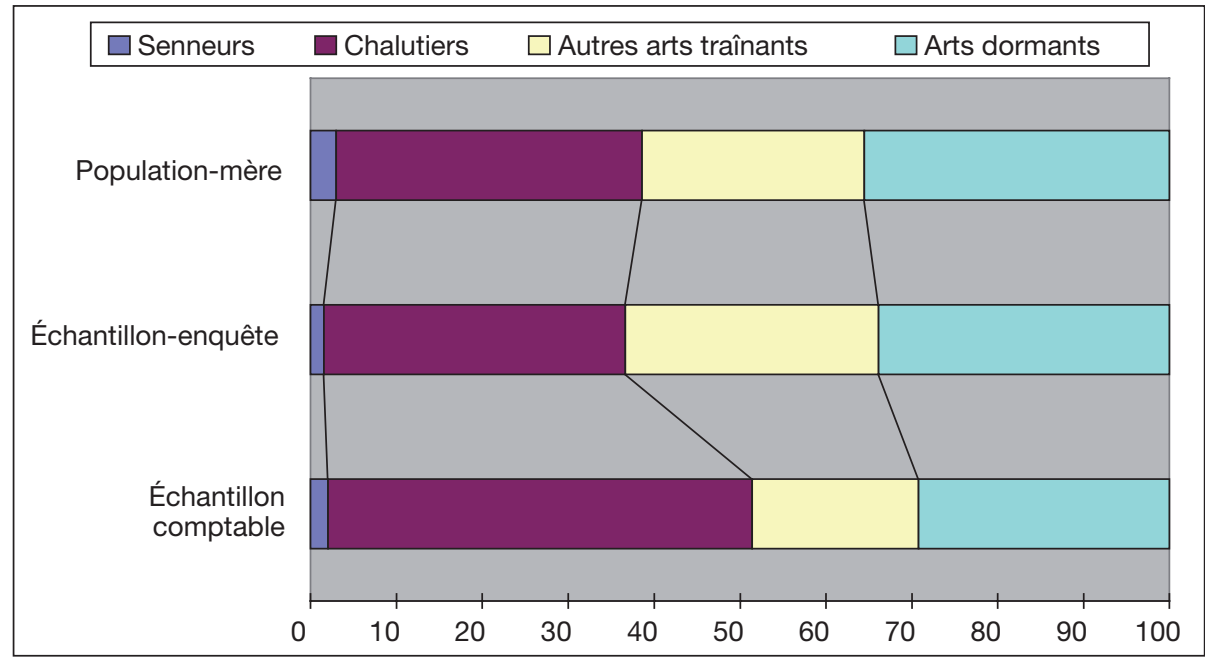

Figure 121. Structure comparée de la population mère et des échantillons par flottille (en \%). 


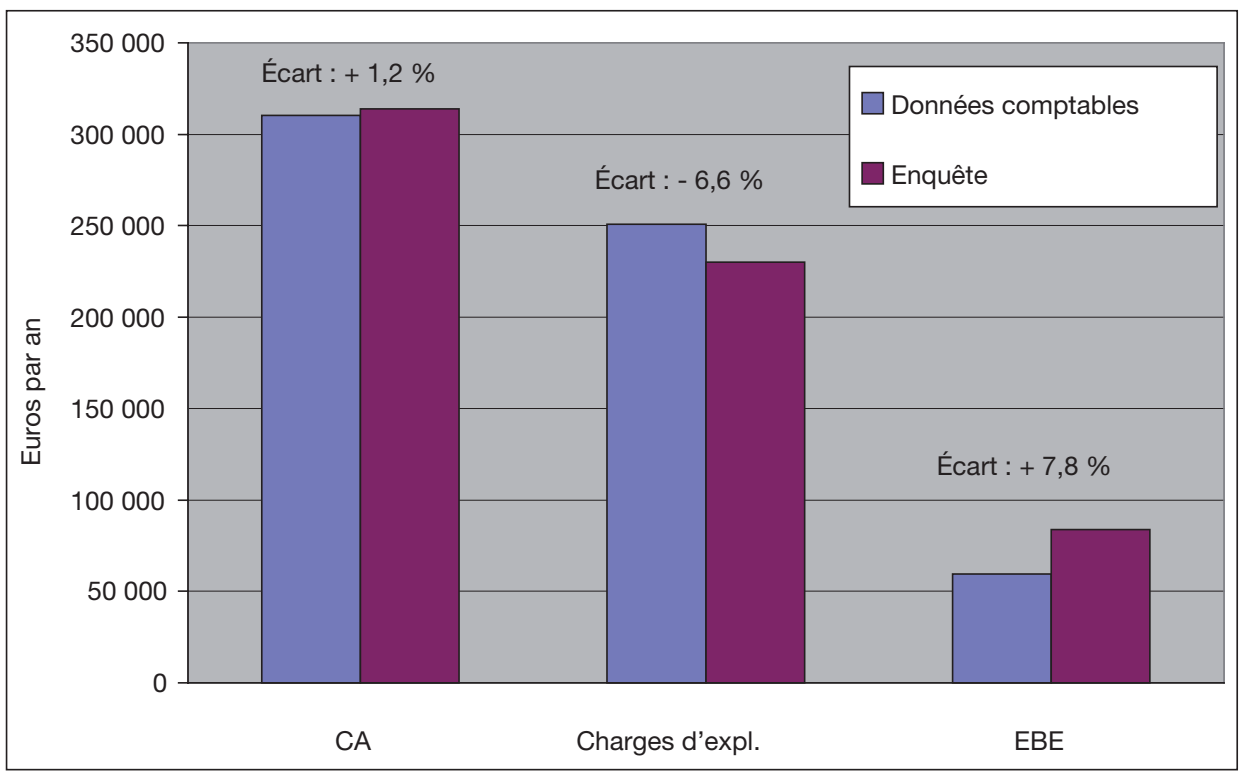

Figure 123. Comparaison des données économiques - Vue globale des valeurs moyennes par bateau (échantillon commun, écarts exprimés en \% du CA comptable).

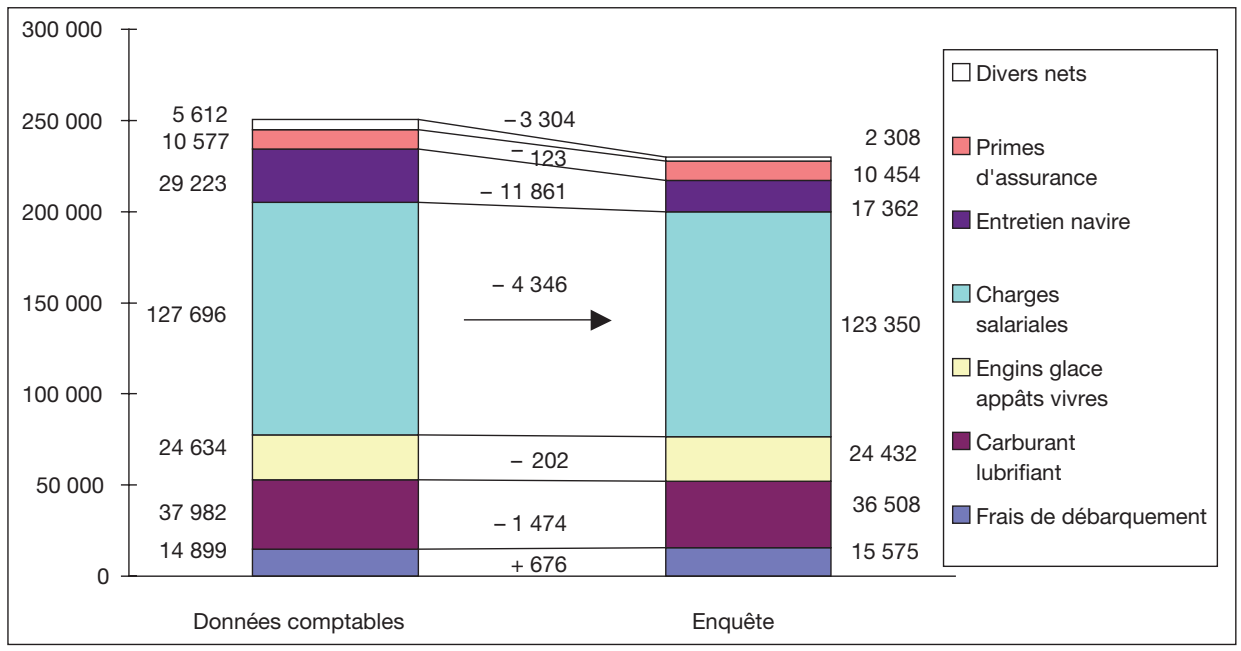

Figure 124. Structure comparée des charges d'exploitation moyennes (hors amortissement) selon les deux sources de données (échantillon commun : unité-euro). 


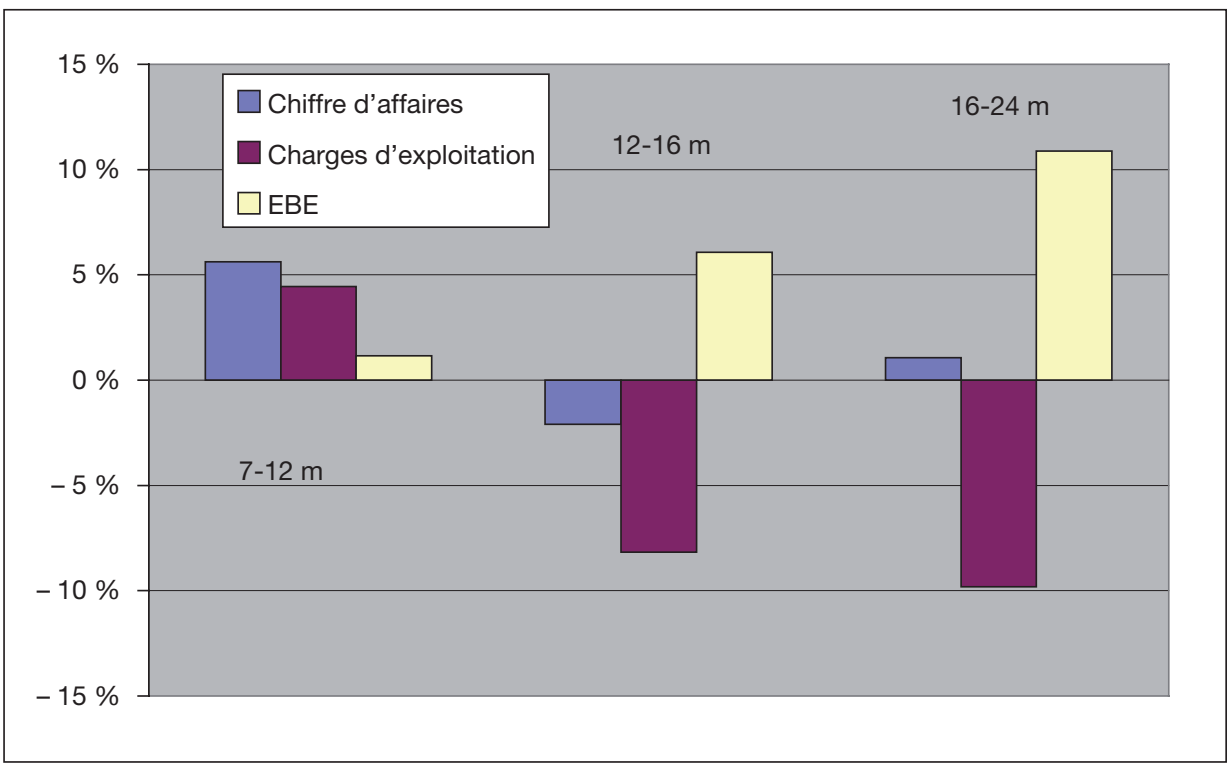

Figure 125. Écarts entre les estimations moyennes du chiffre d'affaires, des charges d'exploitation et de l'EBE, selon la classe de longueur des navires (enquête de terrain, base de données comptables, en \% du CA comptable).

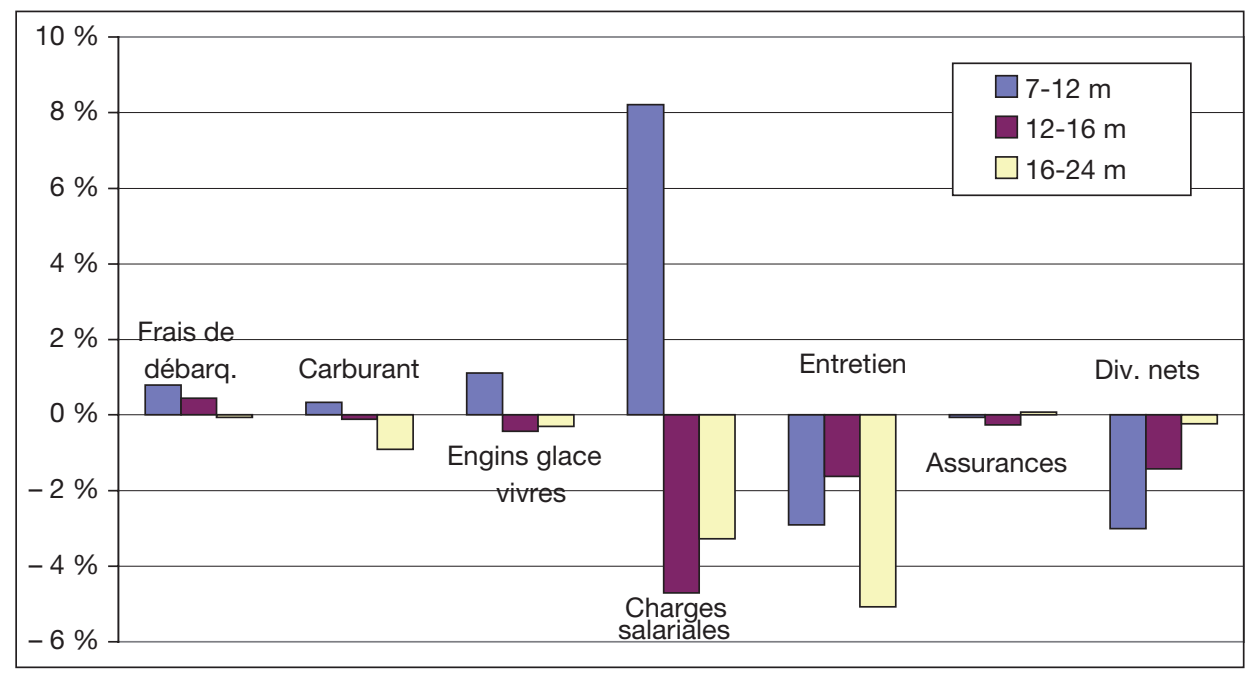

Figure 126. Écart entre les estimations moyennes de charges d'exploitation, selon la classe de longueur du navire (enquête, base de données comptables, en \% du CA comptable). 


\section{Chapitre 5 \\ Conclusion générale}

Le groupe de recherche Ifremer-Cedem-Agrocampus qui a travaillé sur ce projet tient à souligner le soutien important consenti par la Région Bretagne lors de deux CPER successifs (XI et XII). Ce cofinancement a permis notamment de:

- réaliser des opérations supplémentaires de collectes de données sur la pêche bretonne (observation des captures en mer, enquêtes, etc.);

- renforcer le potentiel d'analyse statistique des données halieutiques, afin de produire les synthèses régionales présentées dans ce document;

- mieux faire connaître le groupement de recherche (GdR) Amure, notamment à travers l'organisation d'un atelier international sur la régulation de l'accès aux ressources marines vivantes dans la zone côtière en janvier 2006.

L'aspect méthodologique est un point fort de ce projet de recherche: les développements en matière de collecte de données, d'analyse des effets induits de la pêche côtière, de comparaisons de résultats obtenus selon les sources de données utilisées, ont pour nombre d'entre eux, d'abord été testés par des études pilotes à l'échelle de la Bretagne, avant d'être étendus au niveau national ou appliqués à d'autres régions.

Avec près de 30000 emplois en Bretagne, le secteur de la pêche représente un enjeu économique et social essentiel pour la Région. Cependant, cette activité traverse une période de crise européenne et nationale. Elle est confrontée au déclin des ressources par surexploitation, au renforcement des contraintes de la réglementation internationale et communautaire, et notamment des contrôles, et à l'augmentation des coûts de production en particulier le renchérissement du prix du carburant. Au plan de la réglementation, la France s'est engagée dans le concert international à prévenir les pêches illégales, à créer un réseau d'aires marines protégées à horizon 2012, et à restaurer les stocks au niveau de production maximum soutenable (PMS, préservation des géniteurs) pour 2015. De plus, le contrôle se renforce par exemple dans la surveillance des zones de pêches, la mise en place de nouvelles mesures techniques (maillage, répulsifs...). Ces contraintes s'inscrivent dans un contexte économique défavorable dû à l'accroissement de la compétition des usages du domaine côtier par la plaisance et les transports, du changement de la production des 
ressources, conséquences du changement climatique et du changement global, de chocs économiques dus au marché mondial et au cours du pétrole. Face à ces contraintes, une mutation des pratiques de gestion et d'exploitation est rendue indispensable pour restaurer le développement économique durable de ce secteur d'activité et de ses ressources. Relever ce défi passe par le développement d'une politique maritime qui assure au plan régional le développement équilibré et durable du littoral breton, à partir d'une gestion des pêcheries associant la préservation de l'activité et celle de ses ressources. Pour soutenir une telle démarche, la Région Bretagne peut mettre à contribution et s'appuyer sur le pool de compétences et l'acquis de connaissances qu'elle a développés dans l'exercice des $\mathrm{X}^{\mathrm{e}}$ et XII ${ }^{\mathrm{e}}$ contrats de plan État-Région Bretagne. La vocation de la recherche est de soutenir les acteurs institutionnels et professionnels du système pêche par les connaissances et l'analyse des facteurs, mécanismes et mesures pouvant contribuer au développement d'une gestion écosystémique des pêches qui assure la pérennité du secteur. 
Annexe

Questionnaire d'enquête téléphonique sur la pêche récréative 


\begin{tabular}{|c|c|c|}
\hline 010 & Ifremer & $\begin{array}{c}\text { Questionnaire } \\
\text { Téléphonique } \\
\text { VAGUE 3 }\end{array}$ \\
\hline & $\begin{array}{c}\text { Enquête relative à la } \\
\text { pêche récréative }\end{array}$ & \\
\hline
\end{tabular}

Bonjour, je suis XX de l'Institut BVA. Nous réalisons une étude grand public. Toutes les réponses que vous me donnerez resteront totalement anonymes, conformément à l'usage de notre profession. Nous vous assurons que vous ne ferez l'objet d'aucune relance commerciale.

S0. Accepteriez-vous de répondre à nos questions?

Oui $\rightarrow$ Passer en S1

Non $\rightarrow$ Stop inter

S1. Sexe de l'interviewé :

ENQ: Compléter

1. Homme

2. Femme

S2. Pourriez-vous me préciser l'âge du chef de famille?

ENQ: Noter en clair/

Puis recoder

1. 15-24 ans

2. 25-34 ans

3. 35-49 ans

4. 50-64 ans

5. 65 ans et plus

S3. Pourriez-vous me préciser l'activité professionnelle du chef de famille?

ENQ: Lire - Une seule réponse possible

1. Agriculteur

2. Commerçant, artisan, chef d'entreprise

3. Cadre ou profession intellectuelle supérieure

4. Profession intermédiaire

5. Employé

6. Ouvrier

7. Retraité $\rightarrow$ Ancienne profession

8. Autre inactif

S4. De combien de personnes de plus de 15 ans se compose votre foyer y compris vous-même?

ENQ : Noter en clair: / 


\section{A TOUS}

S5. Vous-même ou quelqu'un de votre foyer pratique t-il la pêche de loisir en mer du bord de mer ou à pied - coquillages ou crustacés.

Oui, il y a des pêcheurs

Non, il n'y a pas de pêcheurs $\rightarrow$ Aller en S5b

S5b. Nous parlons de toutes les variantes de pêche en mer y compris le ramassage de coquillages et crustacés, et même si ce n'est qu'occasionnel comme en vacances par exemple. Vous-même ou quelqu'un de votre foyer est - il concerné ?

Oui, il y a des pêcheurs

Non, il n'y a pas de pêcheurs $\rightarrow$ Aller en signalétiques

S5c. Combien de personnes ont pratiqué cette activité de pêche de loisir en mer, du bord de mer ou à pied en 2005 ou au cours des mois de JUIN, JUILLET ou AOÛT 2006.

Nous ne prenons pas en compte les pêcheurs en eau douce (rivière, étang, lac...) ni les pêcheurs professionnels.

S6. Pourriez-vous me préciser l'âge et le prénom de cette ou ces personnes?

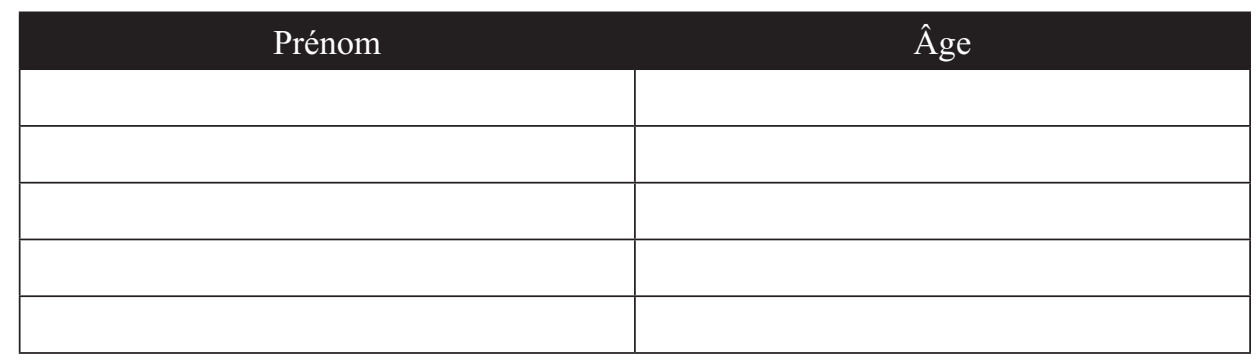

Je vous remercie.

S6c. Je souhaiterai parler à ...... (Personne sélectionnée par tirage aléatoire parmi les 15 ans et plus).

Est-il (elle) disponible?

Oui $\rightarrow$ Passer en S7

Non $\rightarrow$ Si personne non disponible, prendre le pêcheur suivant dans le foyer en S5b

\section{AU RÉPONDANT TIRÉ ALÉATOIREMENT}

S7. Bonjour, je suis XX de l'Institut BVA. Nous réalisons une étude sur les personnes qui pratiquent la pêche en mer à titre de loisir en 2005 ou au cours des mois de JUIN, JUILLET ou AOUT 2006. Accepteriez-vous de répondre à nos questions? Nous garantissons l'anonymat de vos réponses.

Oui $\rightarrow$ Passer en A1

Non $\rightarrow$ Si personne non disponible, prendre le pêcheur suivant tiré aléatoirement 
C1. En 2005, avez-vous personnellement pêché en mer ou du bord de mer ou à pied dans un cadre de loisirs en France métropolitaine ou dans des départements d'outre mer?

ENQ - Une seule réponse possible

1. Oui $\rightarrow$ Aller en A1

2. Non $\rightarrow$ Aller en A1

A1. En 2006 au cours des mois de juin, juillet et août 2006, avez-vous personnellement, pêché en mer ou du bord de mer ou à pied dans un cadre de loisirs?

1. Oui $\rightarrow$ Passer en A2

2. Non $\rightarrow$ Passer en partie C (année 2005)

3. NSP $\rightarrow$ Passer en partie C (année 2005)

SI CODE 1 EN A1: PÈCHE EN 2006 au cours des mois de JUIN, JUILLET, AOUT $\Rightarrow$ Poser parties A et B

\section{Partie A : LES TROIS DERNIERS MOIS}

A2. Quels modes de pêche avez-vous pratiqué au cours des mois de juin, juillet et août 2006 ?

ENQ - Lire les modes - Plusieurs réponses possibles

1. Sous-marine du bord

2. Sous-marine d'un bateau

3. D'un bateau (autre que sous marine)

4. Du bord (canne, lancer)

5. À pied

ENQ - Pour chaque mode de pêche cité par l'interviewé, poser A3 et A3b

A3. Combien de sorties de pêche avez-vous réalisées au cours des mois de juin, juillet et août 2006 ?

A3b. Sur l'ensemble de ces sorties, combien en avez-vous effectué en utilisant une embarcation comme moyen de transport?

\begin{tabular}{|l|c|}
\cline { 2 - 2 } \multicolumn{1}{c|}{} & \multicolumn{1}{c|}{ A3 } \\
\cline { 2 - 2 } \multicolumn{1}{c|}{} & $\begin{array}{c}\text { Combien de sorties sur } \\
\text { juin, juillet et août 2006 }\end{array}$ \\
\hline 1. Sous-marine du bord & \\
\hline 2. Sous-marine d'un bateau & \\
\hline 3. D'un bateau & \\
\hline 4. Du bord & \\
\hline 5. À pied & \\
\hline
\end{tabular}

\begin{tabular}{|c|}
\hline $\mathrm{A} 3 \mathrm{~b}$ \\
\hline $\begin{array}{c}\text { Dont combien de sorties } \\
\text { avec embarcation (comme } \\
\text { moyen de transport)? }\end{array}$ \\
\hline$/$ \\
\hline \\
\hline$/$ \\
\hline
\end{tabular}




\section{Partie B : LA DERNIÈRE SORTIE AU COURS DES TROIS DERNIERS MOIS}

B1. Lors de votre dernière sortie de pêche en mer au cours des mois de juin, juillet et août 2006, dans quel département et commune êtes-vous allé?

ENQ - Sélectionner le numéro de département et saisir la commune

B1. Numéro du département : /

B1b. Commune :

Code INSEE/

(recode)

B2. Pour cette dernière sortie, d'où êtes-vous parti? C'est-à-dire quel est le lieu où vous avez passé la nuit précédant cette dernière sortie? ENQ: Citer - Une seule réponse possible -

Votre résidence principale

Votre résidence secondaire, famille ou amis

Hôtel ou location

Camping

Votre bateau

Autres

$\underline{\text { Si réponse } 1 \text { en B2 (nuitée précédant la dernière sortie dans la résidence principale) }}$

B2b. Quel est le lieu où vous avez passé la nuit suivant votre dernière sortie de pêche? ENQ: Citer - Une seule réponse possible -

Votre résidence principale

$$
1
$$

Votre résidence secondaire, famille ou amis . 2]

Hôtel ou location..............................................................3]

Camping..... 4] $\rightarrow$

Votre bateau $.5]$

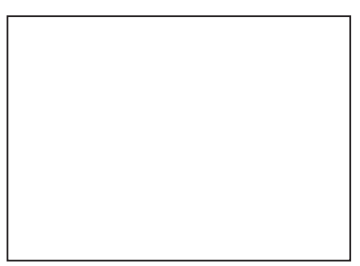

Autres

6

Si réponse 2 à 5 en $\mathrm{B} 2$ ou en $\mathrm{B} 2 \mathrm{~b}$ (hors résidence principale) :

B3A. Quelle a été, en nuitées, la durée de votre séjour? nuitées

B3B. Durant ce séjour, combien avez-vous fait de sorties de pêche en mer? |___ sorties 
B3C. Dans le choix du lieu de ce séjour, la possibilité de pratiquer la pêche en mer a-t-elle joué un rôle? (CITER une seule réponse possible)

1. Essentiel

2. Important

3. Secondaire

4. Négligeable

5. NSP (ne pas citer)

B3D. Vous étiez en séjour sur ce site principalement parce que (citer - UNE seule réponse possible)

1. Vous étiez en vacances durant les vacances scolaires

2. Vous étiez en vacances en dehors des vacances scolaires

3. Vous étiez en week-end

4. C'était une période propice à l'activité

5. Pour d'autres raisons

6. NSP/aucune

\section{A TOUS}

B4. Pour cette dernière sortie, quel mode principal de transport avez-vous utilisé pour vous rendre sur le lieu de pêche ou d'embarquement?

ENQ: Lire - Une seule réponse possible

À pied ou à vélo 1

Deux roues motorisées 2] $\rightarrow$ poser B4b et B4c

Voiture 3]

Bus 4]

Navette bateau 5] $\rightarrow$ poser B4d

Train $6]$

Autres: Préciser: / 7] $\rightarrow$ Passer en B4e

Si code 2 ou 3 en B4

B4b. Combien de kilomètres avez-vous parcouru? |______ B4c. Combien étiez-vous dans le véhicule ? |_Ll

Si code 4, 5 ou 6 en B4 


\section{A TOUS}

B4e. Combien de temps a duré le trajet jusqu'au lieu de pêche ou d'embarquement? |___ $|\mathbf{h}| \_\mid$_ Min.

ENQ: Prendre le temps total de transport au quart d'heure près.

B5. Lors de cette dernière sortie de pêche en mer avez-vous utilisé une embarcation autre qu'une navette, ne serait-ce que pour vous rendre sur votre lieu de pêche? ENQ: Une seule réponse possible

1. Oui $\rightarrow$ poser $\mathrm{B} 5 \mathrm{~b}$

2. Non $\rightarrow$ passer en B6

\section{Si code 1 en B5}

B5b. Combien de temps a duré le trajet en bateau jusqu'au lieu de pêche? |___ $|\mathrm{h}| \ldots|\ldots|$ Min.

ENQ: Prendre le temps total de transport en mer au quart d'heure près.

\section{$\underline{\text { A TOUS }}$}

B6. Lors de cette dernière sortie en mer, quel mode de pêche principal avez-vous pratiqué?

ENQ: Lire les modes - Une seule réponse possible

1. Sous-marine du bord

2. Sous-marine d'un bateau

3. Bateau (autre que sous marine)

4. Du bord (canne, lancer)

5. À pied

6. NSP

Pour cette dernière sortie, pouvez nous indiquer le temps qu'il vous a fallu: ENQ: Lire

\begin{tabular}{|l|l|c|}
\cline { 2 - 3 } \multicolumn{1}{l|}{} & $\begin{array}{c}\text { Temps réel au quart } \\
\text { d'heure près en } \mathrm{h} / \\
\text { Min. }\end{array}$ & Ne sait pas \\
\hline $\begin{array}{l}\text { B7a. Pour préparer la sortie dans sa globalité }: \\
\text { matériel, casse-croûte, pêche des appâts le cas } \\
\text { échéant }\end{array}$ & $\begin{array}{l}\mid \\
\text { B7b. Quel a été le temps effectivement consacré } \\
\text { à la pêche? }\end{array}$ & $\square$ \\
\hline
\end{tabular}


B7c. (Vérification du temps passé)

Au total, pour la préparation, le transport et le temps de pêche, cette sortie vous a occupé durant (Cumul Temps passé : B4e + B5b + B7a + B7b traduit en heures).

Êtes-vous d'accord avec cette estimation?

1. Oui

2. Non (dans ce cas reprendre avec l'interviewé les différents postes temps un à un).

Code 1 en B5 ou code 2 ou 3 en B6 (utilisation d'un bateau)

B8a. Pour la sortie en bateau, pouvez-vous me dire combien de carburant approximativement vous avez utilisé? Litres $\square$ NSP

B8b. Il s'agissait de : ENQ: Une seule réponse possible
1. Gasoil
2. Essence
3. NSP

B8c. Combien de pêcheurs étiez-vous à bord du bateau? Pêcheurs $\square$ NSP

Filtre - Si réponse 2 ou 3 en B6 (pêche à partir d'un bateau) - Valable de la B9 à la B11

B9. Lors de cette dernière sortie en mer, à quelle distance de la côte avez-vous principalement pratiqué votre activité?

ENQ: Lire - Une seule réponse possible

$$
\begin{aligned}
& \text { 1. Moins de } 3 \text { milles } \\
& \text { 2. } 3 \text { à } 6 \text { milles } \\
& \text { 3. } 6 \text { à } 12 \text { milles } \\
& \text { 4. } \quad \text { Plus de } 12 \text { milles }
\end{aligned}
$$

B10. Lors de cette dernière sortie en mer, quel était votre statut à bord du bateau? ENQ: Lire - Une seule réponse possible

1. Vous en êtes propriétaire

2. Vous étiez locataire

3. C'est le bateau d'un club

4. Vous étiez invité à bord

5. C'était un bateau prêté

6. Autre

7. (NSP)

B11. Lors de cette dernière sortie en mer étiez-vous avec un guide de pêche?

ENQ: Une seule réponse possible

1. Oui

2. Non 
Filtre - Si réponse 4 et 5 en B6 (pêche du bord de l'eau ou à pied)

B12. Lors de cette dernière sortie de pêche à pied ou du bord, depuis quel type de lieu avez-vous principalement pêché?

ENQ: Lire les types de lieux - Une seule réponse possible

1. Une plage,

2. Une grève (sablo-vaseux, caillasses)

3. Des rochers

4. Dans un port

5. Une jetée ou une digue (hors d'un port)

6. Autres

7. (NSP)

\section{$\underline{\text { A TOUS }}$}

B13. Quel est ou quels sont les principaux engins de pêche que vous avez personnellement utilisé(s) lors de votre dernière sortie en mer?

ENQ: Ne rien suggérer - Plusieurs réponses possibles

1. Filets

2. Balance

3. Carrelet

4. Casier

5. Fusil sous-marin, arbalète, harpon

6. Trident, fouëne,

7. Palangre

8. Canne

9. Ligne à main

10. Pêche à pied avec outils (couteau, râteau, grapette...)

11. Ramassage à la main sans outils

12. Épuisette (haveneau, dranet)

13. Autres - Préciser:

14. (Aucun de ceux-là)

15. (NSP)

B14. Lors de votre dernière sortie, avez-vous personnellement réalisé au moins une prise?

1. Oui $\rightarrow$ Poser B15a

2. Non $\rightarrow$ Passer à la partie $\mathrm{C}$

B15a. Quelles espèces principales avez-vous personnellement capturées lors de votre dernière sortie en mer? ENQ: NON SUGGERER - Plusieurs réponses possibles Cocher les espèces concernées - Relancer l'interviewé sur les différentes catégories (poissons, coquillages, crustacés) 
B15b. Lors de cette dernière sortie, combien d'individus avez-vous personnellement capturés y compris les remises à l'eau?

B15c. Lors de cette dernière sortie, quel poids en grammes ces captures représentaient y compris les remises à l'eau?

B15d. Toujours lors de cette dernière sortie avez-vous personnellement remis des prises à l'eau?

FAIRE EN HORIZONTAL pour B15b à B15d pour chaque espèce citée en B15a

Les enquêteurs disposent d'un référentiel complet des espèces, y compris les noms vernaculaires.

LISTE COMPLÉTÉE A L'ISSUE DE LA PREMIÈRE VAGUE - ENRICHISSABLE

\begin{tabular}{|c|c|c|c|c|}
\hline & $\begin{array}{l}\text { B15a. } \\
\text { Détail espèces } \\
\text { ENQ- Rechercher } \\
\text { dans la liste } \\
\end{array}$ & $\begin{array}{c}\text { Nombre } \\
\text { d'individus } \\
\text { ENQ - NOTER } \\
\text { B15b } \\
\end{array}$ & $\begin{array}{c}\text { B15c. } \\
\text { Poids total } \\
\text { en grammes } \\
\text { ENQ - NOTER }\end{array}$ & $\begin{array}{l}\text { B15d. Espèces } \\
\text { relâchées }\end{array}$ \\
\hline 1 & $\ldots$ & 1 l_l_l & $1-1-11-1-1 \mathrm{~g}$ & $1-1$ \\
\hline 2 & $\ldots$ & $1 \ldots$ & L_L_l_L_L $g$ & 1 \\
\hline 3 & AUTRES à préciser & $1+1$ & $1-1-1-1-1-1 \mathrm{~g}$ & 1 \\
\hline
\end{tabular}

B16. Comment avez-vous utilisé votre pêche?

ENQ: Lire - Plusieurs réponses possibles

1. En consommation immédiate

2. En conservation pour consommation personnelle

3. En distribution à des amis, des connaissances

4. Non consommation (Ne pas citer)

5. (AUTRES)

6. NSP

B17. Pour cette dernière sortie, sans tenir compte des dépenses liées au transport jusqu'au point de pêche ou d'embarquement, ni des dépenses relatives à l'embarcation (achat, entretien ou utilisation), pourriez-vous nous indiquer approximativement quelles dépenses vous avez réalisées pour:

ENQ: Lire - Il s'agit de la dépense par pêcheur. Si plusieurs pêcheurs ont partagé une dépense, il faut prendre en compte la dépense totale divisée par le nombre de pêcheurs. 


\begin{tabular}{|c|c|c|c|}
\hline & Dépenses en $€$ & Ne sait pas & Non concerné \\
\hline B17a. La préparation: matériel, appâts... & $/ 1 €$ & $\square$ & $\square$ \\
\hline $\begin{array}{l}\text { B17b. La nourriture et les consommations } \\
\text { sur place café, restaurant, pique-nique }\end{array}$ & $/ 1 \ldots$ & $\square$ & $\square$ \\
\hline B17c. L’hébergement & $1 €$ & $\square$ & $\square$ \\
\hline $\begin{array}{l}\text { B17d. Autres dépenses éventuelles. } \\
\text { Préciser: / }\end{array}$ & $-€$ & $\square$ & $\square$ \\
\hline
\end{tabular}

B18. Diriez-vous que cette dernière sortie est atypique par rapport aux autres sorties que vous avez réalisées sur la période juin, juillet et août 2006 :

1. Oui $\rightarrow$ Poser B18a

2. Non $\rightarrow$ Aller en $\mathrm{C} 1$

3. (Ne sait pas) $\rightarrow$ Aller en $\mathrm{C} 1$

\section{B18a. Les différences tiennent:}

ENQ: Citer - Plusieurs réponses possibles

1. Au mode pêche pratiqué

2. À la durée de la sortie

3. Aux espèces capturées

4. Aux quantités capturées

5. Au (x) lieu (x) de pêche

6. Autres éléments

7. (Ne sait pas) Non concerné 
SI CODE 1 EN C1: pèche EN 2005

\section{Partie C : l'année 2005}

\section{PARLONS DE L'ANNÉE 2005}

C2. Quels modes de pêches principaux avez-vous pratiqué au cours de l'année 2005 ? ENQ - Lire les modes - Plusieurs réponses possibles

1. Sous-marine du bord

2. Sous-marine d'un bateau

3. Bateau (autre que sous marine)

4. Du bord (canne, lancer...)

5. À pied

6. (NSP)

C3. Combien de sorties avez-vous réalisées personnellement pour chacune des périodes que je vais vous citer? ENQ : Lire chacune des modalités

1. En janvier, février et mars 2005

2. En avril et mai 2005

3. En juin, juillet et août 2005

4. En septembre et octobre 2005

5. En novembre et décembre 2005
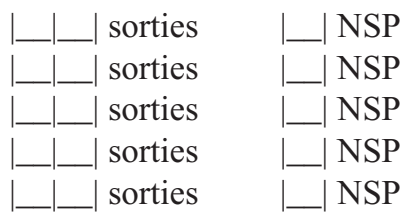

C3b. Au cours de l'année 2005, dans quels départements avez-vous le plus souvent pêché, et combien de sorties y avez-vous effectué dans l'année (max trois départements)?

\begin{tabular}{|c|c|}
\hline Département & Nombre de sorties \\
\hline |-1_l & |_|_ sorties $\quad$ __ NSP \\
\hline |_L & |___ sorties \\
\hline $1-1$ & |___ sorties \\
\hline
\end{tabular}

C4. Au cours de l'année 2005, quelles quantités avez-vous pêchées personnellement: $\mathrm{C} 4 \mathrm{a}$. de poissons toutes espèces confondues et y compris les appâts C4b. de crustacés toutes espèces confondues et y compris les appâts $C 4 c$. de coquillages toutes espèces confondues et y compris les appâts

C4d. de céphalopodes (type seiches, poulpes) toutes espèces confondues et y compris les appâts 


\begin{tabular}{|l|c|c|c|c|}
\cline { 2 - 5 } \multicolumn{1}{c|}{} & $\begin{array}{c}\text { C4a. } \\
\text { Poissons }\end{array}$ & $\begin{array}{c}\text { C4b. } \\
\text { Crustacés }\end{array}$ & $\begin{array}{c}\text { C4c } \\
\text { Coquillages }\end{array}$ & $\begin{array}{c}\text { C4d } \\
\text { Céphalopodes }\end{array}$ \\
\hline 1. Aucune prise & & & & \\
\hline 2. Moins de $2 \mathrm{~kg}$ & & & & \\
\hline 3. Entre 2 et $5 \mathrm{~kg}$ & & & & \\
\hline 4. Entre 6 et $10 \mathrm{~kg}$ & & & & \\
\hline 5. Entre 11 et $20 \mathrm{~kg}$ & & & & \\
\hline 6. Entre 21 et $50 \mathrm{~kg}$ & & & & \\
\hline 7. Entre 51 et $100 \mathrm{~kg}$ & & & & \\
\hline 8. Entre 101 et $150 \mathrm{~kg}$ & & & & \\
\hline 9. $150 \mathrm{~kg}$ et plus & & & & \\
\hline 10. Ne sait pas (ne pas citer) & & & & \\
\hline
\end{tabular}

C5. Au cours de l'année 2005, quelles ont été les trois espèces principales que vous avez recherchées ou pêchées? ENQ: Cocher les trois principales espèces recherchées ou pêchées Les enquêteurs disposent d'un référentiel complet des espèces, y compris les noms vernaculaires.

Ce référentiel s'enrichit automatiquement durant les enquêtes.

\begin{tabular}{|l|c|}
\cline { 2 - 2 } \multicolumn{1}{c|}{} & \multicolumn{1}{c|}{ Espèces citées } \\
\cline { 2 - 2 } \multicolumn{1}{c|}{} & ENQ - Rechercher dans la liste \\
\hline Espèce 1 & \\
\hline Espèce 2 & \\
\hline Espèce 3 & \\
\hline
\end{tabular}

C6. Pouvez-vous me préciser le poids total en kilos de ... ?

\begin{tabular}{|l|l|l|l|}
\cline { 2 - 4 } \multicolumn{1}{c|}{} & $\begin{array}{c}\text { Les Reprendre } \\
\text { <Espèce 1> } \\
\text { pêchées au cours } \\
\text { de l'année 2005 }\end{array}$ & $\begin{array}{c}\text { Les Reprendre } \\
\text { <Espèce 2> } \\
\text { pêchées au cours } \\
\text { de l'année 2005 }\end{array}$ & $\begin{array}{c}\text { Les Reprendre } \\
\text { < Espèce 3> } \\
\text { pêchées au cours } \\
\text { de l'année 2005 }\end{array}$ \\
\hline 1. Aucune prise & & & \\
\hline 2. Moins de $2 \mathrm{~kg}$ & & & \\
\hline 3. Entre 2 et $5 \mathrm{~kg}$ & & & \\
\hline 4. Entre 6 et $10 \mathrm{~kg}$ & & & \\
\hline 5. Entre 11 et $20 \mathrm{~kg}$ & & & \\
\hline 6. Entre 21 et $35 \mathrm{~kg}$ & & & \\
\hline 7. Entre 36 et $50 \mathrm{~kg}$ & & & \\
\hline 8. Entre 51 et $75 \mathrm{~kg}$ & & & \\
\hline 9. Entre 76 et $100 \mathrm{~kg}$ & & & \\
\hline 10. Entre 101 et $150 \mathrm{~kg}$ & & & \\
\hline 11. Plus de $150 \mathrm{~kg}$ & & & \\
\hline 12. Ne sait pas $(n e$ pas citer) & & & \\
\hline
\end{tabular}


C7. Pour l'année 2005, sans tenir compte des dépenses liées au déplacement ou des dépenses relatives à l'embarcation (achat, entretien ou utilisation), pouvez-vous me donner le montant de vos dépenses directement liées à la pratique de la pêche en mer pour chacune des catégories suivantes? (CITER)

\begin{tabular}{|c|c|c|c|}
\hline & Dépenses en $€$ & Ne sait pas & Non concerné \\
\hline Matériel et vêtements de pêche & 1 & $\square$ & $\square$ \\
\hline Location bateau, & $l €$ & $\square$ & $\square$ \\
\hline Appâts, & $l €$ & $\square$ & $\square$ \\
\hline Guide de pêche & $1 €$ & $\square$ & $\square$ \\
\hline Revues spécialisées & $1 €$ & $\square$ & $\square$ \\
\hline Autres (préciser)/ & $l €$ & $\square$ & \\
\hline
\end{tabular}




\section{À TOUS}

\section{SI CODE 1 EN C1 : pèche EN 2005}

\section{Partie D : le bateau}

Aux pêcheurs en 2005 et/ou en 2006 sur la période de référence (avril -mai 2006)

D1. Possédez-vous, à titre personnel, une ou plusieurs embarcations que vous utilisez pour la pratique de la pêche en mer? (CITER) ENQ: Lire - Plusieurs réponses possibles

\begin{tabular}{|l|c|c|}
\cline { 2 - 3 } \multicolumn{1}{c|}{} & D1 & D2 \\
\hline 1. Barque, canot & 1 & 1 \\
\hline 2. Canoë/Kayak & 2 & 2 \\
\hline 3. Pneumatique & 3 & 3 \\
\hline 4. Coque Open (sans cabine) & 4 & 4 \\
\hline 5. Vedette & 5 & 5 \\
\hline 6. Pêche promenade & 6 & 6 \\
\hline 7. Voilier & 7 & 7 \\
\hline 8. Autres & 8 & 8 \\
\hline 9. Aucun & 9 & \\
\hline
\end{tabular}

Si code 9 en D1 passer au chapitre $\mathbf{E}$ - Sinon passer en D2

D2. Quelle est celle que vous utilisez le plus souvent pour la pêche en mer? (Citer une seule réponse possible)

D3. Quelle est sa longueur approximative en mètres?

En clair: / mètres

$\square$ Ne sait pas

Si code 3 à 8 en D2

D4. Quelle est la puissance totale de propulsion du ou des moteur(s) en CV? |_____ CV

D5. Est-il immatriculé en France?

1. Oui

2. Non $\rightarrow$ Dans quel pays est-il immatriculé? PRÉCISER

D6. Avez-vous acheté cette embarcation?

1. Oui, seul

2. Oui, en copropriété

3. Non, on me l'a donnée (héritage, etc., ...) 
D7. En quelle année, avez-vous acquis votre embarcation? ENQ - Noter en clair / Ne sait pas

D8. Votre embarcation a-t-elle été construite, il y a ... ?

1. Moins de 5 ans

2. Entre 6 et 10 ans

3. Entre 11 et 15 ans

4. Entre 16 et 20 ans

5. plus de 20 ans

6. Ne sait pas

À poser uniquement si achat (code 1 ou 2 en D6)

D9. Quel était son prix d'achat (en Euros) en intégrant le moteur et la remorque?

ENQ - Noter en clair : Prix en $€ /$

ENQ - Noter en clair: Prix en Francs /

D10. Pour l'année 2005, pouvez-vous me préciser le montant de vos dépenses pour le bateau et remorque - hors achat du bateau et dépenses de carburant - relatives à :

\begin{tabular}{|c|c|c|c|}
\hline & Dépenses en $€$ & Ne sait pas & Non concerné \\
\hline D10a. L'équipement & 1 & $\square$ & $\square$ \\
\hline D10b. L'entretien & $l €$ & $\square$ & $\square$ \\
\hline $\begin{array}{l}\text { D10c. Les frais portuaires (emplace- } \\
\text { ment, manutention...) }\end{array}$ & $1 \ldots 1 \in$ & $\square$ & $\square$ \\
\hline D10d. Les assurances & $l €$ & $\square$ & $\square$ \\
\hline D10e. Les droits de francisation & $1 €$ & $\square$ & $\square$ \\
\hline D10f. Autres dépenses: Précisez & 1 & $\square$ & $\square$ \\
\hline
\end{tabular}

D11. En 2005, quelle proportion de vos sorties avec cette embarcation avez-vous consacrées à la pêche en mer?

ENQ: Lire - Une seule réponse possible

1. Plus de 3 sorties sur 4

2. Entre 1 sortie sur 2 et 3 sorties sur 4

3. Entre 1 sortie sur 4 et 1 sortie sur 2

4. Moins d'une sortie sur 4

5. Aucune

6. Ne sait pas (Ne pas citer) 
D12. Sur la plus grande partie de l'année, votre bateau est-il ...? (une seule réponse possible)

1. Amarré à un ponton

2. Amarré à une bouée

3. À terre ou sur remorque

4. Autres (précisez) : 


\section{À TOUS LES PÊCHEURS}

\section{Partie E : le pêcheur}

$\underline{\text { À tous }}$

E1. En 2005, avez-vous également pratiqué la pêche récréative en mer dans le cadre d'un séjour à l'étranger?

1. Oui

2. Non $\rightarrow$ Aller en E2

E1a. Pour quelles raisons avez-vous pêché en mer à l'étranger? (NE RIEN SUGGÉRER)

ENQ: Noter en clair

E2. Appartenez-vous à une association de pêche en mer?

1. Oui $\rightarrow$ Poser E2a

2. Non $\rightarrow$ Aller en E 3

\section{Si 1 en E2}

E2a. Pourriez-vous nous préciser de quelle association il s'agit?

ENQ: Cochez liste à l'écran - Sinon inscrire en clair

E2b. Pourriez-vous préciser le montant annuel de vos cotisations y compris les licences? | Euros ou |_____ Francs

E2c. Qu'attendez-vous d'une adhésion à une association?

\section{ENQ : Lire - Plusieurs réponses possibles}

1. Des informations sur la réglementation

2. Des informations sur les techniques de pêche

3. Des informations sur les populations de poissons, crustacés, coquillages

4. Une représentation auprès des pouvoirs publics

5. Une mise en commun des moyens (pour un bateau par exemple)

6. Une gestion des mouillages

7. (NSP)

8. Autres 
E4. Au cours des 5 dernières années, vous diriez que le temps que vous consacrez à la pratique de la pêche en mer ou en bord de mer est:

ENQ: Lire - Une seule réponse possible

1. Constant

2. En Augmentation

3. En diminution

4. Ne sait pas (Ne pas citer)

E5. Pratiquez-vous ou avez-vous pratiqué également la pêche en eau douce et, si oui, avez-vous commencé par la pêche en mer ou par la pêche en eau douce? ENQ: Lire - Une seule réponse possible

1. Oui, j'ai commencé par la pêche en eau douce

2. Oui, j'ai commencé par la pêche en mer

3. Non, je n'ai jamais pêché en eau douce

4. (NSP)

E6. Considérez-vous que la pêche en mer telle que vous la pratiquez est une activité : ENQ: Lire - Une seule réponse possible

1. Très chère

2. Assez chère

3. Peu chère

4. Pas chère du tout

5. (Ne se prononce pas)

6. (Ne sait pas)

E7. Avez-vous le sentiment que la ressource au sens large : poissons, coquillages, crustacés... au cours des cinq dernières années : ENQ : Lire - Une seule réponse possible

1. À beaucoup augmenté

2. À un peu augmenté

3. Est restée stable

4. À un peu diminué

5. À beaucoup diminué

6. Ne sait pas (ne pas citer)

E8. Vous estimez-vous bien informé sur l'évolution de la ressource: poissons, coquillages, crustacés...?

ENQ: Lire - Une seule réponse possible

1. Oui, tout à fait bien informé,

2. Oui, plutôt bien informé

3. Non, je suis peu informé

4. Non, je ne suis pas du tout informé

5. NSP 
E9. Vous estimez-vous bien informé : (CITER - ROTATION)

\begin{tabular}{|c|l|c|c|c|c|}
\cline { 3 - 5 } \multicolumn{2}{c|}{} & $\begin{array}{c}\text { Oui } \\
\text { tout à fait }\end{array}$ & $\begin{array}{c}\text { Oui } \\
\text { assez }\end{array}$ & $\begin{array}{c}\text { Non } \\
\text { Peu }\end{array}$ & $\begin{array}{c}\text { Non } \\
\text { pas du tout }\end{array}$ \\
\hline 1 & Sur les réglementations en vigueur & 1 & 2 & 3 & 4 \\
\hline 2 & $\begin{array}{l}\text { Sur la réglementation relative aux ferme- } \\
\text { tures de la pêche pour des raisons sanitaires }\end{array}$ & 1 & 2 & 3 & 4 \\
\hline 3 & $\begin{array}{l}\text { Sur la taille minimale autorisée des espèces } \\
\text { que vous pêchez }\end{array}$ & 1 & 2 & 3 & 4 \\
\hline
\end{tabular}

E10. Je vais vous citer des mesures pour protéger ou mieux gérer certaines espèces. Pour chacune d'elles dites-moi si vous y êtes favorable?

ENQ: Lire - Plusieurs réponses possibles

\begin{tabular}{|l|c|c|}
\cline { 2 - 3 } \multicolumn{1}{c|}{} & Oui & Non \\
\hline $\begin{array}{l}\text { Une meilleure application de la réglementation actuelle grâce à un renfor- } \\
\text { cement des contrôles à l'encontre des contrevenants. }\end{array}$ & 1 & 2 \\
\hline La mise en place de périodes d'interdiction de pêche: repos biologique & 1 & 2 \\
\hline La mise en place d'un permis & 1 & 2 \\
\hline La mise en place d'un permis pour certaines espèces seulement & 1 & 2 \\
\hline La limitation de prises par sortie & 1 & 2 \\
\hline
\end{tabular}




\section{Signalétique}

Êtes-vous le chef de famille?

1. Oui $\rightarrow$ aller en $\mathrm{G} 3$

2. Non $\rightarrow$ Aller en G0

G0. Sexe

1. Homme

2. Femme

G1. Pourriez-vous me préciser votre âge :

ENQ: Noter en clair/

\section{Puis recoder}

1. 15-24 ans

2. 25-34 ans

3. $35-49$ ans

4. 50-64 ans

5. 65 ans et plus

G2. Pourriez-vous me préciser quelle est votre activité professionnelle (CSP)? ENQ: Lire - Une seule réponse possible

1. Agriculteur

2. Commerçant, artisan, chef d'entreprise

3. Cadre ou profession intellectuelle supérieure

4. Profession intermédiaire

5. Employé

6. Ouvrier

7. Retraité $\rightarrow$ Ancienne profession

8. Autre inactif

G3. Pourriez-vous me préciser dans quelle tranche de revenu mensuel se situe votre foyer?

ENQ: Lire - Une seule réponse possible

1. Moins de $1500 €$

2. De 1500 à $2500 €$

3. De 2500 à $3500 €$

4. Plus de $3500 €$

5. Ne souhaite pas répondre (ne pas citer)

G4. Accepteriez-vous de recevoir un questionnaire complémentaire sur votre pratique de la pêche et d'être contacté ultérieurement?

1. Oui

2. Non 



\section{Références bibliographiques}

Adley J., 2007. Aspects of sustainability of creel fishing for Norway Lobster, Nephrops norvegicus (L.), on the west coast of Scotland, University of Glasgow, $474 \mathrm{p}$.

Alban F., Appéré G., Boncœur J., 2006. Economic Analysis of Marine Protected Areas. A Literature Review, Empafish Project Booklet n 3, Projet européen EMPAFISH SSPB-006539 (6 $\left.6^{\mathrm{e}} \mathrm{PCRD}\right), 51 \mathrm{p}$.

Alban F., Kervarec F., Le Lec G., Le Floc'h P., Boncœur J., 2001. Contraintes socioéconomiques sur l'activité des navires goémoniers de la région Bretagne. Université de Bretagne occidentale/Cedem, $105 \mathrm{p}$.

Al-Masroori H., Al-Oufi H., McIlwain J.L., McLean E., 2004. Catches of lost fish traps (ghost fishing) from fishing ground near Muscat, Sultanate of Oman. Fisheries Research, 69 (3), 407-414.

Alverson D. L., Freeberg M. H., et al., 1994. À global assessement of fisheries bycatch and dicards. Rome, FAO.

Anon, 2001. Data on economic performance of the fisheries sector - Final Report, EC Project $\mathrm{n}^{\circ}$ 00/32.

Anon, 2001. Promotion of common methods for economic assessment of EU fisheries, assessment of economic performance of selected European fishing fleets, SJFI annual report 2001, $155 \mathrm{p}$.

Anon, 2002. Bilan annuel de production des pêches et de l'aquaculture, Ministère de l'Agriculture, de l'Alimentation, de la Pêche et des Affaires Rurales/OFIMER, Paris, 80 p.

Anon, 2002. Évolution de la production de coquille Saint-Jacques sur le gisement classé de Saint-Brieuc. Bilan de la campagne 2001/2002. Côtes-d'Armor Développement, Saint-Brieuc.

Anon, 2003. Economic situation of the Danish Fishery 2003, Fodevareokonomisk Institut, Copenhague.

Anon, 2004. Report of the Working Group on Nephrops Stocks. Lisbon, Portugal, ICES CM 2004/ACFM: 19, 441.

Anon, 2004. Report of the Working Group on the Assessment of Southern Shelf Stocks of Hake, Monk and Megrim, WGHMM, Lisbon, Portugal, ICES CM 2005. 
Anon, 2005. Programme de valorisation des crépidules en Bretagne. Bilan d'activités 2004. AREVAL/Côtes-d'Armor Développement, Saint-Brieuc.

Anon, 2005. Quality HandbookAnnex: WGNSDS-North Minch Nephrops (FU11): Working Group Assessment of Northern Shelf Demersal Stocks, p. 4.

Anon, 2006. Régulation de l'accès aux ressources marines vivantes de la bande côtière: expériences internationales et perspectives pour la Bretagne. Atelier international, IUEM, Plouzané, 20 au 20 janvier 2006 (présentations en ligne sur le site www.gdr-amure.fr).

Anon, 2006. Report of the Working Group on the Assessment of Northern Shelf Demersal Stocks, 10-19 May 2005 (ICES CM 2006/ACFM:13).

Anon, 2006. Report of the Working Group on the Assessment of Northern Shelf Demersal Stocks, 10-19 May 2005 (ICES CM 2006/ACFM:13).

ASH Partnership, 1991. Development Opportunities in the Natural Environment, HRC, HIDB, NCCS, CCS, SO.

Barne J H, Robson C F et al, 1997. Coasts and seas of the United Kingdom. Regions 15\&16. North West Scotland: The Western Isles and West Highland. Peterborough, JNCC.

Ben Mariem S., 1987. Interactions dans les pêches du merlu et de la langoustine. Éléments en vue d'un aménagement des pêcheries du golfe de Gascogne. Thèse Doc. Ingénieur, Sciences agronomiques, Spécialité halieutique, ENSA Rennes, 115 p.

Berkes F., Feeny D., McCay B.J. et Acheson J.M., 1989. The Benefits of the Commons. Nature, 340, p. 391-393.

Berthou P. et al., 1996. "Cadre physique et aspects halieutiques », in Description des pêcheries du golfe Normand-Breton - Analyse du problème de l'aménagement. Rapport $1^{\text {re }}$ année. Programme Amure, Ifremer/UBO-Cedem, Brest, p. 8-78.

Berthou et al., 2003a. Typologies des flottes de pêche: méthodes Ifremer/SIH. Ifremer, rapport interne $\mathrm{DRV} / \mathrm{SIH} / \mathrm{n}^{\circ}$ 4/082003, $26 \mathrm{p}$.

Berthou P. (dir.), 2003b. Synthèse des pêcheries 2002. Flotte mer du Nord-MancheAtlantique. Système d'informations halieutiques. Ifremer, Plouzané.

Berthou P., Daurès F. et Demanèche S., 2005. Some initial comments about Small-Scale Fisheries in Europe. Paper presented at the European Workshop on Small-Scale Fisheries, Kavala, September 12-16th. 2005 (unpublished).

Berthou P., Daurès F., Guyader O., Jézéquel M., Larour M., Leblond E., Talidec C., 2002. Description of the Nephrops fleet in the ICES area VIII, Ifremer-SIH Working paper, Oct. 2002.

Berthou P., Daurès F., Guyader O., Merrien C., Leblond E., Demanèche S., Jézéquel M., Guégan F., Bermell S., Van Iseghem S., 2004. Synthèse des Pêcheries 2002 - Flotte mer du Nord - Manche Atlantique, $80 \mathrm{p}$.

Biseau A., 1996. Étude d'impact d'une augmentation de maillage des chaluts dans le golfe de Gascogne. Rapport Ifremer/DPMA, 73 p.

Biseau A., Forest A., 2002. Nephrops fishery in the Bay of Biscay: simulations of reductions of fishing mortality, improvement of the selection pattern and constant TAC. Working paper, CSTEP, Oct. 2002, 8 p.

Blanchard M., 1995. Origine et état de la population de Crepidula fornicata (Gasteropoda prosobranchia) sur le littoral français. Haliotis, 24, 75-86. 
Blanchard M., Hamon D., 2006. « Bilan du suivi de l'exploitation industrielle de la crépidule en Bretagne Nord (Baies de Saint-Brieuc et du Mont-Saint-Michel), 2002-2005» Rapport de fin de contrat Areval/Ifremer DYNECO/EB/06-01, Ifremer, Brest, France.

Boncœur, J., et B. Le Gallic. 1998. Economic survey of the French fleet operating the English Channel fisheries. FAIR CT 96-1993 Report. Cedem, université de Bretagne occidentale, Brest.

Boncœur J., (Ed.), 2004. Activités halieutiques et activités récréatives dans le cadre d'un espace à protéger: le cas du Parc National de la Mer d'Iroise. projet de recherche cofinancé par le PNEC, le programme « espaces protégés » du MEDD et la Région Bretagne. UBOCedem/Ifremer/UVSQ-C3ED. Rapport final, 516 p.

Boncœur J., (Ed.), 2005. Les revenus à la pêche. Étude pour la région Bretagne. Synthèse de travaux réalisés par le GdR Amure, UBO-Cedem/Ifremer/Agrocampus-Rennes, 58 p. (disponible en ligne sur le site $w w w . g d r$-amure.fr).

Boncœur J., Alban F., Guyader O. Thébaud O., 2002. Fish, fishers, seals and tourists: economic consequences of creating a marine reserve in a multi-species, multi-activity context. Natural Resource Modeling, Vol. 15, n 4, p. 387-411.

Boncœur J., Berthou P., Boude J.P., Curtil O., Daurès F., Guyader O., Le Floc'h P., Talidec C., Thébaud O., 2006. Quel avenir pour la pêche bretonne? Revue Bretagne [s], $\mathrm{n}^{\circ}$ 2, avril-juin 2006, p. 26-33.

Boncœur J., Daurès F., Guyader O., Martin A., Le Floc'h P., Thébaud O., 2004. « Comparing bookkeeping and field survey methods for assessing fishing fleets economic perfoirmance. A case study of Brittany fishing fleets (France) ». 12 conférence biennale de l'IIFET, Tokyo, 20-30 juillet 2004. Proceedings of the 12th Biennial conference of the IIFET, JIFRS/TUMSAT (CD-ROM).

Boncœur J., Fifas S., Le Gallic B., 2000. Un modèle bioéconomique d'évaluation du coût social des rejets au sein d'une pêcherie complexe. Économie et prévision, 143-144 (2-3), p. 185-199.

Boncœur J., Guyader, O., 1996. Management alternatives for a recovery of fishing activity: the case of scallop dredging in the Bay of Brest (France). Proceedings of the 7th annual conference of the EAFE, Cemare Misc. Publication n ${ }^{\circ} 33$, université de Portsmouth (Royaume-Uni), 263-283.

Boncœur J., Le Gallic B., 1998. Enquête sur la pêche professionnelle française dans le golfe Normand-Breton. Programme Amure, UBO-Cedem, Brest.

Boucher J., 1985. Caractéristiques dynamiques du cycle vital de la coquille Saint-Jacques (Pecten maximus) : hypothèses sur les stades critiques pour le recrutement. CIEM, C.M. 1985/ K23/sess.Q: 10 p.

Boucher J., 1987. Déterminisme du recrutement de la coquille Saint-Jacques : programme et résultats actuels. Contr. Bull. info. PNDR, 5: p 3-17.

Boucher J., Arzel P., Buestel D., 1985. Causes probables de variations du recrutement de la coquille Saint-Jacques identifiées en baie de Saint-Brieuc (1975-1982). Réunion PNDR, III 23, ISTPM, Nantes : 9 p.

Boude J.P., (Ed.), 2005. Régulation de l'accès à la ressource. Étude pour la Région Bretagne. Synthèse de travaux réalisés par le GdR Amure, UBO-Cedem/Ifremer/AgrocampusRennes, 55 p. (disponible en ligne sur le site $h t t p: / / w w w . g d r$-amure.fr/). 
Bourillet J.F., Laurans M., Macher C., Le Loc'h F., Guyader O., Le Hir P., Merrien C., Ollitrault A., Talidec C., Vincent B., Vacherot J.P., 2005. Impact des engins de pêche sur le fond. Colloque Défi golfe de Gascogne, Brest, 22-24 mars 2005.

Brook A.M., Price R., Sutherland D., Westerhund N., André C., 2004. Oil prices developments: drivers, economic consequences and policy responses. OECD, Economics Department, Working papers, 412, $51 \mathrm{p}$.

Brown J., Macfadyen G., 2007. Ghost fishing in European waters: Impacts and management responses. Marine Policy, 31 (4), 488-504.

CE, 2001. Livre vert - L'avenir de la politique commune de la pêche. Office des publications officielles des Communautés européennes, Luxembourg, vol. I, 53 p., vol. II, 131 p.

CE, 2003. Guide de l'analyse coût-avantage des projets d'investissement (Fonds structurels FEDER, Fonds de cohésion et ISPA). DG Politique Régionale, Bruxelles.

CE, 2005. Economic assessment of European fisheries, Economic Performance of selected european fishing fleets, annual report 2005, 306 p.

CE, Règlement n ${ }^{\circ}$ 850/98 du Conseil du 30 mars 1998 visant à la conservation des ressources de pêche par le biais de mesures techniques de protection des juvéniles d'organismes marins.

Chapman C.J., et al., 2000. Survival and Growth of the Norway lobster Nephrops norvegicus in relation to light induced eye damage. Marine Biology, 136, 233-241.

Charuau A., 1988a. La langoustine Nephrops norvegicus (Linné 1758) (Nephropsidès). In Les pêcheries du golfe de Gascogne. Bilan des connaissances. J. Dardignac (ed.). Rapp. scient. techn. IFREMER n 9 , 142-151.

Charuau A., 1988b. Les pêcheries mixtes de langoustine et de merlu du golfe de Gascogne. Modélisation bioéconomique et simulation des procédures de gestion. Rapp. CE/Ifremer 88/1219774/BF, 111 p. + annexes.

Chauvaud L., 1998. « La coquille Saint-Jacques en rade de Brest: un modèle biologique d'étude des réponses de la faune benthique aux fluctuations de l'environnement ». Thèse de doctorat, École doctorale des sciences de la mer, Université de Bretagne Occidentale, Brest.

CIEM, 1991. Report of the working group on fishery units in sub-areas VII and VIII. Nantes 29 may-5 june 1991. Cons. int. Explor. Mer, CM 1991/ASSESS : 24, 215 p.

CIEM, 2001. Report of the ICES Advisory Committee on Fishery Management, 2001. ICES Coop. Res. Rep., n² 246, 895 p.

CIEM, 2002a. Report of the ICES Advisory Committee on Fishery Management, 2002. ICES Coop. Res. Rep. (sous presse).

CIEM, 2002b. Report of the Working groups on Nephrops stocks. Lorient, France, 3-9 April 2002. ICES CM 2002/ACFM: 15, 242 p.

Coase R., 1960. « The problem of social cost ». The Journal of Law and Economics, vol. III, p. 1-44.

Cofrepêche, 1999. Emploi et dépendance vis à vis de la pêche, Région F2. in Regional Socio-Economic Studies on Employment and the Level of Dependency on Fishing, CCE, DG XIV, Bruxelles, $101 \mathrm{p}$.

Copes P., 1997. Alternatives in fisheries management. Proceedings of the IXth Annual Conference of the European Association of Fisheries Economists, UBO-CEDEM/ENSAR, Quimper, France, p. 10-35. 
Curtil O., 2001. Le régime juridique de la pêche dans la bande côtière française. Thèse de doctorat. UBO, École doctorale des sciences de la mer, novembre 2001, 506 p.

Dao J.C., 1985. Évaluation de la biomasse de réproducteurs et des recrues du stock de coquille Saint-Jacques de la baie de Saint-Brieuc - Résultats préliminaires. Réun. PNDR, ISTPM, Nantes, 2-4 juil. 1984, contr. No 34: 10 p.

Dao J.C., Laurec A., Buestel D., 1975. Politique et problèmes relatifs aux pêches locales. Application de la dynamique de population au gisement de Coquille Saint-Jacques de la baie de Saint-Brieuc. Recherche d'un modèle bioéconomique. CNEXO/COB, F.I. 159, 23 : 7 p.

Daurès F., Guyader O., Leblond E., Demanèche S., Berthou P., 2002a. Méthodologie de collecte d'informations économiques sur le secteur des pêches professionnelles en France. Rapp. int. DRV-SEM Ifremer, (en préparation).

Daurès F., Guyader O., Leblond E., Jézéquel M., Thébaud O., Demanèche S., 2002b. Méthodologie de traitement des enquêtes économiques dans le secteur des pêches. Rapp. int. DRV-SEM Ifremer (en préparation).

Daurès F., Guyader O., Thébaud O., 2002c. Indicateurs pour l'évaluation de la situation économique des pêcheries. Rapp. int. DRV-SEM Ifremer (en préparation).

Daurès F., Demanèche S., Guyader O., Leblond E., 2003. Methodology for the assessment of aggregated economic indicators in the fishing sector: estimation of a revenue function. EAFE 2003. The Fifteenth Annual Conference of the European Association of Fisheries Economics (EAFE), Brest, Session 5 : Fisheries Economic Impacts and Indicators. Éditions Ifremer, Actes Colloq., 37, 6 p.

DDE du Finistère, 1977. SAUM de la rade de Brest. Étude analytique et méthodologique. 167 p. + hors-texte.

Dupouy H., 1978. L'exploitation de la coquille Saint-Jacques, Pecten maximus (L.) en France. $1^{\text {re }}$ partie : présentation des pêcheries. Science et Pêche, Bull. ISTPM, 276, 11 p.

Dupouy H., De Kargariou G., Latrouite D., 1983. L'exploitation de la coquille SaintJacques, Pecten maximus (L.) en France. $2^{\mathrm{e}}$ partie: baie de Saint-Brieuc. Science et Pêche, Bull. ISTPM, 331, p. 3-11.

Dupouy H., Latrouite D., 1979. Le développement de la crépidule sur le gisement de coquilles Saint-Jacques de la baie de Saint-Brieuc. Science et Pêche, bulletin institutionnel des pêches marines, 292, 13-19.

FAO, 1995. Code of conduct for responsible fisheries, Rome, $41 \mathrm{p}$.

FAO, 2004. The State of World fisheries and Aquaculture 2004. Rome, 153 p.

Faure L., 1965. Les crevettes et les coquilles Saint-Jacques de la baie de Saint-Brieuc (compte rendu de la mission du « Roselys ») (20-31 mars 1965). Sciences et Pêche, bull. Inst. Pêches marit., mai 1965, 137, p. 12-17.

Faure L., 1966. Étude des stocks de coquilles Saint-Jacques de Bretagne en 1966. Sciences et Pêche, bull. Inst. Pêches marit., nov. 1966, 153, p. 1-12.

Fifas S., 1991. Analyse et modélisation des paramètres d'exploitation du stock de coquilles Saint-Jacques (Pecten maximus, L.) en baie de Saint-Brieuc (Manche Ouest, France). Thèse de Doctorat d'Université, Ifremer/UBO, Brest, 422 p.

Fifas S., 1993. Un modèle de capturabilité pour le stock de coquilles Saint-Jacques (Pecten maximus, L.) en baie de Saint-Brieuc (Manche, France) : pp. 141-155. In Les recherches 
françaises etévaluation quantitative etmodélisation des ressources et des systèmes halieutiques. Halieumétrie, Rennes 29/06-1/07/93, Actes du colloque: 405 p.

Fifas S., 1996. La coquille Saint-Jacques (Pecten maximus). In: Augris C., Hamon D. (eds). Atlas thématique de l'environnement marin en baie de Saint-Brieuc (Côtes-d'Armor). Éditions Ifremer, p. 60-63.

Fifas S., 1998. Golfe Normand-Breton: essai de quantification des rejets de pêche occasionnés par le chalutage. Analyse de scénarios d'exploitation de quatre espèces. Document interne, Ifremer DRV/RH, Brest.

Fifas S., 2004. Gisement de coquilles Saint-Jacques de la baie de saint-Brieuc. Résultats de la campagne 2004 d'évaluation directe. Ifremer, DRV/RH, Brest, France.

Fifas S., Arzel P., 2005. Catch capacities of scallop (Pecten maximus, L.) fisheries in Brittany (France). Years 1945-2004. 8th International Conference on Shellfish Restoration, Brest, 2-5 octobre 2005.

Fifas S., Dao J.C., Boucher J., 1990. Un modèle empirique du recrutement. Exemple de la coquille Saint-Jacques (Pecten maximus, L.) en baie de Saint-Brieuc (Manche, France). Aquatic Living Resources, 3 (1), p. 13-28.

Fifas S., Dao, J.C. and J. Boucher, 1990. Un modèle empirique du recrutement pour le stock de coquilles Saint-Jacques, Pecten maximus (L.) en baie de Saint-Brieuc (Manche, France), Aquatic Living Resources, 3, 13-28.

Fifas S., Guyader O., Boucher J., 2003. The Pecten fishery in the Bay of Saint-Brieuc. Productivity and management. Rapp. Sci. Technol., Acad. Sci. Paris, No 17: p. 221-234.

Fifas S., O. Guyader, et J. Boucher. 2003. La pêcherie de coquilles Saint-Jacques en baie de Saint-Brieuc. Productivité et gouvernance, in Laubier, L. (Ed.) Exploitation et surexploitation des ressources marines vivantes, Académie des sciences, RST n ${ }^{\circ}$, Éditions Lavoisier, Paris : 221-234.

FIOM, 1994. Étude des composantes du prix de vente du poisson frais. Juin 1994, Paris.

Fisheries Research Services, Marine Laboratory Aberdeen, 2006. Assessment of Nephrops stocks in the Loch Torridon area for the Scottish Natural Heritage and the Minch Project.

Flaaten, O., 1991. Bioeconomics of sustainable harvest of competing species. Journal of Environmental Economics and Management, 20, 163-180.

Frésard M., Boncœur J., 2006a. Controlling the Biological Invasion of a Commercial Fishery by a Space Competitor: a Bioeconomic Model with Reference to the Bay of St-Brieuc Scallop Fishery. Agricultural and Resource Economics Review, 35 (1), 78-97.

Frésard M., Boncœur J., 2006b. Costs and Benefits of Stock Enhancement and Biological Invasion Control : the Case of the Bay of Brest Scallop Fishery. Aquatic Living Resources, 19 (3) : 299-305.

Fulton G., 1998. Fishery Audit and Assessment for the Loch Torridon Area, Minch project.

Garcia S., Grainger J.R., 2005. Gloom and doom? The future of marine capture fisheries. Phil. Trans. R. Soc. B., 360, p. 21-46.

Gordon H.S., 1954. The economic theory of a common property resource: the fishery. Journal of Political Economy, 62 (2), 124-142.

Guéguen J., Charuau A., 1975. Essai de détermination du taux de survie des langoustines hors taille rejetées lors des opérations de pêche commerciale. ICES, Shellfish Comm., CM 1975/K: 12, 3 p + annexes. 
Guyader O, Fifas S., 2000. Transition to responsible fisheries: modelling the transition to responsible fisheries. Group II case studies France la pêcherie de coquilles Saint-Jacques, rapport OCDE AGR/FI (2000)11/PART2/FINAL. p. 101-110.

Guyader O., et al., 2005b. Typology of management measures for a selection of EU fleets. In: P. Marchal (ed.). Technological developments and tactical adaptations of important EU fleets, Final report of the EU project N Q5RS-2002-019, 512 p.

Guyader O. Daurès F., Jézéquel M. et Thébaud O., 2006. Marché des navires d'occasion et coût d'accès à la ressource. Atelier international «Régulation de l'accès aux ressources marines vivantes de la bande côtière: expériences internationales et perspectives pour la Bretagne » IUEM, Plouzané, 20-21 janvier 2006 (présentation en ligne sur le site $w w w . g d r$ amure.fr).

Guyader O., Daurès F, Fifas S., 2000. A bioeconomic analysis of the impact of decommissioning schemes : application to a limited etry scallop French fichery. Proceedings of the IIFET biannual Conference, Corvallis, Oregon, USA, July $2000: 10$ p.

Guyader O., Daurès F., Fifas S., 2004. A Bioeconomic Analysis of the Impact of Decommissioning Programs: Application to a Limited-Entry French Scallop Fishery. Marine Resource Economics. 2004, 19 (2), 225-242.

Guyader O., Fifas S., 1999. Modélisation bioéconomique de la pêcherie de coquilles SaintJacques de la baie de Saint-Brieuc. Rapports Scientifiques DRV/RH/Ifremer: 113 p.

Guyader O., Fifas S., 2000. Transition to responsible fisheries: modelling the transition to responsible fisheries. Group II case studies France la pêcherie de coquilles Saint-Jacques, AGR/FI (2000)II/PART2/FINAL : 101-110.

Guyader O., Le Pellec L. et Daurès F., 2003. "A hedonistic analysis of capital stock in fisheries: the case of second hand market of the French fishing vessels », XVth EAFE Conference Proceedings, Ifremer, Brest.

Guyader, O., Fifas S., 1999. Modélisation bioéconomique de la pêcherie de coquille SaintJacques de la baie de Saint-Brieuc. Rapports scientifiques DRV/RH/Ifremer, Brest.

Hamon D., Blanchard M., 1994. État de la prolifération de la crépidule (Crepidula fornicata) en baie de Saint-Brieuc. Rapport Ifremer, DEL 94-14, Brest, France.

Hamon D., Blanchard M., Houlgatte A., Blanchet J.D., Gaffet P., Cugier A., Ménesguen P., Bassoulet P., Cann D., Domalain, Haubois A. G., 2002. La crépidule: identifier les mécanismes de sa prolifération et caractériser ses effets sur le milieu pour envisager sa gestion. Chantier Baie de Saint-Brieuc. Rapport final, Programme Liteau, Ifremer DEL, Brest, France.

Hannesson R., 1998. Marine reserves: what do they accomplish. Marine Resource Economics, 13 (3), p. 159-170.

Highland Council, 1997, Highland Trends 1997. Director of Planning, THC, Inverness Marine Fish Farming Policy Paper No 1, 1988, Loch Torridon Fish Farming Framework Plan 1988.

Highlands and Islands Enterprise, 1998. Ross and Cromarty Economic Update, Personal Communication, Market Research \& Development Branch.

Hilborn R., Branch T.A., Ernst B., Magnusson A., Minte-Vera C.V., Scheuerell M.D., Valero J.L., 2003. State of the world's fisheries. Annu. Rev. Environ. Resour., 28 (15), p. 1-15. 
Hilborn R., Walters C.J., 1992. Quantitative Fisheries Stock Assessment. New-York: Chapman and Hall.

Hough A., 2004. Surveillance Report Loch Torridon Nephrops Creel Fishery: Moody Marine Limited, p. 4.

Hough A., 2005. Surveillance Report Loch Torridon Nephrops Creel Fishery: Moody Marine Limited, p. 5.

Hough A., 2006. Surveillance Report Loch Torridon Nephrops Creel Fishery: Moody Marine Limited, p. 6.

Hunter C., Liversedge A. et al., 1997. Tourism and Sustainability in Ross and Cromarty, Aberdeen University.

Ifremer, 1999. La fiche du mois : Langoustine du golfe de Gascogne (Nephrops norvegicus) (subdivision VIIIab du CIEM). Le Marin/Les nouvelles de l'Ifremer, $\mathrm{n}^{\circ} 2714 / \mathrm{n}^{\circ} 6$ - juillet 1999, p. 4.

Ifremer, 2001. Grille sélective à langoustine. Étude préliminaire pour la mise au point et le développement d'un chalut muni d'une grille sélective à langoustine. Rapp. Ifremer/TMSI/ TP 01-34, 26 p.

Integrated Marine Management Ltd, 1996. West of Hebrides Fisheries Study, WIC, WIE.

JORF du 19 novembre 1997, loi n 97-1051 du 18 novembre 1997 d'orientation sur la pêche maritime et les cultures marines.

Knowler D., Barbier B., 2000. The economics of an invading species: a theoretical model and case study application. In Perrings, C., M. Williamson, and S. Dalmazzone, eds., The Economics of Biological Invasions (Part 15 : 70-93). Cheltenham: Edward Elgar Publishing Limited.

Le Floc'h P., Daurès F., Brigaudeau C., Bihel J., 2006. A comparison of economic performance in the fisheries sector: A short and long-term perspective. Marine Policy, 32, 421-431.

Le Foll D., 1993. Biologie et exploitation de l'araignée de mer Maja squinado Herbst en Manche Ouest. Thèse de doctorat, UBO-Ifremer, Brest.

Leblond E., Daurès F., Berthou P., Bermell S., Merrien C., Demaneche S., 2006. Synthèse des flottilles de pêche 2003. Ifremer, Plouzané (http://www.ifremer.fr/drvrhbr/action_ recherche/synthese-pecheries/synthese-flottilles-peche-2003/index.htm).

Macher C., Guyader O., et al., 2006. A cost-benefit analysis of improving selectivity in a fishery with high level of discards, in prep.

Mahé L.P., Ropars C., 2001. L'exploitation régulée d'une ressource renouvelable: inefficacité d'un traitement factoriel et efficacité des quotas individuels transférables, Économie et Prévision, 148 (2), 141-156.

MAP/OFIMER, 2005. Bilan annuel de production 2004 des pêches et de l'aquaculture. Paris, $83 \mathrm{p}$.

Mason, E., Atkinson R.J.A., Hough A., 2002. Certification Report for Loch Torridon Nephrops Creel Fishery: Moody Marine Limited, p. 44.

Maurice J., 2001. Prix du pétrole. Conseil d'analyse économique, Paris, 195 p.

McGoodwin J. R., 2003. Comprendre la culture des communautés de pêcheurs, Element fondamental pour la gestion des pêches et la sécurité alimentaire: FAO Document Technique sur les pêches, 401, $62 \mathrm{p}$. 
Mettling B., Hénaff P., 1995. Rapport d'audit sur la situation financière des navires de pêche artisanale et des organismes d'intervention. Ministère de l'Agriculture et de la Pêche.

Meunier N., 2004. Le mystère des cycles solaires. La recherche, hors série, 15, p. 16-19.

Meuriot E., Cochet Y., Fifas S., Foucher E., Gates J. 1987. Licences de pêche et gestion d'une pêcherie : analyse bio-économique de la pêche de la coquille Saint-Jacques de la baie de Saint-Brieuc. Éditions de l'Ifremer, Rapports économiques et juridiques, 4, 70 p.

Ministère de l'Agriculture et de la Pêche/OFIMER, 2005. Bilan annuel de production 2004 des pêches et de l'agriculture. Paris, $83 \mathrm{p}$.

North Douglas, 1955. Location theory and regional economic growth. Journal of Political Economy, 63 (3), 243-258.

Observatoire économique régional des pêches, 2003. Résultats des flottilles artisanales 2001/2002, Fédération bretonne de la coopération maritime, Quimper, 67 p.

OCDE, 1997. Towards Sustainable Fisheries. Economic Aspects of the Management of Marine Living Resources. Paris, 268 p.

OCDE, 2000. Pour des pêches responsables: implications économiques et politiques. Paris, 298 p.

OCDE, 2006. Using Market Mechanisms to Manage Fisheries. Smoothing the Path. Paris, $325 \mathrm{p}$.

Ofimer, 2005. Consommation des produits de la pêche et de l'aquaculture. Bilan annuel 2004. Paris, 88 p.

Olson L.J., 2006. The economics of terrestrial invasive species: a review of the literature. Agricultural and Resource Economics Review, 35 (1) ; 178-194.

Perrings, C., M. Williamson, Dalmazzone S. (eds.), 2000. The Economics of Biological Invasions. Cheltenham: Edward Elgar Publishing Limited.

Piboubès R., 1973. Pêche et conchyliculture en Bretagne Nord. $1^{\text {re }}$ partie : Bull. CERS, 9 (4), 283-455; 2e partie : Bull. CERS, 10 (1), p. 1-261.

Prat J.L., 1996. Le droit international applicable au golfe Normand-Breton, in Boncœur J. et Prat J.-L. (coordonnateurs), Économie et droit des ressources naturelles renouvelables de la mer-Aspects théoriques et applications à la zone côtière de la manche occidentale française. Programme Amure, UBO-Cedem, Brest, tome II p. 96-121.

Richardson G., 1996. The effect of light-induced eye damage on the behaviour of Nephrops norvegicus. Ph. D., University of Leicester, 263 p.

Riddiford N. J., 1988. «Safeguarding our Heritage - the Fair Isle marine resource : a community proposal for sustainable management $»$ FICA, FIBOT \& NTS, Fair Isle and Inverness.

Ropars C., 2002. «Analyse des politiques de régulation d'une ressource renouvelable: une application sur le gisement de coquilles Saint-Jacques exploité en baie de Saint-Brieuc » Thèse de doctorat en sciences économiques, université de Rennes 1, $220 \mathrm{p}$.

Rural Forum, 1995. Community Profile, Shieldaig, Rural Action, Ross and Cromarty.

Sabatella E., Franquesa R., 2003. Manual of fisheries sampling surveys: methodologies for estimations of socio-economic indicators in the Mediterranean sea. FAO Studies and review, $C G F M, \mathrm{n}^{\circ} 73,37 \mathrm{p}$. 
Salz P., Frost H., 2000. «Model for economic interprétation of the ACFM advice (EIAA), in Promotion of common methods for economic assessment of EU fisheries 》 report of the concerted action FAIR CT97-2541.

Schaefer M.B., 1954. Some aspects of the dynamics of populations important to the management of the commercial marine fisheries. Inter-American Tropical Tuna Commission Bulletin, 1, 27-56.

Schaefer M.B., 1957. Some considerations of population dynamics and economics in relation to the management of marine fisheries. Journal of the Fisheries Research Board of Canada, 14, 669-681.

Scottish Office, 1998. Review of Controls on Inshore Fishing in Scotland, Inshore Fisheries Branch, SFPS, Annual Report 1997, Personal Communications, Fishery Office, Portree, Fisheries Research Services, Scottish Shellfish Farms Annual Production Survey 1997, Classification of Bivalve Mollusc Production Areas Note, 1997, AEFD, Scottish Sea Fisheries Statistics 1997, AEFD, Branch J4, AEFD, Sea Fisheries Division, Notes for Guidance on Regulatory and Several Orders, 1997, Walker A.F., 1997. Salmonid Research Studies in Wester Ross, AEFD, FFL.

Scottish Tourist Board, 1997. Tourism in the Highlands of Scotland 1997, Tourism in Scotland 1997, Fishing Holidays in Scotland 1997, Tourism in Highlands and Islands Enterprise 1997.

Shotton R., (ed.), 2000. Use of property rights in fisheries management. FAO Fisheries Technical Paper 404/1 et 2: 341 p. et 468 p.

Smith S. M., 1985. À survey of the shores and shallow sublittoral of Loch Torridon and Loch Carron (including Loch Kishorn). Report to the Nature Conservancy Council. NCC CSD Report No 610.

Sumaila U. R., Khan A., Teh L., Watson R., Tyedmers P., Pauly D., 2006. Subsidies to high seas bottom trawl fleets. In: U. R. Sumaila, and D. Pauly (Eds), Catching More Bait: A Bottom-up Re-estimation of Global Fisheries Subsidies, Fisheries Centre Research Reports, Vancouver, BC, Canada, 49-53.

Sumaila U. R., Pauly D., 2006. Catching More Bait: A Bottom-up Re-estimation of Global Fisheries Subsidies. Fisheries Centre Research Reports: 14, Vancouver, BC, Canada, 115 p.

Talidec C.; (Ed.), 2005. Scénarios d'aménagement des activités de pêche dans la bande côtière bretonne. Rapport 2004. Projet de recherche cofinancé par la Région Bretagne dans le cadre du XII ${ }^{\mathrm{e}}$ contrat de plan État-Région, Ifremer/UBO-Cedem/Agrocampus-Rennes, 207 p.

Talidec C., Rochet M.-J., et al., 2005. Discards estimates of nephrops and hake in the nephrops trawl fishery of the Bay of Biscay: methodology and preliminary results for 2003 and 2004. ICES Working Group on the Assessment of Southern Shelf Stocks of Hake, Monk and Megrim, WGHMM, Lisbon, Portugal.

TECTAC, 2005. Survey for a selected fleet: The French bottom trawler fleet of the Bay of Biscay, Ifremer Draft 18.10.2005.

TECTAC, 2005. Report on the Typology of Management Measures for Selected Fleets.

Tétard A., Boon M., et al., 1995. Catalogue international des activités des flottilles de la Manche, approche des interactions techniques. Éditions Ifremer, Brest. 
Troadec J.P., 1994. Le nouvel enjeu de la pêche: l'ajustement des institutions aux nouvelles conditions de rareté des ressources. Comptes rendus de l'Académie d'Agriculture, $80(3), 41-60$.

Troadec J.P., Boncœur J., 2003. La régulation de l'accès. In: Laubier L. (éd.). Exploitation et surexploitation des ressources marines vivantes. Académie des sciences, Rapports scientifiques et techniques, 17, 355-394. Éditions Lavoisier, Paris.

Van Isegem S, Demanèche S., Daurès F., et Guyader O., 2004. « Optimization of a sampling plan for economic data collection: application to the Atlantic French Fleet $»$, communication à la $15^{\mathrm{e}}$ conférence annuelle de l'EAFE, Rome, avril 2004.

Veron G., 1979. Pêcherie de la coquille Saint-Jacques en baie de Saint-Brieuc. Éléments de dynamique des populations. DEA Océan. Biol., Station marine d'Endoume, Marseille, 29 p.

Welcome to Shieldaig, 1998. Shieldaig Community Council, Information Pack.

Wester Ross Fisheries Trust, 1998. Annual Review 1998, Butler, Dundonnell. 





\section{Édition}

Acserv

Mise en pages

Desk

Impression

Louis Jean 

L'ouvrage est dédié à la situation des pêches côtières bretonnes au début des années 2000.

La question de la régulation de l'accès aux ressources halieutiques est abordée. L'exploitation des principaux stocks est examinée, les caractéristiques de la flotte, ses activités, son évolution récente et les performances économiques des navires sont analysées. L'impact de l'augmentation du coût du carburant au début de la décennie est mis en évidence. La pêche de loisir est traitée à travers les résultats d'un sondage national permettant de dégager les caractéristiques des plaisanciers pratiquant cette activité en Bretagne. Les sites du golfe du Morbihan et de la mer d'Iroise, et certaines pêcheries (coquilles Saint-Jacques, langoustines) font l'objet d'études particulières.

Cet ouvrage est une référence pour les professionnels et décideurs chargés de l'économie des ressources marines vivantes, soucieux de préserver la biodiversité.

Catherine Talidec, ingénieur halieute, est responsable du département Sciences et technologies halieutiques de l'Ifremer Brest et Lorient.

Jean Boncœur, économiste, est professeur à l'université de Bretagne occidentale (Brest) et directeur de l'unité mixte de recherche Amure (UBO//fremer), centre de recherche regroupant des économistes et des juristes travaillant sur les questions maritimes.

Jean-Pierre Boude, économiste, est professeur à Agrocampus Ouest au pôle halieutique dont la vocation est de former des ingénieurs et des étudiants de Master aux questions de la gestion des pêches et de l'aménagement intégré côtier.

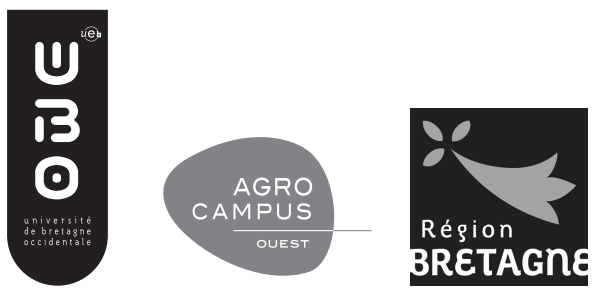

Morbihan Conseil général Cap I'Orient 